\title{
STATISTICAL AND DESCRIPTIVE SUMMARIES OF WATER-RESOURCES DATA FOR THE CANNONBALL RIVER BASIN, NORTH DAKOTA AND SOUTH DAKOTA
}

By Edwin A. Wesolowski, Scott D. Zainhofsky, and Valerie M. Dressler

U.S. GEOLOGICAL SURVEY

Open-File Report 96-108

Prepared in cooperation with the BUREAU OF RECLAMATION 


\title{
U.S. DEPARTMENT OF THE INTERIOR \\ BRUCE BABBITT, Secretary
}

\author{
U.S. GEOLOGICAL SURVEY \\ GORDON P. EATON, Director
}

Any use of trade, product, or firm names is for descriptive purposes only and does not imply endorsement by the U.S. Government.

For additional information write to:

\section{District Chief}

U.S. Geological Survey

Water Resources Division

821 East Interstate Avenue

Bismarck, ND 58501
Copies of this report can be purchased from:

U.S. Geological Survey

Earth Science Information Center

Open-File Reports Section

Box 25286, MS 517

Denver Federal Center

Denver, CO 80225 


\section{CONTENTS}

Abstract
Introduction
Statistical and descriptive summaries of water-resources data and other information from the
U.S. Geological Survey.
Surface-water quality and streamflow data
Ground-water quality and ground-water level data
Water-use data
Bibliographic information
Federal, State, and tribal agencies
Nureau of Reclamation
North Dakota Game and Fish Department Department of Health
North Dakota Parks and Recreation Department Water Commission
Standing Rock Sioux Tribe

\section{FIGURES}

1. Map showing location of Cannonball River Basin, North Dakota and South Dakota 3

2. Map showing location of data-collection sites in the Cannonball River Basin, North Dakota and South Dakota, for which surface-water quality and daily streamflow data were retrieved from the U.S. Geological Survey's National Water Information System

3. Map showing location of data-collection sites in the Cannonball River Basin, North Dakota and South Dakota, for which ground-water quality and ground-water level data were retrieved from the U.S. Geological Survey's National Water Information System. .5

4. Diagram showing latitude-longitude numbering system used for short-term surface-water sites and for ground-water sites 7

5. Diagram showing township-range location-numbering system used for ground-water sites...............................7

6. Map showing location of surface-water data-collection sites monitored by the North Dakota Game and Fish Department, the North Dakota Department of Health, or the Standing Rock Sioux Tribe

7. Map showing location of ground-water data-collection sites monitored by the North Dakota State Water Commission

\section{TABLES}

1. Summary of surface-water quality and daily streamflow data obtained from the U.S. Geological Survey for sites where multiple water-quality samples were collected

2. Summary of daily streamflow data obtained from the U.S. Geological Survey .................................................73

3. Summary of surface-water quality data obtained from the U.S. Geological Survey for sites where one or two water-quality samples were collected

4. Ground-water quality data obtained from the U.S. Geological Survey for sites where one water-quality sample was collected

5. Summary of ground-water quality and periodic ground-water level data obtained from the U.S. Geological Survey for sites where multiple water-quality samples were collected

6. Summary of daily ground-water level data obtained from the U.S. Geological Survey

7. Summary of periodic ground-water level data obtained from the U.S. Geological Survey

8. Summary of periodic ground-water level data obtained from the North Dakota State Water Commission

9. Summary of periodic ground-water quality data obtained from the North Dakota State Water Commission 


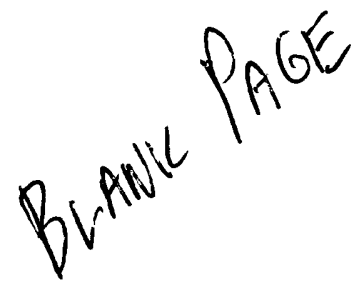




\title{
STATISTICAL AND DESCRIPTIVE SUMMARIES OF WATER-RESOURCES DATA FOR THE CANNONBALL RIVER BASIN, NORTH DAKOTA AND SOUTH DAKOTA
}

\author{
By Edwin A. Wesolowski, Scott D. Zainhofsky, and Valerie M. Dressler
}

\begin{abstract}
Existing hydrologic data and information for the Cannonball River Basin were compiled by the U.S. Geological Survey in cooperation with the Bureau of Reclamation. This report presents a summary of surface-water quality and streamflow data, ground-water quality data, ground-water level data, water-use data, and other information compiled from the U.S. Geological Survey, Bureau of Reclamation, North Dakota Game and Fish Department, North Dakota Department of Health, North Dakota Parks and Recreation Department, North Dakota State Water Commission, and Standing Rock Sioux Tribe data bases. The data are summarized statistically or descriptively, depending on the amount or nature of the data.
\end{abstract}

\section{INTRODUCTION}

The Bureau of Reclamation, in cooperation with the Standing Rock Sioux Tribe and the North Dakota State Water Commission, has begun a water-management study of the Cannonball River Basin. The Cannonball River Basin Water Management (CRBWM) study is part of a larger study known as the Missouri River Basin Tribes Water Management Study (MRBT-WMS). The purpose of the MRBT-WMS is to assist the tribes with the preparation of water-development and water-management plans. The purpose of the CRBWM study is to develop data, information, and analysis techniques to assist Federal, State, local, and tribal agencies in the management of water and other natural resources in the Cannonball River Basin. Of the seven objectives of the CRBWM study, three pertain to the hydrology of the basin. These three objectives are to (1) compile existing hydrologic data and information, describe the distribution and timing of data collection, and identify data gaps; (2) define future baseline hydrologic conditions that could be expected without water-management changes; and (3) develop methods for assessing the effects of various water-management plans.

To accomplish objective 1, the Bureau of Reclamation requested that the U.S. Geological Survey compile existing hydrologic data and information for the Cannonball River Basin. This report presents a summary of surface-water quality and streamflow data, ground-water quality data, ground-water level data, water-use data, and other information compiled from Federal, State, and tribal agency data bases. The data are summarized statistically or descriptively, depending on the amount or nature of the data.

Water-resources data and other information for the Cannonball River Basin were obtained from the U.S. Geological Survey and from other Federal, State, and tribal agencies. Most of the existing waterresources data were retrieved from computerized data bases maintained by the U.S. Geological Survey. The data may be similar to data obtained from other agencies because of the U.S. Geological Survey's cooperative programs with some of those agencies, but no attempt was made to remove duplicate data 
from the report. The data are summarized in two different formats because of incompatibility among U.S. Geological Survey and other agency databases or because the data from other Federal, State, and tribal agencies sometimes were received as hard copy rather than electronic files.

The Cannonball River Basin, located in southwestern North Dakota and north-central South Dakota, drains parts of Adams, Billings, Bowman, Grant, Hettinger, Morton, Sioux, Slope, and Stark Counties, N. Dak., and Corson and Perkins Counties, S. Dak. (fig. 1). The total drainage area is about 4,310 square miles. The basin is divided into three subbasins--the upper Cannonball River (hydrologic unit 10130204), the Cedar Creek (hydrologic unit 10130205), and the lower Cannonball River (hydrologic unit 10130206) (fig. 1). From its headwaters, the Cannonball River flows in a southeasterly direction to its confluence with Cedar Creek and then flows in a northeasterly direction to its confluence with the Missouri River. The entire river is 320 miles long. The basin has a well-developed drainage system, and many impoundments have been created on some of the minor tributaries in the basin.

Most of the land in the Cannonball River Basin is relatively smooth prairie broken at intervals by small sections of rough, deeply eroded, badlands-like plateaus. The Cannonball River and Cedar Creek cut parallel valleys across the basin to their confluence about 75 river miles from the mouth of the Cannonball River. The valleys cut down rapidly near the headwaters and then gradually level off and widen from about one-fourth of a mile wide in the upper reaches to about 2 miles wide in the lower reaches. At the confluence of the Cannonball and Missouri Rivers, the altitude of the flood plain is about 1,590 feet. Little forest growth occurs in the basin; the natural ground cover consists of short grasses on the prairies and brush on the river breaks. A few cottonwood, elm, and boxelder trees fringe the river channels.

\section{STATISTICAL AND DESCRIPTIVE SUMMARIES OF WATER-RESOURCES DATA AND OTHER INFORMATION FROM THE U.S. GEOLOGICAL SURVEY}

This section consists of surface-water quality and streamflow data (tables 1,2, and 3); ground-water quality data (tables 4 and 5); ground-water level data (tables 6 and 7); water-use data; and bibliographic information obtained from the U.S. Geological Survey. Water-quality data were collected periodically, streamflow data were collected daily, and ground-water level data were measured both daily and periodically.

The basic data were retrieved from the National Water Information System (NWIS) computerized data bases maintained by the U.S. Geological Survey. The NWIS consists of several data bases that represent different facets of water resources. These data bases are the Ground-Water Site Inventory (GWSI) system, the Automated Data Processing System (ADAPS), the Water-Quality System (QWDATA), and the SiteSpecific Water-Use Data System (SWUDS). The NWIS system contains descriptive information for all U.S. Geological Survey data-collection sites in North Dakota, except for some water-use sites. The locations of the data-collection sites for which data were retrieved are shown in figures 2 and 3 . Because tables were created by "data dumps", duplication of some constituents may occur, significant figures were not rounded, and some parameters or data fields, such as agency collecting sample, agency analyzing sample, sampling method, conversion factor, etc., do not lend themselves to meaningful descriptive statistics.

\section{Surface-Water Quallty and Streamflow Data}

Long-term surface-water sites are identified by an eight-digit number that was assigned in a downstream order along the mainstream. All sites on tributaries upstream from a mainstream site are assigned numbers smaller than the mainstream site, and all sites on tributaries between two mainstream 


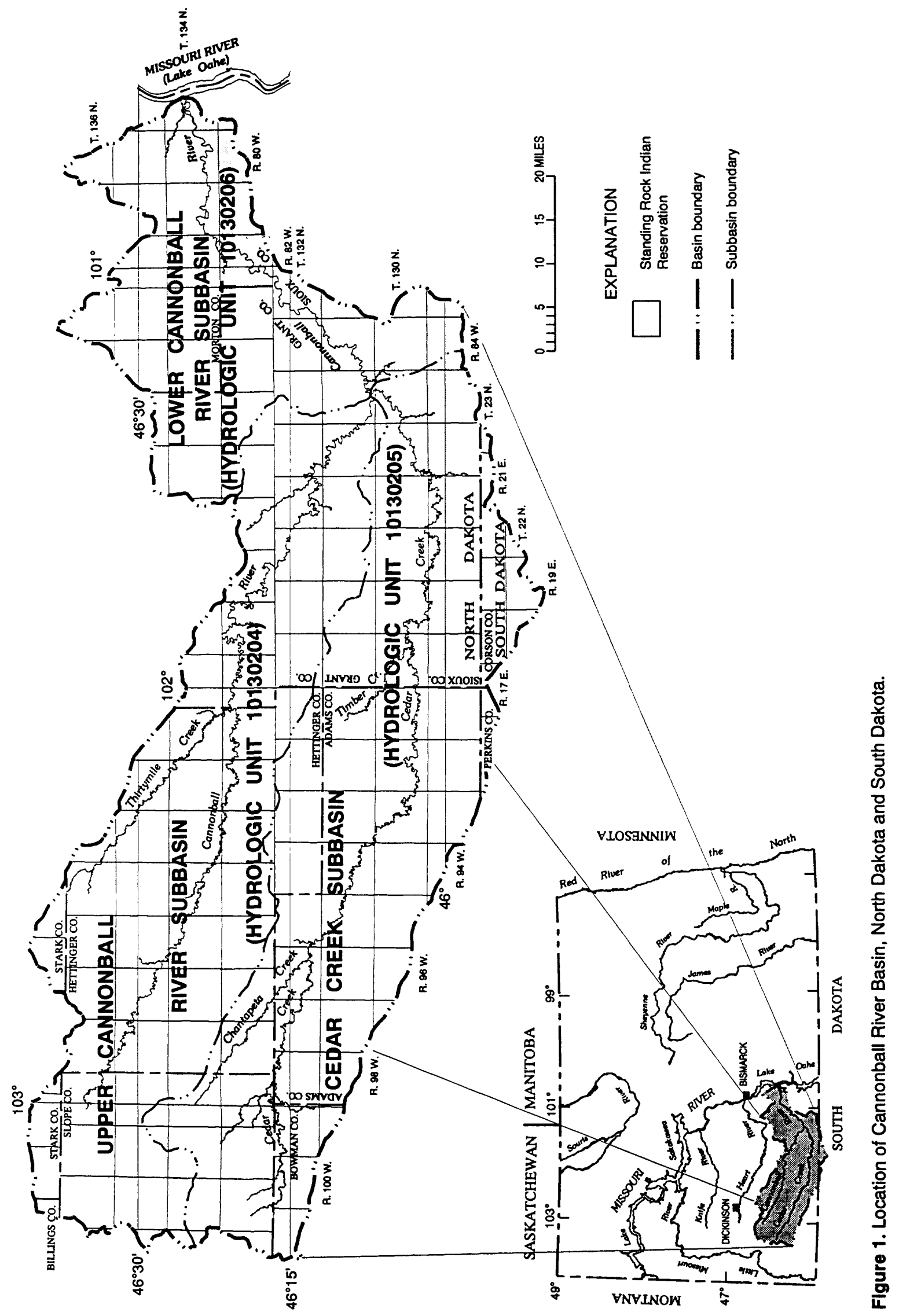




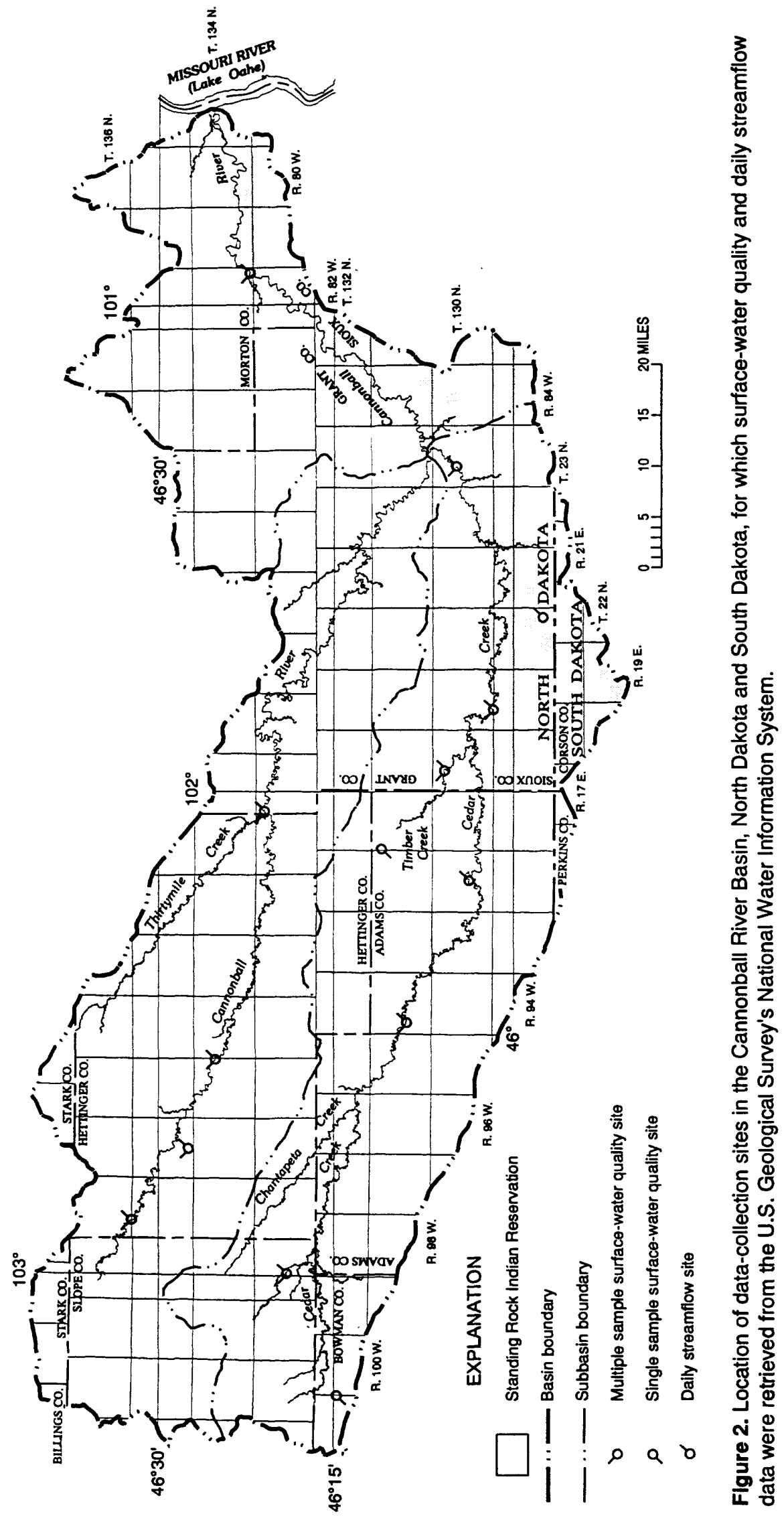




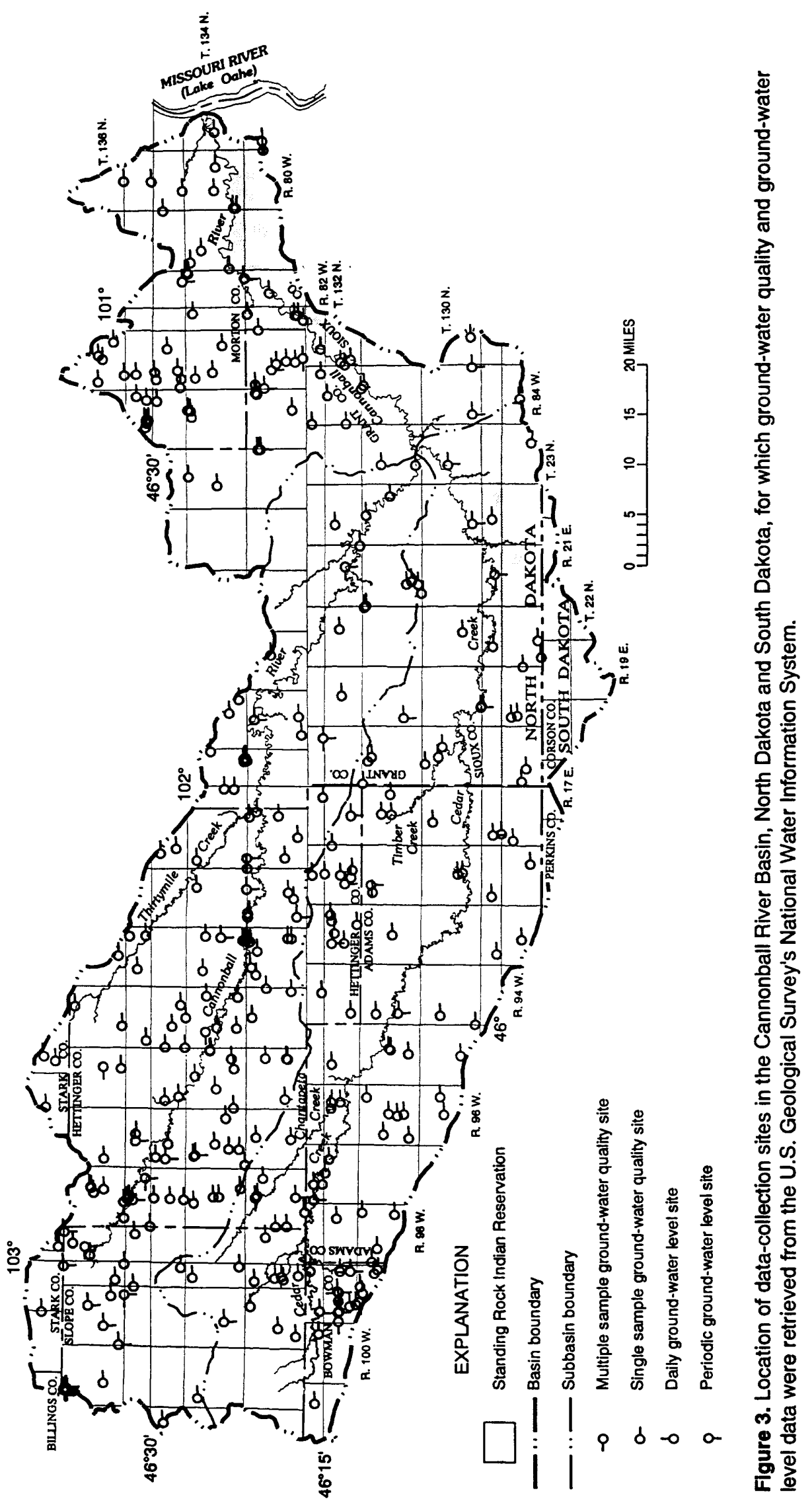

5 
sites are assigned numbers intermediate in magnitude to the mainstream sites. Short-term surface-water sites are identified by a site number that is based on 15-digit latitude-longitude numbering system (fig. 4). The first six digits denote the degrees, minutes, and seconds of latitude; the next seven digits denote the degrees, minutes, and seconds of longitude; and the last two digits (assigned sequentially) differentiate sites within a 1-second grid.

Surface-water quality and daily streamflow data for 10 surface-water sites are summarized in table 1 , daily streamflow data for 2 sites are summarized in table 2 , and surface-water quality data for 2 sites are summarized in table 3 . Statistics shown for surface-water quality data include the:

1. Sample size--the number of data values used to compute the descriptive statistics.

2. Maximum--the largest value in a group of data values.

3. Minimum--the smallest value in a group of data values.

4. Mean--the sum of individual values in a group of data values divided by the total number of values in the group.

5. Standard deviation--a measure of the dispersion of data values about the mean; equal to the square root of the sum of the squares of deviations from a mean of all values in a group divided by the total number of values in the group.

6. Percent [75, 50 (median), and 25] of samples in which values were less than or equal to those shown--except for sites where only one sample was collected, only maximum and minimum values are given if the sample size is less than five.

In addition to the sample size, maximum, minimum, mean, and standard deviation, statistics for streamflow data include the:

1. Coefficient of variation--a dimensionless measure of the dispersion of data values; equal to the percentage the standard deviation is of the mean.

2. Percent of annual runoff--a dimensionless measure of the amount of streamflow that passes the gage house; equal to the percentage monthly streamflow is of annual streamflow.

3. Percent $[95,75,50$ (median), 25, and 5] of days that had values less than or equal to those shown in cubic feet per second.

\section{Ground-Water Quallty and Ground-Water Level Data}

Ground-water sites are identified by a site number. In order to compare ground-water data given in this report to ground-water data given in other publications, the ground-water sites in this report are listed by both site number and local identifier. The local identifier is a township-range location number that is based on the Federal system of rectangular surveys of the public lands (fig. 5). The first number denotes the township north of a base line, the second number denotes the range west of the fifth principal meridian, and the third number denotes the section in which the site is located. The letters A, B, C, and D designate, respectively, the northeast, northwest, southwest, and southeast quarter section, quarter-quarter section, and quarter-quarter-quarter section (10-acre tract). For example, well 134-093-23ADD is in the SE1/4SE1/4NE1/4 sec. 23, T. 134 N., R. 93 W. Consecutive terminal numbers are added if more than one site is located within a 10 -acre tract.

Ground-water quality data for 346 ground-water sites are shown in table 4, and data for 81 sites are summarized in table 5. The sites are shown in ascending order. Generally, data for sites at which one sample was collected are shown in table 4, and data for sites at which multiple samples were collected are summarized in table 5 . However, a few sites at which one sample was collected had data for more constituents than shown in table 4. Therefore, data for those sites are summarized in table 5 , which has a more complete constituent listing. Maximum and minimum ground-water level data for 63 sites are shown 


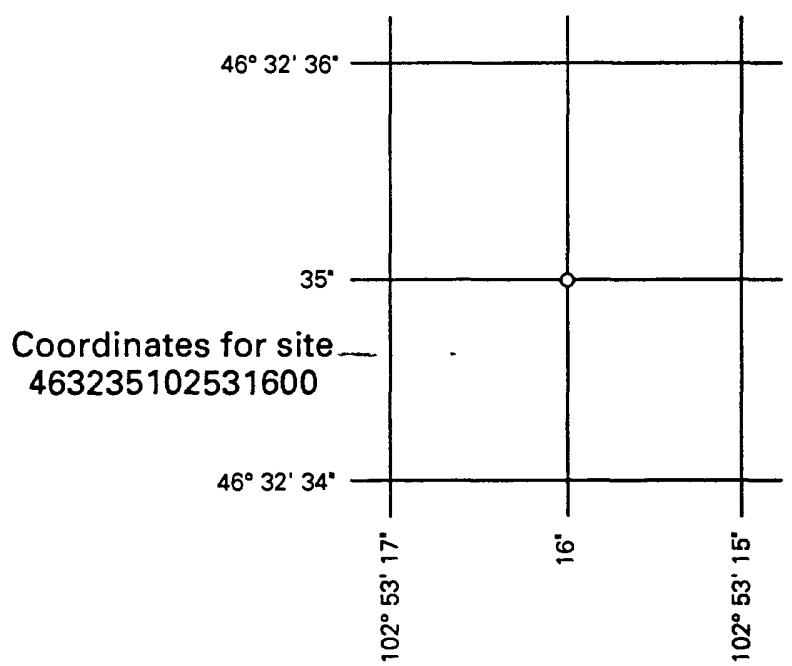

Figure 4. Latitude-longitude numbering system used for short-term surface-water sites and for ground-water sites.

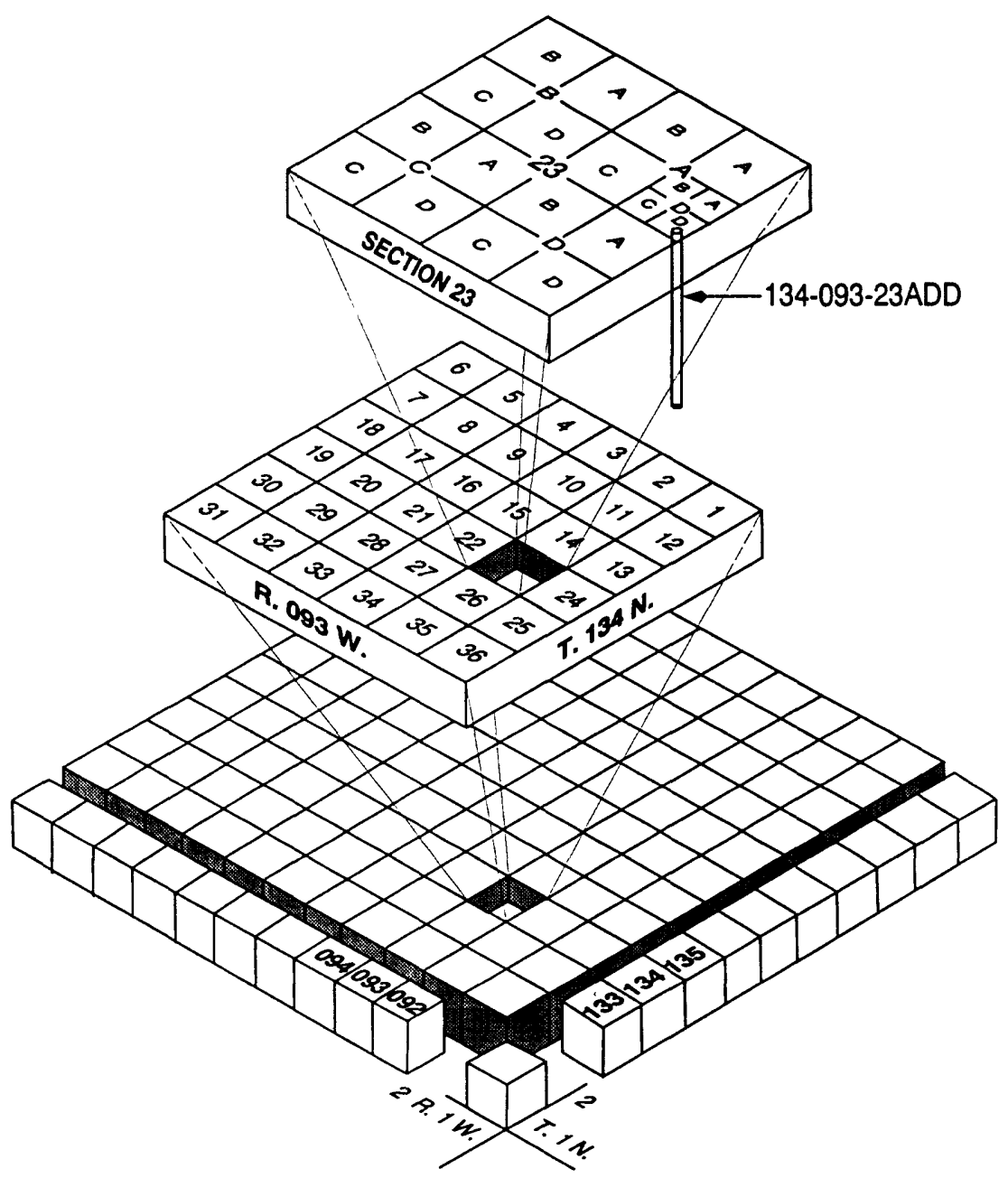

Figure 5. Township-range location-numbering system used for ground-water sites. 
in tables 6 and 7. These sites also are shown in ascending order. Data for sites at which water levels were measured daily are shown in table 6 , and data for sites at which water levels were measured periodically are shown in table 7.

\section{Water-Use Data}

The U.S. Geological Survey canvasses other Federal agencies, State agencies, local agencies, and private individuals and offices for water-use data and stores those data in the SWUDS data base. Much of the water-use data are obtained from the North Dakota State Water Commission. These data are obtained from water-permit applications and from annual reports by water-permit holders. As of August 14, 1994, the North Dakota State Water Commission had issued 124 conditional water-use permits for the Cannonball River Basin. The amount of water appropriated for the 124 permits is about 10,030 acre-feet. The estimated water use for 1990 was calculated on the basis of retrievals from SWUDS. The estimated water use for the three subbasins in the Cannonball River Basin is shown in the following table:

Estimated water use for 1990 for the three subbasins in the Cannonball River Basin

[Population, number of acres irrigated, and number of facilities are reponted in whole numbers; per capita use is reported in gallons per day; all other values are reported in million gallons per day; - , no data]

\begin{tabular}{|c|c|c|c|c|}
\hline Category & $\begin{array}{l}\text { Upper } \\
\text { Cannonball } \\
\text { River }\end{array}$ & Cedar Creek & $\begin{array}{l}\text { Lower } \\
\text { Cannonball } \\
\text { River }\end{array}$ & Total \\
\hline \multicolumn{5}{|c|}{ Domestic } \\
\hline Population, self-supplied, served by ground water & 2,210 & 2,500 & 1,880 & 6,590 \\
\hline Population, self-supplied, served by surface water & 0 & 0 & 0 & 0 \\
\hline Withdrawals, ground-water, fresh & .18 & .19 & .15 & .52 \\
\hline Withdrawals, surface-water, fresh & 0 & $\mathbf{0}$ & $\mathbf{0}$ & 0 \\
\hline Consumptive use & .18 & .19 & .15 & .52 \\
\hline Per capita use & 81 & 76 & 80 & ${ }^{1} 79$ \\
\hline \multicolumn{5}{|c|}{ Public-supplied domestic } \\
\hline Population, public-supplied, served by ground water & 2,810 & -- & 680 & 3,490 \\
\hline Population, public-supplied, served by surface water & 0 & - & 0 & 0 \\
\hline Withdrawals, ground-water, fresh & .26 & - & .05 & .31 \\
\hline Withdrawals, surface-water, fresh & 0 & - & 0 & 0 \\
\hline Deliveries (public use and losses) & .05 & - & .01 & .06 \\
\hline Per capita use & 110 & - & 88 & ${ }^{1} 106$ \\
\hline \multicolumn{5}{|c|}{ Livestock } \\
\hline Withdrawals, ground-water, fresh & 0.35 & 0.40 & 0.29 & 1.04 \\
\hline Withdrawals, surface-water, fresh & .23 & .27 & .19 & .69 \\
\hline Consumptive use & .58 & .67 & .48 & 1.73 \\
\hline \multicolumn{5}{|c|}{ Irrigation } \\
\hline Withdrawals, ground-water, fresh & 0 & 0 & 0.07 & 0.07 \\
\hline Withdrawals, surface-water, fresh & 3.42 & 2.12 & 2.59 & 8.13 \\
\hline Number of acres irrigated by sprinkler methods & 390 & 320 & 510 & 1,220 \\
\hline Number of acres irrigated by gravity/surface methods & 660 & 500 & 860 & 2,020 \\
\hline Conveyance losses & .07 & .05 & .09 & .21 \\
\hline Consumptive use & 3.08 & 1.91 & 2.39 & 7.38 \\
\hline \multicolumn{5}{|c|}{ Sewage treatment } \\
\hline Number of facilities & 6 & 1 & 2 & 9 \\
\hline Retums from municipal systems & .03 & $\mathbf{0}$ & 0 & .03 \\
\hline Reclaimed wastewater from public wastewater facility & 0 & 0 & 0 & 0 \\
\hline \multicolumn{5}{|c|}{ Commercial } \\
\hline Total withdrawals and deliveries & 0.01 & - & - & .01 \\
\hline Consumptive use & 0 & - & -- & 0 \\
\hline
\end{tabular}

${ }^{1}$ Value derived by dividing total withdrawals by total population. 


\section{Bibliographic Information}

Records of stream discharge were first published in a series of U.S. Geological Survey water-supply papers entitled, "Surface Water Supply of the United States." These water-supply papers were published annually through September 30,1960, and then in a multiyear series for 1961-65 and 1966-70. Records of chemical quality, water temperature, and suspended sediment were published annually from 1941 to 1970 in a series of water-supply papers entitled "Quality of Surface Waters of the United States." Records of ground-water levels were published annually from 1935 to 1974 in a series of water-supply papers entitled "Ground-Water Levels in the United States." Beginning with the 1971 water year and continuing to the present (1995), streamflow, surface-water quality, ground-water quality, and ground-water level data for North Dakota have been published annually in a publications series entitled "U.S. Geological Survey Water Resources Data Reports." Almost all data collected through the water-resources data program are stored in the NWIS. Data for a few sites may have been missed, however, when the data were first transformed from hard copy to electronic storage. Data stored in the NWIS can be retrieved in machinereadable format or as computer-printed tables or graphs. Those data also can be retrieved in the form of statistical summaries and various types of plots.

Water-resources data and other information for the Cannonball River Basin also can be obtained from the following U.S. Geological Survey publications:

Armstrong, C.A., 1982, Evaluation of the hydrologic system in the New Leipzig coal area, Grant and Hettinger Counties, North Dakota: U.S. Geological Survey Open-File Report 82-698, 41 p.

Armstrong, C.A., 1984, Evaluation of the hydrologic system and potential effects of mining in the Dickinson lignite area, eastern Slope and western Stark and Hettinger Counties, North Dakota: U.S. Geological Survey Water-Resources Investigations Report 84-4194, 35 p.

Calvert, W.R., Beekly, A.L., Barnett, V.H., and Pishel, M.A., 1914, Geology of the Standing Rock and Cheyenne River Indian Reservations, North and South Dakota: U.S. Geological Survey Bulletin 575, $49 \mathrm{p}$.

Colby, B.R., and Oltman, R.E., 1948, Discharge and runoff in the Missouri River Basin: U.S. Geological Survey Circular 37, 11 p.

Crosby, O.A., 1975, Magnitude and frequency of floods in small drainage basins in North Dakota: U.S. Geological Survey Water-Resources Investigations 19-75, 24 p.

Howells, Lewis, 1982, Geohydrology of the Standing Rock Indian Reservation, North and South Dakota: U.S. Geological Survey Hydrologic Investigations Atlas HA-644, 5 sheets.

Lloyd, R.E., 1914, The Cannonball River lignite field, Morton, Adams, and Hettinger Counties, North Dakota: U.S. Geological Survey Bulletin 541, p. 243-291.

Oltman, R.E., and Tracy, H.J., 1951, Trends in climate and in precipitation-runoff relation in Missouri River Basin: U.S. Geological Survey Circular 98, 113 p.

Wesolowski, E.A., 1990, North Dakota: Water supply and use, in National Water Summary 1987-Hydrologic events and water supply and use: U.S. Geological Survey Water-Supply Paper 2350, p. 401-408.

Wesolowski, E.A., 1991, Estimated use of water in North Dakota in 1985 and trends during 1960-85: U.S. Geological Survey Water-Resources Investigations Report 89-4003, 1 sheet. 


\section{STATISTICAL AND DESCRIPTIVE SUMMARIES OF WATER-RESOURCES DATA AND OTHER INFORMATION FROM OTHER FEDERAL, STATE, AND TRIBAL AGENCIES}

This section consists of water-resources data and other information obtained from agencies that were cooperating in the CRBWM study. The data and information were summarized and compiled in various formats, depending on the nature of the data and information.

The stream and location, period of record, and sampling frequency are shown in most tables in this section. Unless otherwise specified, the latitude and longitude shown for each site were generated for location purposes only and represent the approximate sampling location.

The physical properties heading is used in the tables if data consist of values for temperature, $\mathrm{pH}$, specific conductance, etc. The major ions heading is used if data consist of values for major cations and anions, including nutrients, unless otherwise specified, in solution. The nutrients heading is used if data consist of values for nitrates and phosphates. The metals heading is used if data consist of values for trace metals, including the more common metals, such as iron and manganese, unless otherwise specified. The pesticides heading is used if data consist of insecticide and herbicide values. The microbiological heading is used if data consist of coliform values. The biological heading is used if data consist of values for coliform, algae, biomass, etc. The sediment heading is used if data consist of suspended-sediment and bed-material values.

\section{Bureau of Reclamation}

The Dakota Areas Office of the Bureau of Reclamation has on file unpublished documents that may be of interest to water-resource planners. Data given in these documents were collected as part of the . Bureau's water-resource development studies in the Cannonball River Basin. Ground-water levels that were measured in connection with the drilling and augering of holes for soils and drainage studies also are available.

Water-resources data and other information on the Cannonball River Basin also can be obtained from the following Bureau of Reclamation publications:

Appraisal report on western Dakota basins study, Missouri River Basin Project, Bismarck, North Dakota, March 1975.

Cannonball River Basin streamflow depletion analysis, North/South Dakota, Missouri Basin States Association, September 1984.

Drill hole logs and auger hole permeability tests, Cannonball Division, Missouri River Basin Project, Bismarck, North Dakota, 1962.

Quality of water study, Cannonball Division, Missouri River Basin Project, Bismarck, North Dakota, January 1950.

Reconnaissance report on Cannonball River Basin, Cannonball Division, Missouri River Basin Project, Bismarck, North Dakota, September 1940.

Review report on flood control, Cannonball River and tributaries, North and South Dakota, Cannonball Division, Missouri River Basin Project, Bismarck, North Dakota, December 1954. 
Supporting data to reconnaissance report on Cannonball River Basin, Cannonball Division, Missouri River Basin Project, Bismarck, North Dakota, 1940.

Tailwater study for Mott Dam, Mott Unit (extended), Cannonball Division, Missouri River Basin Project, Bismarck, North Dakota, March 1965.

Water supply study for Cannonball Division, Missouri River Basin Project, Bismarck, North Dakota, March 1953.

\section{North Dakota Game and Fish Department}

The North Dakota Game and Fish Department collects physical-property data on selected lakes and reservoirs in the Cannonball River Basin (fig. 6) as part of its fish netting program. Physical-property data collected before the 1980's included dissolved oxygen and possibly temperature. Physical-property data collected since the 1980's include dissolved oxygen and temperature and may include $\mathrm{pH}$ and specific conductance. The stream and location, period of record, and sampling frequency for data-collection sites monitored by the North Dakota Game and Fish Department are shown in the following table:

Data-collection sites monitored by the North Dakota Game and Fish Department

[Physical properties were sampled once seasonally; the latitude and longitude shown for each site were generated for location purposes only and represent the approximate sampling location]

\begin{tabular}{|c|c|c|}
\hline Location & Period of record & $\begin{array}{l}\text { Sampling } \\
\text { trequency } \\
\text { for physical } \\
\text { proporties }\end{array}$ \\
\hline $\begin{array}{l}\text { Blickenderfer Dam, Hettinger County, T. } 133 \text { N., R. } 92 \text { W., sec. } 39 \\
\text { (lat. 46 } 20^{\prime} 10^{\prime \prime}, \text { long. 102 } 16^{\prime} 06^{\prime \prime} \text { ) }\end{array}$ & $\begin{array}{l}1979-80,82,85,88 \\
1977-80,85-86,89\end{array}$ & $\begin{array}{l}\text { Summer } \\
\text { Winter }\end{array}$ \\
\hline $\begin{array}{l}\text { Castle Rock Dam, Hettinger County, T. } 133 \text { N., R. } 93 \text { W., sec. } 17 \\
\text { (lat. } 46^{\circ} 20^{\prime} 10^{\prime \prime}, \text { long. } 102^{\circ} 16^{\prime} 06^{\prime \prime} \text { ) }\end{array}$ & $\begin{array}{l}1979-81,85,89 \\
1971-75,77-80,85-86,89\end{array}$ & $\begin{array}{l}\text { Summer } \\
\text { Winter }\end{array}$ \\
\hline $\begin{array}{l}\text { Indian Creek, Hettinger County, T. } 133 \text { N., R. } 95 \text { W., sec. } 16 \\
\text { (lat. } 46^{\circ} 20^{\prime} 09^{\prime \prime} \text {, long. } 102^{\circ} 37^{\prime} 06^{\prime \prime} \text { ) }\end{array}$ & $\begin{array}{l}1980-85,88,91 \\
1980,82,86,89\end{array}$ & $\begin{array}{l}\text { Summer } \\
\text { Winter }\end{array}$ \\
\hline $\begin{array}{l}\text { Kilzer Dam, Hettinger County, T. } 132 \text { N., R. } 91 \text { W., sec. } 15 \\
\text { (lat. } 46^{\circ} 15^{\prime} 28^{\prime \prime}, \text { long. } 102^{\circ} 02^{\prime} 45^{\prime} \text { ) }\end{array}$ & $\begin{array}{l}1982 \\
1982\end{array}$ & $\begin{array}{l}\text { Summer } \\
\text { Winter }\end{array}$ \\
\hline $\begin{array}{l}\text { Larsen Lake, Hettinger County, T. } 134 \text { N., R. } 94 \text { W., secs. } 17 \text { and } 18 \\
\text { (lat. } 46^{\circ} 25^{\prime} 35^{\prime \prime}, \text { long. } 102^{\circ} 30^{\prime} 24^{\prime \prime} \text { ) }\end{array}$ & $\begin{array}{l}1979-80,85,90 \\
1950-56,59-62,66-80,82,86\end{array}$ & $\begin{array}{l}\text { Summer } \\
\text { Winter }\end{array}$ \\
\hline $\begin{array}{l}\text { Mot Dam, Hettinger County, T. } 134 \text { N., R. } 93 \text { W., secs. } 26 \text { and } 35 \\
\text { (lat. } 46^{\circ} 22^{\prime} 50^{\prime \prime}, \text { long. } 102^{\circ} 19^{\prime} 37^{\prime \prime} \text { ) }\end{array}$ & $\begin{array}{l}1979-82,85 \\
1971-74,77-80,82,85,86,89,91\end{array}$ & $\begin{array}{l}\text { Summer } \\
\text { Winter }\end{array}$ \\
\hline $\begin{array}{l}\text { North Lemmon Lake, Adams County, T. } 129 \text { N., R. } 92 \text { W., secs. } 10 \text { and } 11 \\
\text { (lat. } 46^{\circ} 00^{\prime} 46^{\prime \prime} \text {, long. } 102^{\circ} 09^{\prime} 36^{\prime \prime} \text { ) }\end{array}$ & $\begin{array}{l}1980-82,84-85,88-91 \\
1952-62,64-73,75,77-80,84,89\end{array}$ & $\begin{array}{l}\text { Summer } \\
\text { Winter }\end{array}$ \\
\hline $\begin{array}{l}\text { Raleigh Reservoir, Grant County, T. } 133 \text { N., R. } 85 \text { W., sec. } 9 \\
\text { (lat. } 46^{\circ} 20^{\prime} 56^{\prime \prime} \text {, long. } 101^{\circ} 22^{\prime} 13^{\prime \prime} \text { ) }\end{array}$ & $\begin{array}{l}1979-82,85,92 \\
1952-54,56-60,63-74,77-80,82\end{array}$ & $\begin{array}{l}\text { Summer } \\
\text { Winter }\end{array}$ \\
\hline $\begin{array}{l}\text { Sheep Creek Dam, Grant County, T. } 133 \text { N., R. } 89 \text { W., secs. } 15 \text { and } 22 \\
\text { (lat. } 46^{\circ} 20^{\prime} 29^{\prime \prime}, \text { long. } 101^{\circ} 50^{\prime} 58^{\prime \prime} \text { ) }\end{array}$ & $\begin{array}{l}1979-83,84-85,89,91 \\
1973-74,77-79,82,89,91,92\end{array}$ & $\begin{array}{l}\text { Summer } \\
\text { Winter }\end{array}$ \\
\hline
\end{tabular}




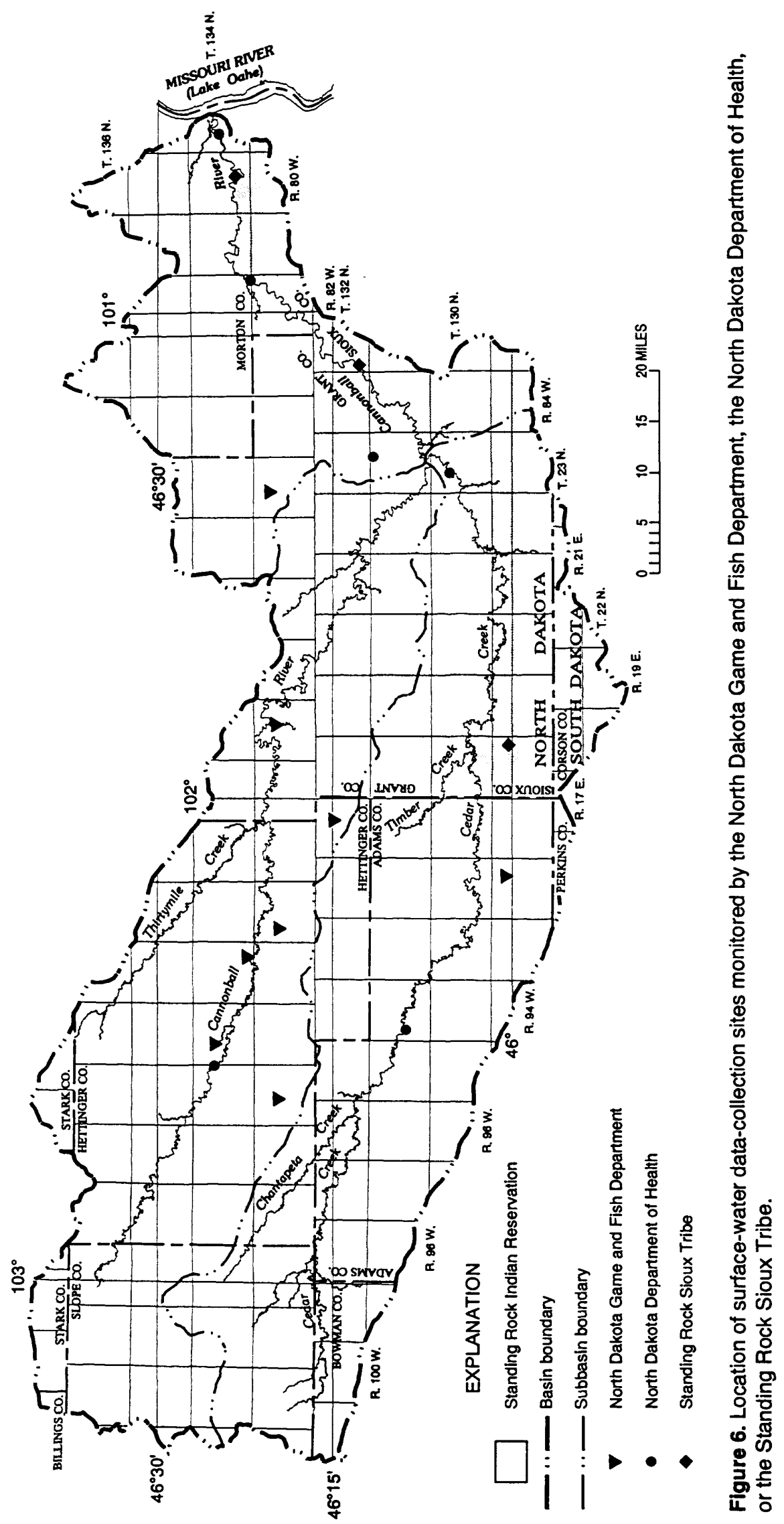




\section{North Dakota Department of Health}

Since 1975, the North Dakota Department of Health has operated an ongoing water-quality network of surface-water data-collection sites in the Cannonball River Basin (fig. 6). Samples are collected seasonally (in the spring), quarterly, or periodically and analyzed for selected physical properties and constituents. The stream and location, period of record, and category for which samples were analyzed or sampling frequency for data-collection sites monitored by the North Dakota Department of Health are shown in the following table:

Data-collection sites monitored by the North Dakota Department of Health

[USGS, U.S. Geological Survey; X, sampled for one or more representative physical properties or constituents in category; --, no data; the latitude and longitude shown for each site were generated for location purposes only and represent the approximate sampling location; $Q$, sampled quarterly; $\mathrm{P}$, sampled periodically; $\mathrm{S}$, sampled in the spring]

\begin{tabular}{|c|c|c|c|c|c|c|c|}
\hline \multirow[b]{2}{*}{ Stream and location } & \multirow[b]{2}{*}{$\begin{array}{l}\text { Period of } \\
\text { record }\end{array}$} & \multicolumn{6}{|c|}{ Category for which samplos wore analyzed or sampling frequency } \\
\hline & & $\begin{array}{l}\text { Physical } \\
\text { properties }\end{array}$ & $\begin{array}{l}\text { Major } \\
\text { ions }\end{array}$ & Metais & $\begin{array}{l}\text { Pestl- } \\
\text { cides }\end{array}$ & Blological & Sediment \\
\hline 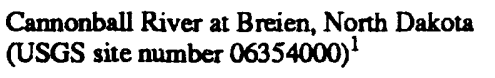 & $1975-93$ & $\mathrm{X}$ & $\mathbf{X}$ & $\mathbf{x}$ & - & $\mathbf{X}$ & $\mathbf{X}$ \\
\hline $\begin{array}{l}\text { Cannonball River at Highway } 1806 \text { bridge } \\
\text { Storet number } 380104 \\
\left.\text { (lat. } 46^{\circ} 24^{\prime} 55^{\prime \prime}, \text { long. } 100^{\circ} 38^{\prime} 05^{\prime \prime}\right)\end{array}$ & 1990-92 & Q & $\mathbf{Q}$ & Q & $\mathbf{P}$ & $\mathbf{Q}$ & -- \\
\hline $\begin{array}{l}\text { Cannonball River at Highway } 31 \text { bridge } \\
\text { Storet number } 380077 \\
\left.\text { (lat. } 46^{\circ} 05^{\prime} 30^{\prime \prime}, \text { long. } 101^{\circ} 20^{\prime} 00^{\prime \prime}\right)\end{array}$ & $1990-92$ & Q & $\mathbf{Q}$ & Q & $\mathbf{P}$ & $\mathbf{Q}$ & - \\
\hline $\begin{array}{l}\text { Cannonball River at Regent, North Dakota } \\
\text { (USGS site number } 06350000)^{1}\end{array}$ & $1975-93$ & $\mathrm{x}$ & $\mathbf{x}$ & $\mathbf{x}$ & - & $\mathbf{X}$ & $\mathrm{x}$ \\
\hline $\begin{array}{l}\text { Cedar Creek at Highway } 31 \text { bridge } \\
\text { Storet number } 380105 \\
\left.\text { (lat. } 46^{\circ} 11^{\prime} 60^{\prime \prime}, \text { long. } 101^{\circ} 18^{\prime} 00^{\prime \prime}\right)\end{array}$ & $1975-92$ & $\mathbf{S}$ & $\mathbf{S}$ & $\mathbf{S}$ & - & $\mathbf{S}$ & -- \\
\hline 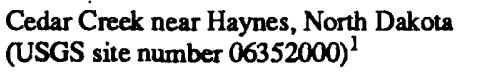 & $1975-93$ & $\mathbf{X}$ & $\mathbf{X}$ & $\mathrm{X}$ & -. & - & - \\
\hline $\begin{array}{l}\text { Cedar Creek near Raleigh, North Dakota } \\
\text { (USGS site number 06353000) }^{1}\end{array}$ & 1975-93 & $X$ & $x$ & $x$ & -- & -- & -- \\
\hline
\end{tabular}

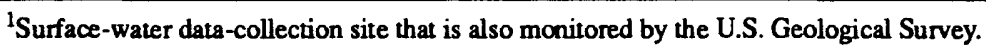

Some of the surface-water data-collection sites are monitored by both the North Dakota Department of Health and the U.S. Geological Survey. However, no attempt was made to determine which physical properties and constituents were analyzed for by the U.S. Geological Survey and which were analyzed for by the North Dakota Department of Health. Therefore, rather than showing a sampling frequency for sites monitored by both agencies, an $\mathrm{X}$ is shown to indicate that the site was sampled for one or more representative physical properties or constituents in that category. For sites monitored only by the North Dakota Department of Health, a sampling frequency is shown. Water-quality data for sites monitored by both agencies are summarized in table 1, but the statistical summaries for these sites do not include data for physical properties and constituents analyzed for by the North Dakota Department of Health because North Dakota Department of Health data are not stored in the NWIS. 
The North Dakota Department of Health archive ground-water data base contains data on water samples collected between 1948 and 1986. These data were not subject to the quality assurance protocol that began in 1986 and are not included in the table. Data and other information available in the data base include major-ion values, well owner, and well and aquifer description. Data also may be available through the Division of Municipal Facilities' drinking-water program and from communities that have been required to have their public water supplies tested periodically.

Water-resources data and other information for the Cannonball River Basin also can be obtained from the following North Dakota Department of Health publications:

North Dakota State Department of Health, 1980, Chemical analysis of public water supplies in North Dakota.

North Dakota State Department of Health and Consolidated Laboratories, 1988, The status of water quality in the state of North Dakota, 1986-87.

North Dakota State Department of Health and Consolidated Laboratories, 1992, North Dakota waterquality assessment, 1990-91.

\section{North Dakota Parks and Recreation Department}

Water-resources data and other information available from the North Dakota Parks and Recreation Department are included in the following publications:

North Dakota Parks and Recreation Department, December 1987, North Dakota rivers study.

North Dakota Parks and Recreation Department, 1990, North Dakota outdoor recreation plan, 1991-95.

Issues such as (1) access to and development of rivers, (2) unstable water quality and quantity, and (3) preservation of rivers are addressed in the "North Dakota Outdoor Recreation Plan, 1991-95." Geologic/ hydrologic features of the Cannonball River and Cedar Creek, botanical resources, zoological resources, unique ecological communities, historic/prehistoric sites, sport fishing, recreational resources, water quality, forest resources, and breeding of waterfowl and white-tail deer are evaluated in the "North Dakota Rivers Study," Section V, "River Related Natural, Cultural, and Recreational Values."

\section{North Dakota State Water Commission}

The North Dakota State Water Commission supports the statewide operation of streamflow gages and the monitoring of ground-water wells both independently and in cooperation with the U.S. Geological Survey. The ground-water well monitoring program began as part of the county ground-water investigations program.

Ground-water resources investigations of all counties within the Cannonball River Basin were completed during the 1970's and 1980's. The investigations were made by the U.S. Geological Survey in cooperation with the North Dakota State Water Commission, the North Dakota Geological Survey, and respective county water management agencies. Results of each of five investigations were published by the North Dakota State Water Commission in a three-part series of reports. Part I is an interpretive report 
describing the geology of the study area, part II is a compilation of the geologic and hydrologic data collected during the investigation, and part III is an interpretive report describing ground-water resources of the study area.

The ongoing water-resources data-collection program includes ground-water sites in the Cannonball River Basin. The locations of these sites are shown in figure 7. Ground-water level data for 19 sites are summarized in table 8, and ground-water quality data for 368 sites are summarized in table 9. The period of record and category for which samples were analyzed are shown for the 368 sites. Data for some of the sites also may be included in tables 4 through 7, but no attempt was made to eliminate duplicate data. Rather, all data provided by the North Dakota State Water Commission were summarized in tables 8 and 9.

Water-resources data and other information for the Cannonball River Basin also can be obtained from the following North Dakota State Water Commission publications:

Chemical analysis of surface water of Cannonball River Basin, North Dakota, 1945-49, 1945-50, 1945-51: U.S. Department of the Interior, U.S. Geological Survey.

Low flow frequency curves Cannonball River Basin, 1962: U.S. Department of the Interior, U.S. Geological Survey.

Ackerman, D.J., 1977, Ground-water basic data for Morton County, North Dakota: North Dakota State Water Commission County Ground-Water Studies 27, pt. II, and North Dakota Geological Survey Bulletin 72, pt. II, 592 p.

Ackerman, D.J., 1980, Ground-water resources of Morton County, North Dakota: North Dakota State Water Commission County Ground-Water Studies 27, pt. III, and North Dakota Geological Survey Bulletin 72, pt. III, 51 p.

Anna, L.O., 1980, Ground-water data for Billings, Golden Valley, and Slope Counties, North Dakota: North Dakota State Water Commission County Ground-Water Studies 29, pt. II, and North Dakota Geological Survey Bulletin 76, pt. II, 241 p.

Anna, L.O., 1981, Ground-water resources of Billings, Golden Valley, and Slope Counties, North Dakota: North Dakota State Water Commission County Ground-Water Studies 29, pt. III, and North Dakota Geological Survey Bulletin 76, pt. III, 56 p.

Bader, C.D., 1987, Trends in water use and estimated water use for North Dakota, 1985: North Dakota State Water Commission Information Series No. 34, 12 p.

Carlson, C.G., 1979, Geology of Adams and Bowman Counties, North Dakota: North Dakota Geological Survey Bulletin 65, pt. I, and North Dakota State Water Commission County Groundwater Studies 22, pt. I, 29 p.

Carlson, C.G., 1982, Geology of Grant and Sioux Counties, North Dakota: North Dakota Geological Survey Bulletin 67, pt. I, and North Dakota State Water Commission County Groundwater Studies 24, pt. I, 32 p.

Carlson, C.G., 1983, Geology of Billings, Golden Valley, and Slope Counties, North Dakota: North Dakota Geological Survey Bulletin 76, pt. I, and North Dakota State Water Commission County Groundwater Studies 29, pt. I, 40 p. 


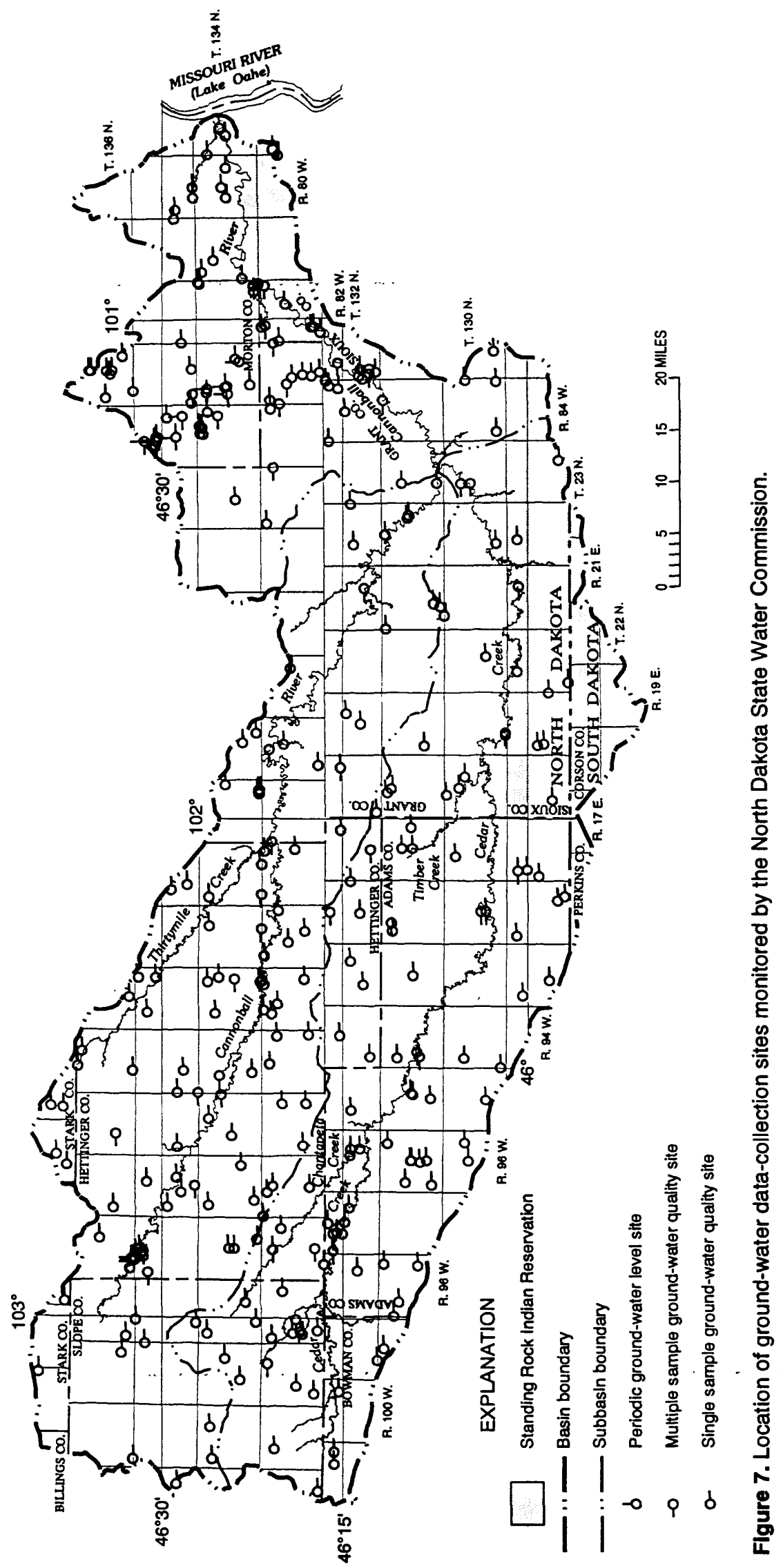


Carlson, C.G., 1983, Geology of Morton County, North Dakota: North Dakota Geological Survey Bulletin 72, pt. I, and North Dakota State Water Commission County Groundwater Studies 27, pt. I, 37 p.

Croft, M.G., 1974, Ground-water basic data for Adams and Bowman Counties, North Dakota: North Dakota State Water Commission County Ground-Water Studies 22, pt. II, and North Dakota Geological Survey Bulletin 65, pt. II, 294 p.

Croft, M.G., 1978, Ground-water resources of Adams and Bowman Counties, North Dakota: North Dakota State Water Commission County Ground-Water Studies 22, pt. III, and North Dakota Geological Survey Bulletin 65, pt. III, 54 p.

North Dakota State Water Commission, 1934, Cannonball, Grand, and Moreau Rivers, North Dakota and South Dakota.

North Dakota State Water Commission, 1972, Cannonball River Basin plan and appendix.

North Dakota State Water Commission, 1975, West River study: An analysis of altematives for developing and managing the West River area's water and related land resources.

North Dakota State Water Commission, 1983, 1983 State water plan.

North Dakota State Water Commission, 1992, 1992 North Dakota State water management plan.

North Dakota State Water Conservation Commission, March 1966, Drainage area data for Cannonball River Basin.

Patch, J.C., and Haffield, N.D., 1982, Estimated use of water for North Dakota: North Dakota State Water Commission Information Series No. 33, 1 p.

Randich, P.G., 1975, Ground-water basic data for Grant and Sioux Counties, North Dakota: North Dakota State Water Commission County Ground-Water Studies 24, pt. II, and North Dakota Geological Survey Bulletin 67, pt. II, 303 p.

Randich, P.G., 1979, Ground-water resources of Grant and Sioux Counties, North Dakota: North Dakota State Water Commission County Ground-Water Studies 24, pt. III, and North Dakota Geological Survey Bulletin 67, pt. III, 49 p.

Ripley, David, 1990, An overview of North Dakota's water resources, in Proceedings, North Dakota Water Quality Symposium, Fargo, North Dakota, March 20-21, 1990: North Dakota State University Extension Service, p. 1.

Smith, M.L., and Harkness, R.E., 1982, Water use in North Dakota, 1980: North Dakota State Water Commission Information Series No. 31, 1 p.

Trapp, Henry, Jr., 1971, Ground water basic data, Hettinger and Stark Counties, North Dakota: North Dakota State Water Commission County Ground Water Studies'16, pt. II, 455 p.

Trapp, Henry, Jr., and Croft, M.G., 1975, Geology and ground-water resources of Hettinger and Stark Counties, North Dakota: North Dakota State Water Commission County Ground-Water Studies 16, pt. I, $51 \mathrm{p}$. 
In addition to these publications, data and other information can be obtained from the following unpublished reports on file at the North Dakota State Water Commission:

Adams County road drainage, Project number 1000

Adams County small projects, Project number 1263

Adams County Water Management District, Project number 701

Adams-Bowman Counties ground-water survey, Project number 940

Cedar River flow control problem (Adams, Bowman, Hettinger, and Slope Counties),

Project number 939

Duck Creek watershed, Adams County, Project number 837

North Lemmin Lake Dam, Adams County, Project number 543

Wolf Butte Dam, Adams County, Project number 359

Billings County drainage general, Project number 1578

Billings County road drainage, Project number 1003

Billings County Water Management District, Project number 1555

Billings, Golden Valley, and Slope Counties ground-water study, Project number 942

Bowman County drainage general, Project number 1580

Bowman County road drainage, Project number 1005

Bowman County small projects, Project number 1268

Bowman County Water Management District, Project number 821

Cannonball and Cedar River development (Grant, Hettinger, Morton, and Sioux Counties),

Project number 262

Grant County road drainage, Project number 1018

Grant County small project, Project number 1281

Grant County Water Management District, Project number 708

Grant and Sioux Counties ground-water survey, Project number 951

Louse Creek watershed, Grant County, Project number 840

New Raleigh Dam, Grant County, Project number 507-2

Raleigh Dam, Grant County, Project number 507-1

Sheep Creek Dam, Grant County, Project number 1358

Tietz Dam, Grant County, Project number 577

Hettinger County road drainage, Project number 1020

Hettinger County small projects, Project number 1283

Hettinger County Water Management District, Project number 1426

Hettinger and Stark Counties ground-water survey, Project number 953

Indian Creek Dam, Hettinger County, Project number 1556

Karey Dam, Hettinger County, Project number 1453

Mott Dam and flood control, Hettinger County, Project number 249

Mott watershed, Hettinger County, Project number 1457

New England ground-water survey, Project number 787

Regent Dam, Hettinger County, Project number 350

Squaw Creek Dam, Hettinger County, Project number 417

Thirty Mile Creek Basin, Hettinger County, Project number 1497

Flasher critical area treatment, Morton County, Project number 1778

Flasher Dam, Morton County, Project number 1292

Flasher ground-water survey, Morton County, Project number 755

Morton County ground-water survey, Project number 960

Morton County road drainage, Project number 1029

Morton County small projects, Project number 1292

Morton County Water Management District, Project number 944 
Selfridge West Dam, Sioux County, Project number 1305-1

SI's Dam, Sioux County, Project number 1305-2

Sioux County road drainage, Project number 1042

Sioux County small projects, Project number 1305

Sioux County Water Management District, Project number 718

Cedar Dam, Slope County, Project number 353

Slope County drainage general, Project number 718

Slope County road drainage, Project number 1043

Slope County small projects, Project number 1306

Slope County Water Management District, Project number 1575

Stark County road drainage, Project number 1044

Stark County small projects, Project number 1307

Stark County Water Management District, Project number 1429

\section{Standing Rock Sloux Trlbe}

The Department of Water and Natural Resources of the Standing Rock Sioux Tribe has a "water users data base," which is contained in dBase files. Data were included for 2,034 data-collection sites--1,146 in Sioux County, North Dakota, and 888 in Corson County, South Dakota. Much of the data, which were obtained from Federal, State, local, and tribal agencies, are shown in this report. The categories for which data are stored are shown in the following table:

\begin{tabular}{|c|c|c|}
\hline \multicolumn{3}{|c|}{ Category for which data are stored } \\
\hline Acreage & Notes & Stream \\
\hline Appropriation date & Owner's address & Structure amount \\
\hline Basin number & Owner's name ${ }^{1}$ & Structure name ${ }^{1}$ \\
\hline Casing type & Power source & Structure owner \\
\hline Construction date & Principal meridian & Structure type $e^{1}$ \\
\hline County ${ }^{1}$ & Priority date & Structure use \\
\hline Dam height & Quadrangle name & Surface area \\
\hline Depth to water ${ }^{1}$ & Range $^{1}$ & Township ${ }^{1}$ \\
\hline Drill date ${ }^{1}$ & Reservoir storage (active) & Use $^{1}$ \\
\hline East/west coordinate & Reservoir storage (total) & Well depth ${ }^{1}$ \\
\hline Formation & Section ${ }^{1}$ & Well diameter ${ }^{1}$ \\
\hline Hazard classification & Standing Rock Sioux Tribe permit number & Well number \\
\hline North coordinate & State designation & Yield \\
\hline
\end{tabular}

${ }^{1}$ Likely to have data.

Information regarding a specific category can be obtained by contacting the Department of Water and Natural Resources.

The Indian Health Service in Aberdeen, S. Dak., provided well-log data for private wells. Information given on the well logs varies, but the most complete well logs include the well owner; the driller's log of the borehole; well-construction information; pump information; test-pump data, including the static water level; and a water analysis to determine if the water is safe for use. Because the well locations and well identifiers or both were inconsistent or nonexistent, no attempt was made to list these wells. 
The U.S. Environmental Protection Agency has approved a water-quality assurance plan, including extensive surface-water sampling for the Reservation. Work has been started to develop a ground-water monitoring network throughout the Reservation.

The Department of Water and Natural Resources has begun a water-quality sampling program for three data-collection sites. The period of record and sampling frequencies for the three sites are shown in the following table:

Data-collection sites monitored by the Standing Rock Sioux Tribe

[The latitude and longitude shown for each site were generated for location purposes only and represent the approximate sampling location; $P$, sampled periodically]

\begin{tabular}{|c|c|c|c|c|c|c|}
\hline \multirow[b]{2}{*}{ Stream and location } & \multirow[b]{2}{*}{$\begin{array}{l}\text { Period of } \\
\text { record }\end{array}$} & \multicolumn{5}{|c|}{ Sampllng frequency } \\
\hline & & $\begin{array}{l}\text { Physical } \\
\text { propertices }\end{array}$ & $\begin{array}{l}\text { Major } \\
\text { ions }\end{array}$ & Nutrients & Motals & $\begin{array}{l}\text { Micro- } \\
\text { biological }\end{array}$ \\
\hline $\begin{array}{l}\text { Cannonball River, Sioux County, T. } 132 \text { N., R. } 83 \text { W., sec. 31, } \\
\left.\text { south of bridge (lat. } 46^{\circ} 13^{\prime} 08^{\prime \prime}, \text { long. } 101^{\circ} 06^{\prime} 43^{\prime \prime}\right)\end{array}$ & 1994 & $\mathbf{P}$ & $\mathbf{P}$ & $\mathbf{P}$ & $\mathbf{P}$ & $\mathbf{P}$ \\
\hline $\begin{array}{l}\text { Cannonball River, Sioux County, T. } 134 \text { N., R. } 80 \text { W., sec. } 26 \text {, } \\
\text { north of train grade (lat. } 46^{\circ} 23^{\prime} 35^{\prime \prime} \text {, long. } 100^{\circ} 43^{\prime} 17^{\prime \prime} \text { ) }\end{array}$ & 1994 & $\mathbf{P}$ & $\mathbf{P}$ & $\mathbf{P}$ & $\mathbf{P}$ & $\mathbf{P}$ \\
\hline $\begin{array}{l}\text { Leaf on the Hill Creek, Sioux County, T. } 130 \text { N., R. } 84 \text { W., sec. } 27 \text {, } \\
\left.\text { north of bridge (lat. } 46^{\circ} 00^{\prime} 31^{\prime \prime} \text {, long. } 101^{\circ} 53^{\prime} 27^{\prime \prime}\right)\end{array}$ & 1994 & $\mathbf{P}$ & $\mathbf{P}$ & $\mathbf{P}$ & $\mathbf{P}$ & $\mathbf{P}$ \\
\hline
\end{tabular}

Water-resources data and other information on the Cannonball River Basin also can be obtained from the following publications:

Morrison-Maierle, Inc., 1977, Water resources evaluation, Phase I, Standing Rock Indian Reservation, North and South Dakota, for U.S. Bureau of Indian Affairs, Aberdeen, South Dakota.

Morrison-Maierle, Inc., 1977, Water resources evaluation, Phase II, Standing Rock Indian Reservation, North and South Dakota, for U.S. Bureau of Indian Affairs, Aberdeen, South Dakota, revised March 1979.

Morrison-Maierle, Inc., 1979, Missouri River Basin water supply and water requirements, for United Sioux Indian Tribes, Pierre, South Dakota.

Morrison-Maierle, Inc., 1980, Water resources evaluation, Phase III, Standing Rock Indian Reservation, North and South Dakota, for U.S. Bureau of Indian Affairs, Aberdeen, South Dakota, revised March 1981.

Native American Natural Resources Development Federation, Standing Rock Sioux inventory, North and South Dakota, 1980.

Standing Rock Sioux Tribe assistance from Technical Resource Corporation standards and criteria, Garrison Diversion Unit authorization for irrigation of 2,380 acres (PL 99-294), November 29, 1988, revised December 21, 1988.

Wright Water Engineers, Inc., 1993, Interim report of irrigable lands adjacent to Lake Oahe and in the Cannonball Basin, for the Standing Rock Sioux Tribe, Fort Yates, North Dakota. 
Table 1. Summary of surface-water quality and daily streamflow data obtained from the U.S. Geological Survey for sites where multiple water-quality samples were collected

[Certain properties or constituents may be listed more than once; however, no distinction is made between field and laboratory values]

\section{Abbreviatlons and symbols}

$\mathrm{ft}$, feet

$\mathrm{ft}^{3} / \mathrm{s}$, cubic feet per second

$\mathrm{g} / \mathrm{kg}$, grams per kilogram

JCU, Jackson candle unit

LSD, land surface datum

$\mathrm{mg} / \mathrm{L}$, milligrams per liter

mi, mile

$\mathrm{mi}^{2}$, square miles

$\mathrm{mL}$, milliliter

$\mathrm{mm}$, millimeter

NGVD, National Geodetic Vertical Datum

NTU, nephelometric turbidity unit

$\mathrm{pCi} / \mathrm{L}$, picocurie per liter

$\mu \mathrm{g} / \mathrm{g}$, micrograms per gram

$\mu \mathrm{g} / \mathrm{L}$, micrograms per liter

$\mu \mathrm{m}$, micrometer

$\mu \mathrm{S} / \mathrm{cm}$, microsiemens per centimeter at 25 degrees Celsius

,-- no data 


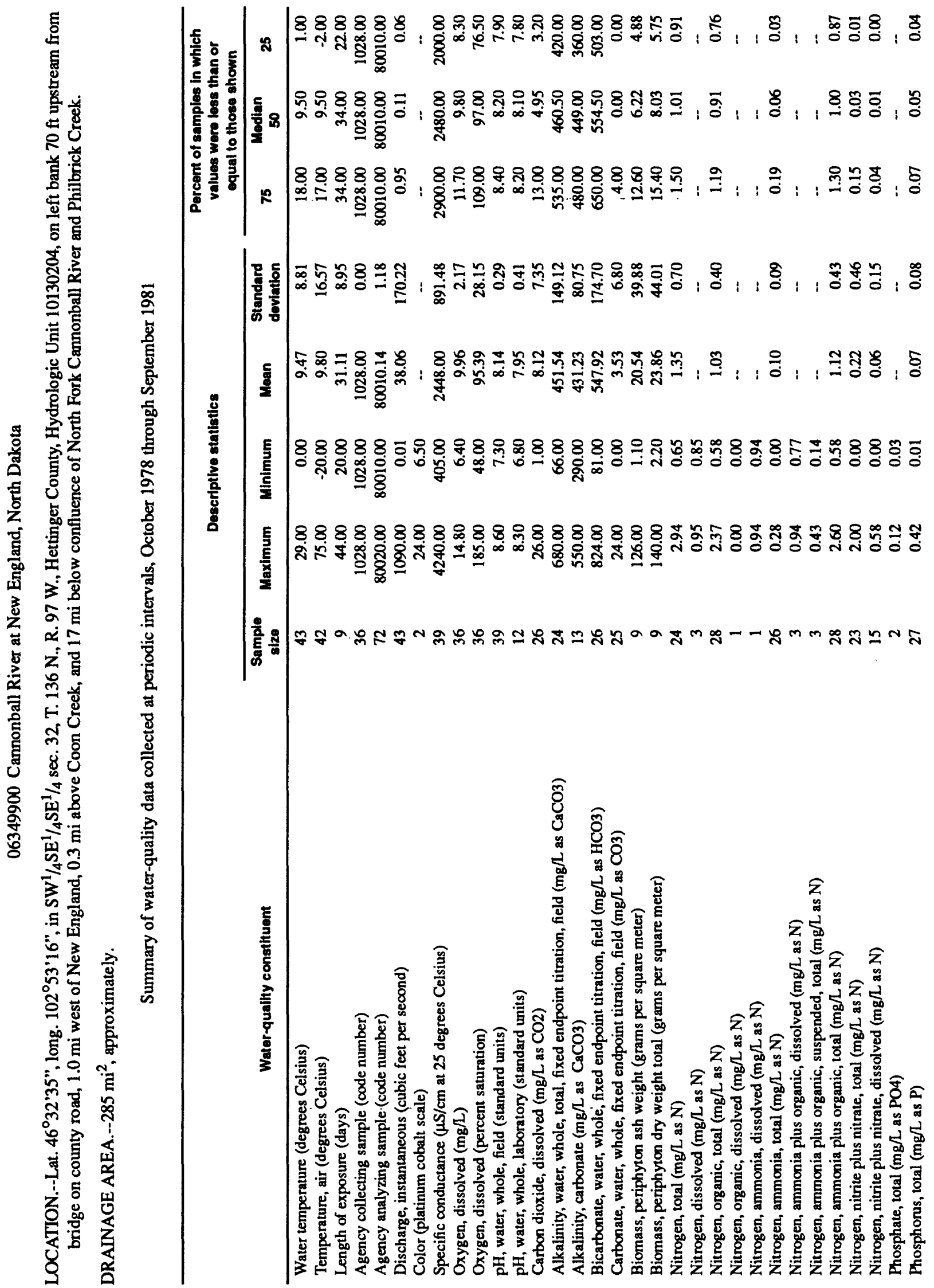




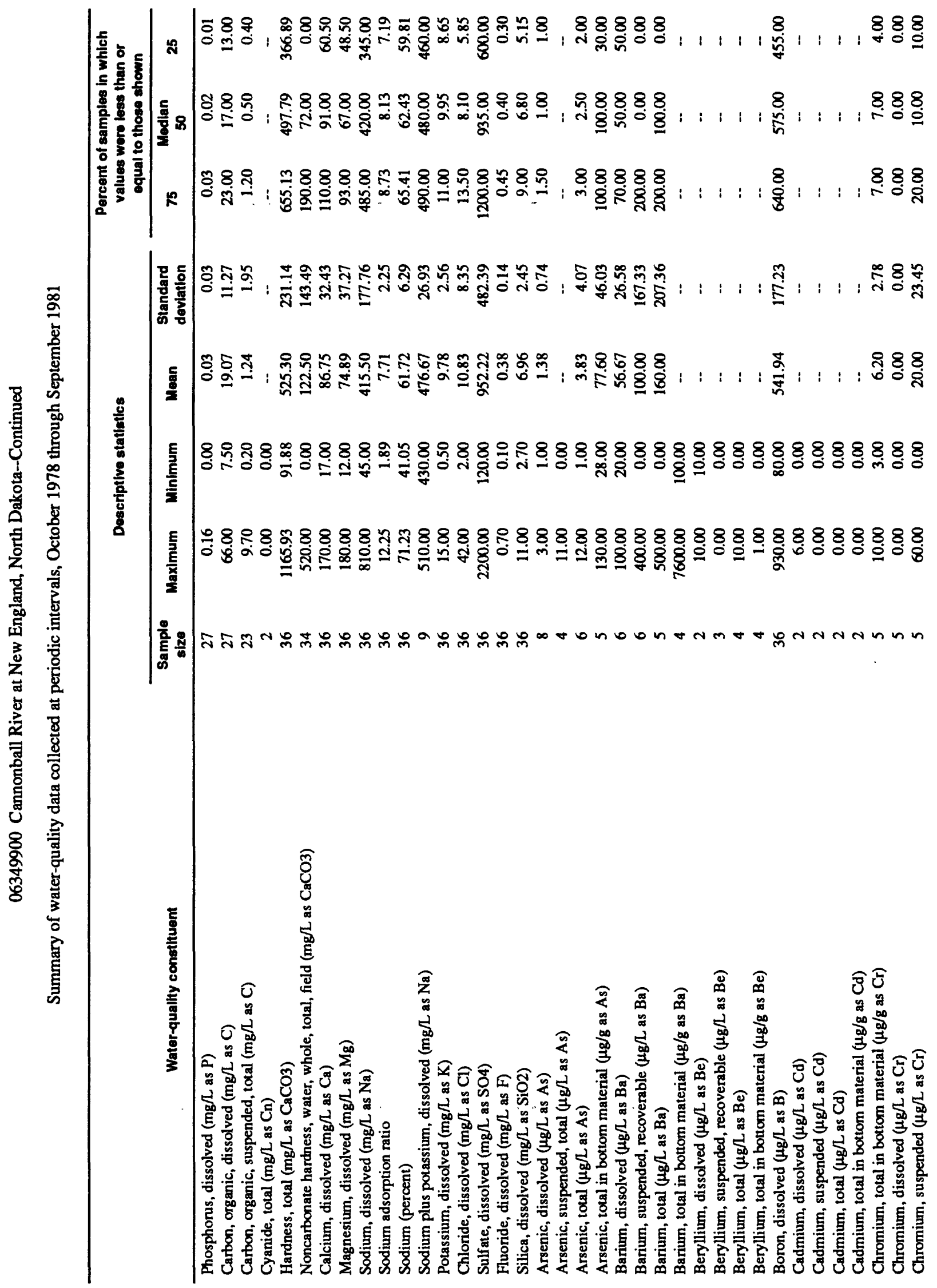




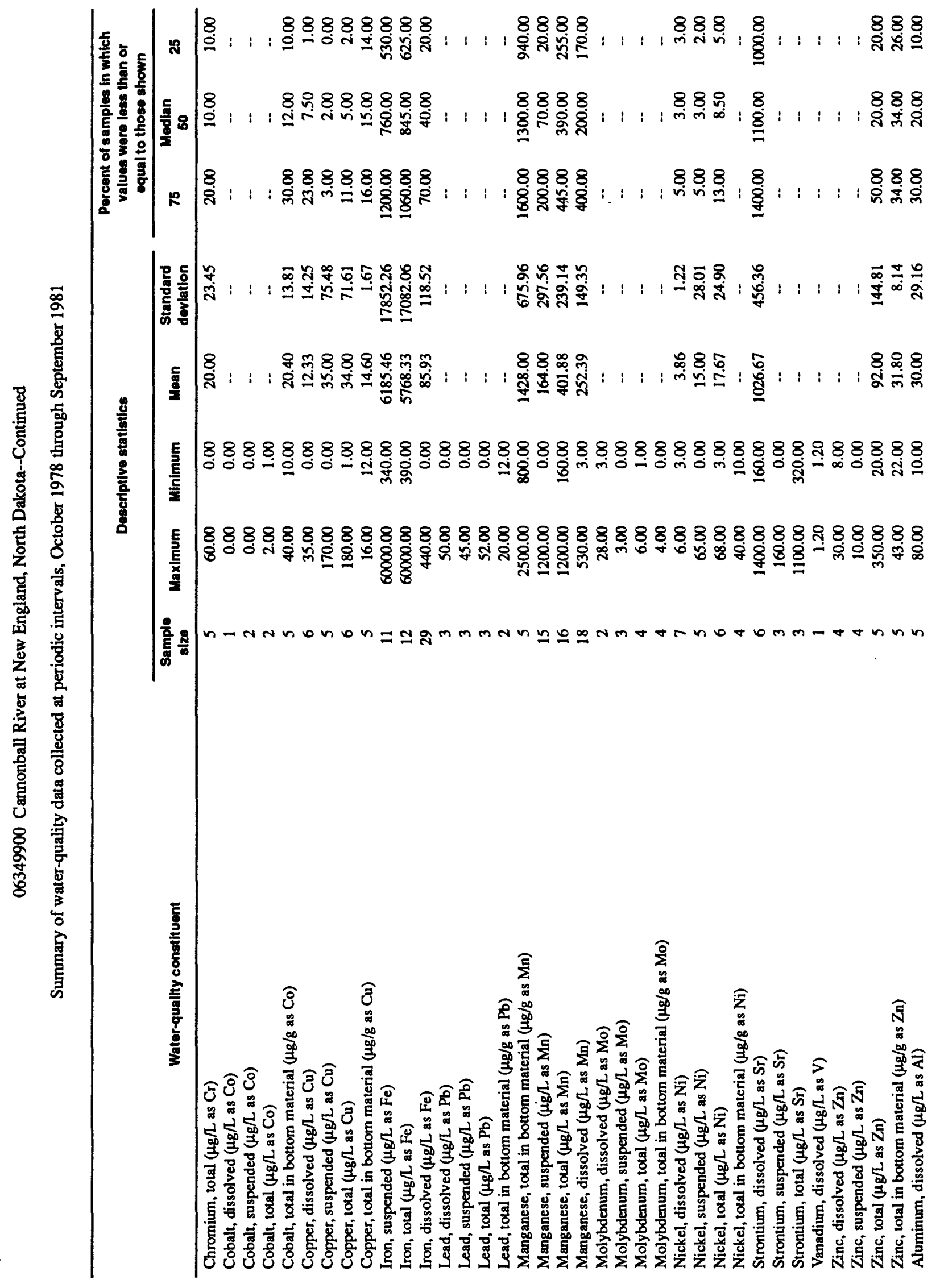




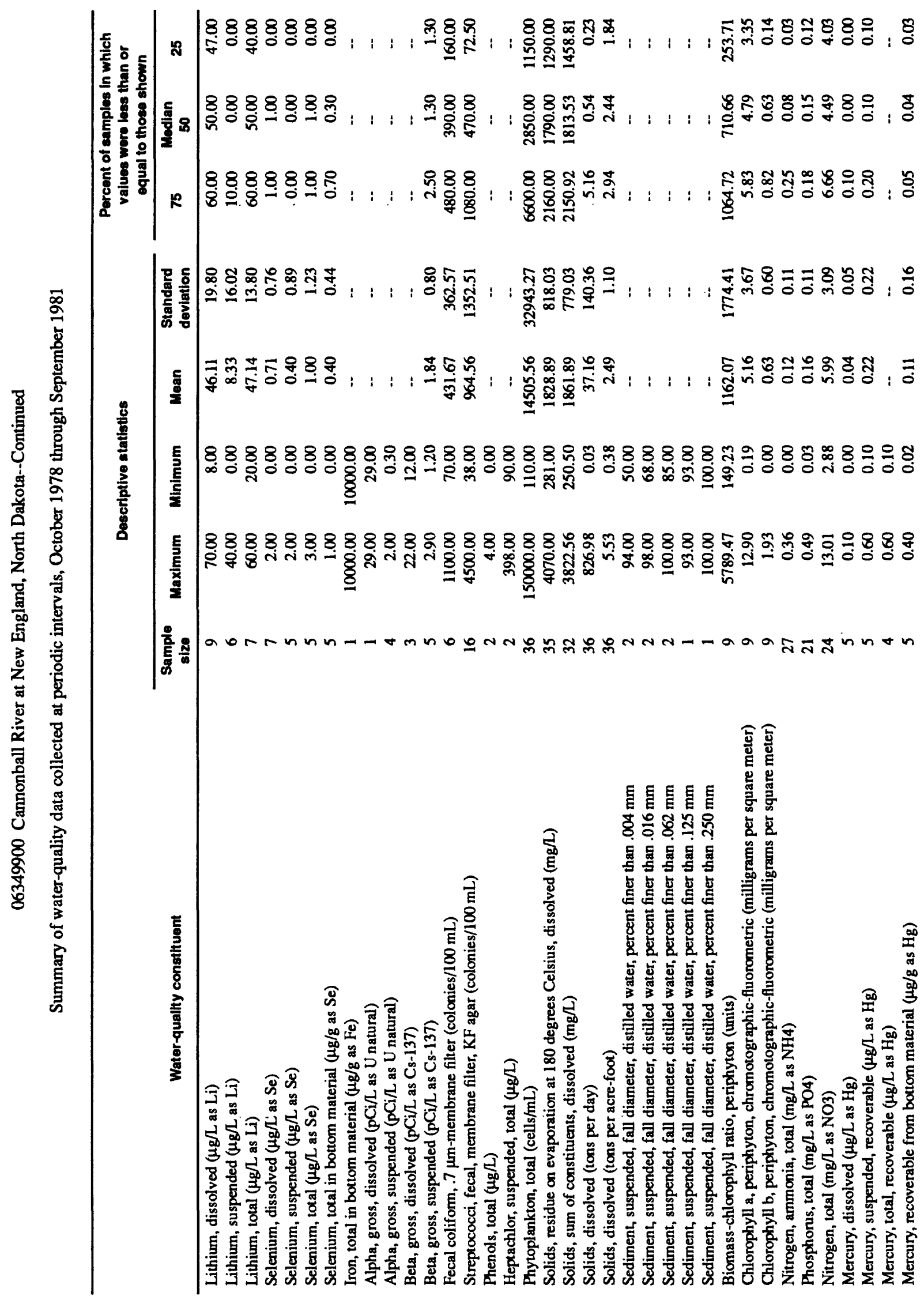




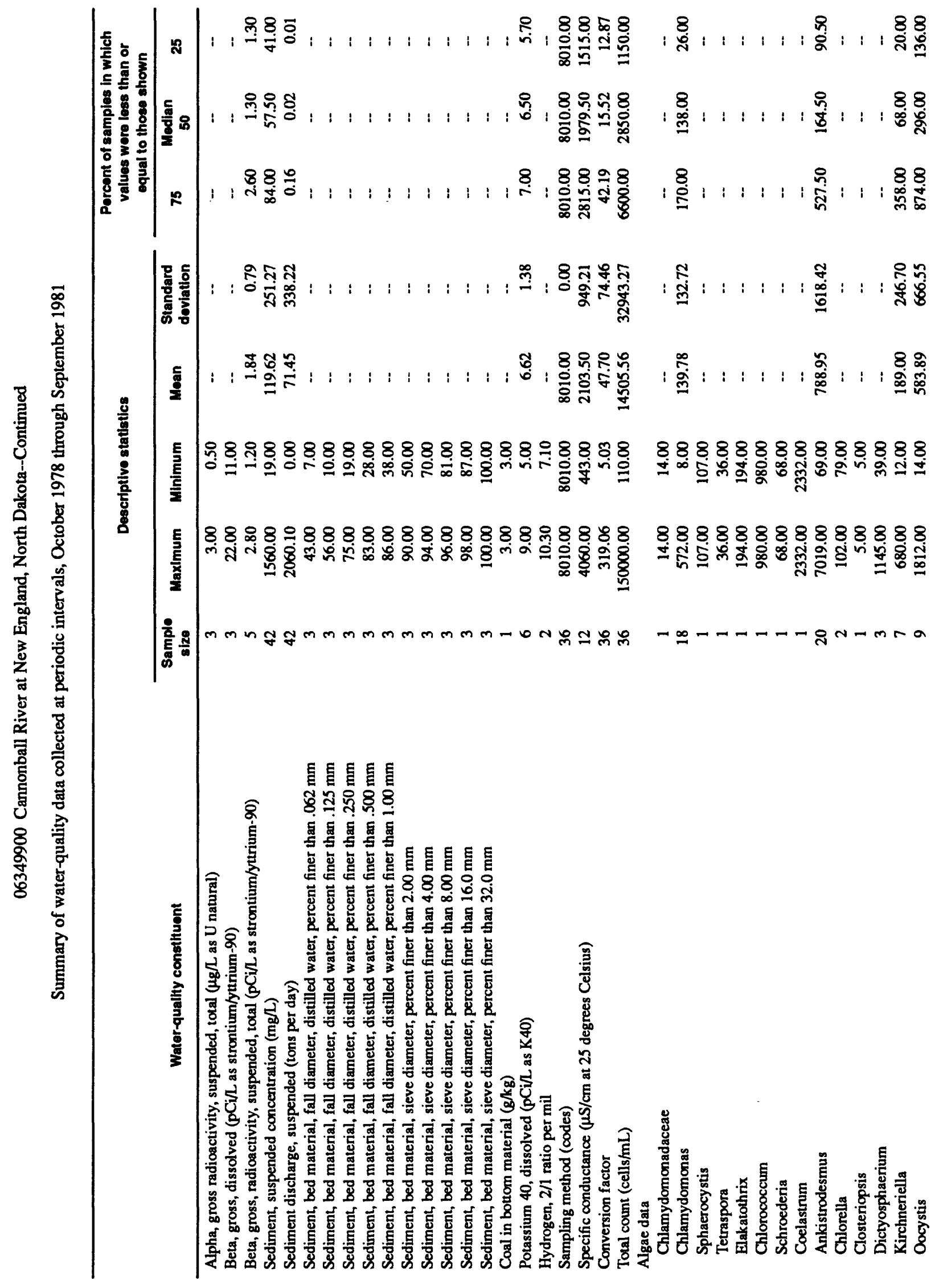




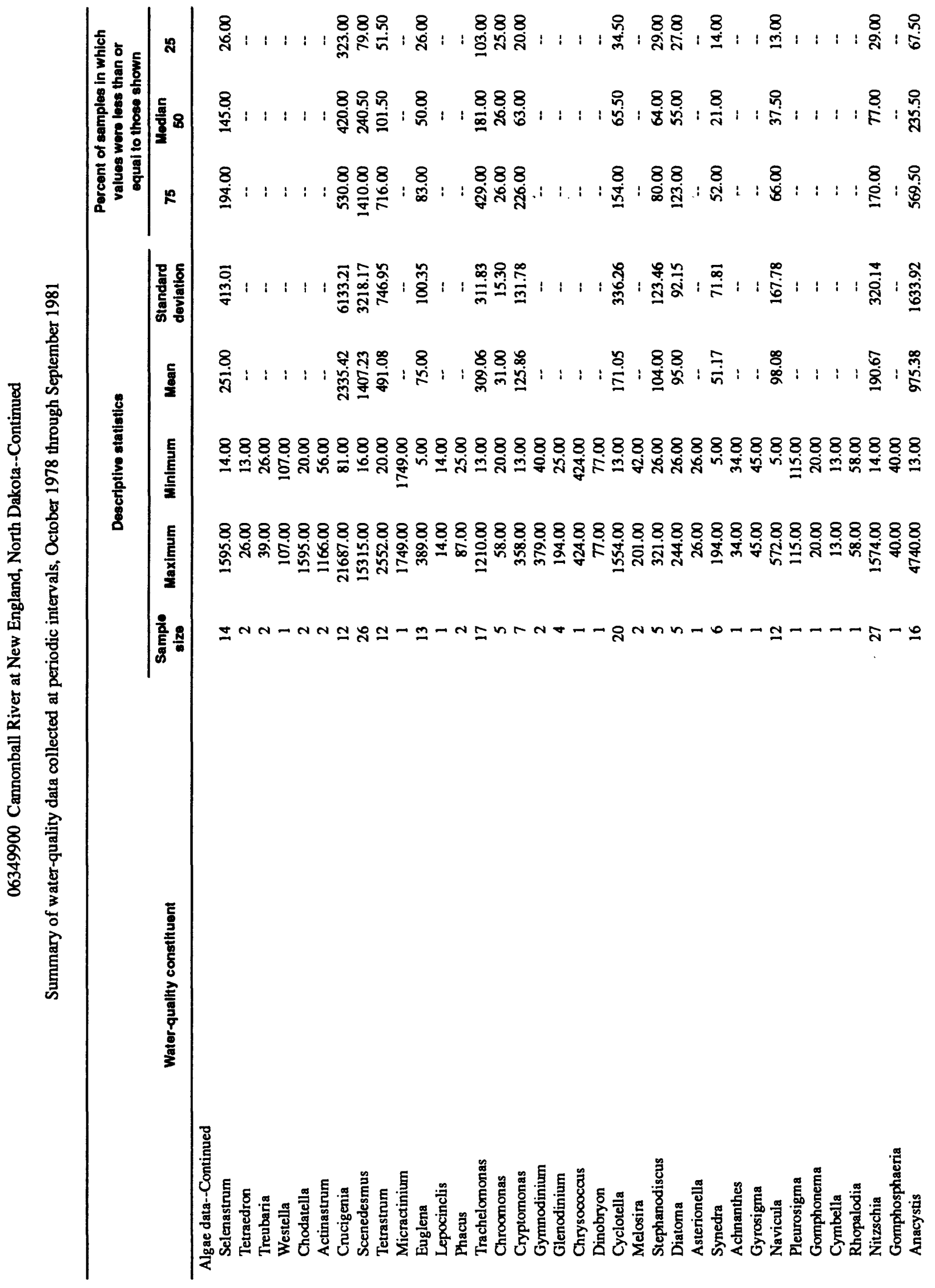



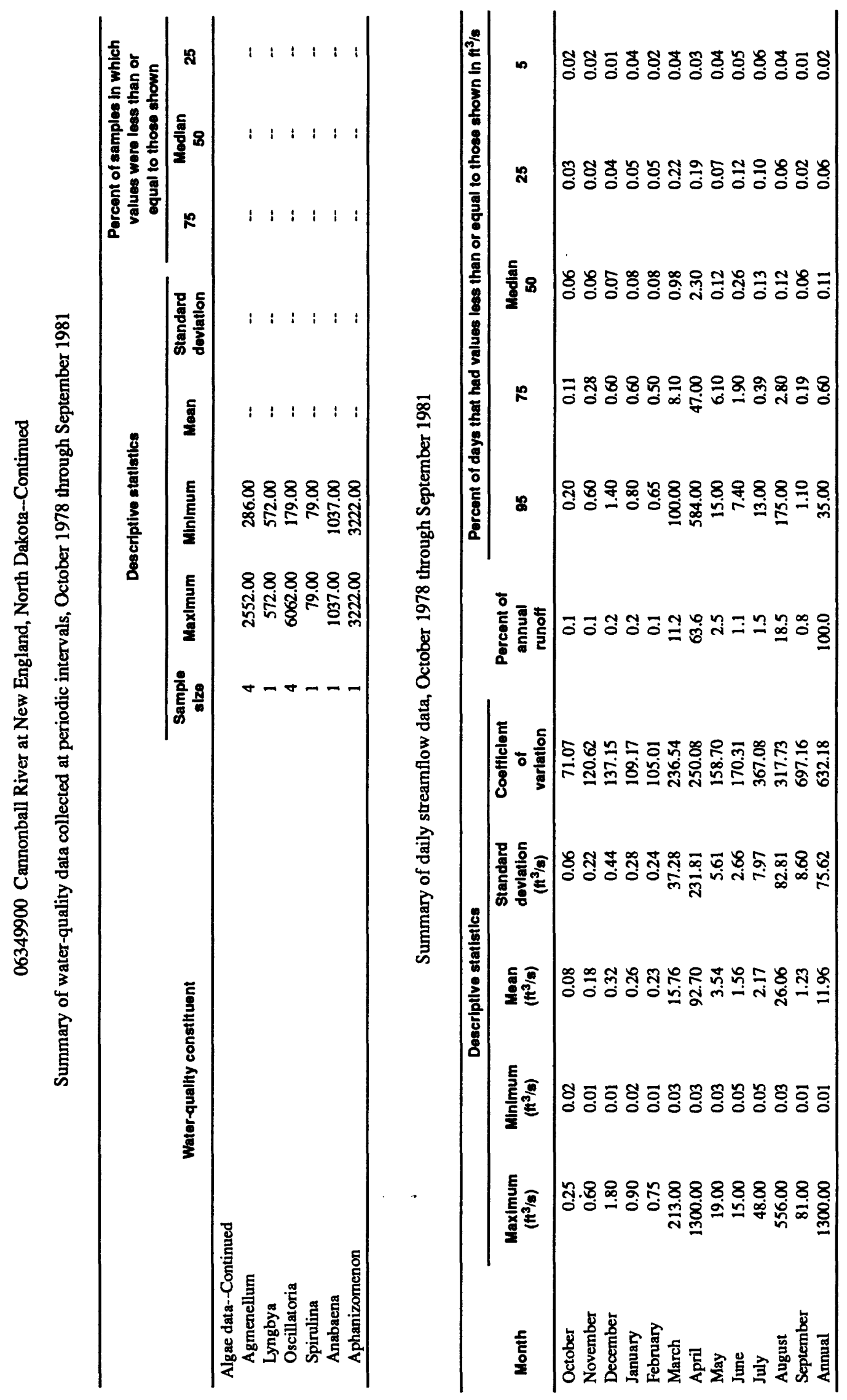


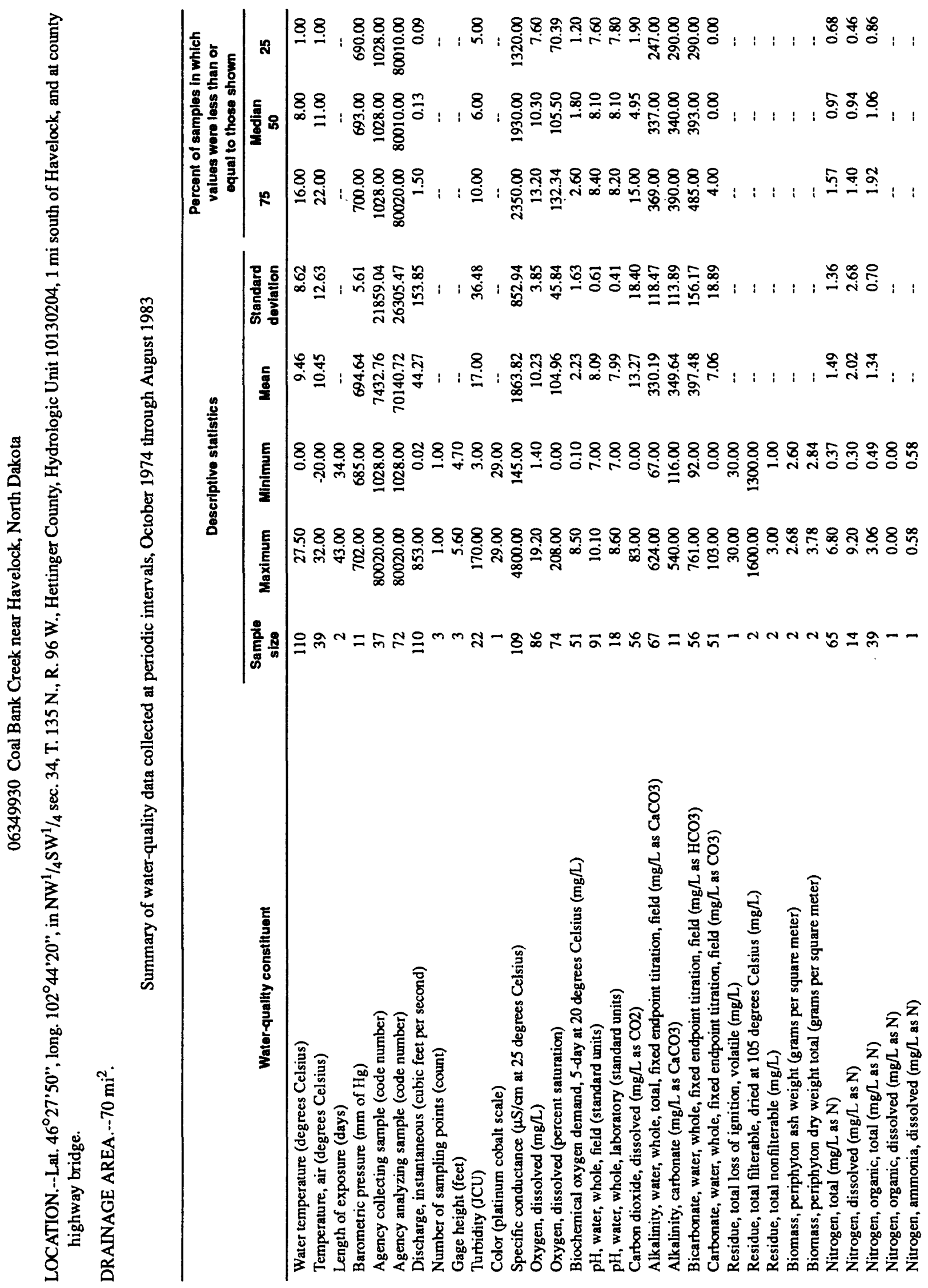




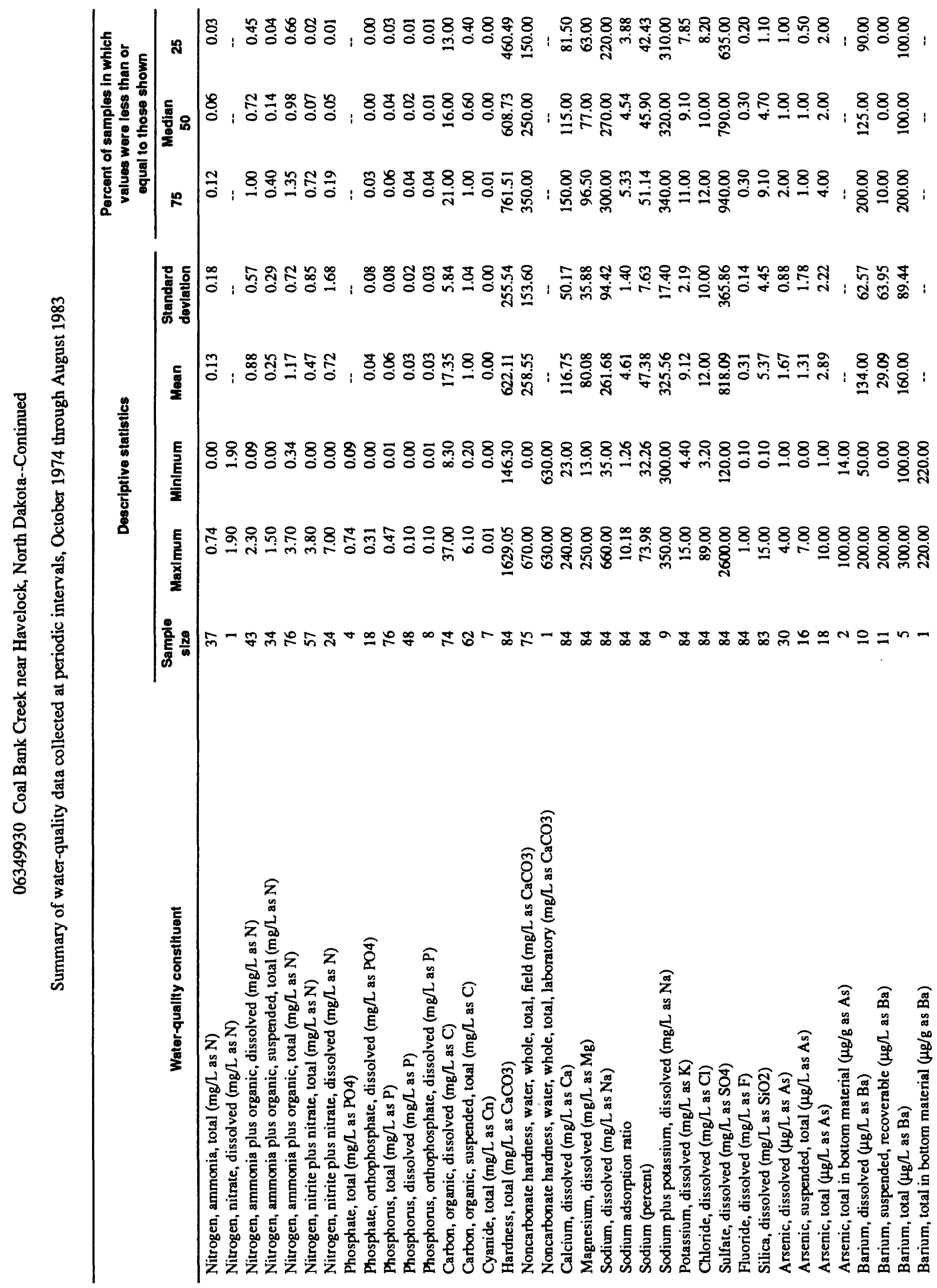




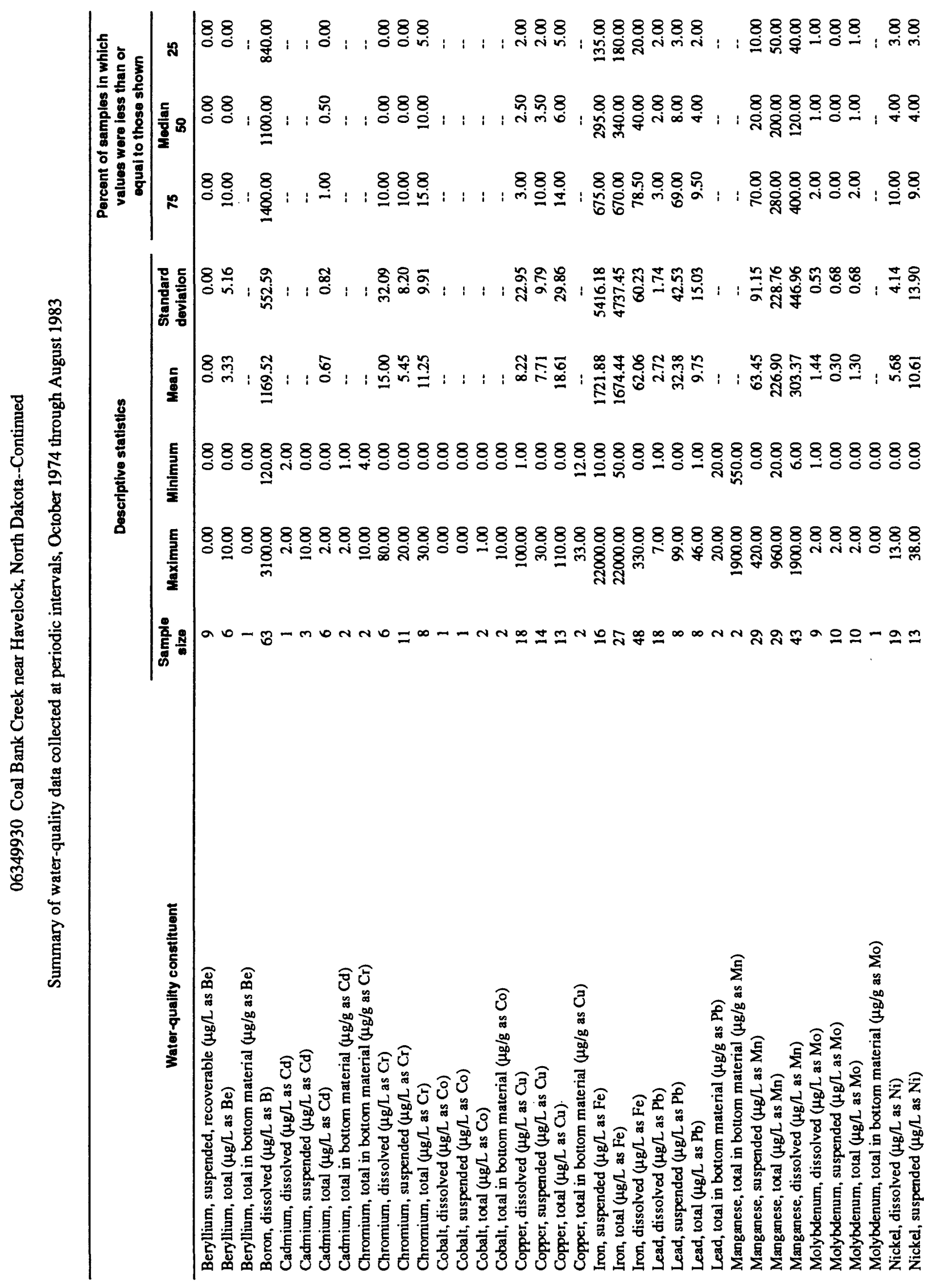




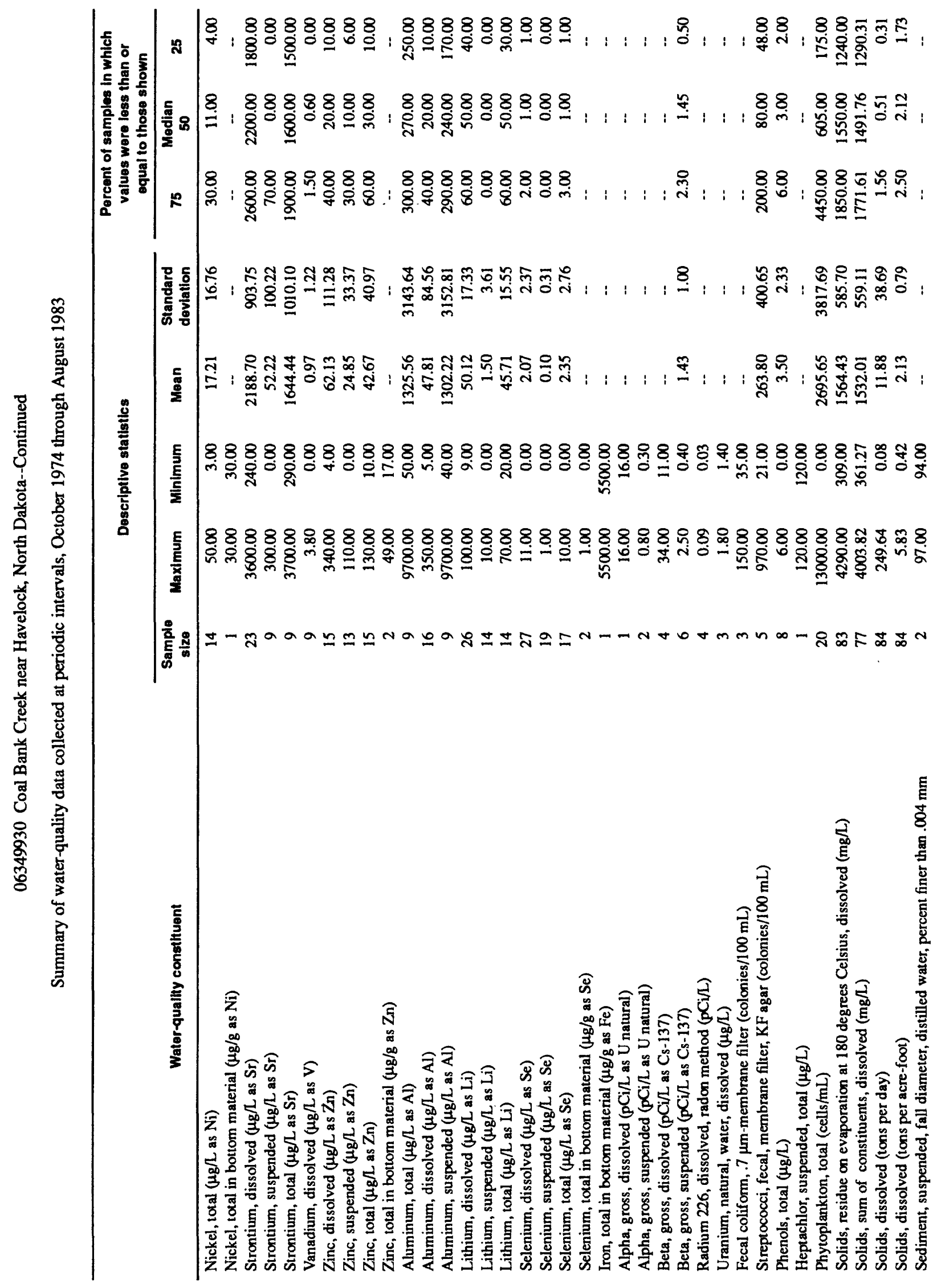




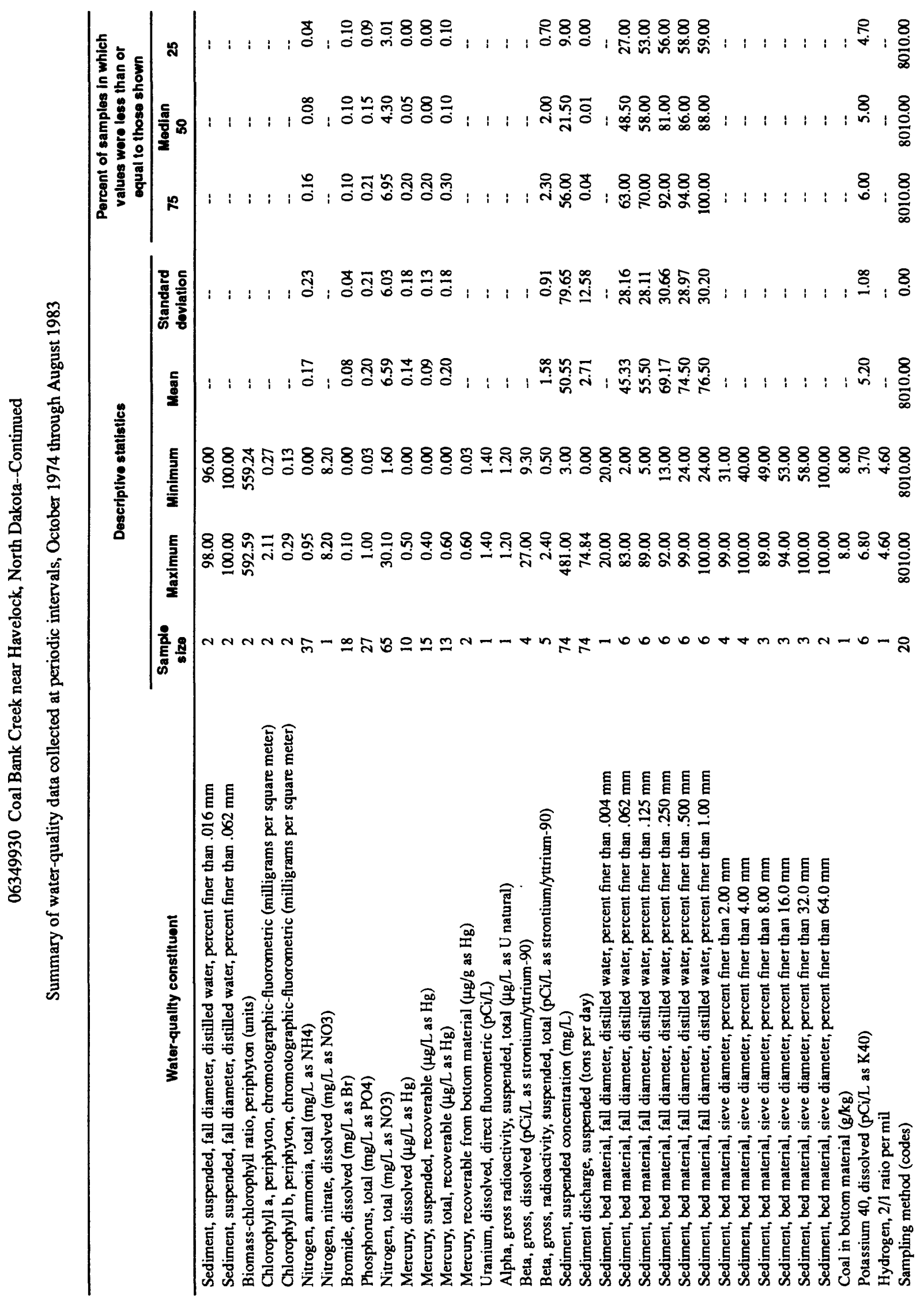




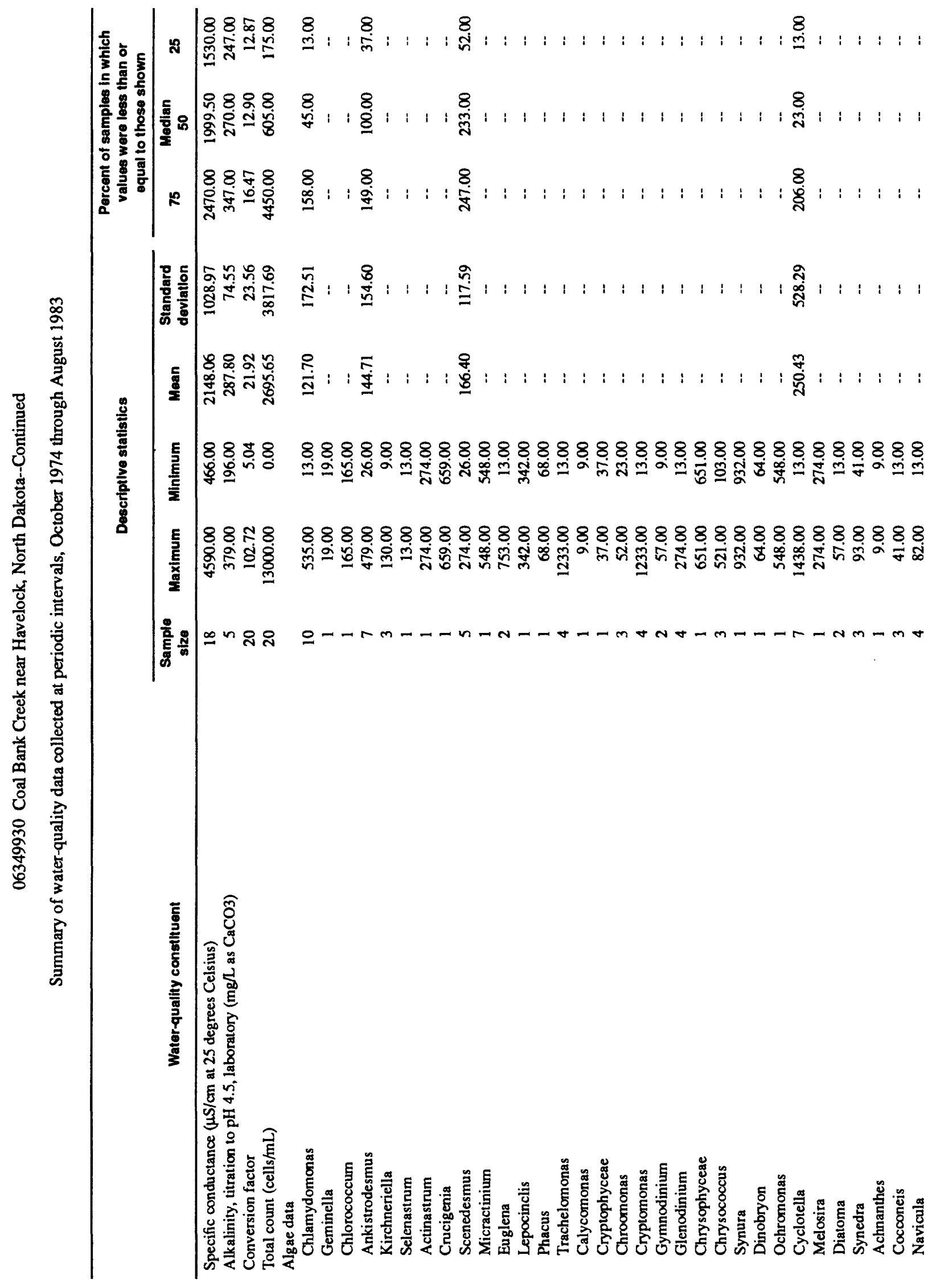




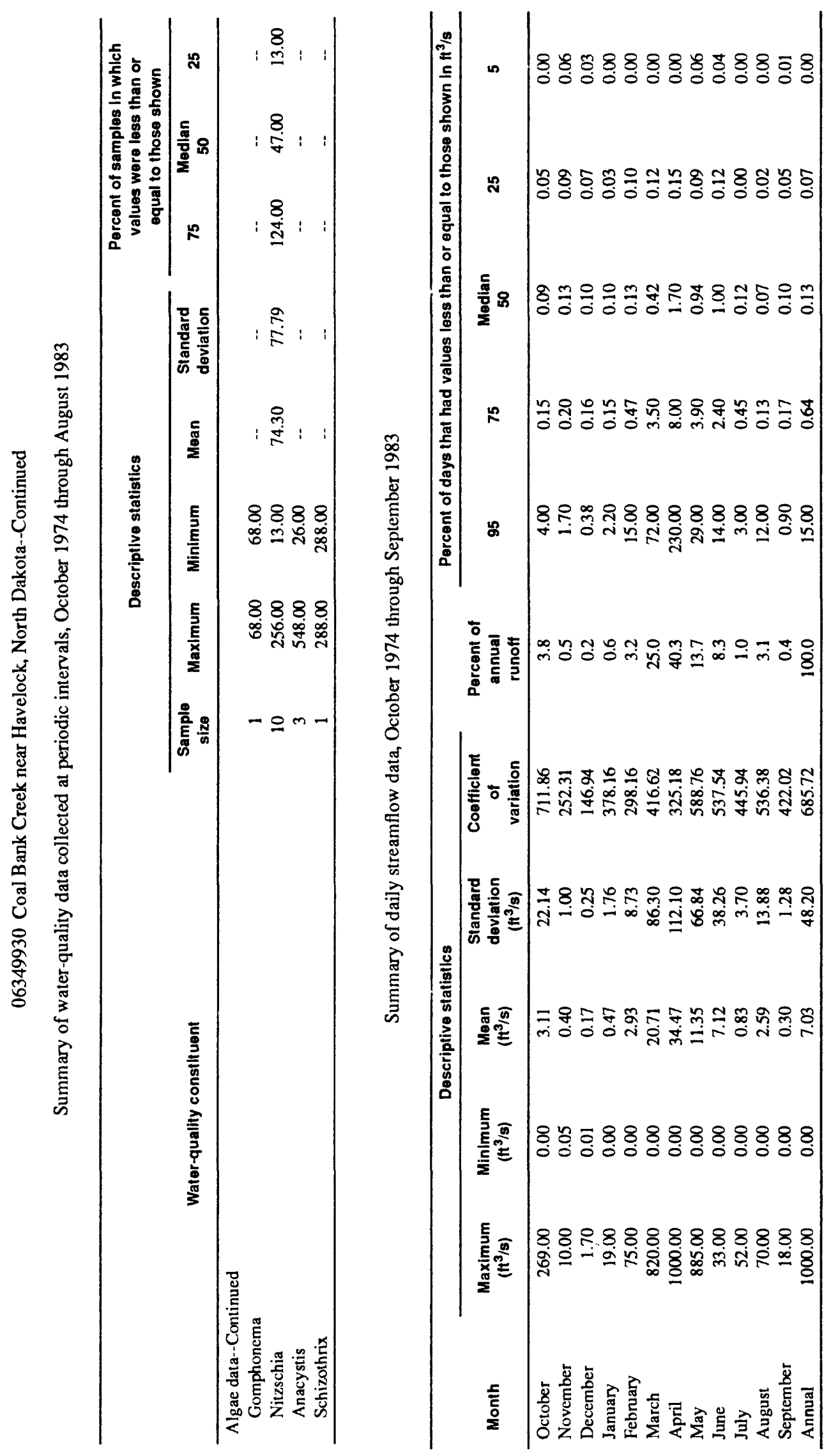




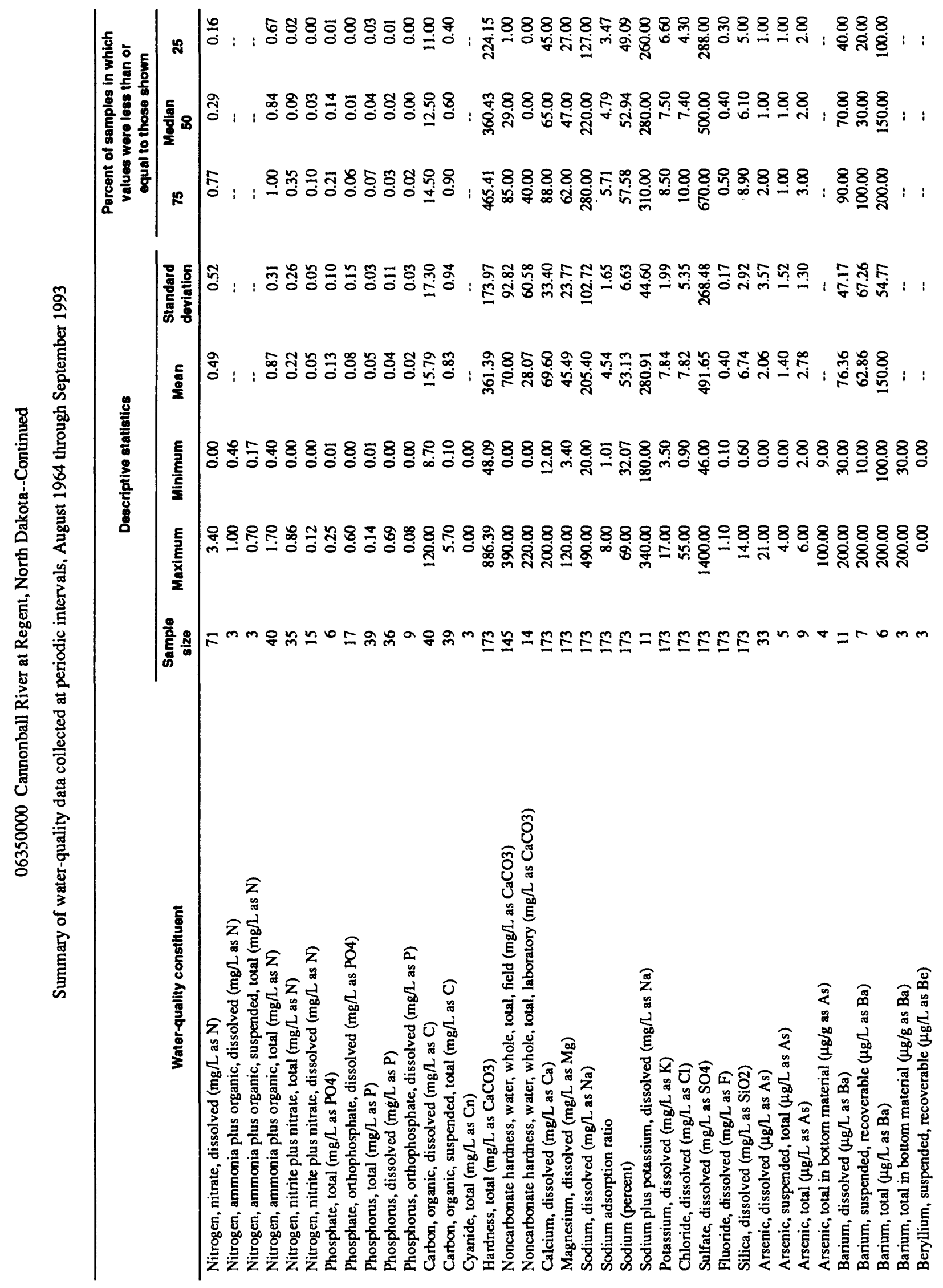




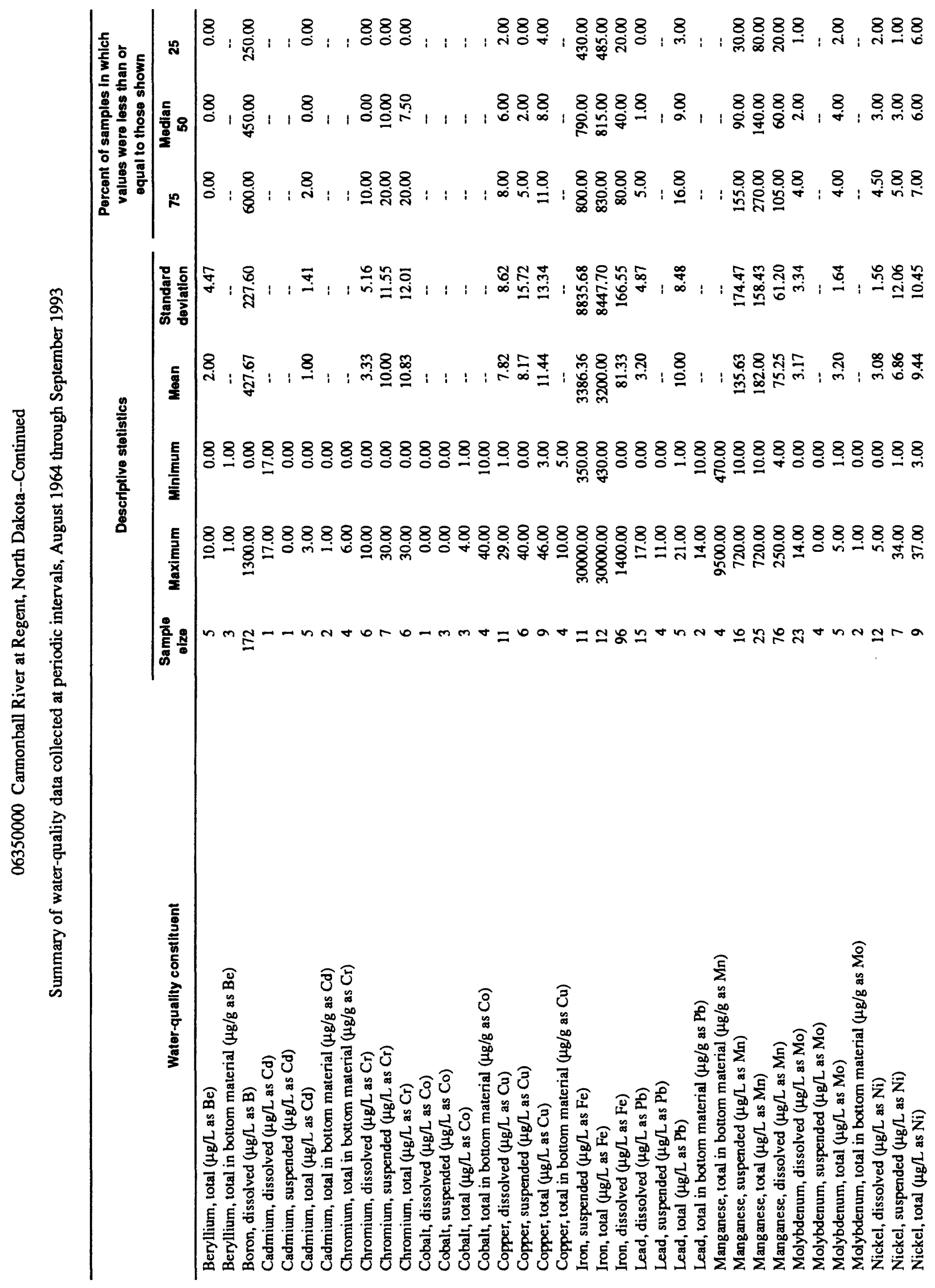




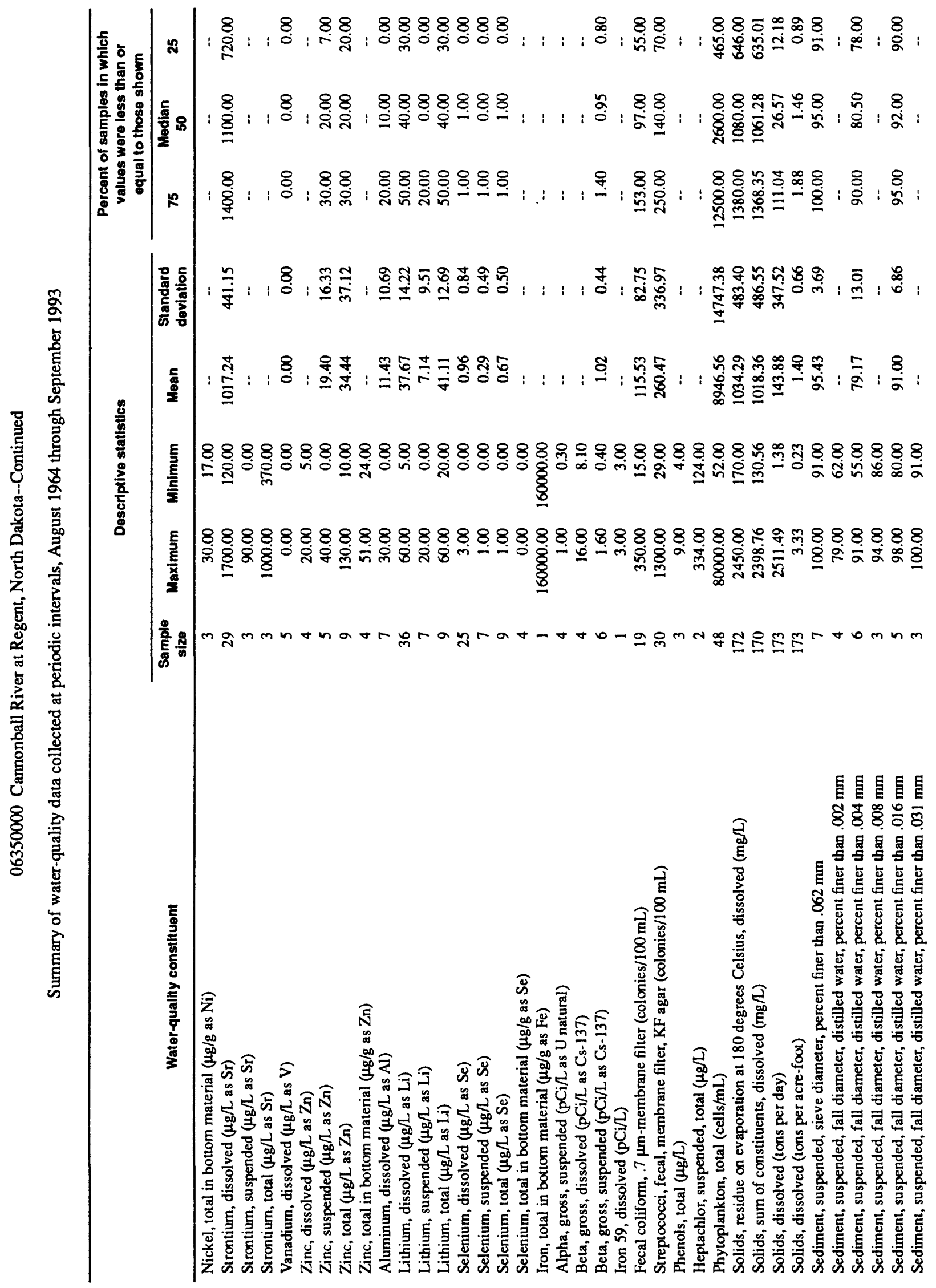




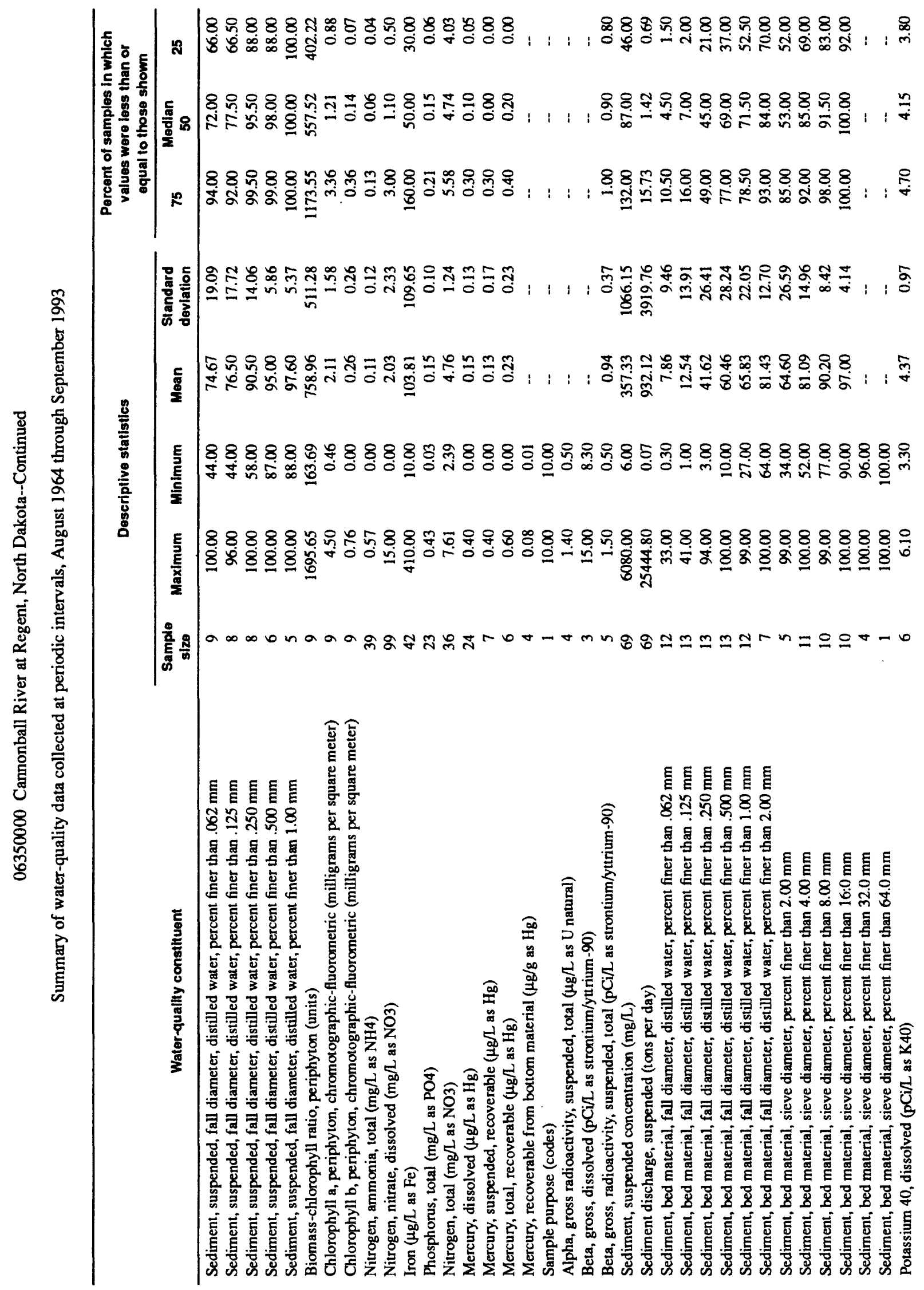




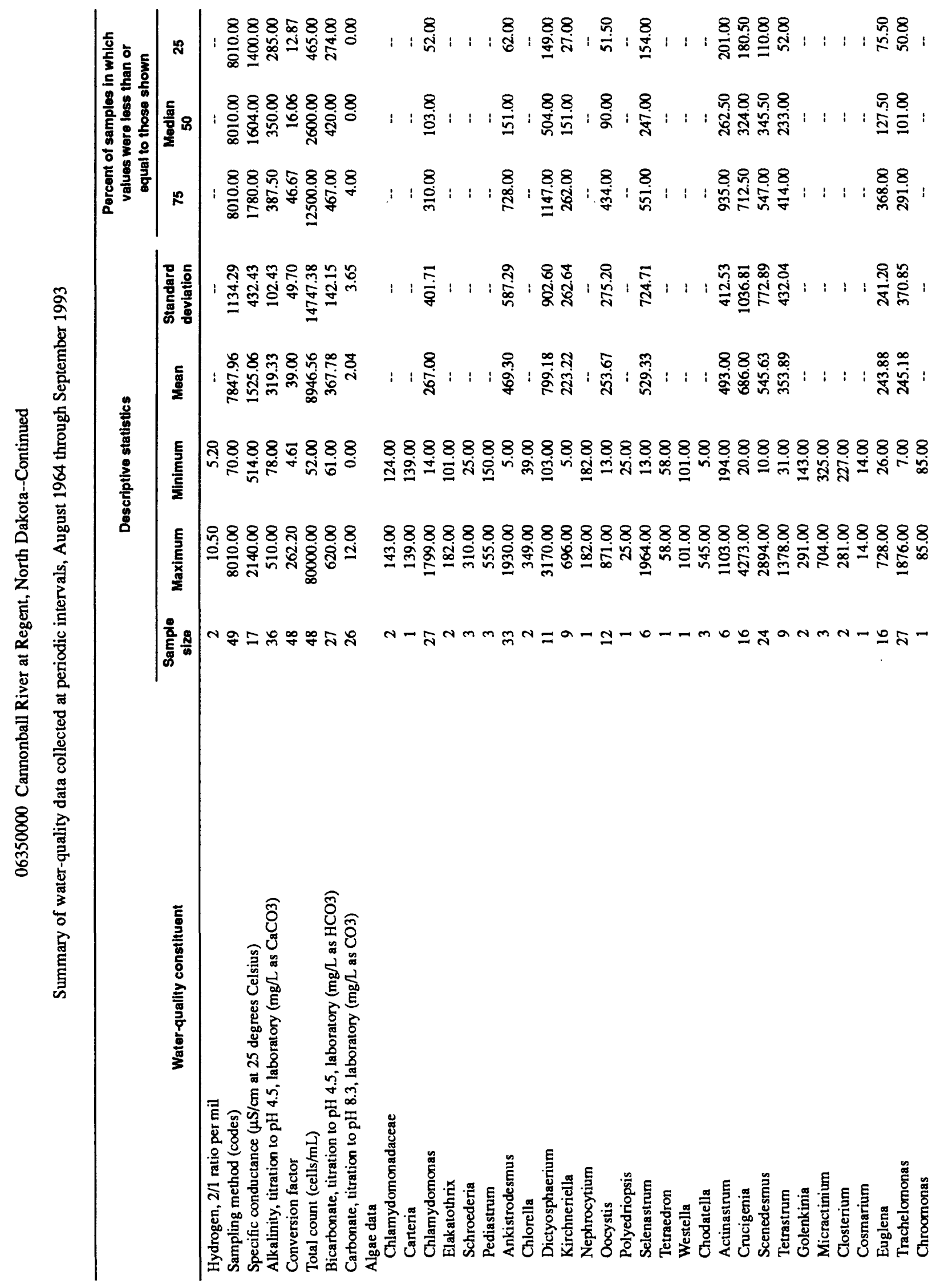




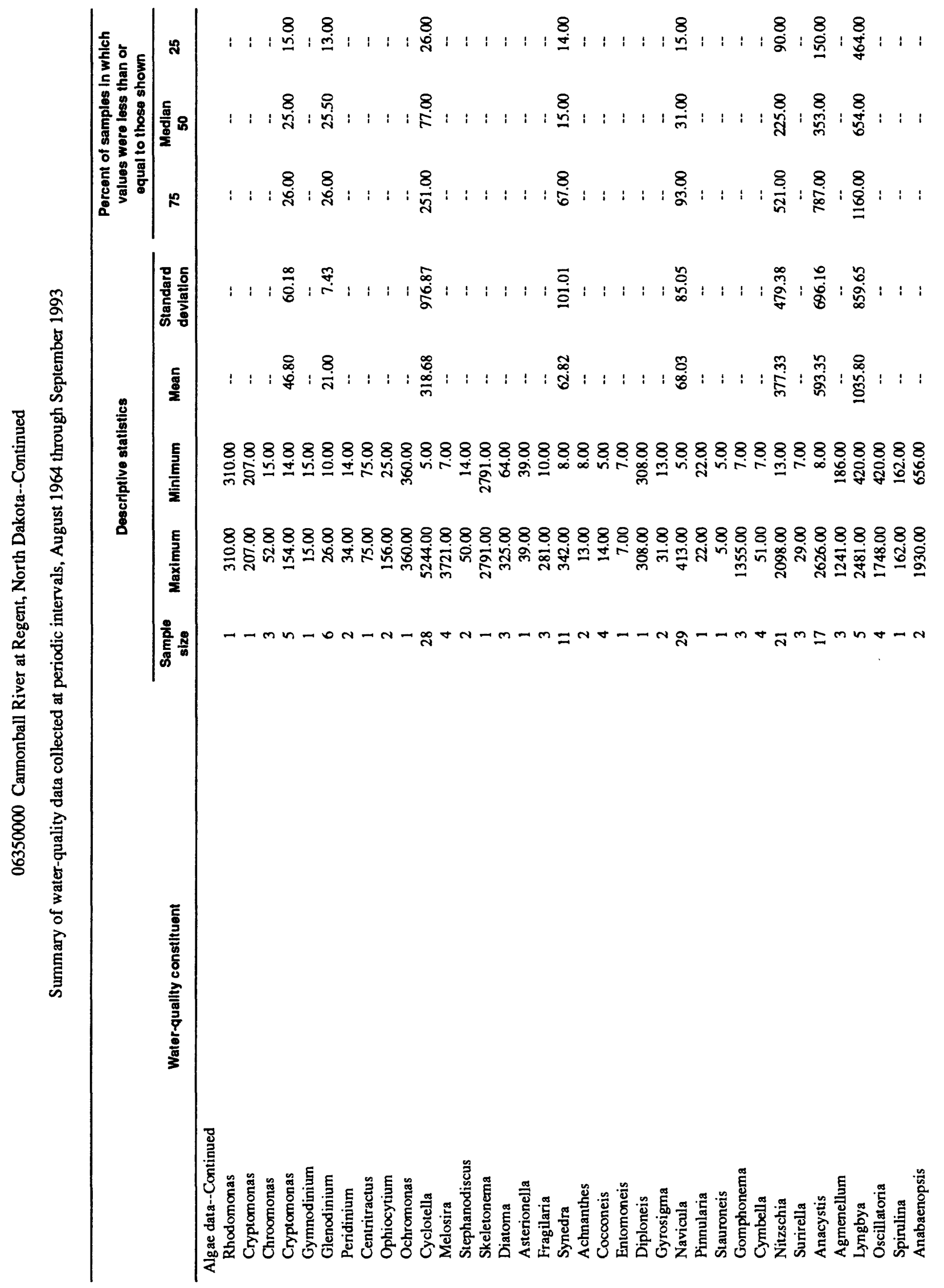




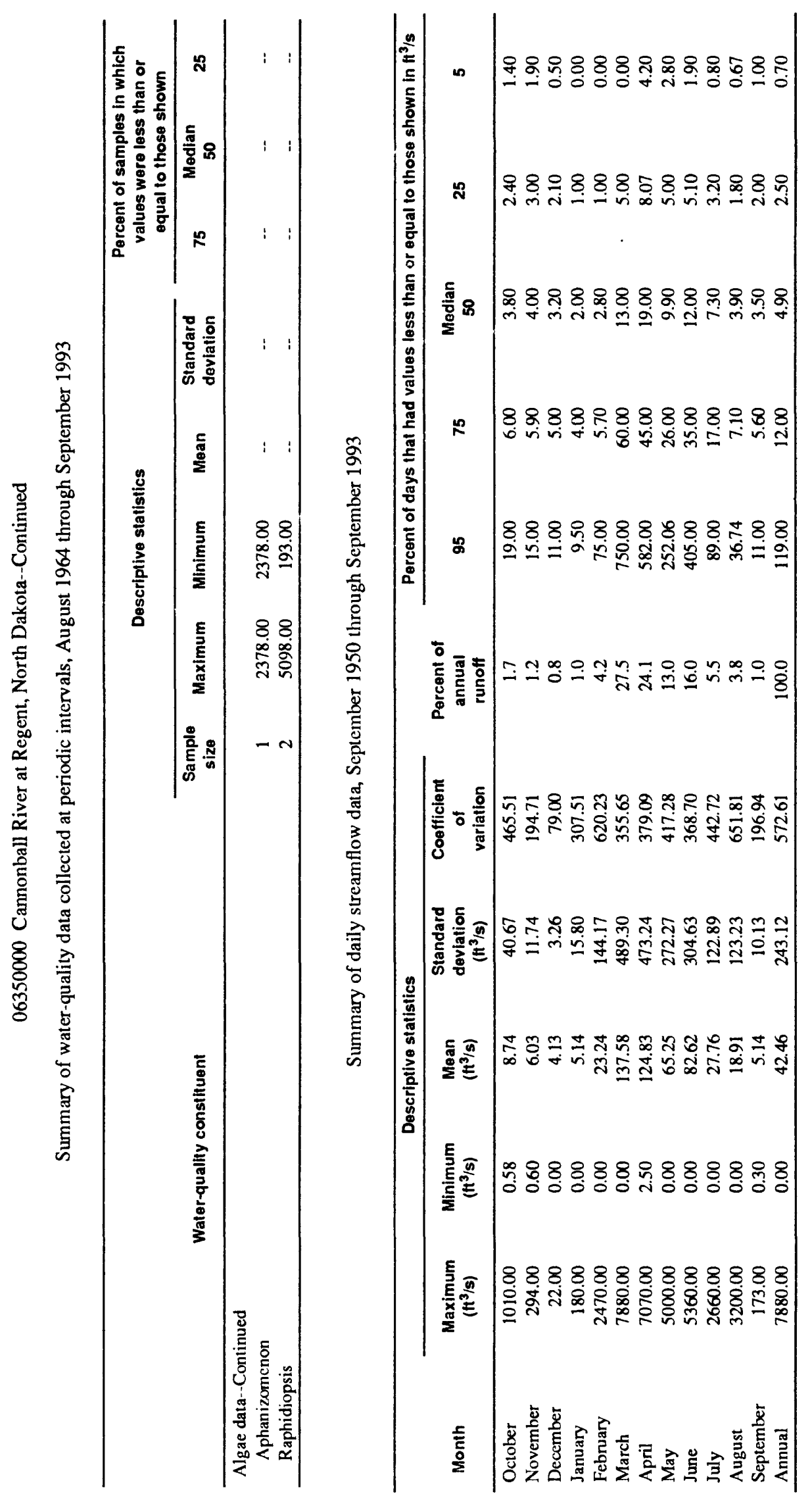




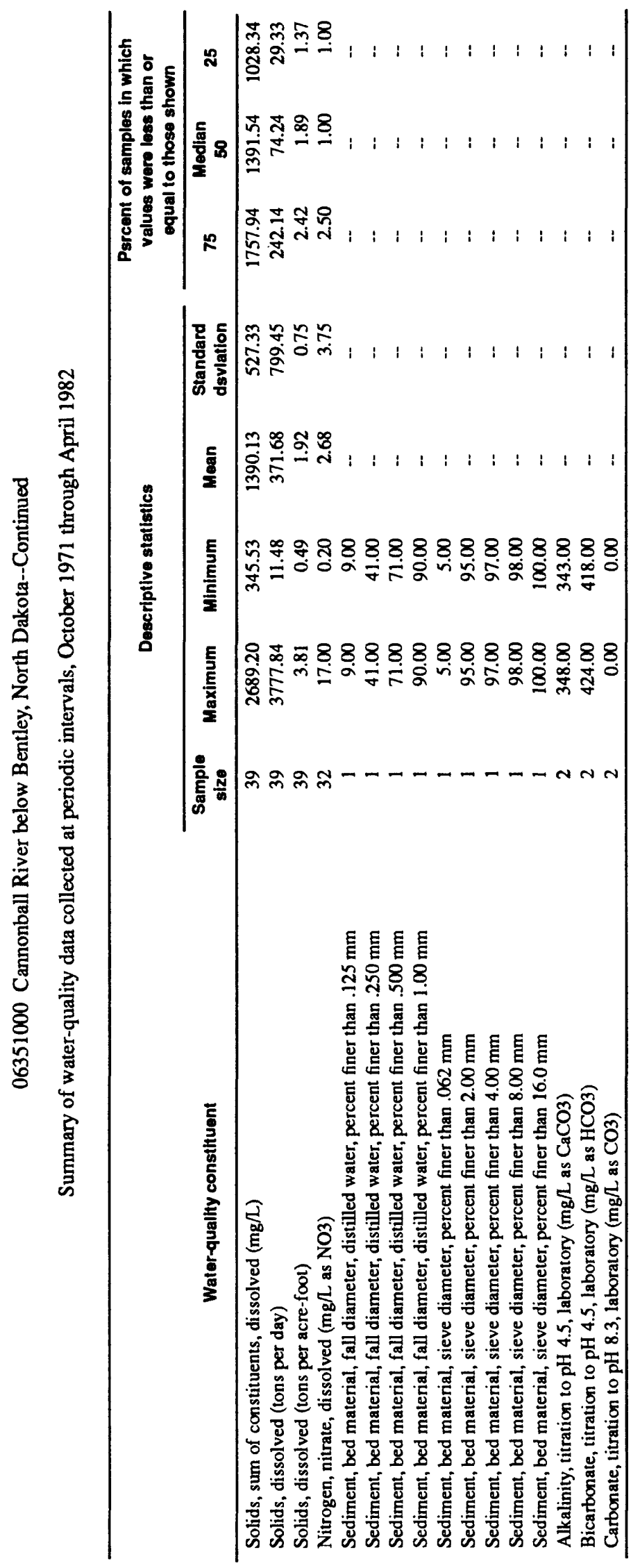




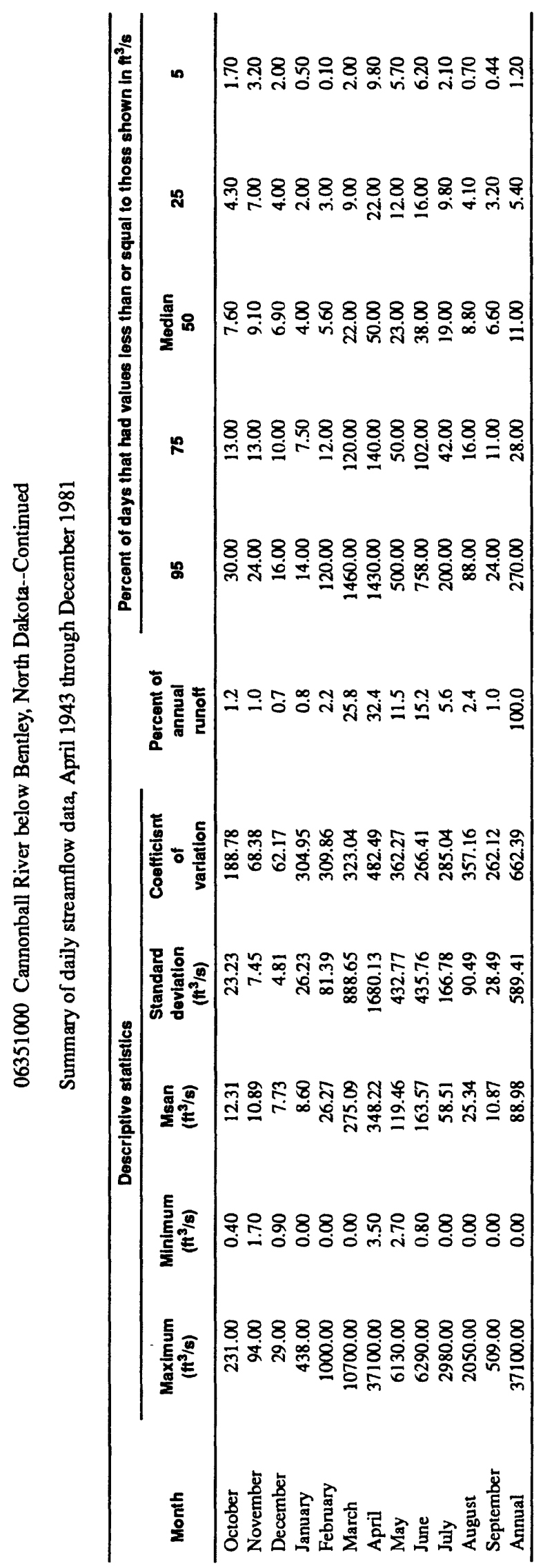




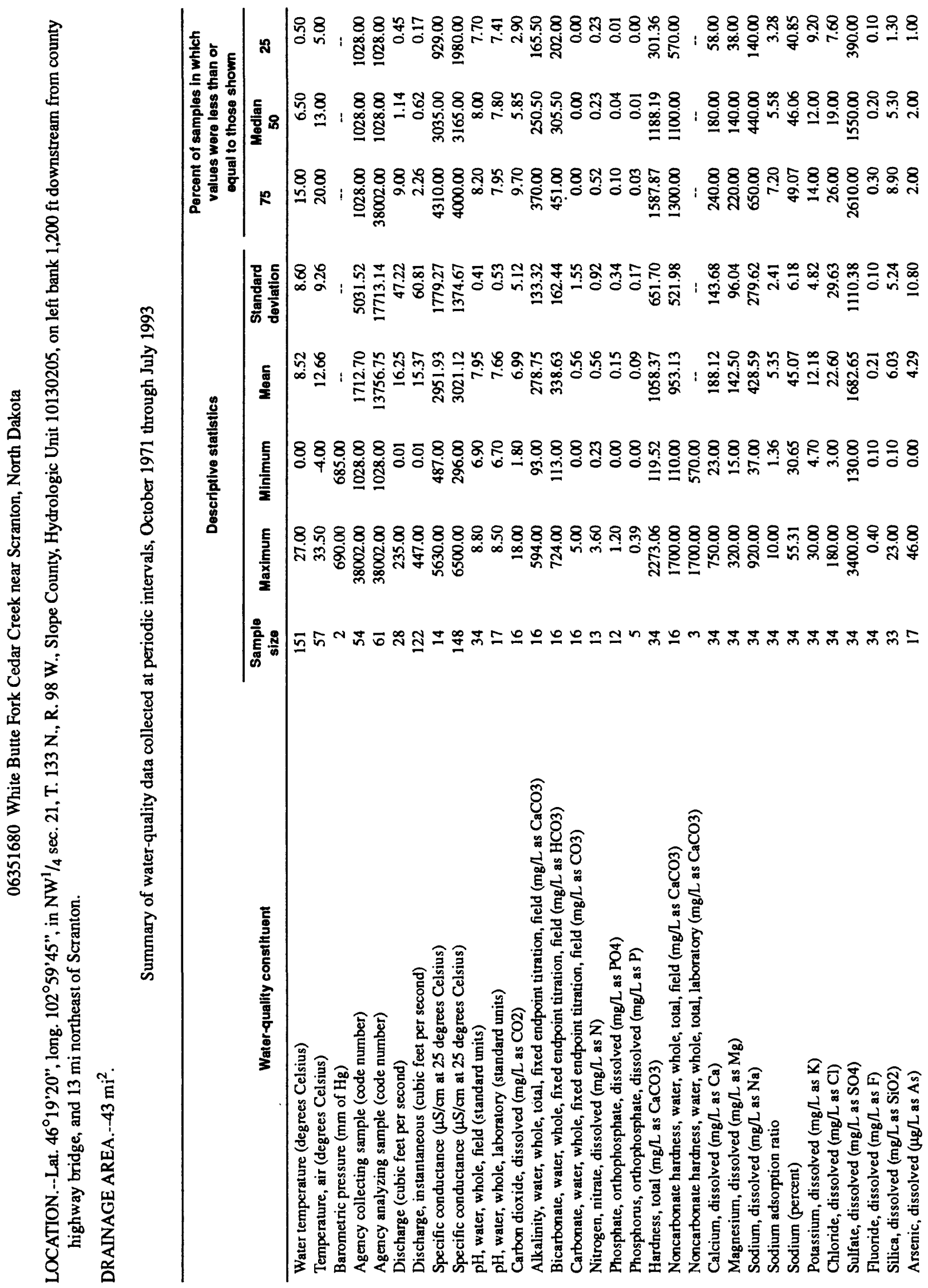




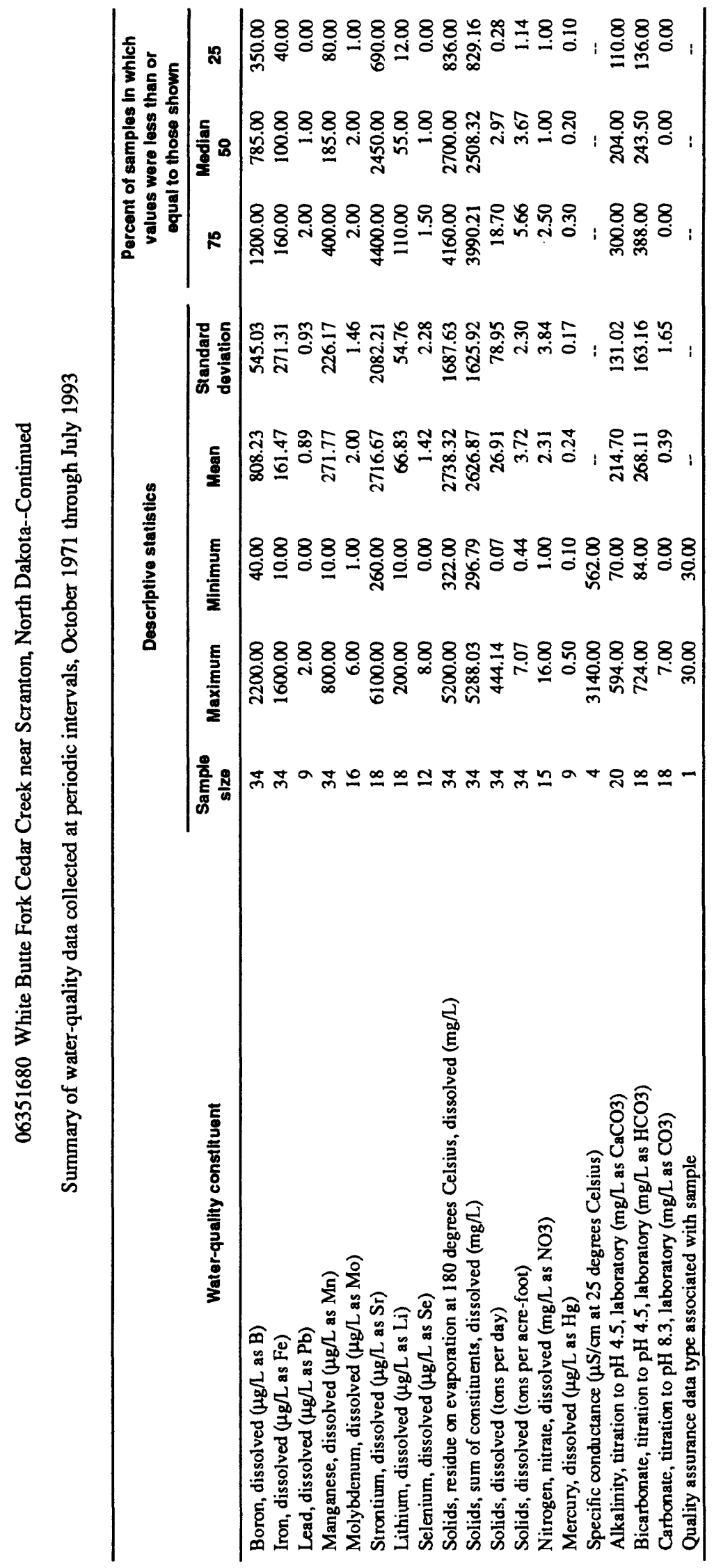




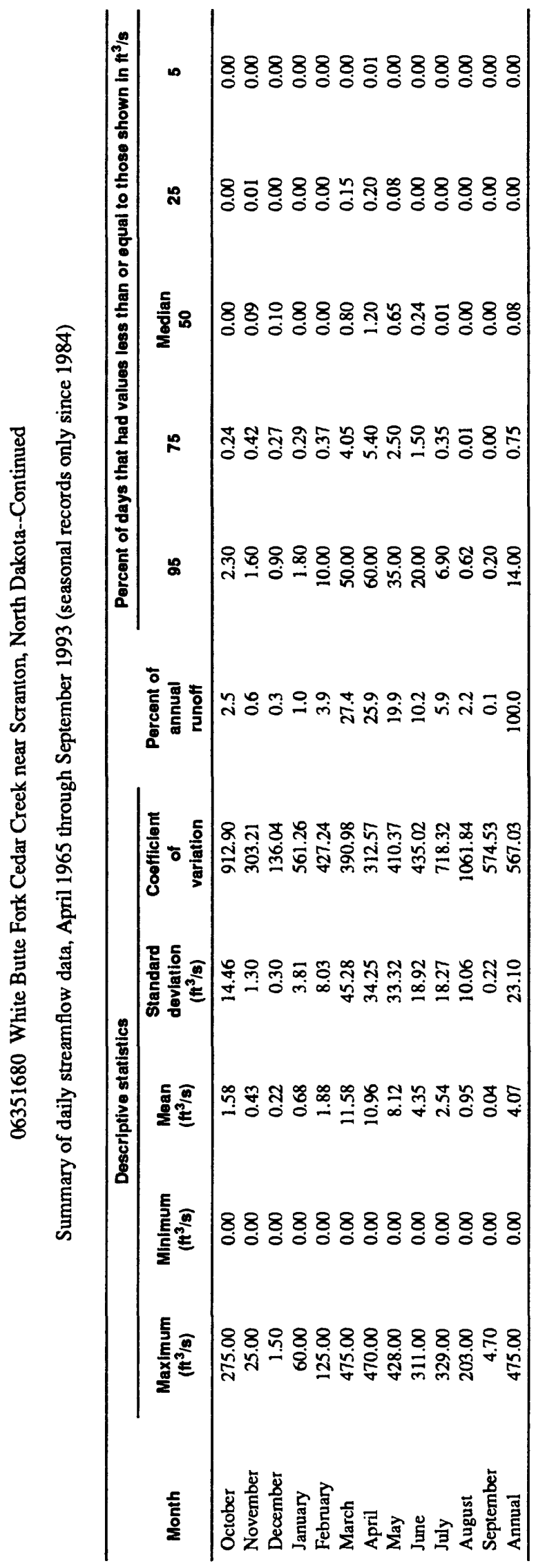




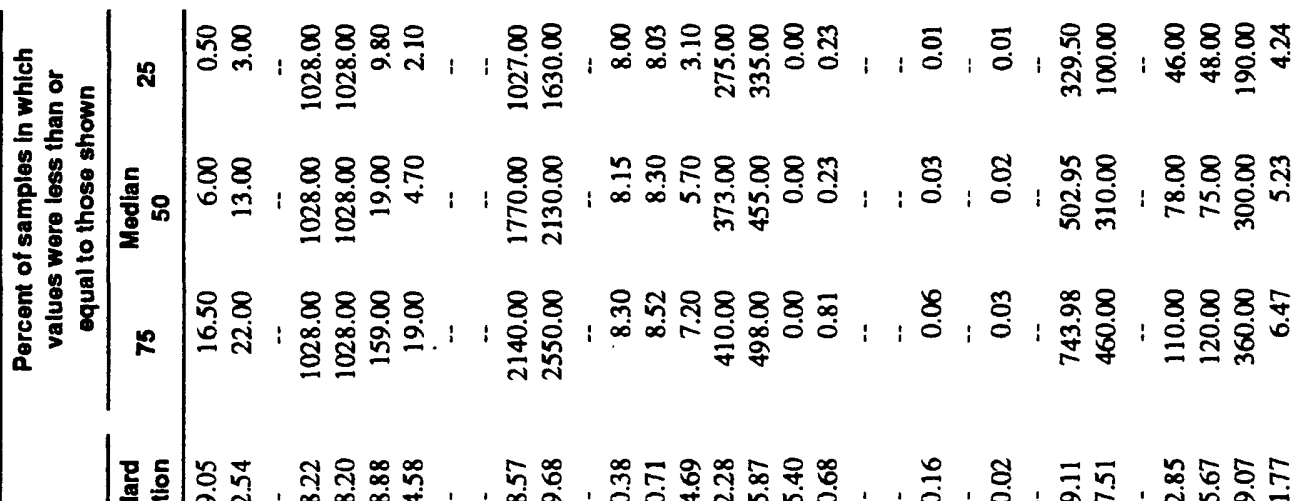

焉帝

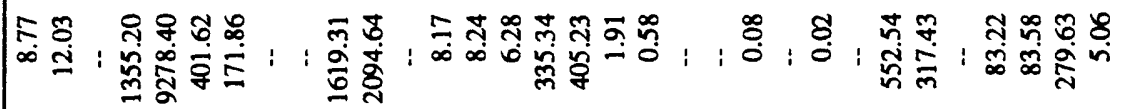

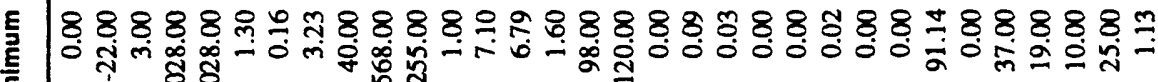

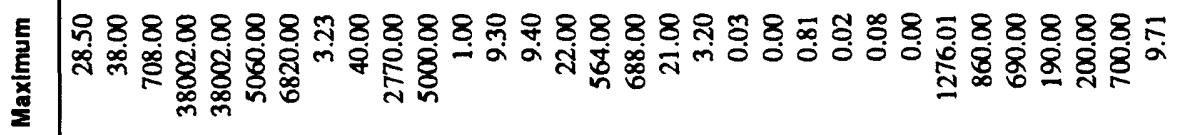

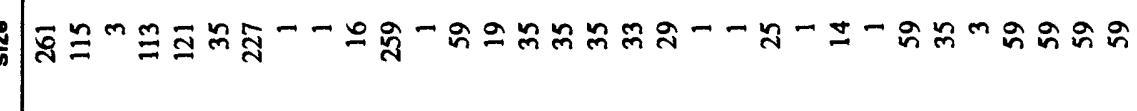




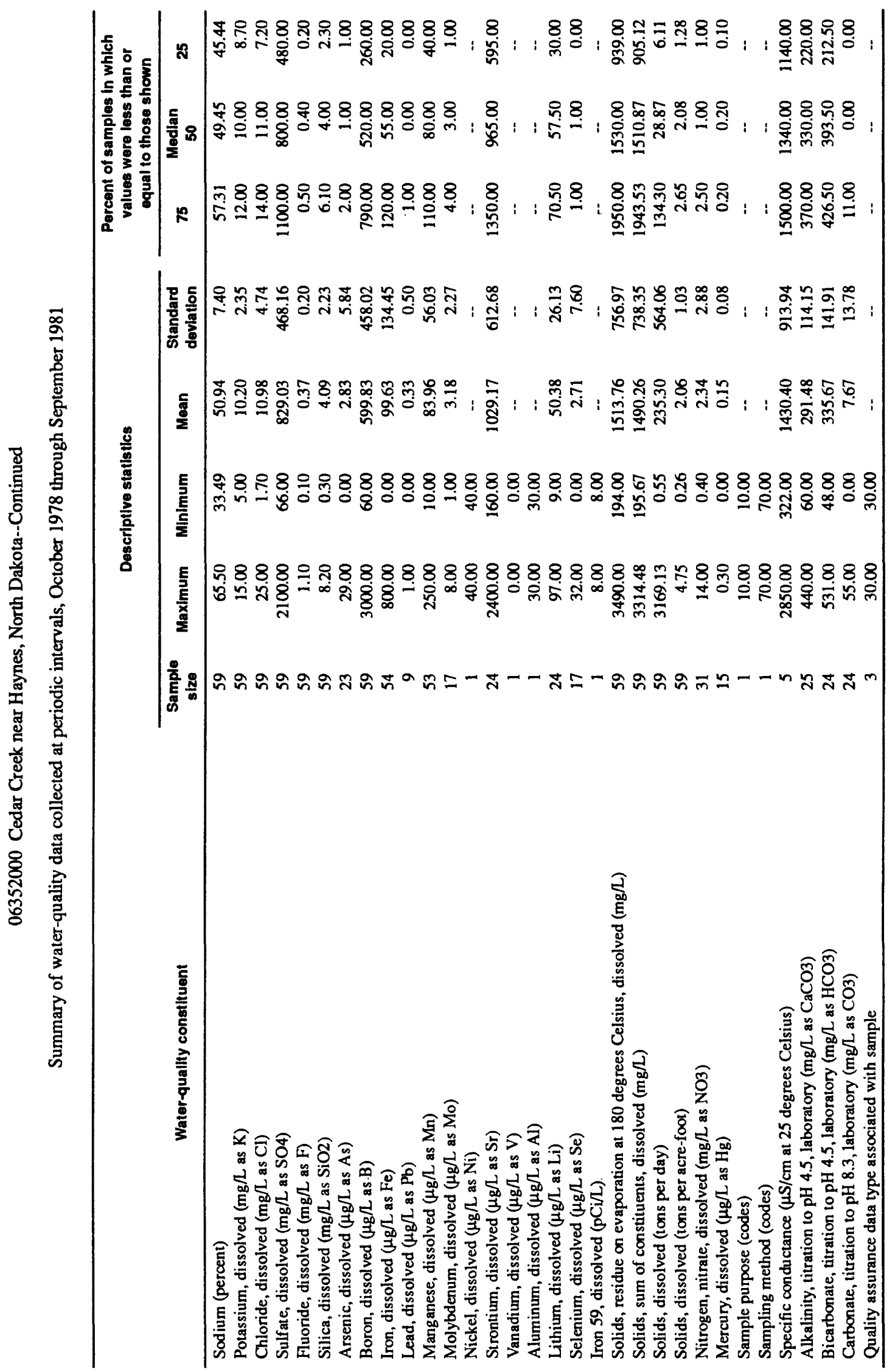




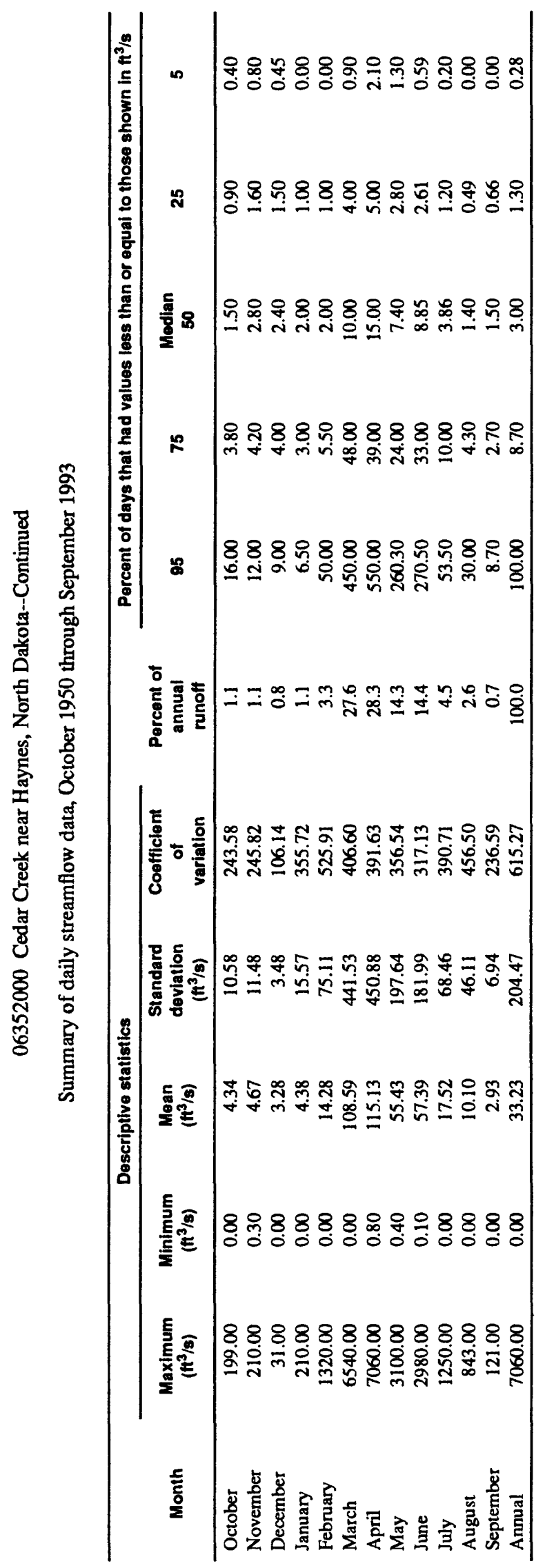




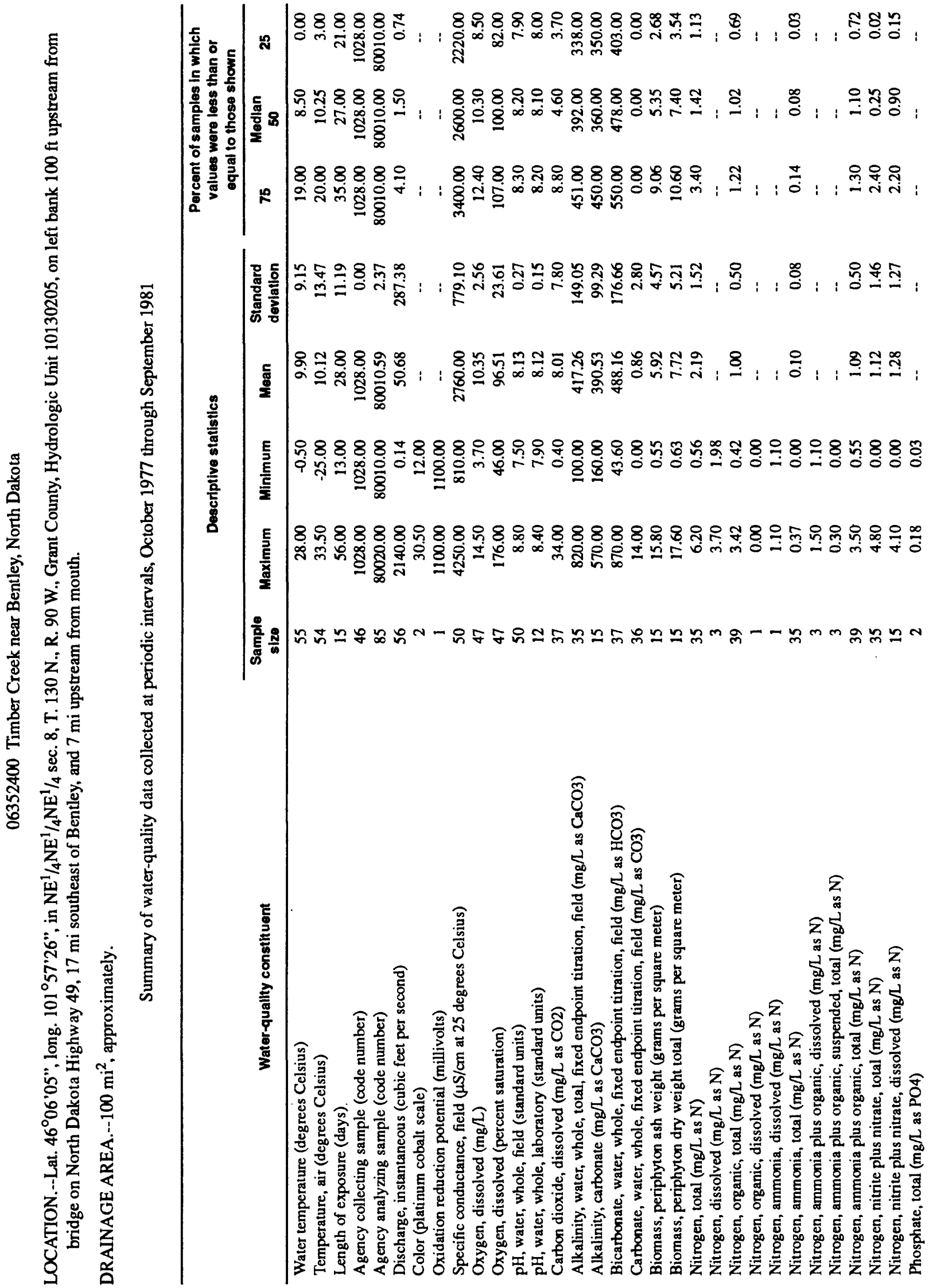




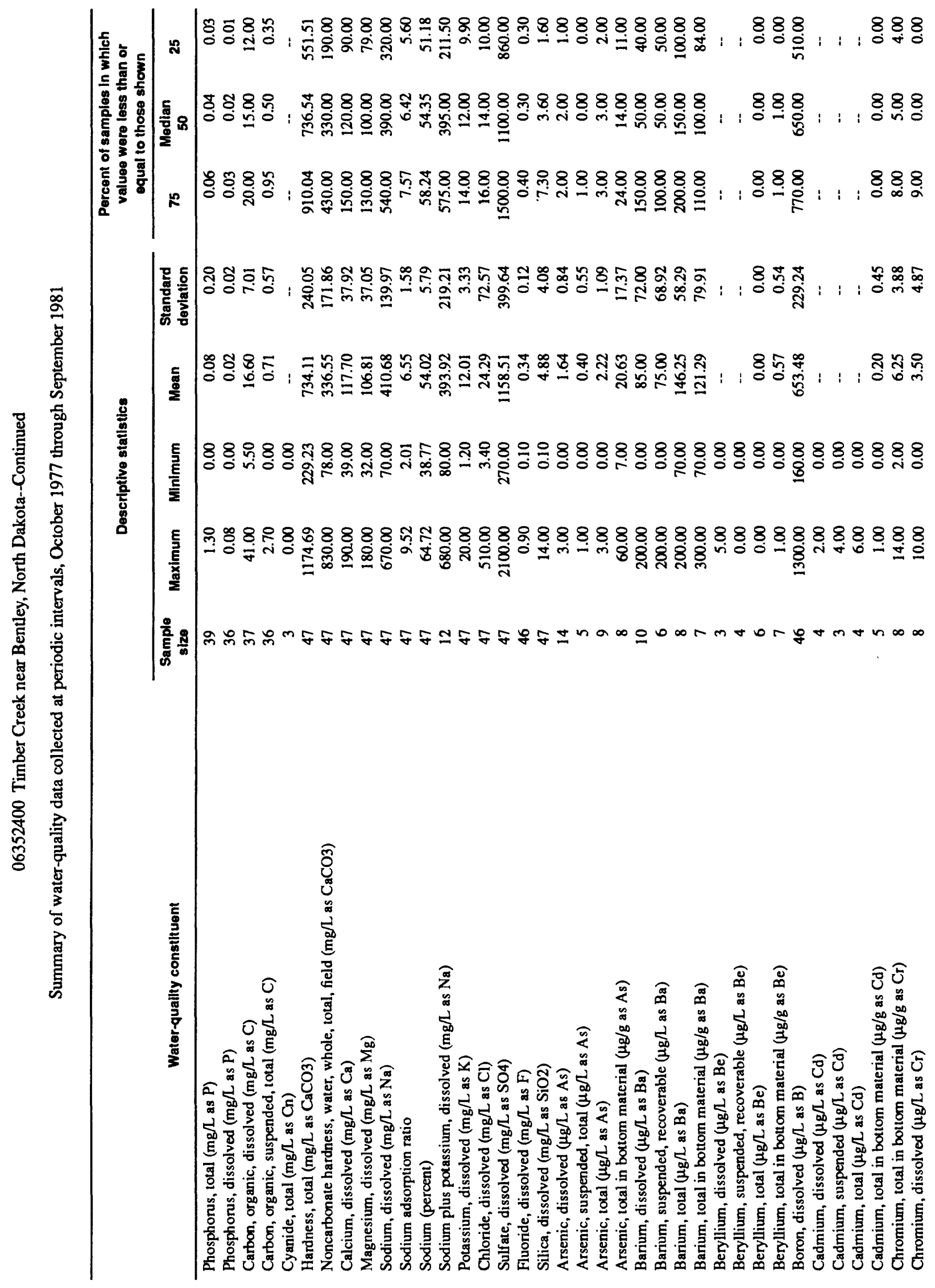




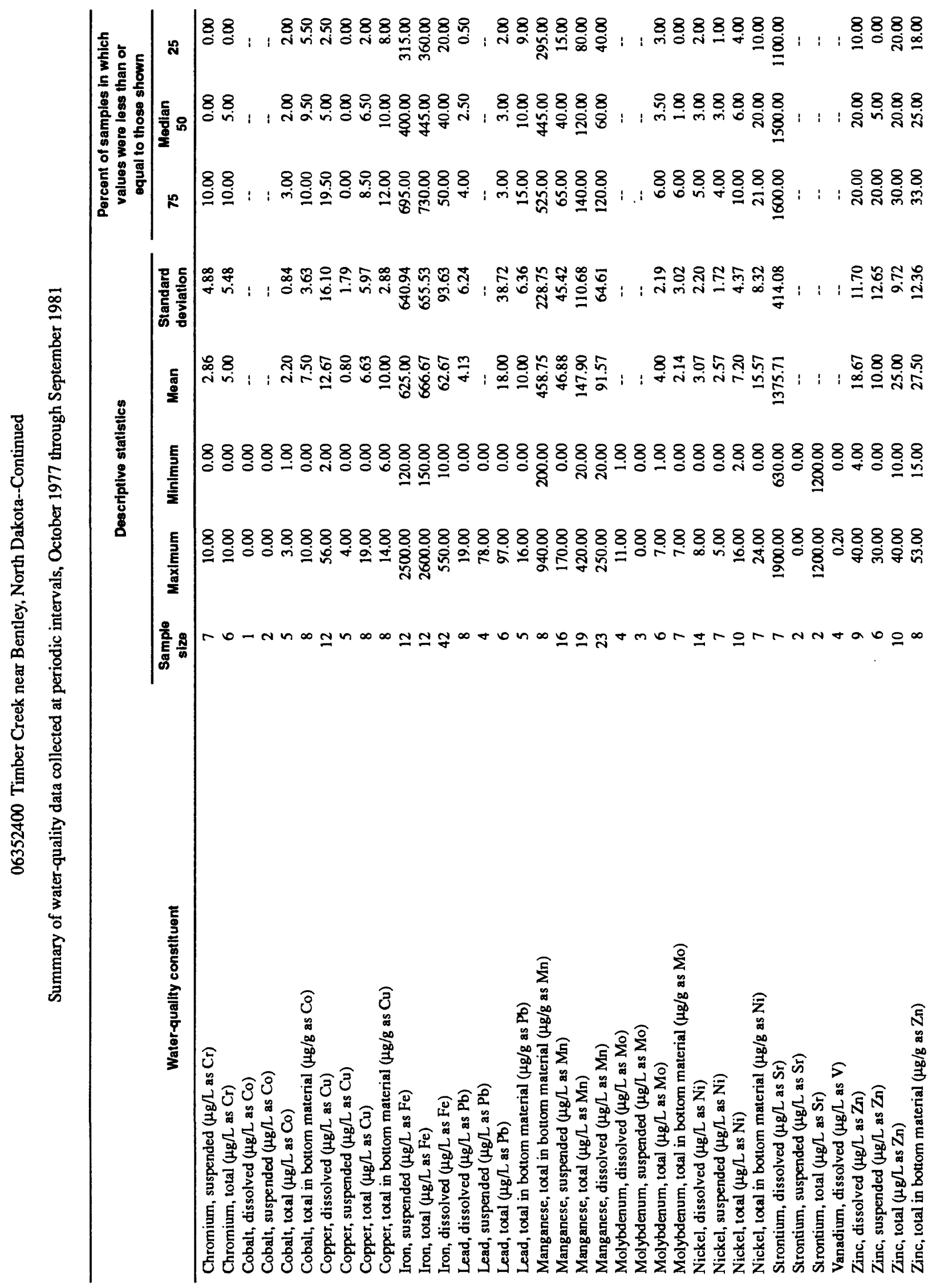




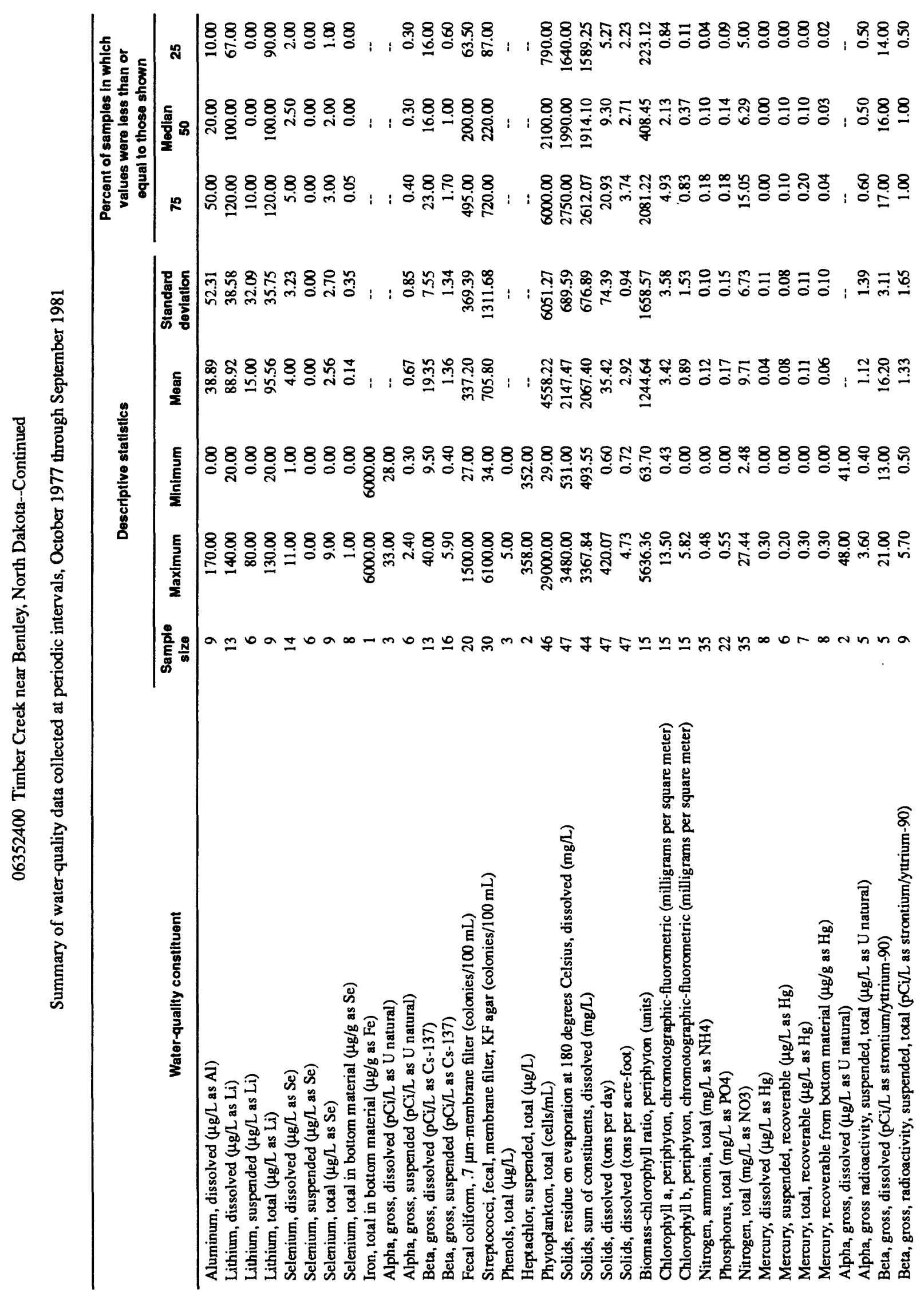




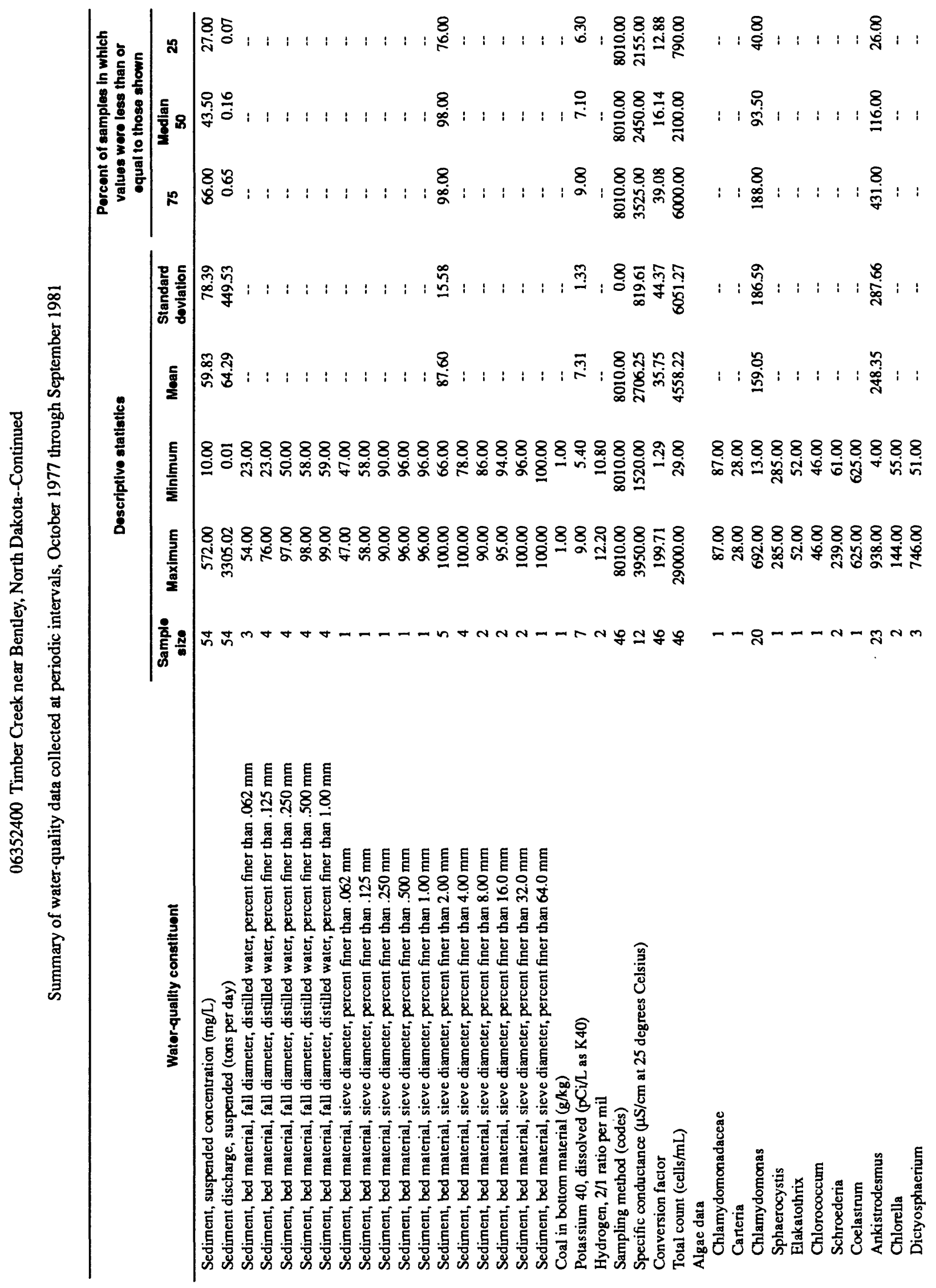




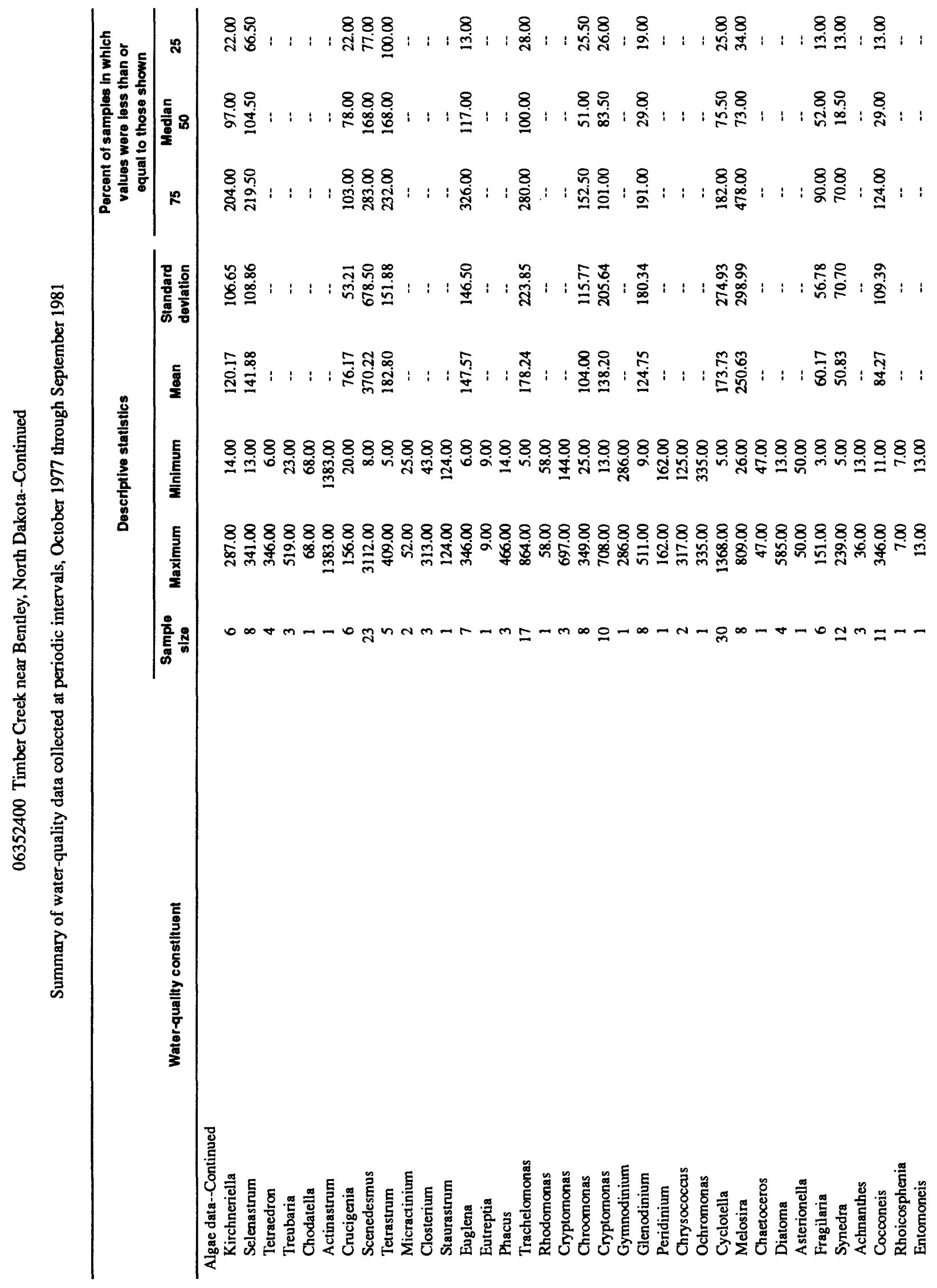



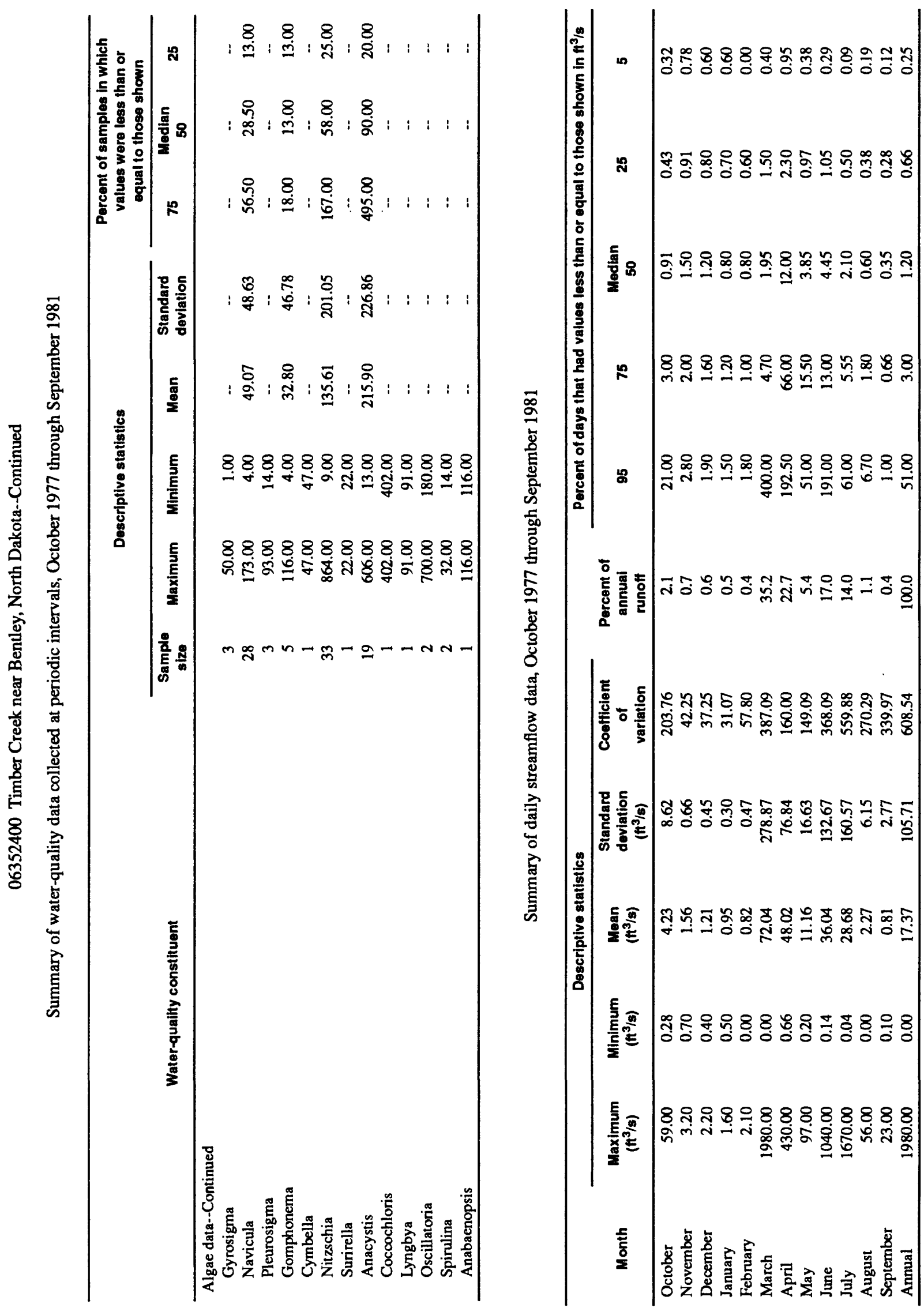


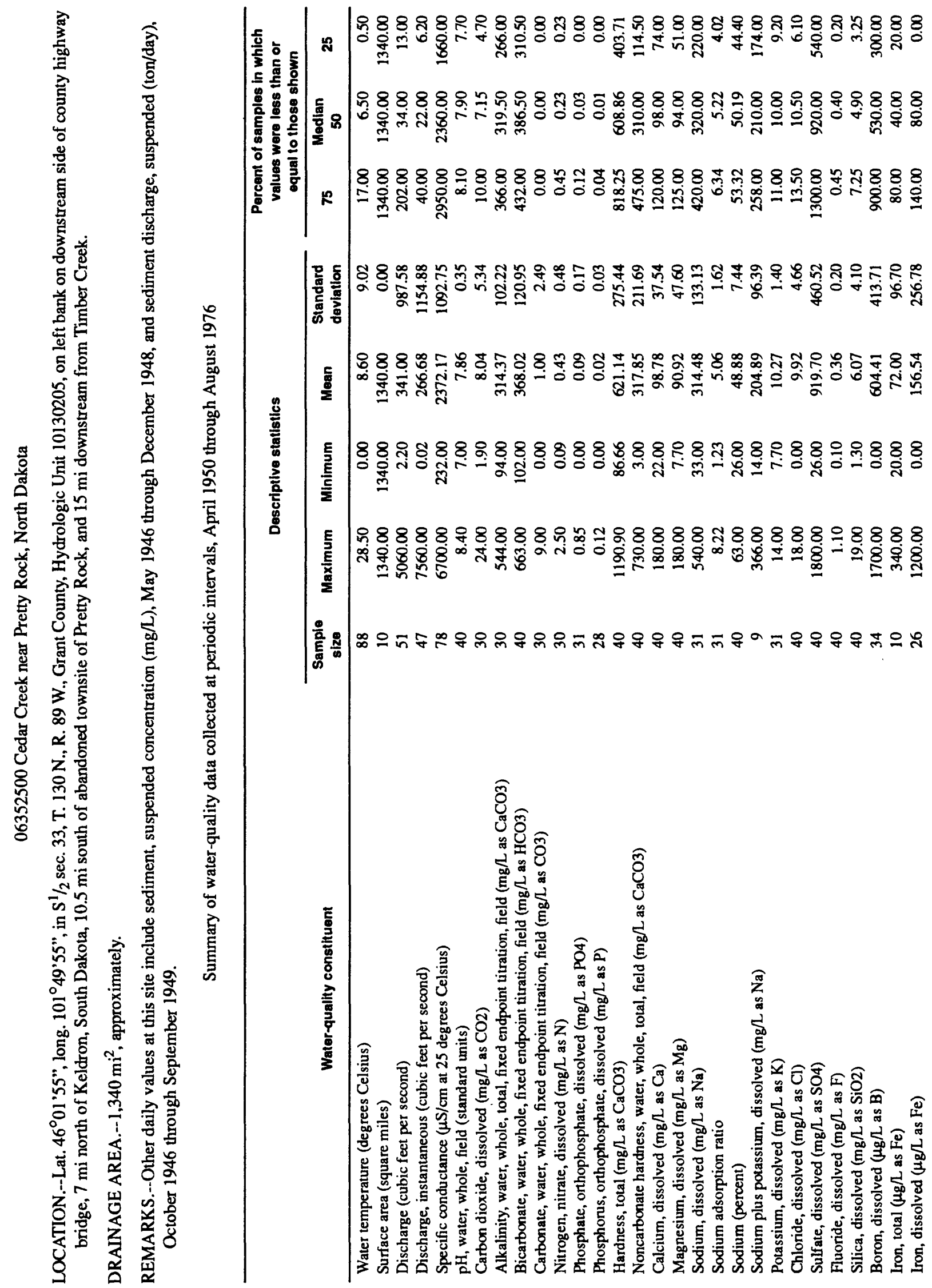



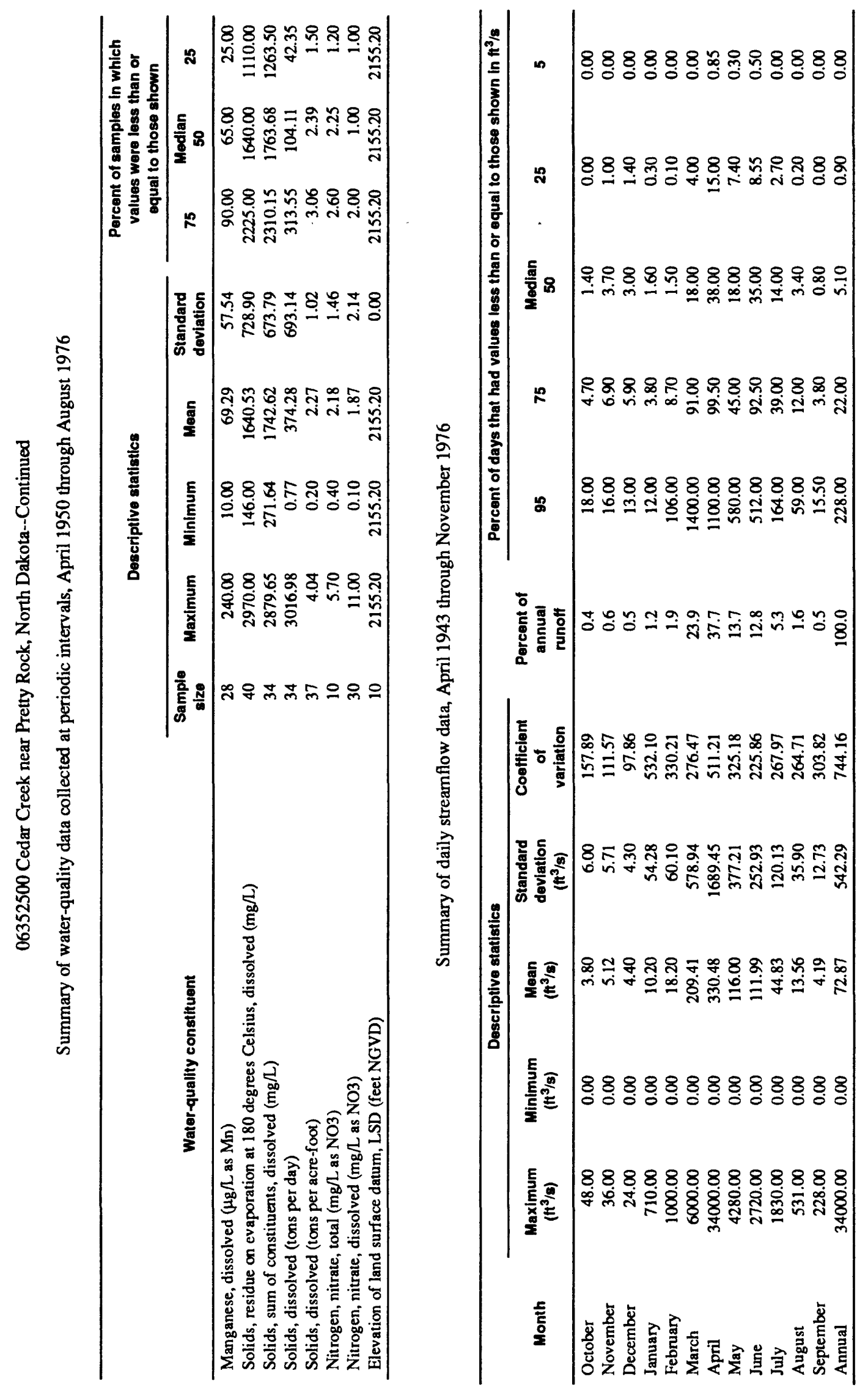


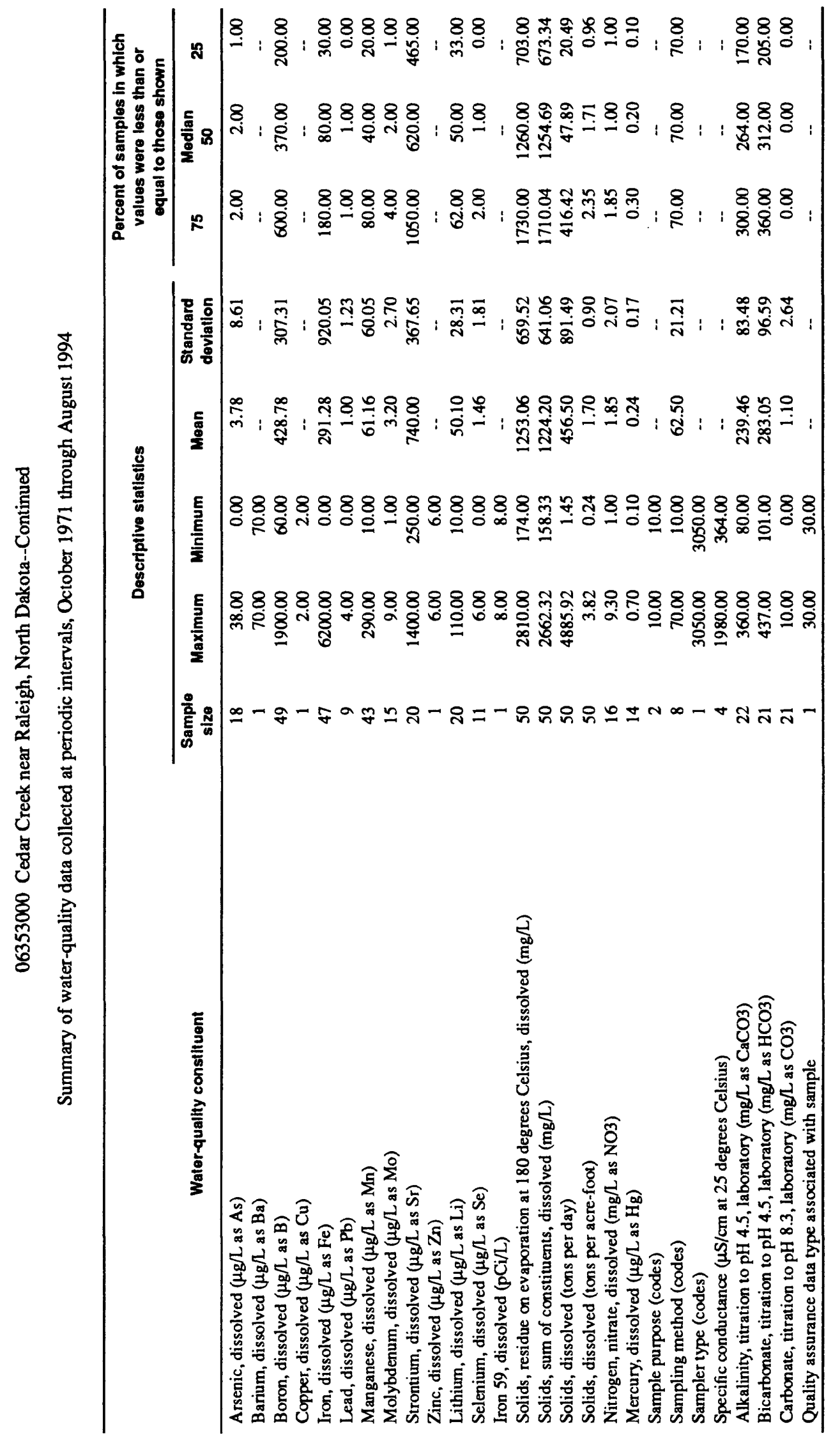




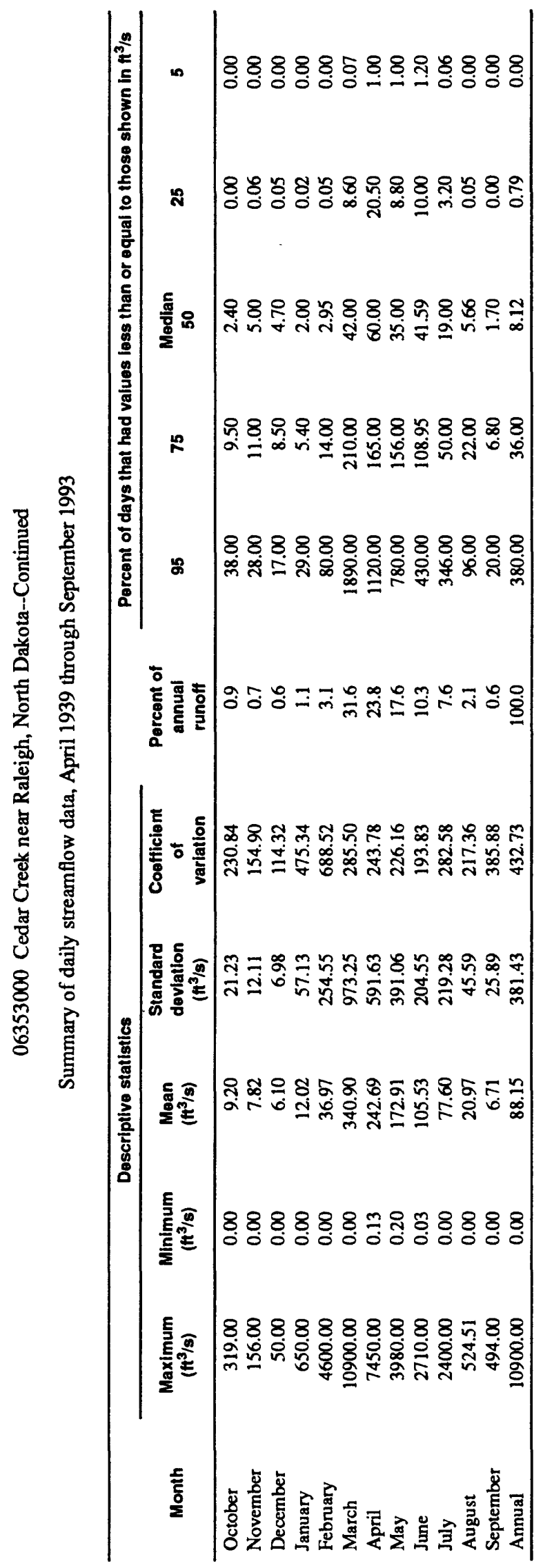




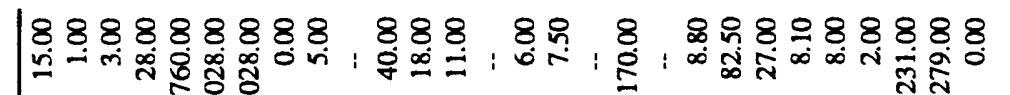

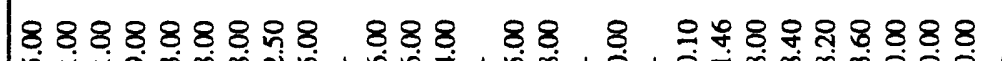

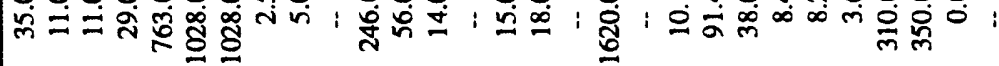

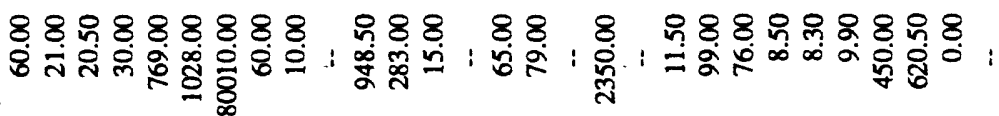

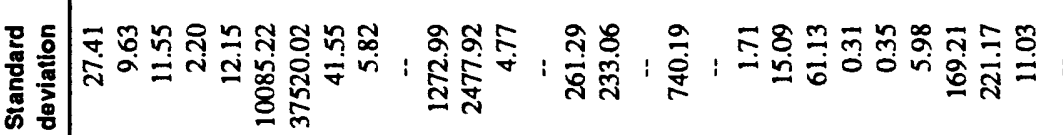

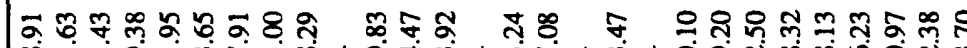

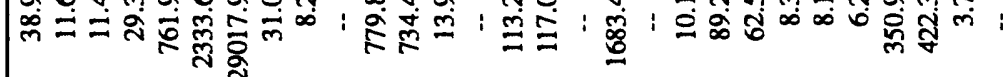

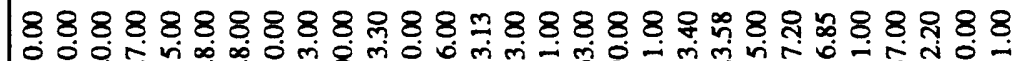

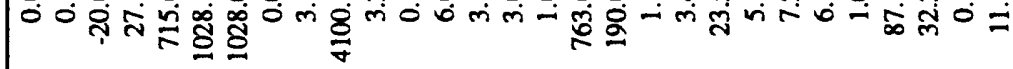

$8088888888889888888 \% 88.88888$

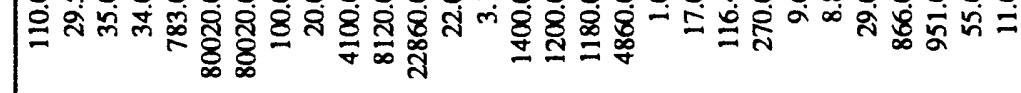

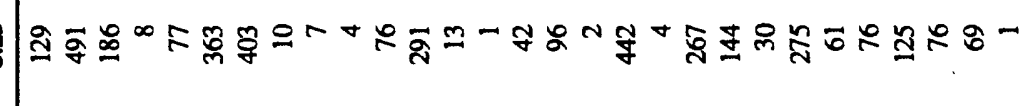




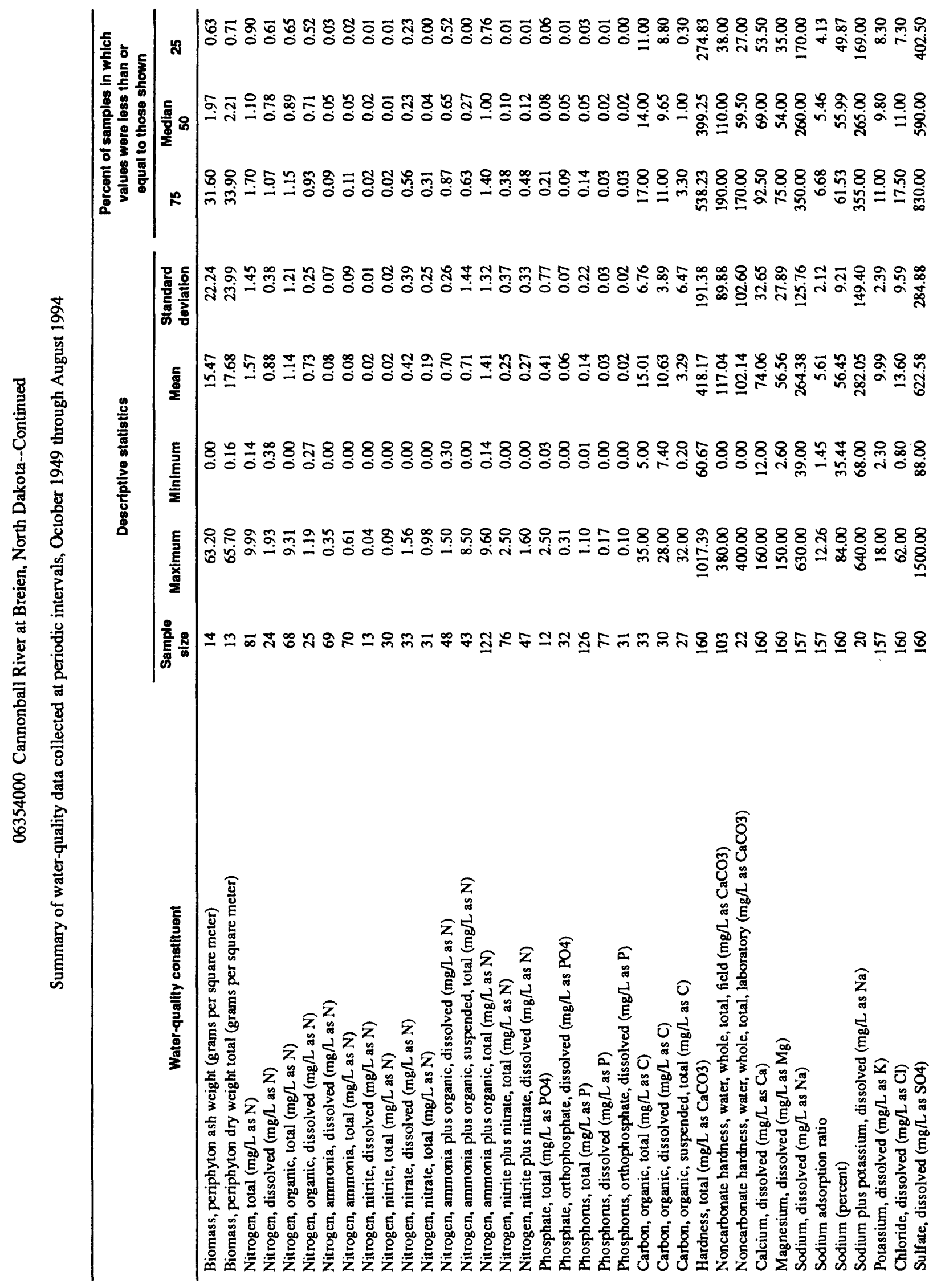




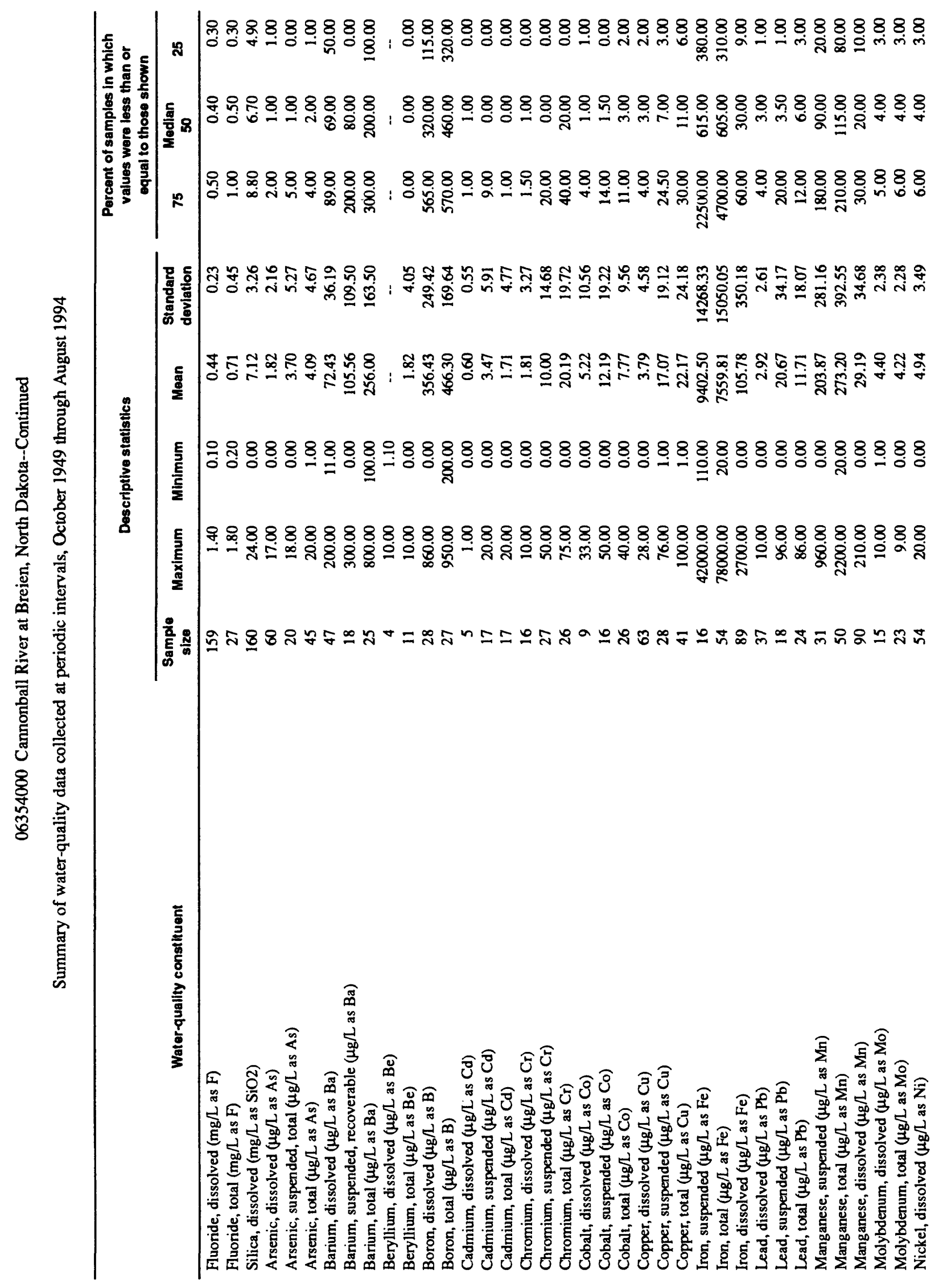




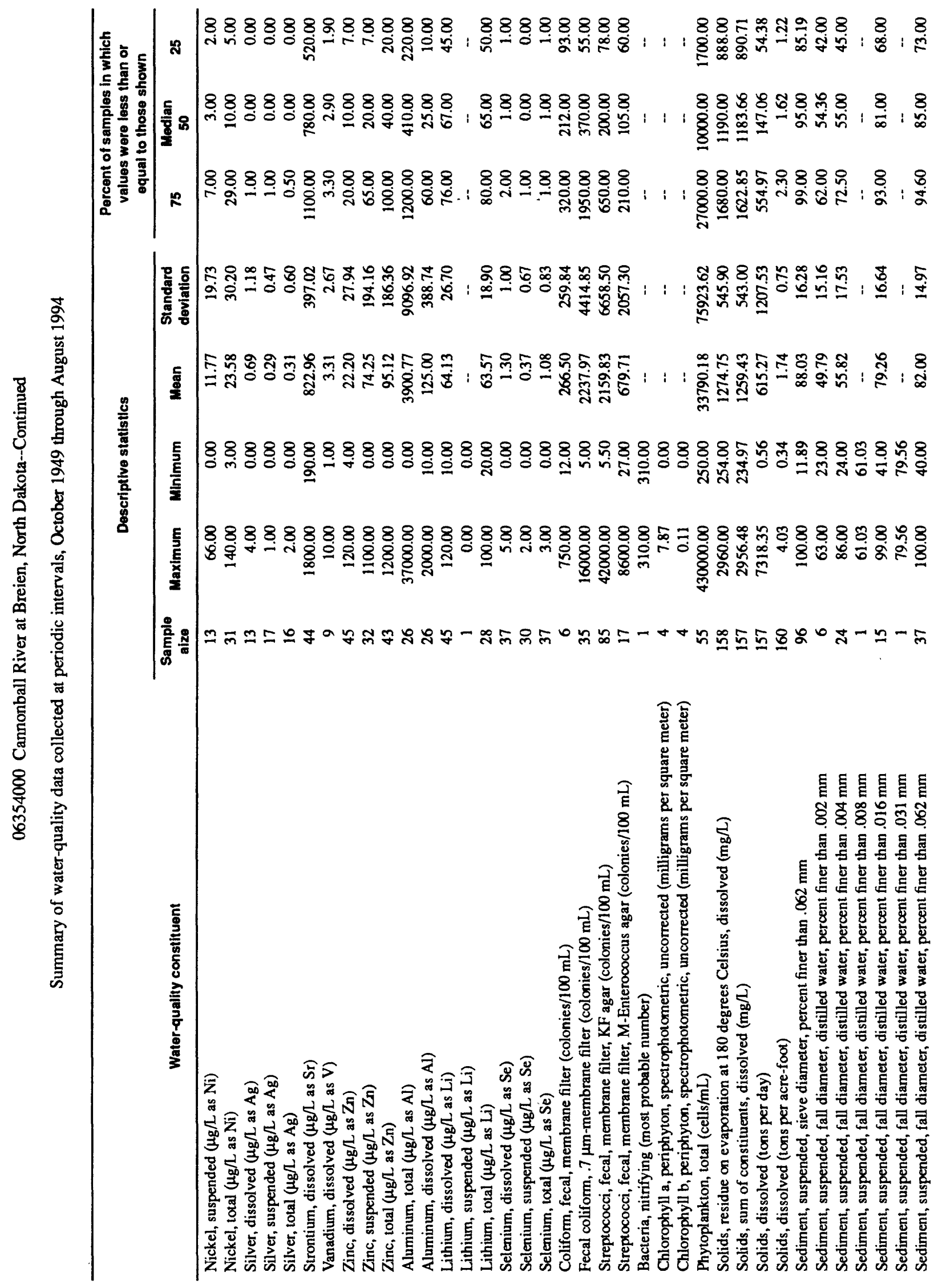




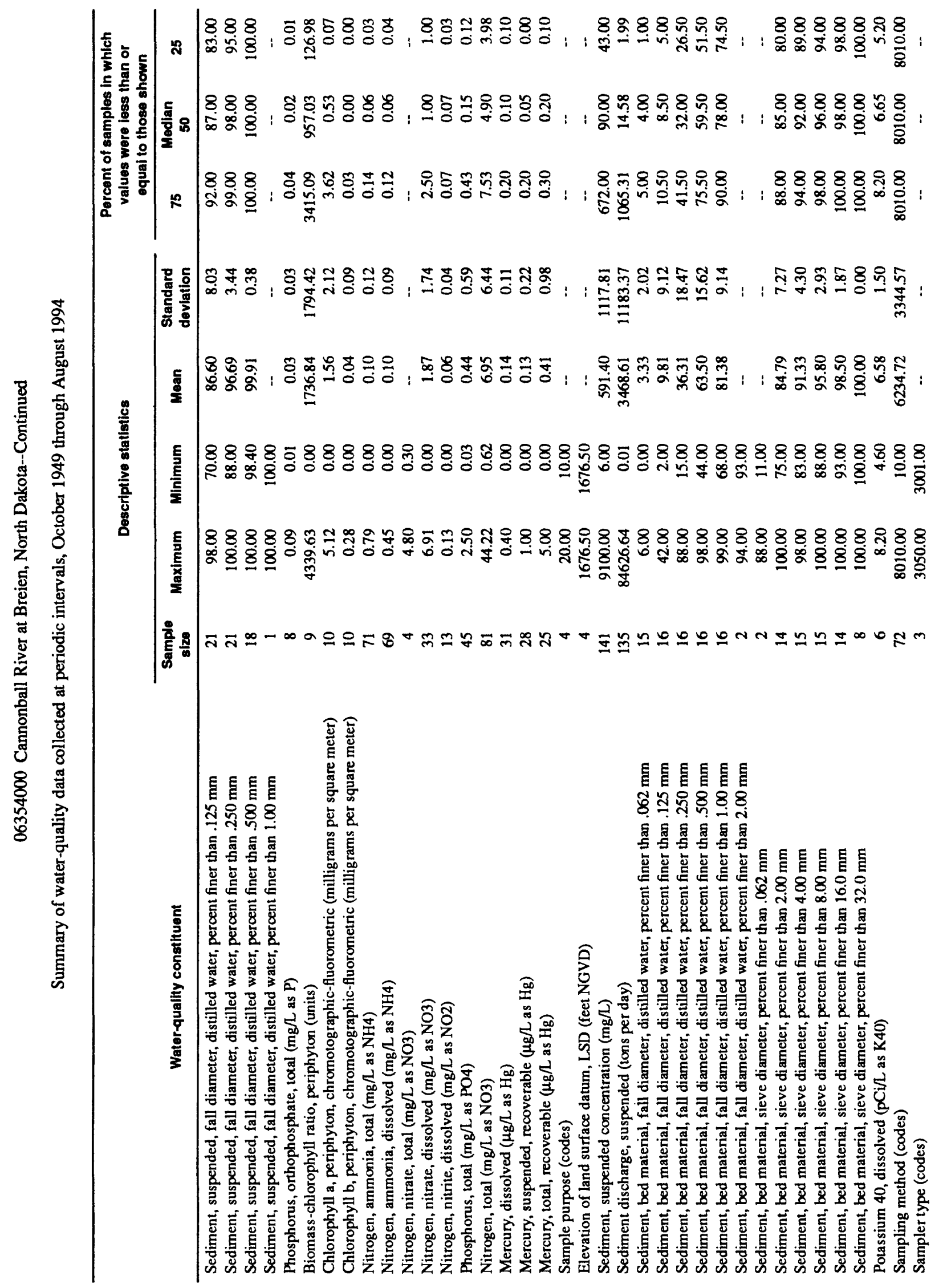




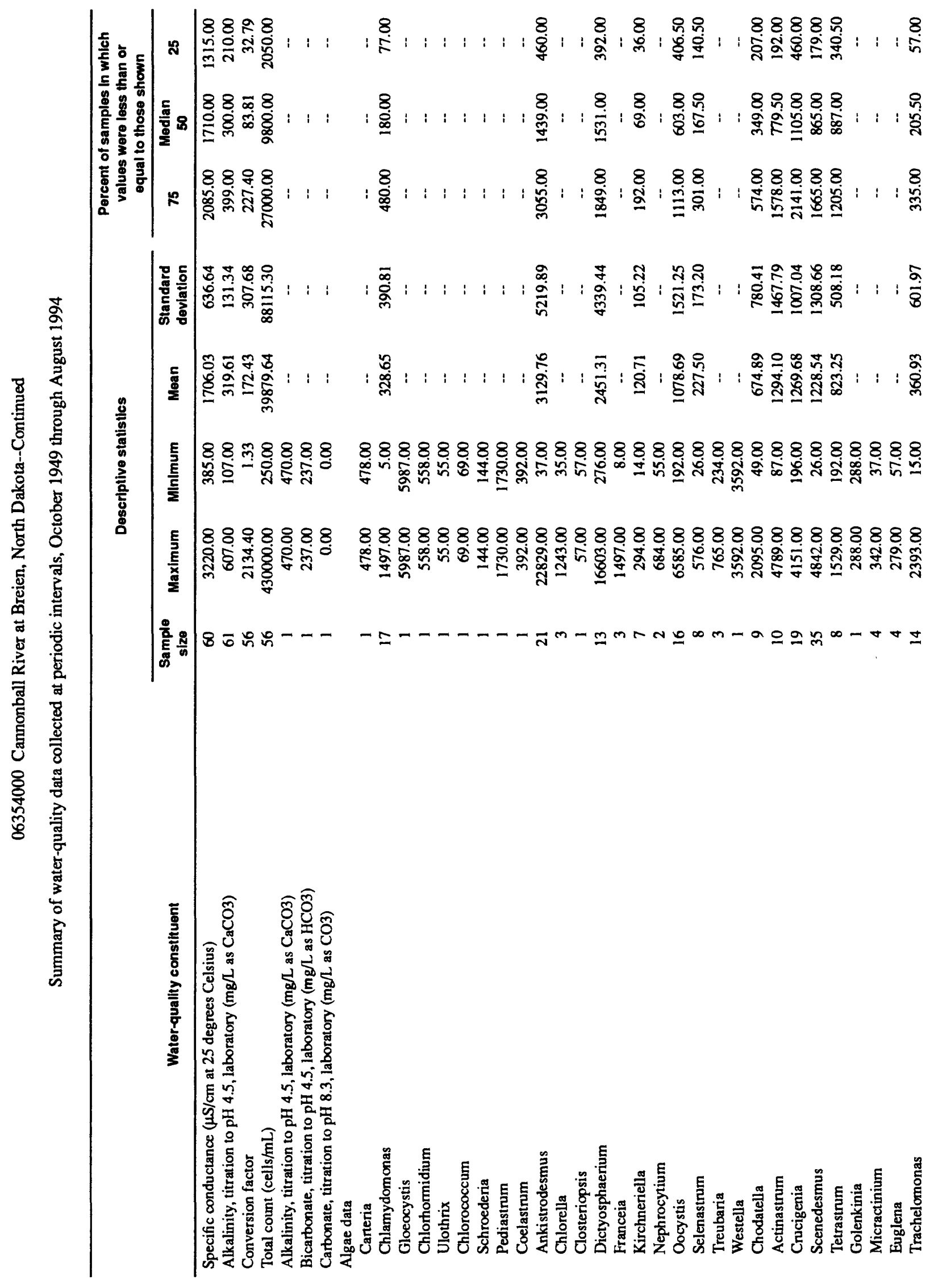




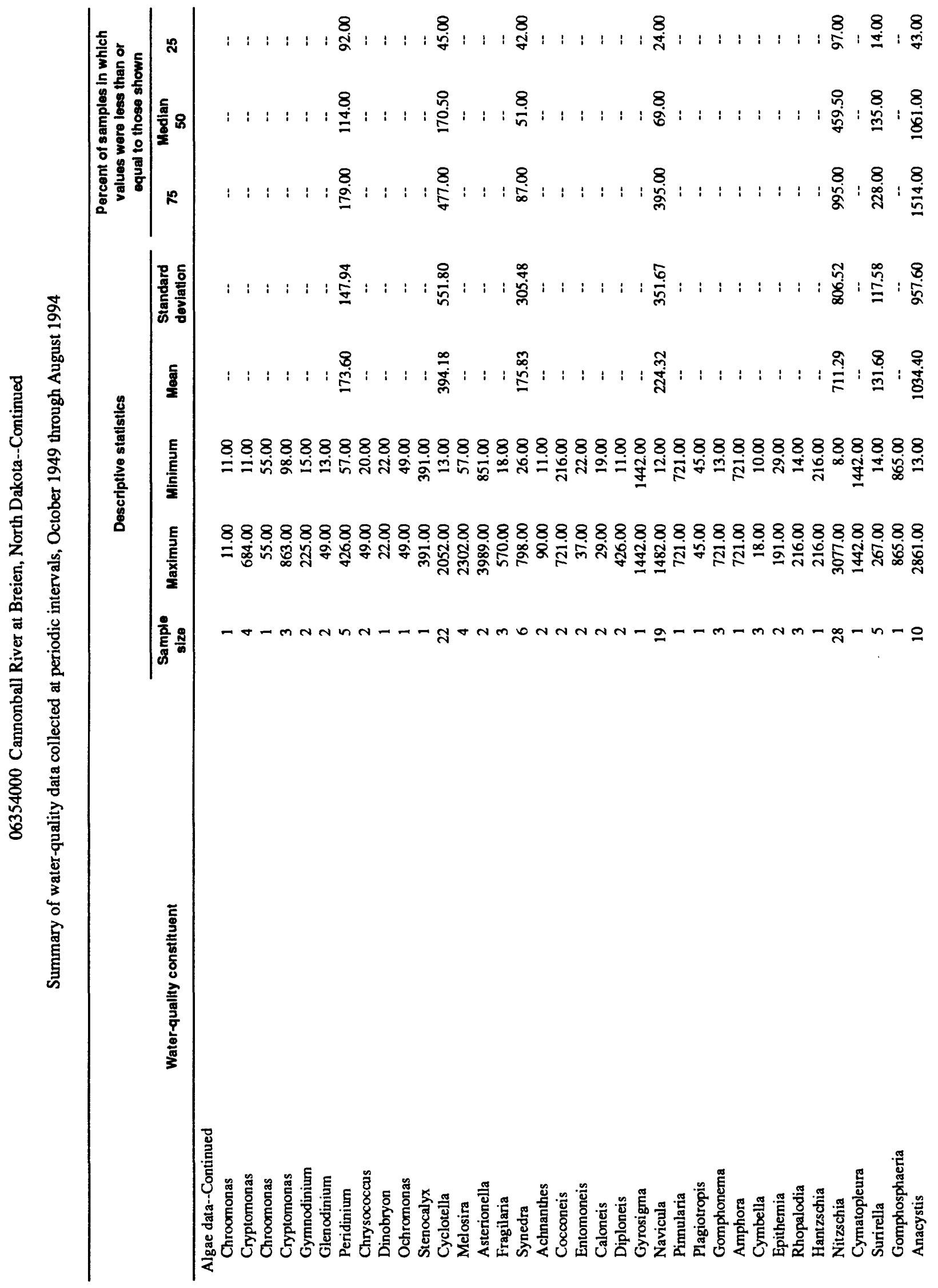



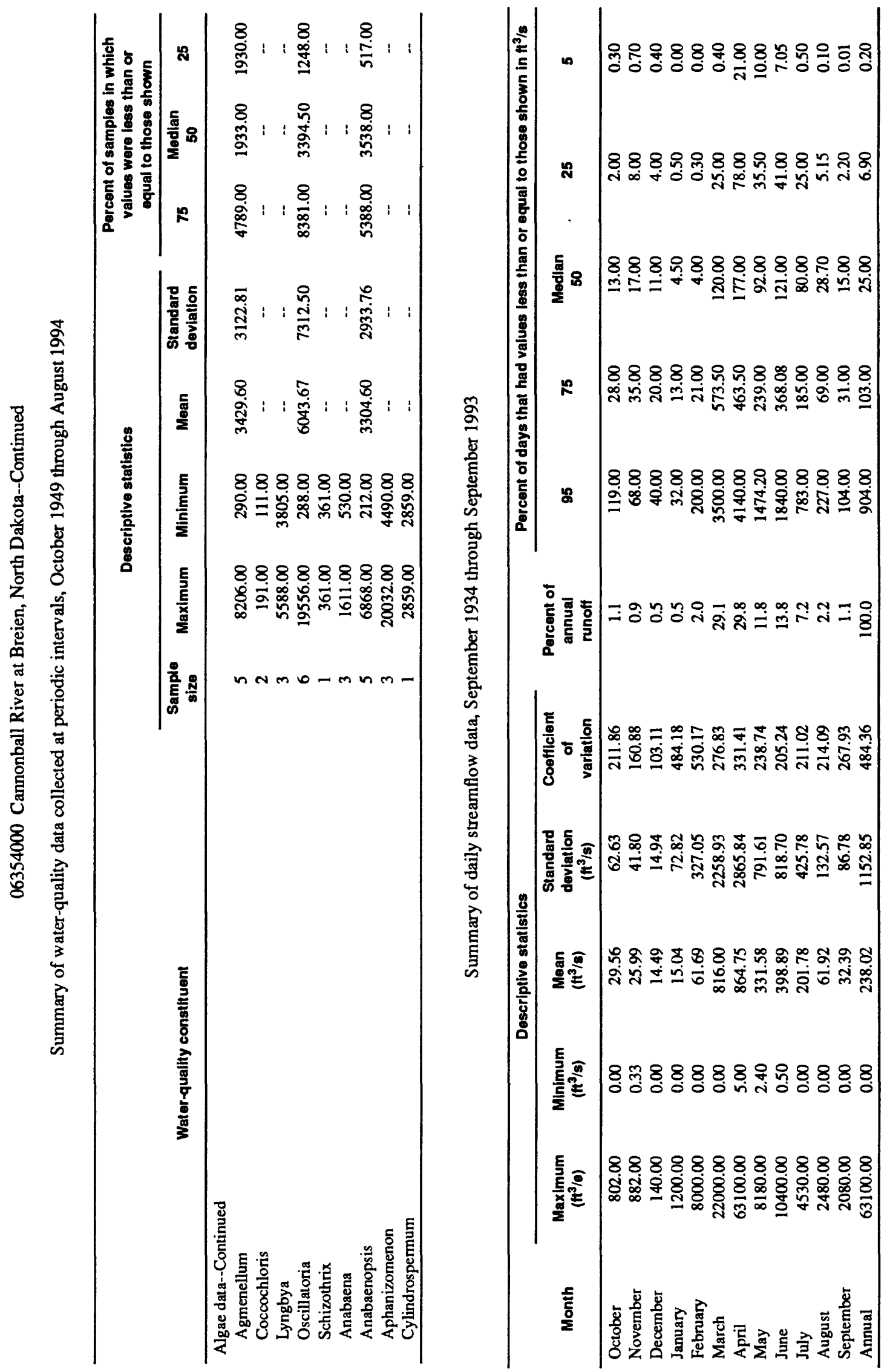
Table 2. Summary of daily streamflow data obtained from the U.S. Geological Survey

\section{Abbreviatlons and symbols}

$\mathrm{ft}^{3} / \mathrm{s}$, cubic feet per second

$\mathrm{mi}^{2}$, square miles

,-- no data 


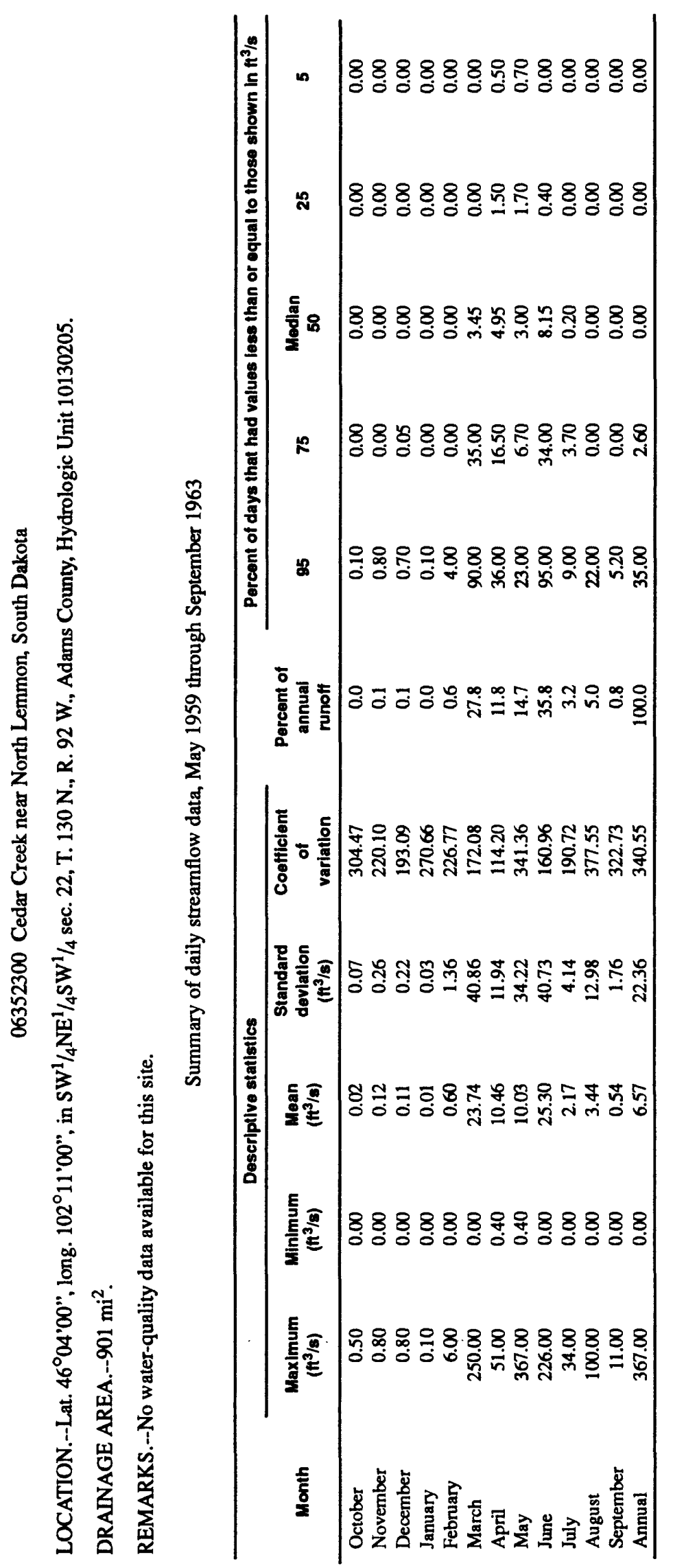




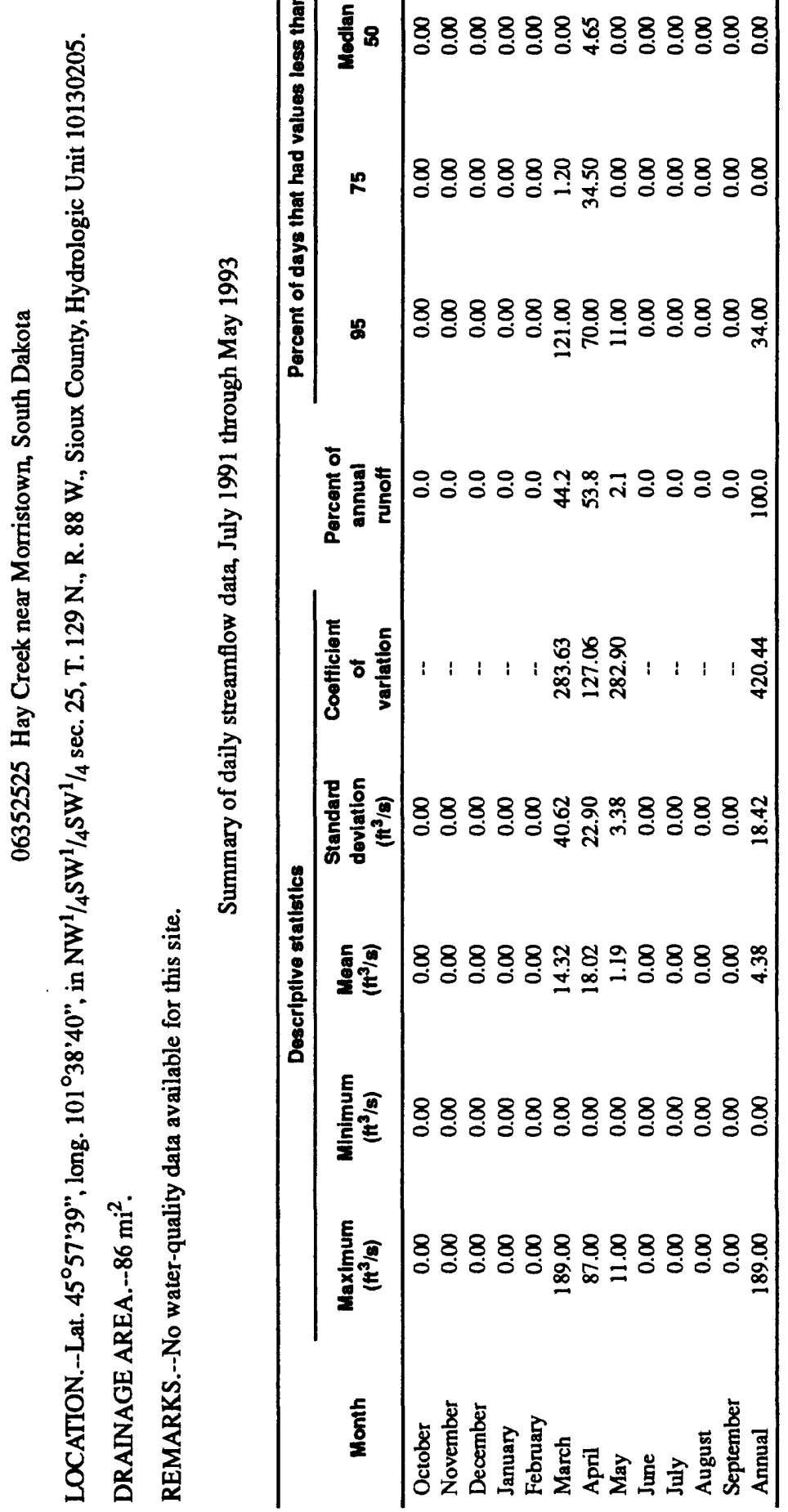


Table 3. Summary of surface-water quality data obtained from the U.S. Geological Survey for sites where one or two water-quality samples were collected

[Centain properties or constituents may be listed more than once; however, no distinction is made between field and laboratory values]

\section{Abbrevlations and symbols}

JCU, Jackson candle unit $\mathrm{mg} / \mathrm{L}$, milligrams per liter

$\mu \mathrm{g} / \mathrm{L}$, micrograms per liter

$\mu \mathrm{S} / \mathrm{cm}$, microsiemens per centimeter at 25 degrees Celsius

,-- no data 


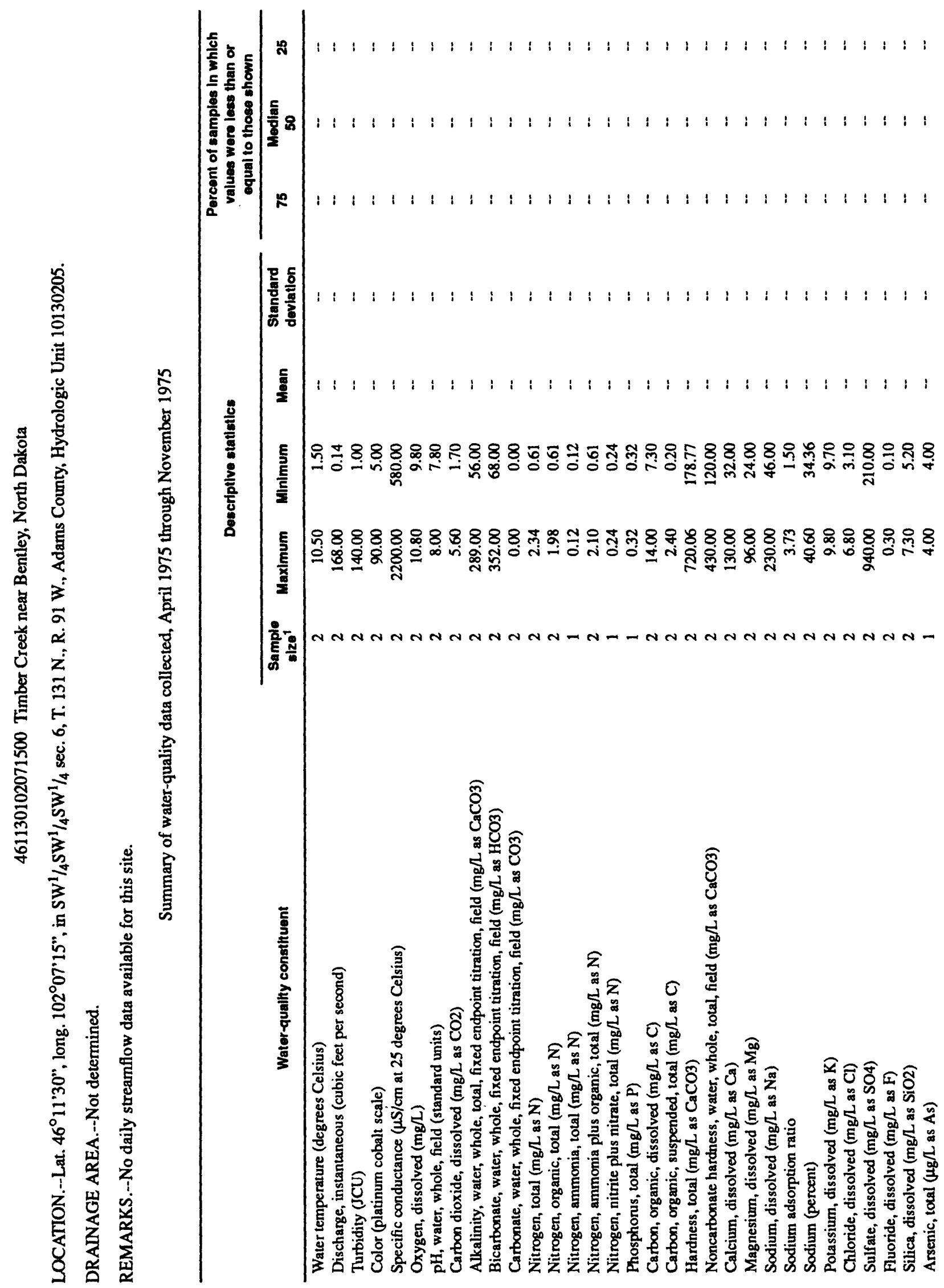




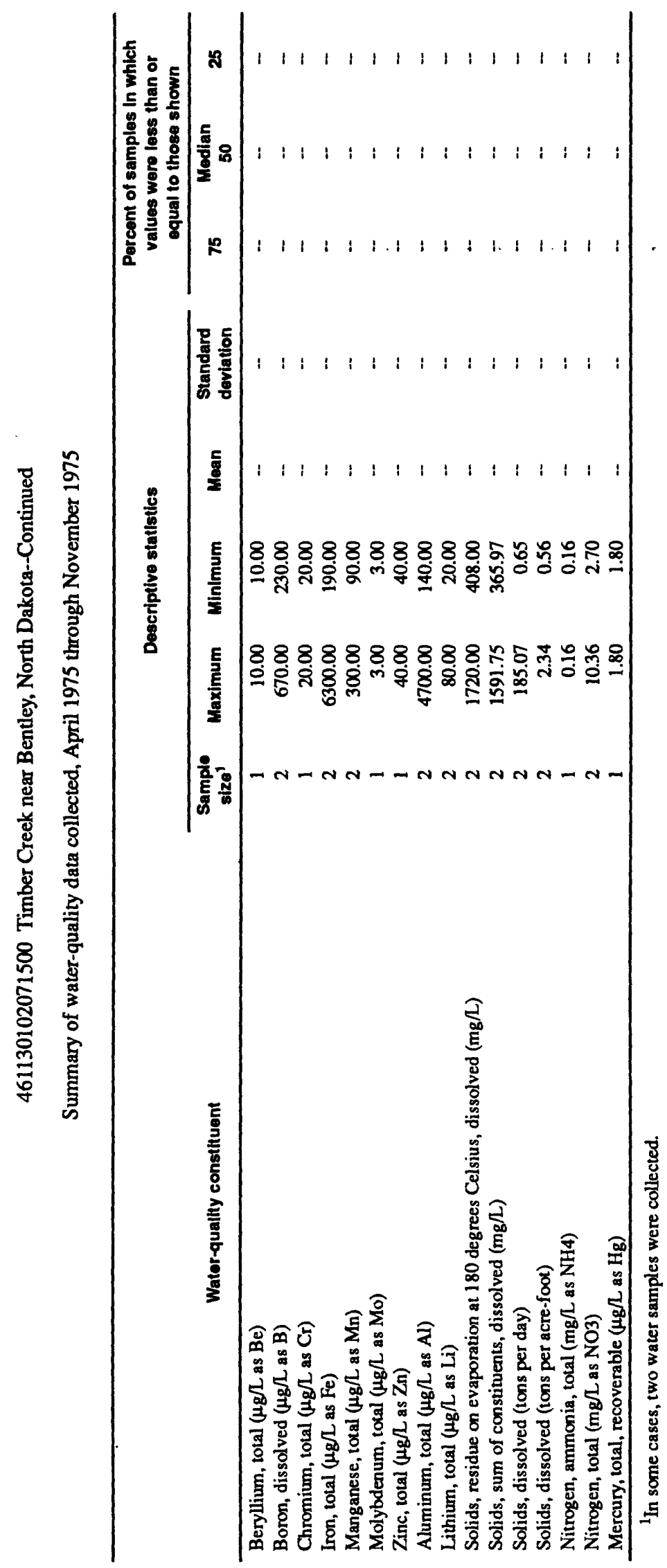




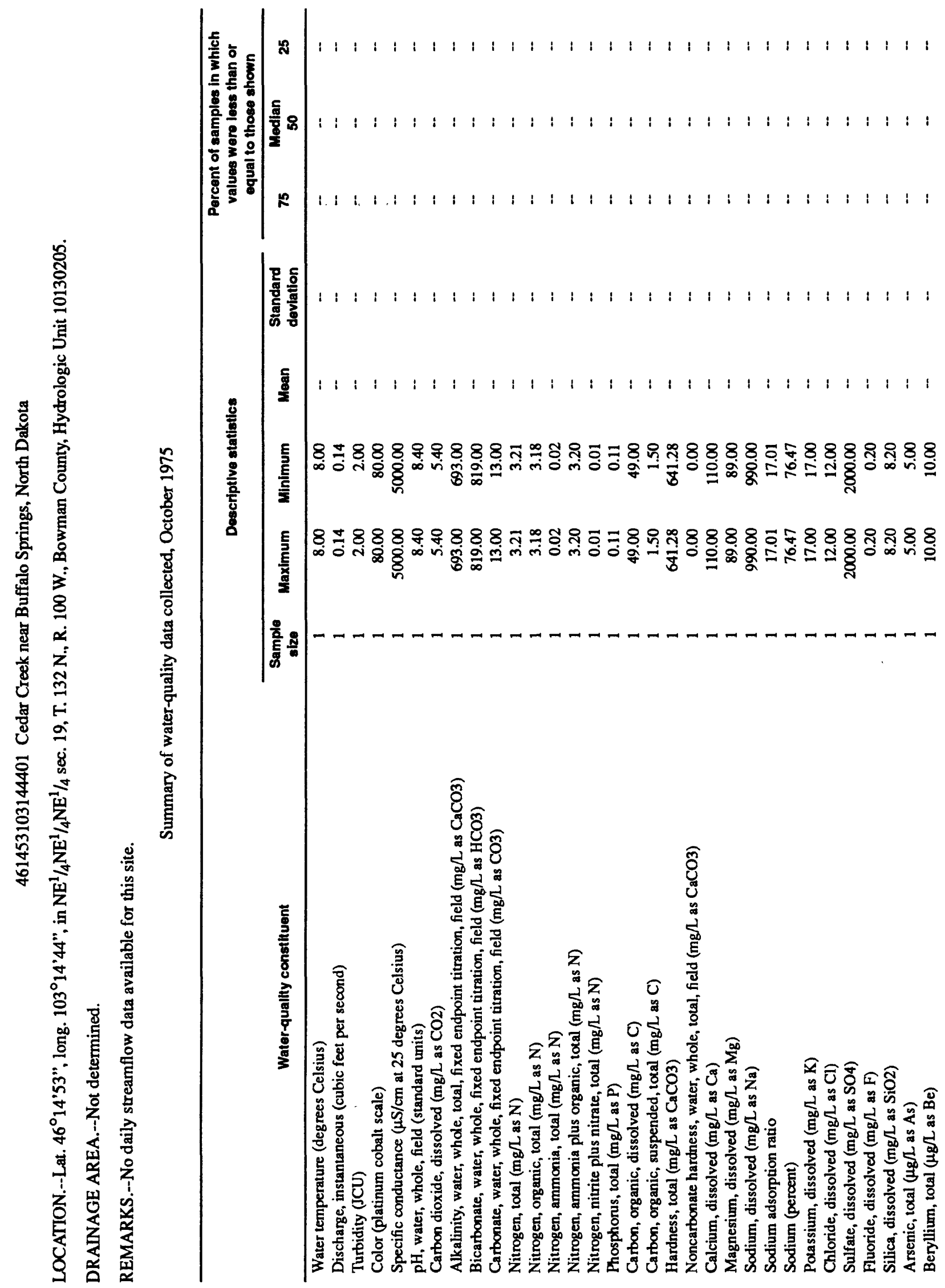




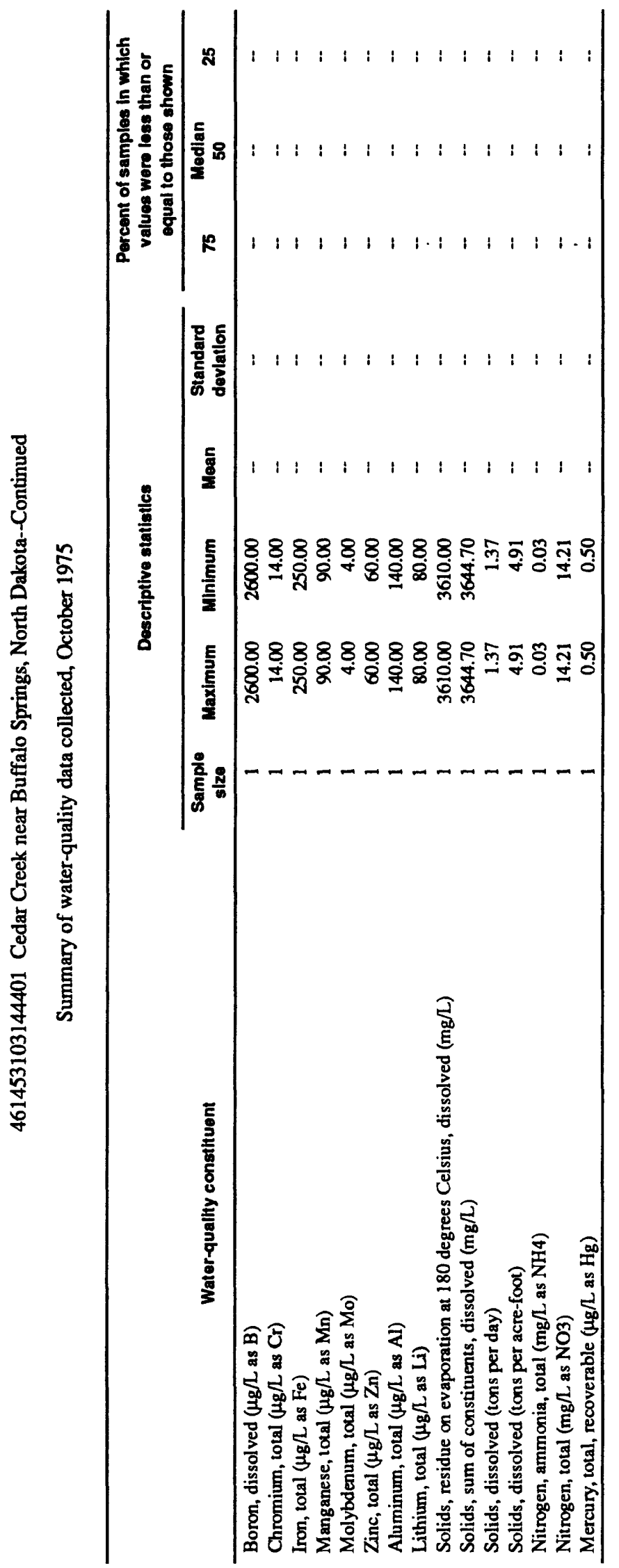


Table 4. Ground-water quality data obtained from the U.S. Geological Survey for sites where one water-quality sample was collected

\section{Abbrevlatlons and symbols}

$\mathrm{mg} / \mathrm{L}$, milligrams per liter

$\mu \mathrm{g} / \mathrm{L}$, micrograms per liter

$\mu \mathrm{S} / \mathrm{cm}$, microsiemens per centimeter at 25 degrees Celsius

,-- no data

\section{County code}

001, Adams County

011, Bowman County

037, Grant County

041, Hettinger County

059, Morton County

085, Sioux County

087, Slope County

089, Stark County

\section{Geologic unlt}

$112 \quad$ Pleistocene

125 Paleocene

211 Upper Cretaceous

BGFV Buried glaciofluvial deposits

CBLD Cannonball-Ludlow Members of Fort Union Formation

CNBL Cannonball Member of Fort Union Formation

EMCK Elm Creek aquifer

FXHL Fox Hills Sandstone

HCFH Hell Creek Formation-Fox Hills Sandstone

HLCK Hell Creek Formation

HRMN Harmon lignite aquifer

LDLW Ludlow Member of Fort Union Formation

LHCK Ludlow Member of Fort Union Formation-Hell Creek Formation

LTHR Little Heart aquifer

SBTR Sentinel Butte-Tongue River Members of Fort Union Formation

SJMS St. James aquifer

SLDS Shields aquifer

SNLB Sentinel Butte Member of Fort Union Formation

TGRV Tongue River Member of Fort Union Formation

TRVL Tongue River-Ludlow Members of Fort Union Formation 


\begin{tabular}{|c|c|c|c|c|c|c|c|c|c|c|}
\hline Stte number & Local identifier & $\begin{array}{l}\text { County } \\
\text { code }\end{array}$ & $\begin{array}{c}\text { Hydrologic } \\
\text { unit }\end{array}$ & $\begin{array}{l}\text { Geologic } \\
\text { unit }\end{array}$ & Date & $\begin{array}{l}\text { Depth } \\
\text { bolow } \\
\text { Iand } \\
\text { surface, } \\
\text { water } \\
\text { lovel } \\
\text { (feot) }\end{array}$ & $\begin{array}{l}\text { Depth } \\
\text { of } \\
\text { woll, } \\
\text { total } \\
\text { (teet) }\end{array}$ & $\begin{array}{l}\text { Attitude } \\
\text { of land } \\
\text { surface, } \\
\text { datum } \\
\text { (feet) }\end{array}$ & 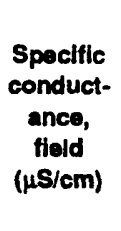 & 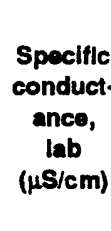 \\
\hline 455645101434801 & 129-088-31DDA & 085 & 10130205 & $125 \mathrm{CNBL}$ & $08-16-71$ & -- & 312 & - & 2150 & .- \\
\hline 455704101414501 & 129-088-33DBB & 085 & 10130205 & $1.25 \mathrm{CNBL}$ & $06-02-69$ & - & 180 & -. & 4660 & -- \\
\hline 455733101172701 & $129-085-26 C C C 1$ & 085 & 10130205 & 211HLCK & $08-19-71$ & - & 280 & - & 2020 & - \\
\hline 455742102094601 & $129-092-26 \mathrm{CCB}$ & 001 & 10130205 & 125LDLW & $06-23-71$ & - & 222 & 2642 & 3660 & -- \\
\hline 455805101574601 & $129-090-29 \mathrm{ACA}$ & 085 & 10130205 & 211HLCK & $08-11-71$ & - & 360 & - & 2050 & - \\
\hline 455822102190802 & 129-093-28ABB2 & $\infty 01$ & 10130205 & 125TRVL & $06-15-71$ & -- & 65 & - & 1950 & -- \\
\hline 455822102190803 & $129-093-28 \mathrm{ABB} 3$ & 001 & 10130205 & 125LDLW & $06-24-71$ & - & 240 & - & 1670 & - \\
\hline 455823101450301 & 129-089-24DDD1 & 085 & 10130205 & $125 \mathrm{CNBL}$ & $08-16-71$ & - & 222 & - & 2160 & -- \\
\hline 455823101450302 & 129-089-24DDD2 & 085 & 10130205 & $125 \mathrm{CNBL}$ & $08-16-71$ & - & 210 & - & 2000 & -- \\
\hline 455828101592301 & $129-090-19 \mathrm{CD}$ & 085 & 10130205 & 211HLCK & $04-12-59$ & -- & 335 & -- & 1910 & -- \\
\hline 455832101120001 & 129-084-21CDA & 085 & 10130206 & $211 \mathrm{HLCK}$ & $12-09-71$ & - & 300 & - & 1750 & - \\
\hline 455850101510701 & $129-089-20 \mathrm{BCC} 1$ & 085 & 10130205 & $125 \mathrm{CNBL}$ & $08-12-71$ & - & 225 & - & 2260 & $\ldots$ \\
\hline 455850101510702 & $129-089-20 \mathrm{BCC} 2$ & 085 & 10130205 & $125 \mathrm{CNBL}$ & $08-12-71$ & - & 250 & - & 2900 & - \\
\hline 455912102064701 & 129-091-19BAA & 001 & 10130205 & 125TRVL & $06-21-71$ & - & 80 & - & 935 & - \\
\hline 455922101511701 & 129-089-18DDA & 085 & 10130205 & $125 \mathrm{CNBL}$ & $08-12-71$ & - & 245 & - & 2730 & -- \\
\hline 460010102060001 & $129-091-8 \mathrm{CCC}$ & 001 & 10130205 & 125LDLW & $06-15-71$ & - & 261 & 2450 & 1790 & - \\
\hline 460032102210001 & $129-093-8 \mathrm{CBB} 1$ & 001 & 10130205 & 125LHCK & $06-14-72$ & - & 363 & 2460 & 1870 & - \\
\hline 460032102210002 & 129-093-8CBB2 & 001 & 10130205 & 125LDLW & $06-13-72$ & -- & 207 & 2460 & 1760 & - \\
\hline 460034103133201 & 129-100-7DAA & 011 & 10130205 & 125LHCK & $08-20-70$ & - & 206 & 2820 & 2570 & - \\
\hline 460056102061001 & 129-091-7AAA1 & 001 & 10130205 & $125 \mathrm{LHCK}$ & $09-27-71$ & - & 348 & 2422 & 1770 & - \\
\hline 460059101423201 & 129-088-5DDD 1 & 085 & 10130205 & 211HLCK & $07-04-73$ & -. & 466 & 2200 & 2120 & -- \\
\hline 460059101423202 & 129-088-5DDD2 & 085 & 10130205 & 211HLCK & $07-04-73$ & - & 348 & 2200 & 1670 & -- \\
\hline 460059101423203 & 129-088-5DDD3 & 085 & 10130205 & - & $07-04-73$ & - & 180 & 2200 & 1710 & - \\
\hline 460101101265701 & $129-086-4 \mathrm{CDD} 1$ & 085 & 10130205 & 211HLCK & $08-18-71$ & - & 56 & - & 1320 & -- \\
\hline 460102102135001 & 129-092-6DDC & 001 & 10130205 & $125 \mathrm{CNBL}$ & $08-11-70$ & -- & 80 & -- & 3190 & -- \\
\hline 460223102294601 & $130-094-31 B C C$ & 001 & 10130205 & 125TRVL & $06-18$ & -- & 116 & -- & 6490 & - \\
\hline 460239101135301 & 130-084-31AAA1 & 085 & 10130206 & $211 \mathrm{HLCK}$ & $07-10-73$ & - & 466 & 2238 & 4260 & -- \\
\hline 460240101075501 & 130-084-36ABA & 085 & 10130206 & $211 \mathrm{FXHL}$ & $10-10-73$ & - & 417 & 2148 & 2470 & -- \\
\hline 460244101272701 & $130-086-28 \mathrm{CCC} 1$ & 085 & 10130205 & 211FXHL & $07-05-73$ & - & 424 & 2062 & 2130 & -- \\
\hline 460246101041201 & 130-083-28DCD1 & 085 & 10130206 & 211HLCK & $09-24-69$ & -- & 274 & -- & 2030 & - \\
\hline 460246101041202 & $130-083-28 D C D 2$ & 085 & 10130206 & $211 \mathrm{HLCK}$ & $09-23-69$ & -- & 390 & - & 2530 & - \\
\hline 460332102105202 & 130-092-27BBA2 & 001 & 10130205 & 125LDLW & $10-10-71$ & - & 204 & 2383 & 1820 & - \\
\hline 460332102105203 & 130-092-27BBA3 & 001 & 10130205 & $125 \mathrm{LHCK}$ & $06-15-72$ & - & 374 & 2383 & 1670 & - \\
\hline 460336102334001 & 130-095-28AAA & 001 & 10130205 & 125TRVL & $07-08-71$ & - & 257 & - & 3190 & - \\
\hline 460337101404201 & $130-088-22 C D D$ & 037 & 10130205 & 211HLCK & $11-03-71$ & - & 350 & - & 1680 & - \\
\hline 460358102110101 & 130-092-22CBВ & 001 & 10130205 & $125 \mathrm{CNBL}$ & $06-29-71$ & - & 31 & 2330 & 1780 & -- \\
\hline 460449101200702 & 130-085-17DAA & 085 & 10130205 & 211FXHL & $04-18-72$ & - & 361 & - & 1860 & -- \\
\hline 460501102405801 & $130-096-15 B C C$ & 001 & 10130205 & 125TRVL & $07-02-71$ & - & 135 & -- & 1430 & -- \\
\hline 460521102384801 & $130-096-14 \mathrm{AAB}$ & 001 & 10130205 & $125 \mathrm{TRVL}$ & $07-02-71$ & - & 198 & - & 1840 & - \\
\hline 460522101550201 & 130-090-10DDD & 037 & 10130205 & $125 \mathrm{CNBL}$ & $09-08-71$ & -- & 210 & - & 3100 & - \\
\hline 460526102284101 & 130-094-7DDD1 & 001 & 10130205 & 125TRVL & $06-15-72$ & - & 390 & 2570 & 1670 & -- \\
\hline 460526102284102 & 130-094-7DDD2 & 001 & 10130205 & 125TRVL & $06-15-72$ & - & 253 & 2570 & 2100 & -- \\
\hline 460541101562201 & 130-090-9ADA & 037 & 10130205 & 211HLCK & $06-16-67$ & - & 233 & - & 2280 & -- \\
\hline 460608102042801 & $130-091-9 \mathrm{BAB}$ & 001 & 10130205 & $125 \mathrm{LHCK}$ & $04-14-71$ & - & 340 & 2405 & 1890 & - \\
\hline 460639102570801 & $130-098-4$ СВВ & $\infty 1$ & 10130205 & 125TRVL & $06-30-71$ & - & 95 & -- & 1140 & -- \\
\hline
\end{tabular}




\begin{tabular}{|c|c|c|c|c|c|c|c|c|c|c|c|}
\hline Site number & Date & $\begin{array}{l}\text { pH, } \\
\text { field } \\
\text { (stand- } \\
\text { ard } \\
\text { units }\end{array}$ & $\begin{array}{l}\mathrm{pH}, \\
\text { lab } \\
\text { (stand- } \\
\text { ard } \\
\text { units) }\end{array}$ & $\begin{array}{l}\text { Temper- } \\
\text { ature, } \\
\text { water } \\
\text { (degrees } \\
\text { Colsius) }\end{array}$ & $\begin{array}{l}\text { Color } \\
\text { (platt- } \\
\text { num } \\
\text { cobalt } \\
\text { scale) }\end{array}$ & $\begin{array}{c}\text { Hard- } \\
\text { nose, } \\
\text { total } \\
(\mathrm{mg} / \mathrm{L} \text { as } \\
\left.\mathrm{CaCO}_{3}\right)\end{array}$ & $\begin{array}{l}\text { Noncar- } \\
\text { bonate } \\
\text { hard- } \\
\text { ness, } \\
\text { total } \\
\text { (mgll ae } \\
\left.\mathrm{CaCO}_{3}\right)\end{array}$ & $\begin{array}{l}\text { Alka- } \\
\text { linity, } \\
\text { fleld } \\
(\mathrm{mg} / \mathrm{L} \text { as } \\
\left.\mathrm{CoCO}_{3}\right)\end{array}$ & $\begin{array}{c}\text { Alka- } \\
\text { ilnity, } \\
\text { lab } \\
(\mathrm{mg} / \mathrm{L} \text { as } \\
\left.\mathrm{CaCO}_{3}\right)\end{array}$ & $\begin{array}{l}\text { Solids, } \\
\text { sum of } \\
\text { constit- } \\
\text { uents, } \\
\text { die- } \\
\text { solved } \\
\text { (mg/L) }\end{array}$ & $\begin{array}{l}\text { Solids, } \\
\text { residue } \\
\text { at } 105 \\
\text { degrees } \\
\text { Coleius, } \\
\text { total } \\
\text { (mgll) }\end{array}$ \\
\hline 455645101434801 & $08-16-71$ & 7.9 & - & - & 2000 & 250 & 0 & - & - & 1490 & -- \\
\hline 455704101414501 & $06-02-69$ & 4.3 & - & - & - & 4800 . & - & - & -- & 3160 & - \\
\hline 455733101172701 & $08-19-71$ & 8.1 & -- & -- & - & 450 & 0 & - & - & 1360 & -- \\
\hline 455742102094601 & $06-23-71$ & 7.8 & - & - & - & 730 & 120 & - & - & 2810 & -. \\
\hline 455805101574601 & $08-11-71$ & 8.0 & - & 9.5 & - & 39 & 0 & - & - & 1380 & -- \\
\hline 455822102190802 & $06-15-71$ & 8.1 & - & - & - & 150 & 0 & - & - & 1310 & -- \\
\hline 455822102190803 & $06-24-71$ & 8.4 & - & 9.0 & -- & 15 & 0 & - & - & 1060 & -- \\
\hline 455823101450301 & $08-16-71$ & 8.4 & - & -- & - & 38 & 0 & - & -- & 1390 & - \\
\hline 455823101450302 & $08-16-71$ & 8.3 & - & - & - & 77 & 0 & - & - & 1390 & - \\
\hline 455828101592301 & $04-12-59$ & 8.1 & - & 8.5 & 40 & 19 & 0 & - & - & 1250 & - \\
\hline 455832101120001 & $12-09-71$ & 8.0 & - & - & -- & 30 & 0 & -- & - & 1140 & - \\
\hline 455850101510701 & $08-12-71$ & 8.0 & - & - & -- & 44 & 0 & - & - & 1500 & - \\
\hline 455850101510702 & $08-12-71$ & 8.1 & -- & 9.5 & - & 120 & 0 & - & - & 2010 & -- \\
\hline 455912102064701 & $06-21-71$ & 7.8 & -- & 10.0 & - & 160 & 0 & - & - & 568 & - \\
\hline 455922101511701 & $08-12-71$ & 8.1 & - & - & - & 110 & 0 & -. & - & 1860 & - \\
\hline 460010102060001 & $06-15-71$ & 8.4 & - & 10.0 & -- & 27 & 0 & - & - & 1170 & -- \\
\hline 460032102210001 & $06-14-72$ & 8.3 & - & 11.0 & - & 20 & 0 & - & - & 1150 & -- \\
\hline 460032102210002 & $06-13-72$ & 8.2 & -- & 10.0 & - & 27 & 0 & - & - & 1100 & - \\
\hline 460034103133201 & $08-20-70$ & 8.4 & -- & 12.0 & - & 9 & 0 & - & - & 1690 & -- \\
\hline 460056102061001 & $09-27-71$ & 8.7 & -- & 10.0 & - & 18 & 0 & - & - & 1080 & - \\
\hline 460059101423201 & $07-04-73$ & 8.5 & - & 12.0 & - & 18 & 0 & - & - & 1250 & - \\
\hline 460059101423202 & $07-04-73$ & 8.3 & -- & 11.0 & - & 13 & 0 & - & - & 1060 & -- \\
\hline 460059101423203 & $07-04-73$ & 8.3 & -- & 13.0 & - & 21 & 0 & - & - & 1080 & - \\
\hline 460101101265701 & $08-18-71$ & 8.5 & - & - & - & 13 & 0 & -- & - & 831 & -- \\
\hline 460102102135001 & $08-11-70$ & 8.3 & -- & 9.0 & - & 160 & 0 & - & - & 2340 & - \\
\hline 460223102294601 & $06-18-71$ & 6.9 & - & 9.5 & -- & 2000 & 1700 & - & - & 5950 & -- \\
\hline 460239101135301 & $07-10-73$ & 8.0 & -- & 13.5 & - & 500 & 95 & - & - & 3340 & -- \\
\hline 460240101075501 & $10-10-73$ & 8.4 & - & 10.0 & - & 22 & 0 & - & $\ldots$ & 1450 & - \\
\hline 460244101272701 & $07-05-73$ & 8.5 & $\cdots$ & 11.0 & -- & 16 & 0 & - & - & 1260 & - \\
\hline 460246101041201 & $09-24-69$ & 8.9 & - & -- & -- & 32 & - & - & - & 1200 & - \\
\hline 460246101041202 & $09-23-69$ & 8.6 & -- & - & - & 46 & -- & - & -- & 1540 & - \\
\hline 460332102105202 & $10-10-71$ & 8.4 & -- & 10.0 & - & 61 & 0 & - & - & 1180 & -- \\
\hline 460332102105203 & $06-15-72$ & 8.7 & -- & - & - & 23 & 0 & - & - & 991 & - \\
\hline 460336102334001 & $07-08-71$ & 8.2 & - & - & - & 62 & 0 & - & - & 2210 & - \\
\hline 460337101404201 & $11-03-71$ & 8.5 & - & - & - & 17 & 0 & - & -- & 997 & - \\
\hline 460358102110101 & $06-29-71$ & 8.2 & -. & 9.5 & - & 17 & 0 & - & - & 1150 & -- \\
\hline 460449101200702 & $04-18-72$ & 8.1 & -- & - & - & 580 & 340 & - & - & 1380 & - \\
\hline 460501102405801 & $07-02-71$ & 8.2 & -- & - & - & 56 & 0 & -- & - & 936 & - \\
\hline 460521102384801 & $07-02-71$ & 8.0 & - & - & -- & 100 & 0 & - & -- & 1230 & -- \\
\hline 460522101550201 & $09-08-71$ & 8.2 & -- & -- & - & 100 & 0 & - & -- & 2210 & -- \\
\hline 460526102284101 & $06-15-72$ & 8.2 & - & 11.5 & - & 62 & 0 & - & -. & 1060 & -- \\
\hline 460526102284102 & $06-15-72$ & 8.6 & - & - & - & 94 & 0 & - & -. & 1370 & -- \\
\hline 460541101562201 & $06-16-67$ & 7.8 & - & -- & - & 24 & $\mathbf{0}$ & - & -- & 1500 & -- \\
\hline 460608102042801 & $04-14-71$ & 8.2 & - & -- & -- & 18 & 0 & - & -- & 1190 & -- \\
\hline 460639102570801 & $06-30-71$ & 7.7 & -- & - & -- & 280 & 0 & - & - & 744 & - \\
\hline
\end{tabular}




\begin{tabular}{|c|c|c|c|c|c|c|c|c|c|c|c|}
\hline Stte number & Date & $\begin{array}{l}\text { Solids, } \\
\text { residue } \\
\text { at } 180 \\
\text { degrees } \\
\text { Colsius, } \\
\text { dis- } \\
\text { solved } \\
\text { (mg/L) }\end{array}$ & $\begin{array}{l}\text { Solids, } \\
\text { dis- } \\
\text { solved } \\
\text { (tons per } \\
\text { acre- } \\
\text { toot) }\end{array}$ & $\begin{array}{l}\text { Calcium, } \\
\text { dia- } \\
\text { solved } \\
\text { (mg/L } \\
\text { as C) }\end{array}$ & $\begin{array}{l}\text { Magne- } \\
\text { slum, } \\
\text { dis- } \\
\text { solved } \\
\text { (mg/L } \\
\text { as Mg) }\end{array}$ & $\begin{array}{l}\text { Sodium, } \\
\text { dis- } \\
\text { solved } \\
\text { (mg/L } \\
\text { as Na) }\end{array}$ & $\begin{array}{l}\text { Sodium } \\
\text { (percent) }\end{array}$ & $\begin{array}{l}\text { Sodlum } \\
\text { adsorp- } \\
\text { tion } \\
\text { ratio }\end{array}$ & $\begin{array}{l}\text { Rosid- } \\
\text { ual, } \\
\text { sodium, } \\
\text { car- } \\
\text { bonate } \\
\text { (mg/L as } \\
\text { CaCO3) }\end{array}$ & $\begin{array}{l}\text { Potas- } \\
\text { slum, } \\
\text { dls- } \\
\text { solved } \\
\text { (mg/L } \\
\text { as K) }\end{array}$ & $\begin{array}{c}\text { Blcar- } \\
\text { bonate } \\
\text { (mg/L as } \\
\mathrm{HCO}_{3} \text { ) }\end{array}$ \\
\hline 455645101434801 & $08-16-71$ & 1460 & 1.99 & 62 & 22 & 430 & 79 & 12 & 5.00 & 3.9 & 590 \\
\hline 455704101414501 & $06-02-69$ & 3680 & - & 1100 & 470 & 150 & -- & 0.9 & - & - & - \\
\hline 455733101172701 & $08-19-71$ & 1430 & 1.94 & 110 & 43 & 320 & 60 & 7 & 2.00 & 4.0 & 660 \\
\hline 455742102094601 & $06-23-71$ & 2850 & 3.88 & 160 & 82 & 660 & 66 & 11 & - & 13 & 750 \\
\hline 455805101574601 & $08-11-71$ & 1330 & 1.81 & 11 & 2.8 & 480 & 96 & 33 & 9.00 & 3.4 & 590 \\
\hline 455822102190802 & $06-15-71$ & 1320 & 1.80 & 24 & 22 & 420 & 86 & 15 & - & 3.9 & 640 \\
\hline 455822102190803 & $06-24-71$ & 1050 & 1.43 & 3.8 & 1.3 & 420 & 98 & 47 & - & 2.2 & 890 \\
\hline 455823101450301 & $08-16-71$ & 1390 & 1.89 & 12 & 1.9 & 500 & 96 & 36 & 12.0 & 2.0 & 740 \\
\hline 455823101450302 & $08-16-71$ & 1330 & 1.81 & 23 & 4.7 & 470 & 93 & 23 & 11.0 & 2.3 & 730 \\
\hline 455828101592301 & $04-12-59$ & 1270 & 1.73 & 5.1 & 1.6 & 460 & 98 & 46 & - & 2.4 & 720 \\
\hline 455832101120001 & $12-09-71$ & 1140 & 1.55 & 10 & 1.2 & 420 & 97 & 33 & 9.00 & 1.4 & 710 \\
\hline 455850101510701 & $08-12-71$ & 1530 & 2.08 & 13 & 2.8 & 510 & 96 & 34 & 10.0 & 3.4 & 670 \\
\hline 455850101510702 & $08-12-71$ & 2010 & 2.73 & 34 & 9.2 & 640 & 91 & 25 & 8.00 & 5.5 & 610 \\
\hline 455912102064701 & $06-21-71$ & 556 & 0.76 & 41 & 14 & 150 & 66 & 5 & - & 4.3 & 410 \\
\hline 455922101511701 & $08-12-71$ & 1920 & 2.61 & 31 & 6.7 & 610 & 92 & 26 & 8.00 & 5.3 & 600 \\
\hline 460010102060001 & $06-15-71$ & 1180 & 1.60 & 7.2 & 2.2 & 420 & 97 & 35 & - & 2.9 & 590 \\
\hline 460032102210001 & $06-14-72$ & 1140 & 1.55 & 4.7 & 2.1 & 470 & 98 & 45 & 19.0 & 2.5 & 1140 \\
\hline 460032102210002 & $06-13-72$ & 1120 & 1.52 & 5.2 & 3.4 & 430 & 97 & 36 & 15.0 & 2.4 & 940 \\
\hline 460034103133201 & $08-20-70$ & 1730 & 2.35 & 0.60 & 1.9 & 610 & 99 & 86 & - & 1.9 & 720 \\
\hline 460056102061001 & $09-27-71$ & 1090 & 1.48 & 4.7 & 1.6 & 440 & 98 & 45 & 17.0 & 2.4 & 970 \\
\hline 460059101423201 & $07-04-73$ & 1290 & 1.75 & 3.4 & 2.3 & 500 & 98 & 51 & 14.0 & 2.9 & 840 \\
\hline 460059101423202 & $07-04-73$ & 1100 & 1.50 & 3.3 & 1.1 & 440 & 99 & 54 & 14.0 & 0.80 & 870 \\
\hline 460059101423203 & $07-04-73$ & 1080 & 1.47 & 4.6 & 2.3 & 410 & 97 & 39 & 13.0 & 2.7 & 780 \\
\hline 460101101265701 & $08-18-71$ & 816 & 1.11 & 4.7 & 0.40 & 330 & 98 & 39 & 11.0 & 1.2 & 680 \\
\hline 460102102135001 & $08-11-70$ & 2220 & 3.02 & 29 & 21 & 740 & 91 & 25 & - & 5.8 & 640 \\
\hline 460223102294601 & $06-18-71$ & 6150 & 8.36 & 400 & 240 & 1100 & 54 & 10 & - & 25 & 400 \\
\hline 460239101135301 & $07-10-73$ & 3320 & 4.52 & 120 & 49 & 900 & 79 & 17 & 0.0 & 6.4 & 500 \\
\hline 460240101075501 & $10-10-73$ & 1470 & 2.00 & 7.4 & 0.90 & 540 & 98 & 50 & -- & 2.0 & 680 \\
\hline 460244101272701 & $07-05-73$ & 1310 & 1.78 & 3.8 & 1.6 & 510 & 98 & 55 & 15.0 & 3.2 & 870 \\
\hline 460246101041201 & $09-24-69$ & 1350 & - & -- & - & -- & - & -- & - & -- & -- \\
\hline 460246101041202 & $09-23-69$ & 1770 & - & -- & -- & -- & - & - & -. & - & -. \\
\hline 460332102105202 & $10-10-71$ & 1250 & 1.70 & 14 & 6.3 & 450 & 93 & 25 & 15.0 & 5.9 & 940 \\
\hline 460332102105203 & $06-15-72$ & 1100 & 1.50 & 6.0 & 1.9 & 420 & 97 & 38 & 17.0 & 3.0 & 980 \\
\hline 460336102334001 & $07-08-71$ & 2170 & 2.95 & 13 & 7.2 & 760 & 96 & 42 & - & 4.3 & 730 \\
\hline 460337101404201 & $11-03-71$ & 1040 & 1.41 & 4.0 & 1.7 & 410 & 98 & 43 & 15.0 & 1.2 & 890 \\
\hline 460358102110101 & $06-29-71$ & 1170 & 1.59 & 4.2 & 1.6 & 460 & 98 & 48 & - & 2.1 & 920 \\
\hline 460449101200702 & $04-18-72$ & 1420 & 1.93 & 100 & 77 & 220 & 44 & 4 & 0.0 & 9.2 & 300 \\
\hline 460501102405801 & $07-02-71$ & 913 & 1.24 & 11 & 6.9 & 340 & 92 & 20 & - & 5.2 & 630 \\
\hline 460521102384801 & $07-02-71$ & 1240 & 1.69 & 20 & 13 & 410 & 89 & 18 & - & 6.7 & 620 \\
\hline 460522101550201 & $09-08-71$ & 2170 & 2.95 & 27 & 8.4 & 760 & 94 & 33 & 15.0 & 4.5 & 1050 \\
\hline 460526102284101 & $06-15-72$ & 1080 & 1.47 & 10 & 9.0 & 430 & 93 & 24 & 17.0 & 2.9 & 1120 \\
\hline 460526102284102 & $06-15-72$ & 1400 & 1.90 & 18 & 12 & 480 & 91 & 22 & 14.0 & 3.8 & 880 \\
\hline 460541101562201 & $06-16-67$ & 1520 & -- & - & - & 590 & - & - & - & - & 1060 \\
\hline 460608102042801 & $04-14-71$ & 1110 & 1.51 & 4.6 & 1.6 & 480 & 98 & 50 & -- & 2.2 & 1180 \\
\hline 460639102570801 & $06-30-71$ & 768 & 1.04 & 59 & 31 & 160 & 54 & 4 & -- & 11 & 410 \\
\hline
\end{tabular}




\begin{tabular}{|c|c|c|c|c|c|c|c|c|c|c|c|}
\hline Slte number & Date & $\begin{array}{c}\text { Car- } \\
\text { bonate } \\
\text { (mg/L as } \\
\left.\mathrm{CO}_{3}\right)\end{array}$ & $\begin{array}{l}\text { Carbon } \\
\text { dioxide, } \\
\text { dis- } \\
\text { solved } \\
(\mathrm{mg} / \mathrm{L} \text { as } \\
\left.\mathrm{CO}_{2}\right)\end{array}$ & $\begin{array}{l}\text { Sulfate, } \\
\text { die- } \\
\text { solved } \\
\left(\mathrm{mgll}_{\mathrm{L}} \mathrm{s}\right. \\
\left.\mathrm{SO}_{4}\right)\end{array}$ & $\begin{array}{l}\text { Chlo- } \\
\text { ride, } \\
\text { dis- } \\
\text { solved } \\
\text { (mglL } \\
\text { as Cl) }\end{array}$ & $\begin{array}{l}\text { Fluoride, } \\
\text { dis- } \\
\text { solved } \\
\text { (mg/L } \\
\text { as F) }\end{array}$ & $\begin{array}{l}\text { Silica, } \\
\text { dle- } \\
\text { solved } \\
\text { (mg/L as } \\
\left.\text { SiO }_{2}\right)\end{array}$ & $\begin{array}{l}\text { Nitro- } \\
\text { gen, } \\
\text { nitrate, } \\
\text { dis- } \\
\text { solved } \\
\text { (mg'L } \\
\text { as N) }\end{array}$ & $\begin{array}{l}\text { Nitro- } \\
\text { gen, } \\
\text { nitrate, } \\
\text { total } \\
(\mathrm{mg} / \mathrm{L} \\
\left.\text { as } \mathrm{NO}_{3}\right)\end{array}$ & $\begin{array}{l}\text { Nitro- } \\
\text { gen, } \\
\text { nitrate, } \\
\text { dis- } \\
\text { solved } \\
\text { (mglL } \\
\text { as } \mathrm{NO}_{3} \text { ) }\end{array}$ & $\begin{array}{c}\text { Boron, } \\
\text { dis- } \\
\text { solved } \\
(\mu g / L \\
\text { as B) }\end{array}$ \\
\hline 455645101434801 & $08-16-71$ & 0 & -- & 670 & 6.4 & 0.30 & 15 & 0.045 & 0.20 & - & 620 \\
\hline 455704101414501 & $06-02-69$ & -- & -- & 2000 & 17 & - & -- & 11.3 & 50 & - & - \\
\hline 455733101172701 & $08-19-71$ & 0 & -- & 540 & 7.8 & 0.30 & 5.4 & 1.69 & 7.5 & -. & 1400 \\
\hline 455742102094601 & $06-23-71$ & 0 & -- & 1500 & 29 & 0.70 & 9.3 & - & - & 8.1 & 730 \\
\hline 455805101574601 & $08-11-71$ & $\mathbf{0}$ & -- & 580 & 3.3 & 1.3 & 9.0 & 0.226 & 1.0 & -- & 1500 \\
\hline 455822102190802 & $06-15-71$ & 0 & 8.2 & 460 & 24 & 1.0 & 10 & 5.90 & - & 26 & 340 \\
\hline 455822102190803 & $06-24-71$ & 14 & -- & 150 & 9.6 & 8.9 & 8.0 & - & - & 1.0 & 1200 \\
\hline 455823101450301 & $08-16-71$ & 14 & - & 480 & 1.5 & 1.2 & 9.8 & 0.565 & 2.5 & - & 890 \\
\hline 455823101450302 & $08-16-71$ & 6 & - & 510 & 2.6 & 1.1 & 10 & 0.565 & 2.5 & - & 1100 \\
\hline 455828101592301 & $04-12-59$ & $\mathbf{0}$ & -- & 400 & 9.6 & 1.4 & 8.8 & 0.316 & 1.4 & - & 1200 \\
\hline 455832101120001 & $12-09-71$ & $\mathbf{0}$ & - & 340 & 0.0 & 0.60 & 10 & 0.226 & 1.0 & - & 890 \\
\hline 455850101510701 & $08-12-71$ & $\mathbf{0}$ & -- & 620 & 5.4 & 0.70 & 11 & 0.452 & 2.0 & -- & 1800 \\
\hline 455850101510702 & $08-12-71$ & 0 & -- & 1000 & 5.3 & 0.20 & 10 & 0.045 & 0.20 & - & 1000 \\
\hline 455912102064701 & $06-21-71$ & $\mathbf{0}$ & -- & 150 & 2.1 & 0.50 & 11 & - & - & 0.0 & 310 \\
\hline 455922101511701 & $08-12-71$ & 0 & - & 890 & 7.5 & 0.50 & 10 & 0.565 & 2.5 & -- & 2000 \\
\hline 460010102060001 & $06-15-71$ & 12 & -- & 420 & 5.3 & 1.3 & 9.7 & - & - & 1.0 & 240 \\
\hline 460032102210001 & $06-14-72$ & 19 & - & 14 & 67 & 3.2 & 8.1 & - & - & 1.0 & 820 \\
\hline 460032102210002 & $06-13-72$ & 0 & - & 170 & 13 & 5.5 & 8.0 & -- & - & 1.0 & 1200 \\
\hline 460034103133201 & $08-20-70$ & 14 & - & 680 & 8.5 & 11 & 7.7 & - & - & 1.3 & 1000 \\
\hline 460056102061001 & $09-27-71$ & 51 & - & 24 & 58 & 3.0 & 7.7 & - & -- & 1.0 & 750 \\
\hline 460059101423201 & $07-04-73$ & 24 & 4.5 & 23 & 270 & 3.2 & 9.1 & 0.230 & 1.0 & 1.0 & 2200 \\
\hline 460059101423202 & $07-04-73$ & 0 & 7.0 & 110 & 60 & 4.3 & 11 & 0.010 & 1.0 & 0.04 & 2200 \\
\hline 460059101423203 & $07-04-73$ & 7 & 6.4 & 210 & 39 & 3.0 & 8.2 & 0.560 & 2.5 & 2.5 & 1900 \\
\hline 460101101265701 & $08-18-71$ & 13 & - & 130 & 1.7 & 2.9 & 10 & 0.226 & 1.0 & - & 2100 \\
\hline 460102102135001 & $08-11-70$ & 4 & - & 1200 & 1.6 & 1.3 & 9.1 & -- & - & 0.0 & 740 \\
\hline 460223102294601 & $06-18-71$ & $\mathbf{0}$ & - & 4000 & 34 & 0.80 & 21 & -- & -- & 21 & 1200 \\
\hline 460239101135301 & $07-10-73$ & 0 & 7.9 & 1900 & 83 & 0.30 & 7.3 & 6.30 & 28 & 28 & 1200 \\
\hline 460240101075501 & $10-10-73$ & 11 & 4.4 & 410 & 130 & 2.0 & 13 & 0.050 & - & 0.20 & 2600 \\
\hline 460244101272701 & $07-05-73$ & 20 & 4.6 & 30 & 250 & 3.2 & 11 & 0.050 & 0.20 & 0.20 & 2300 \\
\hline 460246101041201 & $09-24-69$ & -- & - & 600 & 8.8 & - & -- & 0.00 & 0.0 & -- & - \\
\hline 460246101041202 & $09-23-69$ & -- & -- & 850 & 21 &.- & - & 0.00 & 0.0 & - & -- \\
\hline 460332102105202 & $10-10-71$ & 17 & - & 180 & 20 & 5.1 & 7.0 & -- & -- & 2.0 & 1000 \\
\hline 460332102105203 & $06-15-72$ & 53 & - & 13 & 0.30 & 2.7 & 7.3 & -- & - & 1.0 & 1100 \\
\hline 460336102334001 & $07-08-71$ & 0 & -- & 1000 & 6.5 & 3.0 & 9.1 & - & - & 0.40 & 1000 \\
\hline 460337101404201 & $11-03-71$ & 19 & - & 7.9 & 97 & 4.4 & 11 & 0.407 & 1.8 & - & 2000 \\
\hline 460358102110101 & $06-29-71$ & 0 & - & 210 & 7.3 & 7.2 & 8.2 & - & - & 0.0 & 1500 \\
\hline 460449101200702 & $04-18-72$ & 0 & - & 810 & 6.3 & 0.10 & 4.9 & 0.565 & 2.5 & -- & 360 \\
\hline 460501102405801 & $07-02-71$ & 0 & - & 250 & 3.1 & 0.30 & 9.4 & - & - & 1.2 & 450 \\
\hline 460521102384801 & $07-02-71$ & 0 & -- & 450 & 4.2 & 1.1 & 8.7 & - & - & 1.0 & 550 \\
\hline 460522101550201 & $09-08-71$ & 0 & - & 870 & 3.5 & 0.20 & 12 & 0.655 & 2.9 & - & 1100 \\
\hline 460526102284101 & $06-15-72$ & 0 & -- & 12 & 23 & 6.5 & 8.9 & - & - & 1.0 & 1400 \\
\hline 460526102284102 & $06-15-72$ & 40 & -- & 360 & 10 & 6.3 & 7.3 & - & - & 1.0 & 1300 \\
\hline 460541101562201 & $06-16-67$ & 0 & - & 400 & 14 & -- & - & 0.00 & 0.0 & -- & -- \\
\hline 460608102042801 & $04-14-71$ & 0 & - & 49 & 48 & 4.8 & 9.8 & -- & - & 1.0 & 830 \\
\hline 460639102570801 & $06-30-71$ & 0 & - & 270 & 4.5 & 0.30 & 8.4 & - & - & 2.5 & 900 \\
\hline
\end{tabular}




\begin{tabular}{|c|c|c|c|c|c|c|c|c|c|c|c|}
\hline Site number & Date & $\begin{array}{l}\text { iron, } \\
\text { dis- } \\
\text { solved } \\
(\mu g / L \\
\text { as Fe) }\end{array}$ & $\begin{array}{l}\text { Lead, } \\
\text { dis- } \\
\text { solved } \\
(\mu g / L \\
\text { as } \mathrm{Pb})\end{array}$ & $\begin{array}{l}\text { Lithium, } \\
\text { dis- } \\
\text { solved } \\
(\mu g / L \\
\text { as } L)\end{array}$ & $\begin{array}{l}\text { Manga- } \\
\text { nose, } \\
\text { dis- } \\
\text { solved } \\
\text { ( } \mu g / L \\
\text { as Mn) }\end{array}$ & $\begin{array}{c}\text { Mercury, } \\
\text { dis-- } \\
\text { solved } \\
(\mu g / L \\
\text { as } \mathrm{Hg})\end{array}$ & $\begin{array}{c}\text { Molyb- } \\
\text { denum, } \\
\text { dis- } \\
\text { solved } \\
\text { ( } \mu g / L \\
\text { as Mo) }\end{array}$ & $\begin{array}{l}\text { Selo- } \\
\text { nium, } \\
\text { dis:- } \\
\text { solved } \\
(\mu g / L \\
\text { as So) }\end{array}$ & $\begin{array}{l}\text { Stron- } \\
\text { tlum, } \\
\text { dis- } \\
\text { solved } \\
\text { ( } \mu g / L \\
\text { as Sr) }\end{array}$ & $\begin{array}{l}\text { Vana- } \\
\text { dium, } \\
\text { dis- } \\
\text { solved } \\
(\mu g / L \\
\text { as V) }\end{array}$ & $\begin{array}{l}\text { Zinc, } \\
\text { dise- } \\
\text { solved } \\
(\mu g / L \\
\text { as Zn) }\end{array}$ \\
\hline 455645101434801 & 08-16-71 & 240 & -- & -- & 140 & -- & -- & - & -- & .. & .- \\
\hline 455704101414501 & $06-02-69$ & 3200 & -- & -- & -- & -- & -- & -- & -- & -- & .- \\
\hline 455733101172701 & $08-19-71$ & 0 & -- & .- & 50 & -. & -- & .- & -. & .- & -- \\
\hline 455742102094601 & $06-23-71$ & 70 & -- & -- & 30 & -. & -- & -. & .- & .- & -- \\
\hline 455805101574601 & $08-11-71$ & 0 & -- & -- & 140 & -- & - & -- & - & -- & - \\
\hline 455822102190802 & $06-15-71$ & 80 & - & -- & - & -- & -- & -- & -- & -- & .- \\
\hline 455822102190803 & $06-24-71$ & 480 & -- & -- & 10 & -- & - & -. & -- & -- & -- \\
\hline 455823101450301 & $08-16-71$ & 0 & -- & - & 30 & -- & - & .. & -. & -. & -- \\
\hline 455823101450302 & 08-16-71 & 0 & -- & -- & 20 & -- & - & -- & -- & .- & - \\
\hline 455828101592301 & $04-12-59$ & 220 & -- & -- & 10 & -- & - & -- & -- & -. & -- \\
\hline 455832101120001 & $12-09-71$ & 0 & -- & -- & 20 & -- & - & - & .- & -- & -- \\
\hline 455850101510701 & $08-12-71$ & 0 & -. & -- & 10 & -- & - & -- & -- & -. & -- \\
\hline 455850101510702 & $08-12-71$ & 0 & -- & -- & 40 & -. & - & - & -- & -- & -- \\
\hline 455912102064701 & $06-21-71$ & 100 & -- & - & 20 & .- & -- & -. & -- & -- & -- \\
\hline 455922101511701 & $08-12-71$ & 0 & -- & -- & 40 & -- & -- & - & - & -. & -- \\
\hline 460010102060001 & $06-15-71$ & 50 & -- & -- & 20 & -- & -- & -- & -- & -- & -- \\
\hline 460032102210001 & $06-14-72$ & 90 & -- & -- & 50 & -- & -- & - & -- & -- & -- \\
\hline 460032102210002 & $06-13-72$ & 140 & -- & -- & 30 & -- & -- & -- & -- & -- & -- \\
\hline 460034103133201 & $08-20-70$ & 0 & -- & -- & 20 & -- & - & - & -- & -- & -- \\
\hline 460056102061001 & $09-27-71$ & 120 & -. & - & 40 & -- & - & -- & -- & -. & -- \\
\hline 460059101423201 & $07-04-73$ & 260 & - & -. & 20 & -- & - & -- & -- & -- & - \\
\hline 460059101423202 & $07-04-73$ & 1100 & -- & - & 20 & -- & -- & -- & -- & .- & _- \\
\hline 460059101423203 & $07-04-73$ & 40 & - & -- & $<10$ & -- & - & -- & -- & -- & -- \\
\hline 460101101265701 & 08-18-71 & 0 & -- & - & 10 & -. & - & -. & .- & .- & -- \\
\hline 460102102135001 & $08-11-70$ & 320 & -- & -- & 20 & -- & - & - & - & -- & - \\
\hline 460223102294601 & $06-18-71$ & 4800 & - & -- & 3000 & -. & -- & - & - & .- & -- \\
\hline 460239101135301 & $07-10-73$ & 150 & -- & -- & 70 & -- & - & - & - & .- & .- \\
\hline 460240101075501 & $10-10-73$ & 1200 & - & -- & 80 & .- & -- & _- & - & .- & -- \\
\hline 460244101272701 & $07-05-73$ & $<10$ & - & -- & $<10$ & -- & -- & -- & - & -- & -- \\
\hline 460246101041201 & 09-24-69 & 690 & -. & -- & -- & -- & -- & -- & .- & -. & -- \\
\hline 460246101041202 & 09-23-69 & 2100 & -- & -- & - & -. & - & - & -- & -- & -- \\
\hline 460332102105202 & $10-10-71$ & 0 & -- & -- & 30 & -- & -- & -- & -- & -- & - \\
\hline 460332102105203 & $06-15-72$ & 90 & -- & -- & 50 & -. & -- & -- & -- & -- & -- \\
\hline 460336102334001 & $07-08-71$ & $\mathbf{0}$ & - & -- & 50 & -- & -- & -- & -- & -- & -- \\
\hline 460337101404201 & $11-03-71$ & 0 & - & - & 10 & -- & - & - & - & -- & -- \\
\hline 460358102110101 & $06-29-71$ & 1700 & - & - & 10 & -- & - & -- & -- & -- & -- \\
\hline 460449101200702 & 04-18-72 & 0 & -- & -- & 80 & -- & -- & - & -- & -- & -- \\
\hline 460501102405801 & $07-02-71$ & 0 & -- & - & 20 & -- & -- & - & -- & -- & -- \\
\hline 460521102384801 & $07-02-71$ & 0 & - & -- & 30 & -- & -- & -- & -- & -- & -- \\
\hline 460522101550201 & 09-08-71 & 0 & - & - & 80 & -- & - & -- & - & -- & -- \\
\hline 460526102284101 & $06-15-72$ & 160 & -- & -- & 40 & -- & - & -- & -- & -- & - \\
\hline 460526102284102 & $06-15-72$ & 0 & -- & -- & 60 & -. & -- & -- & -- & -- & -- \\
\hline 460541101562201 & $06-16-67$ & 1900 & - & -- & - & -- & -- & -- & -- & -. & -- \\
\hline 460608102042801 & $04-14-71$ & 280 & - & -- & 10 & -- & -- & -- & -- & -- & -- \\
\hline 460639102570801 & $06-30-71$ & 0 & -- & - & 30 & -- & -- & -- & -- & .. & -- \\
\hline
\end{tabular}




\begin{tabular}{|c|c|c|c|c|c|c|c|c|c|c|}
\hline Site number & Local identifler & $\begin{array}{l}\text { County } \\
\text { code }\end{array}$ & $\begin{array}{c}\text { Hydrologle } \\
\text { unlt }\end{array}$ & $\begin{array}{c}\text { Goologic } \\
\text { unit }\end{array}$ & Date & $\begin{array}{l}\text { Depth } \\
\text { below } \\
\text { land } \\
\text { surface, } \\
\text { water } \\
\text { level } \\
\text { (feet) }\end{array}$ & $\begin{array}{l}\text { Depth } \\
\text { of } \\
\text { well, } \\
\text { total } \\
\text { (teot) }\end{array}$ & $\begin{array}{l}\text { Alttude } \\
\text { of land } \\
\text { surface, } \\
\text { datum } \\
\text { (feet) }\end{array}$ & $\begin{array}{l}\text { Speclfic } \\
\text { conduct- } \\
\text { ance, } \\
\text { field } \\
(\mu S / c m)\end{array}$ & $\begin{array}{c}\text { Speclfle } \\
\text { conduct- } \\
\text { ance, } \\
\text { lab } \\
(\mu \mathrm{S} / \mathrm{cm})\end{array}$ \\
\hline 460653101571101 & $130-090-4 \mathrm{BBD}$ & 037 & 10130205 & $125 \mathrm{CNBL}$ & $09-07-71$ & - & 65 & - & 2510 & - \\
\hline 460707101355201 & $131-087-32 C D C$ & 037 & 10130205 & $125 \mathrm{CNBL}$ & $11-05-71$ & -- & 327 & - & 897 & -- \\
\hline 460726101344601 & 131-087-33CBA & 037 & 10130205 & $125 \mathrm{CNBL}$ & $11-05-71$ & - & 317 & - & 1120 & -- \\
\hline 460737101200701 & 131-085-32ADA & 037 & 10130204 & -- & $08-31-72$ & - & 394 & - & 1310 & - \\
\hline 460804102435401 & $131-096-30 \mathrm{DCD}$ & 001 & 10130205 & 125TRVL & $06-08-71$ & -- & 122 & -- & 1330 & -- \\
\hline 460810102332801 & $131-095-27 \mathrm{CCB}$ & 001 & 10130205 & 125TRVL & $08-07-70$ & - & 125 & -- & 1640 & - \\
\hline 460825101344601 & $131-087-28 B C D$ & 037 & 10130204 & $125 \mathrm{CNBL}$ & $03-06-70$ & - & 246 & - & 1210 & -- \\
\hline 460830102405702 & $131-096-27 \mathrm{BCC} 2$ & 001 & 10130204 & 125TRVL & $06-08-71$ & - & 140 & - & 2920 & - \\
\hline 460830103044503 & 131-099-29ADD3 & 011 & 10130205 & $125 \mathrm{HRMN}$ & $06-24-76$ & -- & 80 & 2796 & 1520 & -- \\
\hline 460845101512001 & 131-089-30AAA & 037 & 10130205 & 211FXHL & $07-02-73$ & -- & 809 & 2395 & 2330 & -- \\
\hline 460903102410701 & 131-096-21DDA & 001 & 10130205 & 125TRVL & $06-08-71$ & -- & 100 & -- & 1760 & -- \\
\hline 460908102282701 & $131-094-20 \mathrm{CBC} 1$ & 001 & 10130205 & $211 \mathrm{HCFH}$ & $07-22-71$ & - & 1045 & 2500 & 1930 & -- \\
\hline 460908102282702 & $131-094-20 \mathrm{CBC} 2$ & 001 & 10130205 & 125LHCK & $10-14-71$ & -- & 537 & 2500 & 2020 & - \\
\hline 460908102282703 & $131-094-20 \mathrm{CBC} 3$ & 001 & 10130205 & 125LDLW & $10-12-71$ & -- & 224 & 2500 & 1590 & - \\
\hline 460910102533301 & 131-098-23DAD1 & 001 & 10130205 & $125 \mathrm{CNBL}$ & $06-21-72$ & - & 356 & 2882 & 1760 & - \\
\hline 460910102533302 & 131-098-23DAD2 & 001 & 10130205 & $125 \mathrm{TRVL}$ & $06-21-72$ & - & 248 & 2882 & 1610 & -- \\
\hline 460939102183901 & 131-093-21AAA1 & 001 & 10130205 & 125LDLW & $06-13-72$ & - & 334 & 2549 & 1600 & - \\
\hline 460939102183902 & 131-093-21AAA2 & 001 & 10130205 & 125LDLW & $06-14-72$ & -- & 253 & 2552 & 1630 & - \\
\hline 460939102183903 & 131-093-21 AAA3 & 001 & 10130205 & 125TRVL & $06-12-72$ & - & 143 & 2549 & 3500 & - \\
\hline 460941102330001 & 131-095-22BAA & 001 & 10130205 & 125TRVL & $07-07-71$ & -- & 31 & - & 2160 & - \\
\hline 460944102033101 & $131-091-15 C C C$ & 001 & 10130205 & 125LHCK & $06-09-72$ & - & 537 & 2360 & 1860 & - \\
\hline 460948102325101 & $131-095-15 \mathrm{DCC}$ & 001 & 10130205 & 125TRVL & $07-07-71$ & - & 63 & - & 1140 & - \\
\hline 460948102405701 & $131-096-15 \mathrm{CCC} 1$ & 001 & 10130205 & 125TRVL & $06-11-71$ & - & 60 & - & 1240 & -- \\
\hline 460951102010001 & $131-091-13 \mathrm{CCB}$ & 001 & 10130205 & 125LDLW & $08-07-70$ & - & 345 & 2489 & 1870 & - \\
\hline 460954101240201 & 131-086-14DAC & 037 & 10130204 & 211HLCK & $00-00-65$ & -- & 127 & - & 673 & - \\
\hline 461015102433501 & 131-096-18ADD & 001 & 10130205 & 125TRVL & $06-08-71$ & - & 202 & -- & 803 & - \\
\hline 461033101200701 & 131-085-8DDD1 & 037 & 10130204 & 211HLCK & $11+17-71$ & -- & 394 & - & 1740 & -- \\
\hline 461037102033101 & $131-091-10 \mathrm{CCC}$ & 001 & 10130205 & 125LDLW & $09-22-71$ & - & 408 & 2450 & 1780 & - \\
\hline 461040102575401 & $131-098-8 C D D$ & 001 & 10130205 & 125TRVL & $06-15-71$ & - & 170 & - & 2030 & -- \\
\hline 461050103010001 & 130-099-11ADD & 011 & 10130205 & 125TRVL & $05-16-74$ & -- & 79 & - & 3790 & - \\
\hline 461059102283701 & 131-094-7DAA & 001 & 10130205 & -- & $08-21-70$ & -- & 101 & -- & 9430 & -- \\
\hline 461106102593701 & $131-098-7 \mathrm{BCC}$ & 001 & 10130205 & $125 \mathrm{CNBL}$ & $06-15-71$ & -- & 352 & 2959 & 1440 & -- \\
\hline 461123102132201 & $131-092-8 B B A$ & 001 & 10130205 & 125TRVL & $08-07-70$ & - & 202 & - & 3080 & -- \\
\hline 461129101562401 & $131-090-4 \mathrm{DDCl}$ & 037 & 10130205 & 125TGRV & $09-09-71$ & -- & 210 & - & 581 & -- \\
\hline 461145102385701 & $131-096-2 \mathrm{DBD}$ & 001 & 10130205 & 125TRVL & $06-11-71$ & - & 73 & - & 1370 & - \\
\hline 461148101565102 & $131-090-4 \mathrm{CAA}$ & 037 & 10130205 & 125TGRV & $09-09-71$ & - & 230 & - & 1040 & -. \\
\hline 461151103033101 & 131-099-4DAA & 011 & 10130205 & 125TRVL & $04-14-71$ & - & 390 & 2955 & 1450 & -- \\
\hline 461158101262201 & 131-086-4ADA & 037 & 10130204 & $125 \mathrm{CNBL}$ & $11-15-71$ & - & -- & - & 680 &.- \\
\hline 461159102532304 & $131-098-1 B C C 4$ & 001 & 10130205 & 125TRVL & $06-15-71$ & -- & 81 & - & 1580 & - \\
\hline 461201101372501 & $131-087-6 \mathrm{BCB} 1$ & 037 & 10130204 & 211FXHL & $11-05-71$ & -- & 560 & - & 1770 & - \\
\hline 461207101373501 & 131-088-1AAD & 037 & 10130204 & $125 \mathrm{CNBL}$ & $02-03-71$ & - & 560 & - & 1750 & - \\
\hline 461216101594301 & $132-090-31 \mathrm{CCD} 1$ & 037 & 10130205 & 125TGRV & $00-00-59$ & - & 150 & - & 1210 & - \\
\hline 461219101103202 & $132-083-35 D D C 2$ & 085 & 10130206 & $112 S L D S$ & $08-25-71$ & - & 93 & 1822 & 858 & - \\
\hline 461226103045401 & 132-099-32DDCl & 011 & 10130205 & 125LHCK & $10-11-71$ & -- & 572 & 2900 & 2600 & -- \\
\hline 461226103045402 & $132-099-32 D D C 2$ & 011 & 10130205 & 125TRVL & $09-11-71$ & - & 110 & 2900 & 1130 & - \\
\hline
\end{tabular}




\begin{tabular}{|c|c|c|c|c|c|c|c|c|c|c|c|}
\hline Stte number & Dete & $\begin{array}{c}\text { pH, } \\
\text { field } \\
\text { (stand- } \\
\text { ard } \\
\text { unite }\end{array}$ & $\begin{array}{l}\text { pH, } \\
\text { lab } \\
\text { (stand- } \\
\text { ard } \\
\text { unite) }\end{array}$ & $\begin{array}{l}\text { Tomper- } \\
\text { ature, } \\
\text { water } \\
\text { (degrees } \\
\text { Colsius) }\end{array}$ & $\begin{array}{l}\text { Coior } \\
\text { (plat1- } \\
\text { num } \\
\text { cobalt } \\
\text { scalo) }\end{array}$ & $\begin{array}{l}\text { Hard- } \\
\text { ness, } \\
\text { total } \\
\text { (mgll as } \\
\text { Cacos) }\end{array}$ & $\begin{array}{l}\text { Noncar- } \\
\text { bonate } \\
\text { hard- } \\
\text { ness, } \\
\text { total } \\
\text { (mgll as } \\
\left.\mathrm{CaCO}_{3}\right)\end{array}$ & $\begin{array}{l}\text { Alka- } \\
\text { linity, } \\
\text { fieid } \\
(\mathrm{mg} / \mathrm{L} \text { as } \\
\left.\mathrm{CaCO}_{3}\right)\end{array}$ & $\begin{array}{c}\text { Aika- } \\
\text { Ilnity, } \\
\text { lab } \\
\text { (mg/L as } \\
\text { CaCO }\end{array}$ & $\begin{array}{l}\text { Solids, } \\
\text { sum of } \\
\text { constit- } \\
\text { uents, } \\
\text { dis- } \\
\text { eoived } \\
\text { (mgll) }\end{array}$ & $\begin{array}{c}\text { Solids, } \\
\text { residue } \\
\text { at } 105 \\
\text { degrees } \\
\text { Colsius, } \\
\text { total } \\
\text { (mgll) }\end{array}$ \\
\hline 460653101571101 & $09-07-71$ & 8.2 & - & - & -- & 240 & $\mathbf{0}$ & -- & - & 1720 & - \\
\hline 460707101355201 & $11-05-71$ & 8.2 & -- & 7.5 & - & 210 & 0 & - & - & 570 & -- \\
\hline 460726101344601 & $11-05-71$ & 8.0 & - & 7.0 & -- & 61 & $\mathbf{0}$ & -- & - & 717 & - \\
\hline 460737101200701 & $08-31-72$ & 7.4 & - & 17.5 & - & 360 & 170 & - & - & 914 & - \\
\hline 460804102435401 & $06-08-71$ & 7.5 & - & - & -- & 540 & 68 & - & - & 813 & - \\
\hline 460810102332801 & $08-07-70$ & 8.0 & - & 12.0 & - & 680 & 300 & - & - & 1170 & - \\
\hline 460825101344601 & $03-06-70$ & 7.9 & -- & -- & - & 200 & - & - & - & 694 & - \\
\hline 460830102405702 & $06-08-71$ & 8.0 & -- & - & - & 160 & 0 & -- & - & 2020 & -- \\
\hline 460830103044503 & $06-24-76$ & 7.6 & -- & 8.0 & 4 & 230 & 0 & - & 466 & 1160 & - \\
\hline 460845101512001 & $07-02-73$ & 8.5 & -- & 13.0 & -- & 12 & 0 & -- & -- & 1370 & -- \\
\hline 460903102410701 & $06-08-71$ & 7.5 & - & 8.5 & -- & 590 & 150 & - & - & 1260 & - \\
\hline 460908102282701 & $07-22-71$ & 7.8 & -- & 19.0 & - & 25 & 0 & - & - & 1250 & -- \\
\hline 460908102282702 & $10-14-71$ & 8.4 & - & 9.5 & - & 50 & 0 & - & - & 1260 & - \\
\hline 460908102282703 & $10-12-71$ & 8.4 & - & 9.5 & - & 64 & 0 & - & - & 1010 & -- \\
\hline 460910102533301 & $06-21-72$ & 8.6 & - & 10.5 & - & 110 & 0 & - & -- & 1250 & -- \\
\hline 460910102533302 & $06-21-72$ & 8.2 & - & 9.5 & - & 98 & 0 & - & - & 1160 & - \\
\hline 460939102183901 & $06-13-72$ & 8.2 & -- & 11.5 & - & 40 & 0 & - & -- & 989 & - \\
\hline 460939102183902 & $06-14-72$ & 8.3 & -- & 10.5 & -- & 32 & 0 & -- & - & 1000 & -- \\
\hline 460939102183903 & $06-12-72$ & 7.7 & - & 11.0 & - & 140 & 0 & - & - & 2510 & -. \\
\hline 460941102330001 & $07-07-71$ & 7.8 & - & - & - & 840 & 430 & - & - & 1140 & - \\
\hline 460944102033101 & $06-09-72$ & 8.3 & - & 10.5 & - & 19 & 0 & - & -- & 1200 & - \\
\hline 460948102325101 & $07-07-71$ & 8.1 & - & 9.0 & - & 180 & 0 & -- & - & 753 & -- \\
\hline 460948102405701 & $06-11-71$ & 7.5 & - & -- & - & 520 & 76 & -- & - & 825 & -- \\
\hline 460951102010001 & $08-07-70$ & 8.3 & - & 13.0 & - & 17 & 0 & -- & - & 1160 & -- \\
\hline 460954101240201 & $00-00-65$ & 7.7 & - & - & - & 91 & - & - & - & 299 & - \\
\hline 461015102433501 & $06-08-71$ & 7.6 & - & 10.0 & -- & 290 & 0 & -- & -- & 513 & - \\
\hline 461033101200701 & $11-17-71$ & 8.4 & -- & - & -- & 18 & 0 & -- & - & 1040 & - \\
\hline 461037102033101 & $09-22-71$ & 8.5 & - & 1.0 & -- & 19 & 0 & - & -- & 1150 & - \\
\hline 461040102575401 & $06-15-71$ & 7.4 & - & -. & -- & 750 & 30 & - & - & 1480 & - \\
\hline 461050103010001 & $05-16-74$ & 9.3 & - & 8.0 & 10 & 100 & 0 & - & 896 & 2510 & - \\
\hline 461059102283701 & $08-21-70$ & 6.9 & - & - & - & 5900 & 5700 & - & - & 10000 & - \\
\hline 461106102593701 & $06-15-71$ & 8.5 & 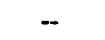 & 9.5 & -- & 52 & 0 & - & -- & 954 & - \\
\hline 461123102132201 & $08-07-70$ & 7.9 & -- & 11.0 & -- & 54 & 0 & - & -- & 2170 & - \\
\hline 461129101562401 & $09-09-71$ & 8.0 & -- & 9.0 & - & 290 & 59 & -- & -- & 364 & -- \\
\hline 461145102385701 & $06-11-71$ & 9.1 & - & 8.0 & - & 31 & 0 & -- & - & 866 & - \\
\hline 461148101565102 & $09-09-71$ & 7.7 & - & - & - & 240 & 0 & - & - & 668 & - \\
\hline 461151103033101 & $04-14-71$ & 8.3 & -- & - & - & 37 & 0 & - & - & 932 & - \\
\hline 461158101262201 & $11-15-71$ & 8.0 & - & 9.5 & -- & 190 & 0 & - & - & 429 & - \\
\hline 461159102532304 & $06-15-71$ & 8.0 & -- & -- & -- & 82 & 0 & - & - & 1060 & -- \\
\hline 461201101372501 & $11-05-71$ & 8.5 & - & - & - & 21 & 0 & -- & - & 1130 & - \\
\hline 461207101373501 & $02-03-71$ & - & - & - & -- & 10 & -- & - & - & 1140 & - \\
\hline 461216101594301 & $00-00-59$ & 8.0 & - & - & -- & 470 & - & - & -- & 684 & - \\
\hline 461219101103202 & $08-25-71$ & 7.9 & - & 7.0 & - & 270 & 0 & -- & - & 561 & -- \\
\hline 461226103045401 & $10-11-71$ & 8.7 & -- & 11.0 & - & 170 & 0 & - & - & 1790 & - \\
\hline 461226103045402 & $09-11-71$ & 8.0 & -- & 8.5 & -- & 480 & 120 & - & -- & 762 & - \\
\hline
\end{tabular}




\begin{tabular}{|c|c|c|c|c|c|c|c|c|c|c|c|}
\hline Site number & Date & $\begin{array}{l}\text { Solids, } \\
\text { residue } \\
\text { at } 180 \\
\text { degrees } \\
\text { Calsius, } \\
\text { dis- } \\
\text { solved } \\
\text { (mg/L) }\end{array}$ & $\begin{array}{l}\text { Solids, } \\
\text { dis- } \\
\text { solved } \\
\text { (tons per } \\
\text { acre- } \\
\text { foot) }\end{array}$ & $\begin{array}{l}\text { Calcium, } \\
\text { dis- } \\
\text { solved } \\
\text { (mg/L } \\
\text { as Ca) }\end{array}$ & $\begin{array}{l}\text { Magne- } \\
\text { sium, } \\
\text { dis- } \\
\text { solved } \\
(\mathrm{mg} / \mathrm{L} \\
\text { as Mg) }\end{array}$ & $\begin{array}{l}\text { Sodium, } \\
\text { dis- } \\
\text { solved } \\
\text { (mg/L } \\
\text { as Na) }\end{array}$ & $\begin{array}{l}\text { Sodlum } \\
\text { (percent) }\end{array}$ & $\begin{array}{l}\text { Sodium } \\
\text { adsorp- } \\
\text { tion } \\
\text { ratio }\end{array}$ & $\begin{array}{l}\text { Resid- } \\
\text { ual, } \\
\text { sodium, } \\
\text { car- } \\
\text { bonate } \\
\text { (mg/L as } \\
\mathrm{CaCO}_{3} \text { ) }\end{array}$ & $\begin{array}{l}\text { Potas- } \\
\text { sium, } \\
\text { dis- } \\
\text { solved } \\
\text { (mg/L } \\
\text { as K) }\end{array}$ & $\begin{array}{c}\text { Bicar- } \\
\text { bonate } \\
\text { (mgle as } \\
\left.\mathrm{HCO}_{3}\right)\end{array}$ \\
\hline 460653101571101 & $09-07-71$ & 1700 & 2.31 & 38 & 36 & 520 & 82 & 15 & 6.00 & 3.9 & 690 \\
\hline 460707101355201 & $11-05-71$ & 546 & 0.74 & 58 & 16 & 110 & 53 & 3 & 1.00 & 4.2 & 340 \\
\hline 460726101344601 & $11-05-71$ & 691 & 0.94 & 16 & 5.1 & 240 & 89 & 14 & 7.00 & 2.7 & 480 \\
\hline 460737101200701 & $08-31-72$ & 950 & 1.29 & 70 & 44 & 160 & 48 & 4 & 0.0 & 9.2 & 220 \\
\hline 460804102435401 & $06-08-71$ & 827 & 1.12 & 110 & 67 & 83 & 25 & 2 & - & 9.9 & 570 \\
\hline 460810102332801 & $08-07-70$ & 1270 & 1.73 & 130 & 84 & 130 & 28 & 2 & - & 9.3 & 460 \\
\hline 460825101344601 & $03-06-70$ & 818 & -- & - & - & 240 & - & -. & - & - & -- \\
\hline 460830102405702 & $06-08-71$ & 2110 & 2.87 & 30 & 21 & 640 & 89 & 22 & - & 5.5 & 720 \\
\hline 460830103044503 & $06-24-76$ & 1130 & 1.54 & 42 & 30 & 310 & 73 & 9 & - & 14 & 570 \\
\hline 460845101512001 & $07-02-73$ & 1380 & 1.88 & 3.9 & 0.60 & 550 & 99 & 68 & 15.0 & 2.6 & 880 \\
\hline 460903102410701 & $06-08-71$ & 1270 & 1.73 & 110 & 75 & 200 & 42 & 4 & - & 11 & 540 \\
\hline 460908102282701 & $07-22-71$ & 1330 & 1.81 & 6.2 & 2.3 & 500 & 97 & 44 & -- & 3.0 & 890 \\
\hline 460908102282702 & $10-14-71$ & 1340 & 1.82 & 8.0 & 7.3 & 520 & 96 & 32 & 20.0 & 1.6 & 1250 \\
\hline 460908102282703 & $10-12-71$ & 1020 & 1.39 & 20 & 3.4 & 410 & 93 & 22 & 15.0 & 2.2 & 970 \\
\hline 460910102533301 & $06-21-72$ & 1260 & 1.71 & 19 & 16 & 430 & 89 & 18 & 9.00 & 1.9 & 640 \\
\hline 460910102533302 & $06-21-72$ & 1240 & 1.69 & 21 & 11 & 390 & 89 & 17 & 7.00 & 2.7 & 570 \\
\hline 460939102183901 & $06-13-72$ & 1030 & 1.40 & 10 & 3.6 & 410 & 95 & 28 & 16.0 & 2.3 & 1030 \\
\hline 460939102183902 & $06-14-72$ & 1040 & 1.41 & 6.6 & 3.8 & 410 & 96 & 31 & 16.0 & 3.6 & 960 \\
\hline 460939102183903 & $06-12-72$ & 2510 & 3.41 & 28 & 17 & 770 & 92 & 28 & 5.00 & 6.4 & 500 \\
\hline 460941102330001 & $07-07-71$ & 1610 & 2.19 & 150 & 110 & 170 & 31 & 3 & 0.0 & 12 & 5 \\
\hline 460944102033101 & $06-09-72$ & 1250 & 1.70 & 5.0 & 1.6 & 490 & 98 & 49 & 19.0 & 2.3 & 1170 \\
\hline 460948102325101 & $07-07-71$ & 692 & 0.94 & 21 & 31 & 200 & 69 & 6 & - & 9.3 & 370 \\
\hline 460948102405701 & $06-11-71$ & 844 & 1.15 & 90 & 71 & 91 & 27 & 2 & - & 10 & 540 \\
\hline 460951102010001 & $08-07-70$ & 1120 & 1.52 & 3.4 & 2.1 & 470 & 98 & 50 & - & 2.2 & 1150 \\
\hline 460954101240201 & $00-00-65$ & 397 & -- & -- & -- & $-\cdot$ & -- & - & - & -- & 280 \\
\hline 461015102433501 & $06-08-71$ & 505 & 0.69 & 58 & 36 & 71 & 34 & 2 & - & 8.1 & 410 \\
\hline 461033101200701 & $11-17-71$ & 1040 & 1.41 & 4.7 & 1.6 & 420 & 98 & 43 & 14.0 & 1.2 & 830 \\
\hline 461037102033101 & $09-22-71$ & 1170 & 1.59 & 3.8 & 2.3 & 470 & 98 & 47 & 19.0 & 1.8 & 1130 \\
\hline 461040102575401 & $06-15-71$ & 1470 & 2.00 & 130 & 100 & 230 & 39 & 4 & -- & 16 & 880 \\
\hline 461050103010001 & $05-16-74$ & 2650 & 3.60 & 32 & 5.4 & 880 & 94 & 38 & - & 11 & 730 \\
\hline 461059102283701 & $08-21-70$ & 10900 & 14.8 & 540 & 1100 & 830 & 24 & 5 & - & 9.6 & $120^{\circ}$ \\
\hline 461106102593701 & $06-15-71$ & 935 & 1.27 & 7.9 & 7.9 & 380 & 94 & 23 & 14.0 & 2.6 & 860 \\
\hline 461123102132201 & $08-07-70$ & 2150 & 2.92 & 0.30 & 13 & 710 & 96 & 42 & - & 3.9 & 460 \\
\hline 461129101562401 & $09-09-71$ & 368 & 0.50 & 71 & 27 & 12 & 8 & 0.3 & 0.0 & 3.6 & 280 \\
\hline 461145102385701 & $06-11-71$ & 873 & 1.19 & 6.3 & 3.8 & 310 & 95 & 24 & - & 3.1 & 500 \\
\hline 461148101565102 & $09-09-71$ & 665 & 0.90 & 61 & 21 & 140 & 55 & 4 & 2.00 & 13 & 410 \\
\hline 461151103033101 & $04-14-71$ & 953 & 1.30 & 6.8 & 4.9 & 360 & 95 & 26 & 12.0 & 1.5 & 770 \\
\hline 461158101262201 & $11-15-71$ & 412 & 0.56 & 59 & 9.2 & 77 & 47 & 2 & 1.00 & 2.1 & 290 \\
\hline 461159102532304 & $06-15-71$ & 1040 & 1.41 & 17 & 9.6 & 340 & 89 & 16 & - & 5.8 & 440 \\
\hline 461201101372501 & $11-05-71$ & 1100 & 1.50 & 4.4 & 2.4 & 430 & 98 & 40 & 12.0 & 1.3 & 740 \\
\hline 461207101373501 & $02-03-71$ & - & -- & -- & - & - & -. & - & - & - & - \\
\hline 461216101594301 & $00-00-59$ & 882 & - & -- & - & - & -- & -- & - & - & 230 \\
\hline 461219101103202 & $08-25-71$ & 525 & 0.71 & 71 & 23 & 94 & 42 & 2 & 2.00 & 4.5 & 440 \\
\hline 461226103045401 & $10-11-71$ & 1700 & 2.31 & 18 & 30 & 600 & 88 & 20 & 14.0 & 3.3 & 950 \\
\hline 461226103045402 & $09-11-71$ & 753 & 1.02 & 92 & 61 & 79 & 26 & 2 & 0.0 & 7.5 & 440 \\
\hline
\end{tabular}




\begin{tabular}{|c|c|c|c|c|c|c|c|c|c|c|c|}
\hline Ste number & Date & $\begin{array}{c}\text { Car- } \\
\text { bonate } \\
\text { (mg/l as } \\
\mathrm{CO}_{3} \text { ) }\end{array}$ & $\begin{array}{c}\text { Carbon } \\
\text { dioxide, } \\
\text { dis- } \\
\text { solved } \\
(\mathrm{mg} / \mathrm{Las} \\
\left.\mathrm{CO}_{2}\right)\end{array}$ & $\begin{array}{l}\text { Sulfate, } \\
\text { dis- } \\
\text { solved } \\
\text { (mg/l as } \\
\mathrm{SO}_{4} \text { ) }\end{array}$ & $\begin{array}{l}\text { Chlo- } \\
\text { ride, } \\
\text { dis- } \\
\text { solved } \\
\text { (mgll } \\
\text { as Cl) }\end{array}$ & $\begin{array}{l}\text { Fluoride, } \\
\text { dis- } \\
\text { solved } \\
\text { (mg/l } \\
\text { as F) }\end{array}$ & $\begin{array}{l}\text { Sillca, } \\
\text { dis- } \\
\text { solved } \\
(\mathrm{mgll} \mathrm{as} \\
\left.\mathrm{SiO}_{2}\right)\end{array}$ & $\begin{array}{l}\text { Nitro- } \\
\text { gen, } \\
\text { nitrate, } \\
\text { dis- } \\
\text { solved } \\
\text { (mg/L } \\
\text { as N) }\end{array}$ & $\begin{array}{c}\text { Nitro- } \\
\text { gen, } \\
\text { nitrate, } \\
\text { total } \\
\text { (mgll } \\
\left.\text { as } \mathrm{NO}_{3}\right)\end{array}$ & $\begin{array}{l}\text { Nitro- } \\
\text { gon, } \\
\text { nitrate, } \\
\text { dis- } \\
\text { solved } \\
\text { (mg/L } \\
\text { as } \mathrm{NO}_{3} \text { ) }\end{array}$ & $\begin{array}{c}\text { Boron, } \\
\text { dis- } \\
\text { solved } \\
(\mu g / L \\
\text { as B) }\end{array}$ \\
\hline 460653101571101 & $09-07-71$ & 0 & -- & 750 & 13 & 0.20 & 13 & 1.94 & 8.6 & - & 580 \\
\hline 460707101355201 & $11-05-71$ & 0 & -- & 150 & 7.8 & 0.60 & 13 & 8.81 & 39 & - & 40 \\
\hline 460726101344601 & $11-05-71$ & 0 & -- & 200 & 1.3 & 0.40 & 6.4 & 0.226 & 1.0 & - & 890 \\
\hline 460737101200701 & $08-31-72$ & 0 & -- & 510 & 0.0 & 0.20 & 5.4 & 0.565 & 2.5 & - & 470 \\
\hline 460804102435401 & $06-08-71$ & 0 & - & 250 & 4.0 & 0.40 & 14 & -- & -- & 1.0 & 0 \\
\hline 460810102332801 & $08-07-70$ & $\mathbf{0}$ & -- & 570 & 2.0 & 0.50 & 10 & - & - & 1.0 & 180 \\
\hline 460825101344601 & $03-06-70$ & - & - & 210 & 7.8 & -- & -- & 4.52 & 20 & - & 800 \\
\hline 460830102405702 & $06-08-71$ & 0 & - & 940 & 14 & 0.80 & 9.0 & - & - & 1.0 & 970 \\
\hline 460830103044503 & $06-24-76$ & 0 & 23 & 460 & 7.6 & 0.40 & 14 & - & - & - & 1000 \\
\hline 460845101512001 & $07-02-73$ & 21 & -- & 10 & 330 & 3.4 & 9.8 & 0.226 & 1.0 & - & 2200 \\
\hline 460903102410701 & $06-08-71$ & 0 & - & 580 & 5.6 & 0.60 & 11 & -- & - & 2.5 & 450 \\
\hline 460908102282701 & $07-22-71$ & 0 & -- & 44 & 240 & 3.6 & 9.7 & -- & -- & 1.0 & 1600 \\
\hline 460908102282702 & $10-14-71$ & 26 & -- & 9.5 & 65 & 3.4 & 10 & -- & - & 2.0 & 1400 \\
\hline 460908102282703 & $10-12-71$ & 21 & -- & 44 & 16 & 7.2 & 8.2 & - & - & 1.0 & 2300 \\
\hline 460910102533301 & $06-21-72$ & 24 & -- & 430 & 1.1 & 5.5 & 9.6 & - & -- & 2.0 & 680 \\
\hline 460910102533302 & $06-21-72$ & 0 & -- & 450 & 4.6 & 3.4 & 8.3 & -- & - & 0.0 & 540 \\
\hline 460939102183901 & $06-13-72$ & 0 & - & 23 & 20 & 5.3 & 9.0 & - & -- & 1.0 & 1400 \\
\hline 460939102183902 & $06-14-72$ & 15 & - & 62 & 17 & 5.5 & 7.3 & - & - & 1.0 & 1100 \\
\hline 460939102183903 & $06-12-72$ & 0 & - & 1400 & 1.8 & 0.80 & 8.0 & - & - & 1.0 & 390 \\
\hline 460941102330001 & $07-07-71$ & 0 & - & 370 & 67 & 0.30 & 9.3 & -- & - & 270 & 140 \\
\hline 460944102033101 & $06-09-72$ & 10 & -- & 99 & 0.60 & 3.2 & 9.2 & - & -- & 1.0 & 1400 \\
\hline 460948102325101 & $07-07-71$ & 0 & - & 300 & 2.7 & 0.10 & 5.0 & -- & - & 1.6 & 210 \\
\hline 460948102405701 & $06-11-71$ & 0 & - & 280 & 4.6 & 0.30 & 14 & -- & - & 1.2 & 450 \\
\hline 460951102010001 & $08-07-70$ & 12 & - & 42 & 50 & 2.7 & 10 & - & -- & 1.0 & 1300 \\
\hline 460954101240201 & $00-00-65$ & 0 & -- & 77 & 6.0 & -- & -- & 0.00 & 0.0 & -- & - \\
\hline 461015102433501 & $06-08-71$ & 0 & - & 120 & 2.5 & 0.40 & 13 & - & -- & 2.5 & 350 \\
\hline 461033101200701 & $11-17-71$ & 13 & - & 19 & 150 & 3.2 & 18 & 0.226 & 1.0 & - & 1700 \\
\hline 461037102033101 & $09-22-71$ & 25 & - & 33 & 50 & 2.1 & 9.4 & - & - & 1.0 & 1600 \\
\hline 461040102575401 & $06-15-71$ & 0 & - & 540 & 8.1 & 0.10 & 15 & - & - & 1.0 & 1900 \\
\hline 461050103010001 & $05-16-74$ & 180 & 0.9 & 1000 & 11 & 1.2 & 28 & - & -- & - & - \\
\hline 461059102283701 & $08-21-70$ & 0 & - & 7500 & 20 & 0.60 & 19 & - & -- & 84 & 4200 \\
\hline 461106102593701 & $06-15-71$ & 18 & - & 85 & 17 & 5.9 & 4.9 & - & -- & 0.30 & 840 \\
\hline 461123102132201 & $08-07-70$ & 0 & - & 1200 & 6.6 & 1.4 & 7.7 & - & - & 2.5 & 640 \\
\hline 461129101562401 & $09-09-71$ & 0 & - & 61 & 5.4 & 0.10 & 16 & 7.00 & 31 & -- & 40 \\
\hline 461145102385701 & $06-11-71$ & 44 & - & 240 & 3.7 & 0.70 & 1.2 & - & - & 1.0 & 450 \\
\hline 461148101565102 & $09-09-71$ & 0 & - & 210 & 0.90 & 0.10 & 11 & 0.226 & 1.0 & -. & 350 \\
\hline 461151103033101 & $04-14-71$ & 12 & -- & 150 & 6.3 & 3.4 & 9.2 & -- & - & 0.10 & 1200 \\
\hline 461158101262201 & $11-15-71$ & 0 & 4.6 & 120 & 0.30 & 0.60 & 16 & 0.230 & 1.0 & 1.0 & 40 \\
\hline 461159102532304 & $06-15-71$ & 0 & - & 460 & 6.3 & 1.0 & 7.6 & - & - & 0.0 & 1800 \\
\hline 461201101372501 & $11-05-71$ & 14 & - & 290 & 13 & 2.3 & 9.9 & 0.565 & 2.5 & -- & 1600 \\
\hline 461207101373501 & $02-03-71$ & - & - & -- & - & -. & - & -- & -- & -- & -- \\
\hline 461216101594301 & $00-00-59$ & 0 & - & 350 & 24 & -- & - & 21.5 & 95 & - & - \\
\hline 461219101103202 & $08-25-71$ & 0 & -- & 130 & 1.8 & 0.60 & 20 & 0.226 & 1.0 & - & 440 \\
\hline 461226103045401 & $10-11-71$ & 41 & - & 600 & 15 & 8.7 & 9.1 & - & - & 1.0 & 1100 \\
\hline 461226103045402 & $09-11-71$ & 0 & - & 290 & 3.2 & 0.40 & 15 & -- & - & 5.0 & 0 \\
\hline
\end{tabular}




\begin{tabular}{|c|c|c|c|c|c|c|c|c|c|c|c|}
\hline Stte number & Dete & $\begin{array}{l}\text { Iron, } \\
\text { dls- } \\
\text { solved } \\
\text { ( } \mu g / \mathrm{l} \\
\text { as Fo) }\end{array}$ & $\begin{array}{l}\text { Lead, } \\
\text { dis- } \\
\text { solved } \\
\text { ( } \mu g / \mathrm{L} \\
\text { as Pb) }\end{array}$ & $\begin{array}{l}\text { Lithium, } \\
\text { dls- } \\
\text { solved } \\
\text { ( } \mu g / L \\
\text { as Li) }\end{array}$ & $\begin{array}{l}\text { Manga- } \\
\text { nese, } \\
\text { dis- } \\
\text { solved } \\
\text { ( } \mu g / \mathrm{L} \\
\text { as Mn) }\end{array}$ & $\begin{array}{l}\text { Morcury, } \\
\text { dis- } \\
\text { solved } \\
(\mu g / L \\
\text { as Hg) }\end{array}$ & $\begin{array}{l}\text { Molyb- } \\
\text { donum, } \\
\text { dis- } \\
\text { solved } \\
(\mu g / \mathrm{L} \\
\text { as Mo) }\end{array}$ & $\begin{array}{l}\text { Sele- } \\
\text { nium, } \\
\text { dls- } \\
\text { solved } \\
(\mu g / L \\
\text { as So) }\end{array}$ & $\begin{array}{l}\text { Stron- } \\
\text { tlum, } \\
\text { dis- } \\
\text { solved } \\
(\mu g / L \\
\text { as Sr) }\end{array}$ & $\begin{array}{l}\text { Vana- } \\
\text { dium, } \\
\text { die- } \\
\text { solved } \\
(\mu g / L \\
\text { as V }\end{array}$ & $\begin{array}{l}\text { Zlnc, } \\
\text { dis- } \\
\text { soived } \\
\text { ( } \mu g / \mathrm{l} \\
\text { as } \mathrm{Zn} \text { ) }\end{array}$ \\
\hline 460653101571101 & $09-07-71$ & 600 & - & -- & 50 & -- & -- & - & - & -- & - \\
\hline 460707101355201 & $11-05-71$ & 0 & -- & - & 0 & - & - & -- & - & - & - \\
\hline 460726101344601 & $11-05-71$ & 0 & -- & -- & 60 & -- & -- & -- & - & -- & - \\
\hline 460737101200701 & $08-31-72$ & 340 & - & -- & 50 & -- & -- & - & - & -- & - \\
\hline 460804102435401 & $06-08-71$ & 1000 & -- & -- & 60 & -- & - & -- & - & - & -- \\
\hline 460810102332801 & $08-07-70$ & 180 & -- & -- & 470 & - & - & - & -- & - & - \\
\hline 460825101344601 & $03-06-70$ & 650 & - & - & -- & - & - & - & -- & - & -- \\
\hline 460830102405702 & $06-08-71$ & 0 & -- & - & 70 & -- & - & -- & - & - & - \\
\hline 460830103044503 & $06-24-76$ & -- & - & - & -- & - & -- & - & - & -- & - \\
\hline 460845101512001 & $07-02-73$ & 980 & -- & - & 40 & -- & -- & -- & - & - & -- \\
\hline 460903102410701 & $06-08-71$ & 180 & - & -- & 130 & -- & - & -- & -- & -- & - \\
\hline 460908102282701 & $07-22-71$ & 260 & -- & -- & 50 & - & -- & - & - & - & - \\
\hline 460908102282702 & $10-14-71$ & 680 & -- & - & 10 & - & - & - & -- & - & -- \\
\hline 460908102282703 & $10-12-71$ & 0 & -- & - & 20 & - & -- & -- & - & -- & -- \\
\hline 460910102533301 & $06-21-72$ & 40 & - & - & 20 & - & -- & - & -- & -- & -- \\
\hline 460910102533302 & $06-21-72$ & 0 & -- & - & 20 & -- & -- & - & -- & - & - \\
\hline 460939102183901 & $06-13-72$ & 40 & - & -. & 30 & -- & -- & - & - & - & - \\
\hline 460939102183902 & $06-14-72$ & 440 & - & -- & 40 & - & -- & - & -- & -- & -- \\
\hline 460939102183903 & $06-12-72$ & 0 & - & - & 40 & - & -- & - & - & -- & - \\
\hline 460941102330001 & $07-07-71$ & 0 & - & - & 30 & -- & -- & -- & - & -- & -- \\
\hline 460944102033101 & $06-09-72$ & 80 & - & - & 30 & - & - & - & - & - & - \\
\hline 460948102325101 & $07-07-71$ & 0 & - & -- & 10 & - & - & - & - & -- & -- \\
\hline 460948102405701 & $06-11-71$ & 0 & -- & - & 40 & -- & - & -- & -- & - & - \\
\hline 460951102010001 & $08-07-70$ & 160 & - & - & 20 & - & - & - & - & -- & - \\
\hline 460954101240201 & $00-00-65$ & 1000 & -- & - & -- & - & - & -- & - & - & -- \\
\hline 461015102433501 & $06-08-71$ & 180 & - & -. & 40 & - & - & -- & -- & - & -- \\
\hline 461033101200701 & $11-17-71$ & 280 & -- & - & 0 & - & - & 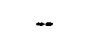 & -- & -- & -- \\
\hline 461037102033101 & $09-22-71$ & 320 & -- & -- & 10 & - & -- & - & -- & -- & - \\
\hline 461040102575401 & $06-15-71$ & - & - & - & - & - & - & - & - & - & - \\
\hline 461050103010001 & $05-16-74$ & 30 & -- & - & 20 & - & - & - & - & -- & -- \\
\hline 461059102283701 & $08-21-70$ & - & - & - & - & - & - & -- & -- & -- & -- \\
\hline 461106102593701 & $06-15-71$ & 530 & -- & - & 20 & - & - & -- & -- & -- & -- \\
\hline 461123102132201 & $08-07-70$ & 200 & - & -- & 20 & - & - & - & - & - & -- \\
\hline 461129101562401 & $09-09-71$ & 0 & - & - & 40 & - & -- & - & - & -- & - \\
\hline 461145102385701 & $06-11-71$ & 580 & - & - & 20 & - & -- & -- & - & - & -- \\
\hline 461148101565102 & $09-09-71$ & 360 & - & -- & 10 & -- & -- & -- & - & - & -- \\
\hline 461151103033101 & $04-14-71$ & 1700 & -- & - & 30 & - & - & - & - & -- & -- \\
\hline 461158101262201 & $11-15-71$ & 680 & -- & -- & 30 & -- & - & - & -- & - & -- \\
\hline 461159102532304 & $06-15-71$ & 70 & - & - & 80 & - & - & - & - & - & - \\
\hline 461201101372501 & $11-05-71$ & 100 & -- & - & 40 & - & - & - & - & - & - \\
\hline 461207101373501 & $02-03-71$ & -- & -- & - & - & - & - & - & -- & - & - \\
\hline 461216101594301 & $00-00-59$ & 480 & - & - & - & - & -- & - & - & - & -- \\
\hline 461219101103202 & $08-25-71$ & 3300 & - & - & 20 & - & - & - & - & - & - \\
\hline 461226103045401 & $10-11-71$ & 2500 & - & -- & 20 & - & -- & - & - & - & - \\
\hline 461226103045402 & $09-11-71$ & 500 & -- & - & 80 & - & - & - & - & - & - \\
\hline
\end{tabular}




\begin{tabular}{|c|c|c|c|c|c|c|c|c|c|c|}
\hline Stte number & Local identifier & $\begin{array}{l}\text { County } \\
\text { code }\end{array}$ & $\begin{array}{l}\text { Hydrologic } \\
\text { unlt }\end{array}$ & $\begin{array}{l}\text { Geologic } \\
\text { unit }\end{array}$ & Date & $\begin{array}{l}\text { Depth } \\
\text { below } \\
\text { land } \\
\text { surface, } \\
\text { water } \\
\text { level } \\
\text { (feot) }\end{array}$ & $\begin{array}{l}\text { Depth } \\
\text { of } \\
\text { well, } \\
\text { total } \\
\text { (teot) }\end{array}$ & $\begin{array}{l}\text { Altlude } \\
\text { of land } \\
\text { surface, } \\
\text { dotum } \\
\text { (feot) }\end{array}$ & $\begin{array}{l}\text { Specific } \\
\text { conduct- } \\
\text { ance, } \\
\text { fleid } \\
(\mu \mathrm{S} / \mathrm{cm})\end{array}$ & $\begin{array}{c}\text { Specific } \\
\text { conduct- } \\
\text { ance, } \\
\text { lab } \\
\text { ( } \mathrm{S} / \mathrm{cm})\end{array}$ \\
\hline 461230101300501 & $131-087-6 \mathrm{DDB}$ & 037 & 10130204 & $125 \mathrm{CNBL}$ & $11-12-69$ & - & 103 & - & 1310 & - \\
\hline 461242102172602 & 132-093-34DAA2 & 041 & 10130205 & - & $08-11-82$ & - & 111 & -- & 1390 & 1340 \\
\hline 461257103051001 & $131-099-32 \mathrm{ABB}$ & 011 & 10130205 & 125TRVL & $05-01-74$ & - & 39 & 2755 & 2720 & -- \\
\hline 461309102103602 & 132-092-34BAA2 & 041 & 10130205 & -. & $08-11-82$ & -. & 58 & - & 1200 & 1190 \\
\hline 461313102283001 & $132-094-29 \mathrm{CCC}$ & 041 & 10130205 & 125TGRV & $05-13-69$ & - & 204 & 2604 & 1490 & -- \\
\hline 461315102034501 & 132-091-28DDD & 041 & 10130205 & 211FXHL & $08-30-68$ & - & 1050 & 2469 & 2260 & - \\
\hline 461315102113201 & $132-092-28 D C D 1$ & 041 & 10130204 & 125SBTR & $12-03-45$ & - & 60 & 2462 & 3700 & -- \\
\hline 461338101145701 & 132-088-32ABA & 037 & 10130206 & $125 \mathrm{CNBL}$ & $03-20-69$ & -- & 160 & - & 1530 & - \\
\hline 461344101072901 & $132-083-30 \mathrm{BCB}$ & 037 & 10130206 & 112 SLDS & $11-16-71$ & - & 212 & 1814 & 1530 & - \\
\hline 461347102194602 & $132-093-28 \mathrm{BCB} 2$ & 041 & 10130205 & 125TGRV & $04-05-69$ & - & 162 & 2514 & 1490 & - \\
\hline 461349103004901 & $131-099-25 \mathrm{BBB} 1$ & 011 & 10130205 & 125LDLW & $05-09-74$ & - & 417 & -- & 1640 & .. \\
\hline 461349103004902 & $131-099-25 B B B 2$ & 011 & 10130205 & 125TRVL & $04-23-74$ & - & 200 & - & 1280 & - \\
\hline 461402102393402 & $132-096-26 \mathrm{BBA} 2$ & 001 & 10130205 & 125TRVL & $06-11-71$ & -- & 115 & - & 1540 & .. \\
\hline 461404101071101 & $132-083-19 \mathrm{CDC}$ & 037 & 10130206 & 112 SLDS & $12-03-71$ & - & 104 & - & 1670 & - \\
\hline 461405101484301 & $132-089-22 \mathrm{CCC} 1$ & 037 & 10130204 & 125TGRV & $00-00-68$ & - & 112 & - & 1270 & - \\
\hline 461411102541101 & 132-098-23CDD & $\infty$ & 10130205 & 125TRVL & $08-06-70$ & - & 150 & -- & 2340 & -- \\
\hline 461408102111302 & 132-092-21DDD2 & 041 & 10130205 & 125TGRV & $06-13-69$ & - & 70 & 2460 & 924 & - \\
\hline 461416101401701 & $132-088-22 \mathrm{DAC}$ & 037 & 10130204 & $125 \mathrm{CNBL}$ & $07-11-49$ & - & 148 & - & 4680 & - \\
\hline 461438101272901 & $132-086-21 \mathrm{BCB}$ & 037 & 10130204 & 125TGRV & $11-15-71$ & -- & - & - & 1180 & - \\
\hline 461439102183102 & $132-093-22 \mathrm{BCB} 2$ & 041 & 10130205 & - & $08-10-82$ & - & 78 & - & 1600 & 1570 \\
\hline 461448102345401 & 132-095-20AAD & 001 & 10130205 & 125TRVL & $07-07-71$ & -. & 210 & 2686 & 2830 & - \\
\hline 461448102393401 & $132-096-23 \mathrm{BBD}$ & 001 & 10130205 & 125TRVL & $06-11-71$ & - & 158 & - & 404 & - \\
\hline 461448102402101 & $132-096-22 \mathrm{ABC1}$ & 001 & 10130205 & 125LDLW & $06-20-72$ & - & 377 & 2585 & 2040 & - \\
\hline 461448102402102 & $132-096-22 \mathrm{ABC} 2$ & 001 & 10130205 & 125TRVL & $06-19-72$ & - & 188 & 2585 & 1550 & - \\
\hline 461452102165802 & $132-093-23 \mathrm{BAB} 2$ & 041 & 10130205 & 125TGRV & $08-03-67$ & - & 82 & 2500 & 4330 & - \\
\hline 461454102072901 & 132-092-24AAA & 041 & 10130205 & 125TGRV & $05-08-69$ & - & 174 & 2559 & 1610 & - \\
\hline 461455102464401 & 132-097-23BAA & 001 & 10130205 & 125TRVL & $08-06-70$ & - & 70 & $\ldots$ & 994 & -- \\
\hline 461459102161201 & 132-093-14DDD & 041 & 10130205 & 125TGRV & $11-13-56$ & - & 180 & 2488 & - & -- \\
\hline 461514101112301 & 132-084-16DAA & 037 & 10130206 & 211FXHL & $07-11-73$ & - & 396 & 1973 & 1770 & - \\
\hline 461515102482601 & $132-097-15 \mathrm{CBC}$ & 001 & 10130205 & 125TRVL & $08-06-70$ & - & 100 & - & 982 & - \\
\hline 461528102495001 & 132-097-17ADD & 001 & 10130205 & 125TRVL & $12-27-76$ & - & 73 & -- & 1310 & - \\
\hline 461532102110401 & $132-092-15 \mathrm{BCB}$ & 041 & 10130205 & 125TGRV & $11-23-54$ & -- & 125 & 2528 & 876 & - \\
\hline 461533103054801 & $131-099-17 \mathrm{BBB}$ & $\infty 01$ & 10130205 & $125 \mathrm{HRMN}$ & $05-15-74$ & -- & 164 & - & 1670 & - \\
\hline 461536103083901 & $132-100-14 \mathrm{ADB}$ & 011 & 10130205 & 125TRVL & $08-14-70$ & - & 51 & - & 2280 & - \\
\hline 461542101535901 & $132-090-14 A A B 1$ & 037 & 10130204 & $125 \mathrm{CNBL}$ & $07-02-73$ & -- & 314 & 2340 & 1890 & - \\
\hline 461543102012501 & $132-091-14 A A B$ & 041 & 10130204 & 125TGRV & $08-08-67$ & - & 202 & 2439 & 1460 & -. \\
\hline 461543102260001 & $132-094-15 \mathrm{BBB} 1$ & 041 & 10130205 & 125TGRV & $06-17-69$ & - & 143 & 2576 & 569 & - \\
\hline 461543102260002 & $132-094-15 B B B 2$ & 041 & 10130205 & $125 \mathrm{SNLB}$ & $06-16-69$ & - & 40 & 2576 & 480 & - \\
\hline 461548101083501 & $132-084-12 C C D$ & 037 & 10130206 & 112 SLDS & $07-26-72$ & -- & 216 & 1841 & 1610 & - \\
\hline 461552103171401 & 132-101-10DDD & 011 & 10130205 & $125 \mathrm{TRVL}$ & $07-12-72$ & - & 264 & 2925 & 1750 & - \\
\hline 461614102515201 & 132-097-7CAB1 & $\infty 01$ & 10130205 & $211 \mathrm{HCFH}$ & $07-29-71$ & - & 1080 & 2665 & 1790 & ـ \\
\hline 461614102515202 & 132-097-7CAB2 & $\infty$ & 10130205 & $125 \mathrm{LHCK}$ & $10-28-71$ & - & 590 & 2665 & 2110 & - \\
\hline 461614102515204 & $132-097-7 \mathrm{CAB} 4$ & $\infty 01$ & 10130205 & $125 \mathrm{LDLW}$ & $06-20-72$ & - & 335 & 2665 & 2050 & - \\
\hline 461637101145701 & $132-084-6 C C C$ & 037 & 10130206 & $125 \mathrm{CNBL}$ & $11-11-71$ & -- & 170 & 2049 & 2390 & - \\
\hline 461638102111301 & 132-092-9AAA & 041 & 10130205 & 125TGRV & $08-11-67$ & - & 225 & 2569 & 1520 & - \\
\hline
\end{tabular}




\begin{tabular}{|c|c|c|c|c|c|c|c|c|c|c|c|}
\hline Sits number & Dats & $\begin{array}{c}\text { pH, } \\
\text { fisid } \\
\text { (stand- } \\
\text { ard } \\
\text { units }\end{array}$ & $\begin{array}{l}\text { pH, } \\
\text { lab } \\
\text { (stand- } \\
\text { ard } \\
\text { units) }\end{array}$ & $\begin{array}{c}\text { Tamper- } \\
\text { ature, } \\
\text { watsr } \\
\text { (dogrees } \\
\text { Colsius) }\end{array}$ & $\begin{array}{c}\text { Color } \\
\text { (platl- } \\
\text { num } \\
\text { cobalt } \\
\text { scale) }\end{array}$ & $\begin{array}{l}\text { Hard- } \\
\text { ness, } \\
\text { total } \\
\text { (mg/L as } \\
\left.\mathrm{CaCO}_{3}\right)\end{array}$ & $\begin{array}{c}\text { Noncar- } \\
\text { bonate } \\
\text { hard- } \\
\text { ness, } \\
\text { total } \\
\text { (mgll as } \\
\text { CaCO) }\end{array}$ & $\begin{array}{l}\text { Alka- } \\
\text { linity, } \\
\text { field } \\
\left(\mathrm{mg}^{\prime} \mathrm{L} \text { as }\right. \\
\left.\mathrm{CoCO}_{3}\right)\end{array}$ & $\begin{array}{c}\text { Alka- } \\
\text { linity, } \\
\text { lab } \\
\text { (mg/L as } \\
\left.\mathrm{CaCO}_{3}\right)\end{array}$ & $\begin{array}{l}\text { Solids, } \\
\text { sum of } \\
\text { consth- } \\
\text { uents, } \\
\text { dis- } \\
\text { solved } \\
\text { (mgll) }\end{array}$ & $\begin{array}{c}\text { Solids, } \\
\text { residue } \\
\text { at } 105 \\
\text { degrees } \\
\text { Celsius, } \\
\text { total } \\
\text { (mg/L) }\end{array}$ \\
\hline 461230101300501 & $11-12-69$ & 7.7 & -- & - & -- & 630 & - & - & - & 796 & -- \\
\hline 461242102172602 & $08-11-82$ & 8.2 & 7.5 & 12.0 & - & 24 & - & - & 479 & 900 & - \\
\hline 461257103051001 & $05-01-74$ & 9.1 & - & 7.0 & 700 & 140 & $\mathbf{0}$ & - & 671 & 1840 & - \\
\hline 461309102103602 & $08-11-82$ & 7.1 & 7.8 & 11.5 & - & 560 & - & -- & 220 & 800 & -- \\
\hline 461313102283001 & $05-13-69$ & 8.5 & - & 10.5 & 200 & 26 & 0 & - & - & 955 & - \\
\hline 461315102034501 & $08-30-68$ & 8.4 & - & - & -- & 17 & 0 & - & - & 1340 & -- \\
\hline 461315102113201 & $12-03-45$ & -- & - & - & - & 49 & -- & - & -- & 2420 & -. \\
\hline 461338101145701 & $03-20-69$ & 8.4 & - & -- & - & 12 & - & - & - & 884 & -- \\
\hline 461344101072901 & $11-16-71$ & 8.2 & - & 8.0 & - & 290 & 0 & - & - & 997 & - \\
\hline 461347102194602 & $04-05-69$ & 8.2 & - & - & 300 & 20 & 0 & - & - & 988 & - \\
\hline 461349103004901 & $05-09-74$ & - & -- & 19.0 & - & 36 & 0 & - & 869 & 1040 & - \\
\hline 461349103004902 & $04-23-74$ & 8.5 & -. & 9.5 & 2300 & 16 & 0 & - & 577 & 853 & - \\
\hline 461402102393402 & $06-11-71$ & 8.5 & - & 8.0 & - & 42 & 0 & - & - & 978 & - \\
\hline 461404101071101 & $12-03-71$ & 8.1 & -- & -- & - & 27 & 0 & - & - & 1080 & - \\
\hline 461405101484301 & $00-00-68$ & 7.4 & - & - & - & 460 & -- & - & - & 676 & $\cdots$ \\
\hline 461411102541101 & $08-06-70$ & 8.2 & - & 14.0 & - & 97 & 0 & 560 & - & 1584 & - \\
\hline 461408102111302 & $06-13-69$ & 7.9 & - & 7.5 & 10 & 240 & 0 & - & - & 616 & -- \\
\hline 461416101401701 & $07-11-49$ & 8.0 & - & -- & - & 270 & - & - & - & - & - \\
\hline 461438101272901 & $11-15-71$ & 7.7 & - & 9.5 & -- & 370 & 33 & - & -- & 781 & -. \\
\hline 461439102183102 & $08-10-82$ & 7.2 & 7.6 & 11.0 & $\cdots$ & 800 & - & - & 277 & 1180 & - \\
\hline 461448102345401 & $07-07-71$ & 8.0 & - & - & - & 90 & 0 & - & - & 2010 & - \\
\hline 461448102393401 & $06-11-71$ & 8.2 & + & 10.0 & - & 230 & 9 & -- & - & 233 & -- \\
\hline 461448102402101 & $06-20-72$ & 8.4 & - & 9.5 & -- & 16 & 0 & - & -- & 1300 & -. \\
\hline 461448102402102 & $06-19-72$ & 8.3 & - & 9.0 & - & 71 & 0 & - & - & 1000 & -- \\
\hline 461452102165802 & $08-03-67$ & 8.1 & - & 10.0 & - & 810 & 300 & - & - & 3470 & - \\
\hline 461454102072901 & $05-08-69$ & 8.1 & - & 7.0 & 100 & 35 & 0 & - & - & 1060 & - \\
\hline 461455102464401 & $08-06-70$ & 7.9 & -- & 12.0 & - & 440 & 70 & -- & - & 638 & - \\
\hline 461459102161201 & $11-13-56$ & 7.5 & - & -- & - & 1300 & 980 & - & - & 3550 & - \\
\hline 461514101112301 & $07-11-73$ & 8.2 & - & 10.0 & - & 13 & 0 & - & - & 1070 & - \\
\hline 461515102482601 & $08-06-70$ & 8.1 & - & 13.0 & - & 370 & 0 & - & - & 604 & - \\
\hline 461528102495001 & $12-27-76$ & 8.2 & - & - & - & 420 & 53 & -. & - & 614 & - \\
\hline 461532102110401 & $11-23-54$ & 7.9 & $\cdots$ & - & - & 340 & 47 & - & -. & 613 & - \\
\hline 461533103054801 & $05-15-74$ & 8.8 & - & 8.0 & 200 & 63 & 0 & - & 560 & 1040 & + \\
\hline 461536103083901 & $08-14-70$ & 8.3 & - & 9.0 & - & 130 & 0 & - & - & 1550 & -- \\
\hline 461542101535901 & $07-02-73$ & 8.5 & - & 10.0 & - & 23 & 0 & - & - & 1200 & - \\
\hline 461543102012501 & $08-08-67$ & 8.3 & - & 9.0 & -- & 35 & 0 & - & - & 966 & - \\
\hline 461543102260001 & $06-17-69$ & 7.9 & - & 8.5 & - & 160 & 0 & - & - & 358 & -- \\
\hline 461543102260002 & $06-16-69$ & 7.7 & - & 6.5 & 100 & 220 & 19 & - & -- & 298 & - \\
\hline 461548101083501 & $07-26-72$ & 8.1 & -- & 8.0 & - & 200 & 0 & - & - & 1070 & -- \\
\hline 461552103171401 & $07-12-72$ & 8.3 & - & 10.0 & - & 51 & 0 & - & - & 1210 & -- \\
\hline 461614102515201 & $07-29-71$ & 8.0 & $\rightarrow$ & 20.0 & - & 19 & 0 & - & - & 1140 & - \\
\hline 461614102515202 & $10-28-71$ & 8.4 & - & 9.5 & - & 36 & 0 & - & -- & 1380 & - \\
\hline 461614102515204 & $06-20-72$ & 8.7 & - & 11.0 & -- & 9 & 0 & - & - & 1290 & - \\
\hline 461637101145701 & $11-11-71$ & 8.1 & - & 9.0 & - & 100 & 0 & - & - & 1620 & - \\
\hline 461638102111301 & $08-11-67$ & 8.3 & - & 9.0 & - & 160 & 0 & -- & - & 1020 & - \\
\hline
\end{tabular}




\begin{tabular}{|c|c|c|c|c|c|c|c|c|c|c|c|}
\hline Site number & Date & $\begin{array}{l}\text { Solids, } \\
\text { residue } \\
\text { at } 180 \\
\text { degrees } \\
\text { Calsius, } \\
\text { dis- } \\
\text { solved } \\
\text { (mg/L) }\end{array}$ & $\begin{array}{l}\text { Solids, } \\
\text { dis- } \\
\text { solved } \\
\text { (tons per } \\
\text { acre- } \\
\text { foot) }\end{array}$ & $\begin{array}{l}\text { Calclum, } \\
\text { dls- } \\
\text { solved } \\
\text { (mg/L } \\
\text { as Ca) }\end{array}$ & $\begin{array}{l}\text { Magne- } \\
\text { slum, } \\
\text { dls- } \\
\text { solved } \\
\text { (mg/L } \\
\text { as } \mathrm{Mg} \text { ) }\end{array}$ & $\begin{array}{l}\text { Sodium, } \\
\text { dis- } \\
\text { solved } \\
\text { (mg/t } \\
\text { as } \mathrm{Na} \text { ) }\end{array}$ & $\begin{array}{l}\text { Sodium } \\
\text { (porcent) }\end{array}$ & $\begin{array}{l}\text { Sodlum } \\
\text { adsorp- } \\
\text { tion } \\
\text { ratio }\end{array}$ & $\begin{array}{c}\text { Resid- } \\
\text { ual, } \\
\text { sodlum, } \\
\text { car- } \\
\text { bonate } \\
\text { (mg/L as } \\
\text { CaCo }\end{array}$ & $\begin{array}{l}\text { Potas- } \\
\text { sium, } \\
\text { dis- } \\
\text { solved } \\
\text { (mg/L } \\
\text { as K) }\end{array}$ & $\begin{array}{c}\text { Blcar- } \\
\text { bonate } \\
\text { (mg/L as } \\
\left.\mathrm{HCO}_{3}\right)\end{array}$ \\
\hline 3 & & & & & & & & & & & \\
\hline 461230101300501 & $11-12-69$ & 939 & - & -- & -- & - & -- & - & -- & 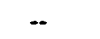 & $\ldots$ \\
\hline 461242102172602 & $08-11-82$ & 898 & 1.22 & 5.5 & 2.4 & 340 & 96 & 30 & - & 3.8 & - \\
\hline 461257103051001 & $05-01-74$ & 1910 & 2.60 & 31 & 14 & 600 & 90 & 22 & - & 9.0 & 510 \\
\hline 461309102103602 & $08-11-82$ & 967 & 1.32 & 120 & 63 & 65 & 20 & 1 & - & 5.0 & -- \\
\hline 461313102283001 & $05-13-69$ & 965 & 1.31 & 4.8 & 3.4 & 350 & 96 & 30 & - & 2.4 & 610 \\
\hline 461315102034501 & $08-30-68$ & 1310 & 1.78 & 4.3 & 1.6 & 550 & 98 & 58 & -- & 2.6 & 1010 \\
\hline 461315102113201 & $12-03-45$ & 2380 & - & -- & - & - & -- & - & - & -- & 380 \\
\hline 461338101145701 & $03-20-69$ & 972 & - & 3.4 & 0.90 & 370 & -- & 47 & - & -- & -- \\
\hline 461344101072901 & $11-16-71$ & 1050 & 1.43 & 110 & 4.4 & 270 & 67 & 7 & 9.00 & 4.4 & 880 \\
\hline 461347102194602 & $04-05-69$ & 955 & 1.30 & 5.6 & 1.5 & 340 & 97 & 33 & - & 2.0 & 530 \\
\hline 461349103004901 & $05-09-74$ & 1150 & 1.56 & 5.1 & 5.7 & 420 & 95 & 30 & - & 5.4 & 970 \\
\hline 461349103004902 & $04-23-74$ & 926 & 1.26 & 4.1 & 1.3 & 320 & 97 & 35 & - & 2.1 & 650 \\
\hline 461402102393402 & $06-11-71$ & 935 & 1.27 & 7.2 & 5.8 & 380 & 95 & 26 & 12.0 & 2.2 & 780 \\
\hline 461404101071101 & $12-03-71$ & 1060 & 1.44 & 7.6 & 1.9 & 410 & 97 & 34 & 13.0 & 1.6 & 820 \\
\hline 461405101484301 & $00-00-68$ & 857 & - & -. & -- & 110 & - & - & -- & - & 310 \\
\hline 461411102541101 & $08-06-70$ & 1640 & 2.23 & 1.0 & 23 & 527 & 92 & 23 & - & 4.9 & 683 \\
\hline 461408102111302 & $06-13-69$ & 647 & 0.88 & 58 & 24 & 120 & 51 & 3 & - & 4.7 & 350 \\
\hline 461416101401701 & $07-11-49$ & 2840 & - & 61 & 28 & 810 & - & 22 & -- & -- & 570 \\
\hline 461438101272901 & $11-15-71$ & 790 & 1.07 & 92 & 34 & 120 & 41 & 3 & 0.0 & 4.4 & 410 \\
\hline 461439102183102 & $08-10-82$ & 1260 & 1.71 & 180 & 84 & 75 & 17 & 1 & -- & 6.9 & -- \\
\hline 461448102345401 & $07-07-71$ & 2050 & 2.79 & 18 & 11 & 650 & 94 & 30 & -- & 4.9 & 610 \\
\hline 461448102393401 & $06-11-71$ & 249 & 0.34 & 44 & 28 & 5.0 & 5 & 0.1 & - & 2.1 & 260 \\
\hline 461448102402101 & $06-20-72$ & 1270 & 1.73 & 2.5 & 2.4 & 540 & 98 & 58 & 23.0 & 2.7 & 1350 \\
\hline 461448102402102 & $06-19-72$ & 1060 & 1.44 & 12 & 10 & 390 & 92 & 20 & 13.0 & 2.6 & 870 \\
\hline 461452102165802 & $08-03-67$ & 3520 & 4.79 & 170 & 94 & 820 & 69 & 13 & -- & 9.1 & 610 \\
\hline 461454102072901 & $05-08-69$ & 1040 & 1.41 & 9.8 & 2.6 & 380 & 95 & 28 & - & 7.1 & 630 \\
\hline 461455102464401 & $08-06-70$ & 650 & 0.88 & 100 & 46 & 57 & 22 & 1 & -- & 5.6 & 450 \\
\hline 461459102161201 & $11-13-56$ & 2710 & - & -- & -- & - & -- & - & -- & - & 420 \\
\hline 461514101112301 & $07-11-73$ & 1120 & 1.52 & 3.6 & 1.0 & 430 & 98 & 52 & 14.0 & 3.8 & 870 \\
\hline 461515102482601 & $08-06-70$ & 620 & 0.84 & 78 & 42 & 86 & 33 & 2 & - & 5.1 & 460 \\
\hline 461528102495001 & $12-27-76$ & 587 & 0.80 & 100 & 41 & 55 & 22 & 1 & - & 3.0 & 450 \\
\hline 461532102110401 & $11-23-54$ & 493 & .- & .. & - & - & 39 & 2 & - & - & 350 \\
\hline 461533103054801 & $05-15-74$ & 1110 & 1.51 & 14 & 6.7 & 360 & 92 & 20 & -- & 5.9 & 620 \\
\hline 461536103083901 & $08-14-70$ & 1500 & 2.04 & 32 & 11 & 500 & 89 & 19 & -- & 4.9 & 630 \\
\hline 461542101535901 & $07-02-73$ & 1230 & 1.67 & 5.0 & 2.6 & 470 & 97 & 42 & 17.0 & 3.6 & 1020 \\
\hline 461543102012501 & $08-08-67$ & 951 & 1.29 & 10 & 2.4 & 340 & 95 & 25 & -. & 3.8 & 540 \\
\hline 461543102260001 & $06-17-69$ & 321 & 0.44 & 36 & 16 & 74 & 50 & 3 & -. & 5.6 & 370 \\
\hline 461543102260002 & $06-16-69$ & 300 & 0.41 & 52 & 22 & 21 & 17 & 0.6 & - & 3.7 & 240 \\
\hline 461548101083501 & $07-26-72$ & 1120 & 1.52 & 47 & 20 & 320 & 77 & 10 & 9.00 & 5.4 & 770 \\
\hline 461552103171401 & $07-12-72$ & 1630 & 2.22 & 9.0 & 6.9 & 430 & 94 & 26 & 10.0 & 5.0 & 630 \\
\hline 461614102515201 & $07-29-71$ & 1110 & 1.51 & 5.2 & 1.5 & 450 & 98 & 45 & 14.0 & 1.7 & 880 \\
\hline 461614102515202 & $10-28-71$ & 1430 & 1.94 & 8.4 & 3.6 & 550 & 97 & 40 & 21.0 & 3.4 & 1250 \\
\hline 461614102515204 & $06-20-72$ & 1310 & 1.78 & 2.2 & 0.90 & 540 & 99 & 77 & 24.0 & 2.1 & 1340 \\
\hline 461637101145701 & $11-11-71$ & 1570 & 2.14 & 11 & 18 & 540 & 92 & 23 & 8.00 & 1.4 & 630 \\
\hline 461638102111301 & $08-11-67$ & 1010 & 1.37 & 39 & 15 & 300 & 79 & 10 & - & 11 & 520 \\
\hline
\end{tabular}




\begin{tabular}{|c|c|c|c|c|c|c|c|c|c|c|c|}
\hline Stte number & Date & $\begin{array}{c}\text { Car- } \\
\text { bonate } \\
\text { (mg/L as } \\
\left.\mathrm{CO}_{3}\right)\end{array}$ & $\begin{array}{c}\text { Carbon } \\
\text { dioxide, } \\
\text { dis- } \\
\text { solved } \\
(\mathrm{mg} / \mathrm{L} \text { as } \\
\left.\mathrm{CO}_{2}\right)\end{array}$ & $\begin{array}{l}\text { Sulfate, } \\
\text { dis- } \\
\text { solved } \\
\text { (mgll as } \\
\mathrm{SO}_{4} \text { ) }\end{array}$ & $\begin{array}{l}\text { Chlo- } \\
\text { ride, } \\
\text { dis- } \\
\text { solved } \\
\text { (mg/L } \\
\text { as CI) }\end{array}$ & $\begin{array}{l}\text { Fluoride, } \\
\text { dis- } \\
\text { solved } \\
\text { (mg/L } \\
\text { as F) }\end{array}$ & $\begin{array}{l}\text { Silica, } \\
\text { dis- } \\
\text { solved } \\
\text { (mg/L as } \\
\text { SiO }_{2} \text { ) }\end{array}$ & $\begin{array}{l}\text { Nitro- } \\
\text { gen, } \\
\text { nitrate, } \\
\text { dis- } \\
\text { solved } \\
\text { (mglL } \\
\text { as N) }\end{array}$ & $\begin{array}{l}\text { Nitro- } \\
\text { gen, } \\
\text { nitrate, } \\
\text { total } \\
\text { (mg/L } \\
\text { as } \mathrm{NO}_{3} \text { ) }\end{array}$ & $\begin{array}{l}\text { Nitro- } \\
\text { gen, } \\
\text { nitrate, } \\
\text { dls- } \\
\text { solved } \\
\text { (mg/L } \\
\text { as } \mathrm{NO}_{3} \text {. }\end{array}$ & $\begin{array}{c}\text { Boron, } \\
\text { dis- } \\
\text { solved } \\
(\mu g / L \\
\text { as B) }\end{array}$ \\
\hline & & & & & & & & & & " & \\
\hline 461230101300501 & $11-12-69$ & - & - & 400 & 10 & -- & - & 0.00 & 0.0 & - & - \\
\hline 461242102172602 & $08-11-82$ & -. & -- & 240 & 10 & 0.60 & 9.3 & -- & - & -- & 760 \\
\hline 461257103051001 & $05-01-74$ & 150 & 1.0 & 770 & 13 & 2.0 & - & -- & -. & - & - \\
\hline 461309102103602 & $08-11-82$ & - & - & 380 & 20 & 0.30 & 14 & - & -. & -- & 230 \\
\hline 461313102283001 & $05-13-69$ & 13 & 3.2 & 270 & 0.0 & 1.1 & 8.6 & 0.560 & -- & 2.5 & 0 \\
\hline 461315102034501 & $08-30-68$ & 18 & 6.7 & 5.7 & 240 & 3.7 & 9.3 & 0.230 & -- & 1.0 & 3 \\
\hline 461315102113201 & $12-03-45$ & 20 & -- & 1300 & 0.0 & -- & -- & - & - & - & - \\
\hline 461338101145701 & $03-20-69$ & - & -- & 330 & 14 & -- & -- & 0.00 & 0.0 & - & - \\
\hline 461344101072901 & $11-16-71$ & 0 & -- & 130 & 5.1 & 3.0 & 26 & 0.226 & 1.0 & -- & 0 \\
\hline 461347102194602 & $04-05-69$ & 0 & 5.4 & 350 & 13 & 1.5 & 8.4 & 0.00 & - & 0.0 & 1 \\
\hline 461349103004901 & $05-09-74$ & 45 & -- & 63 & 6.3 & 6.5 & 3.3 & 0.230 & - & 1.0 & 1300 \\
\hline 461349103004902 & $04-23-74$ & 28 & 3.3 & 140 & 24 & 4.2 & 9.3 & -- & -- & $\ldots$ & -- \\
\hline 461402102393402 & $06-11-71$ & 18 & -- & 160 & 15 & 4.0 & 6.5 & - & -- & 0.20 & 750 \\
\hline 461404101071101 & $12-03-71$ & 0 & -. & 230 & 1.5 & 0.70 & 20 & 0.226 & 1.0 & -- & 1100 \\
\hline 461405101484301 & $00-00-68$ & 0 & - & 360 & 9.0 & -- & - & 0.00 & 0.0 & - & -- \\
\hline 461411102541101 & $08-06-70$ & 0 & 6.9 & 678 & 2.5 & 1.3 & 6.1 & 0.00 & -- & 0.00 & 460 \\
\hline 461408102111302 & $06-13-69$ & 0 & 7.0 & 220 & 1.6 & 0.10 & 13 & 0.560 & - & 2.5 & 0 \\
\hline 461416101401701 & $07-11-49$ & 0 & -- & 1300 & 32 & 0.20 & -- & 1.58 & 7.0 & - & - \\
\hline 461438101272901 & $11-15-71$ & 0 & 13 & 310 & 0.0 & 0.40 & 16 & 0.450 & 2.0 & 2.0 & 530 \\
\hline 461439102183102 & $08-10-82$ & - & -- & 650 & 6.3 & 0.10 & 12 & - & -- & -- & 320 \\
\hline 461448102345401 & $07-07-71$ & 0 & - & 1000 & 5.5 & 0.20 & 7.6 & - & - & 2.5 & 350 \\
\hline 461448102393401 & $06-11-71$ & 0 & - & 18 & 1.4 & 0.30 & 4.3 & - & - & 1.7 & 520 \\
\hline 461448102402101 & $06-20-72$ & 23 & - & 34 & 17 & 4.0 & 9.2 & -- & - & 1.0 & 890 \\
\hline 461448102402102 & $06-19-72$ & 11 & -- & 130 & 12 & 4.6 & 5.9 & -- & - & 1.0 & 460 \\
\hline 461452102165802 & $08-03-67$ & 0 & 7.8 & 2000 & 10 & 0.50 & 7.7 & 2.70 & -- & 12 & 1 \\
\hline 461454102072901 & $05-08-69$ & 0 & 8.0 & 330 & 1.0 & 0.0 & 8.3 & 0.230 & - & 1.0 & 1 \\
\hline 461455102464401 & $08-06-70$ & 0 & -- & 190 & 5.3 & 0.50 & 11 & - & - & 1.0 & 120 \\
\hline 461459102161201 & $11-13-56$ & 0 & 21 & 1800 & 54 & -- & - & -- & - & - & - \\
\hline 461514101112301 & $07-11-73$ & 0 & 8.8 & 17 & 170 & 4.2 & 15 & 0.050 & 0.20 & 0.20 & 1600 \\
\hline 461515102482601 & $08-06-70$ & 0 & - & 140 & 9.2 & 0.50 & 10 & - & -- & 11 & 250 \\
\hline 461528102495001 & $12-27-76$ & 0 & 4.5 & 160 & 13 & 0.10 & 10 & 2.90 & - & 13 & $<20$ \\
\hline 461532102110401 & $11-23-54$ & - & 7.1 & 150 & 0.0 & $\ldots$ & - & -- & - & -. & 1 \\
\hline 461533103054801 & $05-15-74$ & 31 & 1.7 & 290 & 11 & 2.9 & 13 & - & - & - & - \\
\hline 461536103083901 & $08-14-70$ & 6 & - & 670 & 3.6 & 2.3 & 7.5 & -. & - & 1.0 & 300 \\
\hline 461542101535901 & $07-02-73$ & 19 & - & 130 & 52 & 1.7 & 9.1 & 0.226 & 1.0 & - & 1400 \\
\hline 461543102012501 & $08-08-67$ & 7 & 4.5 & 320 & 4.9 & 0.40 & 8.1 & 0.00 & -- & 0.0 & 0 \\
\hline 461543102260001 & $06-17-69$ & 0 & 7.5 & 28 & 0.0 & 0.20 & 13 & 0.560 & - & 2.5 & 0 \\
\hline 461543102260002 & $06-16-69$ & 0 & 7.8 & 49 & 1.5 & 0.40 & 12 & 2.50 & - & 11 & 0 \\
\hline 461548101083501 & $07-26-72$ & 0 & -- & 270 & 0.60 & 0.60 & 24 & 0.226 & 1.0 & - & 1400 \\
\hline 461552103171401 & $07-12-72$ & 7 & - & 430 & 9.2 & 2.5 & 8.0 & - & - & 6.1 & 1100 \\
\hline 461614102515201 & $07-29-71$ & 0 & -- & 170 & 54 & 5.3 & 12 & - & - & 1.0 & 1600 \\
\hline 461614102515202 & $10-28-71$ & 33 & -- & 140 & 15 & 6.5 & 7.7 & - & - & 1.0 & 1600 \\
\hline 461614102515204 & $06-20-72$ & 53 & - & 14 & 7.6 & 6.1 & 7.7 & - & - & 1.0 & 1300 \\
\hline 461637101145701 & $11-11-71$ & 0 & -- & 720 & 0.0 & 1.2 & 16 & 0.226 & 1.0 & - & 2700 \\
\hline 461638102111301 & $08-11-67$ & 5 & 4.3 & 370 & 4.3 & 0.10 & 10 & 0.680 & - & 3.0 & 1 \\
\hline
\end{tabular}




\begin{tabular}{|c|c|c|c|c|c|c|c|c|c|c|c|}
\hline Site number & Date & $\begin{array}{l}\text { Iron, } \\
\text { dis- } \\
\text { solved } \\
\text { ( } \mu g / L \\
\text { as Fe) }\end{array}$ & $\begin{array}{l}\text { Load, } \\
\text { dls- } \\
\text { solved } \\
(\mu g / L \\
\text { as Pb) }\end{array}$ & $\begin{array}{l}\text { Lithium, } \\
\text { dis- } \\
\text { solved } \\
\text { ( } \mu g / L \\
\text { as Li) }\end{array}$ & $\begin{array}{l}\text { Manga- } \\
\text { nese, } \\
\text { dis- } \\
\text { solved } \\
(\mu g / L \\
\text { as Mn) }\end{array}$ & $\begin{array}{c}\text { Mercury, } \\
\text { dis- } \\
\text { solved } \\
(\mu g / L \\
\text { as Hg) }\end{array}$ & $\begin{array}{l}\text { Molyb- } \\
\text { donum, } \\
\text { dis- } \\
\text { solved } \\
\text { ( } \mu g / \mathrm{L} \\
\text { as Mo) }\end{array}$ & $\begin{array}{l}\text { Sole- } \\
\text { nium, } \\
\text { dis- } \\
\text { solved } \\
\text { ( } \mu \text { g/L } \\
\text { as Se) }\end{array}$ & $\begin{array}{l}\text { Stron- } \\
\text { tium, } \\
\text { dia- } \\
\text { solved } \\
(\mu g / L \\
\text { as Sr) }\end{array}$ & $\begin{array}{l}\text { Vana- } \\
\text { dium, } \\
\text { dis- } \\
\text { solved } \\
(\mu g / L \\
\text { as V }\end{array}$ & $\begin{array}{l}\text { Zinc, } \\
\text { dis- } \\
\text { solved } \\
(\mu g / L \\
\text { as Zn) }\end{array}$ \\
\hline 461230101300501 & $11-12-69$ & 360 & - & -- & - & - & - & - & - & - & -- \\
\hline 461242102172602 & $08-11-82$ & 120 & - & - & 28 & - & - & $<1$ & - & - & - \\
\hline 461257103051001 & $05-01-74$ & 170 & - & - & 40 & - & - & - & - & - & - \\
\hline 461309102103602 & $08-11-82$ & 59 &.- & -- & 200 & - & - & 1 & - & - & -- \\
\hline 461313102283001 & $05-13-69$ & 200 & - & - & - & - & - & -- & - & -- & -- \\
\hline 461315102034501 & $08-30-68$ & 300 & - & -- & - & - & - & - & - & -- & - \\
\hline 461315102113201 & $12-03-45$ & - & - & - & - & - & - & -- & - & - & .. \\
\hline 461338101145701 & $03-20-69$ & 680 & - & - & - & - & - & -. & - & - & - \\
\hline 461344101072901 & $11-16-71$ & 13000 & -- & - & 30 & - & - & - & - & -- & - \\
\hline 461347102194602 & $04-05-69$ & 400 & - & - & - & - & -- & - & - & -- & - \\
\hline 461349103004901 & $05-09-74$ & 40 & -- & - & 400 & - & - & -- & - & - & - \\
\hline 461349103004902 & $04-23-74$ & 690 & -- & - & 50 & - & - & -- & - & - & - \\
\hline 461402102393402 & $06-11-71$ & 200 & - & - & 20 & - & - & -- & $\ldots$ & - & - \\
\hline 461404101071101 & $12-03-71$ & 280 & - & - & 90 & - & - & - & - & -- & - \\
\hline 461405101484301 & $00-00-68$ & 6600 & - & - & - & - & - & - & - & - & - \\
\hline 461411102541101 & $08-06-70$ & 4100 & - & - & 60 & - & - & - & -. & -- & - \\
\hline 461408102111302 & $06-13-69$ & 840 & - & - & - & - & - & -- & -- & - & -- \\
\hline 461416101401701 & $07-11-49$ & 1500 & - & - & - & - & - & - & - & - & -- \\
\hline 461438101272901 & $11-15-71$ & 0 & - & -- & 0 & - & - & - & - & - & - \\
\hline 461439102183102 & $08-10-82$ & 660 & -- & - & 430 & - & - & $<1$ & -- & -- & - \\
\hline 461448102345401 & $07-07-71$ & 30 & - & - & 20 & - & -- & - & - & -- & - \\
\hline 461448102393401 & $06-11-71$ & 0 & -- & - & 10 & - & - & - & - & - & - \\
\hline 461448102402101 & $06-20-72$ & 300 & -- & - & 10 & - & - & - & - & - & - \\
\hline 461448102402102 & $06-19-72$ & 90 & - & - & 10 & - & - & - & - & -- & -- \\
\hline 461452102165802 & $08-03-67$ & 80 & - & - & - & - & - & -- & -- & -- & -- \\
\hline 461454102072901 & $05-08-69$ & 12000 & - & -- & - & - & - & - & -- & -- & - \\
\hline 461455102464401 & $08-06-70$ & 400 & - & - & 40 & - & - & - & - & -- & - \\
\hline 461459102161201 & $11-13-56$ & 0 & - & -- & - & - & -- & - & -. & -- & - \\
\hline 461514101112301 & $07-11-73$ & 1200 & - & - & $<10$ & - & - & - & - & -- & - \\
\hline 461515102482601 & $08-06-70$ & 60 & - & - & 0 & - & - & - & -- & - & -- \\
\hline 461528102495001 & $12-27-76$ & 60 & - & - & 40 & - & - & -. & - & -- & - \\
\hline 461532102110401 & $11-23-54$ & 420 & - & - & -- & -- & - & -- & - & - & -- \\
\hline 461533103054801 & $05-15-74$ & 60 & - & - & $<10$ & - & - & -- & - & - & - \\
\hline 461536103083901 & $08-14-70$ & 180 & - & -- & 20 & - & - & - & - & - & -- \\
\hline 461542101535901 & $07-02-73$ & 600 & - & - & 80 & - & - & - & - & - & - \\
\hline 461543102012501 & $08-08-67$ & 130 & - & -- & - & - & - & - & - & - & -- \\
\hline 461543102260001 & $06-17-69$ & 720 & - & - & - & - & - & -- & - & - & - \\
\hline 461543102260002 & $06-16-69$ & 5000 & - & - & - & - & - & - & - & - & - \\
\hline 461548101083501 & $07-26-72$ & 580 & - & - & 130 & - & - & - & - & -- & - \\
\hline 461552103171401 & $07-12-72$ & 1100 & - & - & 30 & - & - & -- & - & - & - \\
\hline 461614102515201 & $07-29-71$ & 2800 & - & -- & 30 & - & - & - & - & - & - \\
\hline 461614102515202 & $10-28-71$ & 0 & - & - & 40 & - & - & - & - & - & - \\
\hline 461614102515204 & $06-20-72$ & 620 & - & - & 10 & - & - & - & - & - & - \\
\hline 461637101145701 & $11-11-71$ & 4800 & - & - & 110 & - & - & - & - & - & -- \\
\hline 461638102111301 & $08-11-67$ & 360 & - & - & - & - & - & - & - & - & - \\
\hline
\end{tabular}




\begin{tabular}{|c|c|c|c|c|c|c|c|c|c|c|}
\hline Ste number & Local identifier & $\begin{array}{c}\text { County } \\
\text { code }\end{array}$ & $\begin{array}{c}\text { Hydrologic } \\
\text { unlt } \\
-\end{array}$ & $\begin{array}{l}\text { Geologic } \\
\text { unit }\end{array}$ & Date & $\begin{array}{l}\text { Depth } \\
\text { below } \\
\text { land } \\
\text { surface, } \\
\text { water } \\
\text { level } \\
\text { (feet) }\end{array}$ & $\begin{array}{c}\text { Depth } \\
\text { of } \\
\text { woil, } \\
\text { total } \\
\text { (teot) }\end{array}$ & $\begin{array}{l}\text { Attitude } \\
\text { of land } \\
\text { surface, } \\
\text { datum } \\
\text { (feet) }\end{array}$ & $\begin{array}{l}\text { Specific } \\
\text { conduct- } \\
\text { ance, } \\
\text { field } \\
(\mu \text { S/cm) }\end{array}$ & 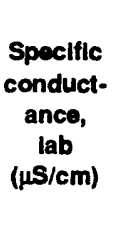 \\
\hline 461700102532603 & $133-097-32 D C C 3$ & 041 & 10130204 & 125TGRV & $08-18-67$ & - & 95 & 2699 & 2310 & -- \\
\hline 461716101014501 & 133-082-31DDA & 059 & 10130206 & $211 \mathrm{FXHL}$ & $06-27-72$ & - & 280 & - & 2150 & -- \\
\hline 461722101063602 & $133-083-34 \mathrm{CBC} 2$ & 037 & 10130206 & $211 \mathrm{FXHL}$ & $12-01-71$ & - & 310 & - & 1560 & - \\
\hline 461727103012701 & 133-098-32BCA & 087 & 10130205 & 125TRVL & $07-01-75$ & - & 106 & - & 2800 & - \\
\hline 461735101533602 & 133-089-32BDA2 & 037 & 10130204 & 125TGRV & $09-15-71$ & - & 146 & $\cdots$ & 2360 & -- \\
\hline 461738103085801 & 133-099-32BBA & 087 & 10130205 & $211 \mathrm{HCFH}$ & $08-28-74$ & - & 1252 & 2871 & 1830 & -- \\
\hline 461749101511503 & 133-089-34BAB3 & 037 & 10130204 & 125TGRV & $04-01-70$ & - & 183 & -. & 1620 & - \\
\hline 461756102062601 & $133-091-27 \mathrm{CCD}$ & 041 & 10130204 & 125TGRV & $05-01-48$ & - & 90 & 2410 & 2880 & - \\
\hline 461801101010701 & $133-082-29 C D D 1$ & 059 & 10130206 & 211FXHL & $06-27-72$ & -- & 280 & - & 2240 & -- \\
\hline 461801101070401 & $133-083-28 D C D$ & 037 & 10130206 & $112 S L D S$ & $11-13-71$ & - & 165 & 1872 & 1850 & - \\
\hline 461815102141202 & 133-092-28DAA2 & 041 & 10130204 & 125SBTR & $07-23-59$ & - & 60 & -- & 1140 & -- \\
\hline 461816102441602 & 133-096-28DAA2 & 041 & 10130205 & 125TGRV & $03-11-69$ & -- & 71 & 2696 & 2310 & -- \\
\hline 461820101131101 & 133-084-27DAB & 037 & 10130206 & $211 \mathrm{HLCK}$ & $07-24-61$ & - & 264 & - & 2720 & -- \\
\hline 461822102190901 & 133-093-26ADD & 041 & 10130204 & - & $08-17-82$ & -- & 462 & - & 760 & 755 \\
\hline 461822102255101 & $133-094-25 A C C$ & 041 & 10130204 & 125TGRV & $06-17-69$ & - & 70 & 2535 & 1820 & - \\
\hline 461833102551401 & $133-097-30 \mathrm{BBC}$ & 041 & 10130205 & 125TGRV & $08-08-69$ & - & 60 & 2760 & 1550 & - \\
\hline 461836102341302 & 133-095-26AAD2 & 041 & 10130204 & 125TGRV & $03-11-69$ & - & 161 & 2659 & 1380 & - \\
\hline 461841102132501 & $133-092-27 A B C$ & 041 & 10130204 & 125TGRV & $06-03-69$ & - & 243 & 2540 & 2560 & -- \\
\hline 461841102190901 & 133-093-26AAA & 041 & 10130204 & 125TGRV & $12-01-67$ & - & 128 & 2505 & 1390 & -- \\
\hline 461845103015601 & 133-098-19DDB & 087 & 10130205 & 125TRVL & $07-01-75$ & - & 60 & -- & 2700 & - \\
\hline 461847101065501 & 133-083-28AAB & 037 & 10130206 & $112 S L D S$ & $11-13-71$ & - & 98 & 1867 & 2210 & $\cdots$ \\
\hline 461849102391601 & 133-095-19DDD & 041 & 10130204 & 125TGRV & $05-14-69$ & - & 214 & 2648 & 1350 & - \\
\hline 461852103013701 & 133-098-20CBC & 087 & 10130205 & 125TRVL & $07-01-75$ & - & 91 & - & 1870 & -- \\
\hline 461901102090501 & $133-091-20 \mathrm{CBC}$ & 041 & 10130204 & 125TGRV & $09-25-47$ & -- & 124 & $\rightarrow$ & 1900 & + \\
\hline 461910103080201 & 133-099-20ADA & 087 & 10130205 & 125TRVL & $06-04-75$ & - & 210 & - & 2290 & -- \\
\hline 461930102551801 & 133-097-19BBВ & 041 & 10130205 & 125TGRV & $03-31-69$ & - & 135 & 2765 & 2330 & - \\
\hline 461931103014601 & 133-098-18DDD & 087 & 10130205 & 125TRVL & $07-01-75$ & -- & 95 & - & 2600 & -- \\
\hline 461934102034701 & 133-091-24BAB & 041 & 10130204 & 125TGRV & $07-25-67$ & -- & 201 & 2352 & 2110 & - \\
\hline 461939101071301 & 133-083-21ABB & 037 & 10130206 & 112SLDS & $11-12-71$ & - & 84 & 1857 & 2290 & -- \\
\hline 462012100581802 & 133-082-15ACD2 & 059 & 10130206 & 211FXHL & $06-28-72$ & - & 260 & - & 2730 & - \\
\hline 462016101432901 & 133-088-15ACB & 037 & 10130204 & 125CNBL & $04-09-69$ & - & 210 & - & 1880 & - \\
\hline 462021102564301 & 133-098-14AAA & 087 & 10130205 & 125LHCK & $07-22-75$ & - & 860 & 2790 & 2200 & - \\
\hline 462032102253201 & 133-094-12DDC & 041 & 10130204 & 125TGRV & $08-25-67$ & - & 458 & 2470 & 2000 & - \\
\hline 462033102341302 & 133-095-11DDD2 & 041 & 10130204 & - & $03-11-69$ & -- & 192 & 2620 & 2360 & 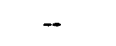 \\
\hline 462038100402801 & $133-080-12 \mathrm{DDD}$ & 085 & 10130206 & 112BGFV & $08-11-71$ & - & 134 & 1730 & 2210 & - \\
\hline 462038102491402 & $133-097-11 \mathrm{DDA} 2$ & 041 & 10130205 & 125TGRV & $05-01-68$ & - & 102 & 2707 & 1310 & - \\
\hline 462041100391701 & $133-079-7 \mathrm{DD}$ & 085 & 10130206 & $211 \mathrm{FXHL}$ & $00-00-52$ & - & 190 & -- & 1640 & - \\
\hline 462103101180301 & 133-085-12AAD & 037 & 10130206 & $211 \mathrm{FXHL}$ & $11-16-72$ & - & 522 & 2020 & 2270 & - \\
\hline 462110101180300 & - & 037 & 10130206 & $211 \mathrm{FXHL}$ & $04-24-73$ & - & 522 & - & 2240 & - \\
\hline 462110102512502 & 133-097-10BBD2 & 041 & 10130205 & 125TGRV & $12-24-68$ & $\rightarrow$ & 75 & 2687 & 910 & -- \\
\hline 462111101030001 & 133-083-12ADA1 & 037 & 10130206 & $211 \mathrm{FXHL}$ & $05-17-73$ & - & 230 & 1764 & 2170 & -- \\
\hline 462111101030002 & 133-083-12ADA2 & 037 & - & 112SLDS & $05-17-73$ & - & 84 & 1764 & 1590 & - \\
\hline 462116101180301 & $133-085-12 A A D$ & 037 & 10130206 & - & $04-24-73$ & - & 522 & - & 2300 & $\rightarrow$ \\
\hline
\end{tabular}




\begin{tabular}{|c|c|c|c|c|c|c|c|c|c|c|c|}
\hline Slte number & Date & $\begin{array}{l}\text { pH, } \\
\text { field } \\
\text { (atand- } \\
\text { ard } \\
\text { units }\end{array}$ & $\begin{array}{l}\mathrm{PH}, \\
\text { lab } \\
\text { (stand- } \\
\text { ard } \\
\text { unlts) }\end{array}$ & $\begin{array}{l}\text { Temper- } \\
\text { ature, } \\
\text { water } \\
\text { (degrees } \\
\text { Celslus) }\end{array}$ & $\begin{array}{l}\text { Color } \\
\text { (plati- } \\
\text { num } \\
\text { cobait } \\
\text { scalo) }\end{array}$ & $\begin{array}{c}\text { Hard- } \\
\text { ness, } \\
\text { total } \\
\text { (mg/L as } \\
\left.\mathrm{CaCO}_{3}\right)\end{array}$ & $\begin{array}{c}\text { Noncar- } \\
\text { bonate } \\
\text { hard- } \\
\text { ness, } \\
\text { total } \\
\text { (mg/L as } \\
\left.\mathrm{CaCO}_{3}\right)\end{array}$ & $\begin{array}{l}\text { Alka- } \\
\text { linity, } \\
\text { field } \\
\text { (mg/L as } \\
\mathrm{CaCO}_{3} \text { ) }\end{array}$ & $\begin{array}{c}\text { Alka- } \\
\text { linity, } \\
\text { lab } \\
\text { (mg/L as } \\
\left.\mathrm{CaCO}_{3}\right)\end{array}$ & $\begin{array}{l}\text { Solids, } \\
\text { sum of } \\
\text { constit- } \\
\text { uents, } \\
\text { dis- } \\
\text { solved } \\
\text { (mg/L) }\end{array}$ & $\begin{array}{c}\text { Solids, } \\
\text { residue } \\
\text { at } 105 \\
\text { degrees } \\
\text { Celsius, } \\
\text { total } \\
\text { (mg/L) }\end{array}$ \\
\hline 461700102532603 & $08-18-67$ & 7.9 & - & 10.0 & - & 750 & 450 & - & -- & 1780 & -- \\
\hline 461716101014501 & $06-27-72$ & 8.2 & - & 10.0 & -- & 17 & 0 & - & -- & 1380 & -- \\
\hline 461722101063602 & $12-01-71$ & 8.0 & - & - & -- & 290 & 0 & -- & - & 1010 & -- \\
\hline 461727103012701 & $07-01-75$ & 8.2 & -- & 11.0 & -- & 80 & 0 & - & -- & 1990 & - \\
\hline 461735101533602 & $09-15-71$ & 7.7 & - & -- & - & 570 & 140 & - & - & 1710 & -- \\
\hline 461738103085801 & $08-28-74$ & 8.7 & - & -- & - & 16 & 0 & - & -- & 1160 & - \\
\hline 461748101010701 & $06-27-72$ & 8.2 & -- & 10.0 & - & 17 & 0 & - & - & 1450 & -- \\
\hline 461749101511503 & $04-01-70$ & 9.1 & - & -- & - & 10 & - & - & - & 946 & - \\
\hline 461756102062601 & $05-01-48$ & - & - & 9.0 & - & 130 & - & - & -- & 1880 & -- \\
\hline 461801101010701 & $06-27-72$ & 8.3 & -- & 10.5 & -- & 26 & 0 & - & - & 1470 & -- \\
\hline 461801101070401 & $11-13-71$ & 7.9 & - & 8.0 & - & 660 & 61 & - & -. & 1290 & -- \\
\hline 461815102141202 & $07-23-59$ & 7.5 & - & - & 200 & 430 & - & - & - & 630 & -- \\
\hline 461816102441602 & $03-11-69$ & 7.6 & - & 8.5 & 20 & 1000 & 600 & - & -- & 1670 & - \\
\hline 461820101131101 & $07-24-61$ & - & - & - & $\ldots$ & 17 & -- & - & - & -. & - \\
\hline 461822102190901 & $08-17-82$ & 7.4 & 7.9 & 17.0 & -- & 340 & - & - & 257 & 427 & -- \\
\hline 461822102255101 & $06-17-69$ & 7.8 & - & 8.5 & -- & 640 & 290 & - & - & 1340 & - \\
\hline 461833102551401 & $08-08-69$ & - & - & -- & -- & 800 & - & - & - & 1240 & - \\
\hline 461836102341302 & $03-11-69$ & 8.2 & - & 8.5 & 200 & 21 & 0 & - & -- & 860 & -- \\
\hline 461841102132501 & $06-03-69$ & 7.9 & - & 15.0 & - & 37 & 0 & - & - & 1750 & -- \\
\hline 461841102190901 & $12-01-67$ & 7.9 & - & 8.5 & 700 & 66 & 0 & - & - & 905 & -- \\
\hline 461842102341301 & $08-18-82$ & 7.6 & 8.0 & 17.0 & -- & 110 & - & $\ldots$ & 781 & 1620 & -- \\
\hline 461845103015601 & $07-01-75$ & 8.0 & - & 10.0 & - & 160 & 0 & -. & - & 1890 & - \\
\hline 461847101065501 & $11-13-71$ & 7.9 & - & 8.0 & -. & 360 & 0 & - &.- & 1560 & - \\
\hline 461849102391601 & $05-14-69$ & 8.4 & - & 10.5 & 2300 & 56 & 0 & - & $\cdots$ & 863 & -. \\
\hline 461852103013701 & $07-01-75$ & 8.7 & - & 14.0 & - & 6 & 0 & -. & - & 1160 & -- \\
\hline 461901102090501 & $09-25-47$ & - & - & -- & - & 69 & -- & -- & -- & 1230 & -- \\
\hline 461910103080201 & $06-04-75$ & 8.3 & -- & 10.5 & -- & 28 & 0 & - & - & 1500 & -- \\
\hline 461930102551801 & $03-31-69$ & - & -- & - & - & 300 & - & -. & - & 1870 & -. \\
\hline 461931103014601 & $07-01-75$ & 6.9 & - & 7.0 & - & 1200 & 860 & - & -- & 1890 & -- \\
\hline 461934102034701 & $07-25-67$ & 8.2 & - & 10.5 & - & 20 & 0 & -- & - & 1420 & -- \\
\hline 461939101071301 & $11-12-71$ & 8.1 & -- & 8.0 & -- & 140 & 0 & - & - & 1520 & - \\
\hline 462012100581802 & $06-28-72$ & 8.2 & -- & 10.0 & - & 24 & 0 & - & - & 1840 & -- \\
\hline 462016101432901 & $04-09-69$ & 7.5 & - & - & - & 200 & - & - & - & 1070 & - \\
\hline 462021102564301 & $07-22-75$ & 8.6 & - & 16.0 & - & 13 & 0 & -- & - & 1400 & -- \\
\hline 462032102253201 & $08-25-67$ & 8.5 & -- & 12.0 & - & 14 & 0 & - & - & 1280 & - \\
\hline 462033102341302 & $03-11-69$ & 8.2 & - & 9.5 & 25 & 54 & 0 & - & -- & 1590 & -- \\
\hline 462038100402801 & $08-11-71$ & 8.3 & - & 7.0 & -- & 86 & 0 & - & - & 1510 & - \\
\hline 462038102491402 & $05-01-68$ & 8.4 & - & 10.0 & 200 & 24 & 0 & -- & - & 863 & - \\
\hline 462041100391701 & $00-00-52$ & 8.0 & - & - & - & 0 & -. & - & - & 800 & - \\
\hline 462103101180301 & $11-16-72$ & 8.2 & - & 7.0 & -- & 12 & 0 & -- & - & 1380 & - \\
\hline 462110101180300 & $04-24-73$ & 8.3 & -- & 10.0 & 8 & 13 & 0 & - & -- & 1370 & -- \\
\hline 462110102512502 & $12-24-68$ & - & -- & - & - & 40 & - & -- & - & 730 & - \\
\hline 462111101030001 & $05-17-73$ & 8.4 & - & 10.0 & - & 18 & 0 & - & - & 1410 & - \\
\hline 462111101030002 & $05-17-73$ & 7.8 & - & 8.0 & - & 300 & 0 & - & -- & 1070 & - \\
\hline 462116101180301 & $04-24-73$ & 8.5 & - & - & - & 13 & 0 & - & -- & 1390 & - \\
\hline
\end{tabular}




\begin{tabular}{|c|c|c|c|c|c|c|c|c|c|c|c|}
\hline Site number & Date & $\begin{array}{l}\text { Solids, } \\
\text { residue } \\
\text { at } 180 \\
\text { degrees } \\
\text { Colsius, } \\
\text { die- } \\
\text { solved } \\
\text { (mg/L) }\end{array}$ & $\begin{array}{l}\text { Sollds, } \\
\text { dis- } \\
\text { solved } \\
\text { (tons per } \\
\text { acre- } \\
\text { took) }\end{array}$ & $\begin{array}{l}\text { Calclum, } \\
\text { dle- } \\
\text { solved } \\
\text { (mg/L } \\
\text { as Ca) }\end{array}$ & $\begin{array}{l}\text { Magno- } \\
\text { sium, } \\
\text { dis- } \\
\text { solved } \\
\text { (mg/L } \\
\text { as Mg) }\end{array}$ & $\begin{array}{l}\text { Sodlum, } \\
\text { dle- } \\
\text { solved } \\
\text { (mg/L } \\
\text { as Na) }\end{array}$ & $\begin{array}{l}\text { Sodium } \\
\text { (percent) }\end{array}$ & $\begin{array}{l}\text { Sodlum } \\
\text { adsorp- } \\
\text { tion } \\
\text { ratio }\end{array}$ & $\begin{array}{l}\text { Resid- } \\
\text { ual, } \\
\text { sodium, } \\
\text { car- } \\
\text { bonate } \\
\text { (mg/L as } \\
\mathrm{CaCO}_{3} \text { ) }\end{array}$ & $\begin{array}{l}\text { Potas- } \\
\text { slum, } \\
\text { dis- } \\
\text { solved } \\
\text { (mg/L } \\
\text { as K) }\end{array}$ & $\begin{array}{c}\text { Blcar- } \\
\text { bonate } \\
\text { (mg/L as } \\
\left.\mathrm{HCO}_{3}\right)\end{array}$ \\
\hline 461700102532603 & $08-18-67$ & 1840 & 2.50 & 190 & 68 & 280 & 45 & 4 & - & 9.0 & 370 \\
\hline 461716101014501 & $06-27-72$ & 1320 & 1.80 & 4.3 & 1.6 & 530 & 98 & 55 & - & 1.9 & 1100 \\
\hline 461722101063602 & $12-01-71$ & 1030 & 1.40 & 77 & 24 & 260 & 66 & 7 & - & 3.7 & 780 \\
\hline 461727103012701 & $07-01-75$ & 1990 & 2.71 & 19 & 7.9 & 660 & 94 & 32 & - & 4.4 & 750 \\
\hline 461735101533602 & $09-15-71$ & 1710 & 2.33 & 120 & 68 & 370 & 58 & 7 & 0.0 & 5.6 & 520 \\
\hline 461738103085801 & $08-28-74$ & 1160 & 1.58 & 2.3 & 2.6 & 450 & 98 & 48 & - & 1.2 & 830 \\
\hline 461748101010701 & $06-27-72$ & 1380 & 1.88 & 4.9 & 1.2 & 570 & 98 & 60 & -- & 2.0 & 1130 \\
\hline 461749101511503 & $04-01-70$ & 1140 & -- & -- & -- & -- & - & - & -- & - & - \\
\hline 461756102062601 & $05-01-48$ & 1580 & - & -. & - & - & - & - & -- & - & 330 \\
\hline 461801101010701 & $06-27-72$ & 1370 & 1.86 & 3.3 & 4.4 & 560 & 98 & 47 & - & 2.0 & 1150 \\
\hline 461801101070401 & $11-13-71$ & 1320 & 1.80 & 160 & 65 & 180 & 37 & 3 & 0.0 & 8.6 & 730 \\
\hline 461815102141202 & $07-23-59$ & -- & - & 95 & 46 & 0.0 & -- & $\mathbf{0}$ & -- & -- & 260 \\
\hline 461816102441602 & $03-11-69$ & 1900 & 2.58 & 260 & 96 & 180 & 27 & 2 & - & 13 & 550 \\
\hline 461820101131101 & $07-24-61$ & 1650 & -- & 6.0 & 0.50 & 480 & - & 50 & - & -- & 710 \\
\hline 461822102190901 & $08-17-82$ & 474 & 0.64 & 77 & 36 & 24 & 13 & 0.6 & - & 5.3 & - \\
\hline 461822102255101 & $06-17-69$ & 1430 & 1.94 & 150 & 64 & 190 & 38 & 3 & - & 13 & 430 \\
\hline 461833102551401 & $08-08-69$ & - & - & -- & -- & -- & - & - & - & - & -- \\
\hline 461836102341302 & $03-11-69$ & 869 & 1.18 & 6.4 & 1.2 & 330 & 97 & 32 & - & 2.4 & 660 \\
\hline 461841102132501 & $06-03-69$ & 1720 & 2.34 & 9.2 & 3.4 & 590 & 97 & 42 & - & 3.8 & 540 \\
\hline 461841102190901 & $12-01-67$ & 947 & 1.29 & 15 & 6.9 & 310 & 90 & 17 & - & 5.4 & 540 \\
\hline 461842102341301 & $08-18-82$ & 1610 & 2.19 & 24 & 11 & 560 & 91 & 24 & -- & 6.6 & - \\
\hline 461845103015601 & $07-01-75$ & 1930 & 2.62 & 41 & 14 & 600 & 89 & 21 & - & 5.9 & 790 \\
\hline 461847101065501 & $11-13-71$ & 1600 & 2.18 & 88 & 35 & 390 & 70 & 9 & 4.00 & 6.8 & 660 \\
\hline 461849102391601 & $05-14-69$ & 1210 & 1.65 & 8.0 & 8.8 & 320 & 92 & 19 & - & 4.0 & 680 \\
\hline 461852103013701 & $07-01-75$ & 1180 & 1.60 & 2.1 & 0.20 & 460 & 99 & 81 & -- & 1.9 & 880 \\
\hline 461901102090501 & $09-25-47$ & 1110 & -- & -- & - & -- & - & - & - & - & 330 \\
\hline 461910103080201 & $06-04-75$ & 1520 & 2.07 & 7.7 & 2.2 & 530 & 97 & 43 & - & 2.3 & 590 \\
\hline 461930102551801 & $03-31-69$ & - & - & - & -- & 400 & - & -- & -- & -- & - \\
\hline 461931103014601 & $07-01-75$ & 2070 & 2.82 & 220 & 160 & 130 & 19 & 2 & - & 7.6 & 430 \\
\hline 461934102034701 & $07-25-67$ & 1320 & 1.80 & 5.0 & 1.8 & 510 & 98 & 49 & - & 2.9 & 590 \\
\hline 461939101071301 & $11-12-71$ & 1520 & 2.07 & 39 & 10 & 500 & 88 & 18 & 11.0 & 3.7 & 820 \\
\hline 462012100581802 & $06-28-72$ & 1770 & 2.41 & 6.6 & 1.8 & 670 & 98 & 60 & -- & 7.6 & 1130 \\
\hline 462016101432901 & $04-09-69$ & 1280 & - & - & - & -- & - & - & - & - & - \\
\hline 462021102564301 & $07-22-75$ & 1430 & 1.94 & 3.7 & 1.0 & 590 & 99 & 70 & -- & 2.1 & 1450 \\
\hline 462032102253201 & $08-25-67$ & 1290 & 1.75 & 3.2 & 1.5 & 550 & 99 & 64 & -- & 1.9 & 1310 \\
\hline 462033102341302 & $03-11-69$ & 1570 & 2.14 & 16 & 3.4 & 570 & 95 & 34 & - & 4.7 & 940 \\
\hline 462038100402801 & $08-11-71$ & 1540 & 2.09 & 22 & 7.5 & 500 & 92 & 23 & 10.0 & 4.7 & 690 \\
\hline 462038102491402 & $05-01-68$ & 885 & 1.20 & 5.7 & 2.4 & 320 & 96 & 28 & - & 2.8 & 610 \\
\hline 462041100391701 & $00-00-52$ & 976 & - & - & - & -- & -- & - & - & - & 880 \\
\hline 462103101180301 & $11-16-72$ & 1390 & 1.89 & 3.3 & 1.0 & 560 & 99 & 69 & 18.0 & 1.8 & 1130 \\
\hline 462110101180300 & $04-24-73$ & 1390 & 1.89 & 3.8 & 0.90 & 540 & 99 & 65 & - & 2.0 & 1120 \\
\hline 462110102512502 & $12-24-68$ & -- & - & -- & - & 240 & - & - & - & - & - \\
\hline 462111101030001 & $05-17-73$ & 1410 & 1.92 & 4.7 & 1.6 & 540 & 98 & 55 & 6.00 & 2.5 & 950 \\
\hline 462111101030002 & $05-17-73$ & 1030 & 1.40 & 66 & 32 & 290 & 67 & 7 & -- & 6.6 & 760 \\
\hline 462116101180301 & $04-24-73$ & 1400 & 1.90 & 3.8 & 0.90 & 570 & 99 & 69 & -- & 2.6 & 1060 \\
\hline
\end{tabular}




\begin{tabular}{|c|c|c|c|c|c|c|c|c|c|c|c|}
\hline Site number & Date & $\begin{array}{c}\text { Car- } \\
\text { bonate } \\
\text { (mg/l as } \\
\left.\mathrm{CO}_{3}\right)\end{array}$ & $\begin{array}{c}\text { Carbon } \\
\text { dioxide, } \\
\text { dis- } \\
\text { solved } \\
(\mathrm{mg} / \mathrm{L} \text { as } \\
\left.\mathrm{CO}_{2}\right)\end{array}$ & $\begin{array}{l}\text { Sulfate, } \\
\text { dis- } \\
\text { solved } \\
\text { (mg/L as } \\
\mathrm{SO}_{4} \text { ) }\end{array}$ & $\begin{array}{l}\text { Chlo- } \\
\text { ride, } \\
\text { dis- } \\
\text { solved } \\
\text { (mg/L } \\
\text { as Cl) }\end{array}$ & $\begin{array}{l}\text { Fluoride, } \\
\text { dis- } \\
\text { solved } \\
\text { (mg/L } \\
\text { as F) }\end{array}$ & $\begin{array}{l}\text { Silica, } \\
\text { dis- } \\
\text { solved } \\
\text { (mg/L as } \\
\left.\mathrm{SiO}_{2}\right)\end{array}$ & $\begin{array}{l}\text { Nitro- } \\
\text { gen, } \\
\text { nitrate, } \\
\text { dis- } \\
\text { solved } \\
\text { (mg/L } \\
\text { as N) }\end{array}$ & $\begin{array}{c}\text { Nitro- } \\
\text { gen, } \\
\text { nitrate, } \\
\text { total } \\
\left(\mathrm{mgll}^{2}\right. \\
\left.\text { as } \mathrm{NO}_{3}\right)\end{array}$ & $\begin{array}{l}\text { Nitro- } \\
\text { gen, } \\
\text { nitrate, } \\
\text { dis- } \\
\text { solved } \\
(\mathrm{mg} / \mathrm{L} \\
\left.\text { as } \mathrm{NO}_{3}\right)\end{array}$ & 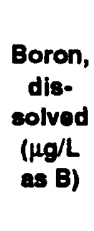 \\
\hline 461700102532603 & $08-18-67$ & 0 & - & 1000 & 15 & 0.10 & 19 & -- & - & 5.5 & 1100 \\
\hline 461716101014501 & $06-27-72$ & 0 & 11 & 240 & 37 & 1.7 & 16 & 0.230 & - & 1.0 & 2000 \\
\hline 461722101063602 & $12-01-71$ & 0 & 12 & 200 & 29 & 1.2 & 24 & 0.560 & - & 2.5 & 930 \\
\hline 461727103012701 & $07-01-75$ & 0 & 7.6 & 910 & 6.5 & 1.2 & 5.6 & 0.230 & - & 1.0 & 790 \\
\hline 461735101533602 & $09-15-71$ & 0 & - & 880 & 8.6 & 0.10 & 11 & 0.226 & 1.0 & - & 270 \\
\hline 461738103085801 & $08-28-74$ & 30 & 2.8 & 180 & 64 & 3.2 & 9.3 & 0.590 & .. & 2.6 & 1300 \\
\hline 461748101010701 & $06-27-72$ & 0 & 11 & 200 & 93 & 1.9 & 16 & 0.230 & -- & 1.0 & 2000 \\
\hline 461749101511503 & $04-01-70$ & - & - & 420 & 13 & -- & $\ldots$ & 0.904 & 4.0 & - & - \\
\hline 461756102062601 & $05-01-48$ & 0 & - & 460 & 0.0 & -- & - & - & $\ldots$ & -- & - \\
\hline 461801101010701 & $06-27-72$ & 7 & 9.3 & 230 & 74 & 1.6 & 16 & 0.230 & -. & 1.0 & 1900 \\
\hline 461801101070401 & $11-13-71$ & 0 & -. & 480 & 1.4 & 0.60 & 25 & 0.226 & 1.0 & - & 710 \\
\hline 461815102141202 & $07-23-59$ & 0 & - & 210 & 9.8 & -- & - & - & -. & 0.0 & $\cdots$ \\
\hline 461816102441602 & $03-11-69$ & 0 & - & 780 & 49 & 0.30 & 15 & - & - & 170 & 700 \\
\hline 461820101131101 & $07-24-61$ & 0 & .. & 450 & 8.0 & $\ldots$ & -- & 0.904 & 4.0 & -. & - \\
\hline 461822102190901 & $08-17-82$ & - & - & 110 & 8.6 & 0.10 & 12 & - & -- & - & 170 \\
\hline 461822102255101 & $06-17-69$ & 0 & -. & 690 & 6.1 & 0.20 & 10 & -. & -. & 4.2 & 440 \\
\hline 461833102551401 & $08-08-69$ & - & - & .. & $\ldots$ &.- & $\ldots$ & $\ldots$ & -- & 20 & -. \\
\hline 461836102341302 & $03-11-69$ & 0 & - & 180 & 3.0 & 1.3 & 8.4 & -- & -. & 0.0 & 560 \\
\hline 461841102132501 & $06-03-69$ & 0 & - & 860 & 2.5 & 2.5 & 8.5 & .. & - & 2.5 & 670 \\
\hline 461841102190901 & $12-01-67$ & 0 & - & 290 & 4.5 & 0.10 & - & - & - & 0.0 & 880 \\
\hline 461842102341301 & $08-18-82$ & - & -- & 530 & 5.0 & 0.60 & 9.9 & - & -. & -. & 950 \\
\hline 461845103015601 & $07-01-75$ & 0 & 13 & 830 & 5.1 & 1.1 & 5.4 & 0.230 & -- & 1.0 & 430 \\
\hline 461847101065501 & $11-13-71$ & 0 & - & 680 & 2.1 & 0.90 & 21 & 0.565 & 2.5 & - & 620 \\
\hline 461849102391601 & $05-14-69$ & 12 & $\ldots$ & 150 & 10 & 5.5 & 11 & - & - & 2.5 & 1500 \\
\hline 461852103013701 & $07-01-75$ & 41 & 3.1 & 130 & 79 & 5.1 & 8.8 & 0.230 & -- & 1.0 & 1500 \\
\hline 461901102090501 & $09-25-47$ & - & -- & 450 & 0.0 & -- & - & - & .. & -- & - \\
\hline 461910103080201 & $06-04-75$ & 7 & 4.8 & 640 & 11 & 0.40 & 7.8 & 0.230 & -- & 1.0 & 340 \\
\hline 461930102551801 & $03-31-69$ & - & $\ldots$ & - & $\ldots$ & -- & - & - & - & 5.0 & - \\
\hline 461931103014601 & $07-01-75$ & 0 & 86 & 1100 & 24 & 0.30 & 16 & 2.30 & -- & 10 & 1900 \\
\hline 461934102034701 & $07-25-67$ & 0 & - & 600 & 4.7 & 0.90 & 7.7 & - & - & 1.0 & 580 \\
\hline 461939101071301 & $11-12-71$ & 0 & - & 530 & 5.6 & 1.9 & 20 & 0.226 & 1.0 & -. & 1200 \\
\hline 462012100581802 & $06-28-72$ & 0 & 11 & 530 & 42 & 1.6 & 16 & 0.230 & - & 1.0 & 2300 \\
\hline 462016101432901 & $04-09-69$ & - & $\ldots$ & 510 & 4.0 & - & - & 0.00 & 0.0 & - & -. \\
\hline 462021102564301 & $07-22-75$ & 48 & 6.2 & 8.6 & 13 & 10 & 10 & 0.200 & - & 0.90 & 1600 \\
\hline 462032102253201 & $08-25-67$ & 36 & $\ldots$ & 7.6 & 24 & 3.9 & 9.3 & - & - & 0.0 & 1400 \\
\hline 462033102341302 & $03-11-69$ & 0 & -- & 520 & 8.0 & 1.1 & 7.3 & - & - & 1.0 & 480 \\
\hline 462038100402801 & $08-11-71$ & 5 & -- & 600 & 3.3 & 0.70 & 19 & 0.813 & 3.6 & - & 1300 \\
\hline 462038102491402 & $05-01-68$ & 5 & -. & 210 & 5.5 & 0.50 & 8.4 & - & - & 0.0 & 880 \\
\hline 462041100391701 & $00-00-52$ & 0 & - & 0.0 & 3.9 & $\ldots$ & - & 0.00 & 0.0 & - & -. \\
\hline 462103101180301 & $11-16-72$ & 0 & -- & 6.2 & 220 & 3.4 & 17 & 0.226 & 1.0 & - & 3000 \\
\hline 462110101180300 & $04-24-73$ & 0 & 9.0 & 15 & 230 & 2.9 & 18 & -. & -. & .. & 3100 \\
\hline 462110102512502 & $12-24-68$ & - & $\ldots$ & - & - & -- & -. & -. & - & 9.0 & - \\
\hline 462111101030001 & $05-17-73$ & 19 & 6.3 & 330 & 24 & 2.1 & 17 & 0.230 & 1.0 & 1.0 & 1900 \\
\hline 462111101030002 & $05-17-73$ & 0 & 19 & 270 & 5.3 & 0.50 & 27 & 0.230 & - & 1.0 & 790 \\
\hline 462116101180301 & $04-24-73$ & 25 & 5.6 & 16 & 230 & 2.1 & 15 & 0.230 & .. & 1.0 & 2600 \\
\hline
\end{tabular}




\begin{tabular}{|c|c|c|c|c|c|c|c|c|c|c|c|}
\hline Stte number & Date & $\begin{array}{l}\text { Iron, } \\
\text { dis- } \\
\text { solved } \\
\text { ( } \mu \mathrm{g} / \mathrm{L} \\
\text { as Fe) }\end{array}$ & $\begin{array}{c}\text { Lead, } \\
\text { dis- } \\
\text { solved } \\
(\mu g / L \\
\text { as Pb) }\end{array}$ & $\begin{array}{l}\text { Lithium, } \\
\text { dis- } \\
\text { eolved } \\
\text { ( } \mu g / \mathrm{L} \\
\text { as Li) }\end{array}$ & $\begin{array}{l}\text { Manga- } \\
\text { nese, } \\
\text { dis- } \\
\text { solved } \\
\text { ( } \mu g / \mathrm{l} \\
\text { as Mn) }\end{array}$ & $\begin{array}{l}\text { Mercury, } \\
\text { dis- } \\
\text { solved } \\
\text { ( } \mu \mathrm{g} / \mathrm{L} \\
\text { as Hg) }\end{array}$ & $\begin{array}{l}\text { Molyb- } \\
\text { denum, } \\
\text { dis- } \\
\text { solved } \\
(\mu g / L \\
\text { as Mo) }\end{array}$ & $\begin{array}{c}\text { Sole- } \\
\text { nium, } \\
\text { dis- } \\
\text { solved } \\
\text { (ug/L } \\
\text { as Se) }\end{array}$ & $\begin{array}{l}\text { Stron- } \\
\text { tium, } \\
\text { dis- } \\
\text { solved } \\
(\mu g / L \\
\text { as Sr) }\end{array}$ & $\begin{array}{l}\text { Vana- } \\
\text { dium, } \\
\text { dis- } \\
\text { solved } \\
\text { ( } \mu g / L \\
\text { as V) }\end{array}$ & $\begin{array}{c}\text { Zinc, } \\
\text { dls- } \\
\text { solved } \\
(\mu g / L \\
\text { as Zn) }\end{array}$ \\
\hline 461700102532603 & $08-18-67$ & 4000 & -- & -- & - & -- & - & - & - & -- & -- \\
\hline 461716101014501 & $06-27-72$ & 220 & -- & -- & 10 & -- & - & -- & - & - & -- \\
\hline 461722101063602 & $12-01-71$ & -- & - & - & - & - & - & - & -- & - & -- \\
\hline 461727103012701 & $07-01-75$ & 370 & -- & -- & 60 & - & - & - & -- & - & -- \\
\hline 461735101533602 & $09-15-71$ & 2800 & - & - & 20 & - & -- & -- & -- & - & - \\
\hline 461738103085801 & $08-28-74$ & 410 & - & -- & 40 & - & -- & -- & -- & - & - \\
\hline 461748101010701 & $06-27-72$ & 0 & -- & - & 0 & -- & -- & -- & - & -- & - \\
\hline 461749101511503 & $04-01-70$ & 960 & -. & -- & - & - & -- & - & -- & -. & -. \\
\hline 461756102062601 & $05-01-48$ & 400 & - & -- & - & - & - & - & -- & -- & - \\
\hline 461801101010701 & $06-27-72$ & 90 & - & -- & 10 & -- & - & - & - & - & - \\
\hline 461801101070401 & $11-13-71$ & 3300 & -- & - & 120 & - & -- & - & - & -- & -- \\
\hline 461815102141202 & $07-23-59$ & 9700 & -- & -. & - & - & -- & -- & - & -- & -. \\
\hline 461816102441602 & $03-11-69$ & 0 & - & - & -- & - & -- & -- & - & $\ldots$ & -- \\
\hline 461820101131101 & $07-24-61$ & 400 & - & - & - & - & - & - & -. & - & -- \\
\hline 461822102190901 & $08-17-82$ & 7 & - & - & 10 & - & - & 1 & - & - & -- \\
\hline 461822102255101 & $06-17-69$ & 1700 & - & - & - & - & - & - & - & -- & -- \\
\hline 461833102551401 & $08-08-69$ & 300 & - & - & - & - & - & - & - & -. & -- \\
\hline 461836102341302 & $03-11-69$ & 280 & - & - & - & - & - & -- & - & - & - \\
\hline 461841102132501 & $06-03-69$ & 500 & $\cdots$ & - & - & -- & -- & -- & - & - & -- \\
\hline 461841102190901 & $12-01-67$ & 4600 & - & - & - & - & - & - & - & - & - \\
\hline 461842102341301 & $08-18-82$ & 220 & - & - & 40 & - & - & $<1$ & - & - & - \\
\hline 461845103015601 & $07-01-75$ & 730 & -- & - & 60 & - & - & - & - & - & - \\
\hline 461847101065501 & $11-13-71$ & 4500 & - & - & 10 & - & -- & -- & - & - & -- \\
\hline 461849102391601 & $05-14-69$ & 780 & -- & - & - & - & -- & - & - & - & - \\
\hline 461852103013701 & $07-01-75$ & 1100 & - & -- & $<10$ & - & -- & -- & -- & - & - \\
\hline 461901102090501 & $09-25-47$ & 700 & - & - & - & - & -- & -- & - & - & -- \\
\hline 461910103080201 & $06-04-75$ & 1300 & - & - & 40 & - & -- & - & -- & -- & -- \\
\hline 461930102551801 & $03-31-69$ & 200 & - & - & - & - & - & - & - & - & - \\
\hline 461931103014601 & $07-01-75$ & 13000 & - & - & 1600 & - & -- & - & - & - & - \\
\hline 461934102034701 & $07-25-67$ & 80 & - & - & -- & - & - & - & - & -- & -- \\
\hline 461939101071301 & $11-12-71$ & 2100 & - & - & 10 & - & - & - & -- & -- & -- \\
\hline 462012100581802 & $06-28-72$ & 860 & - & -- & 40 & - & - & -- & - & -. & -- \\
\hline 462016101432901 & $04-09-69$ & 1200 & - & - & - & -- & -- & - & -- & -. & - \\
\hline 462021102564301 & $07-22-75$ & 190 & - & - & $<10$ & - & -- & - & - & - & - \\
\hline 462032102253201 & $08-25-67$ & 130 & - & - & - & - & -- & - & - & $\ldots$ & - \\
\hline 462033102341302 & $03-11-69$ & 2800 & - & - & -- & - & - & - & -- & - & -. \\
\hline 462038100402801 & $08-11-71$ & 6500 & - & -- & 160 & -- & - & - & - & -. & - \\
\hline 462038102491402 & $05-01-68$ & 3500 & - & - & - & - & -- & - & - & - & -- \\
\hline 462041100391701 & $00-00-52$ & 1500 & - & - & -- & - & -- & - & - & $\ldots$ & - \\
\hline 462103101180301 & $11-16-72$ & 920 & -- & - & $<10$ & - & - & - & - & - & -. \\
\hline 462110101180300 & $04-24-73$ & 200 & 2 & 70 & $<10$ & 0.7 & 9 & 6 & 90 & 5 & 50 \\
\hline 462110102512502 & $12-24-68$ & 450 & - & - & -- & - & - & - & - & -. & - \\
\hline 462111101030001 & $05-17-73$ & 100 & - & -. & 20 & -- & - & - & - & - & -- \\
\hline 462111101030002 & $05-17-73$ & 1300 & - & - & 240 & - & - & - & -- & - & .. \\
\hline 462116101180301 & $04-24-73$ & 300 & - & - & 60 & - & - & - & -- & - & - \\
\hline
\end{tabular}




\begin{tabular}{|c|c|c|c|c|c|c|c|c|c|c|}
\hline Site number & Local Identifier & $\begin{array}{l}\text { County } \\
\text { code }\end{array}$ & $\begin{array}{l}\text { Hydrologic } \\
\text { unit }\end{array}$ & $\begin{array}{c}\text { Geologic } \\
\text { unit }\end{array}$ & Date & $\begin{array}{l}\text { Depth } \\
\text { below } \\
\text { land } \\
\text { surface, } \\
\text { water } \\
\text { iovel } \\
\text { (feot) }\end{array}$ & $\begin{array}{c}\text { Depth } \\
\text { of } \\
\text { weli, } \\
\text { totai } \\
\text { (toot) }\end{array}$ & $\begin{array}{l}\text { Altitude } \\
\text { of land } \\
\text { surface, } \\
\text { datum } \\
\text { (feet) }\end{array}$ & $\begin{array}{l}\text { Specific } \\
\text { conduct- } \\
\text { ance, } \\
\text { field } \\
(\mu S / c m)\end{array}$ & $\begin{array}{c}\text { Specific } \\
\text { conduct- } \\
\text { ance, } \\
\text { lab } \\
(\mu \mathrm{S} / \mathrm{cm})\end{array}$ \\
\hline 462116103021401 & 133-098-6DCC & 087 & 10130205 & 125TRVL & $07-01-75$ & -- & 338 & -- & 2280 & - \\
\hline 462118102205103 & 133-093-10ABB3 & 041 & 10130204 & - & $08-12-82$ & -- & 405 & - & 1880 & 1920 \\
\hline 462119102440601 & 133-096-10BBB & 041 & 10130204 & 125TGRV & $08-17-67$ & - & 141 & 2673 & 2910 & -- \\
\hline 462123101110801 & 133-084-12BAA & 037 & 10130206 & 112SLDS & $05-17-73$ & - & 79 & -- & 728 & - \\
\hline 462124102234901 & $133-093-5 C C D$ & 041 & 10130204 & 12STGRV & $08-29-67$ & - & 207 & 2420 & 1630 & -- \\
\hline 462125102031801 & 133-091-01DCD & 041 & 10130204 & 125TGRV & $04-23-81$ & -- & -- & 2282 & 1325 & 1390 \\
\hline 462129101095301 & 133-083-6CDD & 037 & 10130206 & 211FXHL & $12-03-71$ & - & 307 & -- & 1330 & - \\
\hline 462129101105901 & 133-084-1DCC & 037 & 10130206 & 112SLDS & $05-17-73$ & - & 99 & 1899 & 1170 & - \\
\hline 462144102291002 & 133-094-4DAA2 & 041 & 10130204 & 125TGRV & $08-24-67$ & - & 165 & 2465 & 1920 & -- \\
\hline 462144102291003 & 133-094-4DAA3 & 041 & 10130204 & -- & $08-12-82$ & - & 540 & - & 2100 & 2090 \\
\hline 462150102225301 & 133-093-5ADD1 & 041 & 10130204 & 125TGRV & $07-13-65$ & - & 90 & 2452 & 1360 & - \\
\hline 462157102193701 & $133-093-2 \mathrm{ACB}$ & 041 & 10130204 & 125TGRV & $01-21-60$ & - & 140 & 2385 & -- & - \\
\hline 462203102194601 & 133-093-2BAD & 041 & 10130204 & -- & $06-02-66$ & - & 25 & 2376 & 4560 & - \\
\hline 462204102035601 & 133-091-1BBD & 041 & 10130204 & - & $07-26-67$ & - & 121 & 2322 & 500 & - \\
\hline 462209101005801 & $133-082-5 A B C$ & 059 & 10130206 & 211FXHL & $06-27-72$ & -- & 320 & 1740 & 2300 & - \\
\hline 462210102160302 & 133-092-5BAA2 & 041 & 10130204 & 125TGRV & $08-24-67$ & - & 310 & 2382 & 1720 & - \\
\hline 462210102163001 & 133-092-5BBB1 & 041 & 10130204 & 125TGRV & $08-02-54$ & - & 306 & 2396 &.- & - \\
\hline 462210102191801 & 133-093-2AAB & 041 & 10130204 & 125TGRV & $07-26-67$ & -- & 402 & 2381 & 1910 & - \\
\hline 462211102473201 & 133-096-6BAB & 041 & 10130204 & 125TGRV & $11-12-67$ & - & 212 & 2770 & 2170 & - \\
\hline 462216102102401 & $135-094-31 \mathrm{CCC}$ & 041 & 10130204 & 125SNLB & $10-01-71$ & - & 81 & - & 604 & - \\
\hline 462218101564901 & 134-090-35DDC & 037 & 10130204 & $125 \mathrm{CNBL}$ & $09-26-72$ & - & 420 & - & 2000 & -- \\
\hline 462219102192801 & 134-093-35DCD & 041 & 10130204 & 125TGRV & $07-26-67$ & - & 378 & 2380 & 1920 & - \\
\hline- & - & 037 & 10130204 & 211FXHL & $09-26-72$ & - & 285 & - & 2000 & - \\
\hline 462225101564901 & 134-090-35DDB & 037 & 10130204 & $125 \mathrm{CNBL}$ & $09-26-72$ & - & 560 & - & 2050 & - \\
\hline 462231101563001 & $134-090-36 \mathrm{CBC}$ & 037 & 10130204 & 211FXHL & $09-26-72$ & - & 880 & - & 2000 & - \\
\hline 462231101564901 & 134-090-35DAC & 037 & 10130204 & $125 \mathrm{CNBL}$ & $09-26-72$ & - & 560 & - & 2050 & - \\
\hline 462232102192801 & 134-093-35DBD & 041 & 10130204 & 125TGRV & $07-28-67$ & - & 421 & 2422 & 1890 & - \\
\hline 462235103002701 & 134-098-33BCC & 087 & 10130205 & 125TRVL & $06-04-75$ & - & 200 & - & 2190 & - \\
\hline 462236102503503 & 134-097-34DAA3 & 041 & 10130204 & 125TGRV & $11-17-66$ & - & 189 & 2794 & 1180 & - \\
\hline 462243102453602 & 134-096-32ADD2 & 041 & 10130204 & 125TGRV & $08-23-67$ & - & 240 & 2710 & 1790 & - \\
\hline 462259102264101 & 134-094-35AAA & 041 & 10130204 & - & $08-12-82$ & - & 220 & -- & 1440 & 1440 \\
\hline 462301102303101 & 134-094-32AAA1 & 041 & 10130204 & 125TGRV & $09-01-67$ & -- & 390 & 2465 & 1810 & - \\
\hline- & - & 041 & 10130204 & 125TGRV & $05-16-69$ & - & 390 & 2465 & -- & -- \\
\hline 462303100474002 & 134-080-31 BBA2 & 085 & 10130206 & 211FXHL & $04-23-71$ & - & 168 & - & - & - \\
\hline 462315102353501 & 134-095-27DDA & 041 & 10130204 & - & $08-17-82$ & - & 180 & - & 1550 & 1530 \\
\hline 462316102402701 & $134-095-30 \mathrm{CCB}$ & 041 & 10130204 & - & $08-18-82$ & - & 216 & - & 1080 & 1070 \\
\hline 462323102002601 & 134-090-29DAD & 037 & 10130204 & 125TGRV & $05-21-79$ & - & 63 & - & - & $\ldots$ \\
\hline 462328102341901 & 134-095-26DAA & 041 & 10130204 & - & $08-17-82$ & - & 592 & - & 1830 & 1810 \\
\hline 462336102453601 & 134-096-29ADD1 & 041 & 10130204 & - & $08-18-82$ & - & 280 & 2705 & 1900 & 1900 \\
\hline 462341100551202 & 134-081-30BCD2 & 059 & 10130206 & 211FXHL & $07-13-72$ & - & 200 & - & 2490 & - \\
\hline 462351101505201 & $134-089-27 \mathrm{ABCl}$ & 037 & 10130204 & 125TGRV & $09-25-72$ & - & 69 & - & 1610 & - \\
\hline 462351103071801 & 134-099-21DCC & 087 & 10130205 & 125TRVL & $10-22-76$ & - & 411 & 2885 & 1850 & - \\
\hline 462355102414202 & $134-096-25 B B B 2$ & 041 & 10130204 & 125TGRV & $05-20-69$ & - & 324 & 2610 & 1610 & - \\
\hline 462415102002601 & 134-090-20DAD1 & 037 & 10130204 & 125TGRV & $05-21-79$ & - & 150 & - & 2100 & - \\
\hline 462421101045401 & 134-083-23CAD & 059 & 10130206 & 112EMCK & $07-08-72$ & - & 135 & - & 1410 & -- \\
\hline
\end{tabular}




\begin{tabular}{|c|c|c|c|c|c|c|c|c|c|c|c|}
\hline Site number & Date & $\begin{array}{l}\text { pH, } \\
\text { field } \\
\text { (stand- } \\
\text { ard } \\
\text { units }\end{array}$ & $\begin{array}{c}\text { pH, } \\
\text { lab } \\
\text { (stand- } \\
\text { ard } \\
\text { units) }\end{array}$ & $\begin{array}{l}\text { Temper- } \\
\text { ature, } \\
\text { water } \\
\text { (degrees } \\
\text { Colsius) }\end{array}$ & $\begin{array}{l}\text { Color } \\
\text { (plati- } \\
\text { num } \\
\text { cobalt } \\
\text { scaio) }\end{array}$ & $\begin{array}{c}\text { Hard- } \\
\text { ness, } \\
\text { total } \\
\text { (mg/L as } \\
\left.\mathrm{CaCO}_{3}\right)\end{array}$ & $\begin{array}{l}\text { Noncar- } \\
\text { bonate } \\
\text { hard- } \\
\text { ness, } \\
\text { totai } \\
\text { (mg/L as } \\
\mathrm{CaCO}_{3} \text { ) }\end{array}$ & $\begin{array}{l}\text { Alka- } \\
\text { linity, } \\
\text { field } \\
\text { (mg/L as } \\
\text { CaCO }\end{array}$ & $\begin{array}{c}\text { Aika- } \\
\text { linity, } \\
\text { lab } \\
(\mathrm{mg} / \mathrm{l} \text { as } \\
\left.\mathrm{CaCO}_{3}\right)\end{array}$ & $\begin{array}{l}\text { Solids, } \\
\text { sum of } \\
\text { consth- } \\
\text { uents, } \\
\text { dis- } \\
\text { solved } \\
\text { (mg/L) }\end{array}$ & $\begin{array}{c}\text { Solids, } \\
\text { residue } \\
\text { at } 105 \\
\text { degrees } \\
\text { Colslus, } \\
\text { total } \\
\text { (mg/L) }\end{array}$ \\
\hline 462116103021401 & $07-01-75$ & 8.7 & - & 14.0 & -- & 24 & 0 & -. & - & 1600 & -- \\
\hline 462118102205103 & $08-12-82$ & 8.2 & 8.6 & 15.0 & -- & 10 & -- & -- & 1010 & 1180 & -- \\
\hline 462119102440601 & $08-17-67$ & 8.3 & - & 8.0 & - & 140 & 0 & - & - & 2030 & - \\
\hline 462123101110801 & $05-17-73$ & 7.9 & -- & 8.5 & -- & 230 & 0 & - & - & 445 & -- \\
\hline 462124102234901 & $08-29-67$ & 8.4 & -- & 10.0 & 1000 & 17 & 0 & - & -- & 1030 & - \\
\hline 462125102031801 & $04-23-81$ & 8.4 & 8.4 & 12.5 & - & 434 & 120 & - & 310 & 1005 & - \\
\hline 462129101095301 & $12-03-71$ & 8.0 &.- & -- & -- & 14 & 0 & - & - & 857 & - \\
\hline 462129101105901 & $05-17-73$ & 8.3 & - & 8.0 & - & 120 & 0 & - & - & 756 & - \\
\hline 462144102291002 & $08-24-67$ & 8.4 & -- & 12.0 & 700 & 26 & 0 & - & -- & 1240 & -- \\
\hline 462144102291003 & $08-12-82$ & 8.3 & 8.5 & 13.0 & -- & 19 & - & - & 1150 & 1270 & - \\
\hline 462150102225301 & $07-13-65$ & 7.6 & - & -- & -- & 220 & 0 & -- & - & 884 & -- \\
\hline 462157102193701 & $01-2 I-60$ & -- & -- & -- & 400 & 14 & -- & - & $\ldots$ & 1260 & - \\
\hline 462203102194601 & $06-02-66$ & 7.5 & -- & -- & - & 1200 & 490 & -- & .. & 2960 & -- \\
\hline 462204102035601 & $07-26-67$ & 8.1 & -- & 10.5 & 20 & 89 & 0 & .- & -. & 306 & -- \\
\hline 462209101005801 & $06-27-72$ & 8.3 & - & 9.5 & -. & 18 & 0 & -. & - & 1470 & -- \\
\hline 462210102160302 & $08-24-67$ & 8.4 & - & 11.5 & -- & 10 & -- & -- & -- & 1070 & -- \\
\hline 462210102163001 & $08-02-54$ & - & - & - & -- & 15 & - & -- & - & 1260 & -. \\
\hline 462210102191801 & $07-26-67$ & 8.5 & -- & 13.5 & 100 & 14 & 0 & - & -- & 1180 & - \\
\hline 462211102473201 & $11-12-67$ & 8.0 & -. & - & - & 40 & - & -- & - & 1430 & -- \\
\hline 462216102102401 & $10-01-71$ & 8.1 & - & 9.0 & -- & 91 & 0 & - & .. & 367 & -- \\
\hline 462218101564901 & $09-26-72$ & 7.9 & -- & -- & -- & 8 & 0 & -- & - & 1250 & -. \\
\hline 462219102192801 & $07-26-67$ & 8.4 & - & 11.0 & 90 & 14 & 0 & -- & - & 1200 & - \\
\hline - & $09-26-72$ & 8.0 & -- & - & -- & 12 & 0 & -. & $\ldots$ & 1250 & $\ldots$ \\
\hline 462225101564901 & $09-26-72$ & 8.1 & -- & - & - & 8 & 0 & - & - & 1270 & - \\
\hline 462231101563001 & $09-26-72$ & 8.0 & - & - & - & 12 & 0 & - & - & 1250 & - \\
\hline 462231101564901 & $09-26-72$ & 8.1 & - & - & -- & 8 & 0 & - & - & 1270 & - \\
\hline 462232102192801 & $07-28-67$ & 8.5 & - & - & 100 & 13 & 0 & - & - & 1180 & -- \\
\hline 462235103002701 & $06-04-75$ & 8.4 & - & 10.0 & - & 28 & 0 & - & - & 1440 & - \\
\hline 462236102503503 & $11-17-66$ & 8.0 & - & - & - & 120 & 0 & - & - & 767 & -- \\
\hline 462243102453602 & $08-23-67$ & 8.4 & - & 11.0 & 500 & 22 & 0 & - & -- & 1130 & - \\
\hline 462259102264101 & $08-12-82$ & 8.5 & 8.7 & 11.5 & - & 11 & -- & -- & 717 & 898 & -- \\
\hline 462301102303101 & $09-01-67$ & 8.5 & - & 11.5 & 40 & 12 & 0 & - & - & 1130 & - \\
\hline- & $05-16-69$ & - & - & 10.0 & -- & -- & - & -- & -- & - & -- \\
\hline 462303100474002 & $04-23-71$ & 8.4 & - & - & -- & 7 & -. & - & - & 1730 & -- \\
\hline 462315102353501 & $08-17-82$ & 8.3 & 8.6 & 11.5 & - & 17 & -- & - & 772 & 908 & -- \\
\hline 462316102402701 & $08-18-82$ & 7.5 & 7.9 & 15.0 & - & 130 & - & - & 372 & 694 & - \\
\hline 462323102002601 & $05-21-79$ & 6.9 & - & - & -- & 2200 & - & -- & - & -- & -. \\
\hline 462328102341901 & $08-17-82$ & 8.5 & 8.6 & 15.0 & - & 9 & - & -- & 985 & 1110 & - \\
\hline 462336102453601 & $08-18-82$ & 8.5 & 8.7 & 16.0 & - & 10 & - & -- & 870 & 1140 & -- \\
\hline 462341100551202 & $07-13-72$ & 8.2 & -- & 10.0 & - & 18 & 0 & - & - & 1570 & -- \\
\hline 462351101505201 & $09-25-72$ & 7.8 & - & - & -- & 860 & 490 & - & - & 1180 & - \\
\hline 462351103071801 & $10-22-76$ & 8.7 & - & 11.5 & -- & 60 & 0 & - & .- & 1060 & - \\
\hline 462355102414202 & $05-20-69$ & 8.3 & -- & 10.5 & - & 70 & 0 & - & - & 1010 & - \\
\hline 462415102002601 & $05-21-79$ & 8.2 & - & - & - & 37 & 0 & - & -- & - & - \\
\hline 462421101045401 & $07-08-72$ & 7.1 & - & 11.5 & - & 600 & 110 & -- & .- & 959 & - \\
\hline
\end{tabular}




\begin{tabular}{|c|c|c|c|c|c|c|c|c|c|c|c|}
\hline Slte number & Date & $\begin{array}{l}\text { Solids, } \\
\text { residue } \\
\text { at } 180 \\
\text { degrees } \\
\text { Colsius, } \\
\text { dle- } \\
\text { solved } \\
\text { (mg/L) }\end{array}$ & $\begin{array}{l}\text { Solids, } \\
\text { dis- } \\
\text { solved } \\
\text { (tons per } \\
\text { acre- } \\
\text { foot) }\end{array}$ & $\begin{array}{l}\text { Calclum, } \\
\text { dis- } \\
\text { solved } \\
\text { (mg/L } \\
\text { as Ca) }\end{array}$ & $\begin{array}{l}\text { Magne- } \\
\text { slum, } \\
\text { dis- } \\
\text { solved } \\
\text { (mg/l } \\
\text { as Mg) }\end{array}$ & $\begin{array}{l}\text { Sodium, } \\
\text { dis- } \\
\text { solved } \\
\text { (mg/L } \\
\text { as Na) }\end{array}$ & $\begin{array}{l}\text { Sodium } \\
\text { (percomt) }\end{array}$ & $\begin{array}{l}\text { Sodlum } \\
\text { adsorp- } \\
\text { tlon } \\
\text { ratio }\end{array}$ & $\begin{array}{l}\text { Resid- } \\
\text { ual, } \\
\text { sodium, } \\
\text { car- } \\
\text { bonate } \\
\text { (mg/L ae } \\
\left.\mathrm{CaCO}_{3}\right)\end{array}$ & $\begin{array}{l}\text { Potas- } \\
\text { slum, } \\
\text { dis- } \\
\text { solved } \\
\text { (mg/L } \\
\text { as K) }\end{array}$ & $\begin{array}{c}\text { Bicar- } \\
\text { bonate } \\
\text { (mgll as } \\
\mathrm{HCO}_{3} \text { ) }\end{array}$ \\
\hline 462116103021401 & $07-01-75$ & 1570 & 2.14 & 5.6 & 2.4 & 550 & 98 & 49 & - & 2.1 & 550 \\
\hline 462118102205103 & $08-12-82$ & 1190 & 1.62 & 2.3 & 0.90 & 490 & 99 & 69 & -- & 1.8 & -. \\
\hline 462119102440601 & $08-17-67$ & 1810 & 2.46 & 36 & 12 & 660 & 91 & 24 & - & 6.6 & 790 \\
\hline 462123101110801 & $05-17-73$ & 436 & 0.59 & 58 & 21 & 77 & 41 & 2 & -- & 6.6 & 460 \\
\hline 462124102234901 & $08-29-67$ & 1040 & 1.41 & 4.3 & 1.6 & 400 & 98 & 42 & -- & 1.4 & 730 \\
\hline 462125102031801 & $04-23-81$ & -- & 1.37 & 86 & 53 & 160 & 44 & 3 & - & 7.1 & - \\
\hline 462129101095301 & $12-03-71$ & 855 & 1.16 & 3.6 & 1.2 & 330 & 98 & 38 & 10.0 & 1.0 & 640 \\
\hline 462129101105901 & $05-17-73$ & 736 & 1.00 & 28 & 12 & 240 & 81 & 9 & 6.00 & 3.7 & 510 \\
\hline 462144102291002 & $08-24-67$ & 1280 & 1.74 & 6.0 & 2.7 & 460 & 97 & 39 & - & 2.3 & 740 \\
\hline 462144102291003 & $08-12-82$ & 1310 & 1.78 & 5.4 & 1.3 & 520 & 98 & 52 & - & 2.1 & - \\
\hline 462150102225301 & $07-13-65$ & 888 & - & -- & - & 270 & - & 8 & -- & - & 530 \\
\hline 462157102193701 & $01-21-60$ & - & -. & -- & -- & -- & - & - & - & - & - \\
\hline 462203102194601 & $06-02-66$ & 3650 & - & 250 & 140 & 730 & -- & 9 & - & -- & 890 \\
\hline 462204102035601 & $07-26-67$ & 292 & 0.40 & 20 & 9.5 & 83 & 65 & 4 & -- & 5.8 & 290 \\
\hline 462209101005801 & $06-27-72$ & 1360 & 1.85 & 5.8 & 0.90 & 590 & 98 & 60 & - & 2.2 & 1170 \\
\hline 462210102160302 & $08-24-67$ & 1080 & 1.47 & 2.5 & 1.0 & 450 & 99 & 61 & -- & 1.8 & 1090 \\
\hline 462210102163001 & $08-02-54$ & - & - & - & -- & - & - & - & - & - & - \\
\hline 462210102191801 & $07-26-67$ & 1200 & 1.63 & 4.0 & 1.0 & 500 & 99 & 58 & - & 1.8 & 1220 \\
\hline 462211102473201 & $11-12-67$ & 1420 & - & -- & - & 540 & - & 37 & - & - & 970 \\
\hline 462216102102401 & $10-01-71$ & 378 & 0.51 & 20 & 10 & 100 & 70 & 5 & - & 3.1 & 340 \\
\hline 462218101564901 & $09-26-72$ & 1210 & 1.65 & 2.5 & 0.50 & 530 & 99 & 80 & -- & 1.4 & 1190 \\
\hline 462219102192801 & $07-26-67$ & 1230 & 1.67 & 4.4 & 0.70 & 510 & 99 & 59 & - & 1.8 & 1250 \\
\hline- & $09-26-72$ & 1250 & 1.70 & 2.6 & 1.3 & 520 & 99 & 66 & -- & 1.5 & 1200 \\
\hline 462225101564901 & $09-26-72$ & 1150 & 1.56 & 2.6 & 0.40 & 530 & 99 & 81 & -- & 1.5 & 1240 \\
\hline 462231101563001 & $09-26-72$ & 1250 & 1.70 & 2.6 & 1.3 & 520 & 99 & 66 & 19.0 & 1.5 & 1200 \\
\hline 462231101564901 & $09-26-72$ & 1150 & 1.56 & 2.6 & 0.40 & 530 & 99 & 80 & 20.0 & 1.5 & 1240 \\
\hline 462232102192801 & $07-28-67$ & 1210 & 1.65 & 4.8 & 0.20 & 500 & 99 & 61 & - & 1.8 & 1220 \\
\hline 462235103002701 & $06-04-75$ & 1510 & 2.05 & 8.1 & 1.9 & 510 & 97 & 42 & - & 2.2 & 650 \\
\hline 462236102503503 & $11-17-66$ & 751 & - & -- & - & 250 & -- & 10 & - & - & 630 \\
\hline 462243102453602 & $08-23-67$ & 1170 & 1.59 & 5.0 & 2.3 & 430 & 97 & 40 & -- & 1.9 & 810 \\
\hline 462259102264101 & $08-12-82$ & 957 & 1.30 & 2.8 & 1.0 & 370 & 98 & 48 & - & 1.7 & -- \\
\hline 462301102303101 & 09-01-67 & 1150 & 1.56 & 2.2 & 1.6 & 470 & 99 & 59 & - & 1.9 & 1150 \\
\hline-- & $05-16-69$ & -- & - & -- & -- & - & - & - & -- & -- & - \\
\hline 462303100474002 & $04-23-71$ & -- & -- & 1.0 & 1.0 & 500 & -- & 84 & - & - & 1040 \\
\hline 462315102353501 & $08-17-82$ & 980 & 1.33 & 4.0 & 1.7 & 370 & 98 & 39 & - & 2.3 & - \\
\hline 462316102402701 & $08-18-82$ & 679 & 0.92 & 27 & 14 & 200 & 77 & 8 & - & 4.6 & - \\
\hline 462323102002601 & $05-21-79$ & 3550 & - & 520 & 220 & 250 & - & 2 & -- & - & -- \\
\hline 462328102341901 & $08-17-82$ & 1130 & 1.54 & 2.1 & 0.84 & 460 & 99 & 68 & - & 2.1 & - \\
\hline 462336102453601 & $08-18-82$ & 1150 & 1.56 & 3.1 & 0.44 & 470 & 99 & 66 & - & 1.7 & - \\
\hline 462341100551202 & $07-13-72$ & 1670 & 2.27 & 5.0 & 1.3 & 610 & 98 & 63 & - & 2.5 & 1100 \\
\hline 462351101505201 & $09-25-72$ & 1250 & 1.70 & 200 & 88 & 45 & 10 & 0.7 & 0.0 & 4.7 & 450 \\
\hline 462351103071801 & $10-22-76$ & 1170 & 1.59 & 12 & 7.3 & 390 & 93 & 22 & - & 1.7 & 680 \\
\hline 462355102414202 & $05-20-69$ & 1100 & 1.50 & 5.1 & 14 & 380 & 92 & 20 & - & 1.8 & 930 \\
\hline 462415102002601 & $05-21-79$ & 1450 & -- & 9.2 & 3.5 & 480 & - & 34 & - & - & 300 \\
\hline 462421101045401 & $07-08-72$ & 942 & 1.28 & 140 & 60 & 100 & 26 & 2 & - & 5.9 & 600 \\
\hline
\end{tabular}




\begin{tabular}{|c|c|c|c|c|c|c|c|c|c|c|c|}
\hline SHte number & Date & $\begin{array}{c}\text { Car- } \\
\text { bonate } \\
\text { (mg/L as } \\
\left.\mathrm{CO}_{3}\right)\end{array}$ & $\begin{array}{c}\text { Carbon } \\
\text { dioxide, } \\
\text { dis- } \\
\text { solved } \\
\left(\mathrm{mg}^{2} \mathrm{~L} \mathrm{as}\right. \\
\left.\mathrm{CO}_{2}\right)\end{array}$ & $\begin{array}{l}\text { Sulfate, } \\
\text { dls- } \\
\text { solved } \\
\text { (mg/l as } \\
\left.\mathrm{SO}_{4}\right)\end{array}$ & $\begin{array}{l}\text { Chlo- } \\
\text { ride, } \\
\text { dis- } \\
\text { solved } \\
\text { (mg/L } \\
\text { as Cl) }\end{array}$ & $\begin{array}{l}\text { Fluoride, } \\
\text { dis- } \\
\text { solved } \\
\text { (mg/l } \\
\text { as F }\end{array}$ & $\begin{array}{l}\text { Slllea, } \\
\text { dis- } \\
\text { solved } \\
\text { (mg/l as } \\
\text { sio, }\end{array}$ & $\begin{array}{l}\text { Nitro- } \\
\text { gon, } \\
\text { nitrate, } \\
\text { dis- } \\
\text { solved } \\
\text { (mg/L } \\
\text { as N) }\end{array}$ & $\begin{array}{c}\text { Nitro- } \\
\text { gen, } \\
\text { nitrate, } \\
\text { total } \\
\left(\mathrm{mgll}^{2}\right. \\
\left.\text { as } \mathrm{NO}_{3}\right)\end{array}$ & $\begin{array}{l}\text { Nitro- } \\
\text { gen, } \\
\text { nitrate, } \\
\text { dis- } \\
\text { solved } \\
(\mathrm{mg} / \mathrm{L} \\
\left.\text { as } \mathrm{NO}_{3}\right)\end{array}$ & $\begin{array}{l}\text { Boron, } \\
\text { dls- } \\
\text { solved } \\
(\mu g / L \\
\text { as B) }\end{array}$ \\
\hline 462116103021401 & $07-01-75$ & 27 & 1.9 & 730 & 5.1 & 0.40 & 5.0 & 0.230 & -- & 1.0 & 590 \\
\hline 462118102205103 & $08-12-82$ & - & - & 10 & 54 & 5.1 & 8.7 & -- & - & -- & 2000 \\
\hline 462119102440601 & $08-17-67$ & 16 & -- & 890 & 9.5 & 0.40 & 7.5 & - & - & 0.0 & 880 \\
\hline 462123101110801 & $05-17-73$ & 0 & 9.2 & 33 & 2.8 & 0.50 & 20 & 0.230 & - & 1.0 & 220 \\
\hline 462124102234901 & $08-29-67$ & 13 & -- & 240 & 10 & 0.60 & -- & - & - & 0.0 & 1900 \\
\hline 462125102031801 & $04-23-81$ & -- & - & 430 & 77 & 0.30 & 4.6 & -- & -- & - & 210 \\
\hline 462129101095301 & $12-03-71$ & 0 & -- & 190 & 0.0 & 1.4 & 18 & 0.226 & 1.0 & -- & 620 \\
\hline 462129101105901 & $05-17-73$ & 3 & 4.2 & 200 & 4.5 & 1.4 & 15 & 0.230 & 1.0 & 1.0 & 630 \\
\hline 462144102291002 & $08-24-67$ & 18 & -- & 370 & 11 & 0.20 & - & -- & - & 3.0 & 1600 \\
\hline 462144102291003 & $08-12-82$ & -- & -- & 13 & 23 & 3.4 & 9.0 & - & - & -- & 1500 \\
\hline 462150102225301 & $07-13-65$ & 0 & -- & 350 & 6.0 & -- & -- & -- & - & 0.0 & - \\
\hline 462157102193701 & $01-21-60$ & -- & - & -- &.- & -. & -- & - & -- & - & -. \\
\hline 462203102194601 & $06-02-66$ & 0 & -- & 1900 & 51 & -- & -- & - & -- & 5.0 & -- \\
\hline 462204102035601 & $07-26-67$ & $\mathbf{0}$ & -- & 30 & 1.1 & 0.40 & 11 & - & -- & 0.0 & 100 \\
\hline 462209101005801 & $06-27-72$ & 7 & 9.5 & 170 & 100 & 2.3 & 17 & 0.230 & - & 1.0 & 2000 \\
\hline 462210102160302 & $08-24-67$ & 24 & -- & 17 & 27 & 3.4 & 8.6 & - & - & 1.0 & 1200 \\
\hline 462210102163001 & $08-02-54$ & - & - & -- & - & -- & -- & - & - & -- & -- \\
\hline 462210102191801 & $07-26-67$ & 37 & - & 2.1 & 24 & 5.9 & 7.0 & $\cdots$ & - & 0.0 & 1300 \\
\hline 462211102473201 & $11-12-67$ & $\mathbf{0}$ & - & 390 & 2.9 & - & - & - & - & 1.0 & - \\
\hline 462216102102401 & $10-01-71$ & $\mathbf{0}$ & 4.3 & 50 & 0.70 & 0.60 & 15 & 0.230 & - & 1.0 & 220 \\
\hline 462218101564901 & $09-26-72$ & 0 & 24 & 7.4 & 100 & 4.0 & 10 & 0.430 & - & 1.9 & 2300 \\
\hline 462219102192801 & $07-26-67$ & 24 & - & 5.0 & 24 & 4.9 & 7.2 & -- & - & 0.0 & 1300 \\
\hline- & $09-26-72$ & 0 & 19 & 12 & 100 & 4.2 & 11 & 0.230 & - & 1.0 & 2200 \\
\hline 462225101564901 & $09-26-72$ & 0 & 16 & 13 & 97 & 2.7 & 9.9 & 0.230 & - & 1.0 & 2000 \\
\hline 462231101563001 & $09-26-72$ & 0 & - & 12 & 100 & 4.2 & 11 & 0.226 & 1.0 & - & 2200 \\
\hline 462231101564901 & $09-26-72$ & 0 & - & 13 & 97 & 2.7 & 9.9 & 0.226 & 1.0 & -- & 2000 \\
\hline 462232102192801 & $07-28-67$ & 29 & -- & 2.2 & 24 & 5.0 & 7.1 & - & - & 0.0 & 1300 \\
\hline 462235103002701 & $06-04-75$ & 9 & 4.3 & 560 & 13 & 0.90 & 5.5 & 0.770 & -- & 3.4 & 1000 \\
\hline 462236102503503 & $11-17-66$ & 0 & - & 140 & 3.8 & -- & - & - & -- & 0.0 & -- \\
\hline 462243102453602 & $08-23-67$ & 13 & - & 270 & 5.1 & 0.20 & - & - & - & 2.0 & 980 \\
\hline 462259102264101 & $08-12-82$ & - & - & 64 & 16 & 3.7 & 7.3 & - & - & -- & 1000 \\
\hline 462301102303101 & $09-01-67$ & 29 & - & 32 & 15 & 1.7 & 8.9 & - & -- & 1.0 & 660 \\
\hline- & $05-16-69$ & -- & - & - & -- & -- & - & - & - & - & - \\
\hline 462303100474002 & $04-23-71$ & 36 & - & 10 & 150 & 3.2 & -- & 1.13 & 5.0 & $\ldots$ & - \\
\hline 462315102353501 & $08-17-82$ & - & - & 44 & 11 & 3.5 & 7.3 & - & - & - & 650 \\
\hline 462316102402701 & $08-18-82$ & -- & -- & 210 & 3.3 & 0.30 & 11 & - & -- & - & 370 \\
\hline 462323102002601 & $05-21-79$ & - & -- & 1600 & 44 & - & - & 0.500 & -- & 2.2 & - \\
\hline 462328102341901 & $08-17-82$ & - & -- & 23 & 15 & 2.1 & 8.7 & - & - & - & 930 \\
\hline 462336102453601 & $08-18-82$ & - & - & 45 & 79 & 4.4 & 12 & - & - & - & 1900 \\
\hline 462341100551202 & $07-13-72$ & 0 & 11 & 320 & 69 & 3.2 & 17 & 0.230 & -- & 1.0 & 2300 \\
\hline 462351101505201 & $09-25-72$ & 0 & 11 & 580 & 22 & 0.20 & 13 & 0.450 & 2.0 & 2.0 & 170 \\
\hline 462351103071801 & $10-22-76$ & 25 & 2.3 & 270 & 9.1 & 1.9 & 5.3 & 0.050 & - & 0.20 & 210 \\
\hline 462355102414202 & $05-20-69$ & 7 & -- & 120 & 9.9 & 6.8 & 8.2 & $\ldots$ & - & 0.0 & 200 \\
\hline 462415102002601 & $05-21-79$ & -- & 3.0 & 460 & 11 & -- & -- & -- & -- & $<1.0$ & - \\
\hline 462421101045401 & $07-08-72$ & 0 & 76 & 310 & 14 & 0.50 & 26 & 0.230 & - & 1.0 & 710 \\
\hline
\end{tabular}




\begin{tabular}{|c|c|c|c|c|c|c|c|c|c|c|c|}
\hline Sito number & Date & $\begin{array}{l}\text { Iron, } \\
\text { dis- } \\
\text { solved } \\
(\mu g / L \\
\text { as Fo) }\end{array}$ & $\begin{array}{l}\text { Lead, } \\
\text { dis- } \\
\text { solved } \\
\text { ( } \mu g^{\prime} / \mathrm{L} \\
\text { as Pb) }\end{array}$ & $\begin{array}{l}\text { Lthium, } \\
\text { dis-- } \\
\text { solved } \\
\text { (1/g/L } \\
\text { as LI) }\end{array}$ & $\begin{array}{l}\text { Manga- } \\
\text { nese, } \\
\text { dls- } \\
\text { solved } \\
\text { ( } \mu g / \mathrm{L} \\
\text { as Mn) }\end{array}$ & $\begin{array}{c}\text { Morcury, } \\
\text { dis.- } \\
\text { solvod } \\
(\mu g / L \\
\text { as Hg) }\end{array}$ & $\begin{array}{c}\text { Molyb- } \\
\text { donum, } \\
\text { dis- } \\
\text { solved } \\
\text { ( } \mu g / L \\
\text { as Mo) }\end{array}$ & $\begin{array}{l}\text { Solo- } \\
\text { nium, } \\
\text { dis- } \\
\text { solved } \\
\text { ( } \mu \mathrm{g} / \mathrm{L} \\
\text { as Se) }\end{array}$ & $\begin{array}{l}\text { Stron- } \\
\text { tium, } \\
\text { dis- } \\
\text { solved } \\
\text { ( } \mu \mathrm{g} / \mathrm{L} \\
\text { as Sr) }\end{array}$ & $\begin{array}{l}\text { Vana- } \\
\text { dium, } \\
\text { dle- } \\
\text { solved } \\
(\mu g / L \\
\text { as V) }\end{array}$ & $\begin{array}{l}\text { Zlnc, } \\
\text { dis- } \\
\text { solved } \\
(\mu g / L \\
\text { ss Zn) }\end{array}$ \\
\hline 462116103021401 & $07-01-75$ & 1300 & -- & .- & 40 & - & - & - & - & - & - \\
\hline 462118102205103 & $08-12-82$ & 85 & - & - & 2 & - & - & $<1$ & .- & - & .- \\
\hline 462119102440601 & $08-17-67$ & 900 & -- & -- & -- & -- & -- & -- & -- & -- & - \\
\hline 462123101110801 & $05-17-73$ & 1200 & -- & -- & 180 & - & -- & - & -- & -- & -- \\
\hline 462124102234901 & $08-29-67$ & 220 & - & -- & -- & -- & - & -- & -- & -- & -- \\
\hline 462125102031801 & $04-23-81$ & 30 & .- & 50 & 30 & - & - & - & 1200 & - & - \\
\hline 462129101095301 & $12-03-71$ & 0 & -- & -- & 0 & - & -- & - & - & -- & -- \\
\hline 462129101105901 & $05-17-73$ & $<10$ & _- & _- & 160 & - & _- & .- & .. & _- & _- \\
\hline 462144102291002 & $08-24-67$ & 700 & - & - & - & - & -- & -- & -- & -. & -- \\
\hline 462144102291003 & $08-12-82$ & 90 & - & -- & 10 & - & - & $<1$ & - & -- & -- \\
\hline 462150102225301 & $07-13-65$ & 330 & - & - & -- & - & -- & - & -- & - & - \\
\hline 462157102193701 & $01-21-60$ & -- & .- & -- & - & - & -- & .- & .- & .. & -- \\
\hline 462203102194601 & $06-02-66$ & 800 & - & - & - & - & - & - & - & -- & - \\
\hline 462204102035601 & $07-26-67$ & 180 & -- & -- & -- & - & - & .- & -- & -- & .- \\
\hline 462209101005801 & $06-27-72$ & 270 & -- & - & 30 & - & - & -- & - & -- & -- \\
\hline 462210102160302 & $08-24-67$ & 340 & -- & -- & -- & - & -- & - & -- & -- & -- \\
\hline 462210102163001 & $08-02-54$ & -- & -- & .- & -. & -- & -- & -- & -- & -- & - \\
\hline 462210102191801 & $07-26-67$ & 220 & - & - & - & - & -- & -- & -- & -- & -- \\
\hline 462211102473201 & $11-12-67$ & 1400 & - & -- & - & - & -- & -- & -- & - & -- \\
\hline 462216102102401 & $10-01-71$ & 0 & - & - & 170 & - & - & -- & - & -- & -- \\
\hline 462218101564901 & $09-26-72$ & 820 & - & -- & 10 & - & - & - & - & .- & .- \\
\hline 462219102192801 & $07-26-67$ & 180 & .- & -. & .- & _- & .- & _- & - & .- & .- \\
\hline -- & $09-26-72$ & 1200 & - & - & 20 & - & - & - & - & -- & -. \\
\hline 462225101564901 & $09-26-72$ & 700 & -- & .- & 60 & - & - & -- & -- & _- & .- \\
\hline 462231101563001 & $09-26-72$ & 1200 & -- & -- & 20 & - & - & - & -- & -- & -- \\
\hline 462231101564901 & $09-26-72$ & 700 & - & - & 60 & - & - & -- & - & -- & -- \\
\hline 462232102192801 & $07-28-67$ & 240 & -- & -- & - & - & -- & -- & - & - & -- \\
\hline 462235103002701 & $06-04-75$ & 360 & -- & - & 40 & - & -- & -- & - & -- & -- \\
\hline 462236102503503 & $11-17-66$ & 690 & - & -- & -- & - & -- & -- & - & - & - \\
\hline 462243102453602 & $08-23-67$ & 0 & - & - & - & - & - & - & - & - & -- \\
\hline 462259102264101 & $08-12-82$ & 240 & - & - & 9 & - & - & $<1$ & -- & - & -- \\
\hline 462301102303101 & $09-01-67$ & 90 & .- & -- & -- & - & - & - & - & - & _- \\
\hline - & $05-16-69$ & - & - & - & - & - & - & - & - & _- & - \\
\hline 462303100474002 & $04-23-71$ & 100 & - & - & 0 & -- & - & -- & - & - & - \\
\hline 462315102353501 & $08-17-82$ & 220 & -- & - & 16 & - & - & $<1$ & - & - & -- \\
\hline 462316102402701 & 08-18-82 & 360 & -- & -- & 39 & - & -- & $<1$ & -- & -- & -- \\
\hline 462323102002601 & $05-21-79$ & 1 & - & - & - & -- & - & - & - & -- & - \\
\hline 462328102341901 & $08-17-82$ & 110 & - & -- & 4 & _- & - & $<1$ & - & - & _- \\
\hline 462336102453601 & 08-18-82 & 57 & - & - & 4 & - & -- & $<1$ & - & - & - \\
\hline 462341100551202 & $07-13-72$ & $<20$ & - & - & 10 & -- & - & - & -- & -- & - \\
\hline 462351101505201 & $09-25-72$ & 1900 & - & - & 440 & - & - & - & - & - & - \\
\hline 462351103071801 & $10-22-76$ & 1100 & - & -- & 50 & - & - & - & - & -- & -- \\
\hline 462355102414202 & $05-20-69$ & 250 & - & - & -- & - & - & - & - & - & - \\
\hline 462415102002601 & $05-21-79$ & 1 & - & - & -- & -- & - & -- & -- & -- & -- \\
\hline 462421101045401 & $07-08-72$ & 5800 & -- & - & 500 & - & -- & - & - & - & -- \\
\hline
\end{tabular}




\begin{tabular}{|c|c|c|c|c|c|c|c|c|c|c|}
\hline Site number & Local IdentIfler & $\begin{array}{l}\text { County } \\
\text { code }\end{array}$ & $\begin{array}{l}\text { Hydrologic } \\
\text { unit }\end{array}$ & $\begin{array}{l}\text { Goologlc } \\
\text { unit }\end{array}$ & Date & $\begin{array}{l}\text { Depth } \\
\text { below } \\
\text { land } \\
\text { surface, } \\
\text { water } \\
\text { level } \\
\text {-(feet) }\end{array}$ & $\begin{array}{l}\text { Depth } \\
\text { of } \\
\text { well, } \\
\text { total } \\
\text { (teet) }\end{array}$ & $\begin{array}{l}\text { Altilude } \\
\text { of land } \\
\text { surface, } \\
\text { datum } \\
\text { (feel) }\end{array}$ & $\begin{array}{l}\text { Specific } \\
\text { conduct- } \\
\text { ance, } \\
\text { field } \\
(\mu S / c m)\end{array}$ & $\begin{array}{c}\text { Specific } \\
\text { conduct- } \\
\text { ance, } \\
\text { lab } \\
\text { (HS/cm) }\end{array}$ \\
\hline 462427102514002 & $134-097-22 \mathrm{BCC} 2$ & 041 & 10130204 & 12STGRV & $08-10-67$ & - & 207 & 2701 & 1470 & -- \\
\hline 462445102303101 & 134-094-20AAA & 041 & 10130204 & - & $08-17-82$ & - & 180 & - & 1330 & 1290 \\
\hline 462447102380601 & 134-095-20AAA & 041 & 10130204 & 125TGRV & $09-13-67$ & - & 161 & 2572 & 906 & -- \\
\hline 462447102445902 & 134-096-21BAA2 & 041 & 10130204 & - & $07-22-80$ & - & 1159 & - & - & 2290 \\
\hline 462449100423001 & 134-080-23BAB & 059 & 10130206 & 112SJMS & $08-23-74$ & - & 59 & 1647 & 1940 & - \\
\hline 462450100380501 & $134-079-20 \mathrm{AAB}$ & 059 & 10130206 & - & $08-06-75$ & - & 71 & 1618 & 1150 & - \\
\hline 462451101223703 & 134-085-21BAB3 & 037 & 10130206 & $125 \mathrm{CNBL}$ & $06-28-73$ & -- & 205 & 2204 & 1010 & - \\
\hline 462453102514002 & $134-097-15 C C C 2$ & 041 & 10130204 & 125TGRV & $10-31-67$ & -- & 81 & 2677 & 2260 & - \\
\hline 462456100452801 & 134-080-17DDD & 059 & 10130206 & 112SJMS & $08-22-74$ & - & 204 & 1699 & 1940 & $\cdots$ \\
\hline 462512101082101 & 134-084-17DBD & 059 & 10130206 & 112 SLDS & $07-10-72$ & - & 325 & - & 1610 & - \\
\hline 462519102331301 & 134-095-13ADC & 041 & 10130204 & - & $08-18-82$ & - & 1150 & - & 2150 & 2150 \\
\hline 462519102332301 & $134-095-13 \mathrm{ACD}$ & 041 & 10130204 & $125 \mathrm{CNBL}$ & $01-01-37$ & -- & 546 & 2465 & 2050 & - \\
\hline 462527101554301 & $134-090-13 \mathrm{ACA} 1$ & 037 & 10130204 & 125TGRV & $07-10-68$ & -. & 42 & - & 7990 & - \\
\hline 462527101554302 & $134-090-13 \mathrm{ACA} 2$ & 037 & 10130204 & 125TGRV & $05-28-68$ & - & 160 & - & 4820 & - \\
\hline 462542102263201 & $134-094-12 \mathrm{CCC}$ & 041 & 10130204 & - & $08-17-82$ & - & 672 & $\cdots$ & 2320 & 2340 \\
\hline 462548102190002 & $134-093-12 \mathrm{CCC} 2$ & 041 & 10130204 & 125TGRV & $03-07-69$ & -- & 60 & 2494 & 2830 & - \\
\hline 462600103164902 & $134-100-7 \mathrm{ADD} 2$ & 087 & 10130204 & 125TRVL & $09-08-77$ & - & 304 & 2935 & 1900 & - \\
\hline 462603102232301 & 134-093-8DBB & 041 & 10130204 & 125TGRV & $06-03-69$ & - & 61 & 2528 & 3170 & - \\
\hline 462604100530001 & 134-081-8DAD1 & 059 & 10130206 & 211FXHL & $07-18-72$ & - & 365 & - & 2540 & - \\
\hline 462604100530002 & 134-081-8DAD2 & 059 & 10130206 & 211FXHL & $07-18-72$ & - & 375 & - & 2520 & - \\
\hline 462636103015202 & 134-098-6DDA2 & 087 & 10130205 & 125TRVL & $06-04-75$ & - & 230 & - & 2090 & -- \\
\hline 462637102092702 & 134-091-6DDC2 & 041 & 10130204 & 125TGRV & $08-08-67$ & - & 474 & 2418 & 2330 & - \\
\hline 462637102522701 & 134-097-4CDD & 041 & 10130204 & - & $08-18-82$ & - & 440 & -- & 1850 & 1880 \\
\hline 462638102125102 & $134-092-2 \mathrm{CCC} 2$ & 041 & 10130204 & 125TGRV & $06-02-69$ & - & 131 & 2438 & 1950 & -- \\
\hline 462638102363101 & 134-095-3CCD & 041 & 10130204 & 125TGRV & $08-31-67$ & - & 118 & 2460 & 1670 & - \\
\hline 462638102463202 & $134-096-5 \mathrm{CCD} 2$ & 041 & 10130204 & 125TGRV & $03-11-69$ & - & 160 & 2592 & 1580 & -- \\
\hline 462643101085901 & 134-083-5CCD1 & 059 & 10130206 & 211HLCK & $07-08-72$ & - & 277 & - & 1530 & - \\
\hline 462643101085902 & 134-083-5CCD2 & 059 & 10130206 & 211HLCK & $07-08-72$ & -- & 277 & - & 1640 & - \\
\hline 462654101005001 & 133-082-5DBA & 059 & 10130206 & 112EMCK & $10-17-73$ & - & 174 & 1742 & 2120 & - \\
\hline 462703100542501 & $134-081-6 \mathrm{DAB} 1$ & 059 & 10130206 & 211HLCK & $07-18-72$ & - & 286 & - & 3180 & -- \\
\hline 462703100542502 & 134-081-6DAB2 & 059 & 10130206 & 211FXHL & $07-18-72$ & - & 360 & - & 2700 & - \\
\hline 462709101131301 & 134-084-3ADC & 059 & 10130206 & $211 \mathrm{FXHL}$ & $09-19-75$ & -- & 426 & 1916 & 2240 & -- \\
\hline 462717100554901 & $134-082-1 \mathrm{ACA}$ & 059 & 10130206 & 211HLCK & $07-17-72$ & - & 289 & -- & 1420 & -- \\
\hline 462721101213101 & 134-085-3BBD & 037 & 10130206 & $125 \mathrm{CNBL}$ & $12-19-69$ & - & 280 & - & 1340 & - \\
\hline 462722101130301 & 134-084-3AAD & 059 & 10130206 & 211HLCK & $06-28-72$ & -- & 200 & - & 2020 & -- \\
\hline 462723100554001 & 134-082-1AAC & 059 & 10130206 & 211HLCK & $07-17-72$ & - & 289 & - & 1800 & - \\
\hline 462730102291601 & 135-094-33DDD & 041 & 10130204 & 125SNLB & $06-09-69$ & - & 162 & 2535 & 2920 & - \\
\hline 462730102325701 & $135-094-31 C C C$ & 041 & 10130204 & 125SNLB & $05-15-69$ & - & 81 & 2478 & 628 & 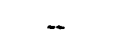 \\
\hline 462739103003801 & 135-098-32DAD1 & 087 & 10130205 & $125 S N L B$ & $07-02-75$ & - & 62 & 2885 & 4010 & - \\
\hline 462742100452601 & 135-080-32DDA & 059 & 10130206 & 211FXHL & $08-07-72$ & - & 330 & - & 1770 & - \\
\hline 462747100564701 & 134-082-35DAA & 059 & 10130206 & 112EMCK & $10-17-73$ & -- & 119 & 1697 & 2370 & - \\
\hline 462755101090601 & $135-083-32 \mathrm{CBB} 1$ & 059 & 10130206 & 211FXHL & $05-27-75$ & - & 466 & 1884 & 3110 & - \\
\hline 462755101090602 & $135-083-32 \mathrm{CBB} 2$ & 059 & 10130206 & 211HLCK & $05-27-75$ & - & 370 & 1884 & 3870 & - \\
\hline 462756102274001 & $135-094-35 \mathrm{BCD}$ & 041 & 10130204 & 125SNLB & $03-31-58$ & - & 180 & 2596 & 3000 & - \\
\hline 462801101101201 & $135-083-31 \mathrm{BCD}$ & 059 & 10130206 & - & $06-14-72$ & - & 500 & - & 1450 & - \\
\hline
\end{tabular}




\begin{tabular}{|c|c|c|c|c|c|c|c|c|c|c|c|}
\hline Slte number & Date & $\begin{array}{l}\mathrm{pH}, \\
\text { field } \\
\text { (stand- } \\
\text { ard } \\
\text { units }\end{array}$ & $\begin{array}{c}\text { pH, } \\
\text { lab } \\
\text { (stand- } \\
\text { ard } \\
\text { units) }\end{array}$ & $\begin{array}{c}\text { Temper- } \\
\text { ature, } \\
\text { water } \\
\text { (degrees } \\
\text { Colsius) }\end{array}$ & $\begin{array}{c}\text { Color } \\
\text { (plati- } \\
\text { num } \\
\text { cobalt } \\
\text { scale) }\end{array}$ & $\begin{array}{l}\text { Hard- } \\
\text { neas, } \\
\text { total } \\
(\mathrm{mg} / \mathrm{L} \text { as } \\
\left.\mathrm{CaCO}_{3}\right)\end{array}$ & $\begin{array}{c}\text { Noncar- } \\
\text { bonate } \\
\text { hard- } \\
\text { ness, } \\
\text { total } \\
\text { (mg/L as } \\
\mathrm{CaCO}_{3} \text { ) }\end{array}$ & $\begin{array}{l}\text { Alka- } \\
\text { Ilnity, } \\
\text { field } \\
\left(\mathrm{mg}^{\prime} \mathrm{L} \text { as }\right. \\
\left.\mathrm{CaCO}_{3}\right)\end{array}$ & $\begin{array}{c}\text { Alka- } \\
\text { linity, } \\
\text { lab } \\
\text { (mg/L as } \\
\text { CaCO }\end{array}$ & $\begin{array}{l}\text { Sollds, } \\
\text { sum of } \\
\text { constlt- } \\
\text { uents, } \\
\text { dls- } \\
\text { solved } \\
\text { (mg/L) }\end{array}$ & $\begin{array}{c}\text { Solids, } \\
\text { residue } \\
\text { at } 105 \\
\text { degrees } \\
\text { Colsius, } \\
\text { total } \\
\text { (mg/L) }\end{array}$ \\
\hline 462427102514002 & $08-10-67$ & 8.5 & - & - & 200 & 26 & 0 & -- & - & 944 & - \\
\hline 462445102303101 & $08-17-82$ & 8.4 & 8.6 & 13.5 & - & 18 & -- & -- & 648 & 802 & - \\
\hline 462447102380601 & $09-13-67$ & 8.4 & -- & 10.0 & 500 & 18 & 0 & - & - & 549 & - \\
\hline 462447102445902 & $07-22-80$ & - & 8.1 & - & - & 12 & -- & - & -- & - & -- \\
\hline 462449100423001 & $08-23-74$ & 8.2 & - & 9.5 & -- & 62 & 0 & - & - & 1260 & -- \\
\hline 462450100380501 & $08-06-75$ & 8.0 & -- & 10.0 & -- & 220 & 0 & -- & -- & 759 & -- \\
\hline 462451101223703 & $06-28-73$ & 7.5 & - & 10.5 & -- & 480 & 140 & - & $\cdots$ & 656 & - \\
\hline 462453102514002 & $10-31-67$ & 8.3 & -- & 11.0 & -- & 150 & 0 & - & -- & 1510 & - \\
\hline 462456100452801 & $08-22-74$ & 8.1 & - & 10.0 & - & 76 & 0 & - & - & 1250 & - \\
\hline 462512101082101 & $07-10-72$ & 7.9 & - & 12.0 & - & 52 & 0 & - & - & 1060 & - \\
\hline 462519102331301 & $08-18-82$ & 8.3 & 8.6 & 12.0 & - & 9 & - & - & 1040 & 1290 & -- \\
\hline 462519102332301 & $01-01-37$ & - & - & -- & -- & 74 & 0 & - & - & 1330 & - \\
\hline 462527101554301 & $07-10-68$ & 5.7 & - & - & - & 3500 & - & -- & -- & 5480 & -. \\
\hline 462527101554302 & $05-28-68$ & 7.9 & -- & - & - & 2000 & - & - & - & 3240 & - \\
\hline 462542102263201 & $08-17-82$ & 8.3 & 8.5 & 17.5 & - & 12 & - & - & 1260 & 1430 & - \\
\hline 462548102190002 & $03-07-69$ & 7.8 & -- & - & 90 & 1200 & 680 & - & - & 2340 & - \\
\hline 462600103164902 & $09-08-77$ & 8.6 & - & 10.0 & - & 39 & 0 & -- & - & 1310 & -. \\
\hline 462603102232301 & $06-03-69$ & 7.8 & -- & 9.0 & 25 & 340 & 0 & - & -- & 2290 & -- \\
\hline 462604100530001 & $07-18-72$ & 7.9 & - & 9.0 & - & 110 & 0 & -- & - & 1760 & - \\
\hline 462604100530002 & $07-18-72$ & 7.9 & -- & 8.0 & - & 32 & 0 & -- & -- & 1780 & -- \\
\hline 462636103015202 & $06-04-75$ & 8.5 & - & 9.0 & -- & 22 & 0 & - & - & 1360 & - \\
\hline 462637102092702 & $08-08-67$ & 8.4 & - & 10.5 & 40 & 17 & 0 & - & - & 1470 & - \\
\hline 462637102522701 & $08-18-82$ & 8.5 & 8.9 & 18.0 & - & 14 & - & - & 532 & 1210 & - \\
\hline 462638102125102 & $06-02-69$ & 7.8 & -- & 10.0 & - & 25 & 0 & - & - & 1280 & - \\
\hline 462638102363101 & $08-31-67$ & 8.6 & -- & 9.5 & 500 & 22 & 0 & -- & -- & 1050 & - \\
\hline 462638102463202 & $03-11-69$ & 8.6 & - & - & 400 & 52 & 0 & -- & - & 1000 & - \\
\hline 462643101085901 & $07-08-72$ & 8.1 & - & 12.0 & - & 6 & 0 & - & - & 1020 & $\cdots$ \\
\hline 462643101085902 & $07-08-72$ & 8.2 & $\cdots$ & 11.0 & - & 5 & 0 & .. & -- & 1070 & -. \\
\hline 462654101005001 & $10-17-73$ & 7.9 & -- & 9.0 & - & 320 & 0 & -- & -- & 1350 & - \\
\hline 462703100542501 & $07-18-72$ & 7.9 & - & 8.0 & - & 45 & 0 & - & - & 2110 & - \\
\hline 462703100542502 & $07-18-72$ & 8.1 & - & 12.0 & - & 41 & 0 & - & - & 1910 & - \\
\hline 462709101131301 & $09-19-75$ & 8.3 & - & 11.0 & - & 20 & 0 & - & - & 1370 & - \\
\hline 462717100554901 & $07-17-72$ & 7.7 & - & 10.0 & - & 20 & 0 & - & - & 927 & - \\
\hline 462721101213101 & $12-19-69$ & 8.2 & - & -. & - & 19 & - & - & - & 721 & - \\
\hline 462722101130301 & $06-28-72$ & 8.3 & - & 11.5 & - & 9 & 0 & - & - & 1380 & - \\
\hline 462723100554001 & $07-17-72$ & 8.1 & - & 9.5 & -- & 20 & $\mathbf{0}$ & - & - & 1180 & - \\
\hline 462730102291601 & $06-09-69$ & 8.3 & -- & - & - & 850 & - & - & - & 3650 & - \\
\hline 462730102325701 & $05-15-69$ & 7.7 & - & 9.5 & 100 & 120 & 0 & - & - & 393 & - \\
\hline 462739103003801 & $07-02-75$ & 7.4 & -. & 8.0 & - & 3200 & 2700 & -- & - & 4050 & -- \\
\hline 462742100452601 & $08-07-72$ & 7.9 & - & - & -- & 26 & 0 & - & - & 1150 & - \\
\hline 462747100564701 & $10-17-73$ & 8.4 & - & 9.0 & - & 36 & 0 & - & - & 1490 & - \\
\hline 462755101090601 & $05-27-75$ & 8.5 & - & 10.0 & - & 24 & 0 & - & - & 2000 & - \\
\hline 462755101090602 & $05-27-75$ & 8.6 & - & 11.0 & - & 50 & 0 & - & - & 2830 & - \\
\hline 462756102274001 & $03-31-58$ & 8.2 & - & - & - & 73 & - & - & - & 2180 & - \\
\hline 462801101101201 & $06-14-72$ & 7.4 & - & 10.5 & - & 69 & 0 & - & - & 908 & - \\
\hline
\end{tabular}




\begin{tabular}{|c|c|c|c|c|c|c|c|c|c|c|c|}
\hline Site number & Dats & $\begin{array}{c}\text { Solids, } \\
\text { residus } \\
\text { at } 180 \\
\text { degrees } \\
\text { Colsius, } \\
\text { dis- } \\
\text { solvsd } \\
\text { (mg/L) }\end{array}$ & $\begin{array}{l}\text { Sollds, } \\
\text { dis- } \\
\text { solved } \\
\text { (tons per } \\
\text { acre- } \\
\text { foot) }\end{array}$ & $\begin{array}{l}\text { Calcium, } \\
\text { dis- } \\
\text { solved } \\
\text { (mglL } \\
\text { as Ca) }\end{array}$ & $\begin{array}{l}\text { Magne- } \\
\text { sium, } \\
\text { dis- } \\
\text { solved } \\
\text { (mg/L } \\
\text { as Mg) }\end{array}$ & $\begin{array}{l}\text { Sodium, } \\
\text { dis- } \\
\text { solved } \\
\text { (mglL } \\
\text { as } \mathrm{Na} \text { ) }\end{array}$ & $\begin{array}{c}\text { Sodium } \\
\text { (percent) }\end{array}$ & $\begin{array}{l}\text { Sodium } \\
\text { adsorp- } \\
\text { tion } \\
\text { ratio }\end{array}$ & $\begin{array}{l}\text { Rssid- } \\
\text { ual, } \\
\text { sodium, } \\
\text { car- } \\
\text { bonats } \\
\text { (mg/L as } \\
\left.\mathrm{CaCO}_{3}\right)\end{array}$ & $\begin{array}{l}\text { Potas- } \\
\text { slum, } \\
\text { dis- } \\
\text { solved } \\
\text { (mg/L } \\
\text { as K) }\end{array}$ & $\begin{array}{c}\text { Bicar- } \\
\text { bonate } \\
\text { (mg/L as } \\
\left.\mathrm{HCO}_{3}\right)\end{array}$ \\
\hline 462427102514002 & $08-10-67$ & 950 & 1.29 & 7.0 & 2.1 & 350 & 96 & 30 & -- & 1.9 & 600 \\
\hline 462445102303101 & $08-17-82$ & 866 & 1.18 & 5.0 & 1.2 & 330 & 97 & 34 & - & 2.5 & -- \\
\hline 462447102380601 & $09-13-67$ & 595 & 0.81 & 5.2 & 1.2 & 220 & 96 & 22 & -- & 1.6 & 480 \\
\hline 462447102445902 & $07-22-80$ & 1830 & -- & 3.0 & 1.0 & - & 99 & 77 & -- & 2.4 & -- \\
\hline 462449100423001 & $08-23-74$ & 1290 & 1.75 & 12 & 7.8 & 450 & 94 & 25 & - & 3.4 & 840 \\
\hline 462450100380501 & $08-06-75$ & 763 & 1.04 & 36 & 32 & 190 & 64 & 6 & -- & 5.6 & 470 \\
\hline 462451101223703 & $06-28-73$ & 709 & 0.96 & 100 & 57 & 43 & 16 & 0.9 & 0.0 & 5.3 & 420 \\
\hline 462453102514002 & $10-31-67$ & 1540 & 2.09 & 24 & 21 & 490 & 88 & 18 & -- & 4.0 & 770 \\
\hline 462456100452801 & $08-22-74$ & 1290 & 1.75 & 19 & 6.9 & 440 & 92 & 22 & -- & 4.8 & 910 \\
\hline 462512101082101 & $07-10-72$ & 1010 & 1.37 & 14 & 4.1 & 380 & 94 & 23 & -- & 2.3 & 720 \\
\hline 462519102331301 & $08-18-82$ & 1320 & 1.80 & 2.5 & 0.70 & 540 & 99 & 78 & - & 1.7 & -- \\
\hline 462519102332301 & $01-01-37$ & -- & - & 16 & 8.3 & 450 & 93 & 23 & - & - & 1230 \\
\hline 462527101554301 & $07-10-68$ & 6250 & -- & 540 & 520 & - & - & - & - & - & 64 \\
\hline 462527101554302 & $05-28-68$ & 3630 & - & 410 & 230 & -- & -- & - & -- & - & 250 \\
\hline 462542102263201 & $08-17-82$ & 1480 & 2.01 & 2.7 & 1.3 & 600 & 99 & 75 & - & 2.7 & -- \\
\hline 462548102190002 & $03-07-69$ & 2400 & 3.26 & 290 & 120 & 310 & 35 & 4 & -- & 15 & 630 \\
\hline 462600103164902 & $09-08-77$ & 1320 & 1.80 & 7.8 & 4.7 & 460 & 96 & 32 & - & 1.1 & 570 \\
\hline 462603102232301 & $06-03-69$ & 2230 & 3.03 & 82 & 34 & 640 & 80 & 15 & - & 10 & 700 \\
\hline 462604100530001 & $07-18-72$ & 1840 & 2.50 & 25 & 11 & 600 & 92 & 25 & - & 3.1 & 800 \\
\hline 462604100530002 & $07-18-72$ & 1760 & 2.39 & 6.6 & 3.8 & 610 & 97 & 47 & - & 1.8 & 760 \\
\hline 462636103015202 & $06-04-75$ & 1400 & 1.90 & 6.1 & 1.7 & 510 & 98 & 47 & -- & 2.2 & 890 \\
\hline 462637102092702 & $08-08-67$ & 1470 & 2.00 & 4.7 & 1.3 & 610 & 99 & 64 & -- & 2.4 & 1470 \\
\hline 462637102522701 & $08-18-82$ & 1260 & 1.71 & 4.2 & 0.90 & 430 & 98 & 50 & -- & 1.7 & -- \\
\hline 462638102125102 & $06-02-69$ & 1260 & 1.71 & 6.7 & 2.1 & 460 & 97 & 39 & - & 2.7 & 700 \\
\hline 462638102363101 & $08-31-67$ & 1050 & 1.43 & 5.4 & 2.1 & 410 & 97 & 38 & - & 1.9 & 830 \\
\hline 462638102463202 & $03-11-69$ & 1010 & 1.37 & 7.5 & 8.1 & 360 & 93 & 21 & - & 1.8 & 620 \\
\hline 462643101085901 & $07-08-72$ & 967 & 1.32 & 2.2 & 0.10 & 380 & 99 & 68 & -. & 0.90 & 700 \\
\hline 462643101085902 & $07-08-72$ & 1040 & 1.41 & 1.8 & 0.10 & 400 & 99 & 79 & - & 1.1 & 740 \\
\hline 462654101005001 & $10-17-73$ & 1340 & 1.82 & 79 & 30 & 410 & 73 & 10 & - & 6.6 & 1170 \\
\hline 462703100542501 & $07-18-72$ & 2180 & 2.96 & 12 & 3.6 & 750 & 97 & 49 & -- & 2.4 & 820 \\
\hline 462703100542502 & $07-18-72$ & 1940 & 2.64 & 9.2 & 4.4 & 680 & 97 & 46 & -- & 2.6 & 890 \\
\hline 462709101131301 & $09-19-75$ & 1450 & 1.97 & 3.5 & 2.8 & 520 & 98 & 50 & -. & 1.8 & 960 \\
\hline 462717100554901 & $07-17-72$ & 958 & 1.30 & 2.6 & 3.3 & 360 & 97 & 35 & -- & 1.1 & 730 \\
\hline 462721101213101 & $12-19-69$ & 818 & - &.- & - & -. & - & - & - & - & -- \\
\hline 462722101130301 & $06-28-72$ & 1360 & 1.85 & 2.5 & 0.70 & 490 & 99 & 71 & - & 1.2 & 620 \\
\hline 462723100554001 & $07-17-72$ & 1190 & 1.62 & 3.2 & 2.9 & 470 & 98 & 46 & - & 1.5 & 1000 \\
\hline 462730102291601 & $06-09-69$ & - & -. & 500 & -- & 280 & - & - & - & - & - \\
\hline 462730102325701 & $05-15-69$ & 388 & 0.53 & 25 & 14 & 100 & 65 & 4 & - & 3.3 & 350 \\
\hline 462739103003801 & $07-02-75$ & 4210 & 5.73 & 420 & 520 & 62 & 4 & 0.5 & - & 5.5 & 570 \\
\hline 462742100452601 & $08-07-72$ & 1180 & 1.60 & 5.9 & 2.8 & 430 & 97 & 37 & -- & 1.9 & 800 \\
\hline 462747100564701 & $10-17-73$ & 1510 & 2.05 & 5.2 & 5.6 & 570 & 97 & 41 & - & 2.3 & 1110 \\
\hline 462755101090601 & $05-27-75$ & 2100 & 2.86 & 5.7 & 2.4 & 750 & 98 & 66 & - & 2.0 & 1160 \\
\hline 462755101090602 & $05-27-75$ & 2990 & 4.07 & 13 & 4.3 & 980 & 98 & 60 & - & 3.2 & 1140 \\
\hline 462756102274001 & $03-31-58$ & 1880 & - & -- & - & - & - & -- & -- & - & 640 \\
\hline 462801101101201 & $06-14-72$ & 970 & 1.32 & 16 & 7.1 & 320 & 91 & 17 & - & 2.4 & 670 \\
\hline
\end{tabular}




\begin{tabular}{|c|c|c|c|c|c|c|c|c|c|c|c|}
\hline Slte number & Date & $\begin{array}{c}\text { Car- } \\
\text { bonate } \\
\text { (mg/l as } \\
\left.\mathrm{CO}_{3}\right)\end{array}$ & $\begin{array}{l}\text { Carbon } \\
\text { dloxide, } \\
\text { dis- } \\
\text { solved } \\
\left(\mathrm{mg}^{2} \mathrm{Las}\right. \\
\left.\mathrm{CO}_{2}\right)\end{array}$ & $\begin{array}{l}\text { Sulfate, } \\
\text { dis- } \\
\text { solved } \\
\text { (mg/L as } \\
\text { so, }\end{array}$ & $\begin{array}{l}\text { Chlo- } \\
\text { ride, } \\
\text { dls- } \\
\text { solved } \\
\text { (mg/h } \\
\text { as Cl) }\end{array}$ & $\begin{array}{l}\text { Fluoride, } \\
\text { dis- } \\
\text { solved } \\
\text { (mg/L } \\
\text { as F) }\end{array}$ & $\begin{array}{l}\text { Silica, } \\
\text { dls- } \\
\text { solved } \\
\text { (mg/L as } \\
\text { sio })\end{array}$ & $\begin{array}{l}\text { Nitro- } \\
\text { gon, } \\
\text { nitrate, } \\
\text { dis- } \\
\text { eolved } \\
\text { (mg/L } \\
\text { as N) }\end{array}$ & $\begin{array}{c}\text { Nitro- } \\
\text { gen, } \\
\text { nitrate, } \\
\text { total } \\
(\mathrm{mg} / \mathrm{L} \\
\left.\text { as } \mathrm{NO}_{3}\right)\end{array}$ & $\begin{array}{l}\text { Nitro- } \\
\text { gen, } \\
\text { nitrato, } \\
\text { dis- } \\
\text { solved } \\
\text { (mg/L } \\
\text { as } \mathrm{NO}_{3} \text { ) }\end{array}$ & $\begin{array}{c}\text { Boron, } \\
\text { dis- } \\
\text { soived } \\
\text { ( } \mu g / L \\
\text { as B) }\end{array}$ \\
\hline 462427102514002 & $08-10-67$ & 14 & - & 270 & 5.2 & 0.80 & 1.7 & -- & -- & 0.0 & 520 \\
\hline 462445102303101 & $08-17-82$ & - & - & 46 & 17 & 3.2 & 7.1 & - & -- & -- & 740 \\
\hline 462447102380601 & $09-13-67$ & 5 & - & 81 & 2.6 & 0.20 & -- & - & -- & 0.0 & 630 \\
\hline 462447102445902 & $07-22-80$ & -- & - & -- & - & -- & -- & -- & -- & -- & -- \\
\hline 462449100423001 & $08-23-74$ & 0 & 8.5 & 350 & 5.0 & 1.3 & 15 & 0.230 & - & 1.0 & 1800 \\
\hline 462450100380501 & $08-06-75$ & 0 & 7.5 & 240 & 7.4 & 0.50 & 15 & 0.00 & -- & 0.0 & 900 \\
\hline 462451101223703 & $06-28-73$ & 0 & 21 & 220 & 4.3 & 0.40 & 18 & 0.200 & 0.40 & 0.89 & 130 \\
\hline 462453102514002 & $10-31-67$ & 6 & - & 570 & 5.3 & 0.60 & 7.8 & -- & -- & 4.0 & 780 \\
\hline 462456100452801 & $08-22-74$ & 0 & 12 & 300 & 10 & 1.4 & 19 & 0.230 & - & 1.0 & 2100 \\
\hline 462512101082101 & $07-10-72$ & 0 & 15 & 270 & 2.3 & 2.1 & 24 & 0.230 & -- & 1.0 & 1000 \\
\hline 462519102331301 & $08-18-82$ & - & - & 7.0 & 100 & 2.6 & 12 & -- & -- & -- & 1900 \\
\hline 462519102332301 & $01-01-37$ & - & -- & 23 & 22 & 2.0 & 13 & 0.050 & - & 0.20 & - \\
\hline 462527101554301 & $07-10-68$ & 0 & - & 3600 & 58 & -- & - & 113 & 500 & -- & -- \\
\hline 462527101554302 & $05-28-68$ & 0 & - & 2200 & 26 & -- & -. & - & -- & - & -- \\
\hline 462542102263201 & $08-17-82$ & - & -. & 21 & 33 & 1.5 & 9.9 & - & - & -- & 1300 \\
\hline 462548102190002 & $03-07-69$ & 0 & - & 1300 & 6.9 & 1.2 & 13 & -- & -- & 0.50 & 810 \\
\hline 462600103164902 & $09-08-77$ & 9 & 2.4 & 530 & 4.4 & 0.60 & 5.7 & 0.230 & - & 1.0 & 190 \\
\hline 462603102232301 & $06-03-69$ & 0 & -- & 1200 & 3.3 & 1.3 & 8.3 & - & - & 3.0 & 590 \\
\hline 462604100530001 & $07-18-72$ & 0 & 16 & 690 & 4.1 & 1.0 & 25 & 0.990 & -- & 4.4 & 1700 \\
\hline 462604100530002 & $07-18-72$ & 0 & 15 & 750 & 1.7 & 0.60 & 25 & 0.560 & - & 2.5 & 1200 \\
\hline 462636103015202 & $06-04-75$ & 16 & 4.6 & 370 & 8.0 & 0.50 & 5.4 & 0.560 & - & 2.5 & 630 \\
\hline 462637102092702 & $08-08-67$ & 28 & - & 48 & 43 & 1.8 & 9.5 & - & - & 0.0 & 1400 \\
\hline 462637102522701 & $08-18-82$ & - & - & 420 & 19 & 1.6 & 8.2 & - & - & - & 970 \\
\hline 462638102125102 & $06-02-69$ & 0 & - & 460 & 3.6 & 4.0 & 7.0 & - & - & 1.0 & 890 \\
\hline 462638102363101 & $08-31-67$ & 23 & - & 190 & 3.2 & 0.30 & - & -- & -- & 0.0 & 930 \\
\hline 462638102463202 & $03-11-69$ & 12 & -- & 300 & 2.9 & 2.9 & 7.6 & - & -- & 0.0 & 200 \\
\hline 462643101085901 & $07-08-72$ & 0 & 8.9 & 260 & 0.0 & 1.0 & 27 & 0.230 & -- & 1.0 & 1200 \\
\hline 462643101085902 & $07-08-72$ & 0 & 7.5 & 270 & 4.6 & 1.0 & 24 & 0.230 & - & 1.0 & 1200 \\
\hline 462654101005001 & $10-17-73$ & 0 & 24 & 130 & 96 & 0.70 & 21 & 0.230 & - & 1.0 & 1500 \\
\hline 462703100542501 & $07-18-72$ & 0 & 17 & 900 & 12 & 1.0 & 27 & 0.160 & - & 0.70 & 1800 \\
\hline 462703100542502 & $07-18-72$ & 0 & 11 & 700 & 41 & 1.4 & 26 & 0.560 & -- & 2.5 & 1800 \\
\hline 462709101131301 & $09-19-75$ & 8 & 7.8 & 250 & 90 & 3.8 & 13 & 0.160 & - & 0.70 & 2600 \\
\hline 462717100554901 & $07-17-72$ & 0 & 23 & 160 & 8.5 & 5.5 & 24 & 0.230 & - & 1.0 & 1900 \\
\hline 462721101213101 & $12-19-69$ & - & -- & 220 & 3.5 & -- & - & 0.00 & 0.0 & - & - \\
\hline 462722101130301 & $06-28-72$ & 10 & 5.1 & 550 & 0.60 & 2.7 & 11 & 0.230 & - & 1.0 & 2500 \\
\hline 462723100554001 & $07-17-72$ & 0 & 13 & 140 & 44 & 3.0 & 22 & 0.560 & - & 2.5 & 2100 \\
\hline 462730102291601 & $06-09-69$ & 40 & - & - & - & -- & - & - & - & 40 & - \\
\hline 462730102325701 & $05-15-69$ & 0 & - & 51 & 0.0 & 0.10 & 15 & - & -- & 2.5 & 70 \\
\hline 462739103003801 & $07-02-75$ & 0 & 37 & 2700 & 16 & 0.50 & 11 & 7.90 & -- & 35 & 1300 \\
\hline 462742100452601 & $08-07-72$ & 0 & 16 & 300 & 0.0 & 0.40 & 16 & 0.320 & - & 1.4 & 1200 \\
\hline 462747100564701 & $10-17-73$ & 18 & 7.3 & 240 & 82 & 2.7 & 16 & 0.230 & - & 1.0 & 2500 \\
\hline 462755101090601 & $05-27-75$ & 28 & 6.2 & 470 & 160 & 2.1 & 8.1 & 0.230 & -- & 1.0 & 2800 \\
\hline 462755101090602 & $05-27-75$ & 44 & 4.9 & 1200 & 14 & 1.1 & 10 & 0.230 & - & 1.0 & 2400 \\
\hline 462756102274001 & $03-31-58$ & 48 & - & 860 & 0.0 & -- & -- & - & -- & -- & -- \\
\hline 462801101101201 & $06-14-72$ & 0 & 43 & 230 & 0.0 & 0.50 & - & 0.110 & - & 0.50 & 1400 \\
\hline
\end{tabular}




\begin{tabular}{|c|c|c|c|c|c|c|c|c|c|c|c|}
\hline Site number & Date & $\begin{array}{l}\text { Iron, } \\
\text { dis- } \\
\text { solved } \\
(\mu g / \mathrm{L} \\
\left.\text { as } \mathrm{Fe}_{\theta}\right)\end{array}$ & $\begin{array}{l}\text { Lead, } \\
\text { dls- } \\
\text { solved } \\
\text { ( } \mu \mathrm{g} / \mathrm{L} \\
\text { as } \mathrm{Pb})\end{array}$ & $\begin{array}{l}\text { Lithium, } \\
\text { dis- } \\
\text { eolved } \\
\text { ( } \mu g / L \\
\text { as Li) }\end{array}$ & 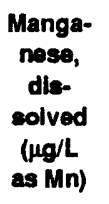 & $\begin{array}{l}\text { Mercury, } \\
\text { dls- } \\
\text { solved } \\
(\mu g / l \\
\text { as } H g)\end{array}$ & $\begin{array}{l}\text { Molyb- } \\
\text { denum, } \\
\text { dis- } \\
\text { solved } \\
\text { ( } \mu g / L \\
\text { as Mo) }\end{array}$ & $\begin{array}{l}\text { Sole- } \\
\text { nium, } \\
\text { dis- } \\
\text { solved } \\
\text { (ug/L } \\
\text { as Se) }\end{array}$ & $\begin{array}{l}\text { Stron- } \\
\text { tlum, } \\
\text { dis- } \\
\text { solved } \\
\text { ( } \mu \text { gll } \\
\text { as Sr) }\end{array}$ & 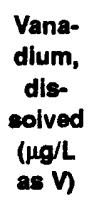 & $\begin{array}{l}\text { Zinc, } \\
\text { dis- } \\
\text { solved } \\
\text { ( } \mu \text { g/L } \\
\text { as Zn) }\end{array}$ \\
\hline 462427102514002 & $08-10-67$ & 220 & - & - & - & - & - & -- & - & - & - \\
\hline 462445102303101 & $08-17-82$ & 220 & - & - & 26 & - & - & $<1$ & - & - & - \\
\hline 462447102380601 & $09-13-67$ & 20 & $\cdots$ & -- & - & - & - & $\cdots$ & - & -- & - \\
\hline 462447102445902 & $07-22-80$ & 0 & - & -- & 0 & - & - & - & - & - & - \\
\hline 462449100423001 & $08-23-74$ & 490 & -- & -- & 240 & - & - & - & - & - & - \\
\hline 462450100380501 & $08-06-75$ & 150 & -- & -- & 260 & - & - & - & - & -- & - \\
\hline 462451101223703 & $06-28-73$ & $<10$ & -- & -- & 40 & -- & - & - & - & - & - \\
\hline 462453102514002 & $10-31-67$ & 3400 & -- & - & -- & - & - & - & - & - & -- \\
\hline 462456100452801 & $08-22-74$ & 200 & -- & - & 80 & - & - & - & -- & -- & -- \\
\hline 462512101082101 & $07-10-72$ & 1300 & - & - & 100 & - & - & - & -- & - & - \\
\hline 462519102331301 & $08-18-82$ & 10 & - & - & $<10$ & - & - & $<1$ & - & - & -- \\
\hline 462519102332301 & $01-01-37$ & - & -- & -- & -- & - & - & - & - & - & -- \\
\hline 462527101554301 & $07-10-68$ & 1400 & -- & - & - & - & - & - & $\rightarrow$ & -- & -- \\
\hline 462527101554302 & $05-28-68$ & 980 & -- & - & -- & - & - & - & -- & -- & - \\
\hline 462542102263201 & $08-17-82$ & 80 & -- & -- & $<10$ & - & -- & $<1$ & -- & - & - \\
\hline 462548102190002 & $03-07-69$ & 3100 & -- & $*$ & - & - & - & -- & -- & -- & -- \\
\hline 462600103164902 & $09-08-77$ & 260 & -- & - & $<10$ & - & - & - & -- & - & -- \\
\hline 462603102232301 & $06-03-69$ & 0 & - & - & - & - & - & -- & -- & -- & -- \\
\hline 462604100530001 & $07-18-72$ & 310 & -- & - & 40 & - & - & - & -- & - & -- \\
\hline 462604100530002 & $07-18-72$ & 80 & - & - & 30 & - & - & - & - & -- & -- \\
\hline 462636103015202 & $06-04-75$ & 550 & - & - & 120 & - & -- & - & - & - & - \\
\hline 462637102092702 & $08-08-67$ & 80 & -- & - & - & - & - & - & - & - & -- \\
\hline 462637102522701 & $08-18-82$ & 460 & - & - & 17 & - & - & $<1$ & - & - & - \\
\hline 462638102125102 & $06-02-69$ & 400 & -- & - & -- & - & - & - & - & - & - \\
\hline 462638102363101 & $08-31-67$ & 220 & - & -- & - & - & - & -- & - & - & - \\
\hline 462638102463202 & $03-11-69$ & 70 & - & - & - & - & - & -- & - & - & - \\
\hline 462643101085901 & $07-08-72$ & $<20$ & - & - & 10 & - & - & - & - & -- & - \\
\hline 462643101085902 & $07-08-72$ & 40 & - & - & 0 & - & - & -- & - & - & - \\
\hline 462654101005001 & $10-17-73$ & 380 & -- & -- & 20 & - & -- & - & - & - & - \\
\hline 462703100542501 & $07-18-72$ & 680 & -- & -- & 50 & - & -- & - & - & - & - \\
\hline 462703100542502 & $07-18-72$ & $<20$ & -. & - & 40 & - & - & - & - & - & - \\
\hline 462709101131301 & $09-19-75$ & 170 & -- & -- & $<10$ & -- & - & - & - & -- & - \\
\hline 462717100554901 & $07-17-72$ & 400 & - & - & 50 & - & - & - & - & - & - \\
\hline 462721101213101 & $12-19-69$ & 820 & -- & -- & - & - & - & - & -- & - & -- \\
\hline 462722101130301 & $06-28-72$ & 230 & -- & - & 60 & - & -- & - & - & - & - \\
\hline 462723100554001 & $07-17-72$ & 90 & -- & -- & 50 & - & - & $-\cdots$ & -- & - & -- \\
\hline 462730102291601 & $06-09-69$ & - & -- & - & - & - & - & - & - & - & - \\
\hline 462730102325701 & $05-15-69$ & 6800 & -- & -- & - & - & - & - & - & - & - \\
\hline 462739103003801 & $07-02-75$ & 80 & - & - & $<10$ & - & - & - & - & -- & - \\
\hline 462742100452601 & $08-07-72$ & 640 & -- & -- & 50 & - & -- & - & - & - & - \\
\hline 462747100564701 & $10-17-73$ & 60 & - & - & $<10$ & - & - & - & - & -- & 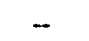 \\
\hline 462755101090601 & $05-27-75$ & $<10$ & - & - & 40 & - & -- & - & - & - & - \\
\hline 462755101090602 & $05-27-75$ & 1300 & - & - & 60 & - & - & - & -- & - & -- \\
\hline 462756102274001 & $03-31-58$ & 480 & -- & - & -- & -- & - & - & - & -- & - \\
\hline 462801101101201 & $06-14-72$ & 940 & - & -- & 60 & - & - & - & -- & - & - \\
\hline
\end{tabular}




\begin{tabular}{|c|c|c|c|c|c|c|c|c|c|c|}
\hline Site number & Local identifier & $\begin{array}{l}\text { County } \\
\text { code }\end{array}$ & $\begin{array}{c}\text { Hydrologic } \\
\text { unit }\end{array}$ & $\begin{array}{l}\text { Geologic } \\
\text { unit }\end{array}$ & Date & $\begin{array}{l}\text { Depth } \\
\text { below } \\
\text { land } \\
\text { surface, } \\
\text { water } \\
\text { lovel } \\
\text { (teet) }\end{array}$ & $\begin{array}{c}\text { Depth } \\
\text { of } \\
\text { woll, } \\
\text { total } \\
\text { (feot) }\end{array}$ & $\begin{array}{l}\text { Altitude } \\
\text { of land } \\
\text { surface, } \\
\text { datum } \\
\text { (feet) }\end{array}$ & $\begin{array}{c}\text { Specific } \\
\text { conduct- } \\
\text { ance, } \\
\text { field } \\
(\mu \mathrm{S} / \mathrm{cm})\end{array}$ & 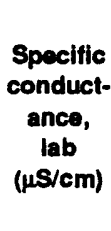 \\
\hline 462802102390401 & $135-095-32 \mathrm{BCA}$ & 041 & 10130204 & 125TGRV & $11-12-65$ & -- & 175 & -- & 1300 & -. \\
\hline 462815101080302 & 134-083-32AAA2 & 059 & 10130206 & 112SLDS & $10-23-73$ & ..- & 99 & 1863 & 1790 & - \\
\hline 462827102075503 & $135-091-28 C C B 3$ & 041 & 10130204 & 125TGRV & $06-03-69$ & -- & 120 & 2500 & 2330 & - \\
\hline 462840102515201 & 135-097-28DAA & 041 & 10130204 & 125TGRV & $07-01-63$ & -- & 350 & -- & 2040 & -- \\
\hline 462842102302302 & $135-094-28 \mathrm{CBB} 2$ & 041 & 10130204 & $125 S N L B$ & $06-04-69$ & - & 60 & 2515 & 1810 & -- \\
\hline 462854102450001 & 135-096-28BDA & 041 & 10130204 & 125TGRV & $04-02-69$ & -- & 196 & 2569 & 1480 & .. \\
\hline 462856103200001 & 135-101-26BAA & 087 & 10130204 & $125 \mathrm{LHCK}$ & $08-28-74$ &.- & 1082 & 2929 & 2000 & - \\
\hline 462907101051401 & 134-083-26BBA & 059 & 10130206 & 112EMCK & $10-25-73$ & - & 144 & 1785 & 2110 & -- \\
\hline 462914102325702 & $135-094-19 \mathrm{CCC} 2$ & 041 & 10130204 & $125 S N L B$ & $09-12-67$ & -- & 81 & 2521 & 920 & - \\
\hline 462914102393202 & 135-095-19DDC2 & 041 & 10130204 & 125TGRV & $06-05-69$ & $\cdots$ & 81 & 2515 & 1670 & - \\
\hline 462921100475801 & 135-081-24DDD & 059 & 10130206 & 211FXHL & $08-07-75$ & -. & 291 & 1844 & 1760 & -- \\
\hline 462946102083201 & $135-091-20 \mathrm{ACB}$ & 041 & 10130204 & 125TGRV & $04-24-69$ & -- & 420 & 2481 & 1980 & -- \\
\hline 462958102464202 & $135-096-20 \mathrm{BBB} 2$ & 041 & 10130204 & 125SBTR & $06-05-69$ & -- & 60 & 2557 & 2410 & - \\
\hline 463004102515201 & 135-097-16DDD & 041 & 10130204 & -- & $08-18-82$ & -- & 80 & -- & 2910 & 2920 \\
\hline 463005101115501 & $135-084-23 \mathrm{AAB}$ & 059 & 10130206 & 211HLCK & $06-14-72$ & -- & 310 & -- & 2170 & - \\
\hline 463006101090901 & $134-083-17 \mathrm{CCC}$ & 059 & 10130206 & 112SLDS & $10-30-73$ & - & 244 & 1830 & 1600 & -- \\
\hline 463012101081201 & 134-083-17DDB1 & 059 & 10130206 & $112 S L D S$ & $10-25-73$ & -- & 244 & 1819 & 1950 & - \\
\hline 463022100441401 & $134-080-16 \mathrm{DAA}$ & 059 & 10130206 & $112 S J M S$ & $10-16-73$ & -- & 169 & 1672 & 1930 & - \\
\hline 463022102552703 & $135-097-18 C B B 3$ & 041 & 10130204 & -- & $08-18-82$ & - & 160 & - & - & 1710 \\
\hline 463050101141601 & 135-084-16AAD & 059 & 10130206 & 112EMCK & $07-25-74$ & -- & 269 & 1887 & 1540 & -- \\
\hline 463052102320901 & $135-094-18 \mathrm{ABA}$ & 041 & 10130204 & -- & $08-18-82$ & -- & 80 & - & 3510 & 3550 \\
\hline 463057101141601 & 135-084-16AAA1 & 059 & 10130206 & 112EMCK & $06-07-74$ & -- & 283 & 1893 & 919 & -- \\
\hline 463057101141602 & 135-084-16AAA2 & 059 & 10130206 & 112EMCK & $07-24-74$ & -- & 174 & 1892 & 889 & - \\
\hline 463057101141603 & $135-084-16 \mathrm{AAA} 3$ & 059 & 10130206 & 112EMCK & $07-25-74$ & -- & 169 & 1890 & 933 & - \\
\hline 463057101141604 & 135-084-16AAA4 & 059 & 10130206 & 112EMCK & $07-25-74$ & -- & 304 & 1888 & 1480 & - \\
\hline 463057101141605 & 135-084-16AAA5 & 059 & 10130206 & 112EMCK & $07-25-74$ & -- & 214 & 1888 & 990 & $\cdots$ \\
\hline 463057101141606 & 135-084-16AAA6 & 059 & 10130206 & 112EMCK & $07-26-74$ & -- & 164 & 1888 & 969 & - \\
\hline 463057101141607 & 135-084-16AAA7 & 059 & 10130206 & 112EMCK & $07-26-74$ & -- & 94 & 1889 & 995 & - \\
\hline 463057101141608 & 135-084-16AAA8 & 059 & 10130206 & 112EMCK & $07-26-74$ & - & 264 & 1888 & 904 & -- \\
\hline 463057101142601 & $135-084-16 \mathrm{AAB}$ & 059 & 10130206 & 112EMCK & $07-26-74$ & -- & 294 & 1891 & 1320 & -- \\
\hline 463057101143501 & $135-084-16 \mathrm{ABA}$ & 059 & 10130206 & 112EMCK & $06-10-74$ & -- & 296 & 1899 & 1950 & -- \\
\hline 463057101144401 & $135-084-16 \mathrm{ABB}$ & 059 & 10130206 & 112EMCK & $06-10-74$ & - & 164 & 1880 & 1620 & -- \\
\hline 463058101114901 & 134-084-11DDD & 059 & 10130206 & 112SLDS & $10-29-73$ & -- & 159 & 1869 & 730 & - \\
\hline 463058102190001 & $135-093-12 \mathrm{CCC}$ & 041 & 10130204 & 125TGRV & $11-30-67$ & -- & 201 & 2438 & 2160 & -- \\
\hline 463103101151301 & $135-084-9 \mathrm{CCD}$ & 059 & 10130206 & 112EMCK & $07-23-74$ & -- & 104 & 1883 & 2330 & - \\
\hline 463139102563301 & $135-098-12 B B A$ & 087 & 10130204 & -- & $08-11-82$ & -- & 150 & - & 3700 & 3830 \\
\hline 463141103030902 & 135-099-1DDD2 & 087 & 10130204 & 125TRVL & $08-29-74$ & -- & 902 & 2820 & 2390 & -- \\
\hline 463143102434501 & 135-096-10BAA & 041 & 10130204 & 125TGRV & $04-02-69$ & -- & 318 & 2589 & 1680 & - \\
\hline 463144102231301 & $135-093-8 \mathrm{ABA}$ & 041 & 10130204 & $125 \mathrm{SNLB}$ & $06-03-69$ & -- & 81 & 2500 & 1300 & - \\
\hline 463150101083101 & 134-083-5DCC & 059 & 10130206 & 112EMCK & $10-30-73$ & -- & 114 & 1835 & 1610 & -- \\
\hline 463150101112101 & $134-084-1 \mathrm{CDC} 1$ & 059 & 10130206 & 112 SLDS & $10-30-73$ & -- & 214 & 1895 & 773 & - \\
\hline 463150101112102 & 134-084-1CDC2 & 059 & 10130206 & 112SLDS & $10-29-73$ & -- & 94 & 1895 & 862 & - \\
\hline 463213102515201 & 135-097-4ADD1 & 041 & 10130204 & 125SNLB & $06-20-67$ & - & 105 & 2593 & 1450 & - \\
\hline 463219102520102 & $135-097-4 \mathrm{ADB} 2$ & 041 & 10130204 & 125SNLB & $11-07-58$ & - & 84 & 2595 & 1350 & - \\
\hline 463223102190002 & $135-093-1 \mathrm{BCB} 2$ & 041 & 10130204 & 125TGRV & $07-26-67$ & -- & 252 & 2532 & 1440 & - \\
\hline
\end{tabular}




\begin{tabular}{|c|c|c|c|c|c|c|c|c|c|c|c|}
\hline Stte number & Date & $\begin{array}{c}\text { pH, } \\
\text { field } \\
\text { (stand- } \\
\text { ard } \\
\text { units }\end{array}$ & $\begin{array}{c}\mathrm{pH}_{1} \\
\text { lab } \\
\text { (stand- } \\
\text { ard } \\
\text { units) }\end{array}$ & $\begin{array}{c}\text { Temper- } \\
\text { ature, } \\
\text { water } \\
\text { (degrees } \\
\text { Colsius) }\end{array}$ & $\begin{array}{l}\text { Color } \\
\text { (platt- } \\
\text { num } \\
\text { cobalt } \\
\text { scalo) }\end{array}$ & $\begin{array}{c}\text { Hard- } \\
\text { ness, } \\
\text { total } \\
(\mathrm{mg} / \mathrm{L} \text { as } \\
\left.\mathrm{CaCO}_{3}\right)\end{array}$ & $\begin{array}{l}\text { Noncar- } \\
\text { bonate } \\
\text { hard- } \\
\text { ness, } \\
\text { total } \\
\text { (mg/L as } \\
\left.\mathrm{CaCO}_{3}\right)\end{array}$ & $\begin{array}{l}\text { Alka- } \\
\text { linity, } \\
\text { field } \\
\text { (mg/L as } \\
\text { CaCO }\end{array}$ & $\begin{array}{c}\text { Alka- } \\
\text { linity, } \\
\text { lab } \\
\text { (mg/L as } \\
\left.\mathrm{CaCO}_{3}\right)\end{array}$ & $\begin{array}{l}\text { Solids, } \\
\text { sum of } \\
\text { constit- } \\
\text { uents, } \\
\text { dis- } \\
\text { solved } \\
\text { (mg/L) }\end{array}$ & $\begin{array}{c}\text { Solids, } \\
\text { residue } \\
\text { at } 105 \\
\text { degrees } \\
\text { Colsius, } \\
\text { total } \\
\text { (mg/L) }\end{array}$ \\
\hline 462802102390401 & $11-12-65$ & 8.8 & - & - & 7000 & 80 & - & -- & - & 1040 & -- \\
\hline 462815101080302 & $10-23-73$ & 8.0 & - & 8.5 & - & 470 & 0 & - & - & 1250 & -- \\
\hline 462827102075503 & $06-03-69$ & 8.2 & - & 9.0 & 35 & 41 & $\mathbf{0}$ & -- & - & 1560 & -- \\
\hline 462840102515201 & $07-01-63$ & 8.2 & - & - & -- & 20 & 0 & -- & - & 1320 & -- \\
\hline 462842102302302 & $06-04-69$ & 6.4 & -- & 8.5 & - & 850 & 760 & - & - & 1370 & $\cdots$ \\
\hline 462854102450001 & $04-02-69$ & 9.6 & - & 11.0 & 400 & 18 & 0 & - & - & 834 & - \\
\hline 462856103200001 & $08-28-74$ & 8.5 & - & - & - & 13 & 0 & - & - & 1250 & -- \\
\hline 462907101051401 & $10-25-73$ & 8.0 & - & 9.0 & - & 310 & 0 & -- & - & 1400 & - \\
\hline 462914102325702 & $09-12-67$ & 8.0 & -- & 9.5 & - & 200 & 0 & - & - & 588 & - \\
\hline 462914102393202 & $06-05-69$ & 7.9 & - & 7.0 & 10 & 450 & 0 & - & - & 1170 & - \\
\hline 462921100475801 & $08-07-75$ & 8.4 & - & 10.5 & -- & 40 & 0 & - & - & 1140 & -- \\
\hline 462946102083201 & $04-24-69$ & 8.4 & - & 12.0 & 200 & 20 & 0 & -- & -- & 1350 & - \\
\hline 462958102464202 & $06-05-69$ & 7.9 & - & 7.5 & 35 & 220 & 0 & - & - & 1700 & - \\
\hline 463004102515201 & $08-18-82$ & 7.4 & 7.9 & 15.0 & -- & 620 & - & -- & 514 & 2060 & -- \\
\hline $46300510111550 \mathrm{I}$ & $06-14-72$ & 8.2 & - & 10.0 & - & 13 & 0 & - & - & 1390 & -- \\
\hline 463006101090901 & $10-30-73$ & 8.1 & - & 9.0 & -- & 93 & 0 & - & - & 1040 & - \\
\hline 463012101081201 & $10-25-73$ & 7.9 & $\cdots$ & 9.0 & -- & 630 & 0 & -- & - & 1370 & -- \\
\hline 463022100441401 & $10-16-73$ & 8.1 & -- & 8.5 & -- & 64 & 0 & - & - & 1260 & -- \\
\hline 463022102552703 & $08-18-82$ & - & 8.6 & -- & - & 17 & - & -- & 541 & 1080 & - \\
\hline 463050101141601 & $07-25-74$ & 8.4 & - & - & -- & 120 & 0 & - & - & 1000 & - \\
\hline 463052102320901 & $08-18-82$ & 7.1 & 8.1 & 13.0 & - & 320 & -- & -- & 594 & 2660 & -- \\
\hline 463057101141601 & $06-07-74$ & 8.1 & - & 9.5 & - & 470 & 16 & - & -- & 574 & -- \\
\hline 463057101141602 & $07-24-74$ & 8.2 & - & - & - & 440 & 13 & - & - & 555 & - \\
\hline 463057101141603 & $07-25-74$ & 8.2 & - & -- & - & 470 & 34 & - & - & 585 & - \\
\hline 463057101141604 & $07-25-74$ & 8.2 & - & - & - & 130 & 0 & - & - & 968 & - \\
\hline 463057101141605 & $07-25-74$ & 8.1 & - & -. & - & 480 & 14 & - & - & 632 & - \\
\hline 463057101141606 & $07-26-74$ & 8.2 & - & - & - & 470 & 17 & - & - & 610 & - \\
\hline 463057101141607 & $07-26-74$ & 8.2 & - & - & - & 320 & 0 & - & - & 624 & -- \\
\hline 463057101141608 & $07-26-74$ & 8.2 & - & - & - & 460 & 1 & - & - & 568 & -- \\
\hline 463057101142601 & $07-26-74$ & 8.3 & - & - & - & 160 & 0 & - & - & 868 & - \\
\hline 463057101143501 & $06-10-74$ & 8.1 & - & - & - & 290 & 0 & - & - & 1330 & - \\
\hline 463057101144401 & $06-10-74$ & 8.0 & - & - & - & 440 & 0 & - & - & 1120 & - \\
\hline 463058101114901 & $10-29-73$ & 7.8 & - & 8.5 & -- & 280 & 2 & - & -- & 463 & - \\
\hline 463058102190001 & $11-30-67$ & 8.1 & - & 9.0 & 100 & 98 & 0 & - & - & 1480 & - \\
\hline 463103101151301 & $07-23-74$ & 8.1 & -- & - & - & 550 & 10 & - & - & 1710 & -- \\
\hline 463139102563301 & $08-11-82$ & 7.5 & 8.1 & 13.0 & - & 120 & -- & - & 702 & 2670 & -- \\
\hline 463141103030902 & $08-29-74$ & 8.4 & - & - & - & 20 & 0 & - & - & 1520 & - \\
\hline 463143102434501 & $04-02-69$ & 8.5 & -- & 11.0 & 600 & 59 & 0 & - & -- & 1080 & -- \\
\hline 463144102231301 & $06-03-69$ & 7.9 & - & 9.0 & 25 & 230 & 0 & - & - & 863 & -- \\
\hline 463150101083101 & $10-30-73$ & 8.2 & - & 8.5 & - & 110 & 0 & - & - & 1060 & -- \\
\hline 463150101112101 & $10-30-73$ & 7.6 & - & 9.0 & - & 290 & 18 & - & - & 501 & -- \\
\hline 463150101112102 & $10-29-73$ & 7.6 & - & 8.0 & -- & 400 & 110 & - & - & 570 & - \\
\hline 463213102515201 & $06-20-67$ & 7.9 & - & 9.0 & - & 270 & 0 & - & - & 964 & - \\
\hline 463219102520102 & $11-07-58$ & 7.9 & - & 8.5 & 400 & 170 & 0 & - & - & 911 & - \\
\hline 463223102190002 & $07-26-67$ & 8.2 & - & - & 40 & 70 & 0 & - & - & 934 & -- \\
\hline
\end{tabular}




\begin{tabular}{|c|c|c|c|c|c|c|c|c|c|c|c|}
\hline Stte number & Date & $\begin{array}{l}\text { Solids, } \\
\text { residue } \\
\text { at } 180 \\
\text { degrees } \\
\text { Caisius, } \\
\text { dis- } \\
\text { solved } \\
\text { (mgll) }\end{array}$ & $\begin{array}{l}\text { Solids, } \\
\text { dis- } \\
\text { solved } \\
\text { (tons per } \\
\text { acre- } \\
\text { toot) }\end{array}$ & $\begin{array}{l}\text { Calcium, } \\
\text { dis- } \\
\text { solved } \\
\text { (mgll } \\
\text { as Ca) }\end{array}$ & $\begin{array}{l}\text { Magno- } \\
\text { sium, } \\
\text { dle- } \\
\text { solved } \\
\text { (mg/L } \\
\text { as Mg) }\end{array}$ & $\begin{array}{l}\text { Sodium, } \\
\text { die- } \\
\text { solved } \\
\text { (mg/L } \\
\text { as Na) }\end{array}$ & $\begin{array}{c}\text { Sodium } \\
\text { (percent) }\end{array}$ & $\begin{array}{l}\text { Sodium } \\
\text { adsorp- } \\
\text { tion } \\
\text { ratio }\end{array}$ & $\begin{array}{l}\text { Resid- } \\
\text { ual, } \\
\text { sodium, } \\
\text { car- } \\
\text { bonats } \\
\text { (mgli as } \\
\mathrm{CaCO}_{3} \text { ) }\end{array}$ & $\begin{array}{l}\text { Potas- } \\
\text { sium, } \\
\text { dis- } \\
\text { solved } \\
\text { (mg/L } \\
\text { as K) }\end{array}$ & $\begin{array}{c}\text { Blcar- } \\
\text { bonate } \\
(\mathrm{mg} / \mathrm{L} \text { as } \\
\left.\mathrm{HCO}_{3}\right)\end{array}$ \\
\hline 462802102390401 & $11-12-65$ & - & - & - & - & 280 & - & - & - & -- & - \\
\hline 462815101080302 & $10-23-73$ & 1260 & 1.71 & 110 & 47 & 250 & 53 & 5 & - & 6.8 & 670 \\
\hline 462827102075503 & $06-03-69$ & 1470 & 2.00 & 9.8 & 4.0 & 580 & 96 & 39 & -- & 4.3 & 1040 \\
\hline 462840102515201 & $07-01-63$ & 1110 & - & - & - & - & - & -- & - & -- & 530 \\
\hline 462842102302302 & $06-04-69$ & 1530 & 2.08 & 180 & 97 & 91 & 19 & 1 & - & 9.7 & 110 \\
\hline 462854102450001 & $04-02-69$ & 943 & 1.28 & 5.4 & 1.1 & 340 & 97 & 35 & -- & 2.1 & 340 \\
\hline 462856103200001 & $08-28-74$ & 1290 & 1.75 & 2.9 & 1.5 & 530 & 99 & 63 & .. & 1.4 & 1300 \\
\hline 462907101051401 & $10-25-73$ & 1410 & 1.92 & 70 & 33 & 420 & 74 & 10 & - & 5.2 & 1050 \\
\hline 462914102325702 & $09-12-67$ & 570 & 0.78 & 38 & 26 & 140 & 59 & 4 & - & 5.6 & 370 \\
\hline 462914102393202 & $06-05-69$ & 1190 & 1.62 & 90 & 54 & 250 & 55 & 5 & - & 4.0 & 660 \\
\hline 462921100475801 & $08-07-75$ & 1160 & 1.58 & 7.9 & 5.0 & 420 & 95 & 29 & - & 2.3 & 800 \\
\hline 462946102083201 & $04-24-69$ & 1310 & 1.78 & 4.8 & 1.9 & 480 & 98 & 47 & - & 2.0 & 880 \\
\hline 462958102464202 & $06-05-69$ & 1810 & 2.46 & 55 & 20 & 520 & 83 & 15 & - & 5.4 & 770 \\
\hline 463004102515201 & $08-18-82$ & 2020 & 2.75 & 140 & 65 & 480 & 63 & 8 & -- & 5.3 & - \\
\hline 463005101115501 & $06-14-72$ & 1430 & 1.94 & 3.9 & 0.90 & 530 & 99 & 63 & - & 1.4 & 930 \\
\hline 463006101090901 & $10-30-73$ & 1060 & 1.44 & 22 & 9.2 & 350 & 89 & 16 & - & 3.3 & 680 \\
\hline 463012101081201 & $10-25-73$ & 1360 & 1.85 & 140 & 68 & 250 & 46 & 4 & -- & 6.8 & 800 \\
\hline 463022100441401 & $10-16-73$ & 1250 & 1.70 & 14 & 7.1 & 450 & 93 & 24 & -- & 3.6 & 880 \\
\hline 463022102552703 & $08-18-82$ & 1080 & 1.47 & 4.7 & 1.3 & 390 & 98 & 41 & -- & 2.6 & -. \\
\hline 463050101141601 & $07-25-74$ & 1040 & 1.41 & 31 & 10 & 350 & 86 & 14 & - & 3.6 & 820 \\
\hline 463052102320901 & $08-18-82$ & 2420 & 3.29 & 78 & 30 & 760 & 83 & 19 & - & 8.5 & -- \\
\hline 463057101141601 & $06-07-74$ & 590 & 0.80 & 130 & 35 & 35 & 14 & 0.7 & - & 2.4 & 550 \\
\hline 463057101141602 & $07-24-74$ & 588 & 0.80 & 110 & 40 & 33 & 14 & 0.7 & -- & 4.5 & 520 \\
\hline 463057101141603 & $07-25-74$ & 616 & 0.84 & 120 & 41 & 34 & 14 & 0.7 & -- & 4.3 & 530 \\
\hline 463057101141604 & $07-25-74$ & 993 & 1.35 & 32 & 12 & 330 & 84 & 13 & - & 3.2 & 830 \\
\hline 463057101141605 & $07-25-74$ & 638 & 0.87 & 130 & 38 & 41 & 16 & 0.8 & -- & 3.9 & 570 \\
\hline 463057101141606 & $07-26-74$ & 635 & 0.86 & 120 & 41 & 40 & 16 & 0.8 & - & 4.5 & 550 \\
\hline 463057101141607 & $07-26-74$ & 621 & 0.84 & 79 & 30 & 110 & 42 & 3 & - & 4.2 & 560 \\
\hline 463057101141608 & $07-26-74$ & 591 & 0.80 & 130 & 33 & 27 & 11 & 0.5 & - & 3.4 & 560 \\
\hline 463057101142601 & $07-26-74$ & 875 & 1.19 & 37 & 16 & 280 & 79 & 10 & - & 4.7 & 700 \\
\hline 463057101143501 & $06-10-74$ & 1330 & 1.81 & 70 & 28 & 370 & 73 & 9 & - & 6.0 & 830 \\
\hline 463057101144401 & $06-10-74$ & 1120 & 1.52 & 97 & 48 & 230 & 53 & 5 & -- & 5.9 & 700 \\
\hline 463058101114901 & $10-29-73$ & 465 & 0.63 & 67 & 27 & 51 & 28 & 1 & - & 3.8 & 340 \\
\hline 463058102190001 & $11-30-67$ & 1530 & 2.08 & 25 & 8.6 & 480 & 91 & 21 & - & 4.8 & 540 \\
\hline 463103101151301 & $07-23-74$ & 1680 & 2.28 & 130 & 55 & 380 & 60 & 7 & - & 6.1 & 660 \\
\hline 463139102563301 & $08-11-82$ & 2690 & 3.66 & 27 & 13 & 880 & 94 & 35 & - & 6.0 & - \\
\hline 463141103030902 & $08-29-74$ & 1550 & 2.11 & 2.7 & 3.3 & 650 & 98 & 63 & - & 1.9 & 1600 \\
\hline 463143102434501 & $04-02-69$ & 1090 & 1.48 & 5.3 & 11 & 380 & 93 & 22 & - & 1.4 & 580 \\
\hline 463144102231301 & $06-03-69$ & 820 & 1.12 & 51 & 24 & 230 & 68 & 7 & -- & 7.7 & 520 \\
\hline 463150101083101 & $10-30-73$ & 1040 & 1.41 & 26 & 11 & 360 & 87 & 15 & - & 1.8 & 800 \\
\hline 463150101112101 & $10-30-73$ & 495 & 0.67 & 70 & 28 & 56 & 29 & 1 & - & 4.0 & 330 \\
\hline 463150101112102 & $10-29-73$ & 572 & 0.78 & 90 & 43 & 39 & 17 & 0.8 & - & 4.2 & 360 \\
\hline 463213102515201 & $06-20-67$ & 975 & 1.33 & 58 & 30 & 250 & 67 & 7 & - & 4.4 & 550 \\
\hline 463219102520102 & $11-07-58$ & 911 & 1.24 & 38 & 18 & 300 & 79 & 10 & - & 3.0 & 560 \\
\hline 463223102190002 & $07-26-67$ & 959 & 1.30 & 18 & 6.1 & 330 & 91 & 17 & $\ldots$ & 2.6 & 640 \\
\hline
\end{tabular}




\begin{tabular}{|c|c|c|c|c|c|c|c|c|c|c|c|}
\hline Stte number & Date & $\begin{array}{c}\text { Car- } \\
\text { bonate } \\
\text { ( } \mathrm{mg} / \mathrm{L} \text { as } \\
\mathrm{CO}_{3} \text { ) }\end{array}$ & $\begin{array}{c}\text { Carbon } \\
\text { dioxide, } \\
\text { dis- } \\
\text { solved } \\
(\mathrm{mg} / \mathrm{L} \mathrm{as} \\
\left.\mathrm{CO}_{2}\right)\end{array}$ & $\begin{array}{l}\text { Sulfate, } \\
\text { dis- } \\
\text { solved } \\
\text { (mg/L as } \\
\left.\mathrm{SO}_{4}\right)\end{array}$ & $\begin{array}{l}\text { Chlo- } \\
\text { ride, } \\
\text { dis- } \\
\text { solved } \\
\text { (mg/L } \\
\text { as Cl) }\end{array}$ & $\begin{array}{l}\text { Fluoride, } \\
\text { dis- } \\
\text { solved } \\
\text { (mg/l } \\
\text { as F) }\end{array}$ & $\begin{array}{l}\text { Sillca, } \\
\text { dis- } \\
\text { solved } \\
(\mathrm{mg} / \mathrm{L} \text { as } \\
\left.\mathrm{SiO}_{2}\right)\end{array}$ & $\begin{array}{l}\text { Nitro- } \\
\text { gen, } \\
\text { nitrate, } \\
\text { dis- } \\
\text { solved } \\
\text { (mg/L } \\
\text { as N) }\end{array}$ & $\begin{array}{c}\text { Nitro- } \\
\text { gen, } \\
\text { nitrate, } \\
\text { total } \\
\text { (mg/L } \\
\text { as } \mathrm{NO}_{3} \text { ) }\end{array}$ & $\begin{array}{l}\text { Nitro- } \\
\text { gen, } \\
\text { nitrate, } \\
\text { dis- } \\
\text { solved } \\
\text { (mg/L } \\
\text { as } \mathrm{NO}_{3} \text { ) }\end{array}$ & $\begin{array}{l}\text { Boron, } \\
\text { dis- } \\
\text { soived } \\
(\mu g / L \\
\text { as B) }\end{array}$ \\
\hline 462802102390401 & $11-12-65$ & -- & - & - & - & - & -- & -- & -- & -- & -- \\
\hline 462815101080302 & $10-23-73$ & 0 & 11 & 470 & 4.2 & 0.50 & 22 & 0.230 & - & 1.0 & 730 \\
\hline 462827102075503 & $06-03-69$ & 0 & - & 440 & 1.0 & 2.0 & 7.5 & -- & -- & 1.0 & 740 \\
\hline 462840102515201 & $07-01-63$ & 40 & 6.2 & 450 & 7.0 & - & - & 0.00 & - & 0.0 & -- \\
\hline 462842102302302 & $06-04-69$ & 0 & - & 910 & 21 & 0.10 & 13 & $\ldots$ & -. & 40 & 440 \\
\hline 462854102450001 & $04-02-69$ & -- & - & 300 & 13 & 1.3 & 2.1 & -. & -. & 0.0 & 630 \\
\hline 462856103200001 & $08-28-74$ & 31 & 6.9 & 2.9 & 24 & 6.1 & 9.0 & 0.560 & -- & 2.5 & 1300 \\
\hline 462907101051401 & $10-25-73$ & 0 & 17 & 300 & 30 & 1.5 & 23 & 0.230 & -- & 1.0 & 1600 \\
\hline 462914102325702 & $09-12-67$ & $\mathbf{0}$ & - & 180 & 5.7 & 0.40 & 14 & - & - & 0.0 & 110 \\
\hline 462914102393202 & $06-05-69$ & $\mathbf{0}$ & -- & 430 & 2.9 & 0.30 & 12 & -- & -- & 2.5 & 300 \\
\hline 462921100475801 & $08-07-75$ & 8 & 5.2 & 270 & 9.4 & 0.60 & 12 & 0.630 & -. & 2.8 & 6100 \\
\hline 462946102083201 & $04-24-69$ & 84 & -- & 330 & 10 & 2.2 & 8.5 & -- & -- & 1.1 & 1100 \\
\hline 462958102464202 & $06-05-69$ & 0 & - & 700 & 16 & 1.1 & 8.7 & -- & - & 2.5 & 480 \\
\hline 463004102515201 & $08-18-82$ & - & - & 1000 & 49 & 0.40 & 11 & - & - & -- & 410 \\
\hline 463005101115501 & $06-14-72$ & 0 & 9.4 & 370 & 17 & 5.9 & - & 0.560 & - & 2.5 & 2700 \\
\hline 463006101090901 & $10-30-73$ & 0 & 8.6 & 280 & 16 & 1.5 & 25 & 0.230 & - & 1.0 & 1400 \\
\hline 463012101081201 & $10-25-73$ & $\mathbf{0}$ & 16 & 460 & 27 & 0.60 & 24 & 0.230 & - & 1.0 & 600 \\
\hline 463022100441401 & $10-16-73$ & 0 & 11 & 310 & 10 & 1.7 & 24 & 0.230 & - & 1.0 & 1900 \\
\hline 463022102552703 & $08-18-82$ & - & - & 340 & 4.3 & 1.1 & 9.4 & -- & - & -- & 820 \\
\hline 463050101141601 & $07-25-74$ & 11 & 5.4 & 160 & 7.9 & 1.0 & 17 & 0.230 & - & 1.0 & 1600 \\
\hline 463052102320901 & $08-18-82$ & -- & -- & 1400 & 14 & 1.5 & 7.3 & -- & - & -- & 560 \\
\hline 463057101141601 & $06-07-74$ & 0 & 7.0 & 73 & 1.0 & 0.20 & 24 & 0.230 & - & 1.0 & $<20$ \\
\hline 463057101141602 & $07-24-74$ & $\mathbf{0}$ & 5.3 & 88 & 2.9 & 0.90 & 15 & 0.230 & - & 1.0 & 430 \\
\hline 463057101141603 & $07-25-74$ & $\mathbf{0}$ & 5.4 & 100 & 3.3 & 0.60 & 15 & 0.230 & - & 1.0 & 240 \\
\hline 463057101141604 & $07-25-74$ & $\mathbf{0}$ & 8.4 & 150 & 8.9 & 0.90 & 19 & 0.230 & - & 1.0 & 1500 \\
\hline 463057101141605 & $07-25-74$ & $\mathbf{0}$ & 7.2 & 110 & 3.4 & 0.60 & 17 & 0.230 & - & 1.0 & 350 \\
\hline 463057101141606 & $07-26-74$ & 0 & 5.6 & 110 & 3.5 & 0.60 & 16 & 0.230 & -- & 1.0 & 390 \\
\hline 463057101141607 & $07-26-74$ & $\mathbf{0}$ & 5.7 & 100 & 3.1 & 0.70 & 15 & 0.230 & - & 1.0 & 870 \\
\hline 463057101141608 & $07-26-74$ & $\mathbf{0}$ & 5.7 & 69 & 2.3 & 0.60 & 23 & 0.230 & - & 1.0 & 240 \\
\hline 463057101142601 & $07-26-74$ & 5 & 5.7 & 150 & 5.9 & 1.0 & 18 & 0.230 & -- & 1.0 & 1200 \\
\hline 463057101143501 & $06-10-74$ & 0 & 11 & 410 & 4.9 & 0.60 & 25 & 0.230 & - & 1.0 & 950 \\
\hline 463057101144401 & $06-10-74$ & 0 & 11 & 360 & 1.4 & 0.60 & 24 & 0.230 & - & 1.0 & 860 \\
\hline 463058101114901 & $10-29-73$ & 0 & 8.5 & 120 & 2.3 & 0.60 & 20 & 0.230 & - & 1.0 & 300 \\
\hline 463058102190001 & $11-30-67$ & $\mathbf{0}$ & -- & 680 & 8.3 & 1.0 & 11 & -- & - & 3.0 & 640 \\
\hline 463103101151301 & $07-23-74$ & 0 & 8.4 & 790 & 3.5 & 0.90 & 14 & 0.230 & - & 1.0 & 630 \\
\hline 463139102563301 & $08-11-82$ & - & - & 1300 & 5.9 & 0.50 & 12 & -. & - &.- & 720 \\
\hline 463141103030902 & $08-29-74$ & 26 & 11 & 19 & 12 & 4.6 & 8.1 & 0.230 & - & 1.0 & 1300 \\
\hline 463143102434501 & $04-02-69$ & 10 & - & 370 & 8.1 & 6.5 & 8.1 & -- & - & 0.0 & 200 \\
\hline 463144102231301 & $06-03-69$ & $\mathbf{0}$ & - & 280 & 1.1 & 0.40 & 17 & -- & -- & 1.0 & 300 \\
\hline 463150101083101 & $10-30-73$ & $\mathbf{0}$ & 8.1 & 230 & 4.1 & 4.0 & 22 & 0.230 & - & 1.0 & 2700 \\
\hline 463150101112101 & $10-30-73$ & $\mathbf{0}$ & 13 & 150 & 2.5 & 0.50 & 24 & 0.230 & -- & 1.0 & 130 \\
\hline 463150101112102 & $10-29-73$ & $\mathbf{0}$ & 14 & 190 & 2.8 & 0.20 & 19 & 0.230 & - & 1.0 & 220 \\
\hline 463213102515201 & $06-20-67$ & $\mathbf{0}$ & - & 330 & 8.8 & 0.60 & 12 & -- & - & 1.0 & 350 \\
\hline 463219102520102 & $11-07-58$ & $\mathbf{0}$ & - & 230 & 26 & 1.4 & 13 & -- & -- & 1.4 & - \\
\hline 463223102190002 & $07-26-67$ & $\mathbf{0}$ & - & 250 & 3.2 & 1.9 & 7.0 & - & - & 0.0 & 610 \\
\hline
\end{tabular}




\begin{tabular}{|c|c|c|c|c|c|c|c|c|c|c|c|}
\hline Slte number & Date & $\begin{array}{l}\text { Iron, } \\
\text { dis- } \\
\text { solved } \\
(\mu g / L \\
\text { as Fe) }\end{array}$ & $\begin{array}{l}\text { Lead, } \\
\text { dis- } \\
\text { solved } \\
\text { ( } \mu g / \mathrm{L} \\
\text { as } \mathrm{Pb})\end{array}$ & $\begin{array}{l}\text { Lthium, } \\
\text { dls- } \\
\text { solved } \\
\text { ( } \mu g / L \\
\text { as Li) }\end{array}$ & $\begin{array}{l}\text { Manga- } \\
\text { ness, } \\
\text { dis- } \\
\text { solved } \\
\text { ( } \mu g / \mathrm{L} \\
\text { ae Mn) }\end{array}$ & $\begin{array}{l}\text { Mercury, } \\
\text { dis- } \\
\text { solved } \\
(\mu g / l \\
\text { as Hg) }\end{array}$ & $\begin{array}{l}\text { Molyb- } \\
\text { denum, } \\
\text { dis- } \\
\text { solved } \\
\text { ( } \mu g / \mathrm{L} \\
\text { as Mo) }\end{array}$ & $\begin{array}{l}\text { Sele- } \\
\text { nium, } \\
\text { dis- } \\
\text { solved } \\
\text { ( } \mu g / L \\
\text { as So) }\end{array}$ & $\begin{array}{l}\text { Stron- } \\
\text { tium, } \\
\text { dis- } \\
\text { solved } \\
(\mu g / L \\
\text { as Sr) }\end{array}$ & $\begin{array}{l}\text { Vana- } \\
\text { dium, } \\
\text { dis- } \\
\text { solved } \\
(\mu g / L \\
\text { as V) }\end{array}$ & $\begin{array}{c}\text { Zinc, } \\
\text { dis- } \\
\text { soived } \\
(\mu g / L \\
\text { as } \mathrm{Zn})\end{array}$ \\
\hline 462802102390401 & $11-12-65$ & - & - & -. & - & - & - & -- & -- & - & -- \\
\hline 462815101080302 & $10-23-73$ & 3600 & -- & -- & 60 & - & - & - & -- & -- & -- \\
\hline 462827102075503 & $06-03-69$ & 0 & -- & - & - & - & - & - & -- & - & - \\
\hline 462840102515201 & $07-01-63$ & - & - & -- & - & - & - & - & - & - & .. \\
\hline 462842102302302 & $06-04-69$ & 100 & - & -- & - & - & -- & - & -- & - & -- \\
\hline 462854102450001 & $04-02-69$ & 740 & - & -- & -- & - & -- & - & - & - & -- \\
\hline 462856103200001 & $08-28-74$ & 270 & -- & - & 20 & - & - & -- & - & - & - \\
\hline 462907101051401 & $10-25-73$ & 270 & - & -- & 20 & -- & - & -- & - & - & - \\
\hline 462914102325702 & $09-12-67$ & 130 & - & -- & -- & -- & - & - & -- & - & -- \\
\hline 462914102393202 & $06-05-69$ & 1100 & - & -- & - & - & - & - & -- & - & - \\
\hline 462921100475801 & $08-07-75$ & 100 & -- & - & $<10$ & - & -- & - & - & - & -- \\
\hline 462946102083201 & $04-24-69$ & 460 & -- & $\ldots$ & - & -- & - & - & -- & - & - \\
\hline 462958102464202 & $06-05-69$ & 400 & - & -- & -- & -- & - & - & -- & -- & -- \\
\hline 463004102515201 & $08-18-82$ & 50 & -- & -- & 30 & - & - & 7 & - & $\ldots$ & -- \\
\hline 463005101115501 & $06-14-72$ & 300 & - & -- & 20 & - & - & -- & - & - & -- \\
\hline 463006101090901 & $10-30-73$ & 920 & - & - & 160 & - & - & - & - & - & -. \\
\hline 463012101081201 & $10-25-73$ & 190 & -. & - & 140 & -- & -- & -- & - & -. & -. \\
\hline 463022100441401 & $10-16-73$ & $<10$ & - & - & 20 & - & -- & -- & -- & -- & - \\
\hline 463022102552703 & $08-18-82$ & 48 & - & - & 15 & - & - & $<1$ & - & - & -- \\
\hline 463050101141601 & $07-25-74$ & 2200 & - & -- & 40 & - & - & -- & -- & -- & - \\
\hline 463052102320901 & $08-18-82$ & 70 & -- & - & 110 & - & -- & $<1$ & - & -- & - \\
\hline 463057101141601 & $06-07-74$ & 1700 & - & - & 340 & - & - & -- & - & -- & - \\
\hline 463057101141602 & $07-24-74$ & 2900 & -- & - & 220 & - & -. & - & - & -- & - \\
\hline 463057101141603 & $07-25-74$ & 4500 & - & -- & 540 & - & -- & - & - & - & -- \\
\hline 463057101141604 & $07-25-74$ & 1200 & - & - & 80 & - & - & - & - & - & - \\
\hline 463057101141605 & $07-25-74$ & 5700 & - & -- & 300 & - & - & - & - & -- & -- \\
\hline 463057101141606 & $07-26-74$ & 2600 & - & -- & 300 & - & - & - & - & -- & - \\
\hline 463057101141607 & $07-26-74$ & 4500 & - & - & 140 & - & - & -- & - & - & -- \\
\hline 463057101141608 & $07-26-74$ & 2700 & - & - & 40 & - & - & -- & - & - & - \\
\hline 463057101142601 & $07-26-74$ & 3300 & - & - & 560 & -- & -- & - & -- & -- & - \\
\hline 463057101143501 & $06-10-74$ & 1400 & - & - & 160 & - & - & - & -- & - & -- \\
\hline 463057101144401 & $06-10-74$ & 5700 & -- & - & 200 & - & - & - & -- & -- & - \\
\hline 463058101114901 & $10-29-73$ & 2100 & -- & -- & 540 & -- & -- & - & - & - & - \\
\hline 463058102190001 & $11-30-67$ & 1400 & - & - & - & - & -- & - & - & - & - \\
\hline 463103101151301 & $07-23-74$ & 7400 & - & - & 220 & - & - & - & -- & - & - \\
\hline 463139102563301 & $08-11-82$ & 70 & - & - & 40 & -- & - & $<1$ & - & - & - \\
\hline 463141103030902 & $08-29-74$ & 410 & -- & - & 40 & - & - & - & - & - & -- \\
\hline 463143102434501 & $04-02-69$ & 150 & -- & - & - & - & - & - & - & - & -- \\
\hline 463144102231301 & $06-03-69$ & 0 & - & - & - & $\ldots$ & - & - & - & - & - \\
\hline 463150101083101 & $10-30-73$ & 610 & - & - & 100 & - & - & - & - & - & - \\
\hline 463150101112101 & $10-30-73$ & 2100 & - & - & 620 & -- & - & - & -- & -- & - \\
\hline 463150101112102 & $10-29-73$ & 3100 & - & - & 520 & - & - & - & - & - & - \\
\hline 463213102515201 & $06-20-67$ & 180 & - & - & - & - & - & - & - & - & - \\
\hline 463219102520102 & $11-07-58$ & 570 & - & - & 100 & - & - & - & - & - & - \\
\hline 463223102190002 & $07-26-67$ & 1100 & - & - & - & - & - & -- & - & - & - \\
\hline
\end{tabular}




\begin{tabular}{|c|c|c|c|c|c|c|c|c|c|c|}
\hline Stte number & Local identifier & $\begin{array}{l}\text { County } \\
\text { code }\end{array}$ & $\begin{array}{c}\text { Hydrologic } \\
\text { unit }\end{array}$ & $\begin{array}{l}\text { Geologic } \\
\text { unit }\end{array}$ & Date & $\begin{array}{l}\text { Depth } \\
\text { below } \\
\text { land } \\
\text { surface, } \\
\text { water } \\
\text { level } \\
\text { (feet) }\end{array}$ & $\begin{array}{l}\text { Depth } \\
\text { of } \\
\text { well, } \\
\text { total } \\
\text { (foet) }\end{array}$ & $\begin{array}{l}\text { Altitude } \\
\text { of land } \\
\text { surface, } \\
\text { datum } \\
\text { (feet) }\end{array}$ & $\begin{array}{l}\text { Specific } \\
\text { conduct- } \\
\text { ance, } \\
\text { field } \\
(\mu \mathrm{S} / \mathrm{cm})\end{array}$ & 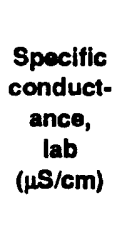 \\
\hline 463232103001901 & $136-098-33 C C D$ & 087 & 10130204 & $125 S \mathrm{NLB}$ & $07-02-75$ & - & 120 & -- & 2390 & -- \\
\hline 463238102542701 & 136-097-31DDD & 041 & 10130204 & -- & $08-19-82$ & - & 92 & -- & 1850 & 1920 \\
\hline 463239102522501 & 136-097-33DCC & 041 & 10130204 & $125 S N L B$ & $06-20-67$ & - & 104 & 2600 & 1910 & - \\
\hline 463245100441101 & 135-080-33DDA & 059 & 10130206 & 112LTHR & $10-12-73$ & - & 169 & 1725 & 2080 & -- \\
\hline 463250101083501 & $136-083-32 C D D 1$ & 059 & 10130206 & 125CBLD & $06-05-72$ & - & 550 & - & 2310 & - \\
\hline 463258102302201 & $136-094-33 \mathrm{CBC}$ & 041 & 10130204 & $125 S N L B$ & $08-21-67$ & - & 50 & 2582 & 10000 & - \\
\hline 463301102353701 & 136-095-34DAA1 & 041 & 10130204 & $125 S N L B$ & $03-20-56$ & - & 48 & 2670 & 1960 & $\cdots$ \\
\hline 463318103021901 & $136-098-31 \mathrm{ABB}$ & 087 & 10130204 & $125 \mathrm{LHCK}$ & $07-22-75$ & - & 960 & - & 2070 & -- \\
\hline 463320102212802 & 136-093-34BCB2 & 041 & 10130204 & $125 S N L B$ & $06-01-69$ & - & 100 & 2515 & 888 & -- \\
\hline 463338103042001 & 136-099-26DAD & 087 & 10130204 & 125TRVL & $10-28-76$ & -- & 534 & 2640 & 2000 & - \\
\hline 463345101042001 & $136-083-26 \mathrm{DDC}$ & 059 & 10130206 & 125CBLD & $06-05-72$ & -- & 105 & .. & 9090 & - \\
\hline 463410103150601 & $136-100-28 \mathrm{BAB}$ & 087 & 10130204 & $125 S N L B$ & $08-12-82$ & - & 54 & - & 1130 & 1140 \\
\hline 463424102505101 & $136-097-22 \mathrm{DDC}$ & 041 & 10130204 & - & $08-19-82$ & - & 120 & -. & 1170 & 1190 \\
\hline 463425102465501 & 136-096-19DDD & 041 & 10130204 & $125 S N L B$ & $08-25-67$ & -- & 132 & 2698 & 864 & -. \\
\hline 463443101063201 & $136-083-22 \mathrm{CCB}$ & 059 & 10130206 & $125 C B L D$ & $07-06-72$ & - & 315 & -- & 1760 & -- \\
\hline 463503101060401 & 136-083-22BDD1 & 059 & 10130206 & 125CBLD & $07-06-72$ & -- & 286 & - & 2160 & -- \\
\hline 463503101060402 & 136-083-22BDD2 & 059 & 10130206 & 125CBLD & $07-06-72$ & - & 290 & -- & 2110 & - \\
\hline 463503101060403 & 136-083-22BDD3 & 059 & 10130206 & $125 \mathrm{CBLD}$ & $07-06-72$ & - & 284 & - & 2980 & -- \\
\hline 463507101092301 & 136-083-19ADB1 & 059 & 10130206 & 125CBLD & $06-06-72$ & - & 500 & -- & 2150 & -- \\
\hline 463516102511901 & $136-097-15 C D D$ & 041 & 10130204 & - & $08-19-82$ & - & 48 & - & 2500 & 2510 \\
\hline 463529102503201 & $136-097-14 \mathrm{CBC1}$ & 041 & 10130204 & -- & $08-19-82$ & -- & 255 & 2790 & 1430 & 1490 \\
\hline 463529102503202 & $136-097-14 C B C 2$ & 041 & 10130204 & - & $08-19-82$ & -- & 263 & -. & 1430 & 1490 \\
\hline 463529102504101 & 136-097-15DAD & 041 & 10130204 & 125SNLB & $12-06-67$ & -- & 201 & 2758 & 1520 & - \\
\hline 463659102574302 & $136-098-2 \mathrm{CDC} 2$ & 087 & 10130204 & -- & $08-12-82$ & - & 160 & - & 1780 & 1760 \\
\hline 463704102275701 & 136-094-3DDD & 041 & 10130204 & $125 \mathrm{SNLB}$ & $11-23-68$ & - & 224 & 2568 & 1990 & - \\
\hline 463817102581001 & 137-097-31BDD & 089 & 10130204 & 125SNLB & $07-17-67$ & - & 255 & 2755 & 1340 & -- \\
\hline 463841102344401 & 137-094-32BBB 1 & 089 & 10130204 & -- & $03-06-69$ & - & 112 & 2670 & 625 & - \\
\hline 463931102403302 & 137-095-28BAA2 & 089 & 10130204 & 125TGRV & $06-30-67$ & -- & 500 & 2686 & 1700 & - \\
\hline 463936103062601 & 137-099-24DDD & 089 & 10130204 & 125SNLB & $06-09-69$ & -- & 224 & 2722 & 2540 & - \\
\hline 463939102341502 & $137-094-20 \mathrm{CDD} 2$ & 089 & 10130204 & $125 S N L B$ & $03-06-69$ & - & 40 & 2633 & 1190 & - \\
\hline
\end{tabular}




\begin{tabular}{|c|c|c|c|c|c|c|c|c|c|c|c|}
\hline Sito number & Date & $\begin{array}{l}\text { pH, } \\
\text { fiold } \\
\text { (stand- } \\
\text { and } \\
\text { units }\end{array}$ & $\begin{array}{l}\text { pH, } \\
\text { lab } \\
\text { (stand- } \\
\text { ard } \\
\text { units) }\end{array}$ & $\begin{array}{c}\text { Temper- } \\
\text { ature, } \\
\text { water } \\
\text { (degrees } \\
\text { Celslus) }\end{array}$ & $\begin{array}{c}\text { Color } \\
\text { (platl- } \\
\text { num } \\
\text { cobalt } \\
\text { scale) }\end{array}$ & $\begin{array}{l}\text { Hard- } \\
\text { ness, } \\
\text { total } \\
\text { (mg/L as } \\
\left.\mathrm{CaCO}_{3}\right)\end{array}$ & $\begin{array}{c}\text { Noncar- } \\
\text { bonate } \\
\text { hard- } \\
\text { ness, } \\
\text { total } \\
\text { (mg/L as } \\
\left.\mathrm{CaCO}_{3}\right)\end{array}$ & $\begin{array}{l}\text { Alka- } \\
\text { linity, } \\
\text { floid } \\
\text { (mg/L as } \\
\mathrm{CaCO}_{3} \text { ) }\end{array}$ & $\begin{array}{l}\text { Alka- } \\
\text { linity, } \\
\text { lab } \\
\text { (mg/L ae } \\
\left.\mathrm{CaCO}_{3}\right)\end{array}$ & $\begin{array}{l}\text { Solids, } \\
\text { sum of } \\
\text { consth- } \\
\text { uents, } \\
\text { dis- } \\
\text { solved } \\
\text { (mg/L) }\end{array}$ & $\begin{array}{c}\text { Solids, } \\
\text { residue } \\
\text { at } 105 \\
\text { degrees } \\
\text { Colslus, } \\
\text { total } \\
\text { (mg/L) }\end{array}$ \\
\hline 463232103001901 & $07-02-75$ & 7.8 & -- & 11.0 & -- & 130 & 0 & - & - & 1700 & - \\
\hline 463238102542701 & $08-19-82$ & 7.4 & 7.9 & 13.5 & -- & 160 & - & - & 514 & 1270 & $\cdots$ \\
\hline 463239102522501 & $06-20-67$ & 7.9 & -- & 9.0 & -- & 130 & 0 & -- & - & 1290 &.- \\
\hline 463245100441101 & $10-12-73$ & 8.0 & -- & 8.5 & - & 280 & 0 & -- & -- & 1400 & -- \\
\hline 463250101083501 & $06-05-72$ & 7.4 & - & 11.0 & - & 1300 & 690 & - & - & 1920 & -- \\
\hline 463258102302201 & $08-21-67$ & 7.3 & -- & 10.5 & - & 6000 & 5900 & -- & -- & 10900 & - \\
\hline 463301102353701 & $03-20-56$ & - & -- & - & $\cdots$ & 26 & - & -- & -- & 1370 & -- \\
\hline 463318103021901 & $07-22-75$ & 8.5 & - & 14.0 & - & 19 & 0 & - & -- & 1290 & -- \\
\hline 463320102212802 & $06-01-69$ & 7.5 & -- & -- & - & 360 & 69 & - & - & 604 & - \\
\hline 463338103042001 & $10-28-76$ & 8.8 & -. & 12.0 & - & 20 & 0 & - & - & 1140 & - \\
\hline 463345101042001 & $06-05-72$ & 7.9 & - & 7.0 & - & 5000 & 4300 & - & - & 9780 & -- \\
\hline 463410103150601 & $08-12-82$ & 7.0 & 7.8 & 9.0 & - & 330 & - & -- & 395 & 739 & - \\
\hline 463424102505101 & $08-19-82$ & 7.7 & 7.9 & 16.0 & - & 51 & -- & - & 292 & 751 & - \\
\hline 463425102465501 & $08-25-67$ & 7.8 & -- & -- & - & 420 & 200 & -- & - & 590 & -- \\
\hline 463443101063201 & $07-06-72$ & 7.9 & -- & 9.0 & - & 13 & 0 & - & - & 1160 & -- \\
\hline 463503101060401 & $07-06-72$ & 8.1 & - & - & - & 21 & 0 & -- & - & 1450 & -- \\
\hline 463503101060402 & $07-06-72$ & 7.9 & -. & 4.5 & - & 62 & 0 & -. & - & 1470 & -- \\
\hline 463503101060403 & $07-06-72$ & 7.7 & - & 9.0 & - & 160 & 0 & - & -- & 2150 & - \\
\hline 463507101092301 & $06-06-72$ & 8.0 & -- & 10.5 & - & 12 & 0 & - & -- & 1340 & - \\
\hline 463516102511901 & $08-19-82$ & 6.7 & 7.3 & 10.0 & - & 1400 & - & - & 541 & 1470 & -- \\
\hline 463529102503201 & $08-19-82$ & 8.2 & 8.5 & 13.0 & -- & 15 & - & - & 585 & 896 & - \\
\hline 463529102503202 & $08-19-82$ & 8.2 & 8.5 & 13.0 & - & 15 & -- & - & 585 & 896 & -- \\
\hline 463529102504101 & $12-06-67$ & 8.2 & - & 7.0 & 100 & 38 & 0 & -- & - & 987 & -. \\
\hline 463659102574302 & $08-12-82$ & 8.0 & 8.5 & 17.0 & -- & 29 & - & -- & 658 & 1150 & - \\
\hline 463704102275701 & $11-23-68$ & 7.9 & - & 8.5 & - & 190 & 0 & - & - & 1350 & - \\
\hline 463817102581001 & $07-17-67$ & 8.2 & -- & 9.5 & 90 & 21 & 0 & - & - & 886 & - \\
\hline 463841102344401 & $03-06-69$ & 7.9 & - & - & 20 & 280 & 110 & - & - & 304 & - \\
\hline 463931102403302 & $06-30-67$ & 8.2 & - & 10.0 & 50 & 20 & 0 & - & -- & 1060 & - \\
\hline 463936103062601 & $06-09-69$ & 8.4 & - & 10.5 & 900 & 160 & 0 & - & -- & 1720 & - \\
\hline 463939102341502 & $03-06-69$ & 7.8 & - & 8.5 & 10 & 610 & 430 & - & - & 904 & - \\
\hline
\end{tabular}




\begin{tabular}{|c|c|c|c|c|c|c|c|c|c|c|c|}
\hline Slte number & Date & $\begin{array}{l}\text { Solids, } \\
\text { reeidue } \\
\text { at } 180 \\
\text { degrees } \\
\text { Celsius, } \\
\text { dis- } \\
\text { solved } \\
\text { (mglh) }\end{array}$ & $\begin{array}{l}\text { Solids, } \\
\text { dio- } \\
\text { solved } \\
\text { (tons per } \\
\text { acre- } \\
\text { foot) }\end{array}$ & $\begin{array}{l}\text { Calcium, } \\
\text { dis- } \\
\text { solved } \\
\text { (mglL } \\
\text { as Ca) }\end{array}$ & $\begin{array}{l}\text { Magne- } \\
\text { sium, } \\
\text { dis- } \\
\text { solved } \\
\text { (mg/L } \\
\text { as Mg) }\end{array}$ & $\begin{array}{l}\text { Sodium, } \\
\text { dis- } \\
\text { solved } \\
\text { (mgll } \\
\text { as Na) }\end{array}$ & $\begin{array}{l}\text { Sodium } \\
\text { (porcent) }\end{array}$ & $\begin{array}{l}\text { Sodlum } \\
\text { adsorp } \\
\text { tion } \\
\text { ratio }\end{array}$ & $\begin{array}{l}\text { Resid- } \\
\text { ual, } \\
\text { sodium, } \\
\text { car- } \\
\text { bonate } \\
(\mathrm{mgl} \text { as } \\
\left.\mathrm{CaCO}_{3}\right)\end{array}$ & $\begin{array}{l}\text { Potas- } \\
\text { sium, } \\
\text { dis- } \\
\text { solved } \\
\text { (mg/L } \\
\text { as K) }\end{array}$ & $\begin{array}{c}\text { Bicar- } \\
\text { bonate } \\
\text { (mg/L as } \\
\left.\mathrm{HCO}_{3}\right)\end{array}$ \\
\hline 463232103001901 & $07-02-75$ & 1660 & 2.26 & 36 & 9.7 & 580 & 90 & 22 & -- & 5.1 & 950 \\
\hline 463238102542701 & $08-19-82$ & 1270 & 1.73 & 38 & 15 & 380 & 84 & 13 & -- & 5.1 & - \\
\hline 463239102522501 & $06-20-67$ & 1290 & 1.75 & 29 & 15 & 430 & 87 & 16 & -- & 4.3 & 670 \\
\hline 463245100441101 & $10-12-73$ & 1450 & 1.97 & 70 & 26 & 400 & 75 & 10 & -- & 6.0 & 720 \\
\hline 463250101083501 & $06-05-72$ & 1840 & 2.50 & 230 & 170 & 130 & 18 & 2 & - & 9.4 & 710 \\
\hline 463258102302201 & $08-21-67$ & 11700 & 15.9 & 480 & 1200 & 1100 & 29 & 6 & - & 21 & 120 \\
\hline 463301102353701 & $03-20-56$ & - & -. & -- & -- & -- & -- & -- &.- & + & - \\
\hline 463318103021901 & $07-22-75$ & 1350 & 1.84 & 2.7 & 3.0 & 540 & 98 & 54 & -- & 2.1 & 1390 \\
\hline 463320102212802 & $06-01-69$ & 646 & 0.88 & 91 & 33 & 63 & 27 & 1 & -- & 4.5 & 360 \\
\hline 463338103042001 & $10-28-76$ & 1150 & 1.56 & 2.6 & 3.3 & 460 & 98 & 45 & -- & 1.9 & 980 \\
\hline 463345101042001 & $06-05-72$ & 10200 & 13.9 & 400 & 980 & 1200 & 34 & 7 &.- & 9.5 & 930 \\
\hline 463410103150601 & $08-12-82$ & 741 & 1.01 & 67 & 40 & 140 & 47 & 3 & -. & 5.6 & - \\
\hline 463424102505101 & $08-19-82$ & 764 & 1.04 & 11 & 5.7 & 240 & 90 & 15 & -- & 5.7 & -- \\
\hline 463425102465501 & $08-25-67$ & 600 & 0.82 & 120 & 30 & 33 & 14 & 0.7 & -- & 3.8 & 270 \\
\hline 463443101063201 & $07-06-72$ & 1210 & 1.65 & 3.3 & 1.2 & 440 & 98 & 53 & - & 1.3 & 810 \\
\hline 463503101060401 & $07-06-72$ & 1450 & 1.97 & 5.6 & 1.7 & 510 & 98 & 48 & -- & 1.6 & 680 \\
\hline 463503101060402 & $07-06-72$ & 1430 & 1.94 & 5.1 & 12 & 500 & 94 & 28 & - & 1.6 & 740 \\
\hline 463503101060403 & $07-06-72$ & 2060 & 2.80 & 37 & 16 & 720 & 91 & 25 & -- & 3.7 & 1070 \\
\hline 463507101092301 & $06-06-72$ & 1360 & 1.85 & 3.5 & 0.90 & 550 & 99 & 68 & - & 1.8 & 1150 \\
\hline 463516102511901 & $08-19-82$ & 1880 & 2.56 & 360 & 120 & 29 & 4 & 0.3 & - & 5.1 & - \\
\hline 463529102503201 & $08-19-82$ & 940 & 1.28 & 3.7 & 1.5 & 340 & 98 & 38 & -- & 2.3 & -- \\
\hline 463529102503202 & $08-19-82$ & 940 & 1.28 & 3.7 & 1.5 & 340 & 98 & 38 & - & 2.3 & - \\
\hline 463529102504101 & $12-06-67$ & 1000 & 1.36 & 9.6 & 3.4 & 350 & 95 & 25 & -- & 3.6 & 540 \\
\hline 463659102574302 & $08-12-82$ & .1150 & 1.56 & 7.1 & 2.7 & 420 & 97 & 34 & -- & 2.2 & -- \\
\hline 463704102275701 & $11-23-68$ & 1350 & 1.84 & 47 & 18 & 390 & 81 & 12 & -- & 6.4 & 410 \\
\hline 463817102581001 & $07-17-67$ & 876 & 1.19 & 5.6 & 1.7 & 330 & 97 & 31 & - & 2.7 & 620 \\
\hline 463841102344401 & $03-06-69$ & 390 & 0.53 & 77 & 21 & 15 & 10 & 0.4 & - & 7.4 & 210 \\
\hline 463931102403302 & $06-30-67$ & 1070 & 1.46 & 5.2 & 1.7 & 440 & 98 & 43 & $\rightarrow$ & 2.5 & 1080 \\
\hline 463936103062601 & $06-09-69$ & 1670 & 2.27 & 8.0 & 35 & 570 & 88 & 19 & -- & 2.5 & 730 \\
\hline 463939102341502 & $03-06-69$ & 948 & 1.29 & 160 & 51 & 48 & 14 & 0.8 & - & 12 & 220 \\
\hline
\end{tabular}




\begin{tabular}{|c|c|c|c|c|c|c|c|c|c|c|c|}
\hline Slto number & Date & $\begin{array}{c}\text { Car- } \\
\text { bonate } \\
\text { (mg/L as } \\
\left.\mathrm{CO}_{3}\right)\end{array}$ & $\begin{array}{c}\text { Carbon } \\
\text { dioxide, } \\
\text { dis- } \\
\text { solved } \\
\text { (mg/L as } \\
\left.\mathrm{CO}_{2}\right)\end{array}$ & $\begin{array}{l}\text { Sulfate, } \\
\text { dis- } \\
\text { solved } \\
\text { (mg/L as } \\
\text { sol) }^{-}\end{array}$ & $\begin{array}{l}\text { Chlo- } \\
\text { ride, } \\
\text { dis- } \\
\text { solved } \\
\text { (mgll } \\
\text { as Cl) }\end{array}$ & $\begin{array}{l}\text { Fluoride, } \\
\text { dis- } \\
\text { solved } \\
\text { (mg/L } \\
\text { as F) }\end{array}$ & $\begin{array}{l}\text { SHica, } \\
\text { dis- } \\
\text { solved } \\
(\mathrm{mg} / \mathrm{L} \text { as } \\
\left.\mathrm{SiO}_{2}\right)\end{array}$ & $\begin{array}{l}\text { Nitro- } \\
\text { gon, } \\
\text { nitrate, } \\
\text { dis- } \\
\text { solved } \\
\text { (mg/L } \\
\text { as N) }\end{array}$ & $\begin{array}{c}\text { Nitro- } \\
\text { gon, } \\
\text { nitrate, } \\
\text { total } \\
\left(\mathrm{mg}^{\prime} \mathrm{L}\right. \\
\left.\text { as } \mathrm{NO}_{3}\right)\end{array}$ & $\begin{array}{l}\text { Nitro- } \\
\text { gen, } \\
\text { nitrate, } \\
\text { dis- } \\
\text { solved } \\
\text { (mg/L } \\
\left.\text { as } \mathrm{NO}_{3}\right)\end{array}$ & $\begin{array}{c}\text { Boron, } \\
\text { dis- } \\
\text { solved } \\
(\mu g / L \\
\text { as B) }\end{array}$ \\
\hline 463232103001901 & $07-02-75$ & $\mathbf{0}$ & 24 & 580 & 4.0 & 1.5 & 9.2 & 0.230 & -. & 1.0 & 630 \\
\hline 463238102542701 & 08-19-82 & -- & - & 500 & 4.5 & 0.50 & 11 & - & - & - & 1100 \\
\hline 463239102522501 & $06-20-67$ & 0 & - & 470 & 6.5 & 1.1 & 11 & - & - & 1.0 & 620 \\
\hline 463245100441101 & $10-12-73$ & 0 & 12 & 510 & 7.4 & 0.60 & 27 & 0.230 & - & 1.0 & 1400 \\
\hline $46325010108350 \mathrm{I}$ & $06-05-72$ & 0 & 45 & 960 & 5.9 & 0.10 & 17 & 0.230 & - & 1.0 & 540 \\
\hline 463258102302201 & $08-21-67$ & $\mathbf{0}$ & - & 8000 & 23 & 0.0 & - & - & - & 0.0 & 930 \\
\hline 463301102353701 & $03-20-56$ & - & - & -- & .. & -- & - & - & - & - & 1300 \\
\hline 463318103021901 & $07-22-75$ & 25 & 7.3 & 6.2 & 14 & 1.5 & 5.9 & 0.900 & -. & 4.0 & 1500 \\
\hline 463320102212802 & $06-01-69$ & 0 & 18 & 210 & 2.5 & 0.20 & 24 & 0.230 & - & 1.0 & 0 \\
\hline 463338103042001 & $10-28-76$ & 47 & 2.7 & 120 & 9.1 & 4.8 & 5.1 & 0.230 & -- & 1.0 & $<20$ \\
\hline 463345101042001 & $06-05-72$ & $\mathbf{0}$ & 19 & 6400 & 150 & 0.70 & 14 & 38.0 & - & 170 & 540 \\
\hline 463410103150601 & $08-12-82$ & -- & - & 220 & 9.2 & 0.50 & 19 & - & - & - & 120 \\
\hline 463424102505101 & $08-19-82$ & - & - & 300 & 1.9 & 0.20 & 10 & - & -- & - & 570 \\
\hline 463425102465501 & $08-25-67$ & $\mathbf{0}$ & - & 260 & 2.5 & 0.20 & 9.1 & - & -- & 2.5 & 40 \\
\hline 463443101063201 & $07-06-72$ & 0 & 16 & 300 & 0.0 & 1.9 & 11 & 0.230 & - & 1.0 & 2000 \\
\hline 463503101060401 & $07-06-72$ & 0 & 8.6 & 580 & 0.0 & 0.50 & 11 & 0.110 & - & 0.50 & 1300 \\
\hline 463503101060402 & $07-06-72$ & $\mathbf{0}$ & 15 & 570 & 0.0 & 0.60 & 11 & 0.00 & - & 0.0 & 1300 \\
\hline 463503101060403 & $07-06-72$ & $\mathbf{0}$ & 34 & 830 & 0.60 & 0.10 & 13 & 0.230 & - & 1.0 & 1100 \\
\hline 463507101092301 & $06-06-72$ & $\mathbf{0}$ & 18 & 61 & 140 & 3.0 & 11 & 0.230 & - & 1.0 & 3100 \\
\hline 463516102511901 & $08-19-82$ & -- & - & 450 & 170 & 0.20 & 13 & - & - & - & 90 \\
\hline 463529102503201 & $08-19-82$ & - & - & 180 & 5.8 & 3.5 & 7.8 & - & -- & - & 580 \\
\hline 463529102503202 & $08-19-82$ & - & - & 180 & 5.8 & 3.5 & 7.8 & - & - & - & 580 \\
\hline 463529102504101 & $12-06-67$ & 0 & - & 330 & 12 & 1.5 & 7.3 & - & - & 2.0 & 340 \\
\hline 463659102574302 & $08-12-82$ & - & - & 300 & 9.4 & 2.9 & 9.6 & - & - & - & 770 \\
\hline 463704102275701 & $11-23-68$ & 0 & - & 650 & 26 & 0.70 & 7.7 & - & - & 17 & 310 \\
\hline 463817102581001 & $07-17-67$ & 0 & - & 230 & 1.5 & 3.1 & 5.3 & - & -- & 0.0 & 590 \\
\hline 463841102344401 & $03-06-69$ & 0 & - & 42 & 26 & 0.20 & 15 & - & - & 82 & 40 \\
\hline 463931102403302 & $06-30-67$ & 0 & .. & 52 & 8.6 & 5.8 & 8.1 & - & - & 0.0 & 590 \\
\hline 463936103062601 & $06-09-69$ & 17 & - & 710 & 6.1 & 6.7 & 7.7 & - & -- & 0.0 & 220 \\
\hline 463939102341502 & $03-06-69$ & 0 & - & 490 & 14 & 0.50 & 14 & - & - & 0.0 & 0 \\
\hline
\end{tabular}




\begin{tabular}{|c|c|c|c|c|c|c|c|c|c|c|c|}
\hline Site number & Date & $\begin{array}{l}\text { Iron, } \\
\text { dis- } \\
\text { solved } \\
\text { ( } \mu \mathrm{g} / \mathrm{L} \\
\text { as Fe) }\end{array}$ & $\begin{array}{l}\text { Lead, } \\
\text { dis- } \\
\text { solved } \\
(\mu g / L \\
\text { as } \mathrm{Pb})\end{array}$ & 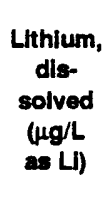 & $\begin{array}{l}\text { Manga- } \\
\text { nose, } \\
\text { dis- } \\
\text { solved } \\
\text { ( } \mu g / L \\
\text { as Mn) }\end{array}$ & $\begin{array}{l}\text { Mercury, } \\
\text { dls- } \\
\text { solved } \\
\text { ( } \mu \mathrm{g} / \mathrm{L} \\
\text { as Hg) }\end{array}$ & $\begin{array}{c}\text { Moiyb- } \\
\text { donum, } \\
\text { dis- } \\
\text { solved } \\
(\mu g / L \\
\text { as Mo) }\end{array}$ & $\begin{array}{l}\text { Sole- } \\
\text { nium, } \\
\text { dis- } \\
\text { solved } \\
(\mu g / L \\
\text { as Se) }\end{array}$ & $\begin{array}{l}\text { Stron- } \\
\text { tium, } \\
\text { dis- } \\
\text { soived } \\
(\mu g / L \\
\text { as Sr) }\end{array}$ & $\begin{array}{l}\text { Vana- } \\
\text { dium, } \\
\text { dis- } \\
\text { solved } \\
(\mu g / L \\
\text { as V) }\end{array}$ & $\begin{array}{c}\text { Zinc, } \\
\text { dls- } \\
\text { solved } \\
(\mu g / L \\
\text { as } \mathrm{Zn})\end{array}$ \\
\hline 463232103001901 & $07-02-75$ & 730 & - & - & 160 & - & -- & - & - & - & - \\
\hline 463238102542701 & $08-19-82$ & 1500 & - & - & 160 & - & - & $<1$ & - & -- & - \\
\hline 463239102522501 & $06-20-67$ & 200 & -- & - & -- & - & - & - & - & - & - \\
\hline 463245100441101 & $10-12-73$ & 100 & - & - & 140 & - & - & - & - & - & - \\
\hline 463250101083501 & $06-05-72$ & 44000 & - & -- & 400 & - & - & - & - & - & -- \\
\hline 463258102302201 & $08-21-67$ & 52000 & - & - & -- & - & - & - & -- & - & - \\
\hline 463301102353701 & $03-20-56$ & -- & - & - & - & -- & - & - & - & -- & -_. \\
\hline 463318103021901 & $07-22-75$ & 400 & - & - & $<10$ & - & -- & - & -- & - & -- \\
\hline 463320102212802 & $06-01-69$ & - & -- & - & - & - & - & - & - & -- & -- \\
\hline 463338103042001 & $10-28-76$ & 2400 & - & - & 30 & - & - & - & - & -- & -- \\
\hline 463345101042001 & $06-05-72$ & 310 & -- & - & 80 & $\ldots$ & - & - & - & -- & -- \\
\hline 463410103150601 & $08-12-82$ & 77 & -- & - & 110 & - & - & 1 & - & - & -- \\
\hline 463424102505101 & $08-19-82$ & 890 & - & -- & 56 & - & - & $<1$ & -- & - & -- \\
\hline 463425102465501 & $08-25-67$ & 2800 & - & - & - & - & - & - & -- & - & -- \\
\hline 463443101063201 & $07-06-72$ & 160 & - & - & 30 & - & - & - & - & - & - \\
\hline 463503101060401 & $07-06-72$ & 90 & -- & - & 20 & - & -- & - & - & - & -- \\
\hline 463503101060402 & $07-06-72$ & 180 & - & $\ldots$ & 20 & - & -- & $\ldots$ & -- & -- & -- \\
\hline 463503101060403 & $07-06-72$ & 900 & - & -- & 90 & - & - & - & - & - & - \\
\hline 463507101092301 & $06-06-72$ & 40 & -- & - & 0 & - & - & - & - & - & - \\
\hline 463516102511901 & $08-19-82$ & 1300 & -- & -- & 70 & - & - & 64 & -- & - & - \\
\hline 463529102503201 & $08-19-82$ & 66 & -. & -. & 9 & - & - & $<1$ & -- & -- & - \\
\hline 463529102503202 & $08-19-82$ & 66 & -- & - & 9 & - & -. & $<1$ & -- & - & - \\
\hline 463529102504101 & $12-06-67$ & 1100 & - & - & - & - & $\cdots$ & - & - & - & - \\
\hline 463659102574302 & $08-12-82$ & 96 & - & - & 17 & - & - & $<1$ & - & - & - \\
\hline 463704102275701 & $11-23-68$ & 720 & - & - & - & - & - & - & -- & -- & -- \\
\hline 463817102581001 & $07-17-67$ & 2500 & - & $\ldots$ & - & - & - & - & - & - & - \\
\hline 463841102344401 & $03-06-69$ & $\mathbf{0}$ & - & -- & - & - & - & -- & - & - & -- \\
\hline 463931102403302 & $06-30-67$ & 340 & - & -- & - & - & - & - & - & - & - \\
\hline 463936103062601 & $06-09-69$ & 510 & -. & -- & - & - & - & - & - & - & -- \\
\hline 463939102341502 & $03-06-69$ & 1500 & - & -- & -- & - & -- & -- & - & - & - \\
\hline
\end{tabular}


Table 5. Summary of ground-water quality and periodic ground-water level data obtained from the U.S. Geological Suney for sites where multiple water-quality samples were collected

[Certain properties or constituents may be listed more than once; however, no distinction is made between field and laboratory values]

\section{Abbreviations and symbols}

$\mathrm{ft}$, feet

$\mathrm{g} / \mathrm{kg}$, grams per kilogram

JCU, Jackson candle unit

LSD, land surface datum

$\mathrm{mg} / \mathrm{kg}$, milligrams per kilogram

$\mathrm{mg} / \mathrm{L}$, milligrams per liter

$\mathrm{mm}$, millimeter

NGVD, National Geodetic Vertical Datum

NTU, nephelometric turbidity unit

$\mathrm{pCi} / \mathrm{L}$, picocurie per liter

$\mu \mathrm{g} / \mathrm{g}$, micrograms per gram

$\mu \mathrm{g} / \mathrm{L}$, micrograms per liter

$\mu \mathrm{S} / \mathrm{cm}$, microsiemens per centimeter at 25 degrees Celsius

,-- no data

\section{County code}

001, Adams County

011, Bowman County

037, Grant County

041, Hettinger County

059, Morton County

085, Sioux County

087, Slope County

\section{Geologic unit}

$112 \quad$ Pleistocene

125 Paleocene

$211 \quad$ Upper Cretaceous

BGFV Buried glaciofluvial deposits

CNBL Cannonball Member of Fort Union Formation

EMCK Elm Creek aquifer

FXHL Fox Hills Sandstone

HCFH Hell Creek Formation-Fox Hills Sandstone

HLCK Hell Creek Formation

HRMN Harmon lignite aquifer

LDLW Ludlow Member of Fort Union Formation

SNLB Sentinel Butte Member of Fort Union Formation

TGRV Tongue River Member of Fort Union Formation

TRVL Tongue River-Ludlow Members of Fort Union Formation 


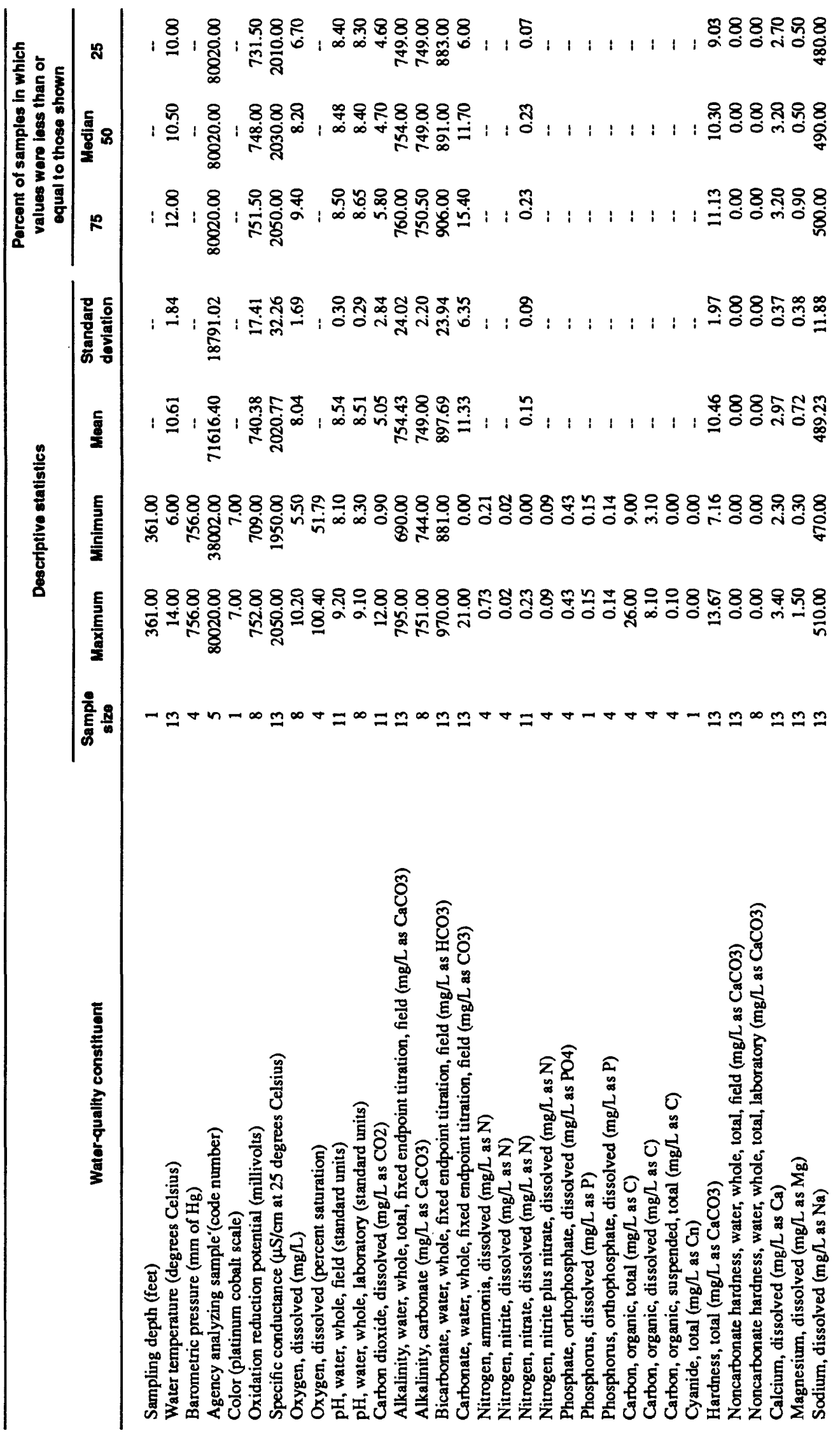




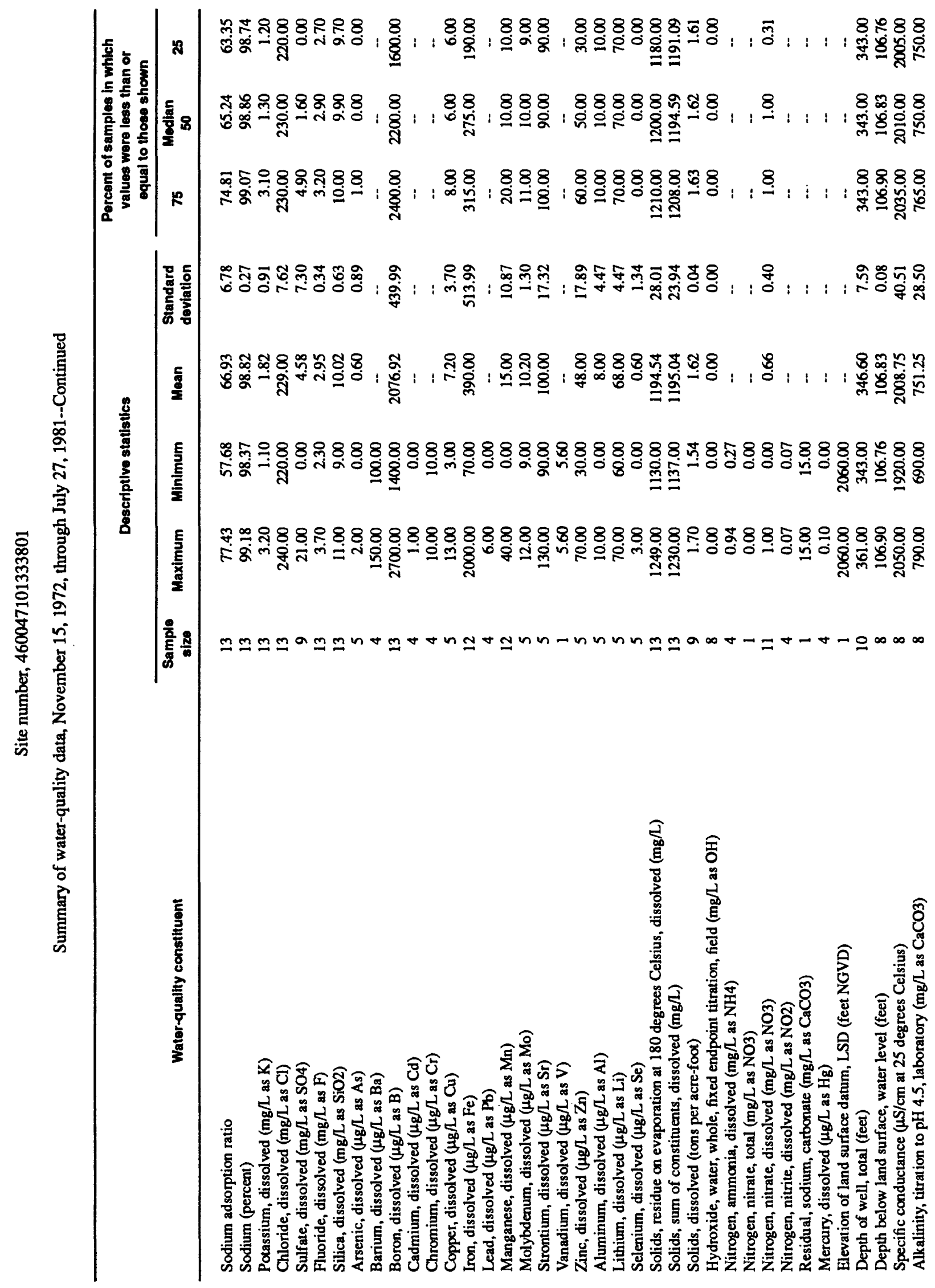




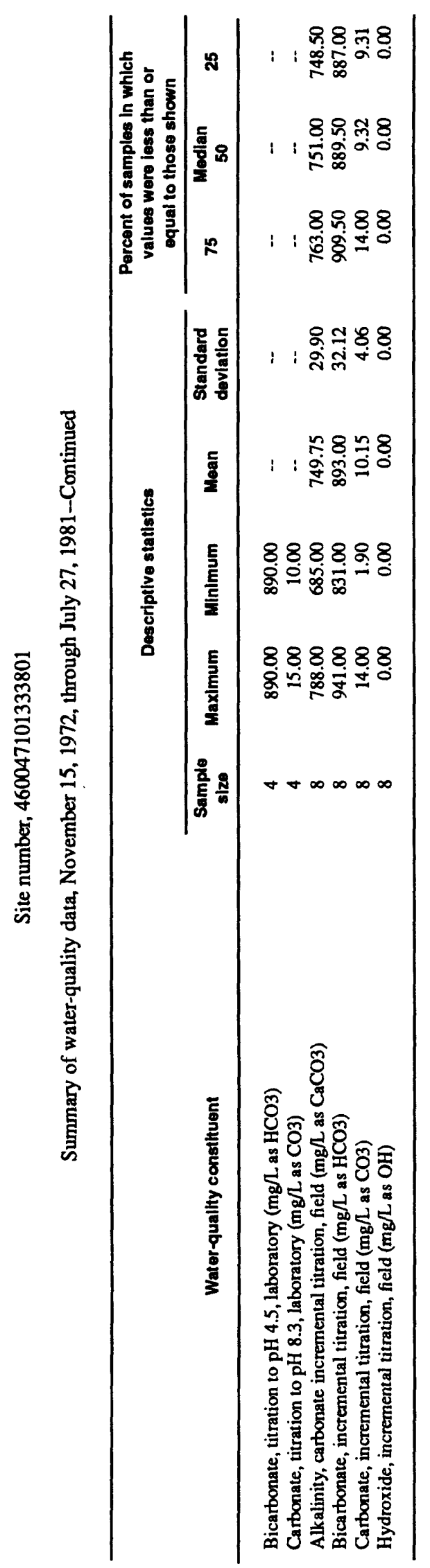




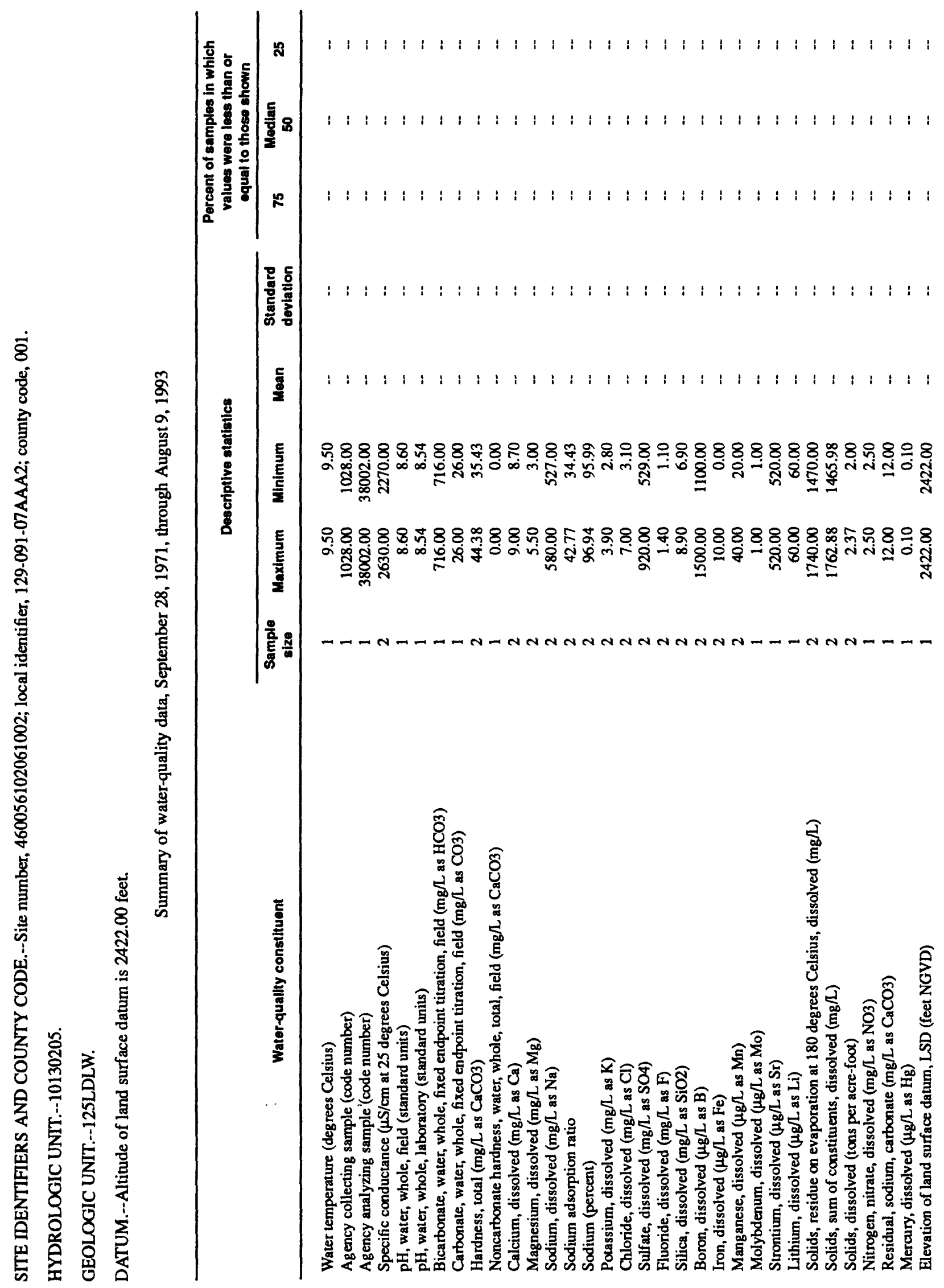




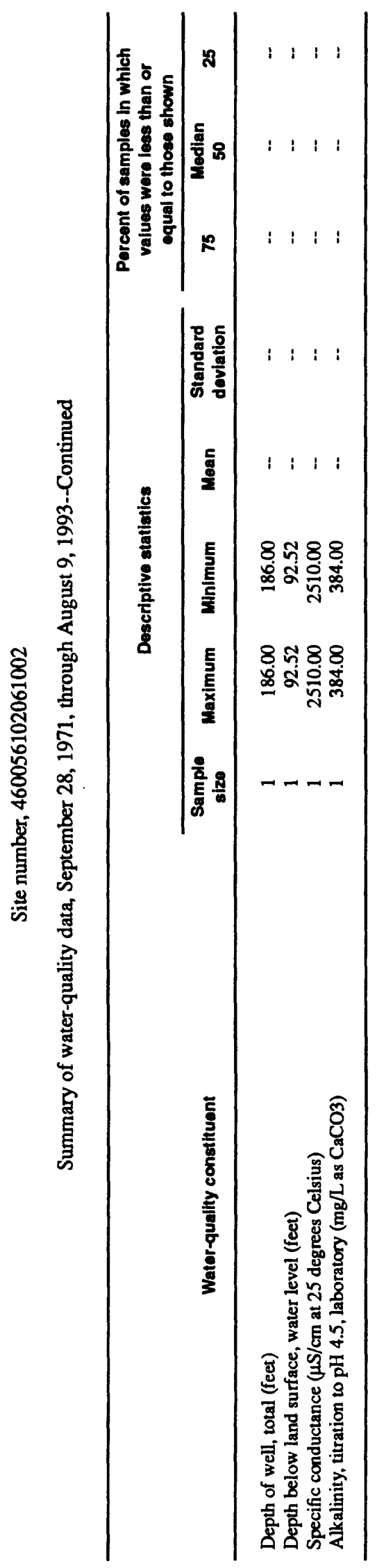




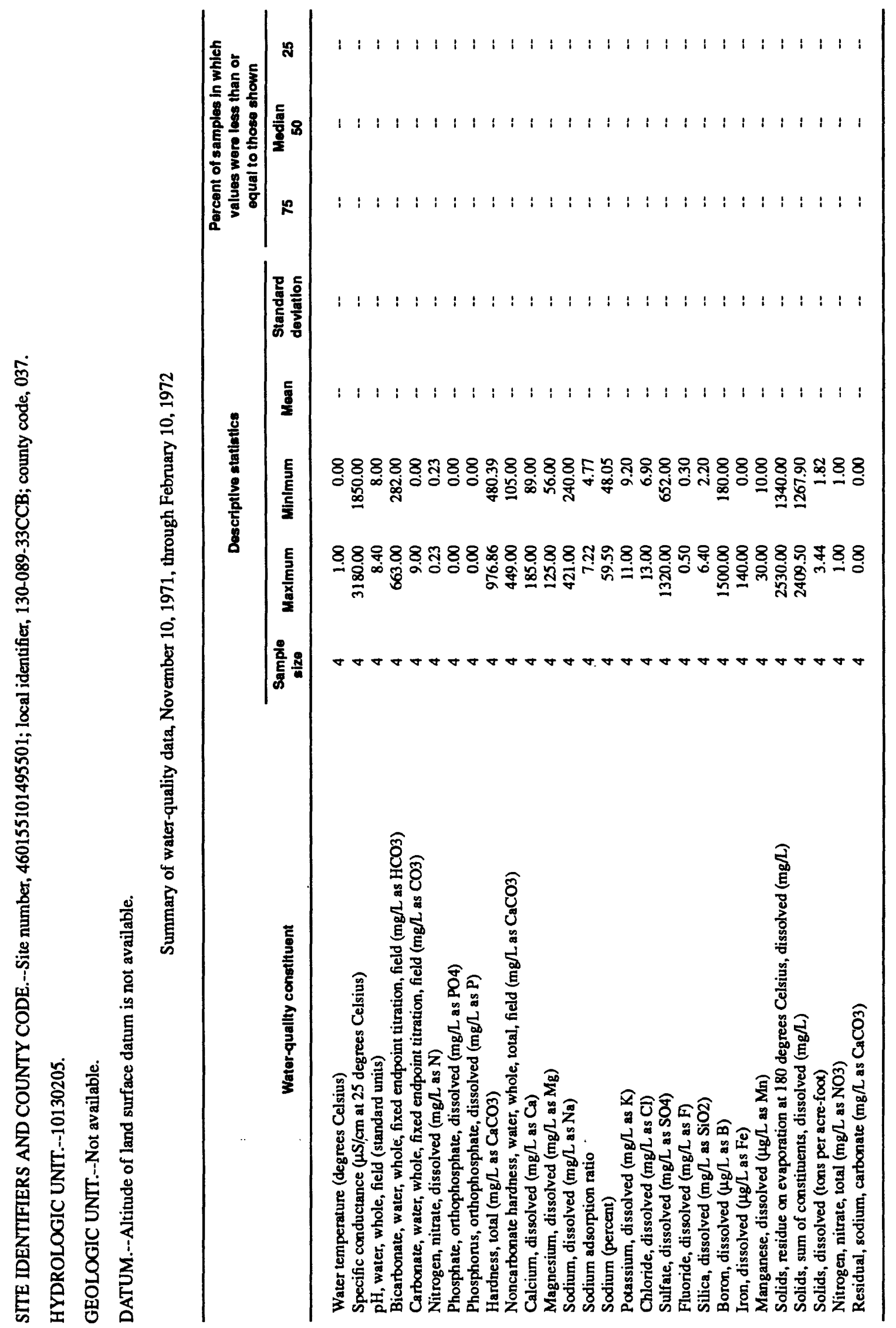




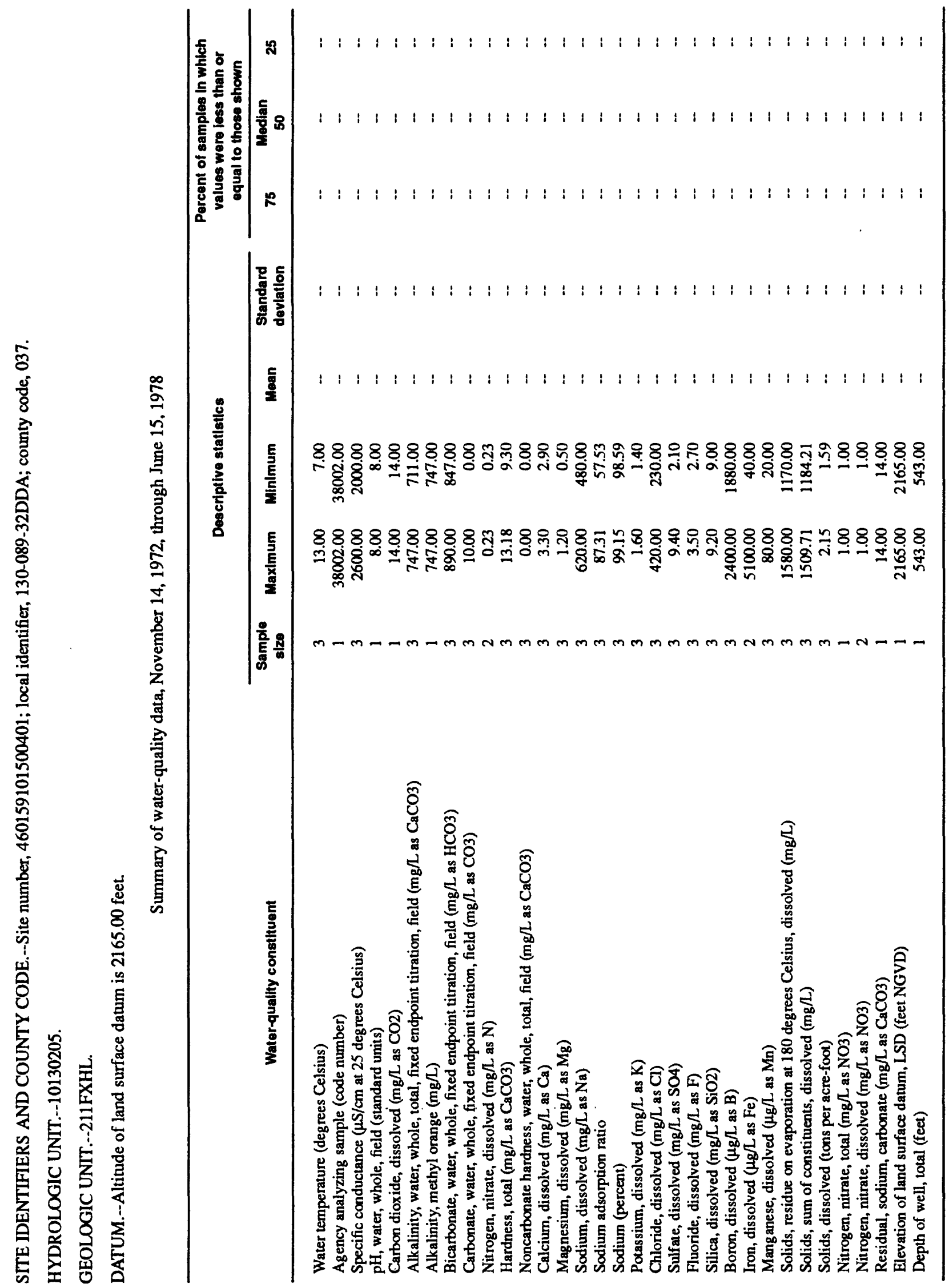




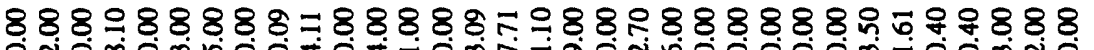

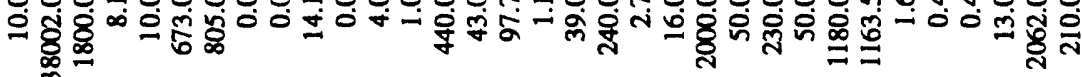

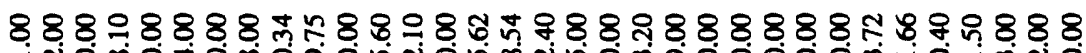
政

ชิ ชิ 要 要窟

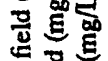
จ

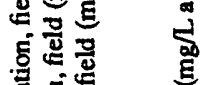

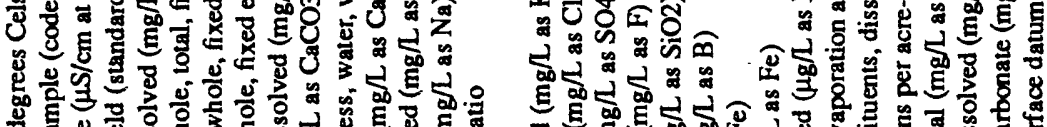

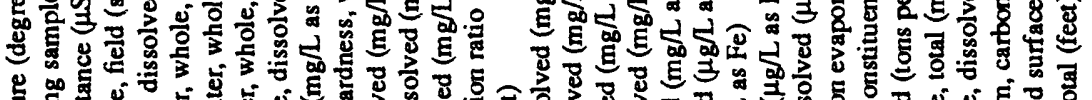

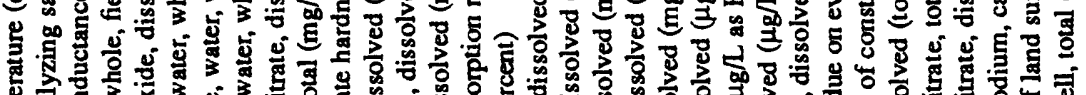

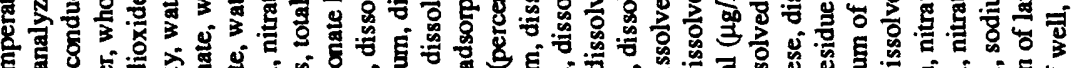
可

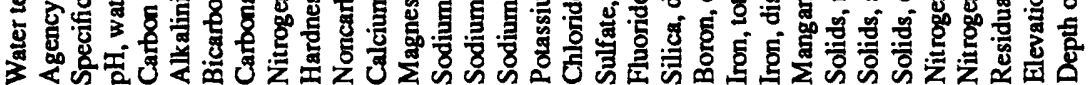




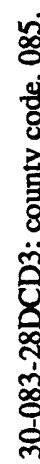

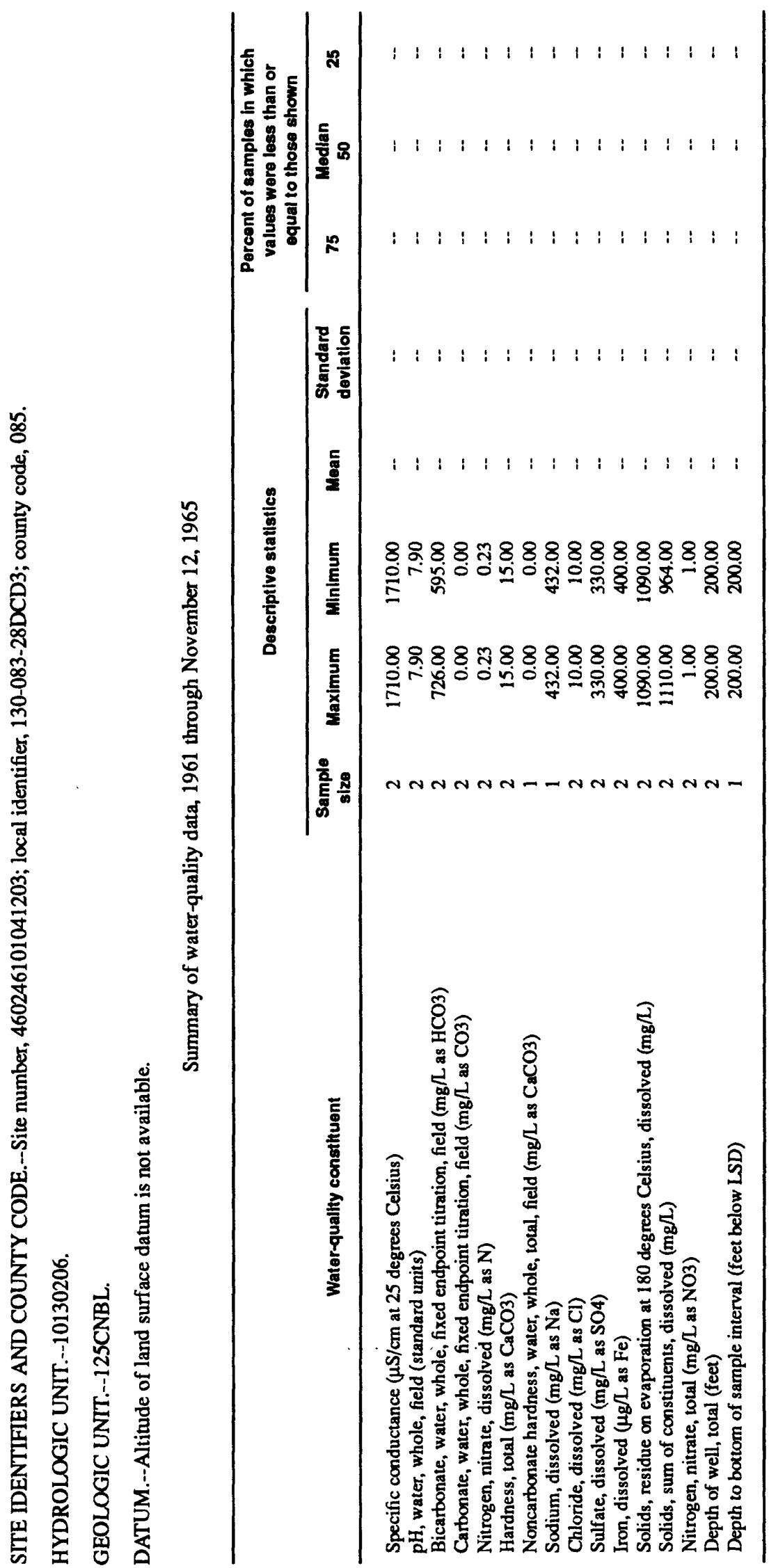




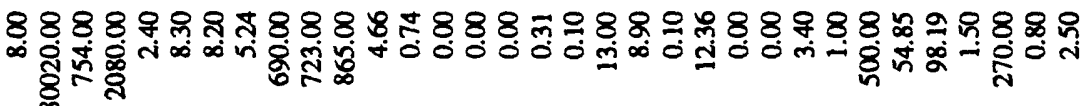
88 8 8 \& 通造

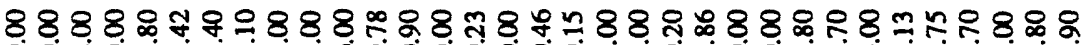

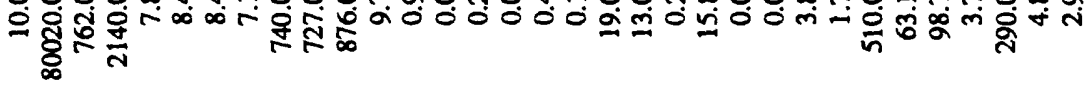

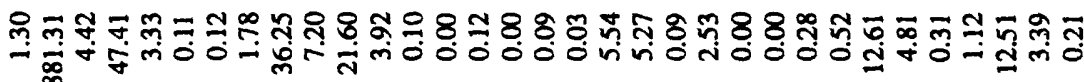
$\stackrel{\infty}{\check{\infty}}$

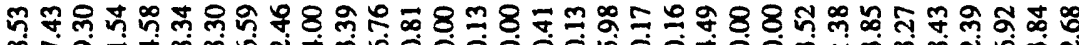

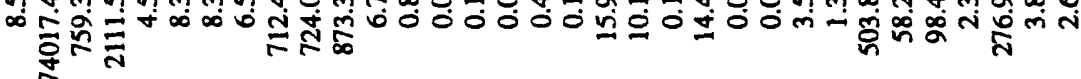

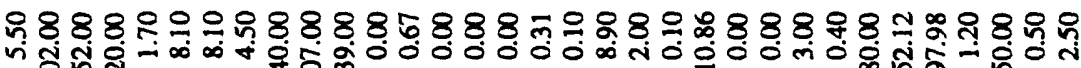

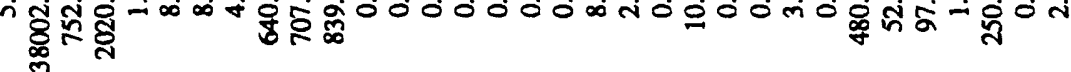

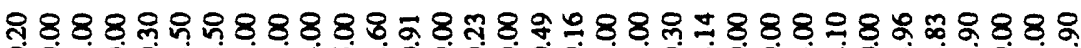
绻

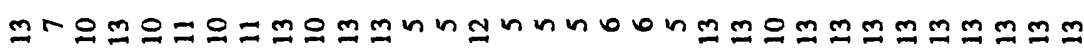

ช్

ริ $\stackrel{2}{3}$

兽

ชิ

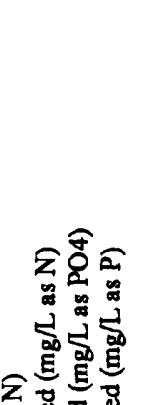

ธิช

ธิธู

망

ర్요

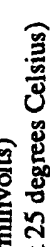

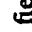

要焉

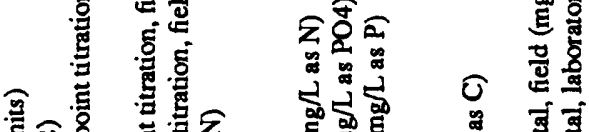

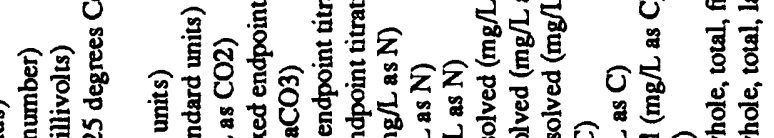

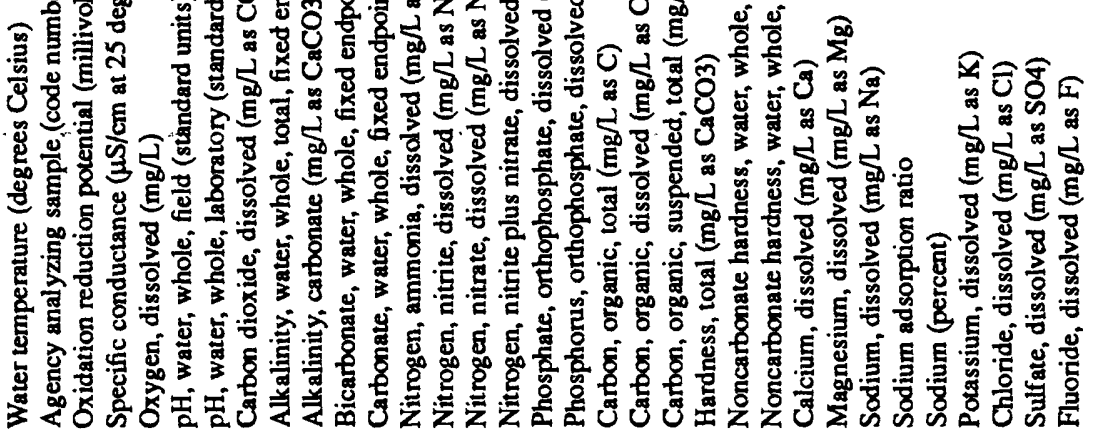




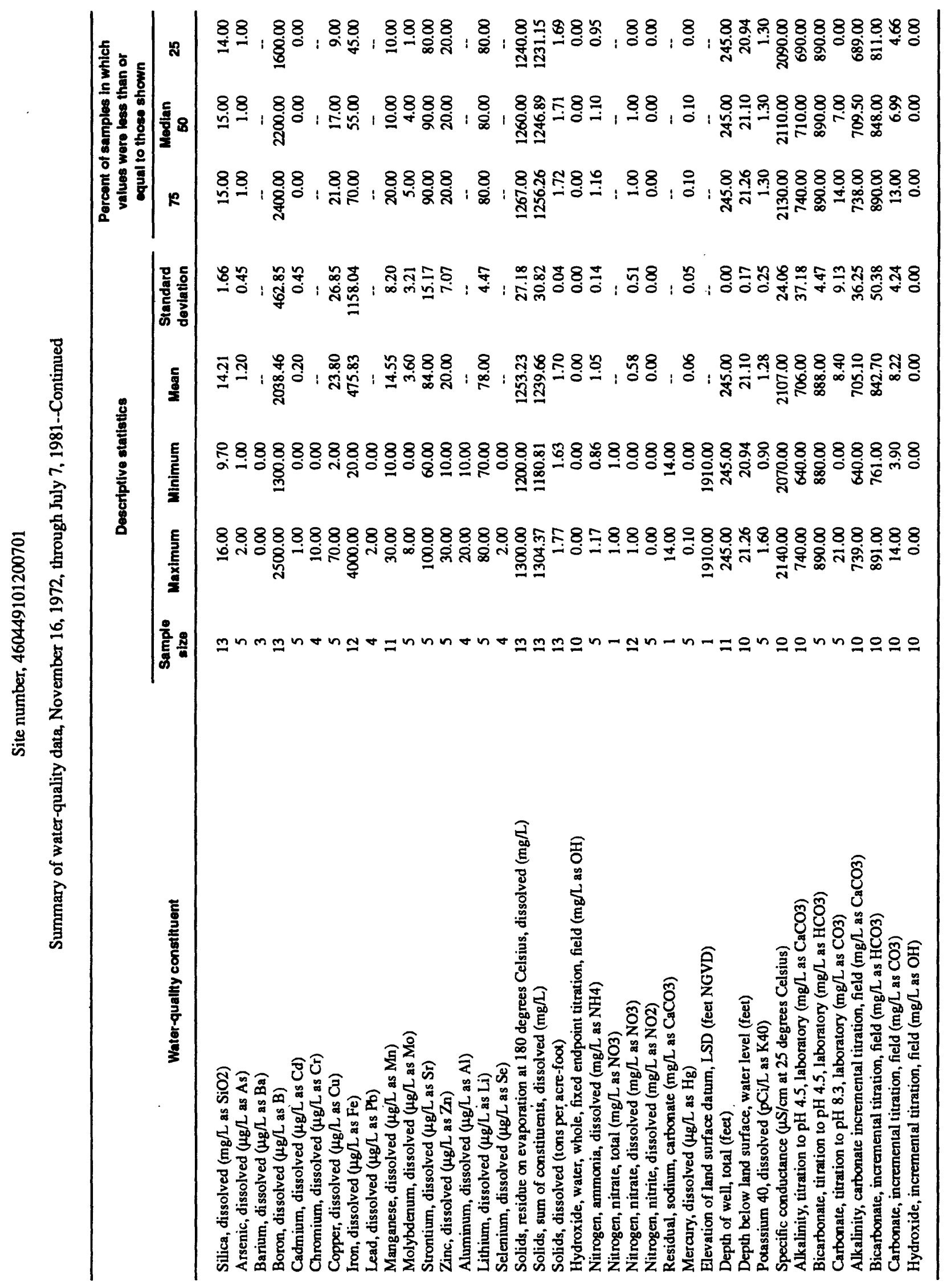




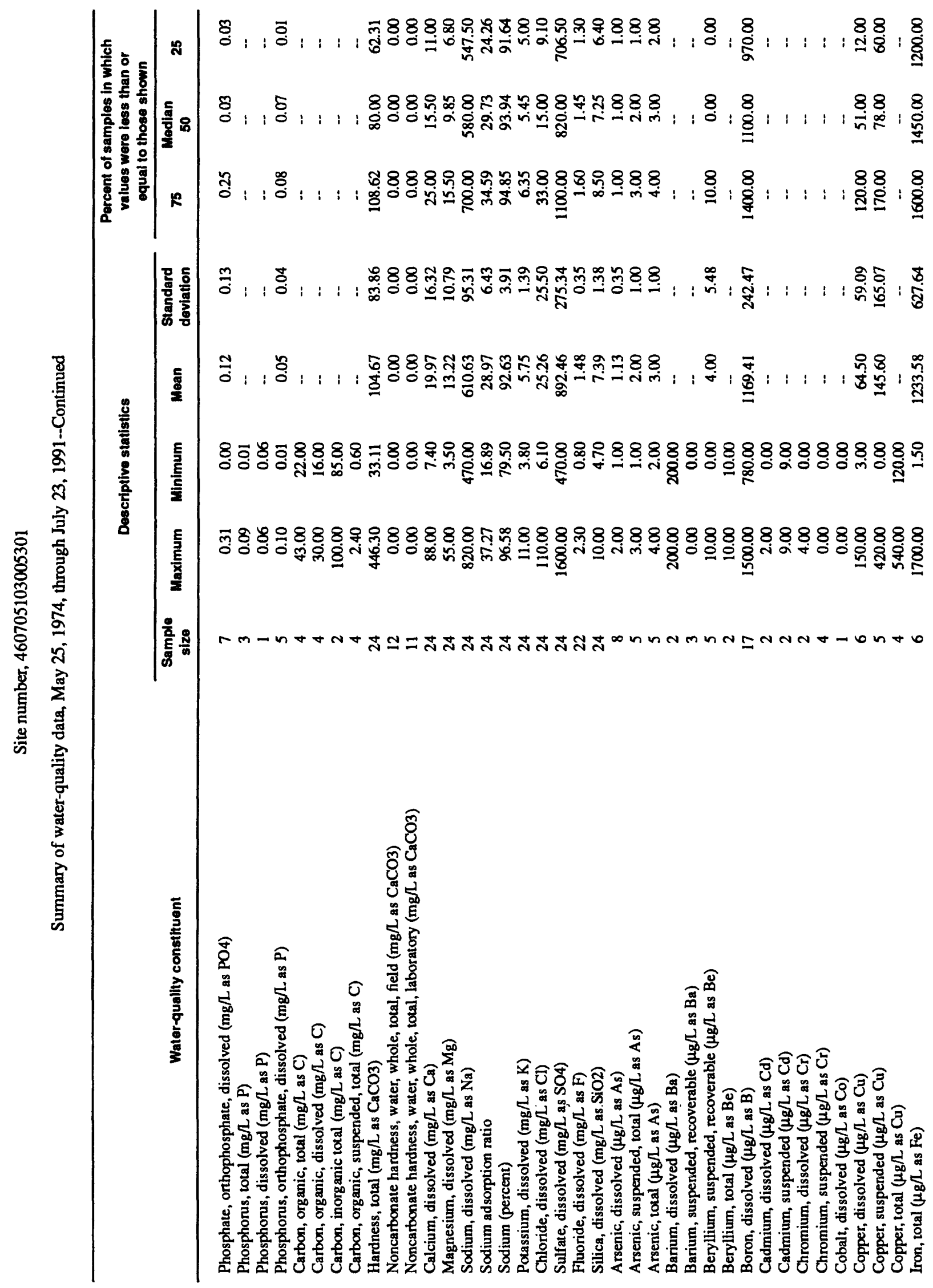




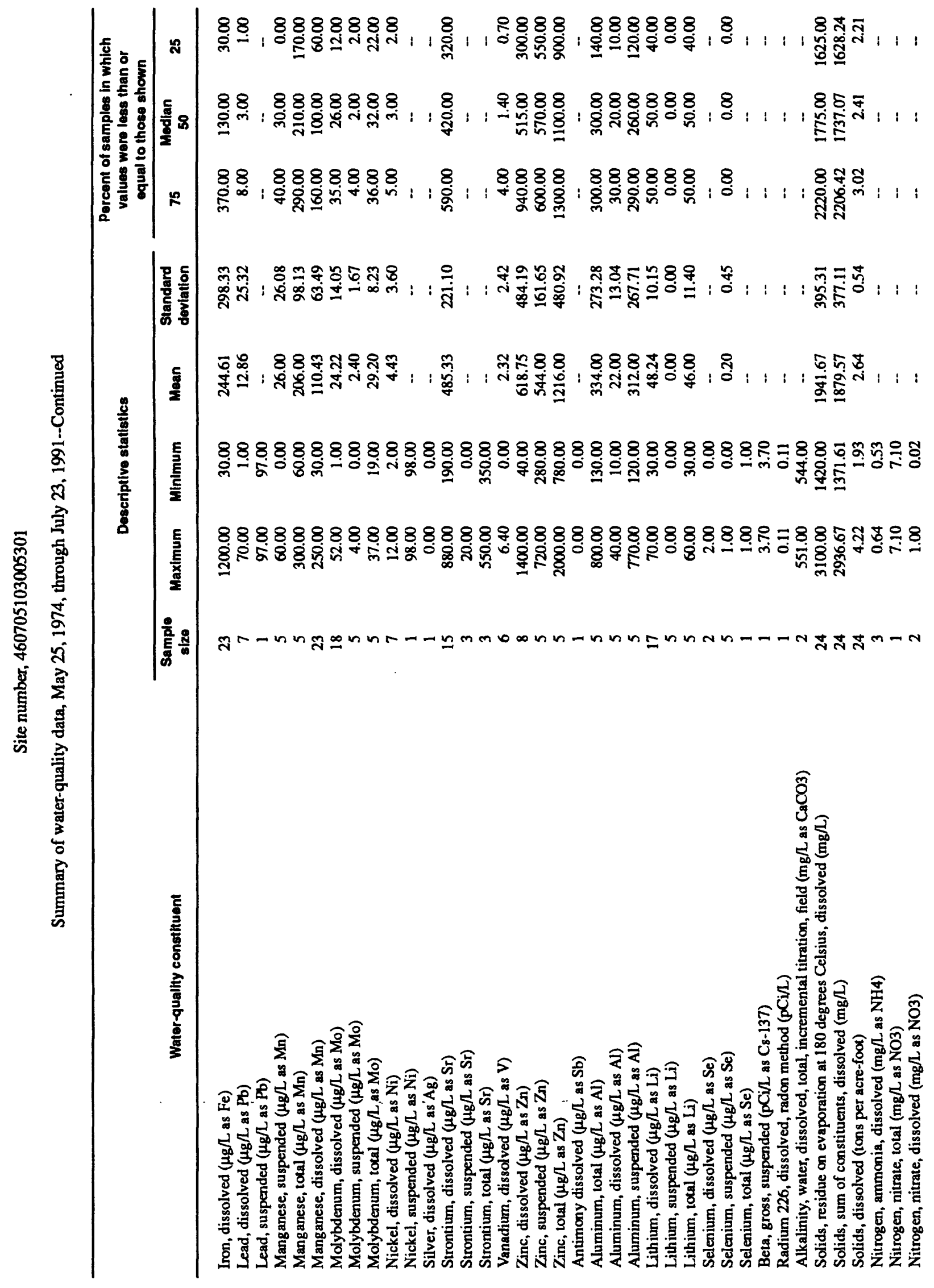




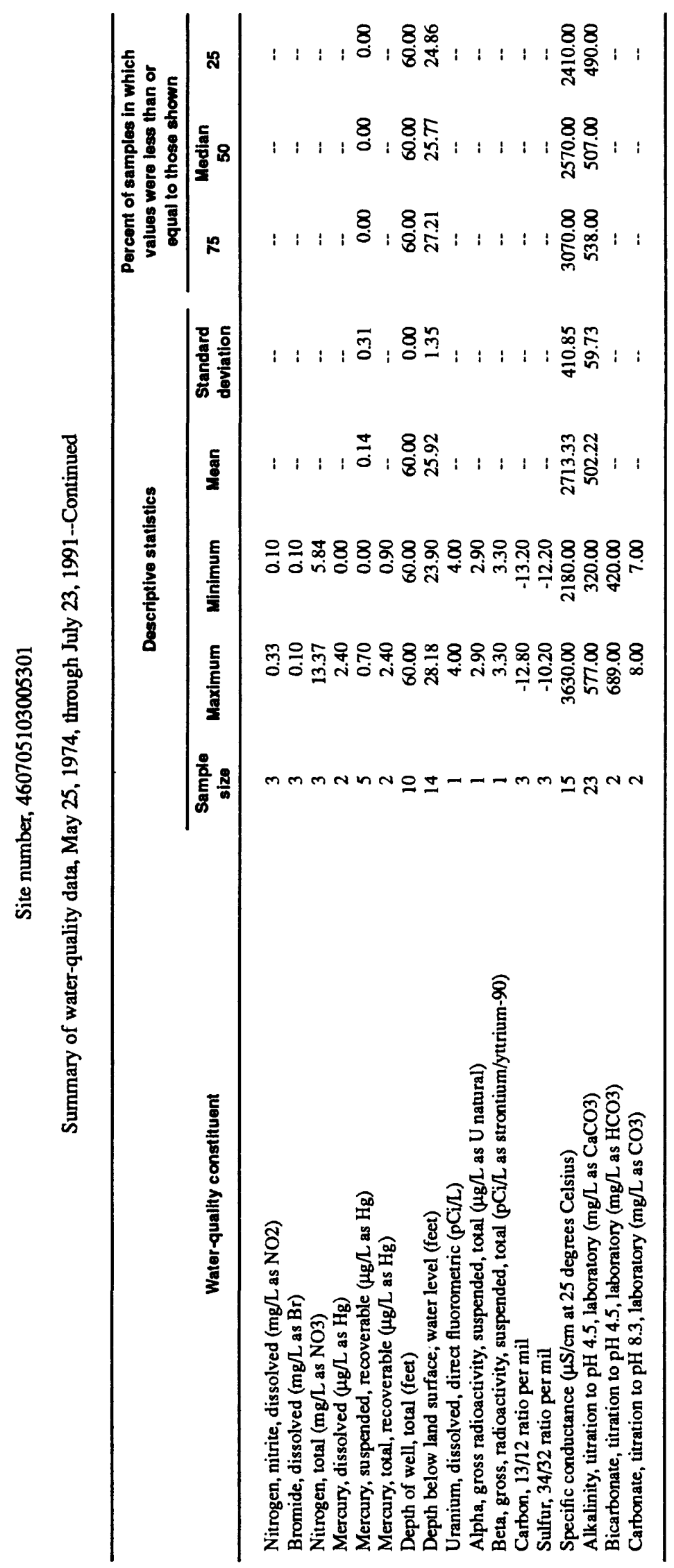




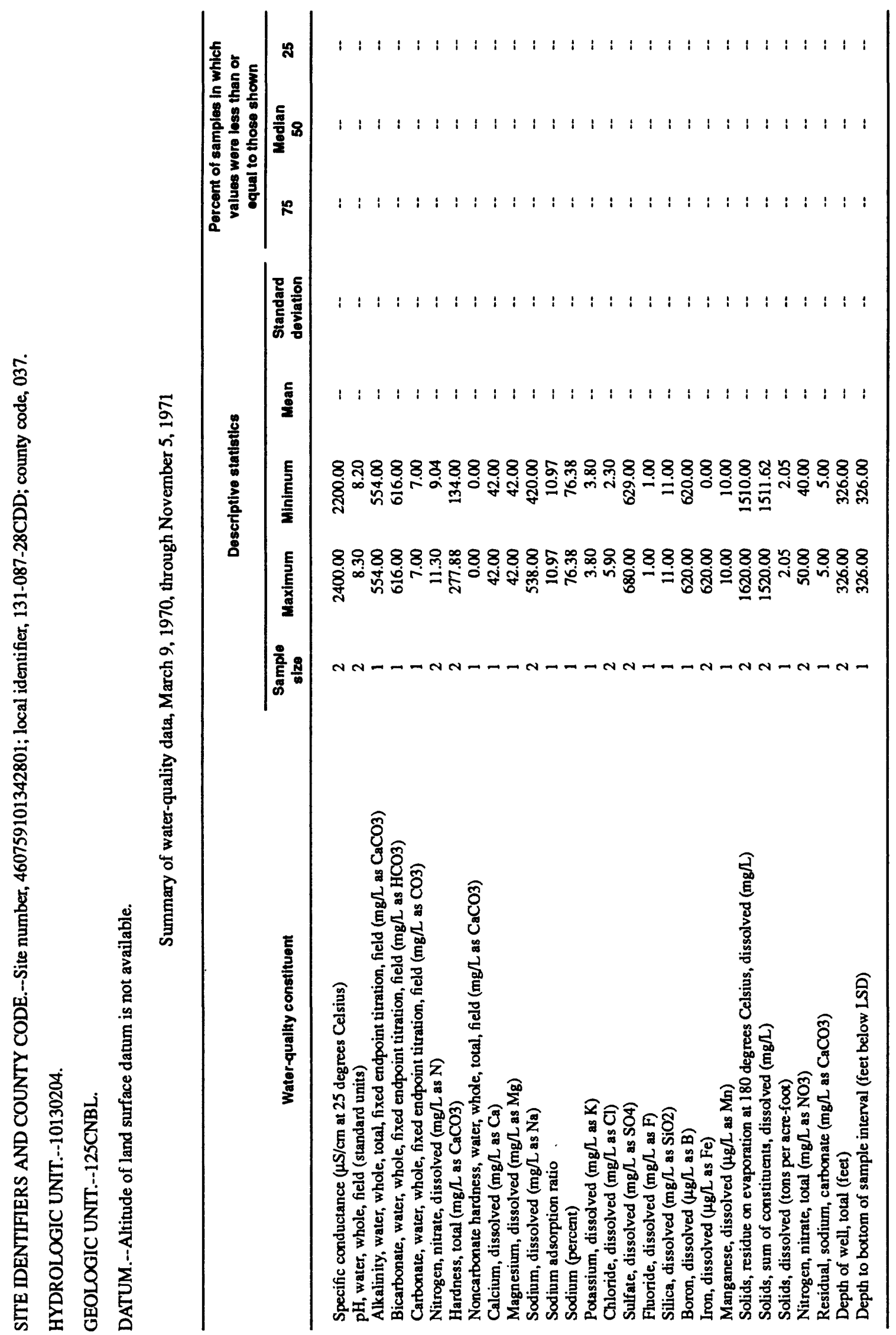




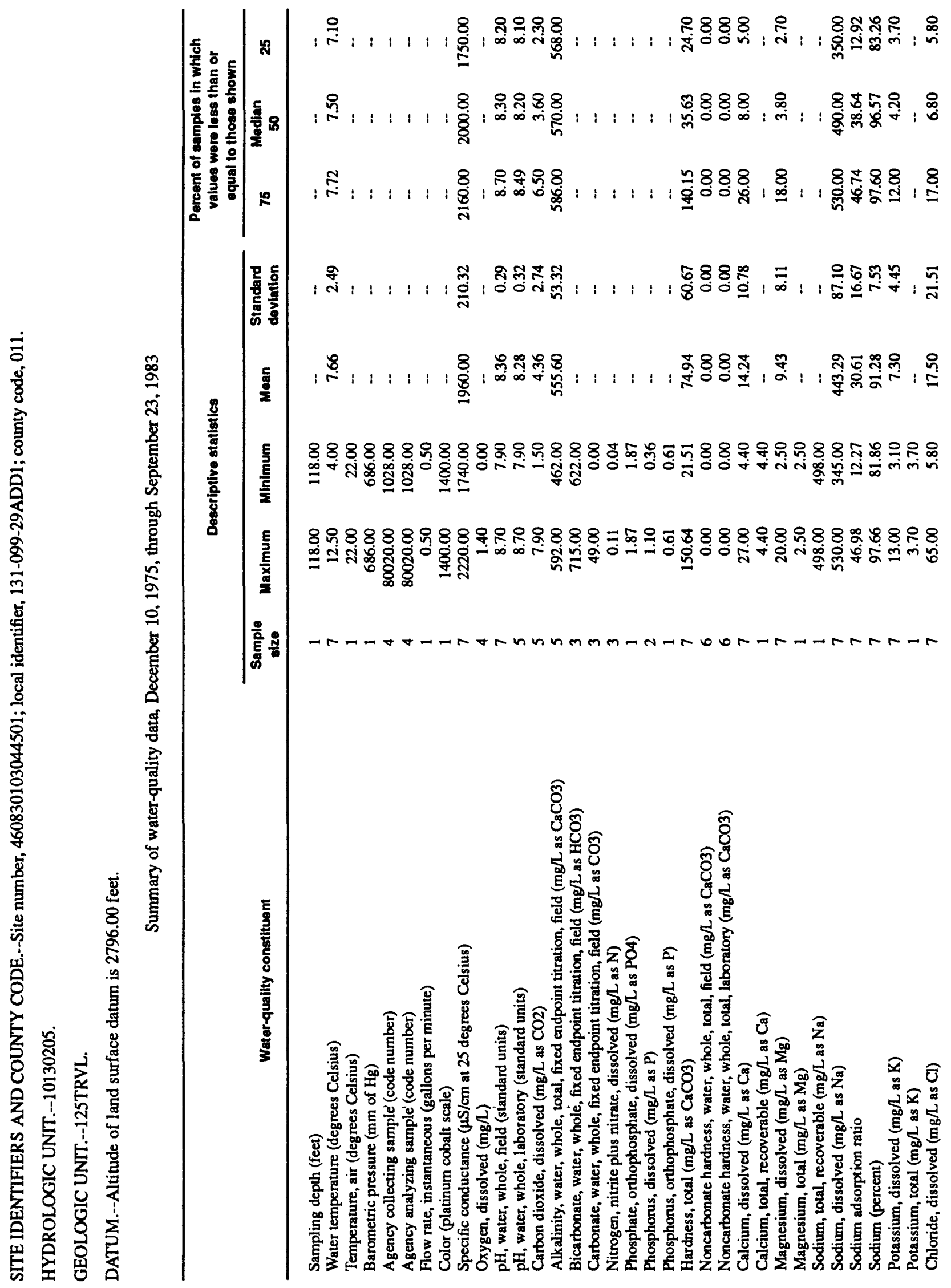




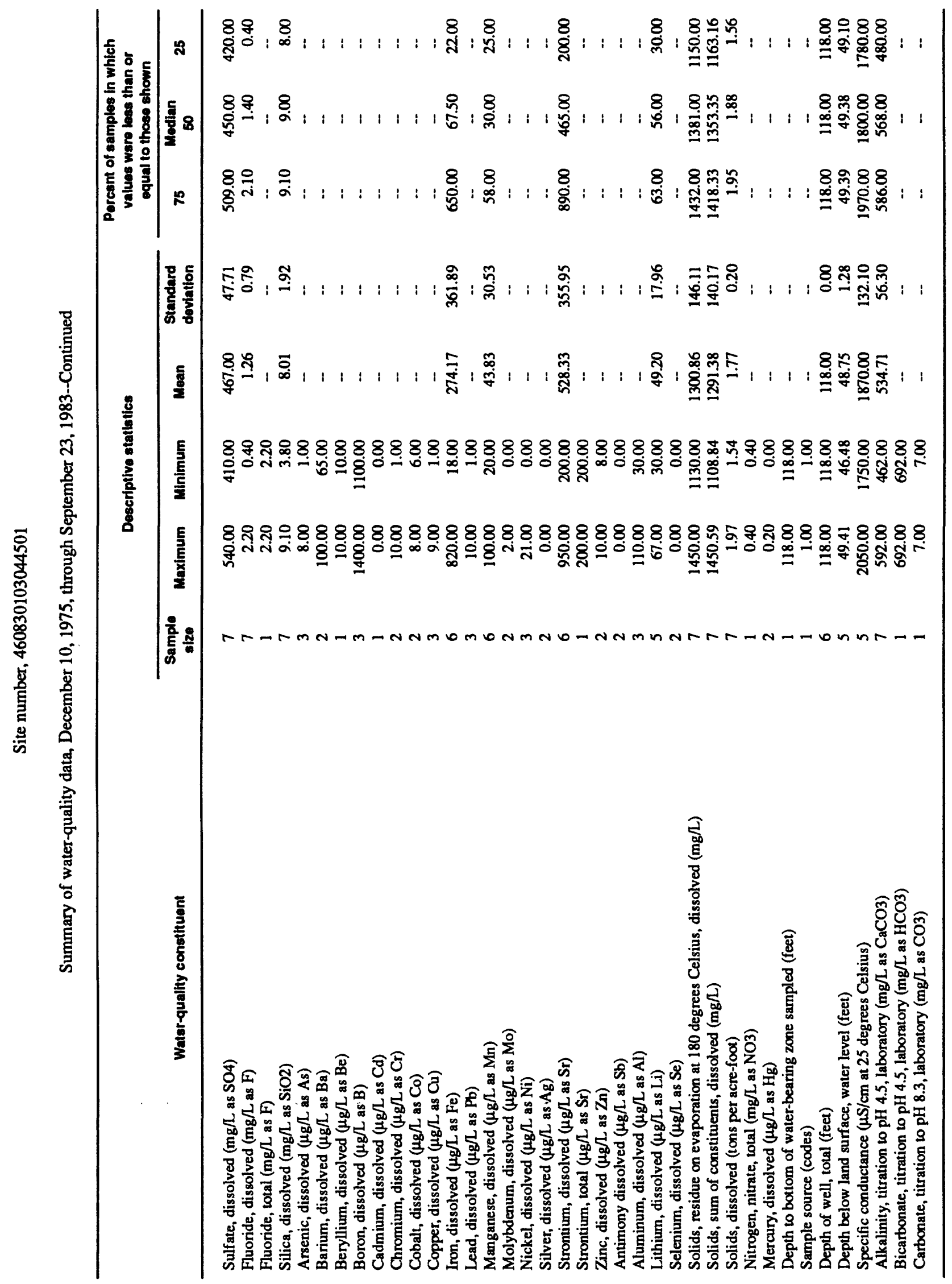




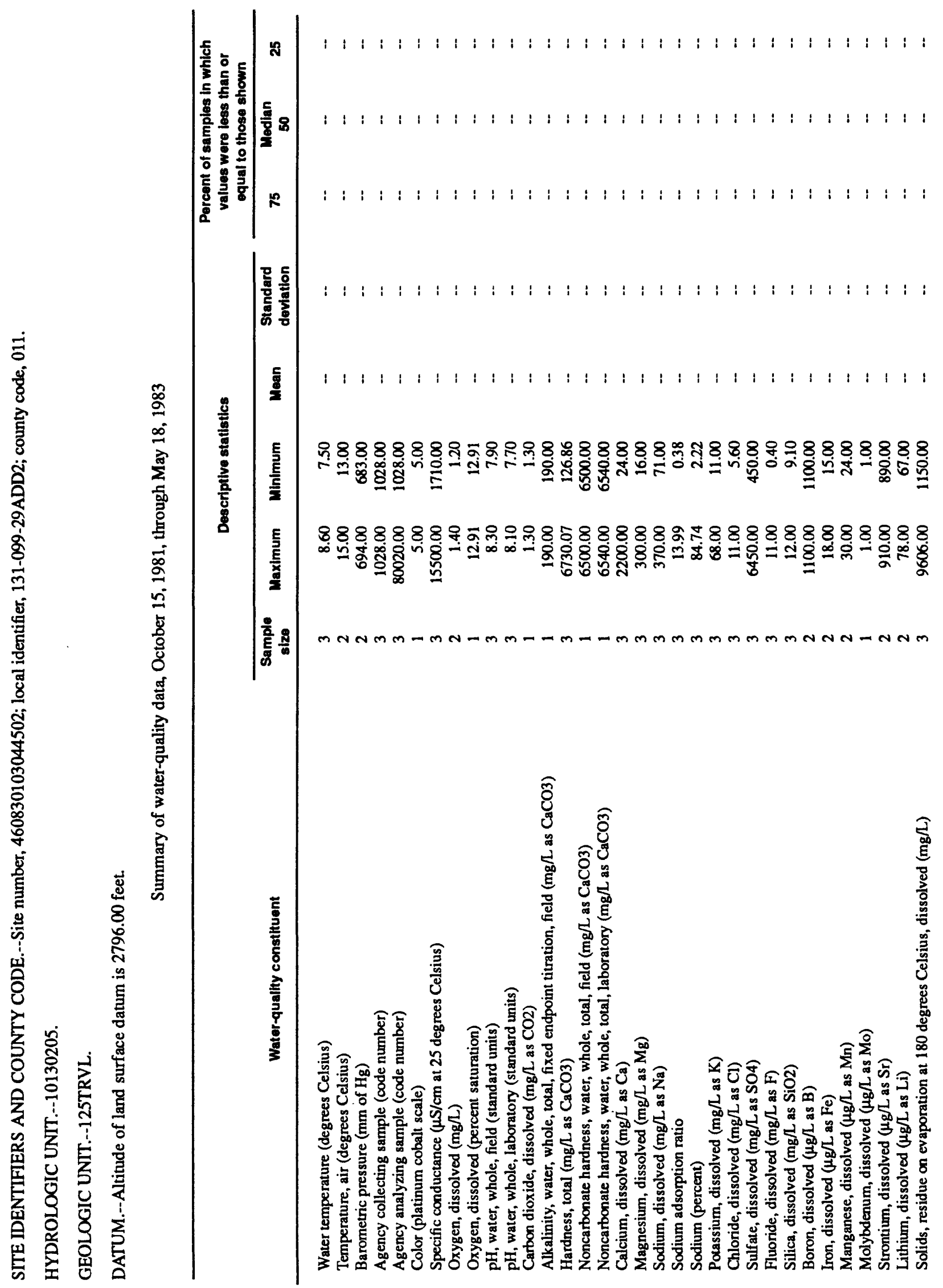




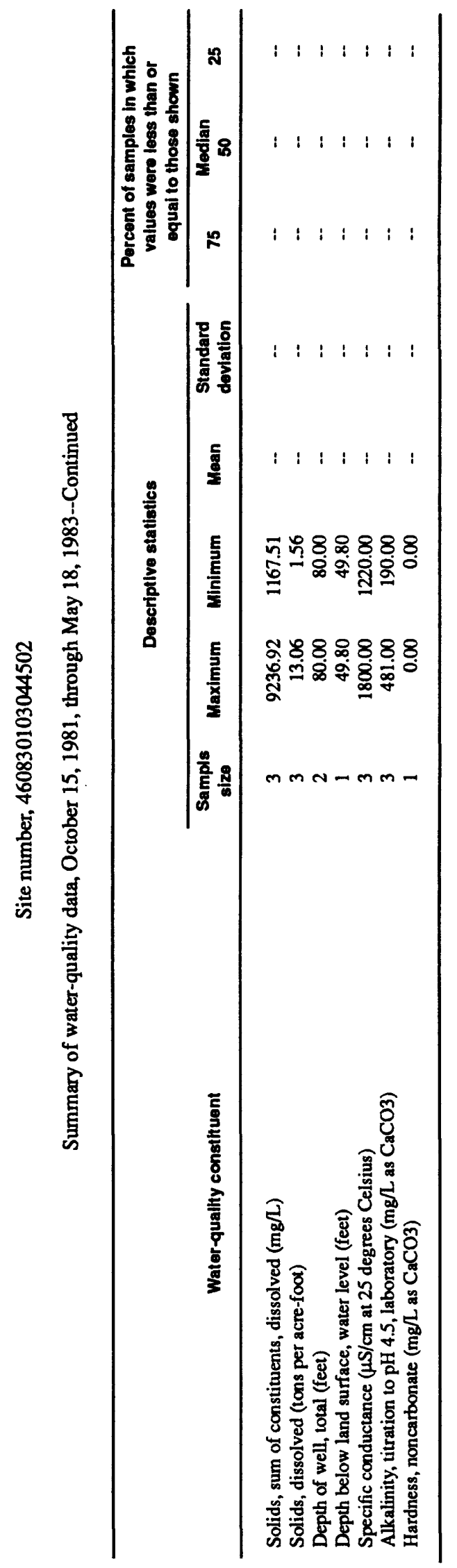




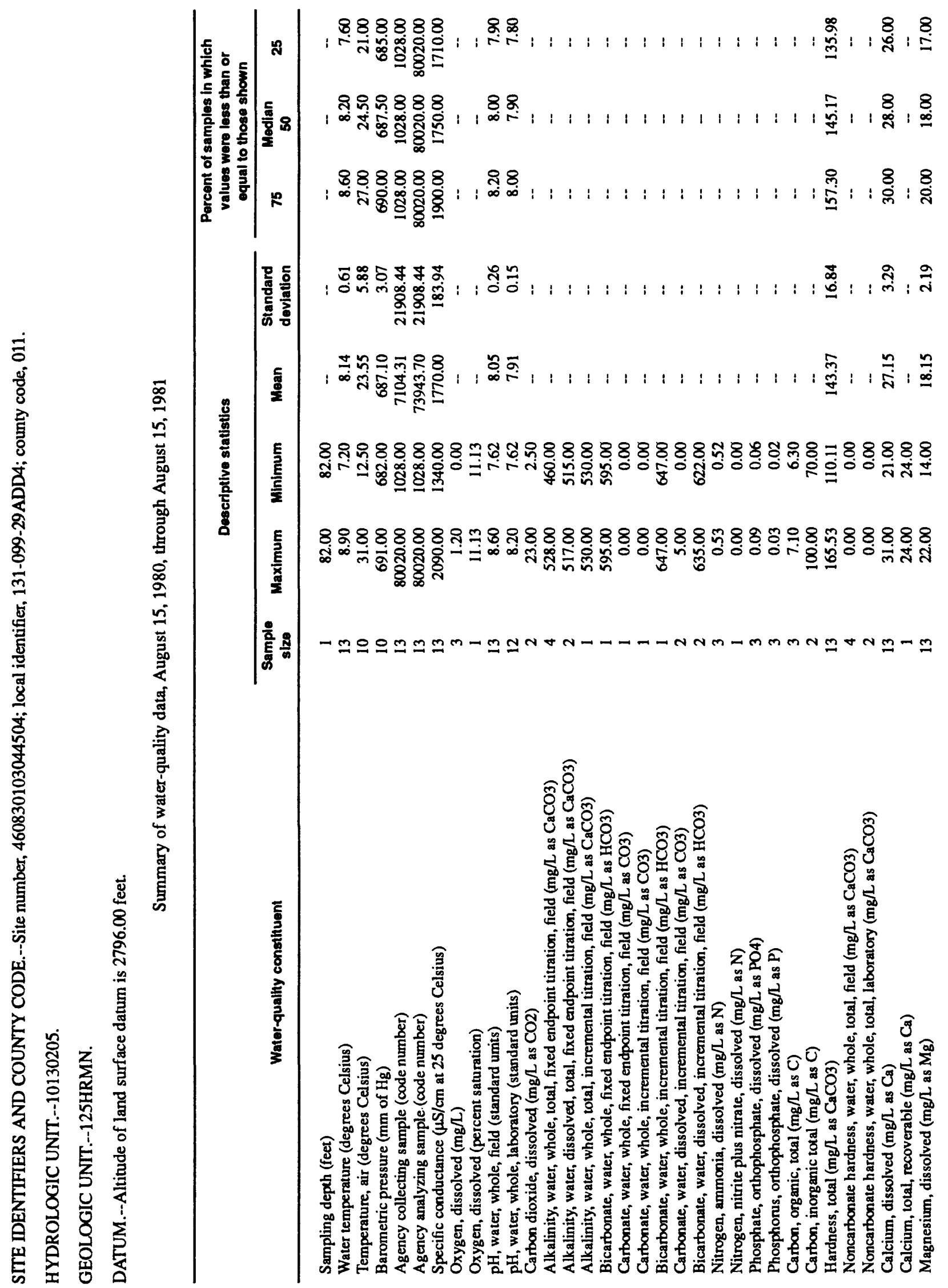




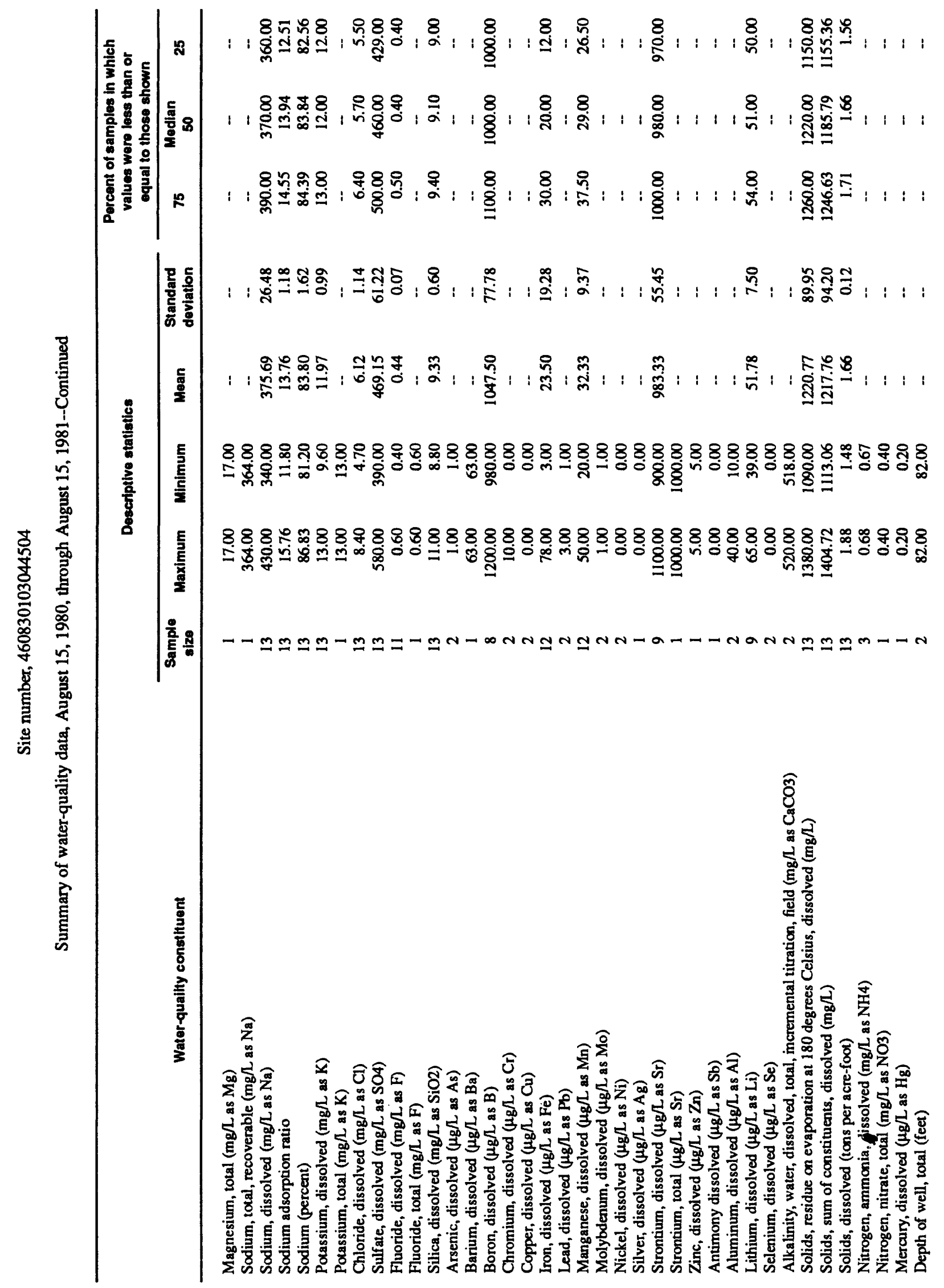




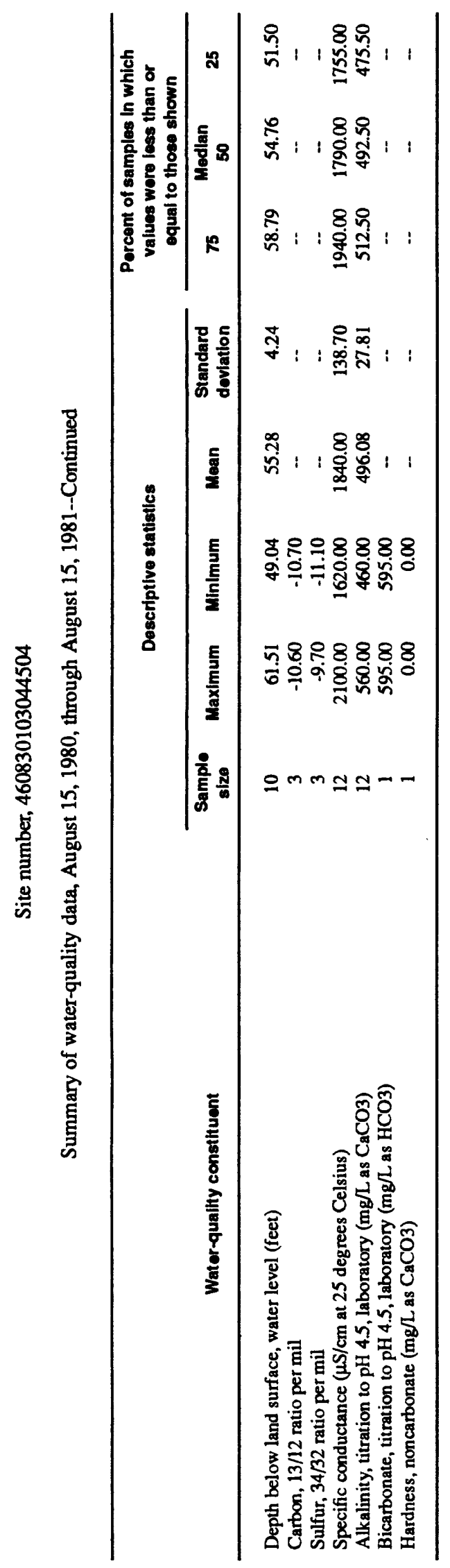




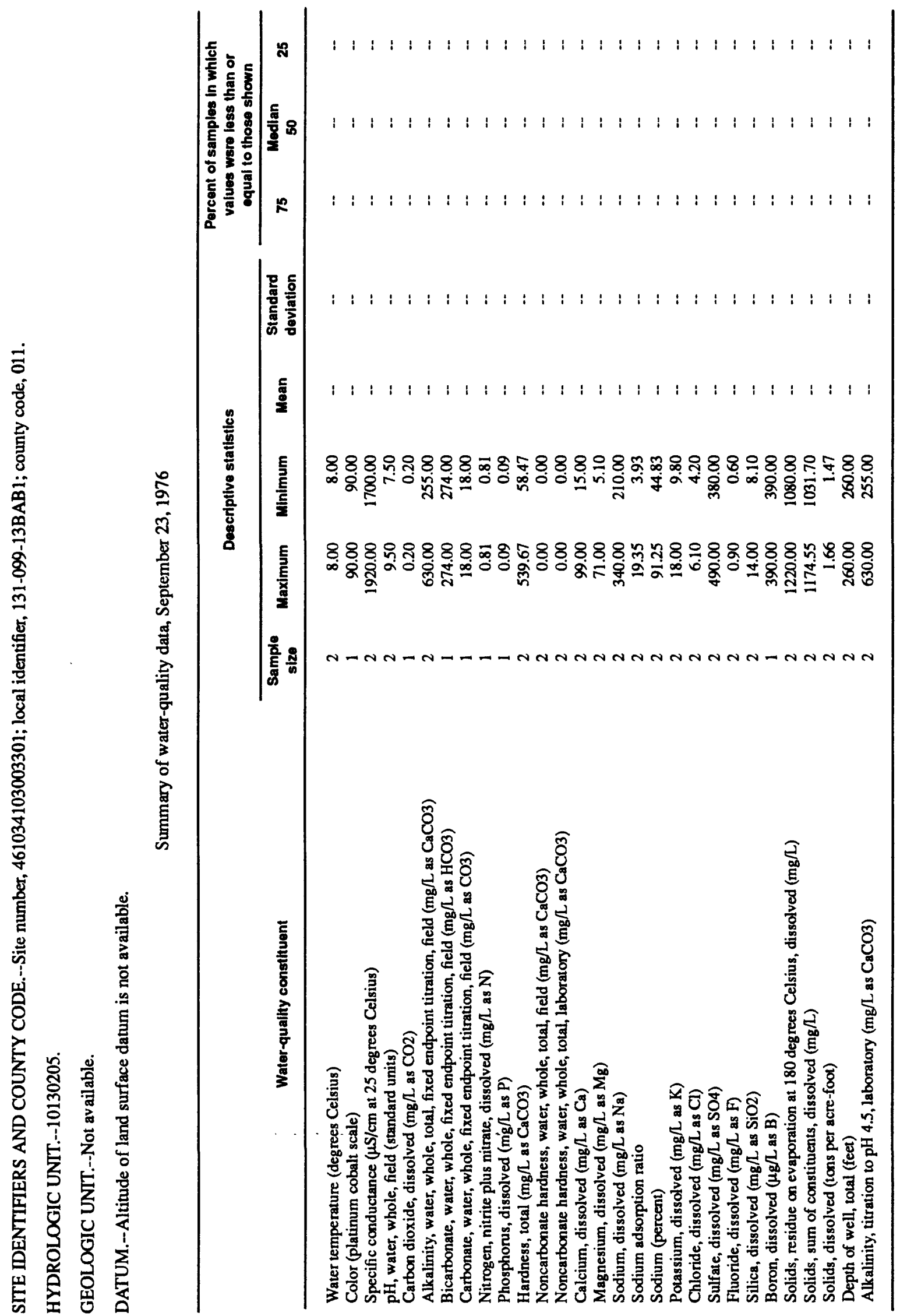




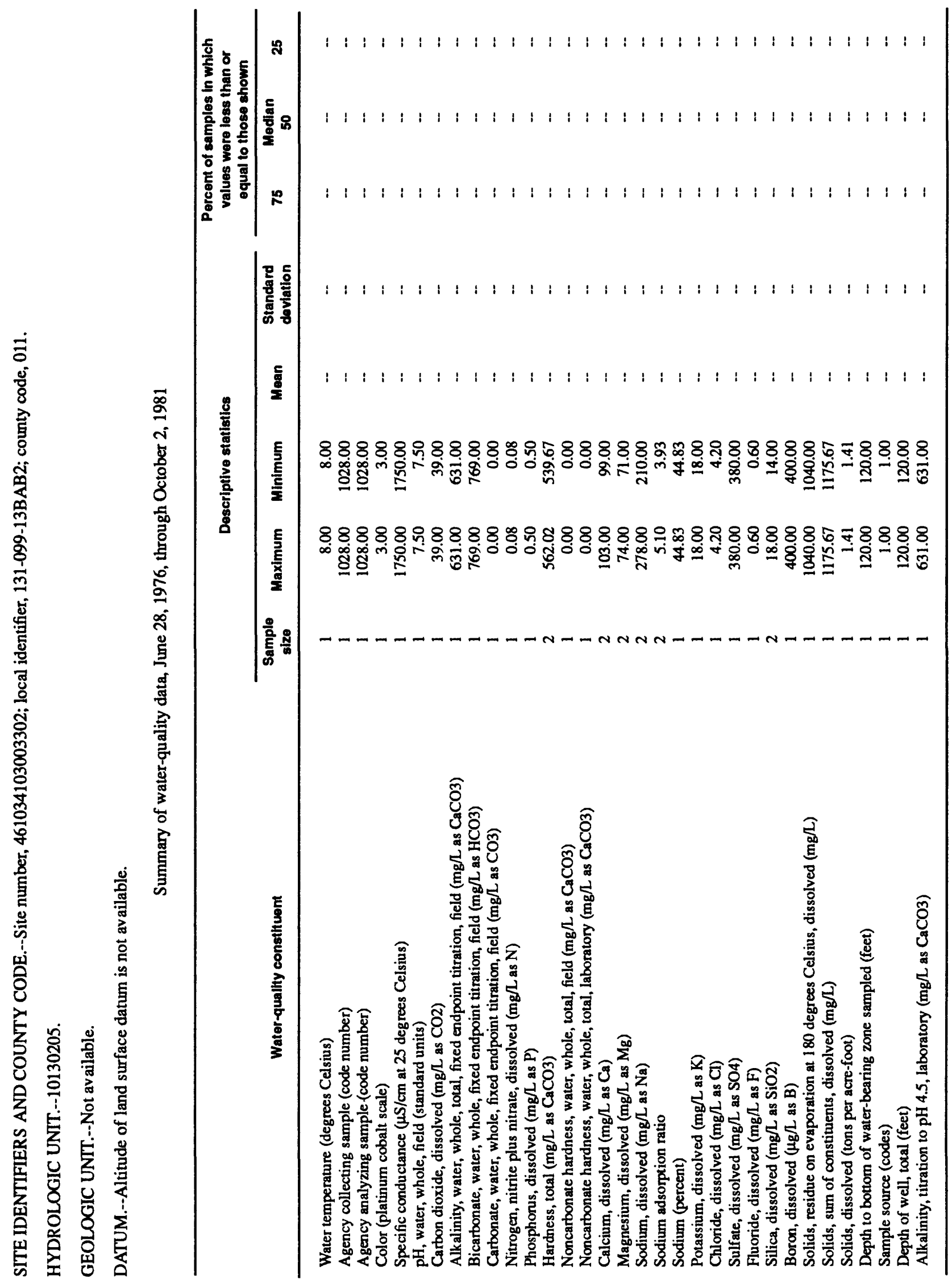




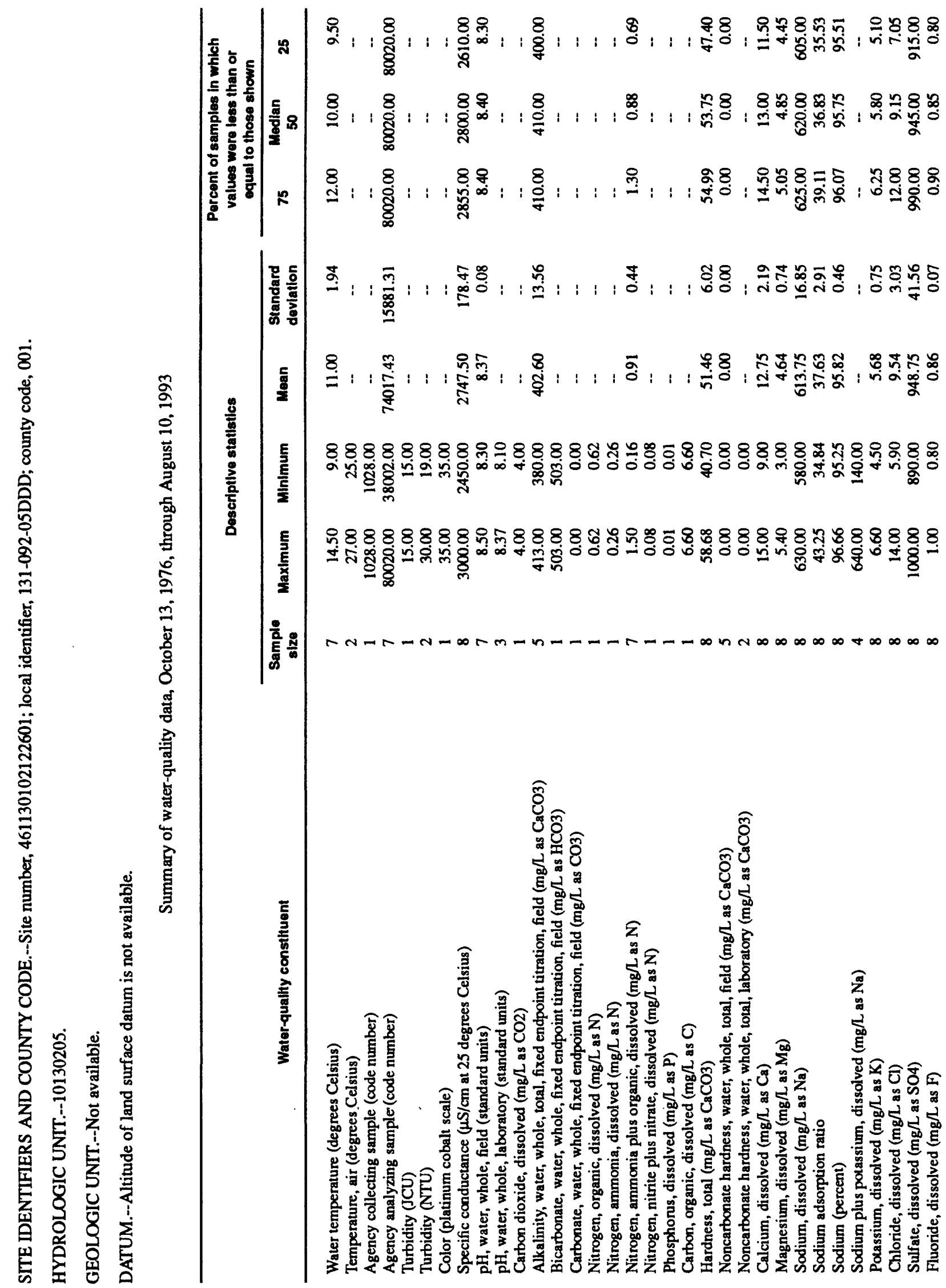




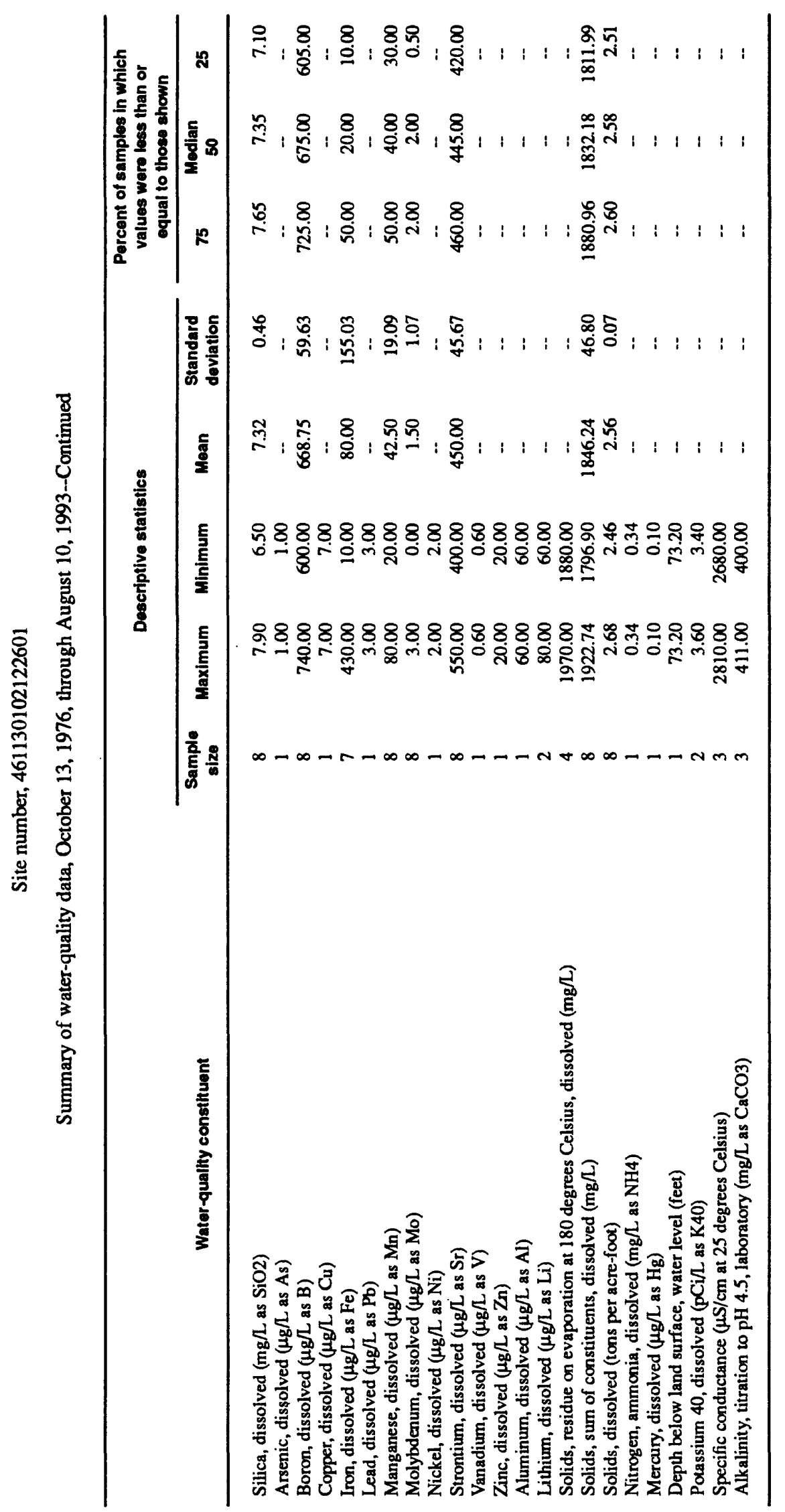




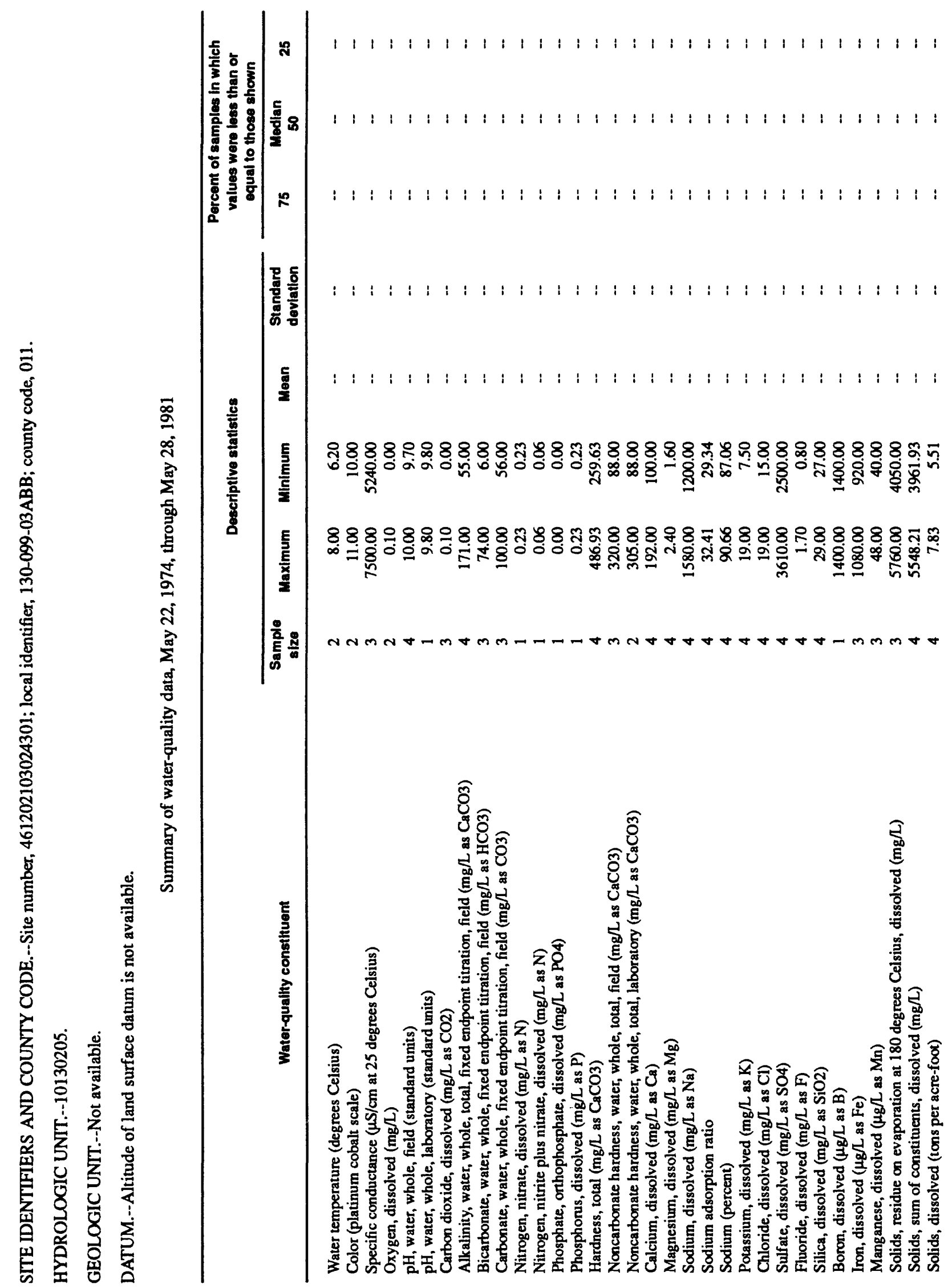




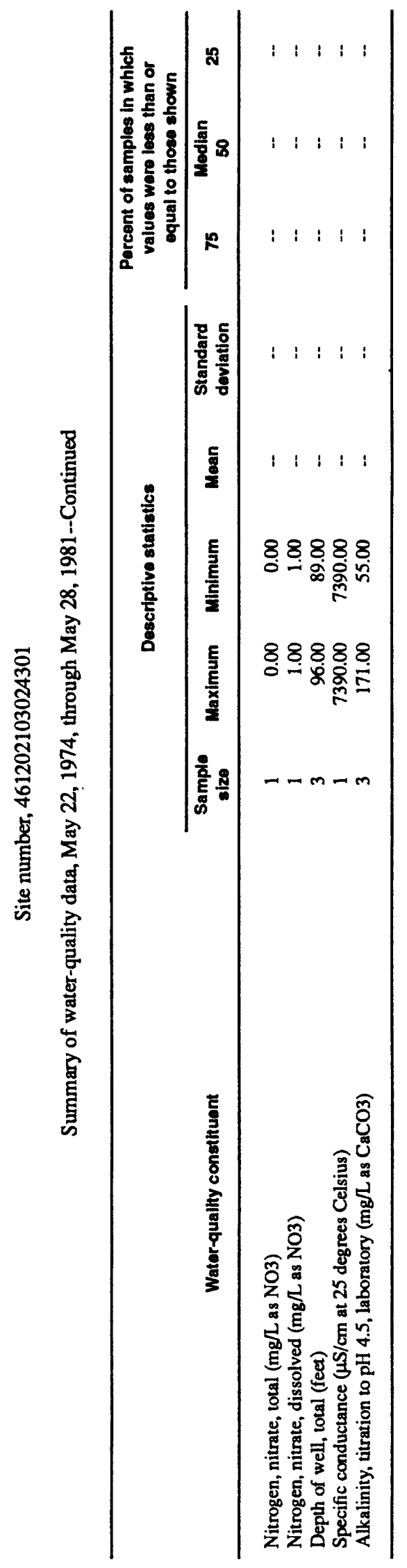




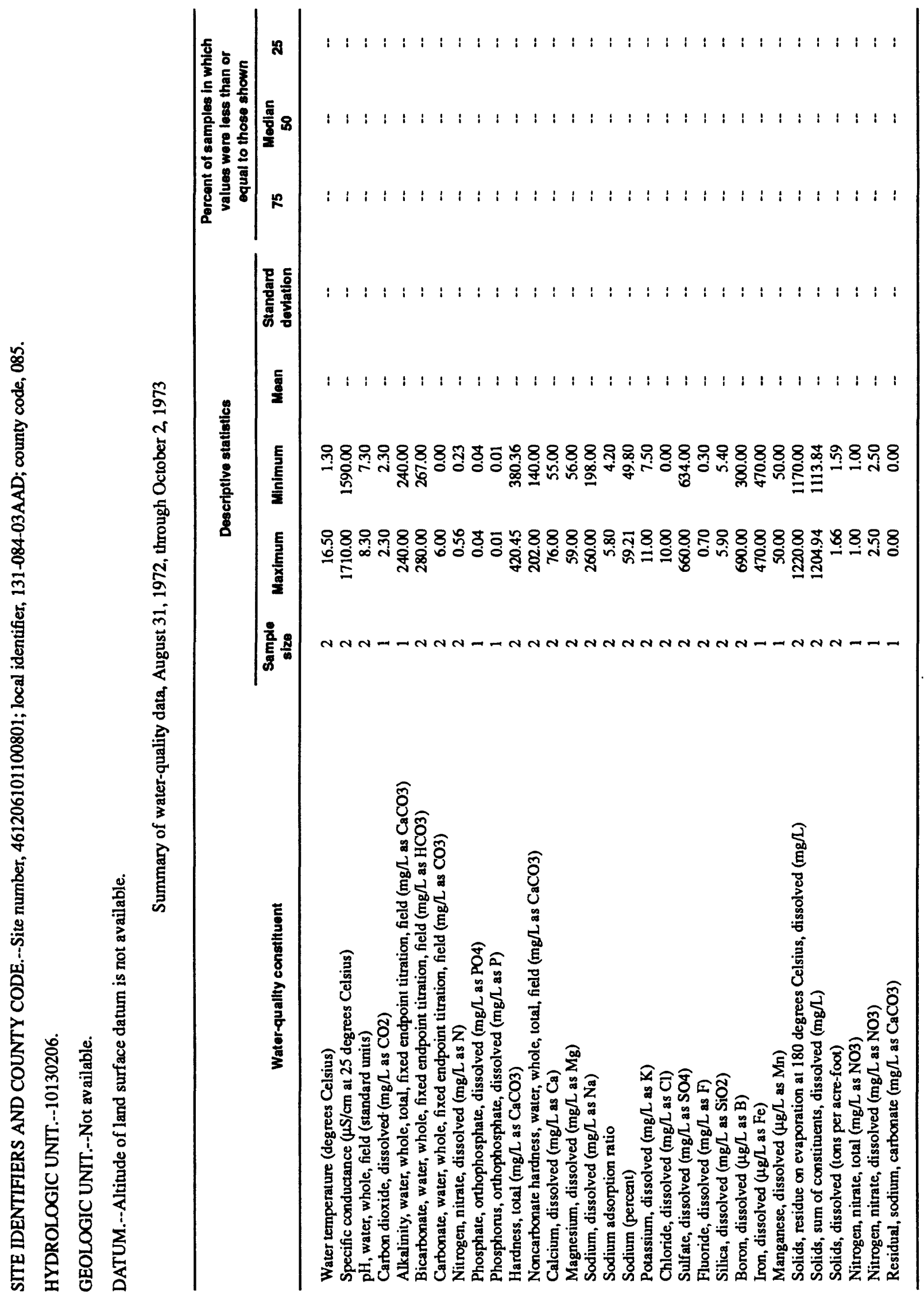




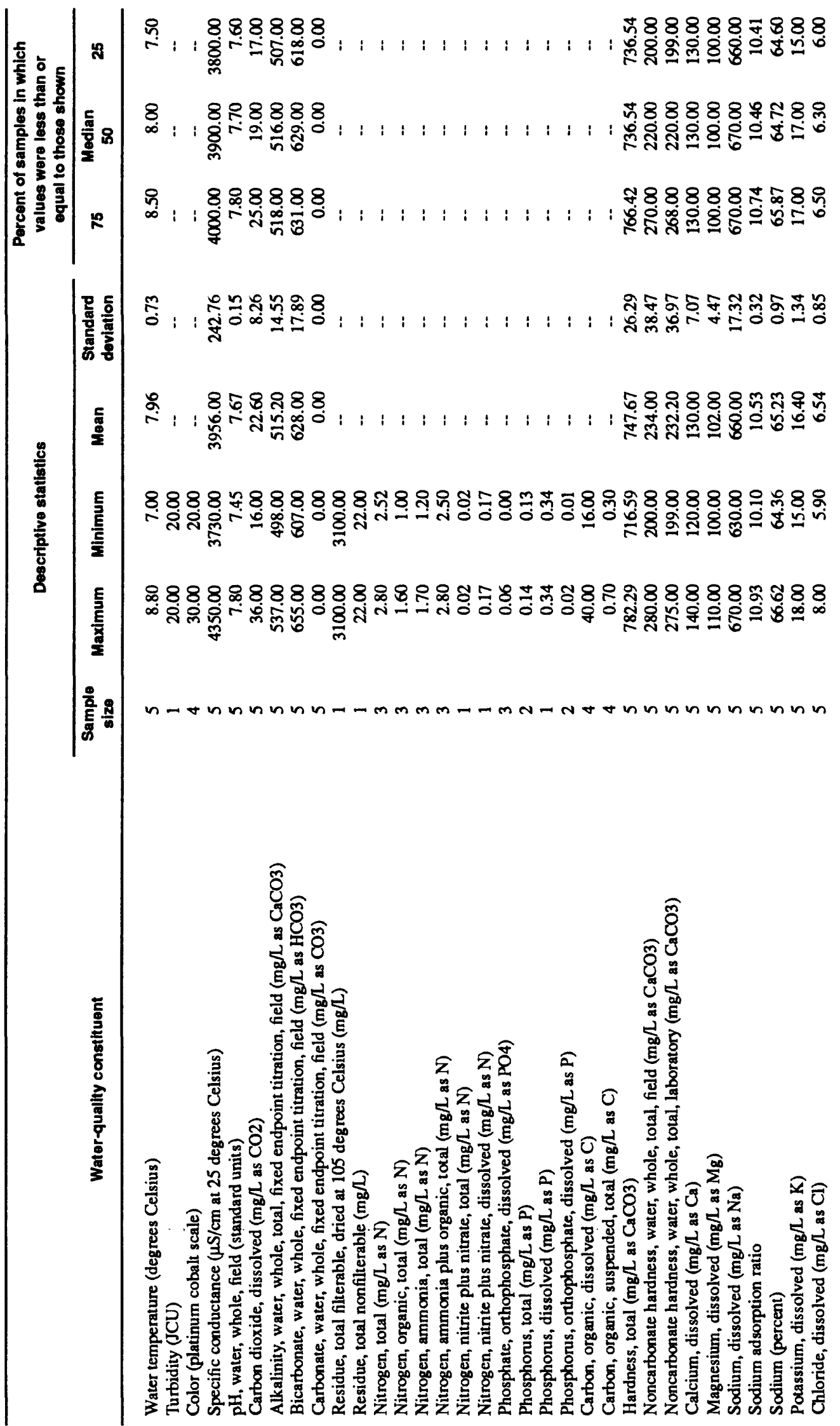




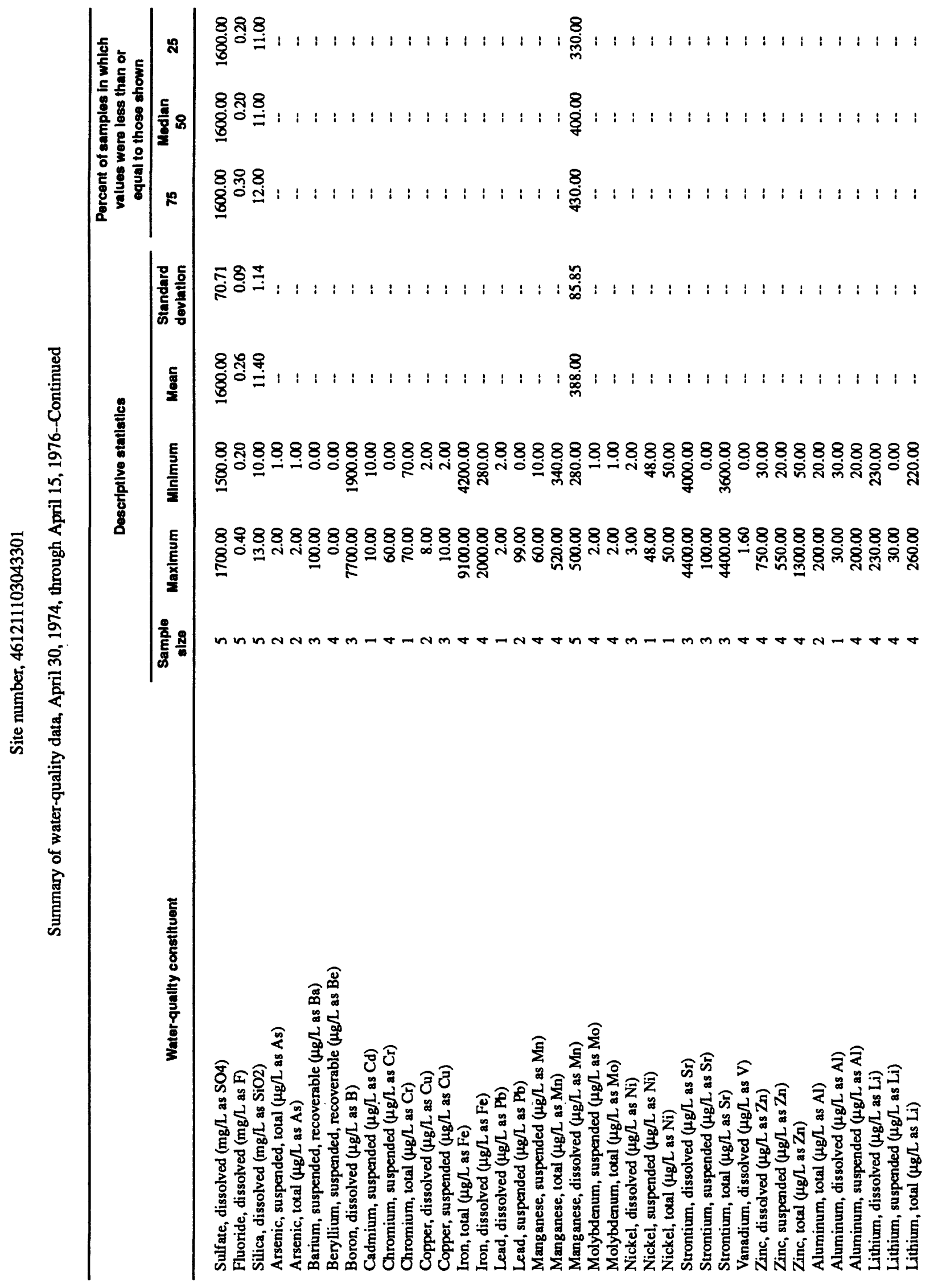




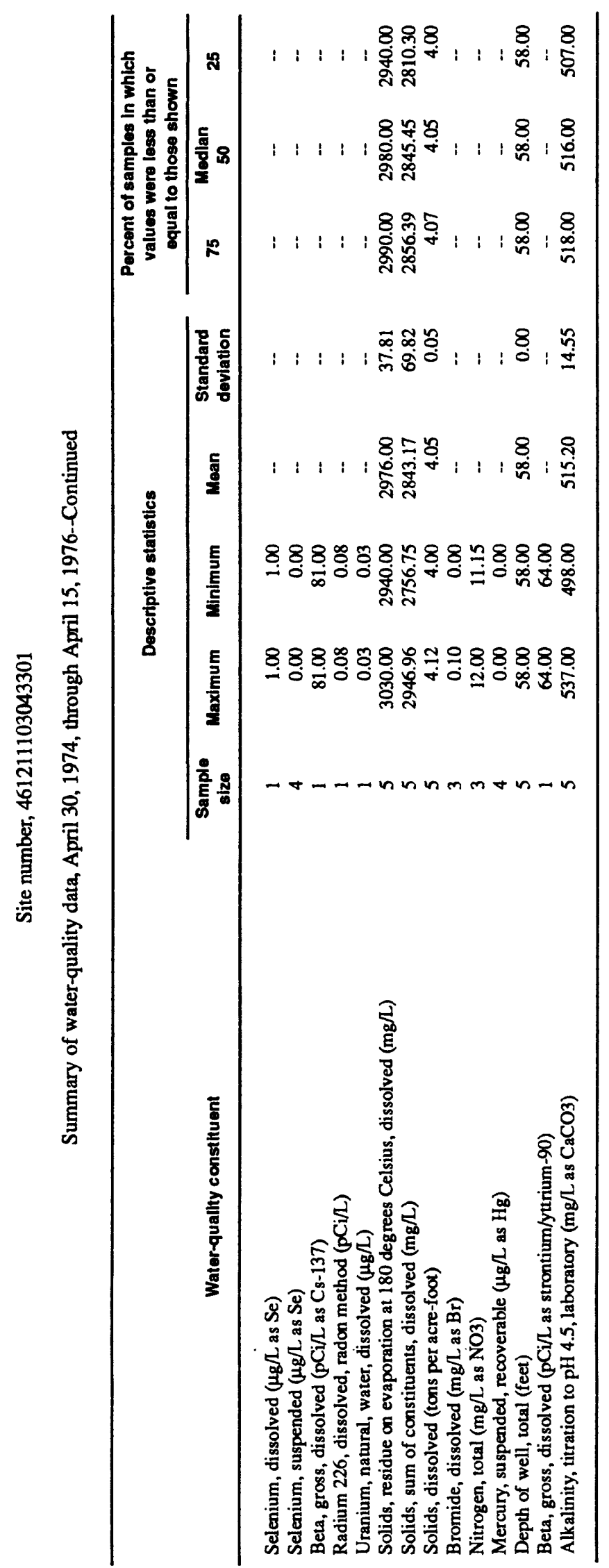




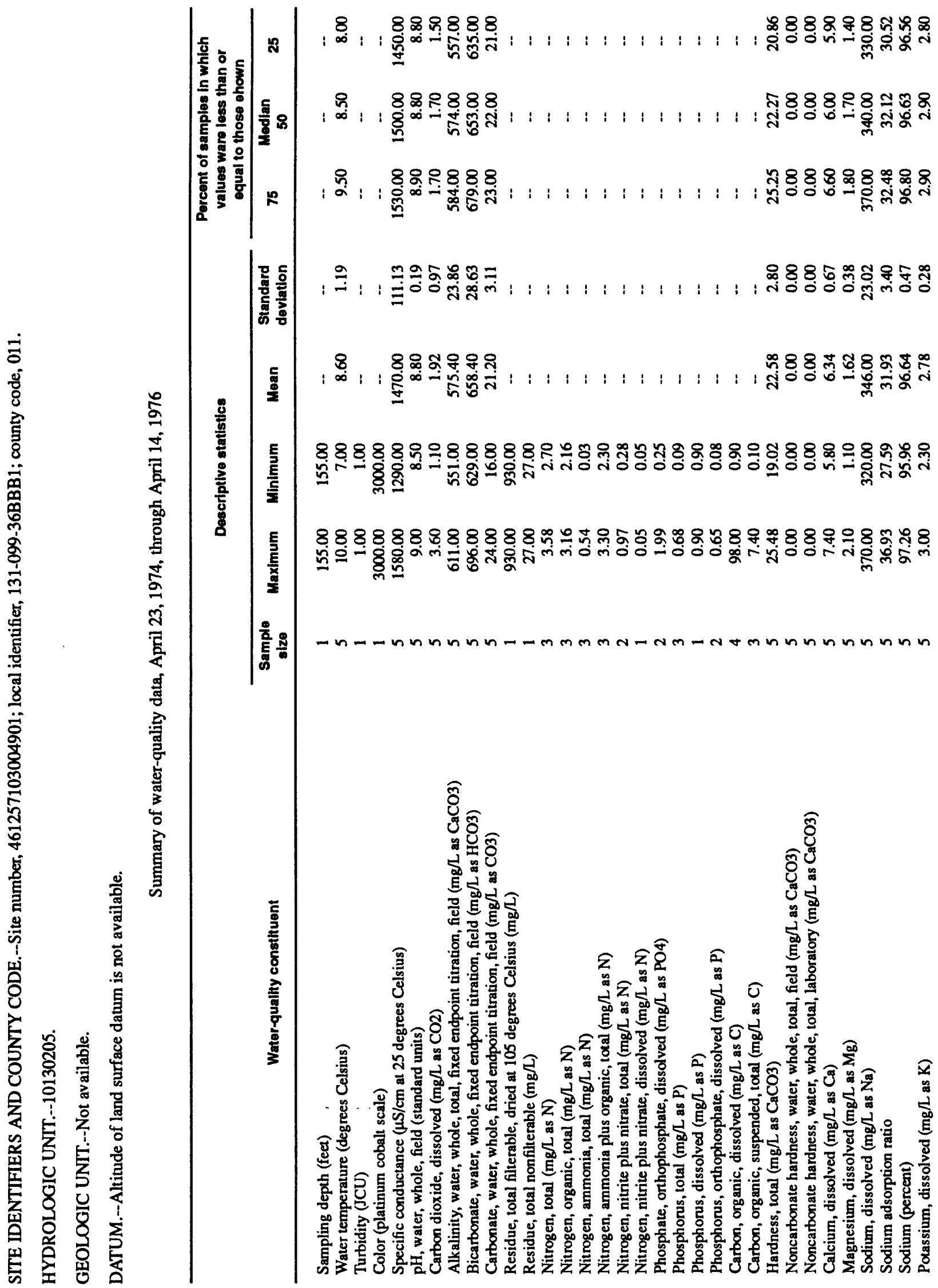




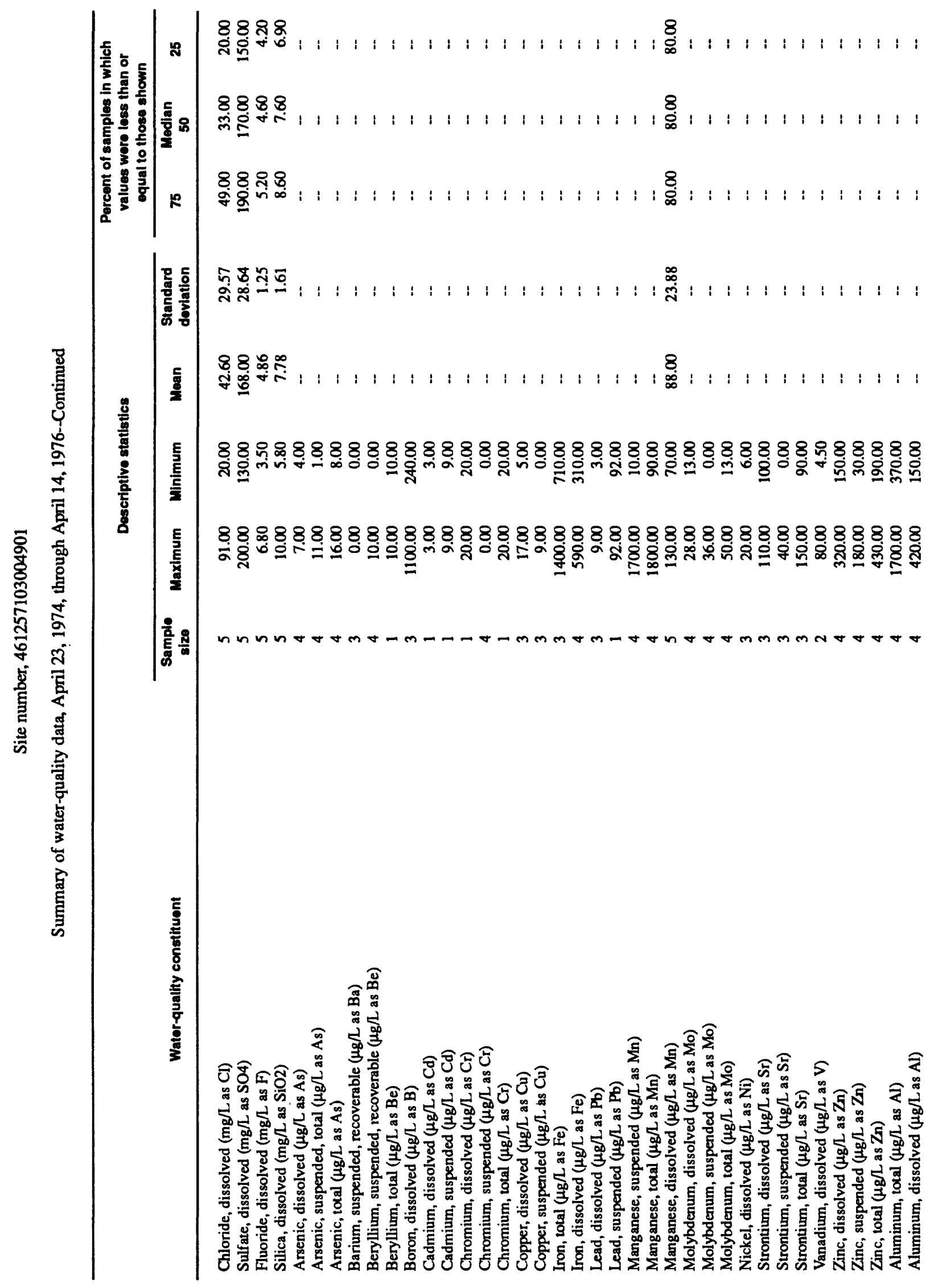




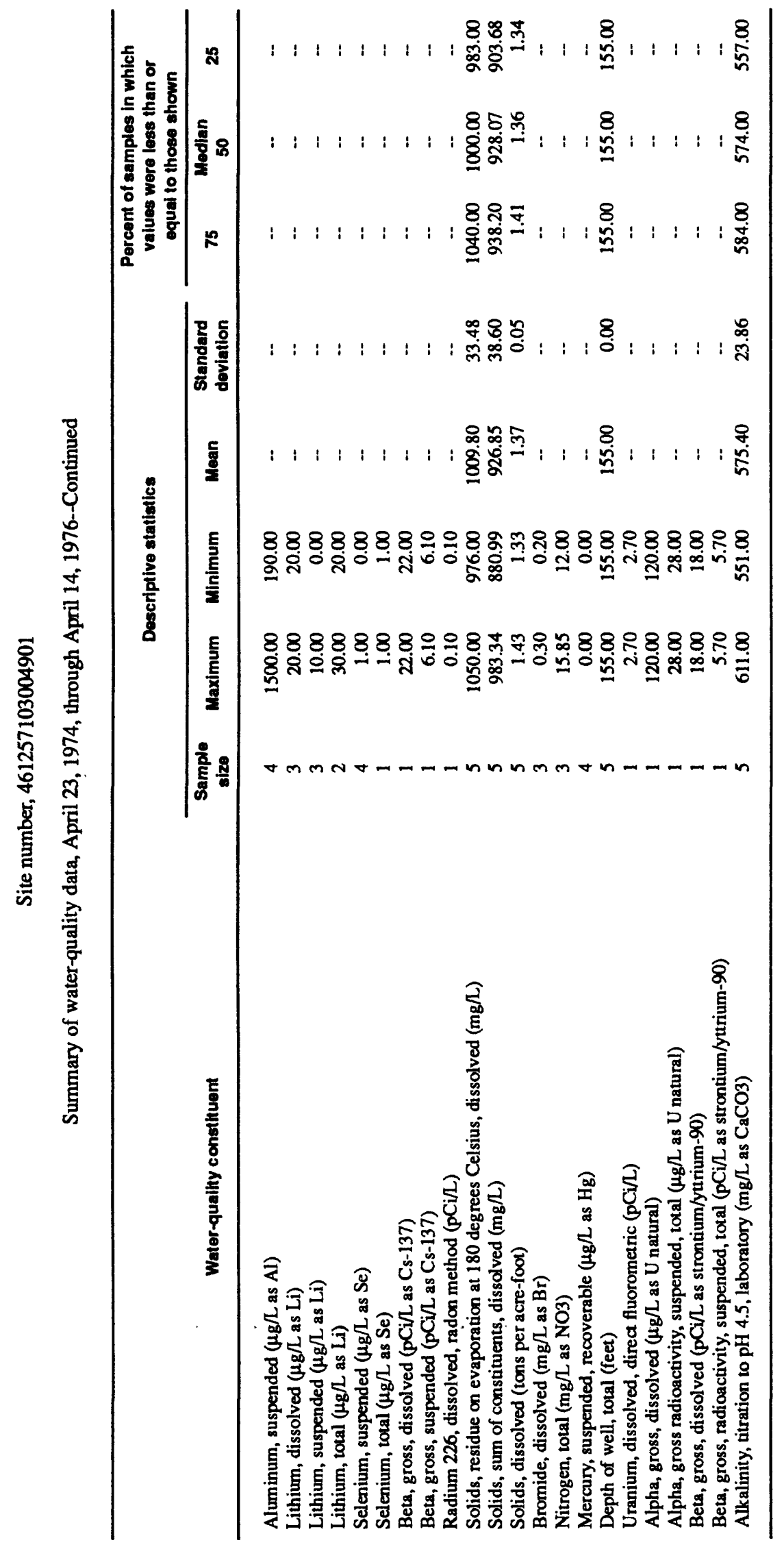




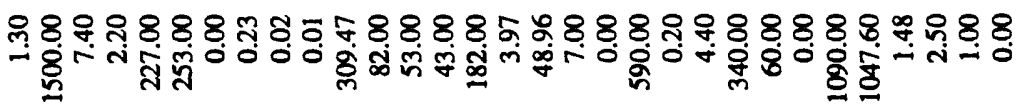

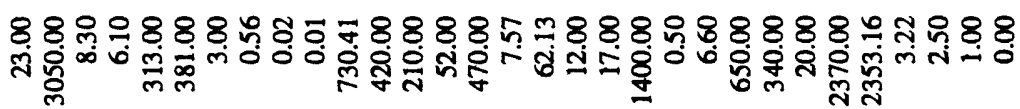

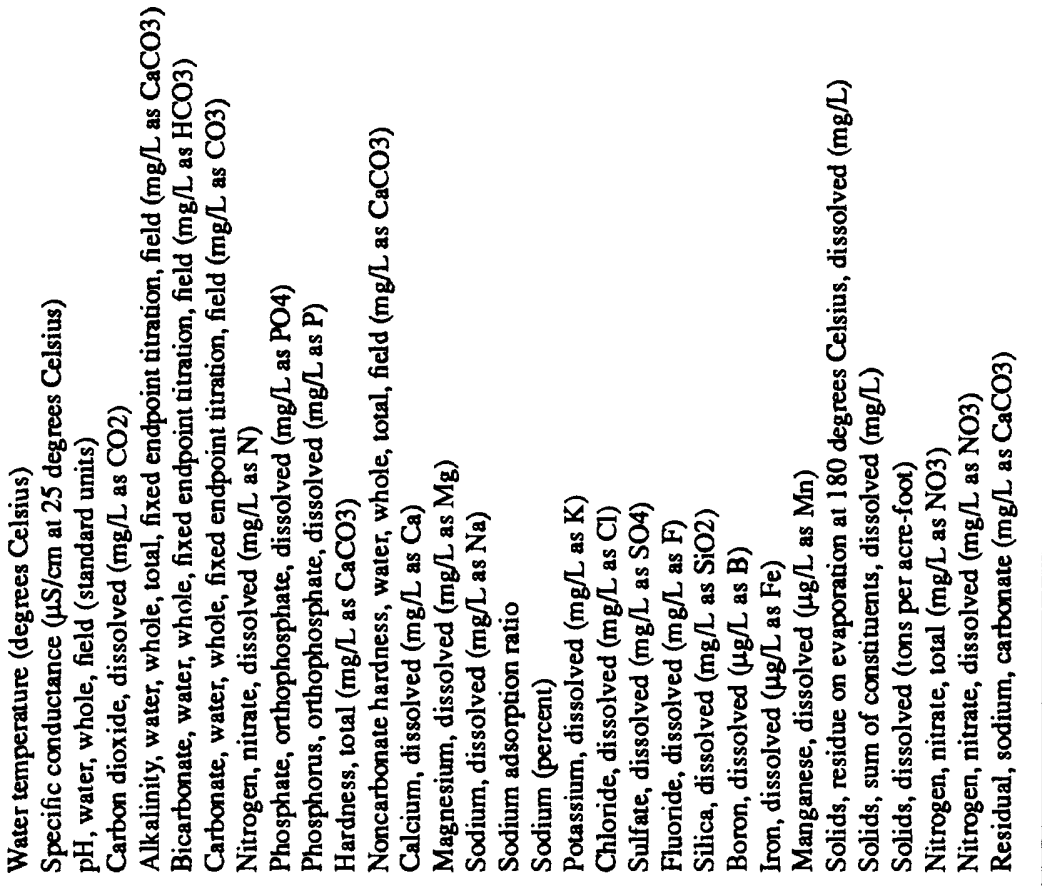




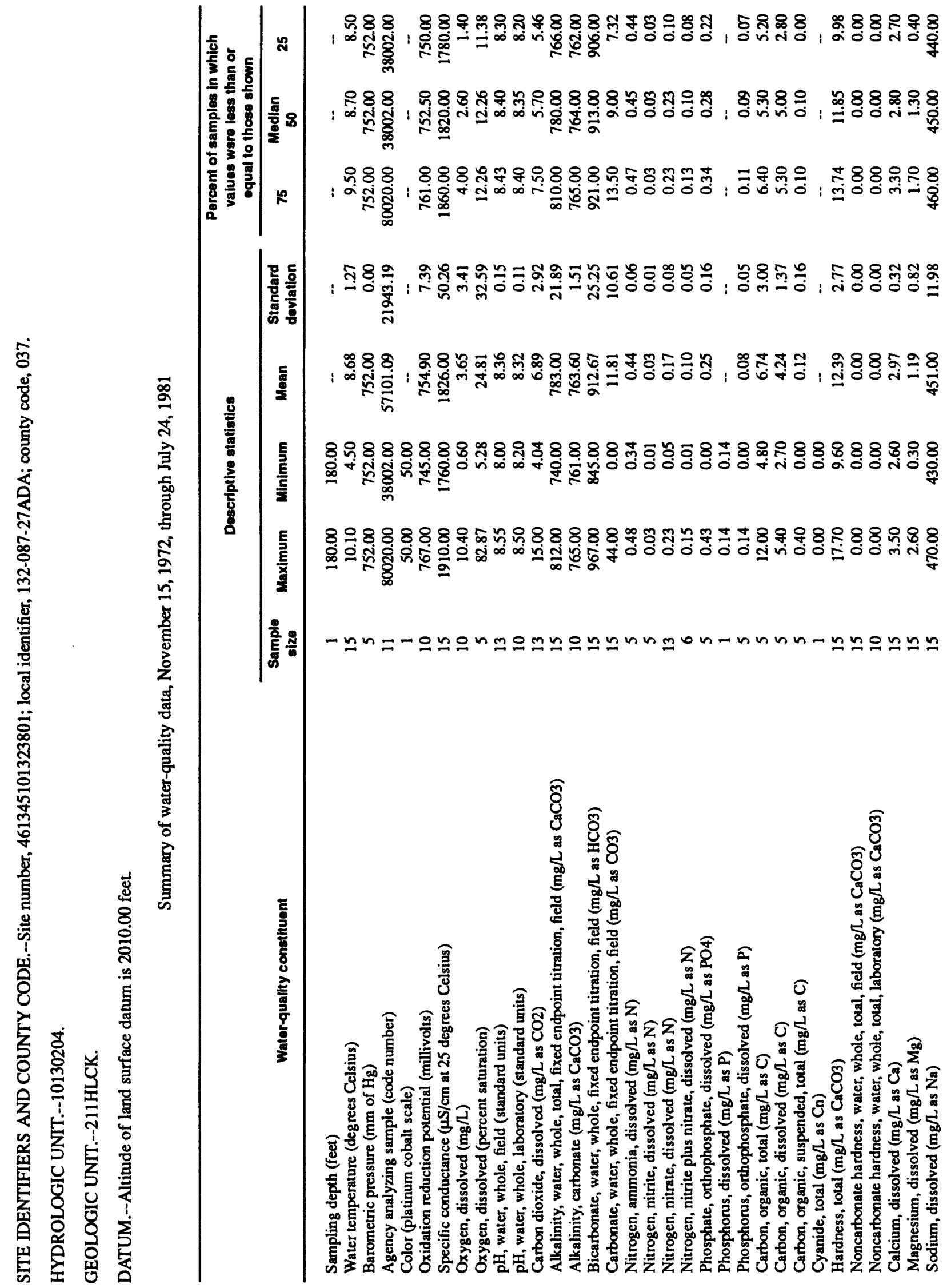




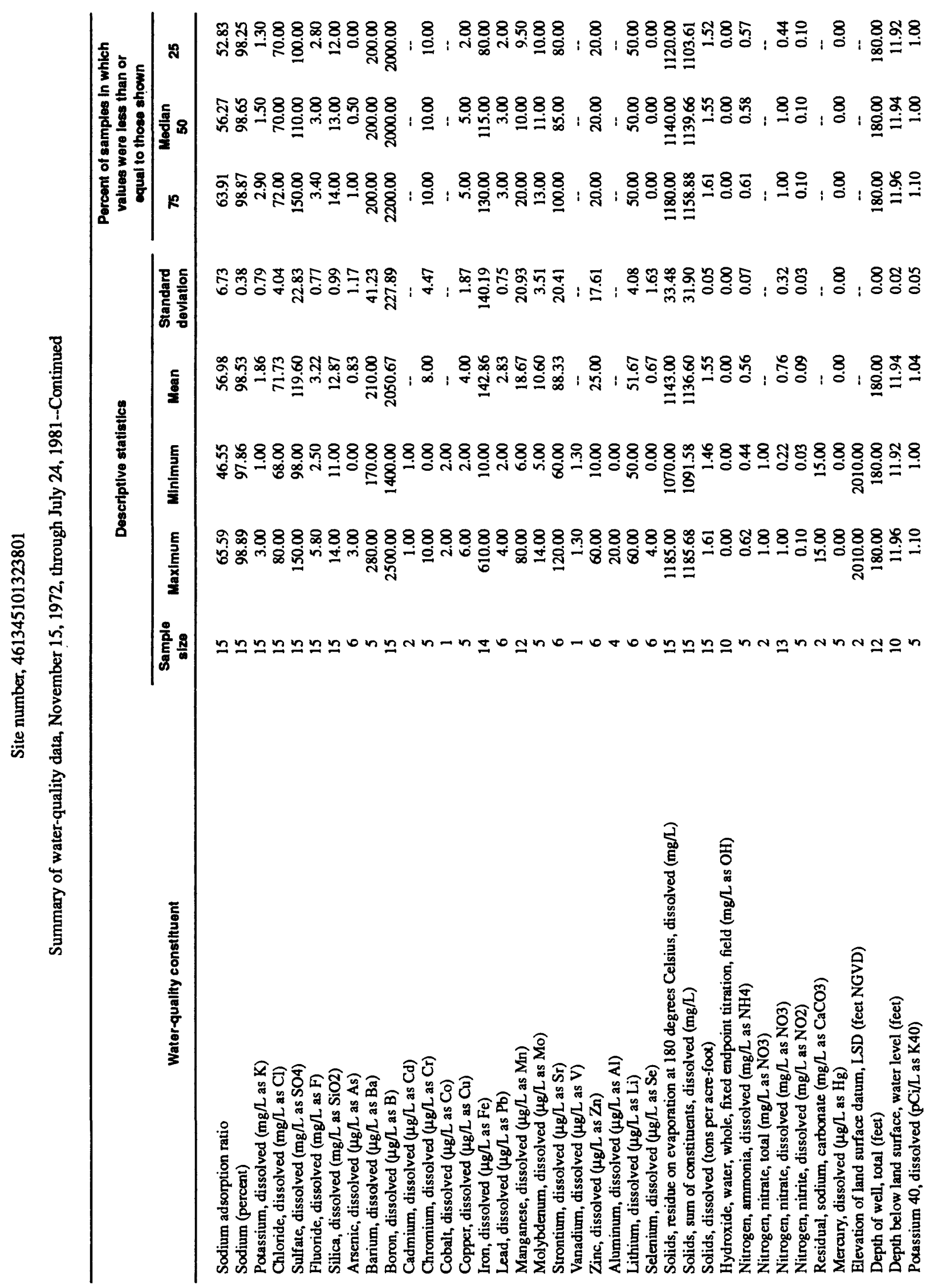




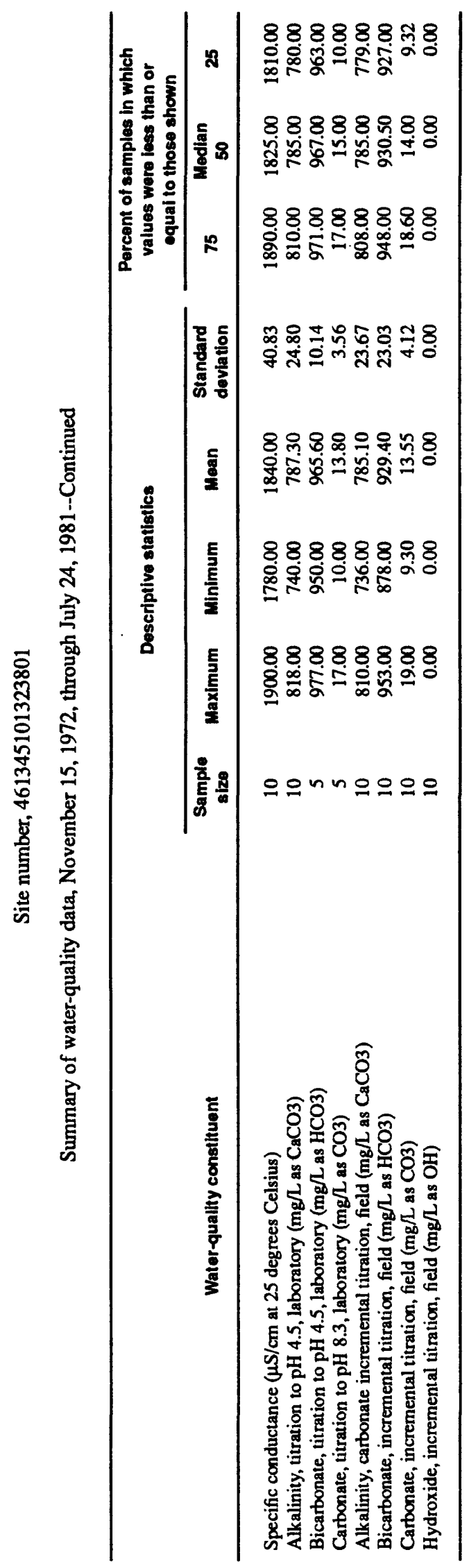




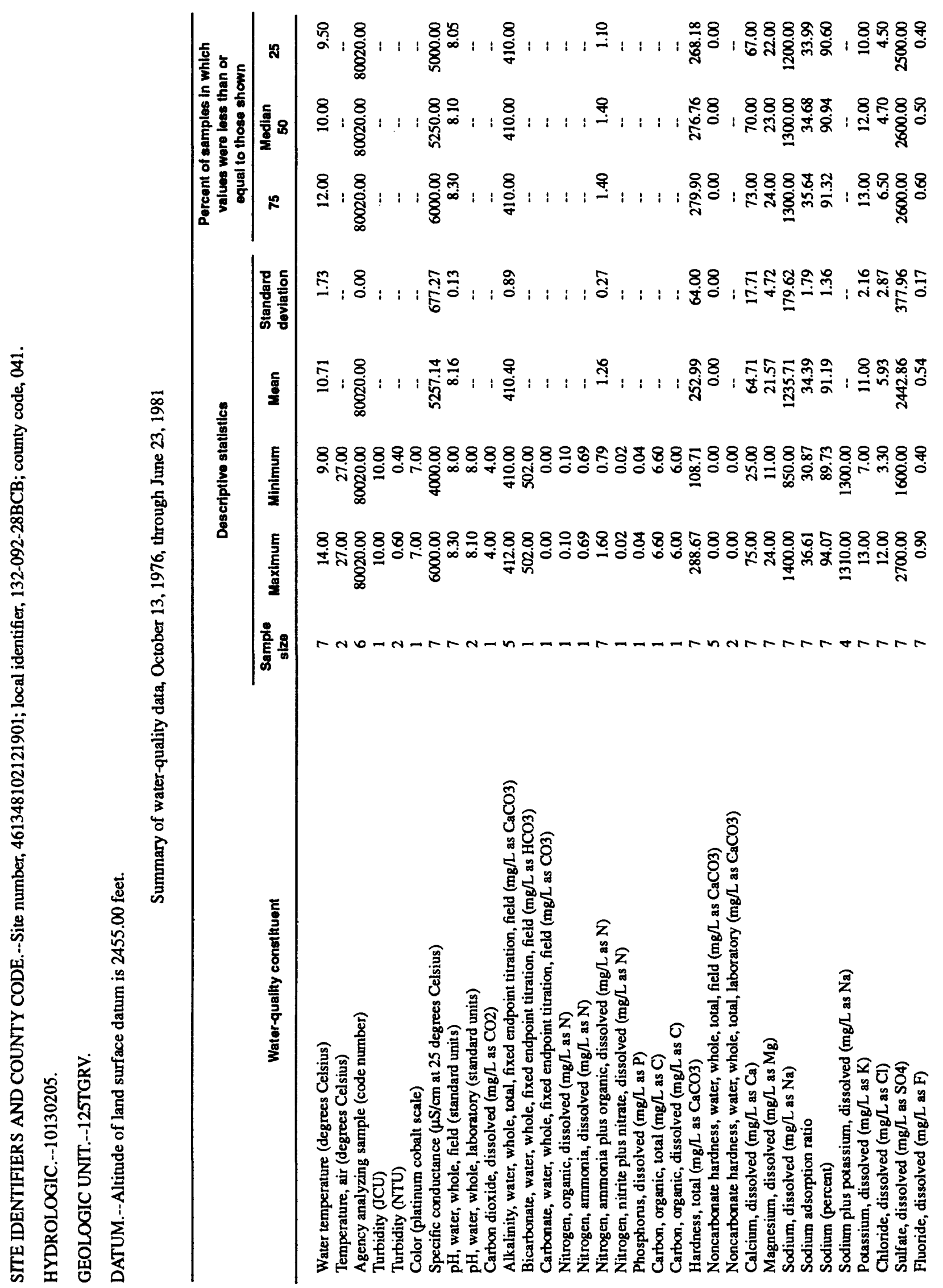




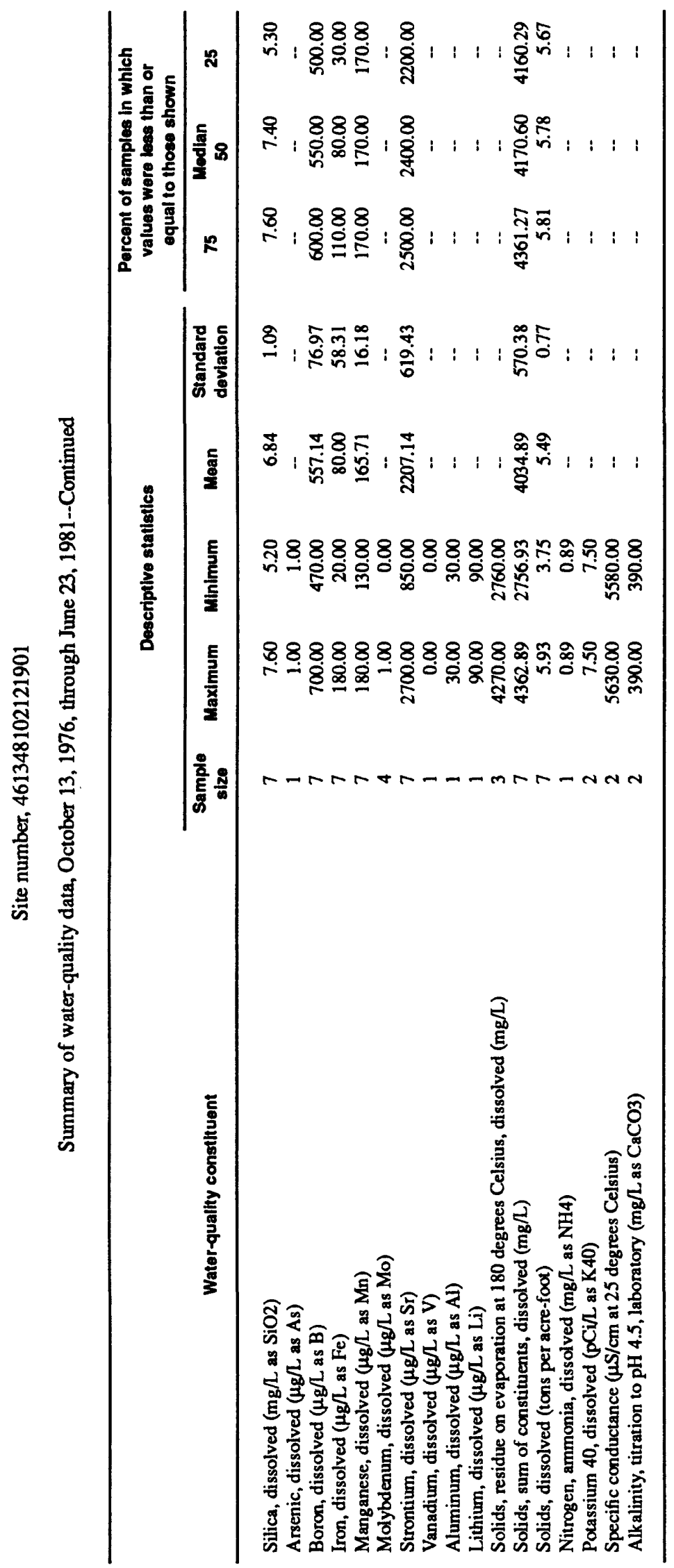




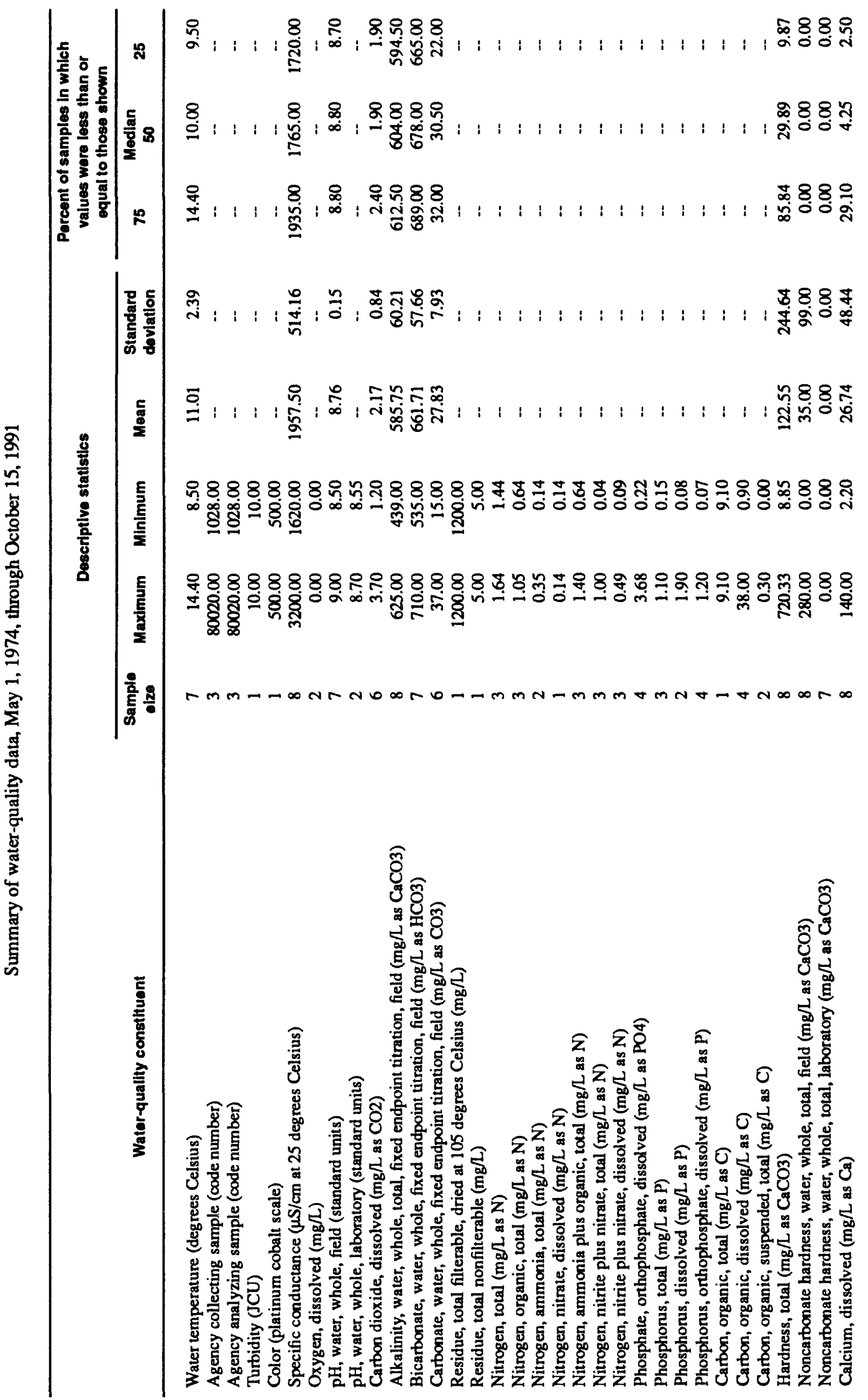




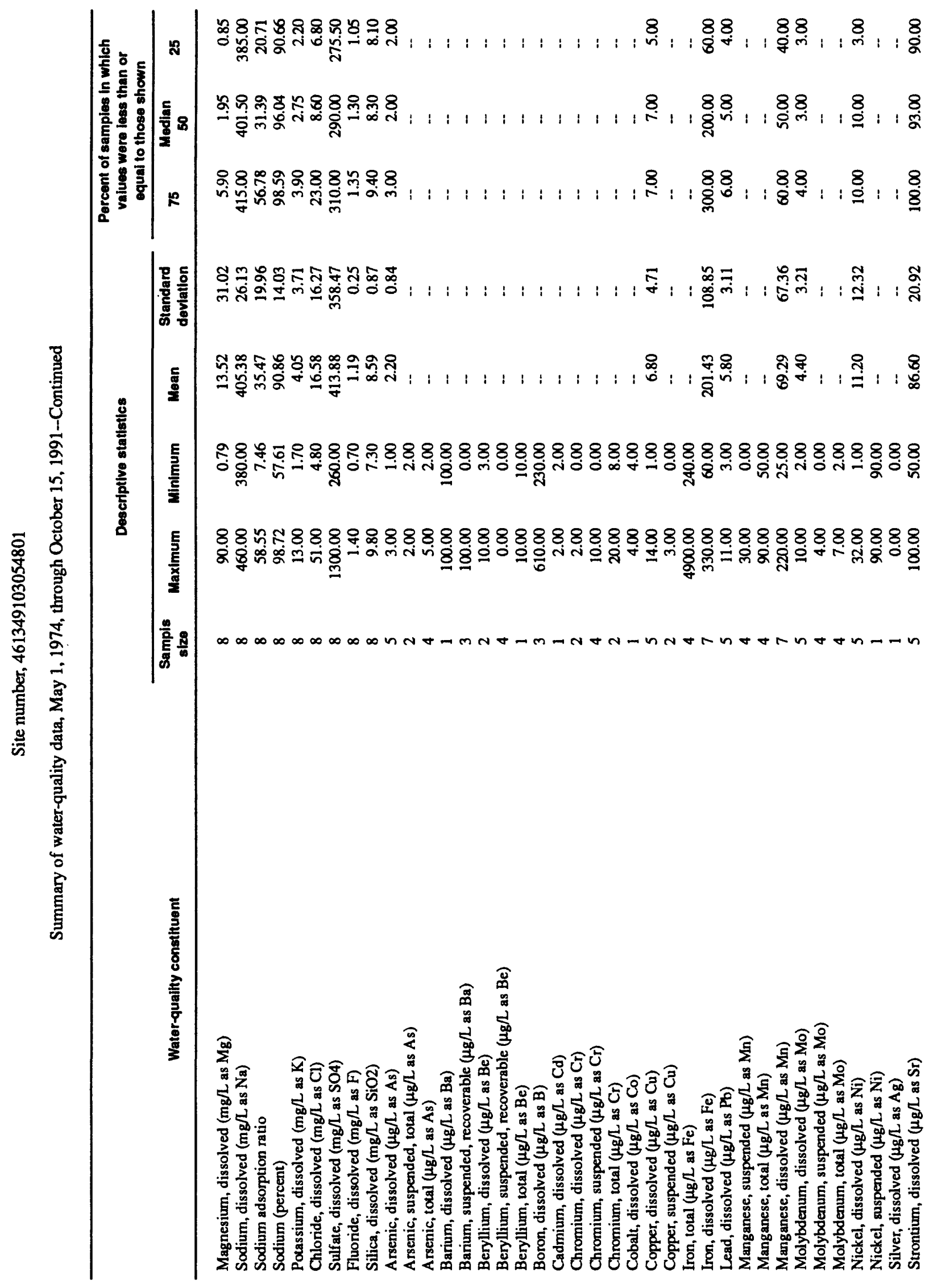




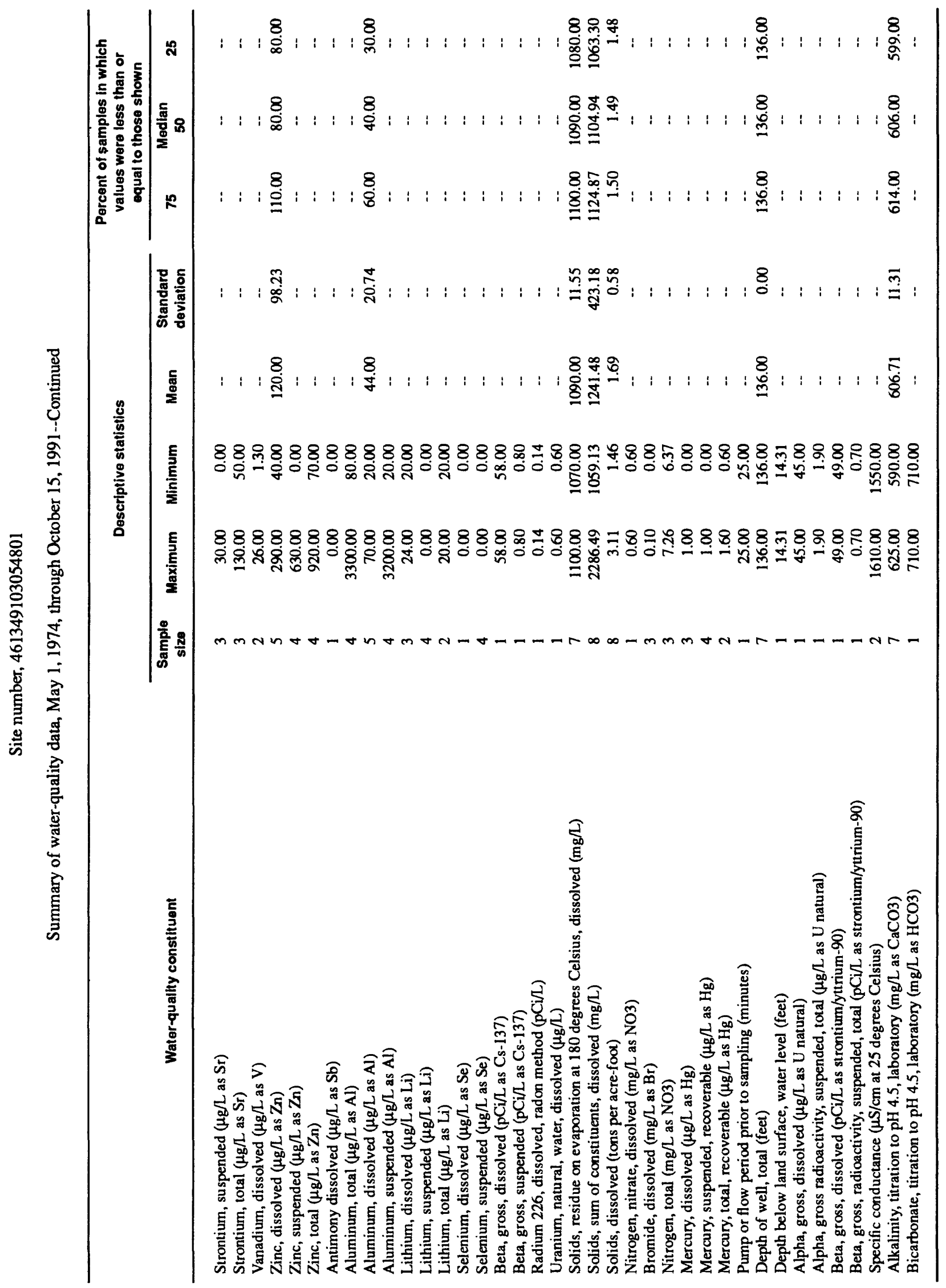




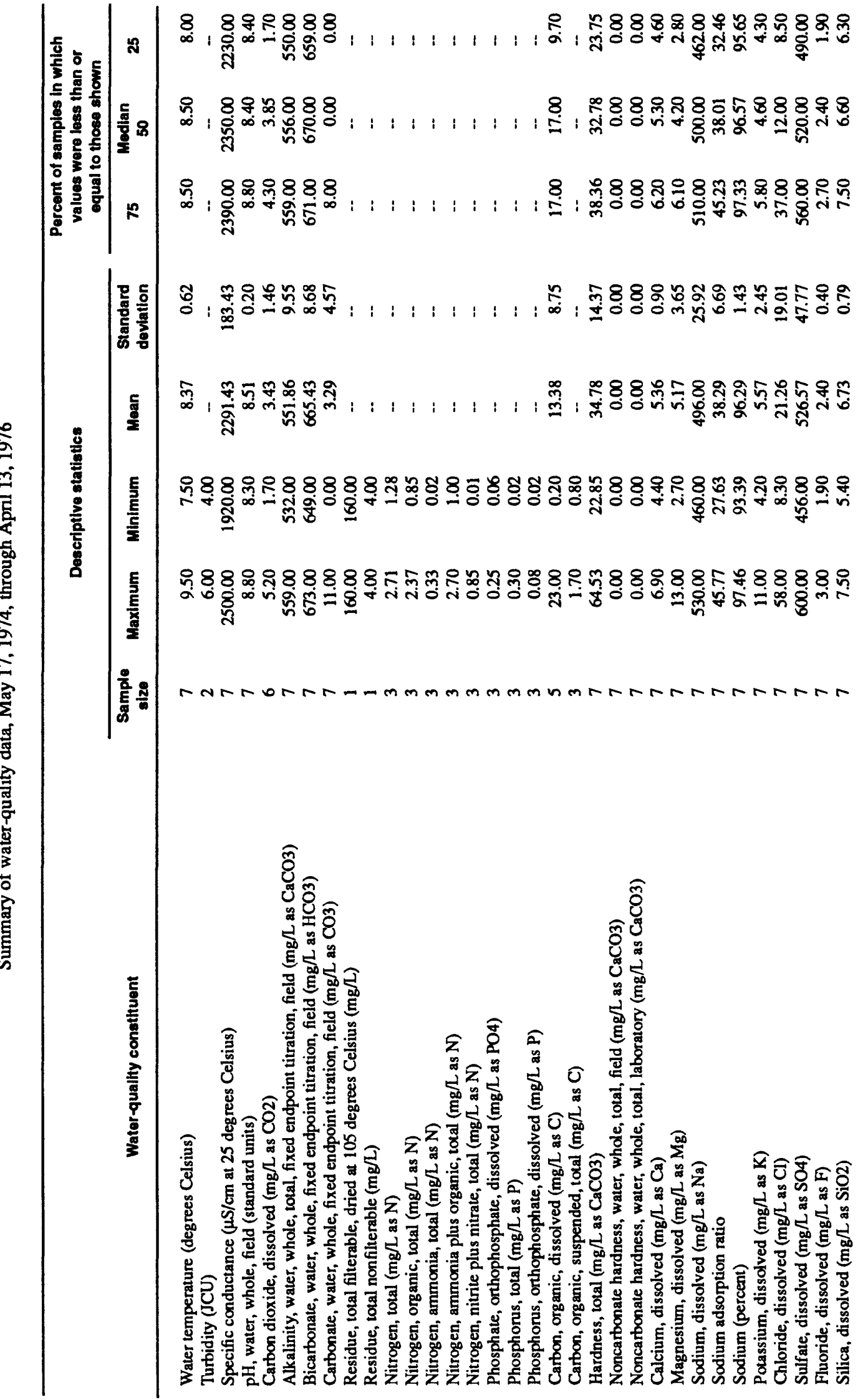




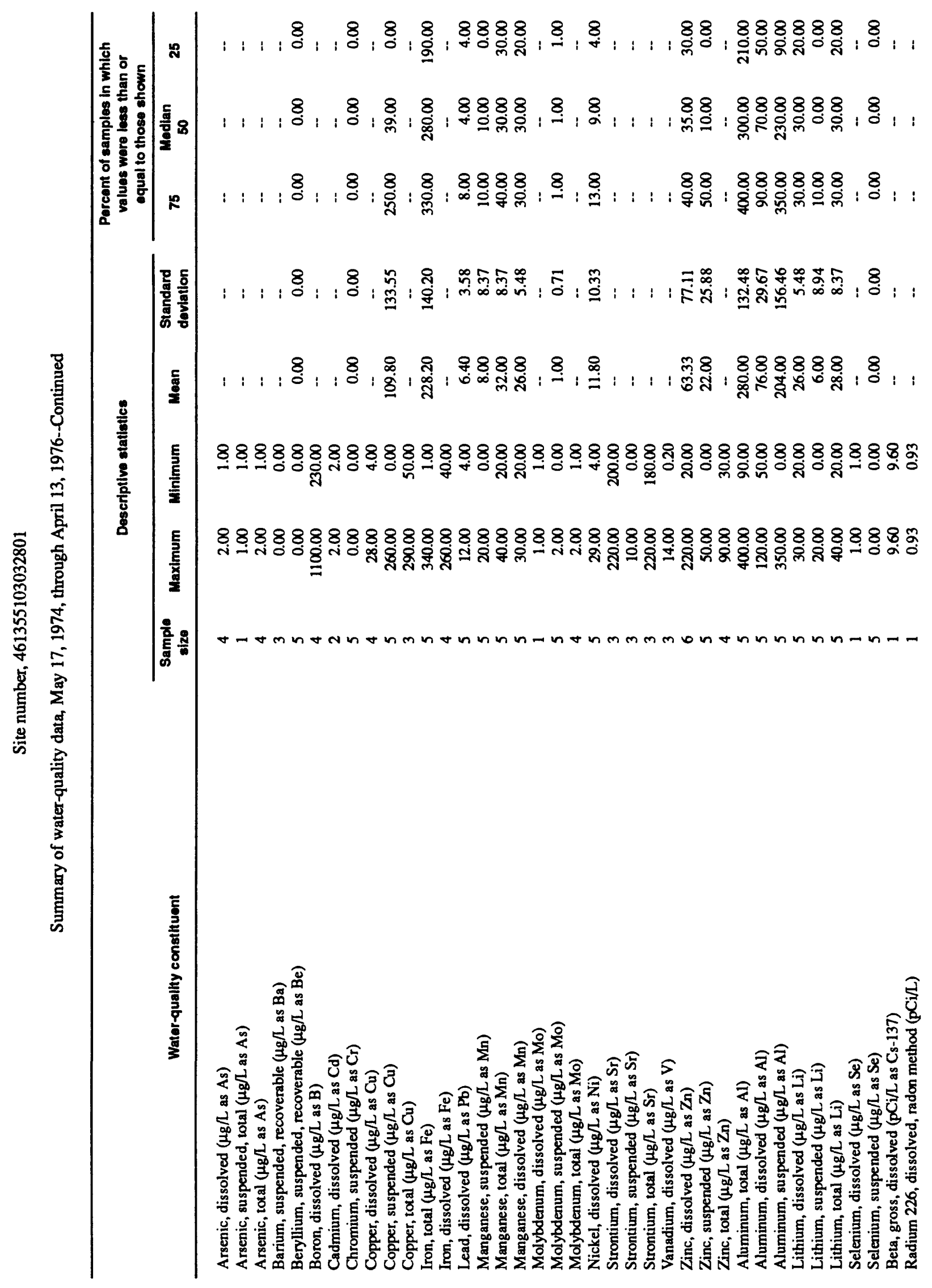




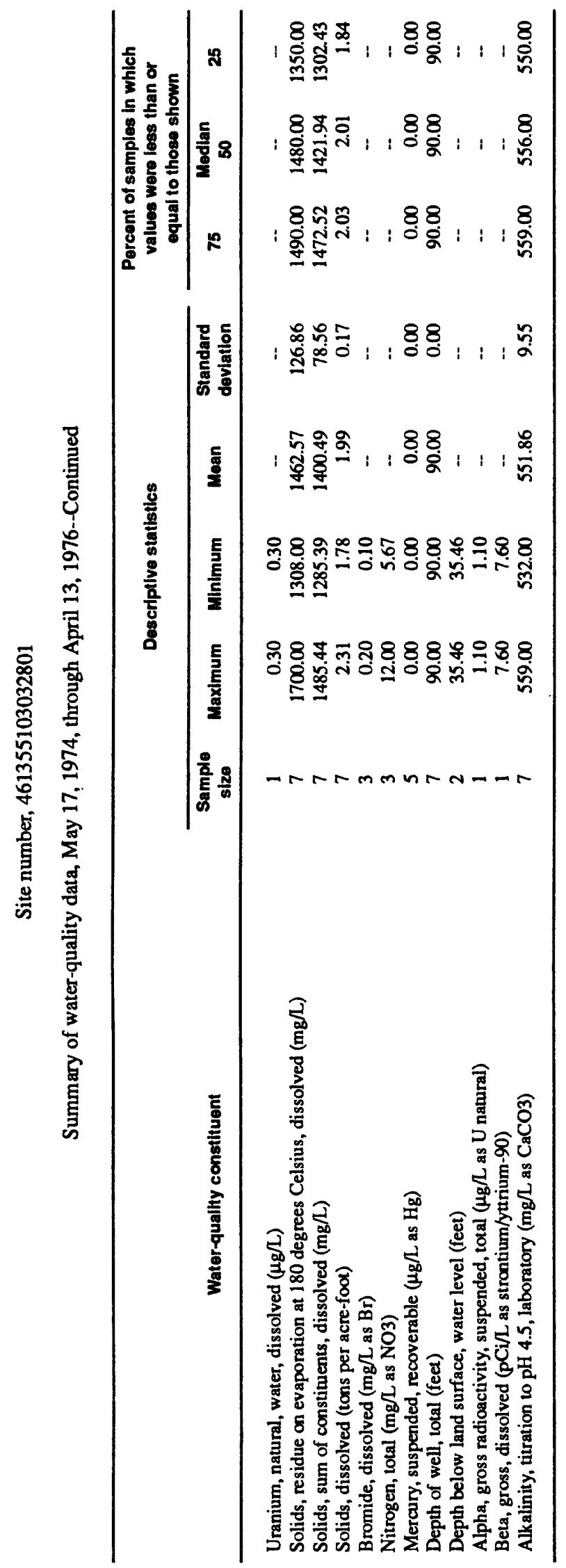




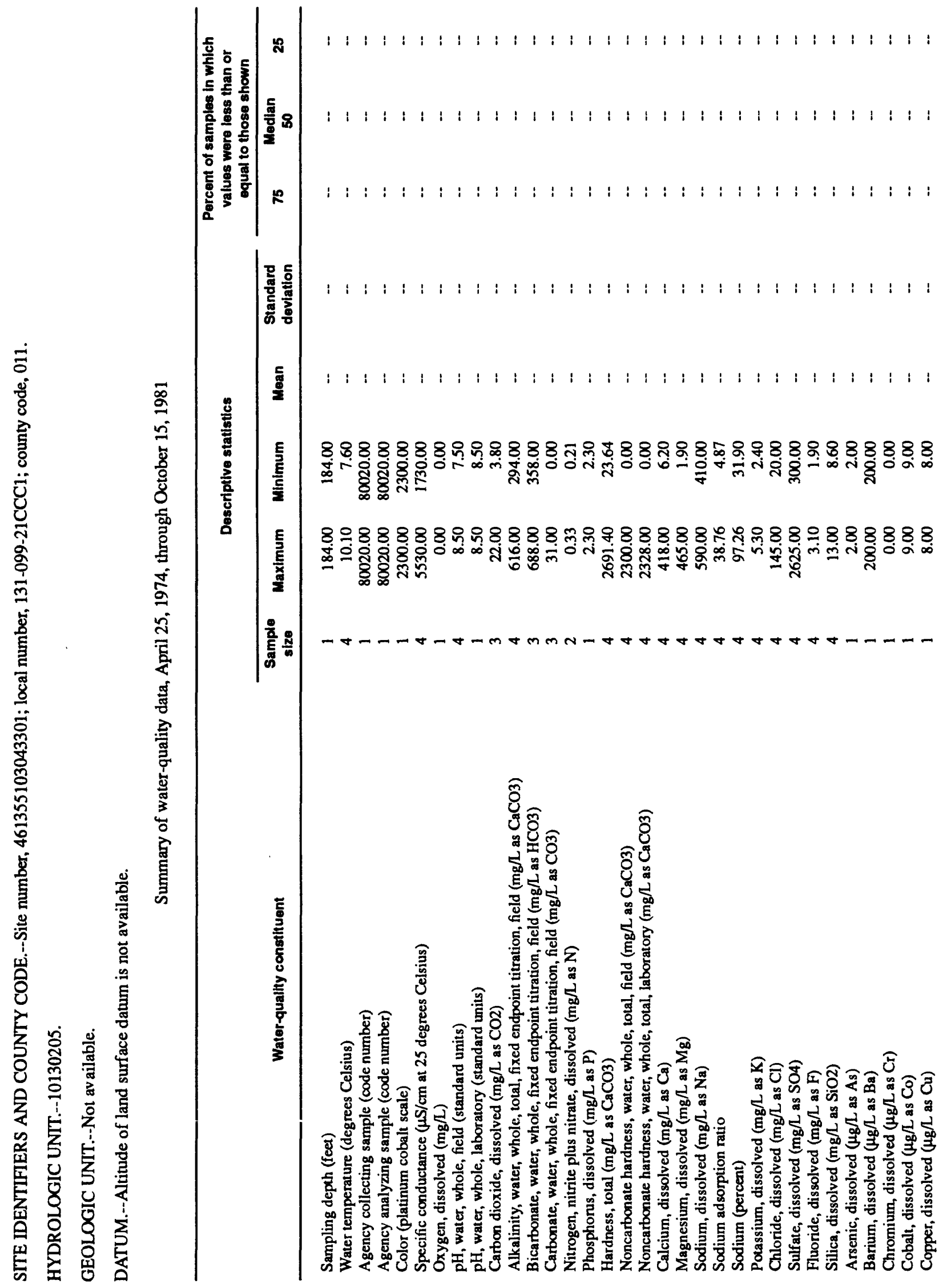




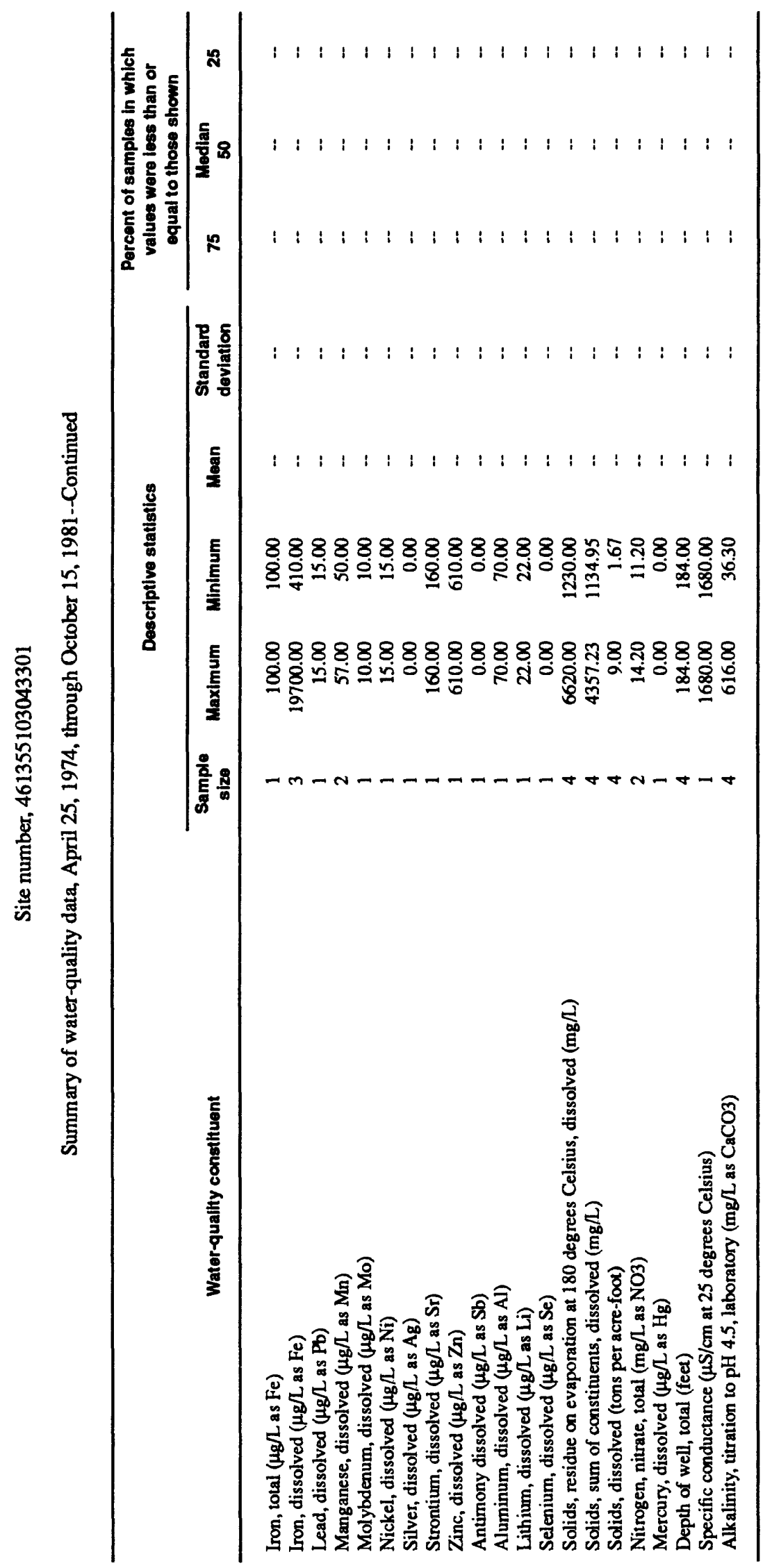




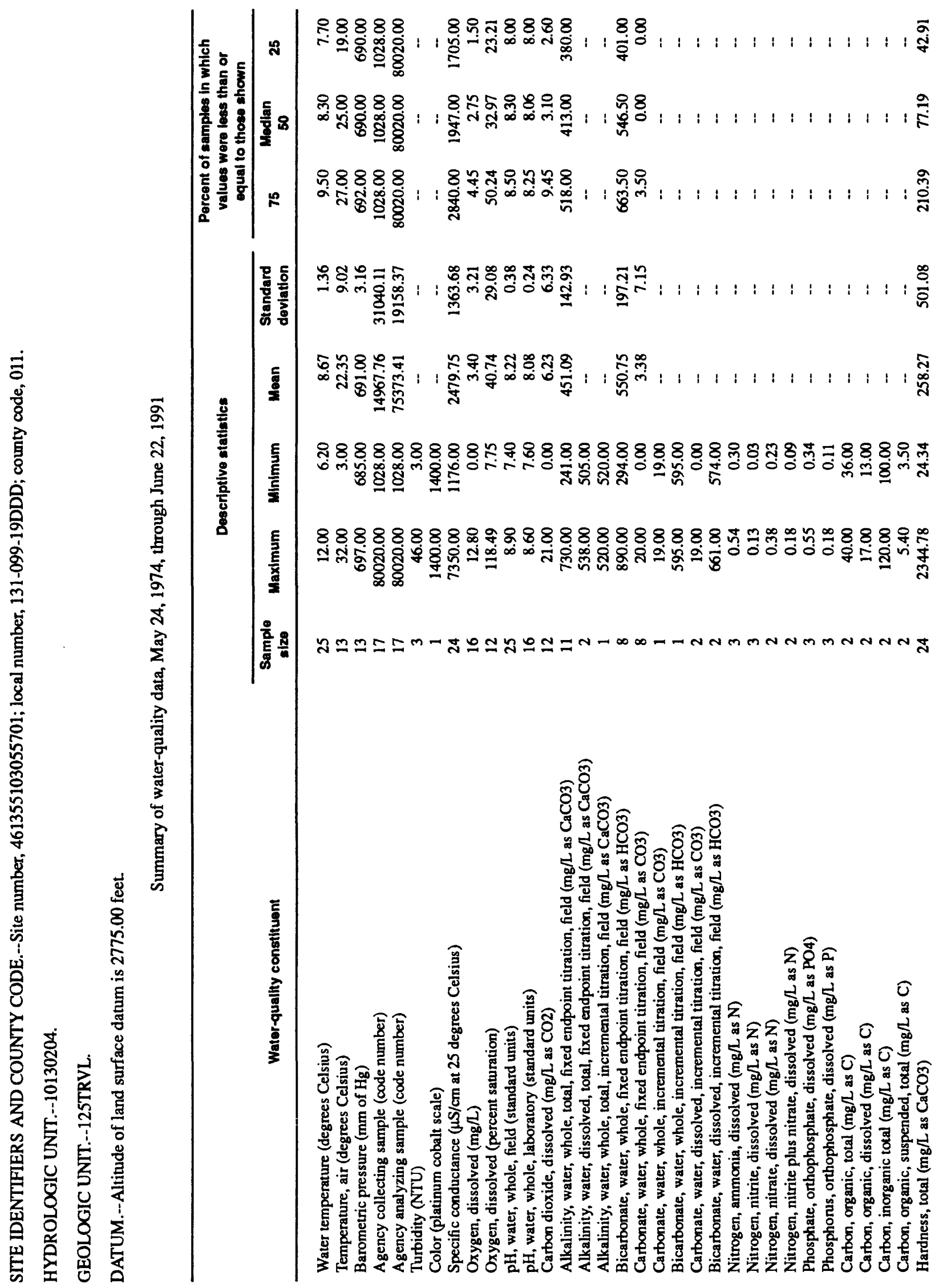




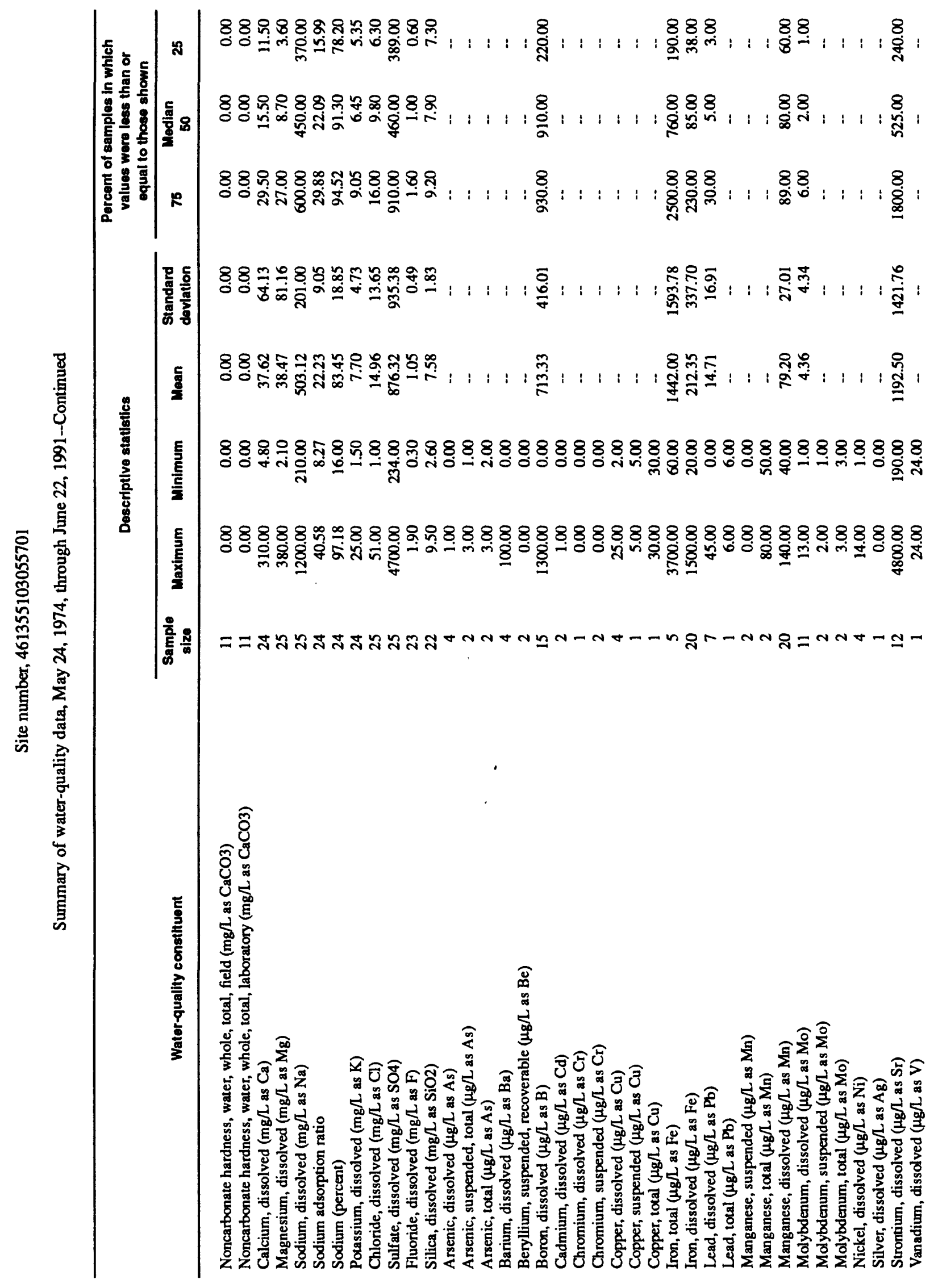




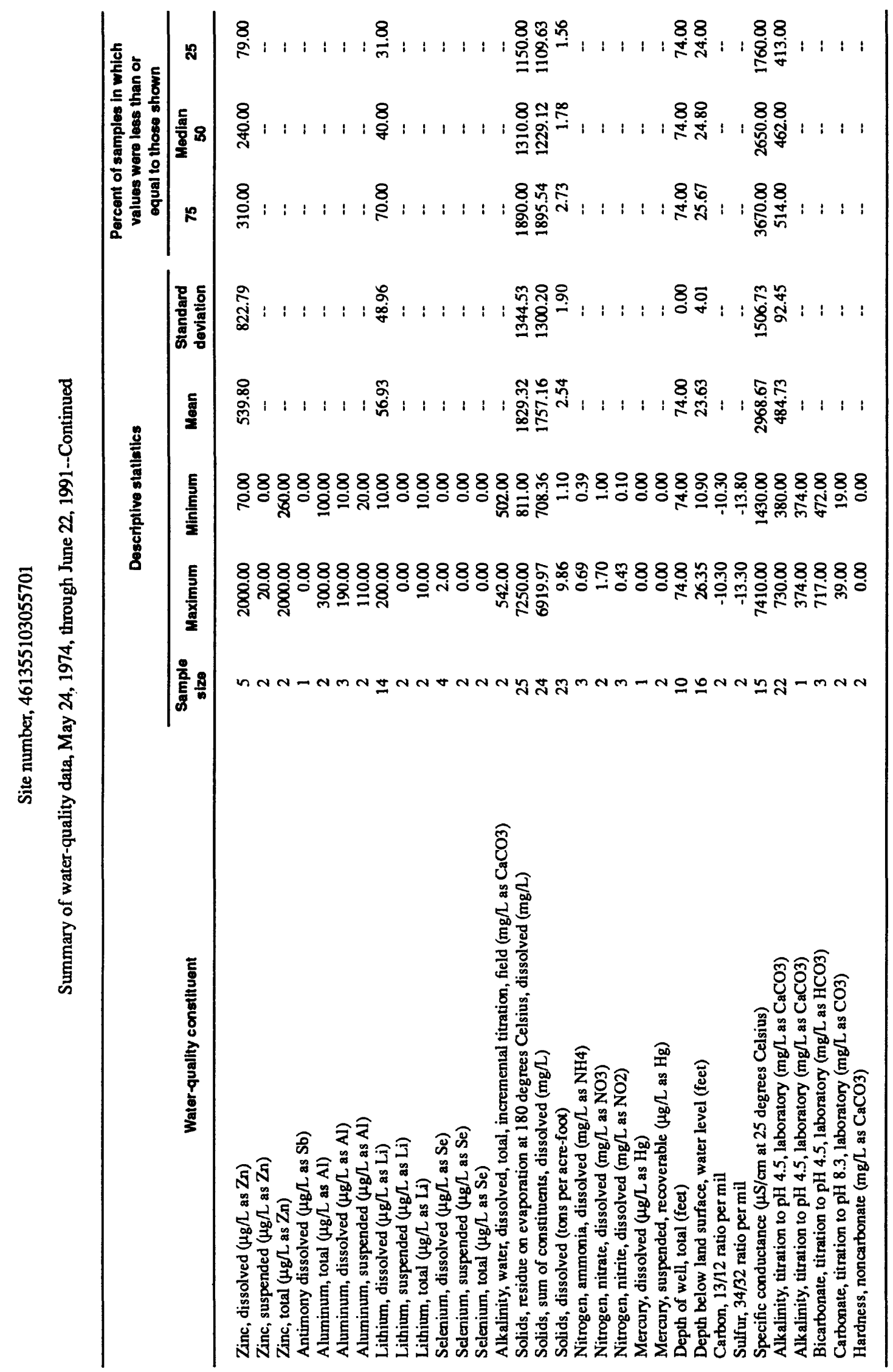




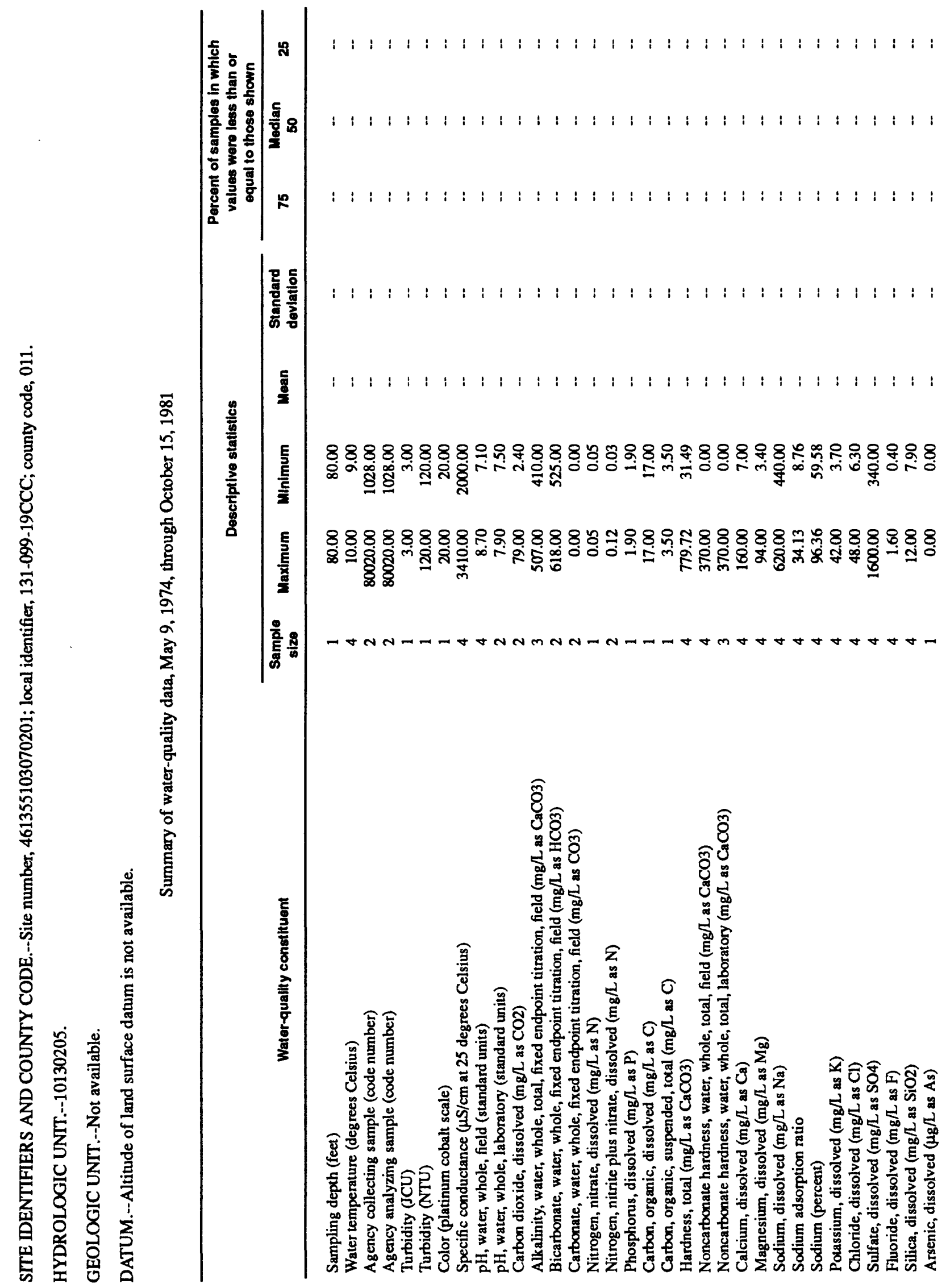




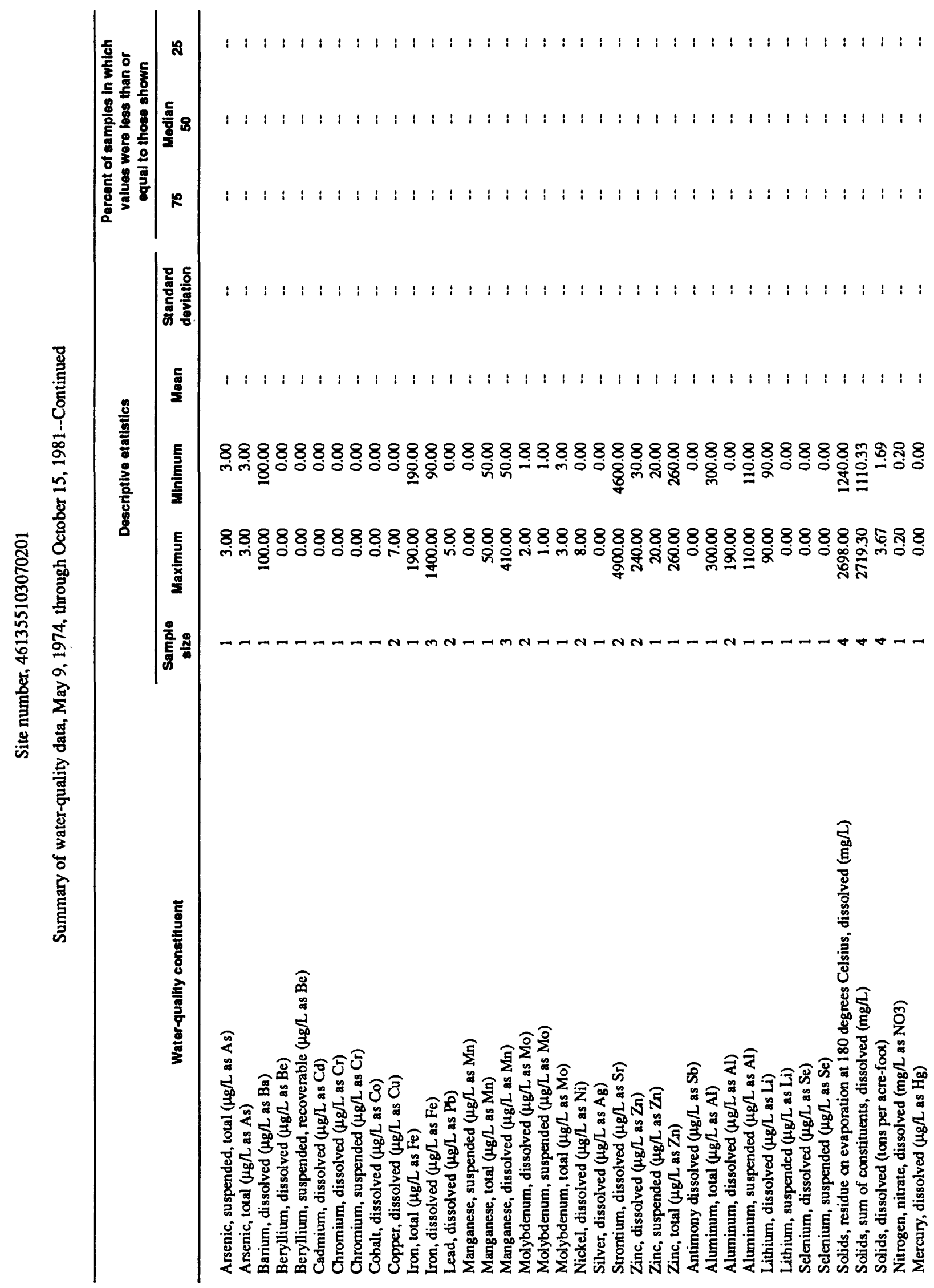




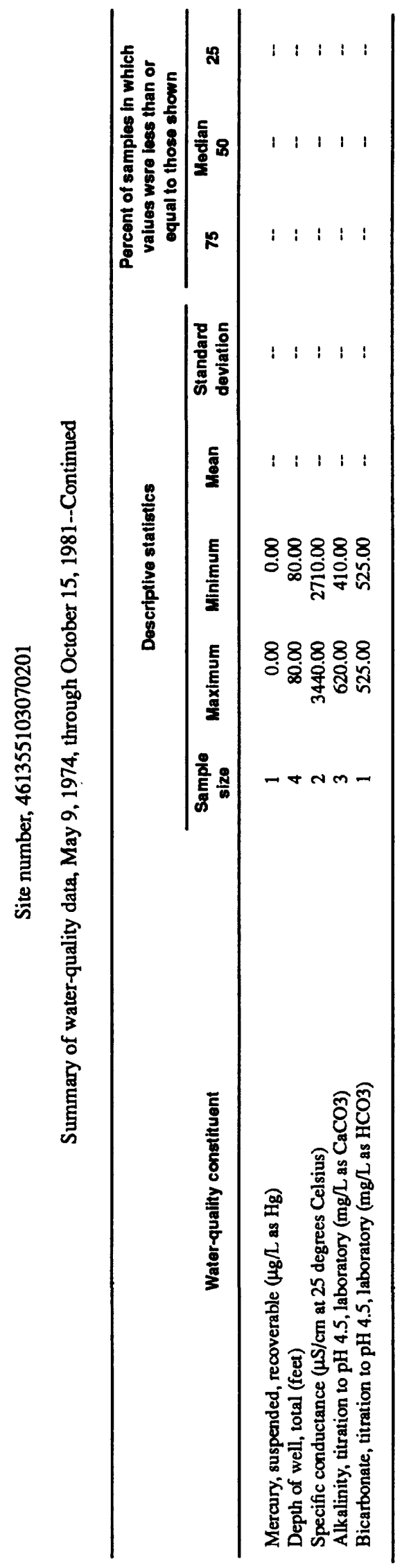




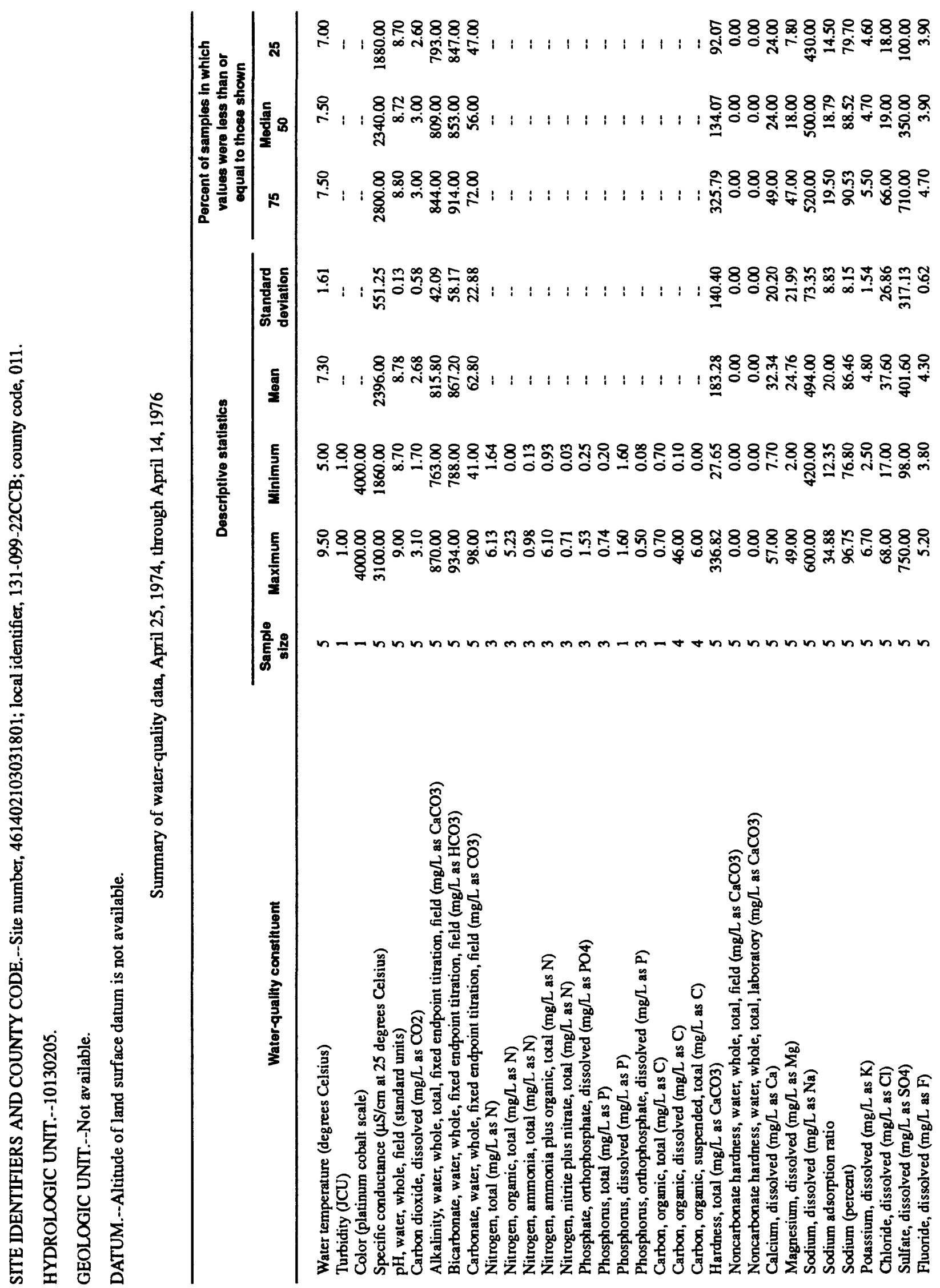




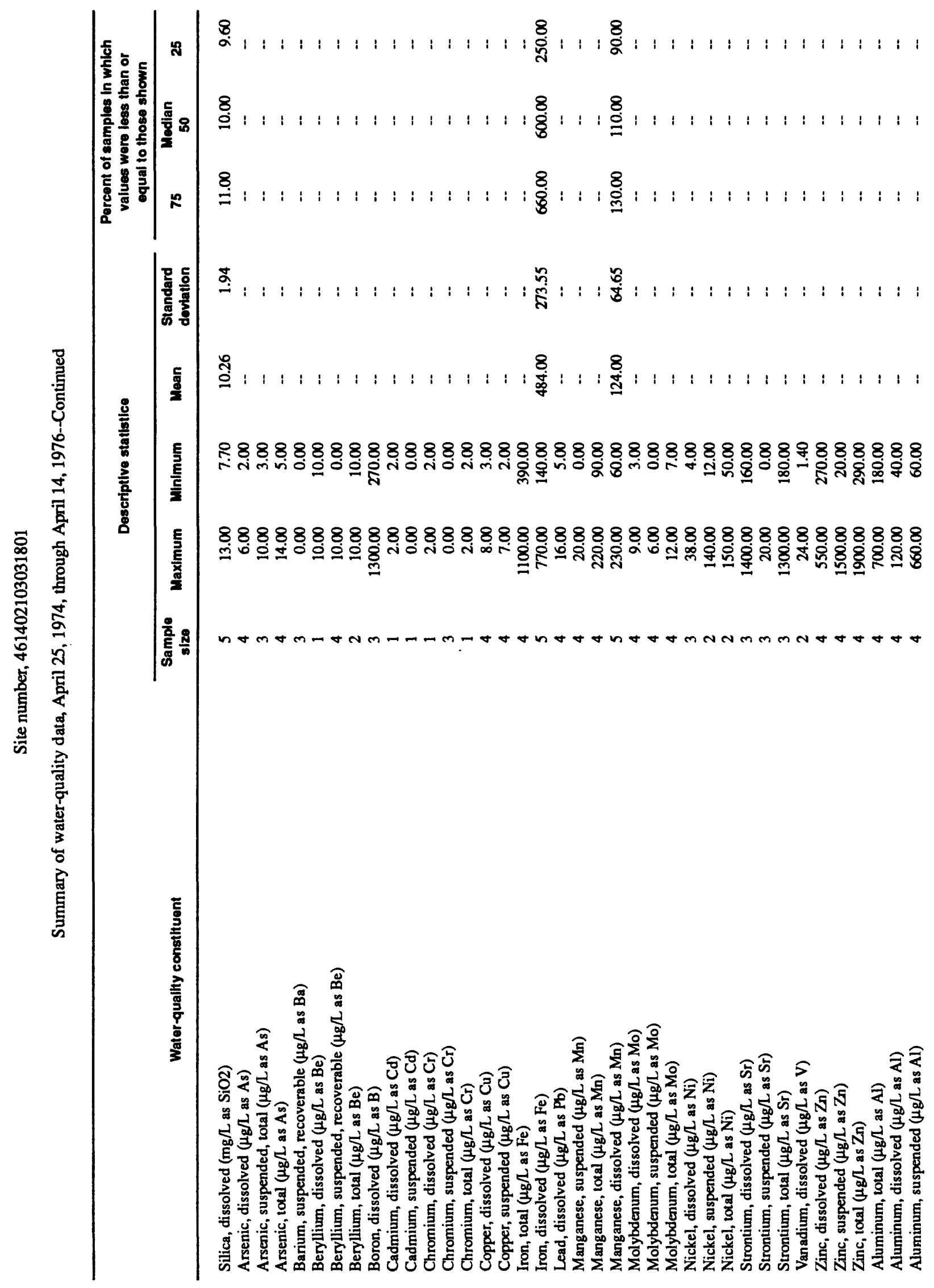




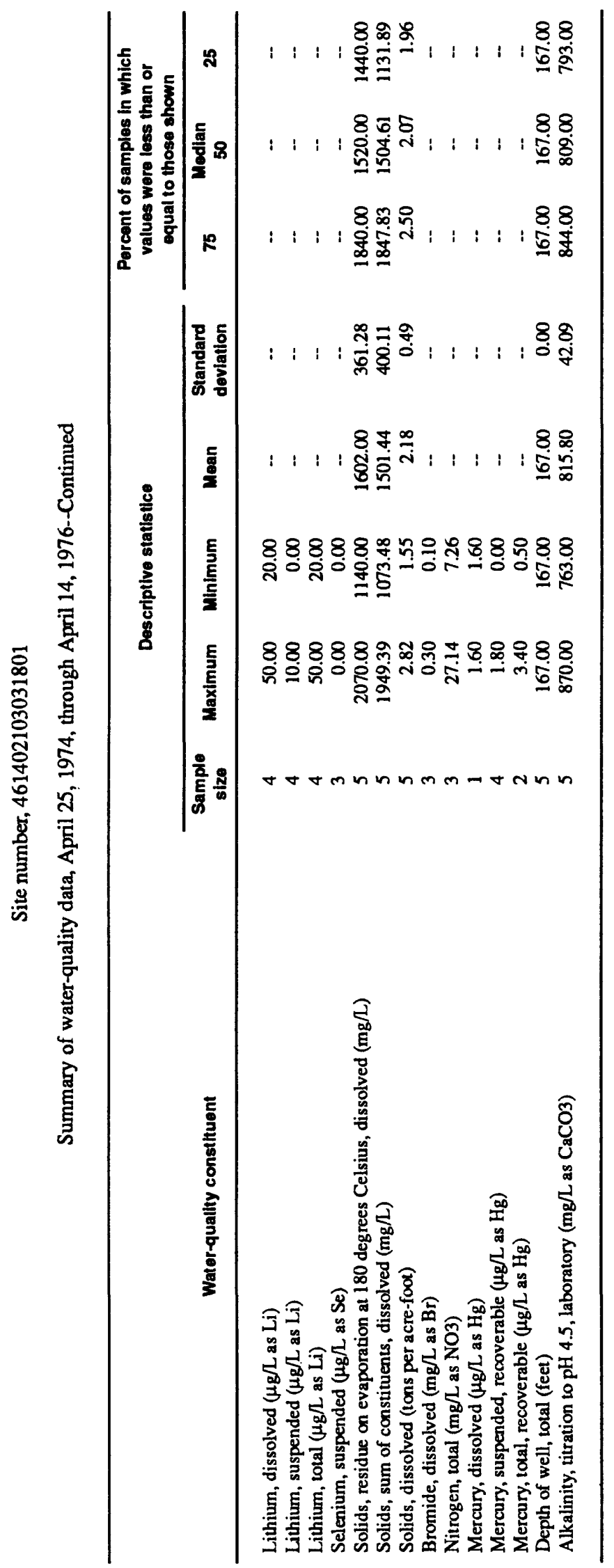




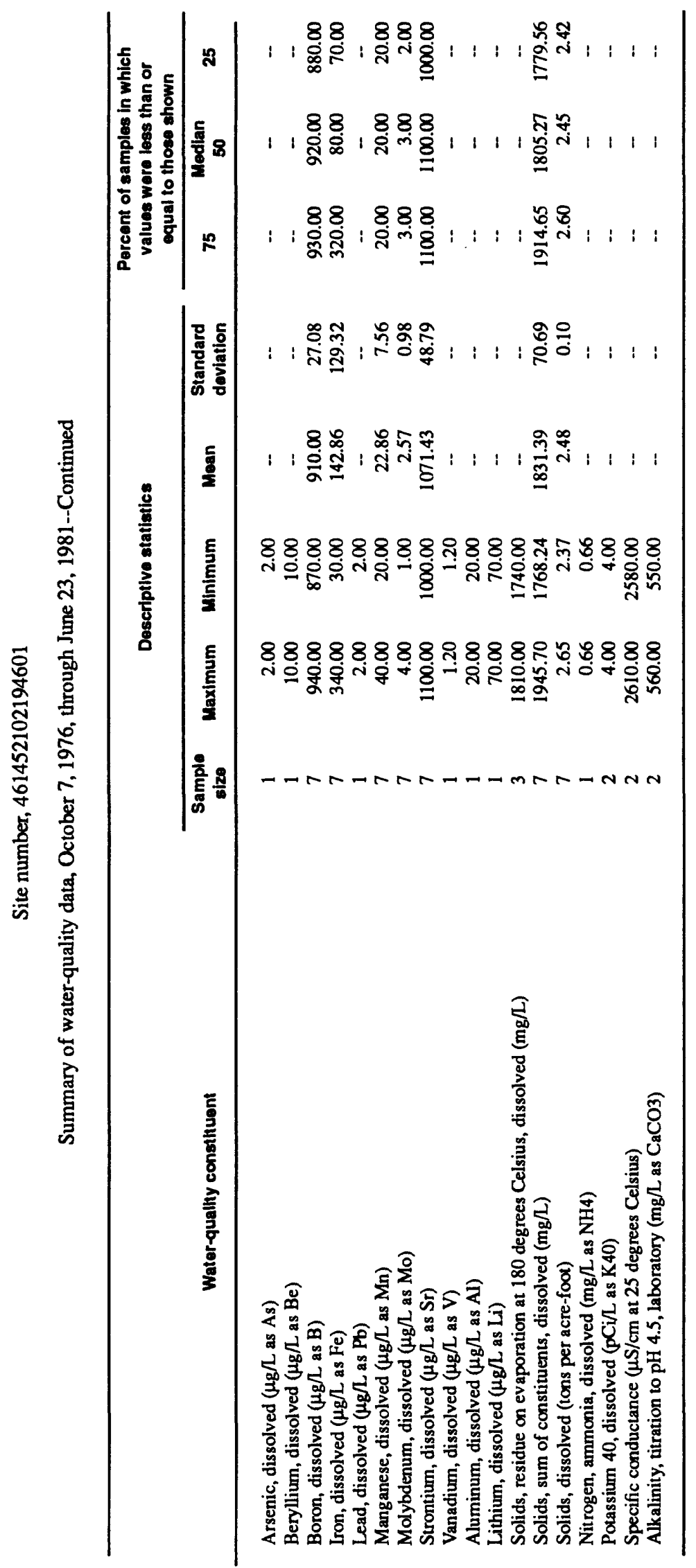




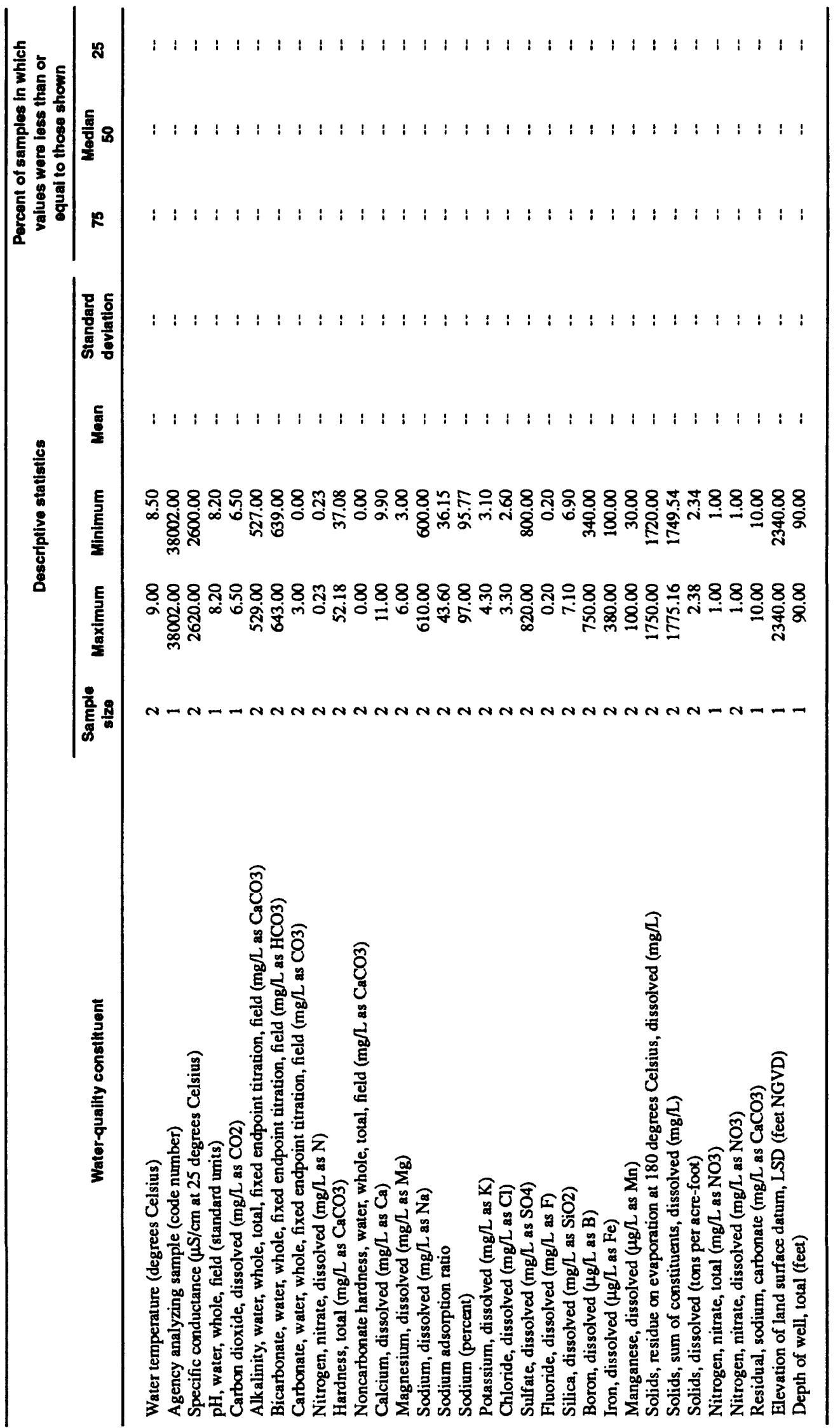




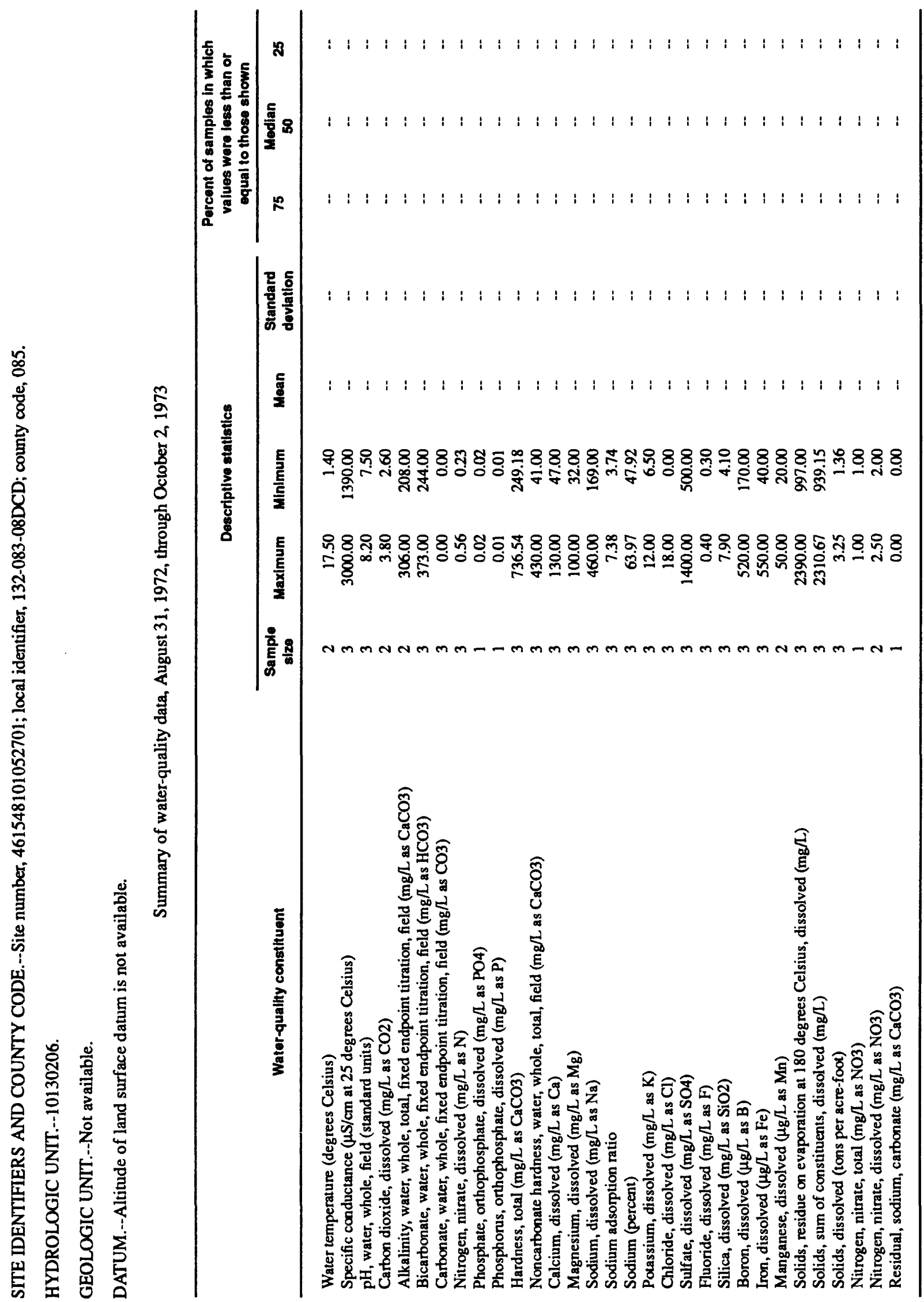




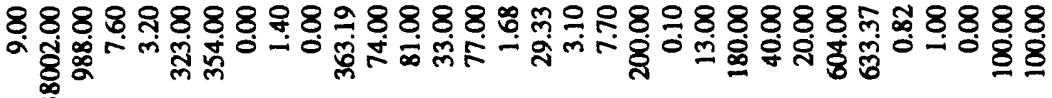

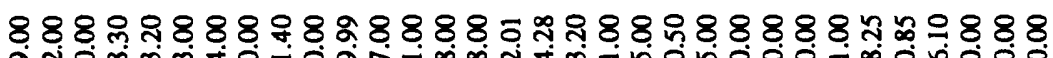

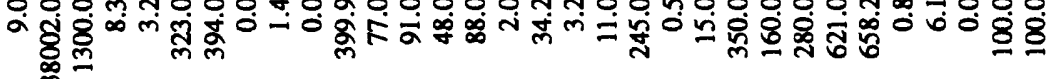

ชิ

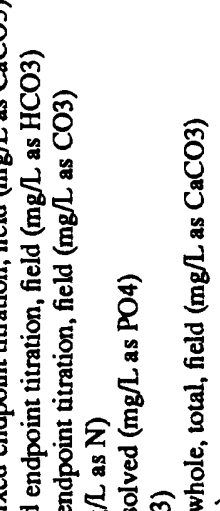

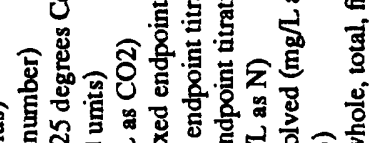

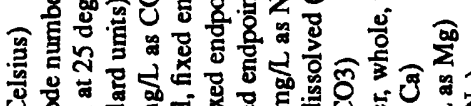

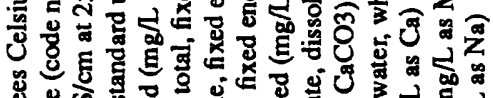

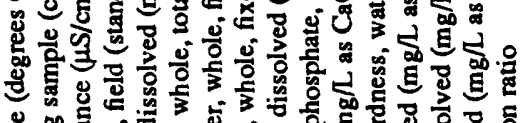

密

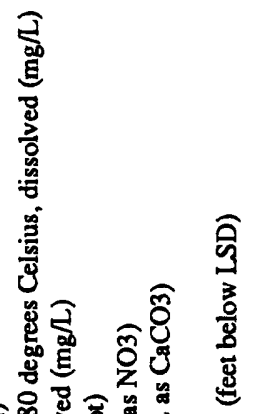

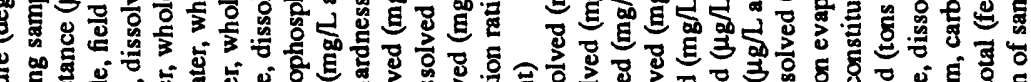

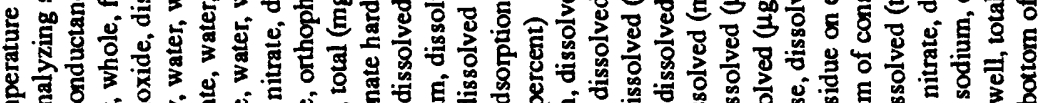

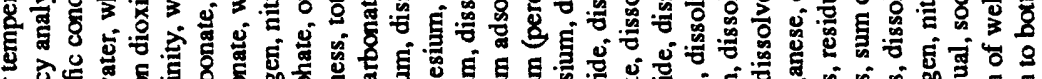

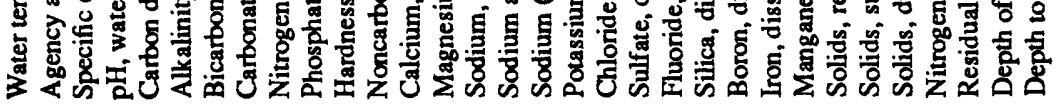




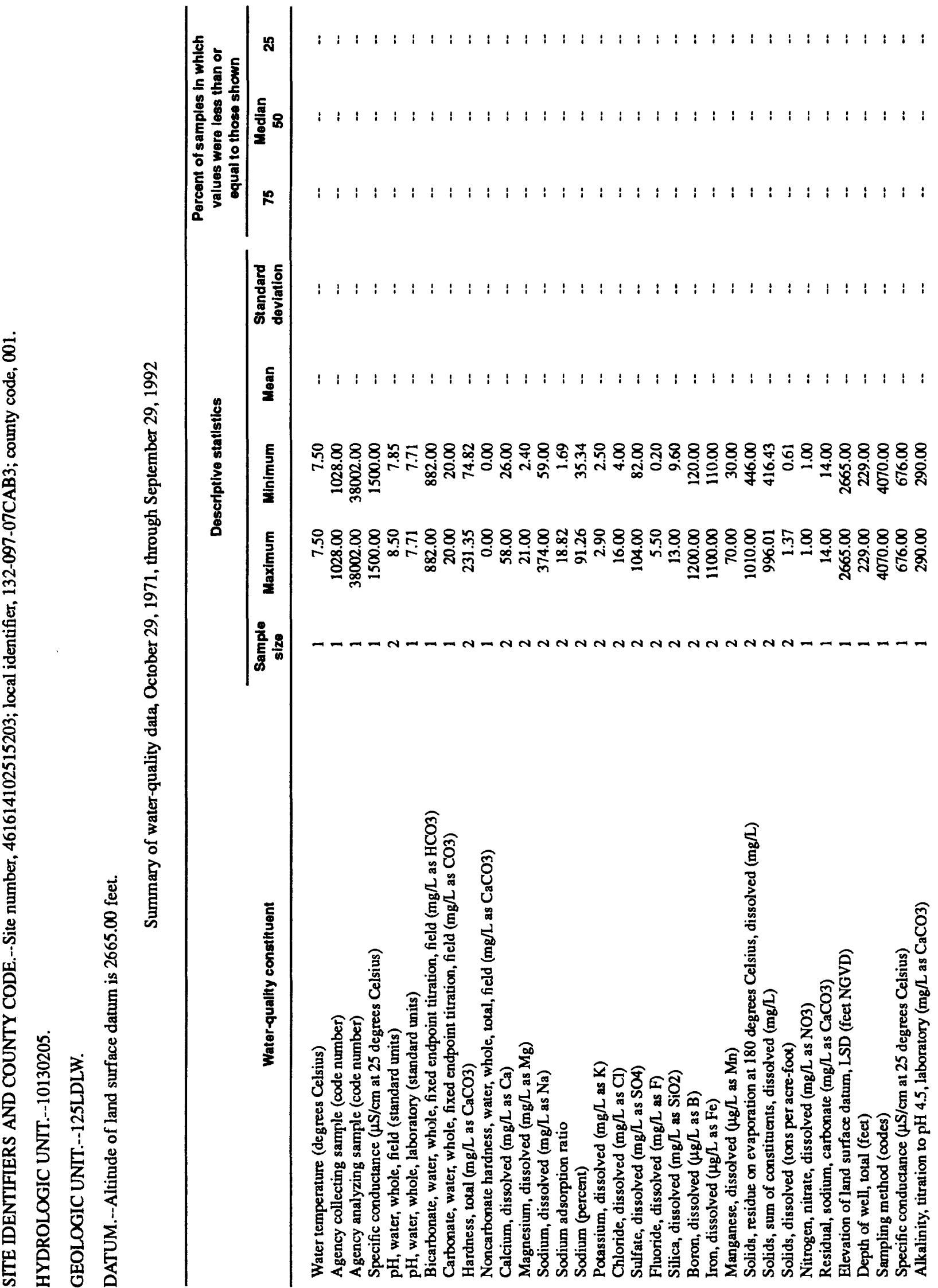




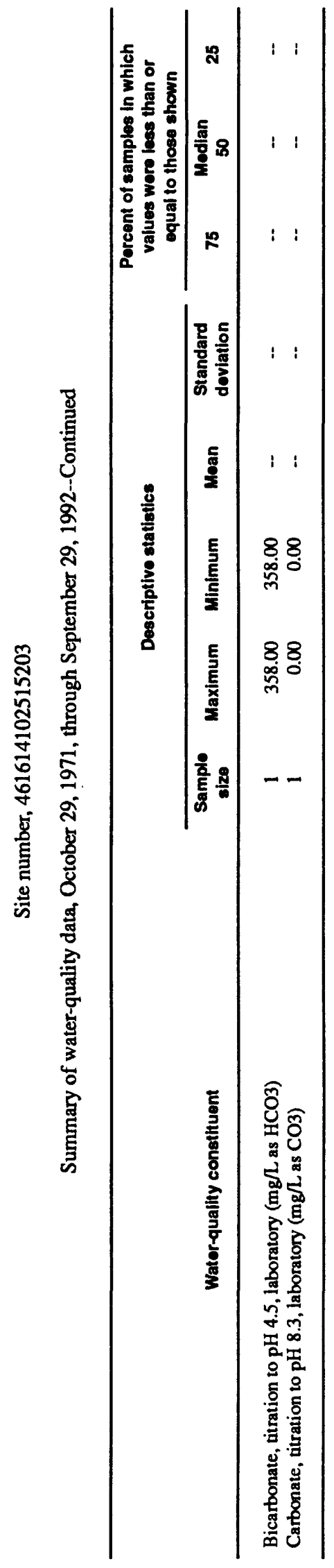




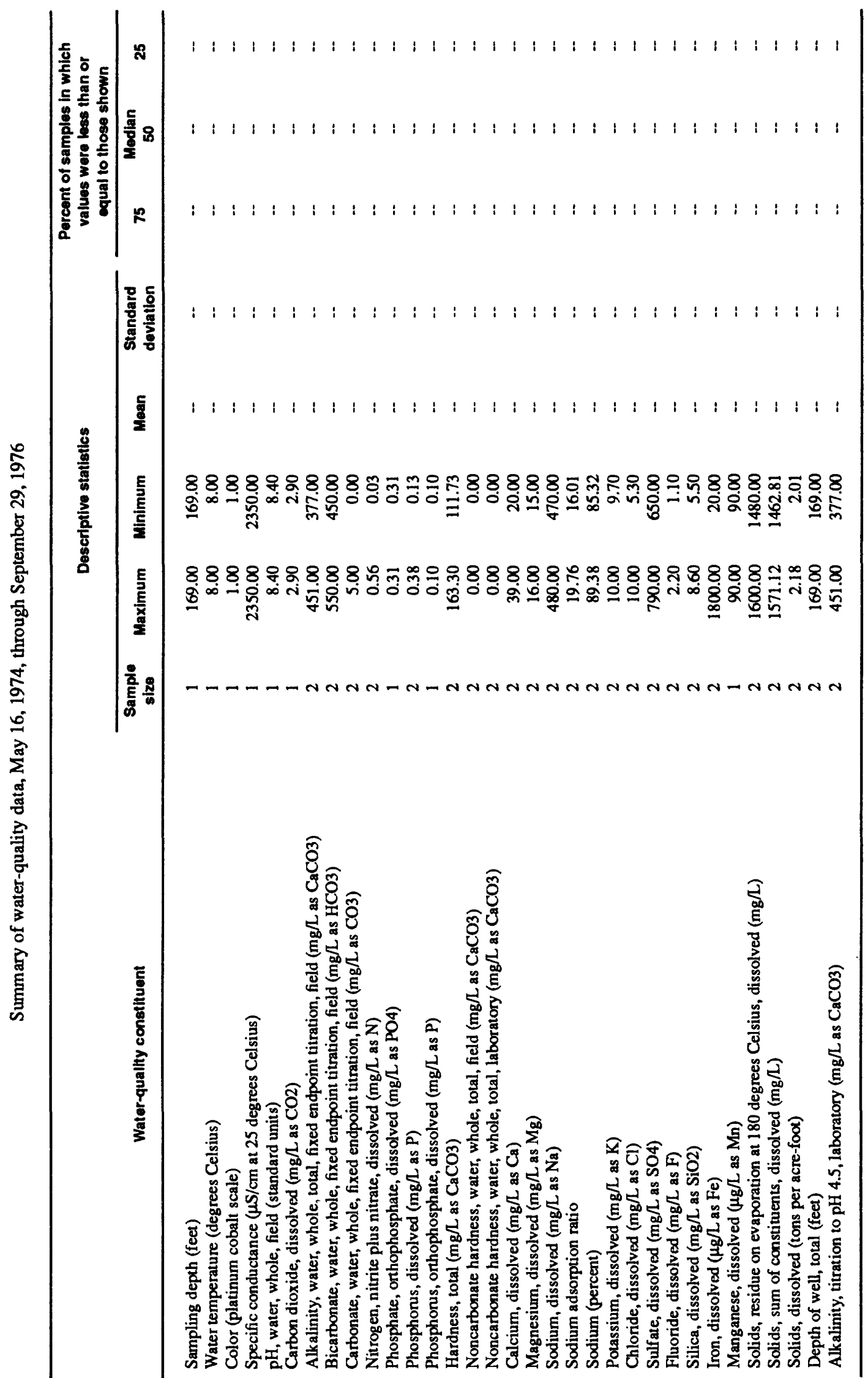




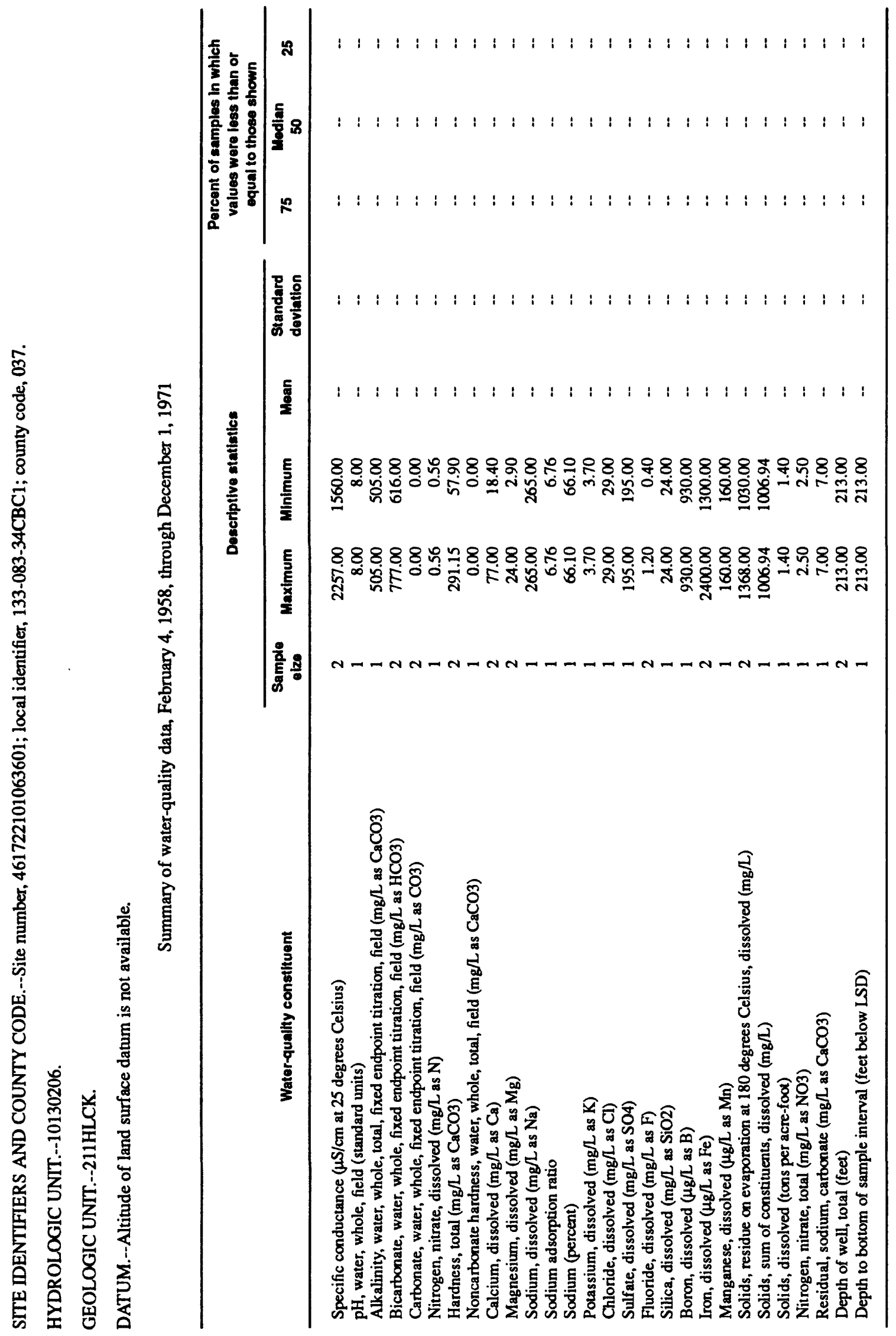




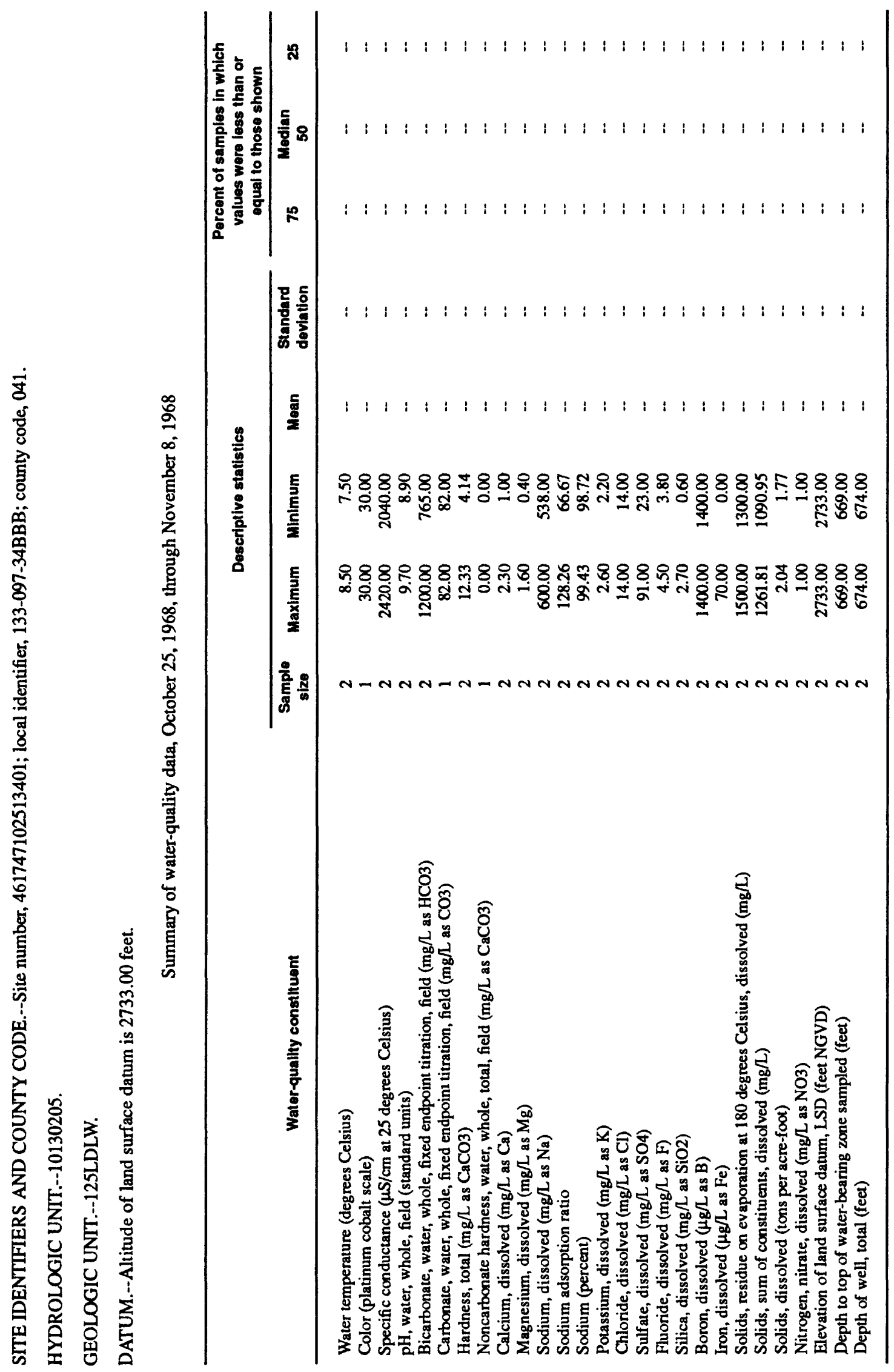




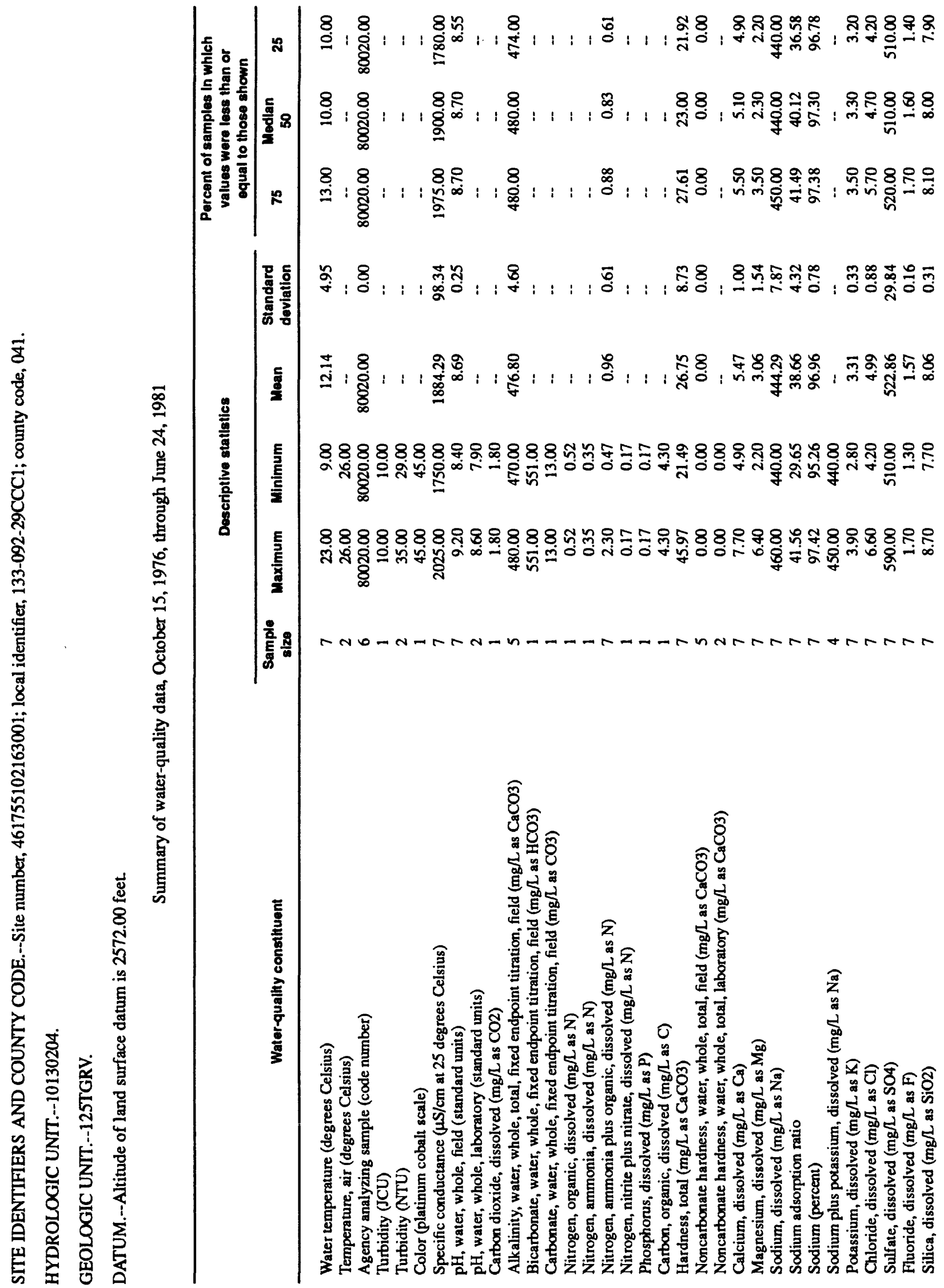




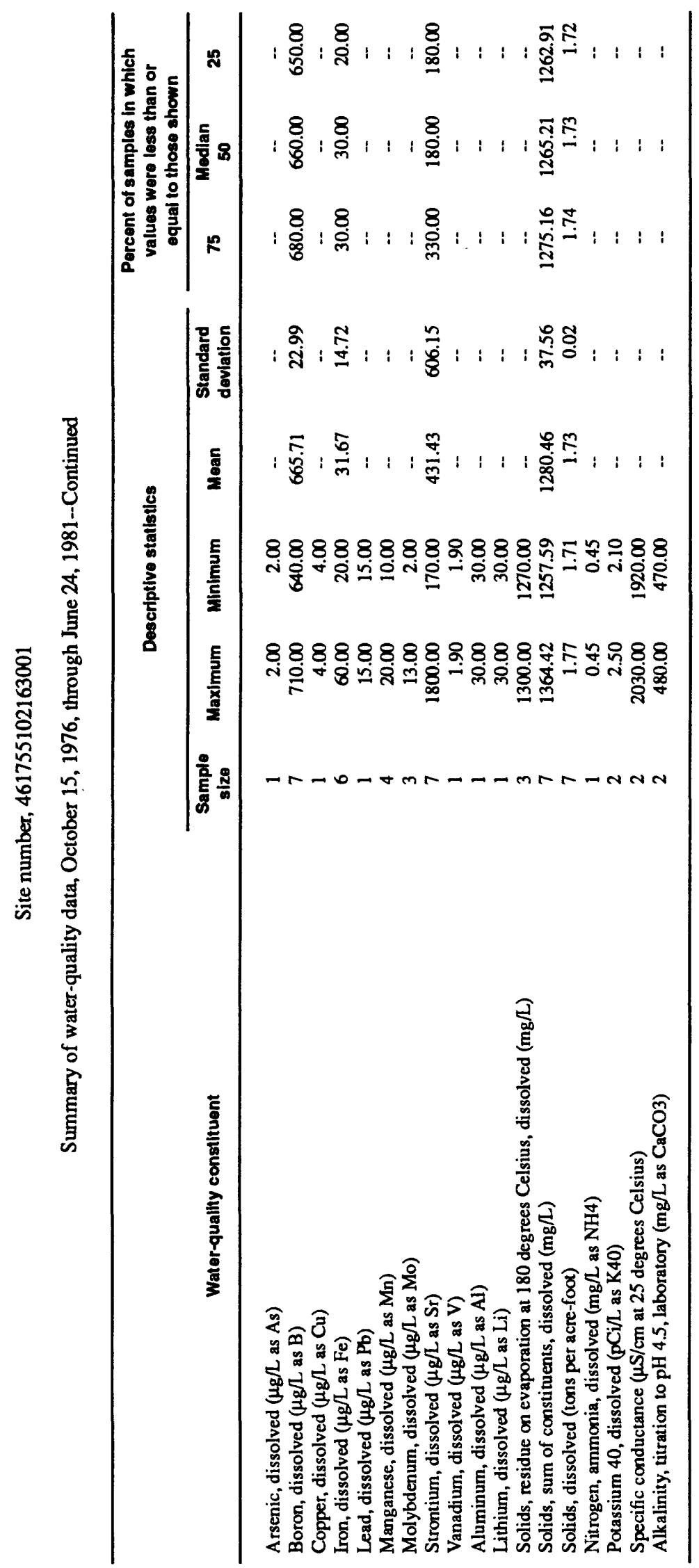




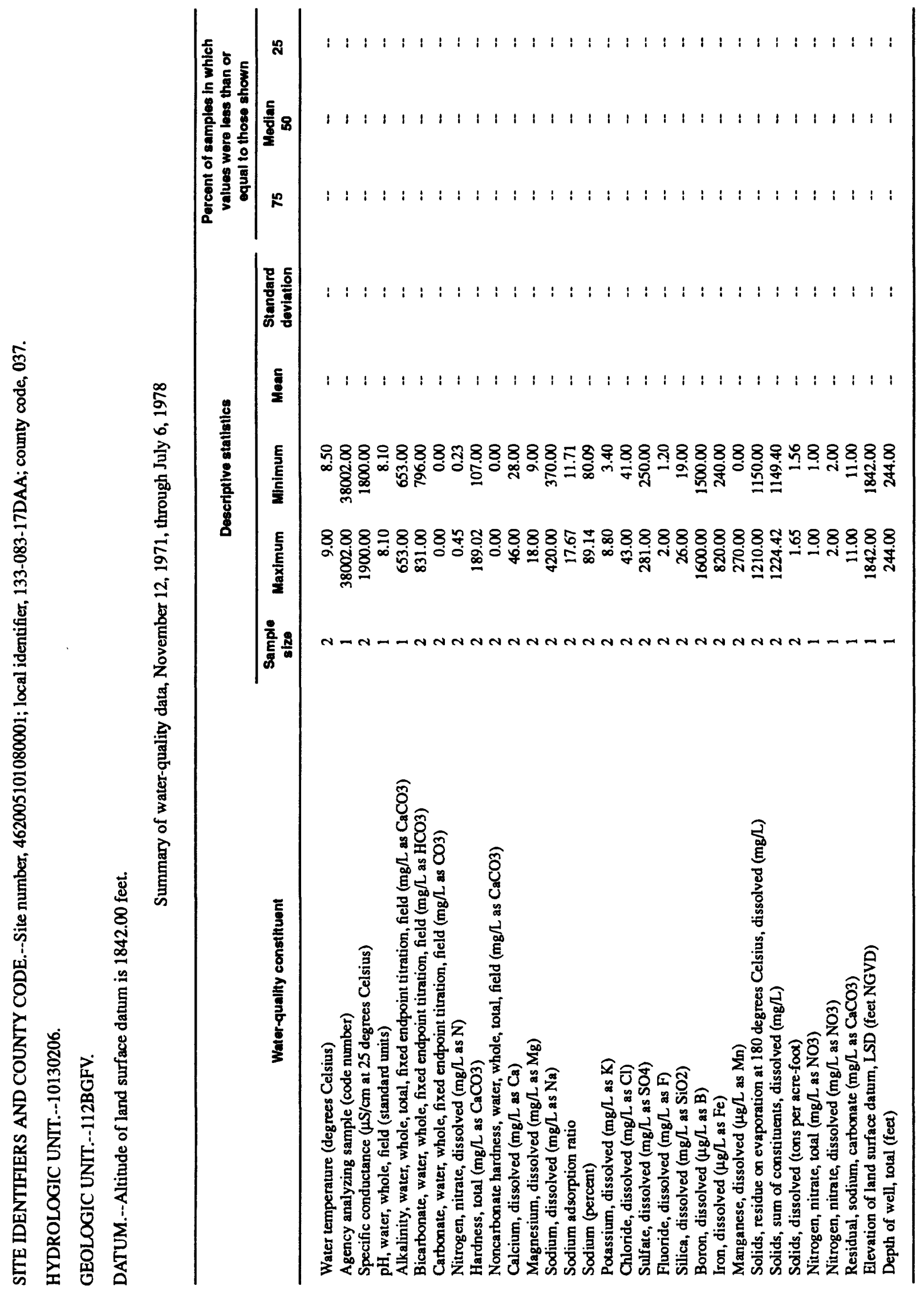




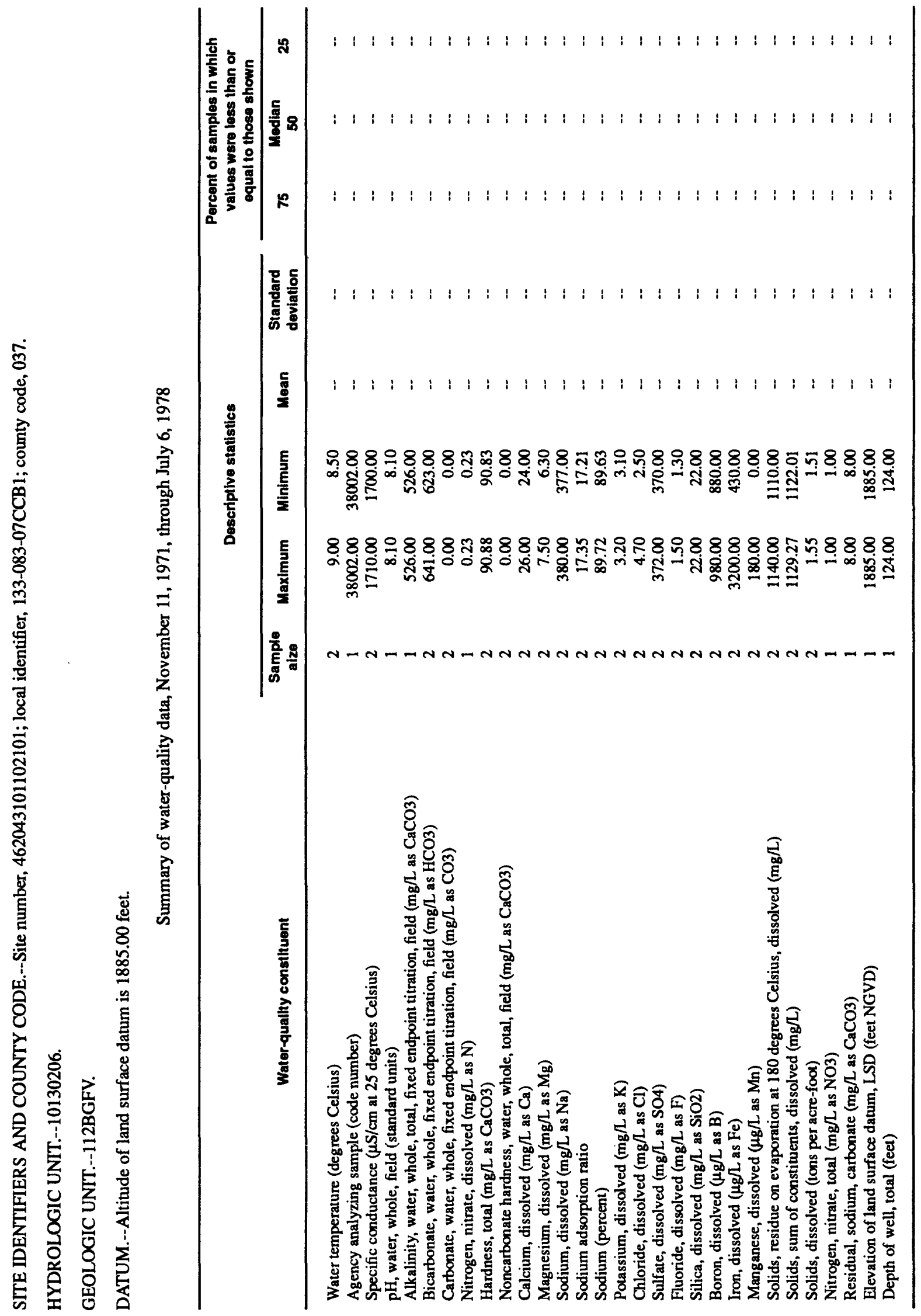




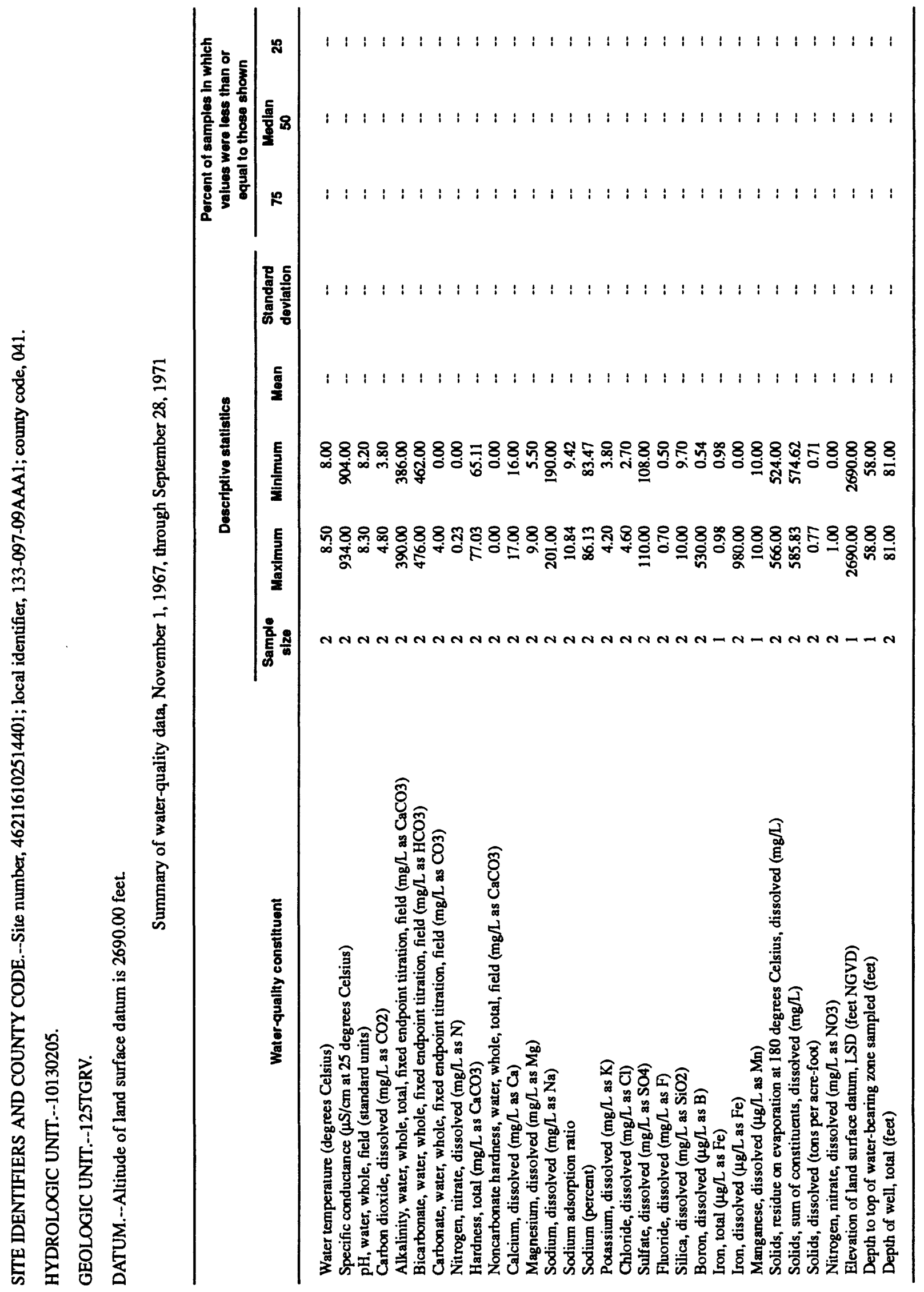




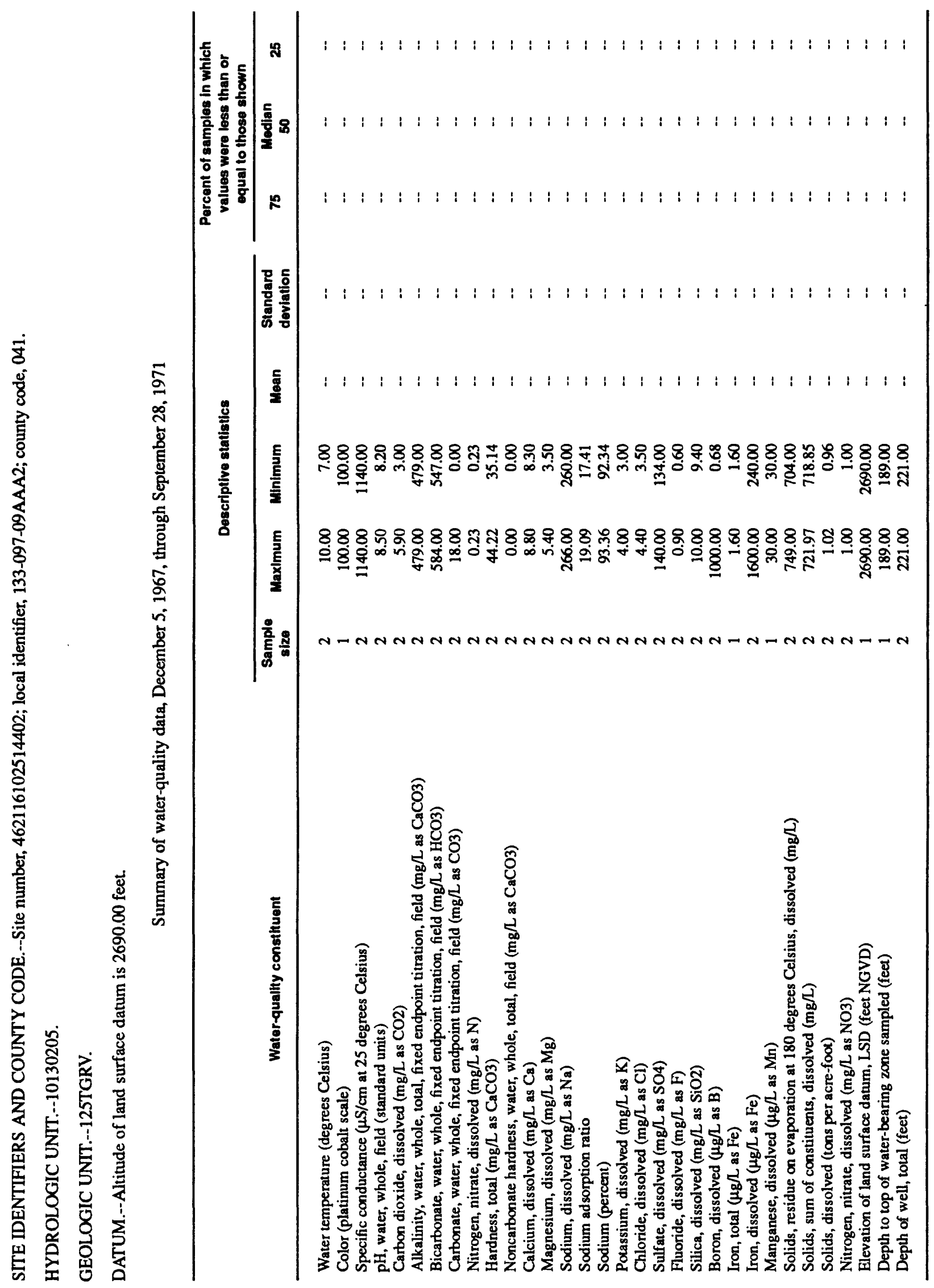




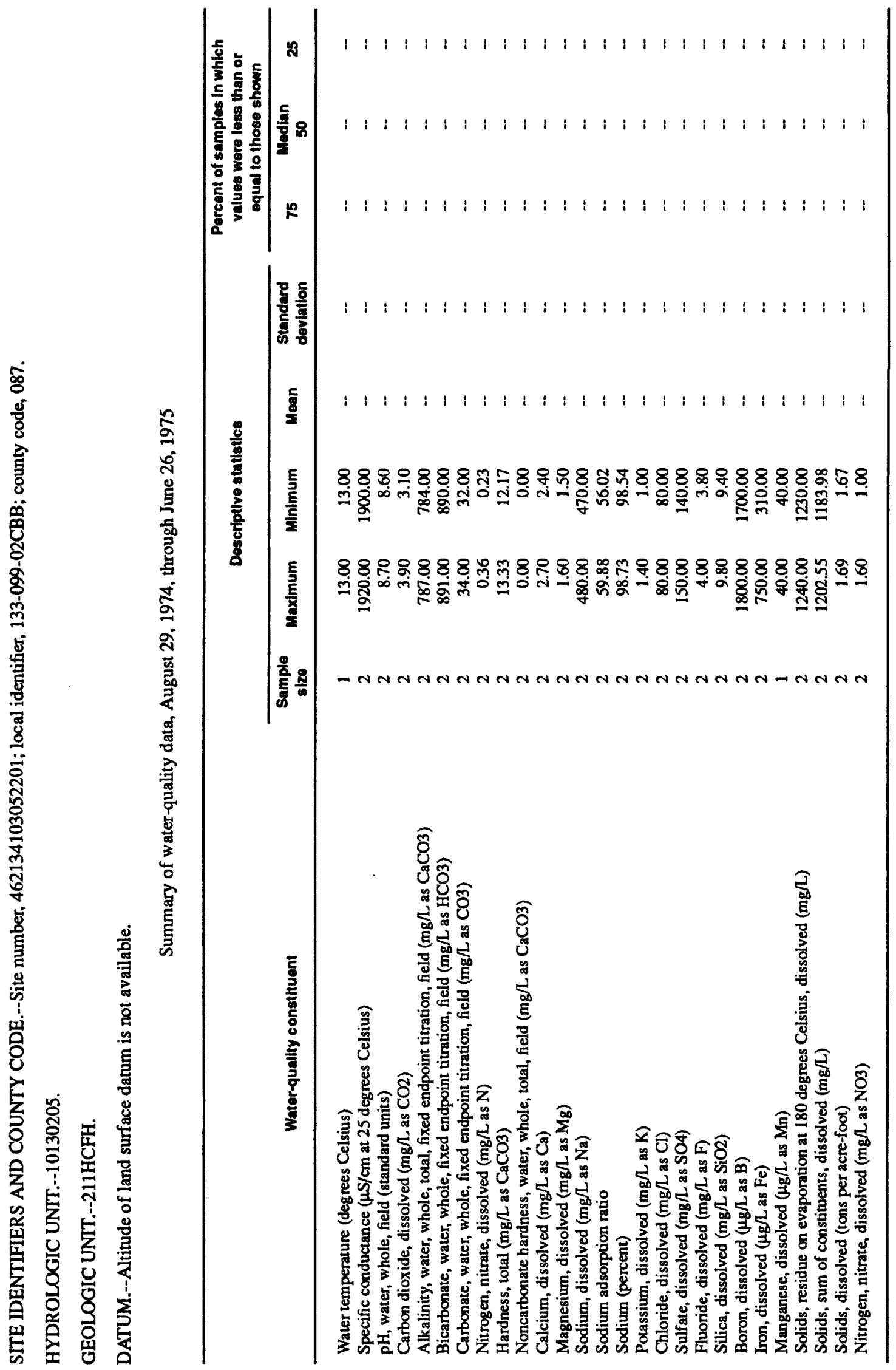




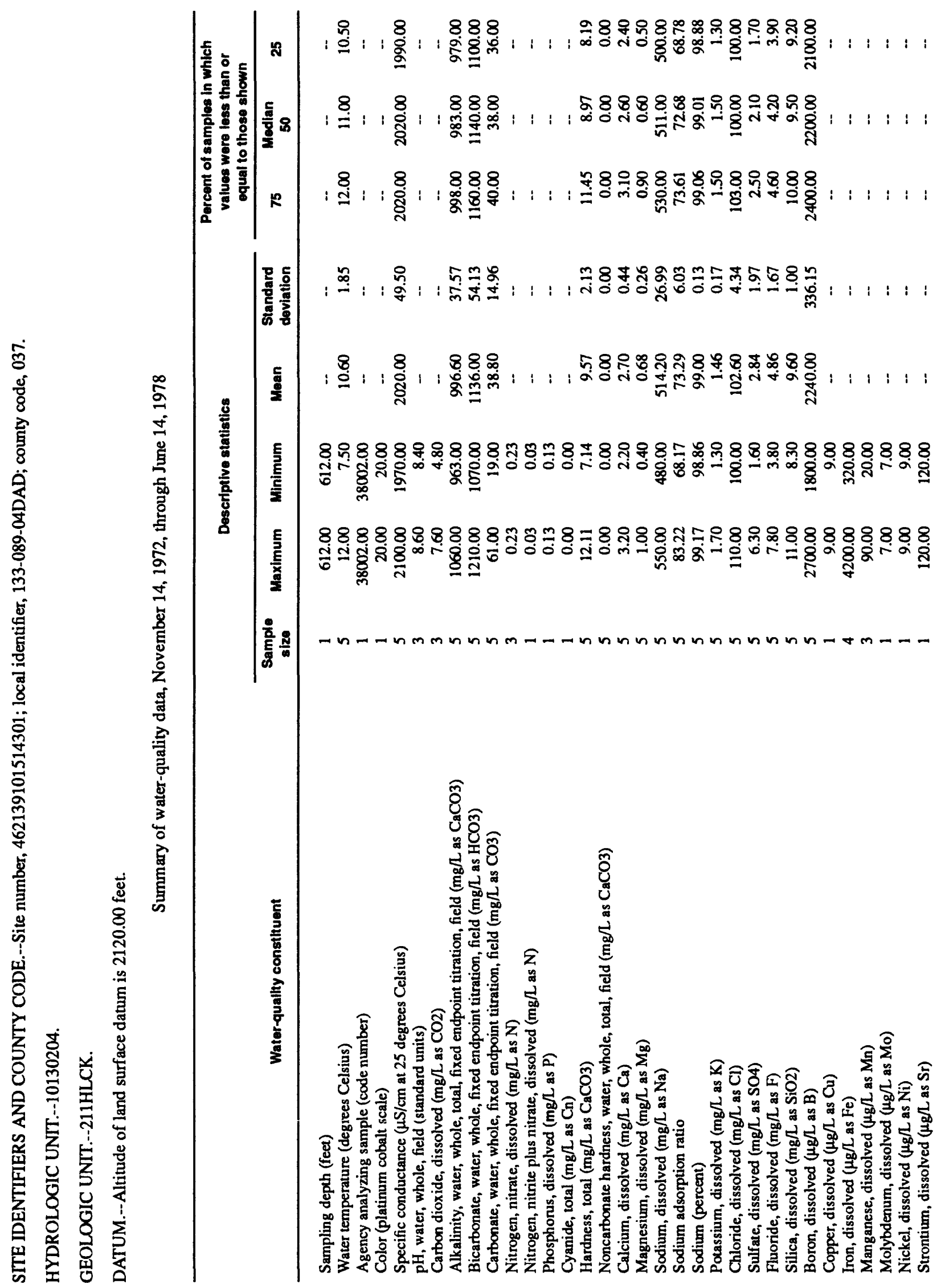




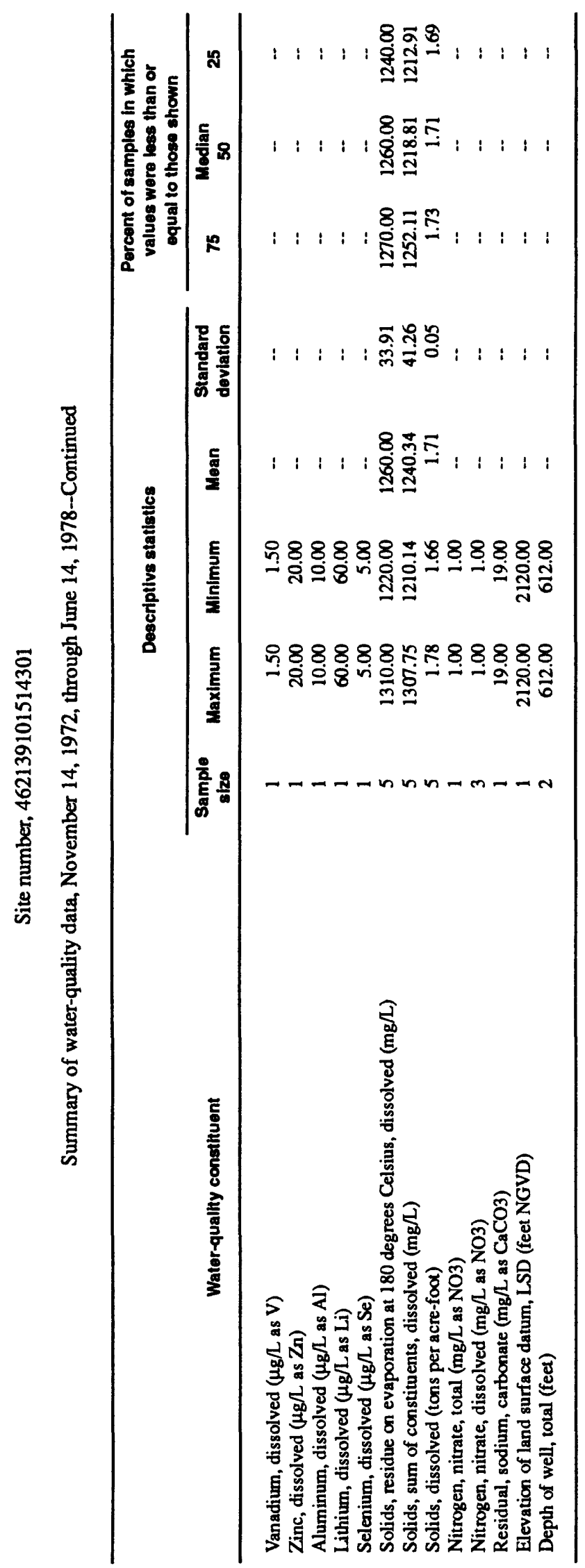




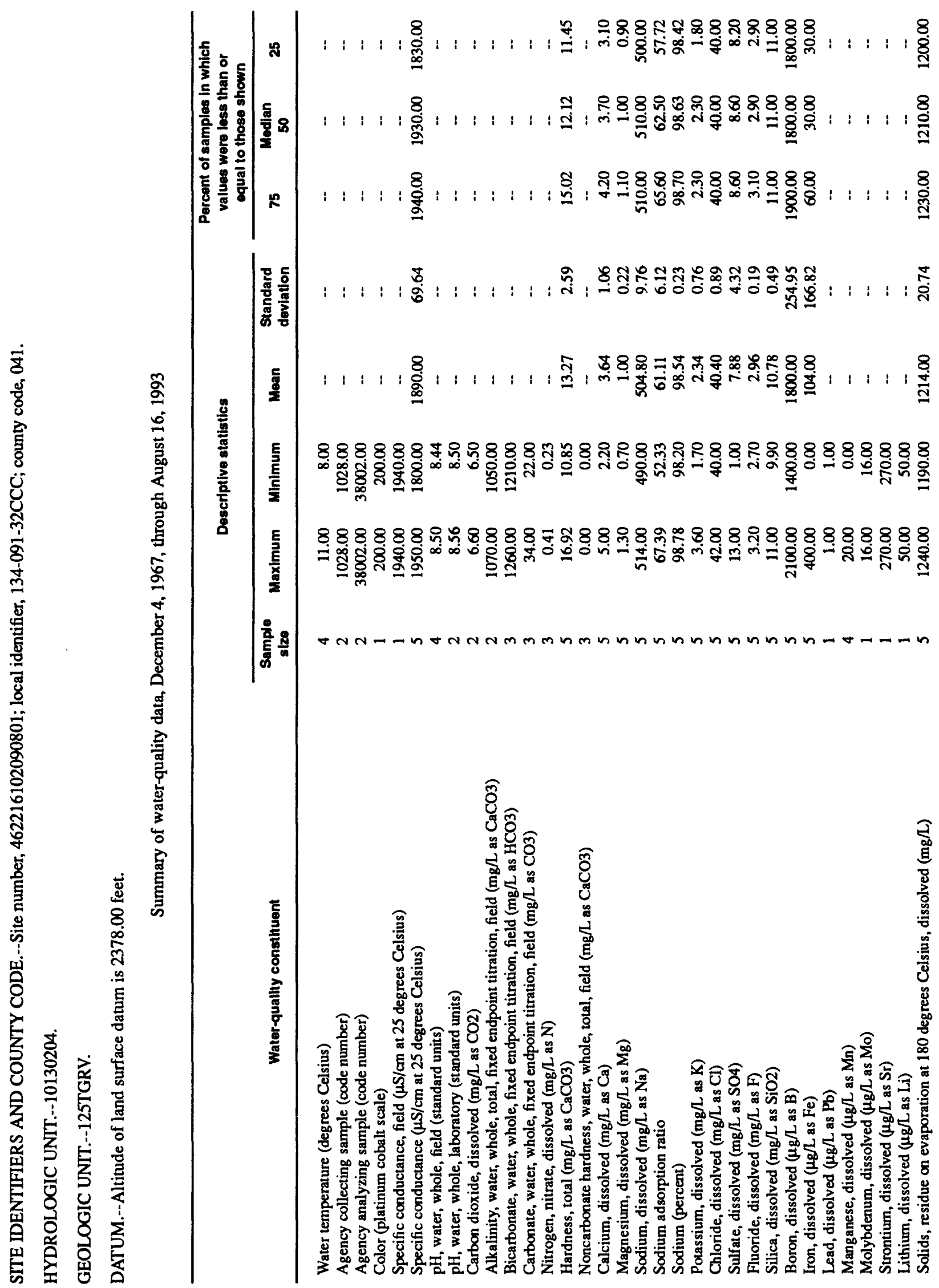




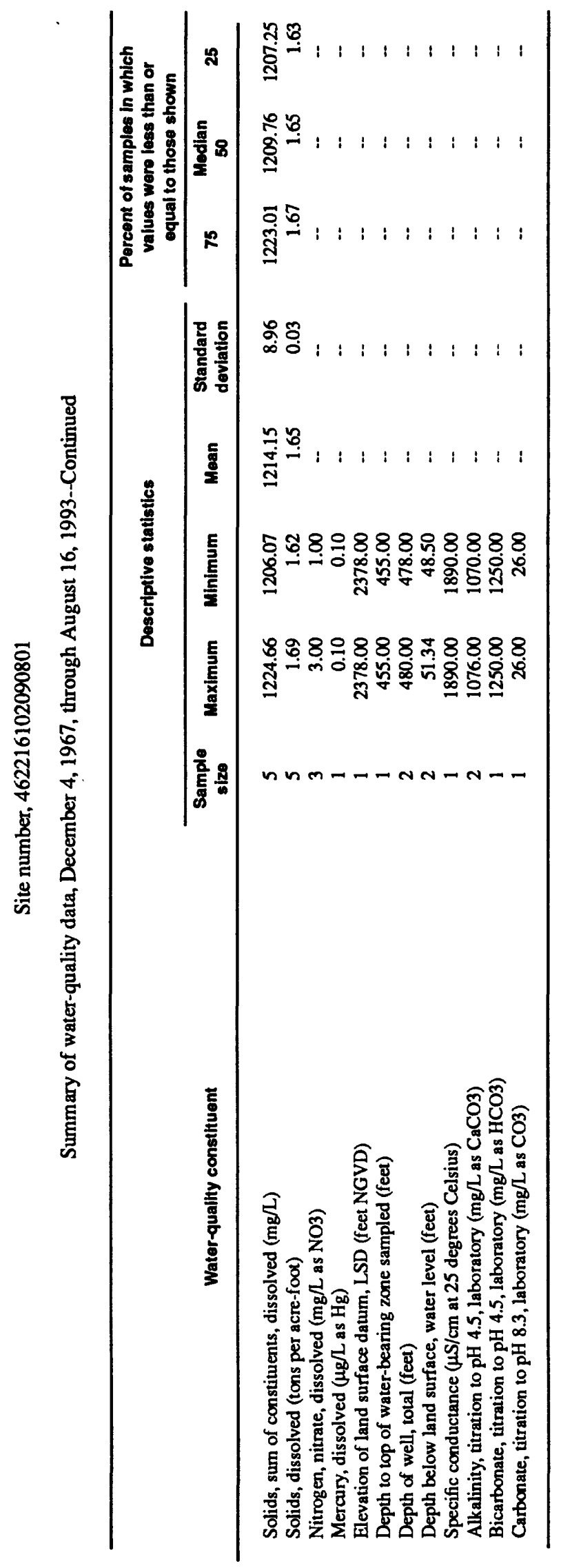




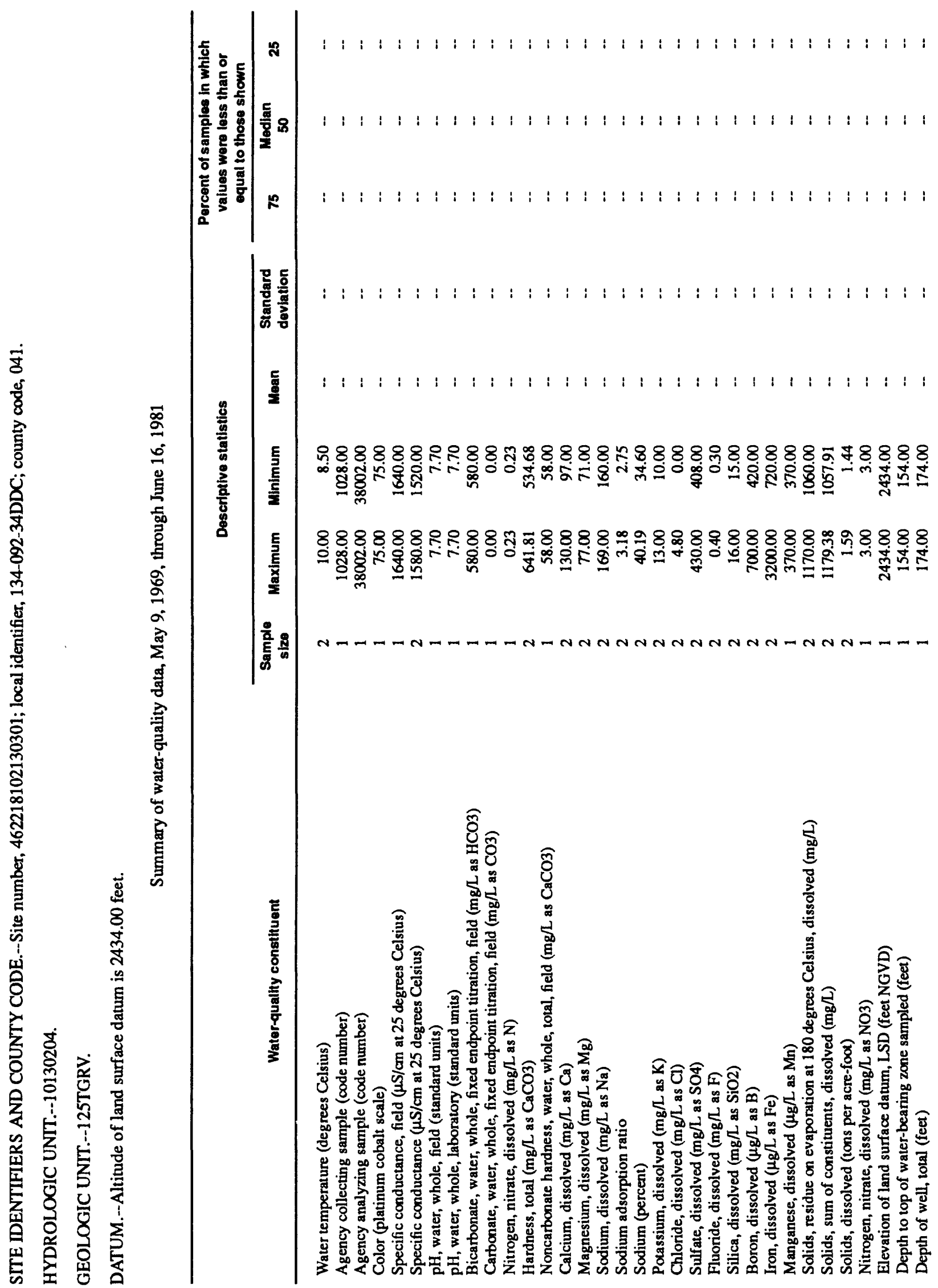




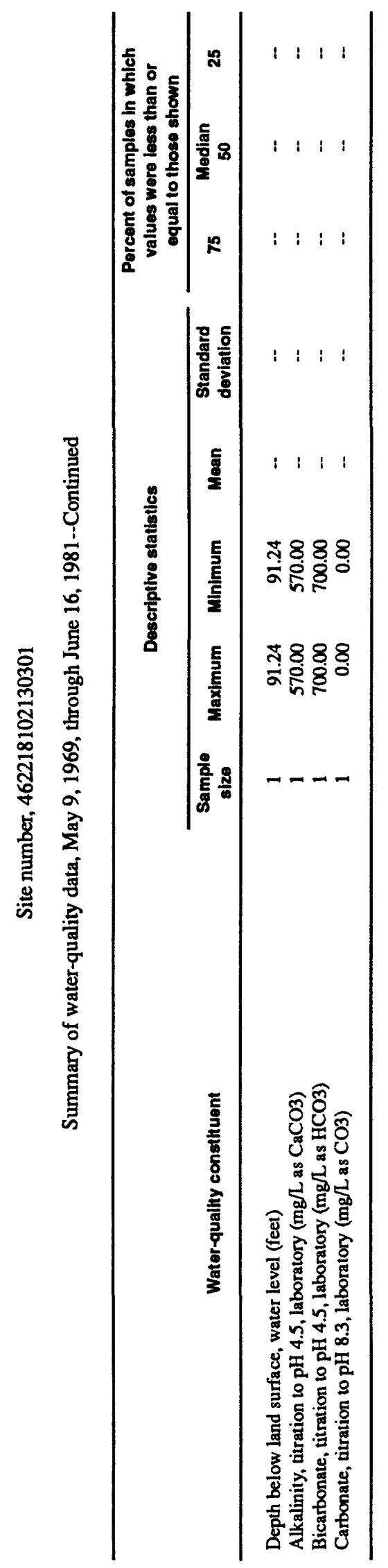




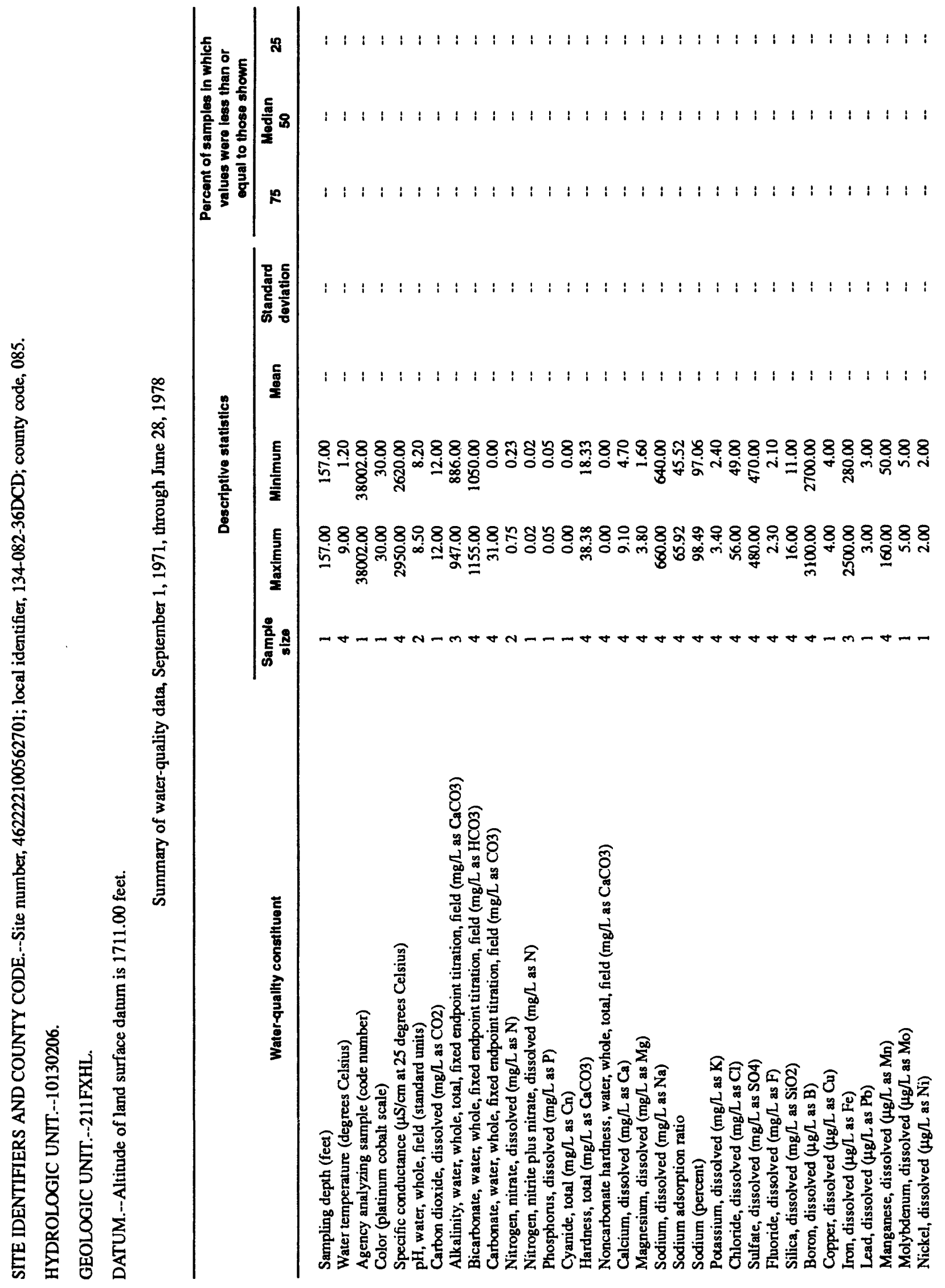




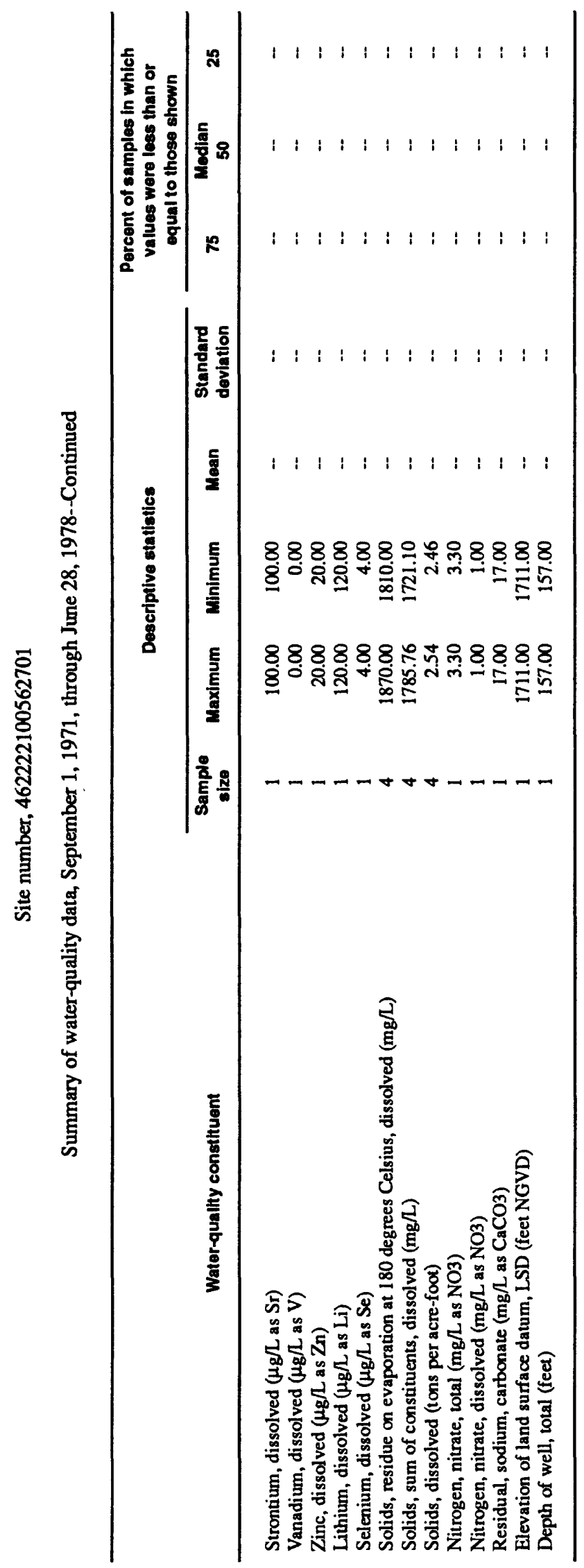




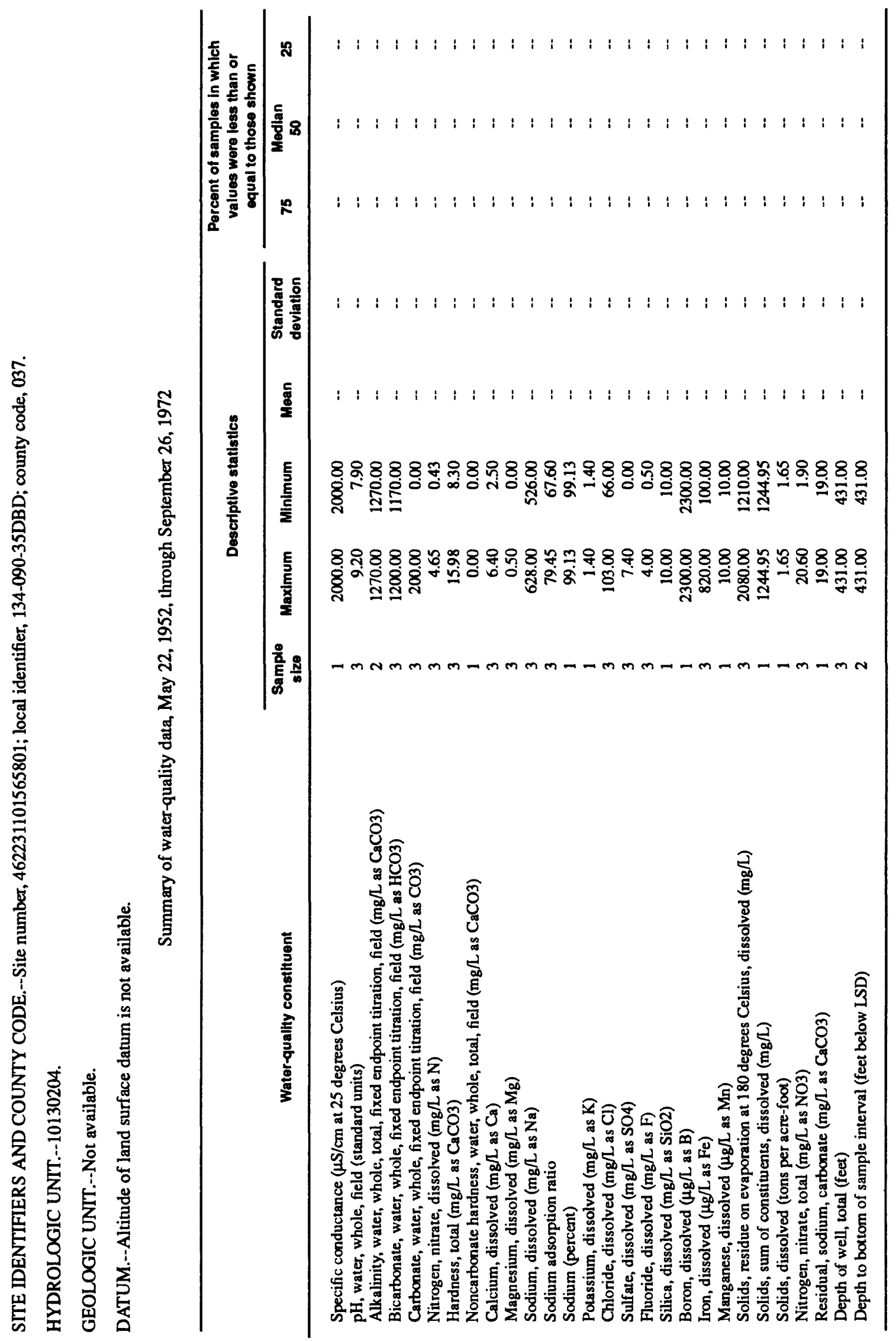




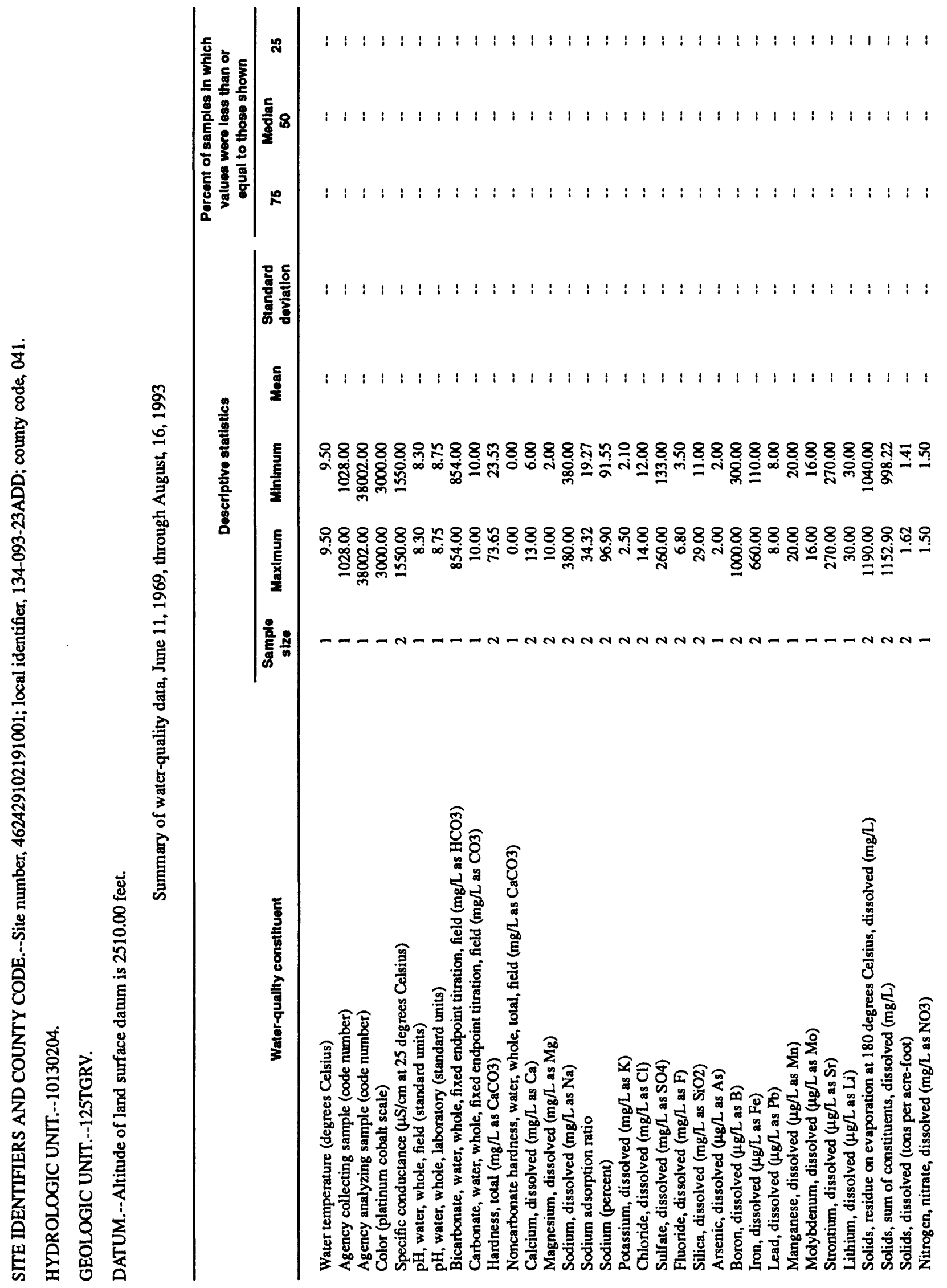




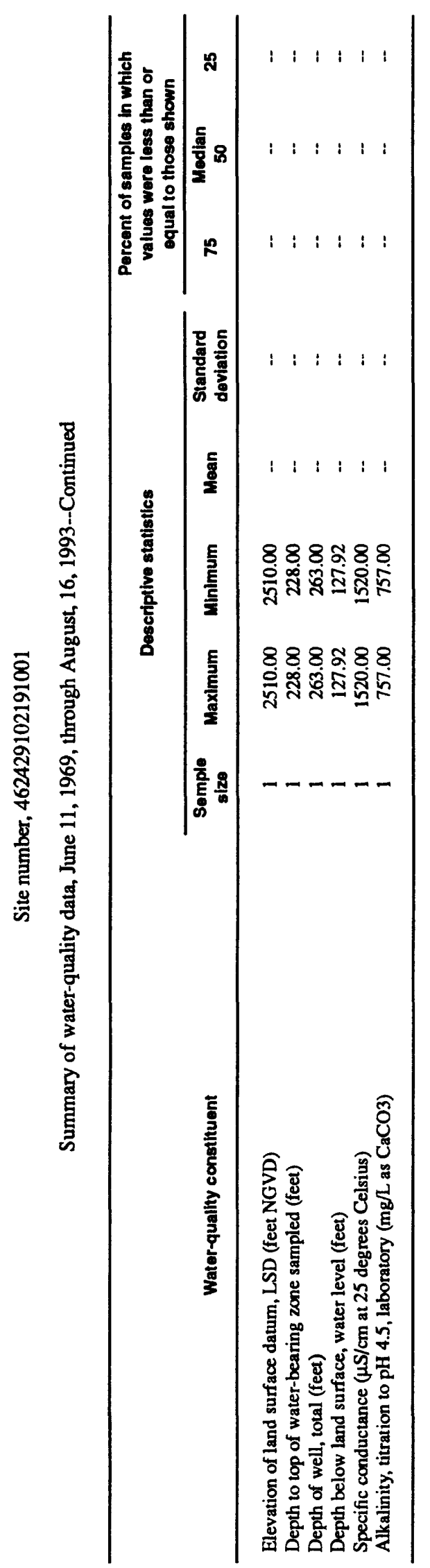




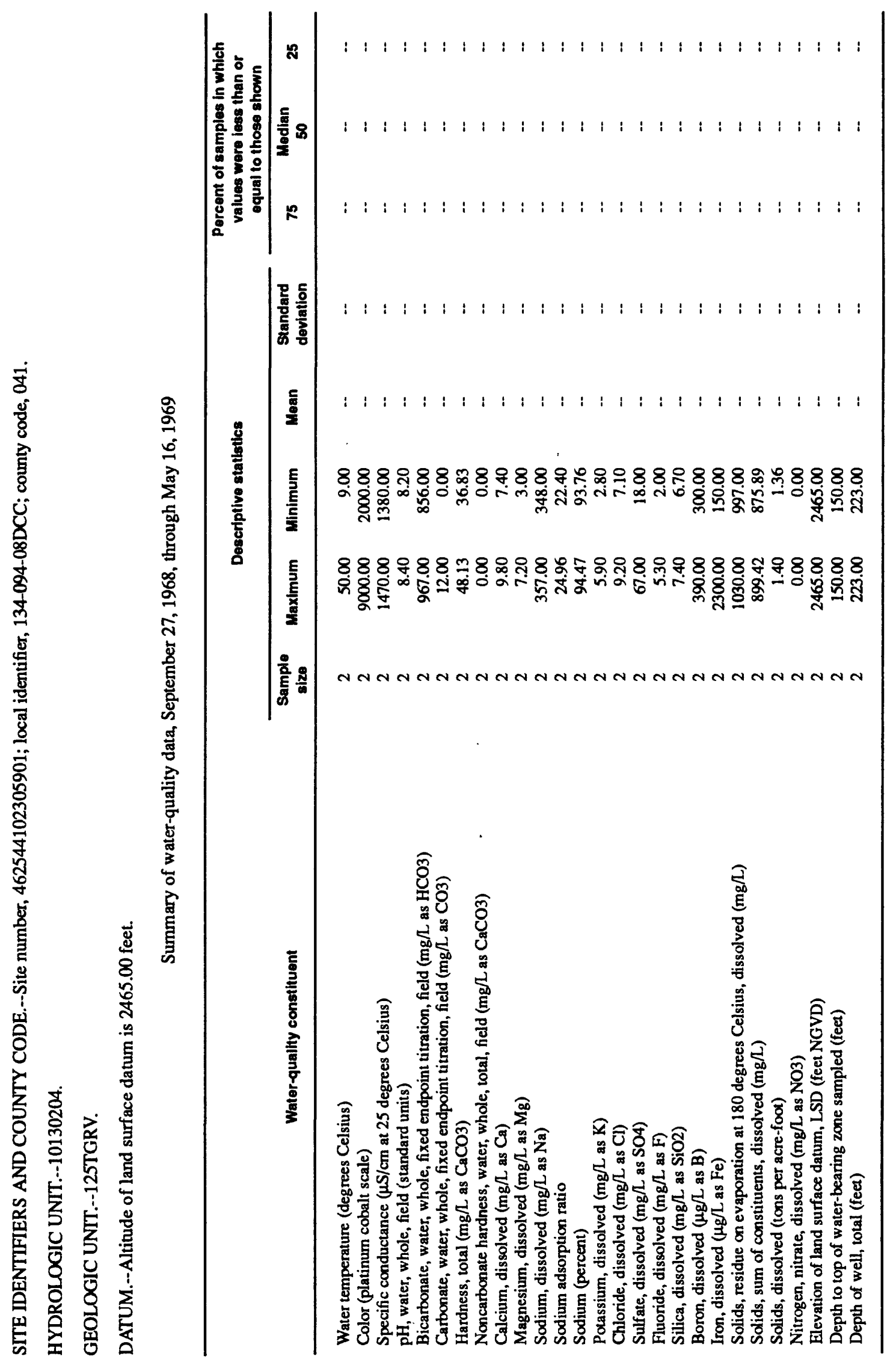




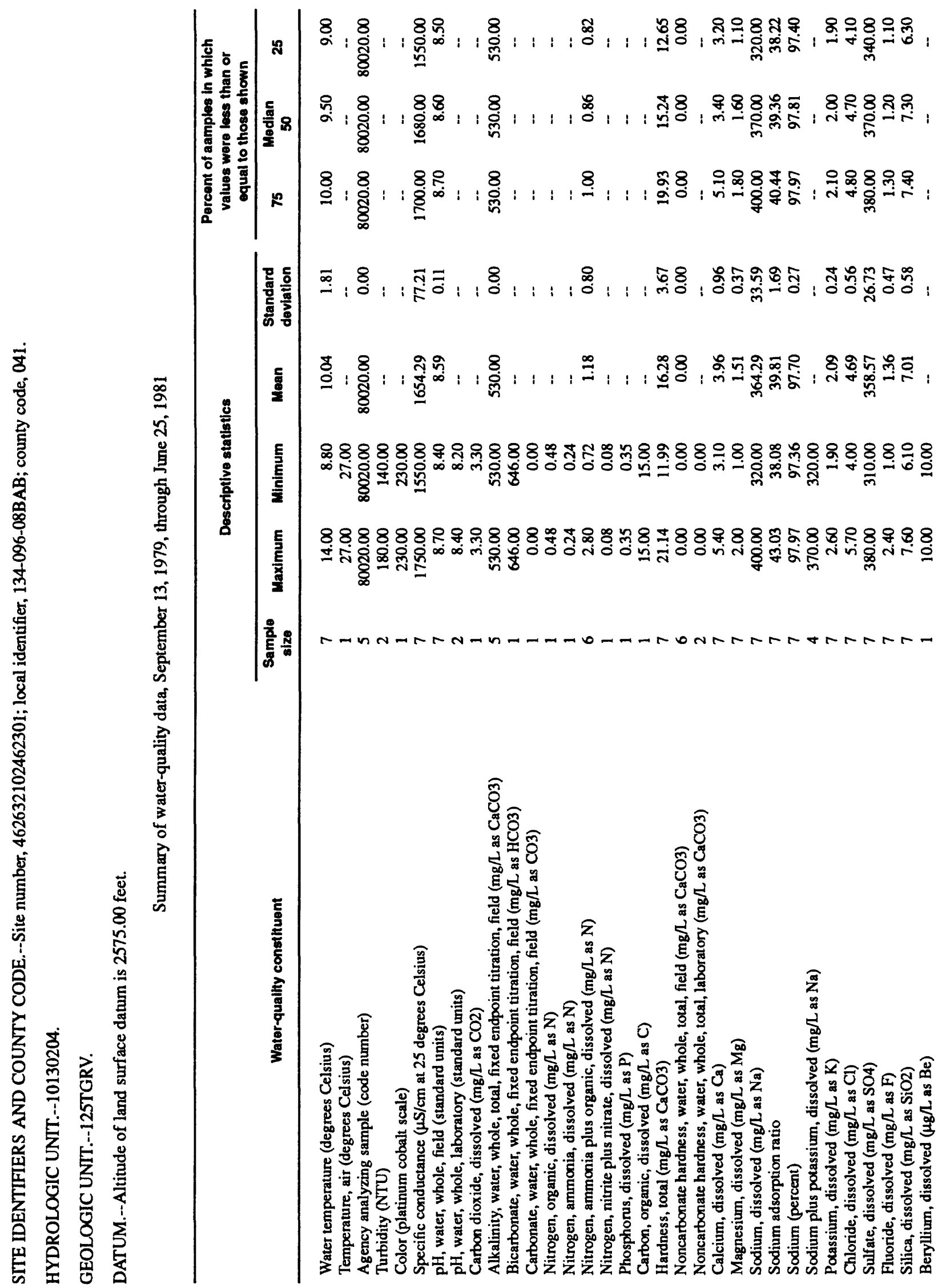




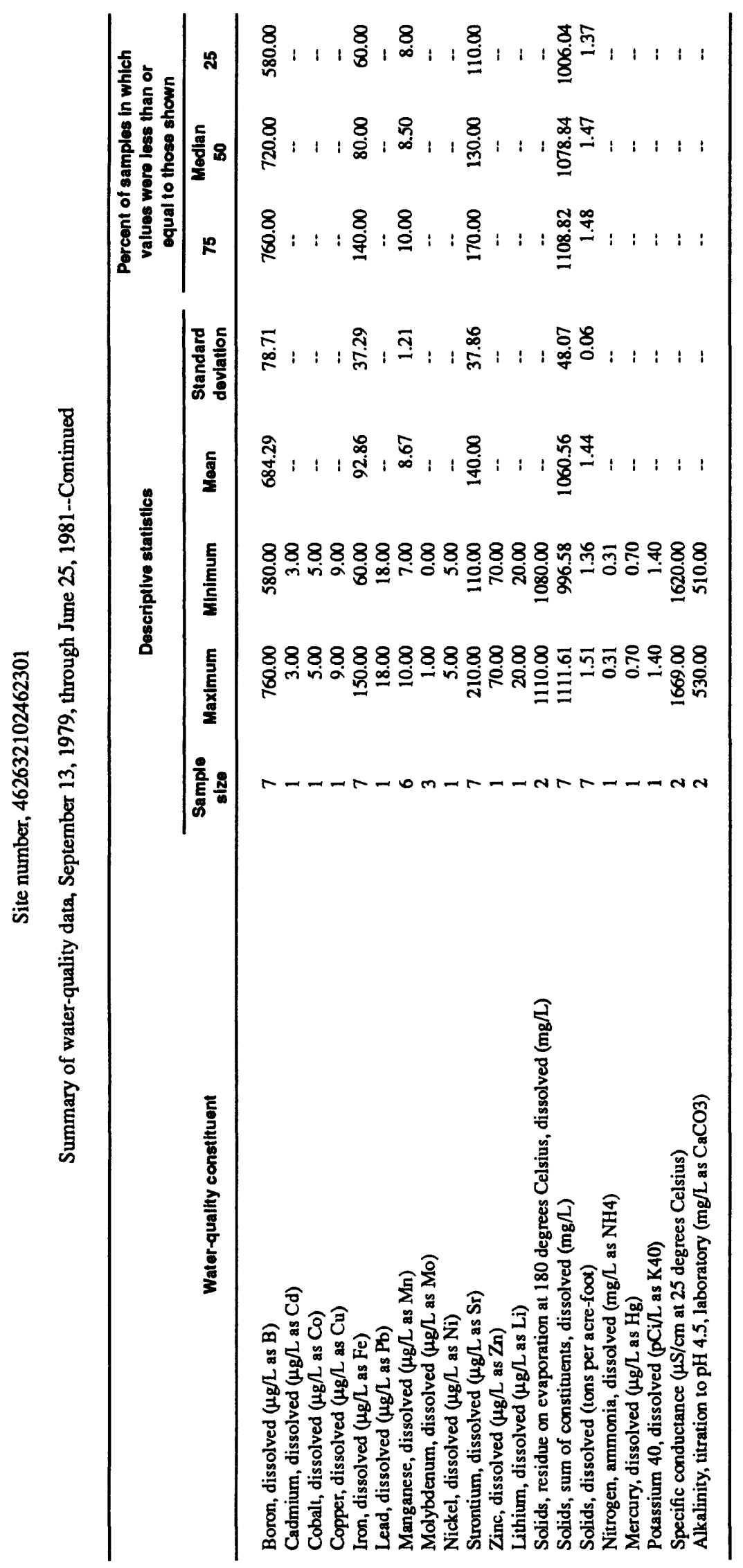



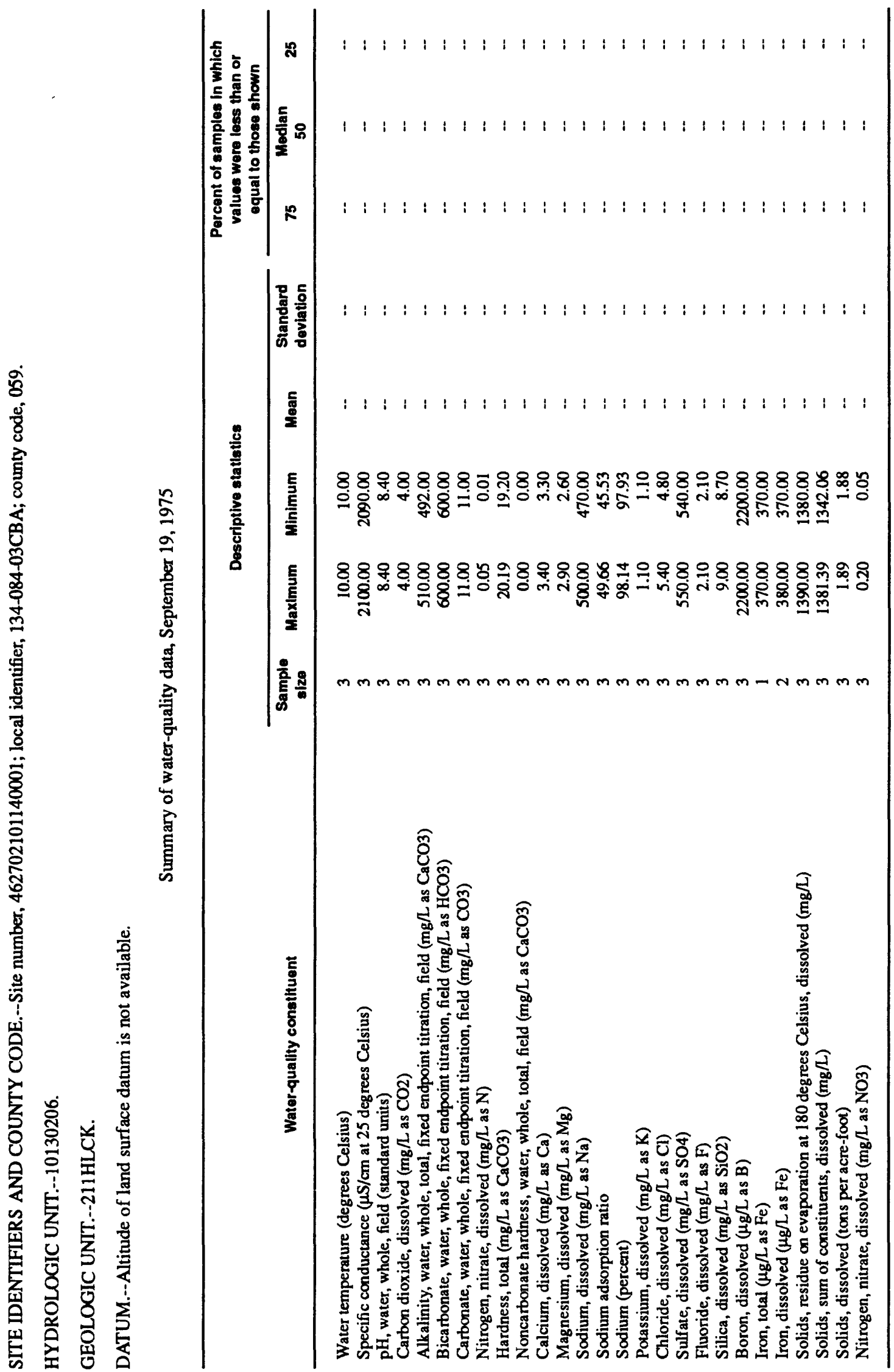


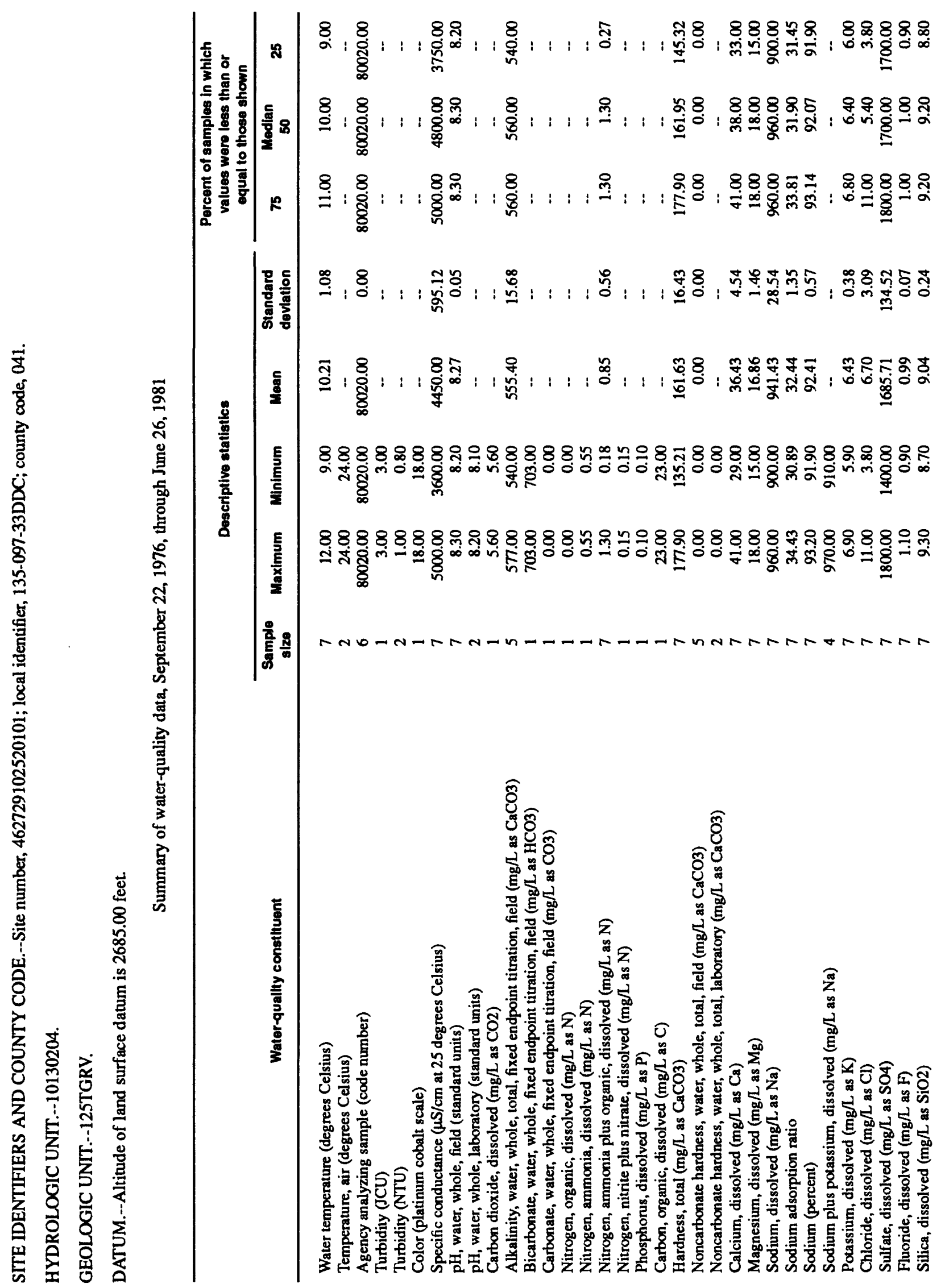




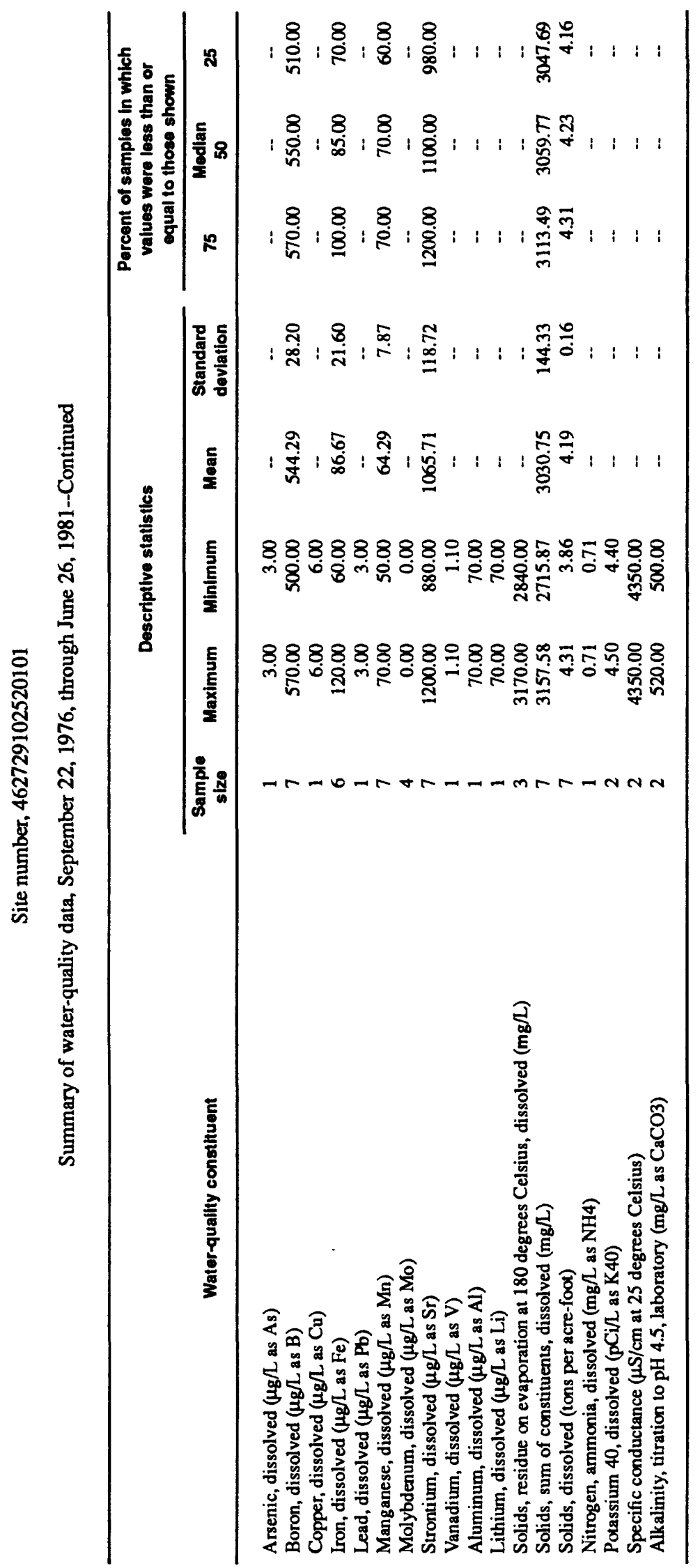




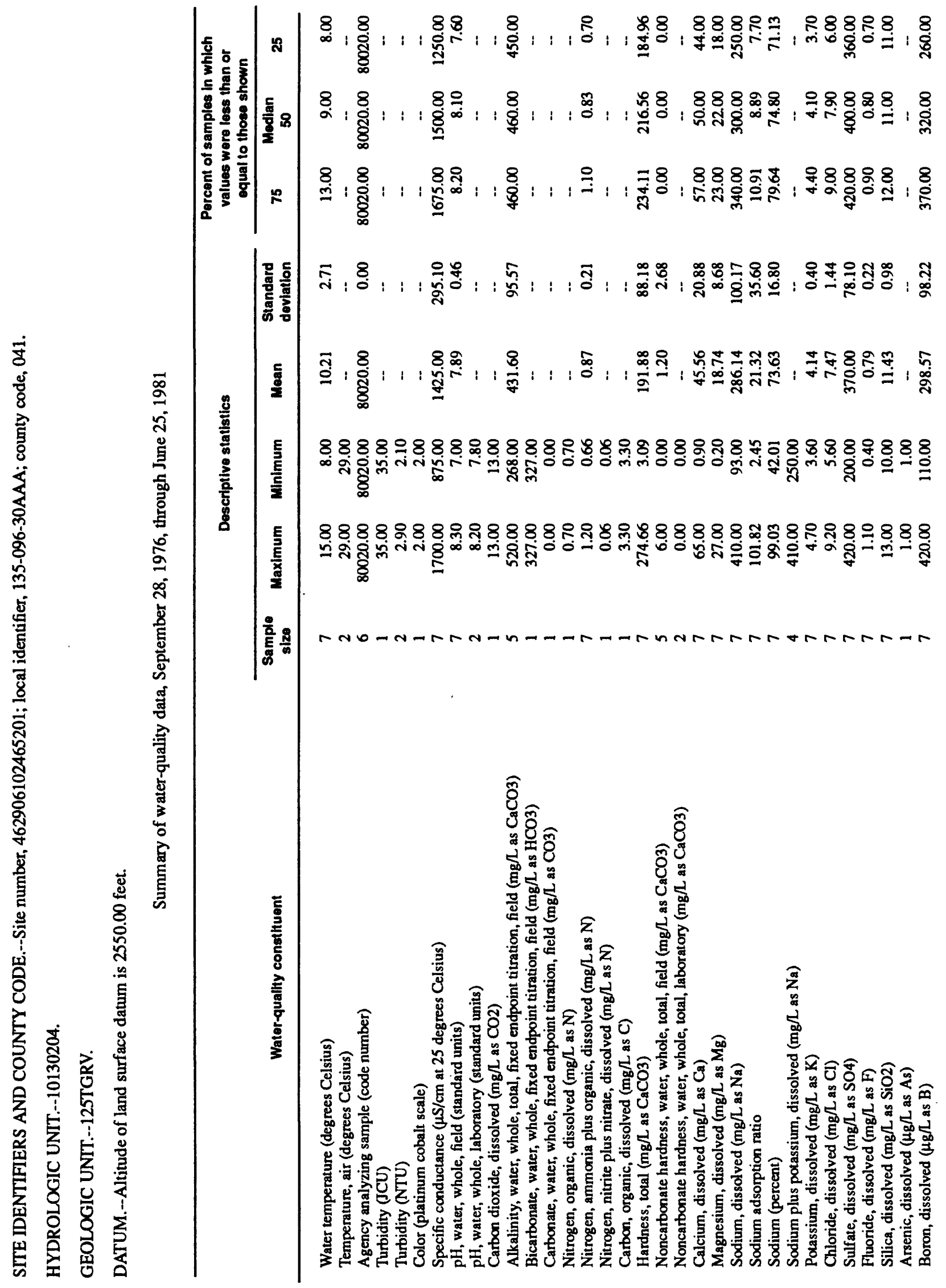




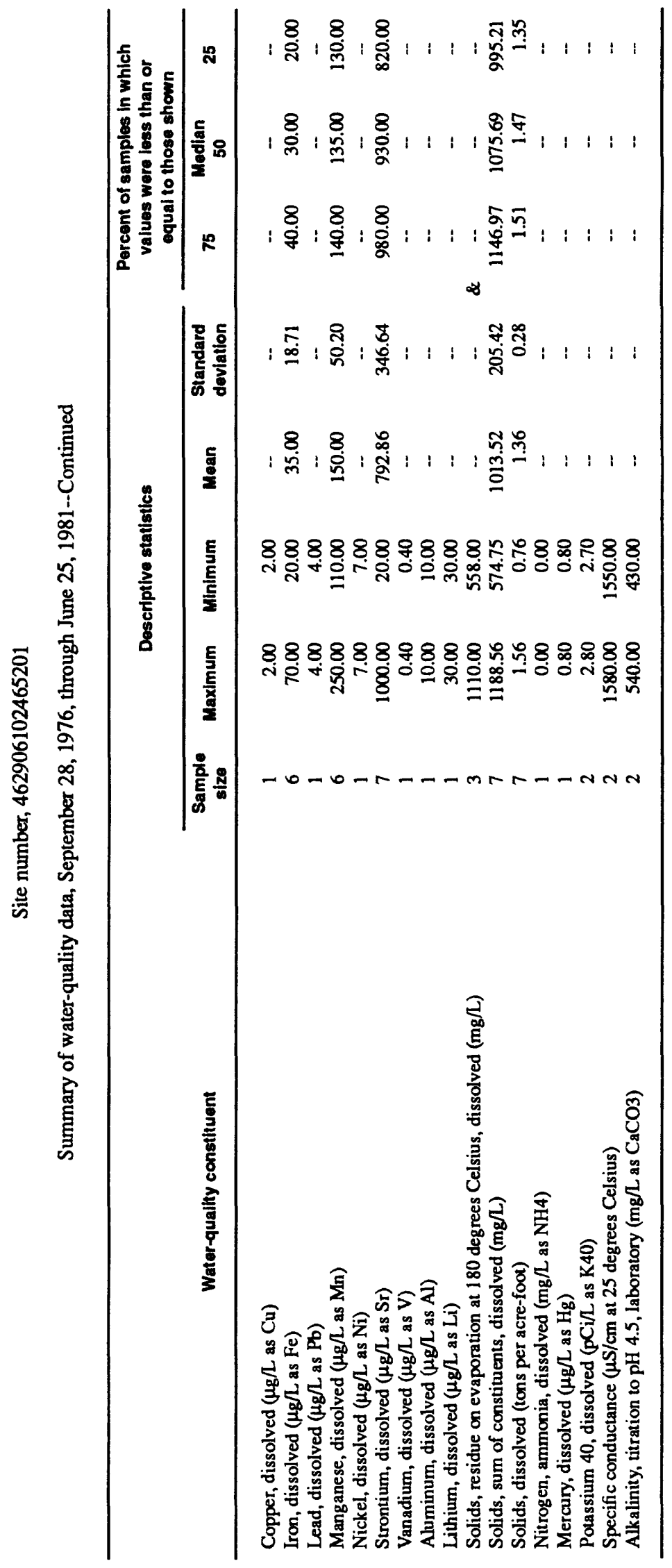




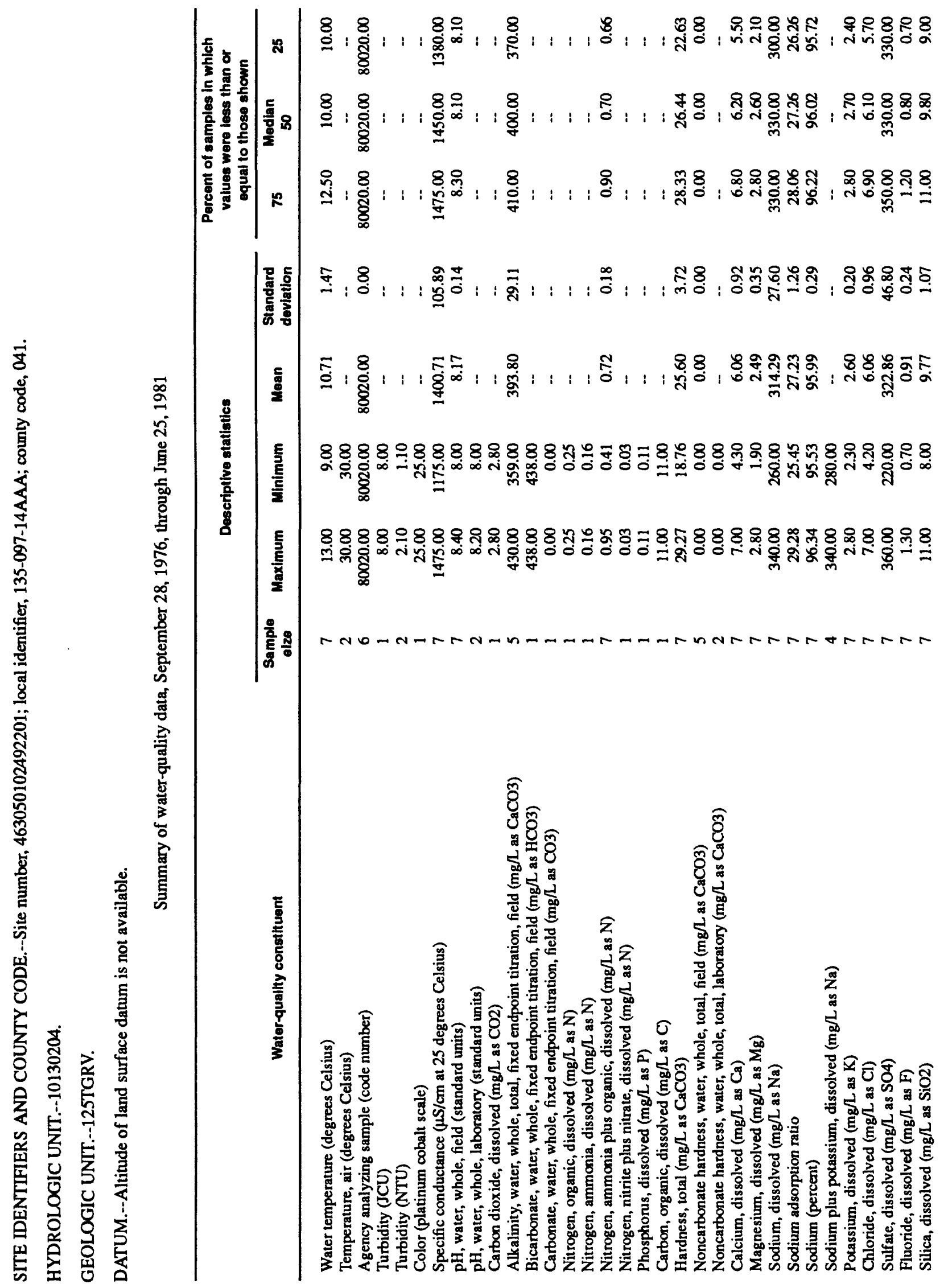




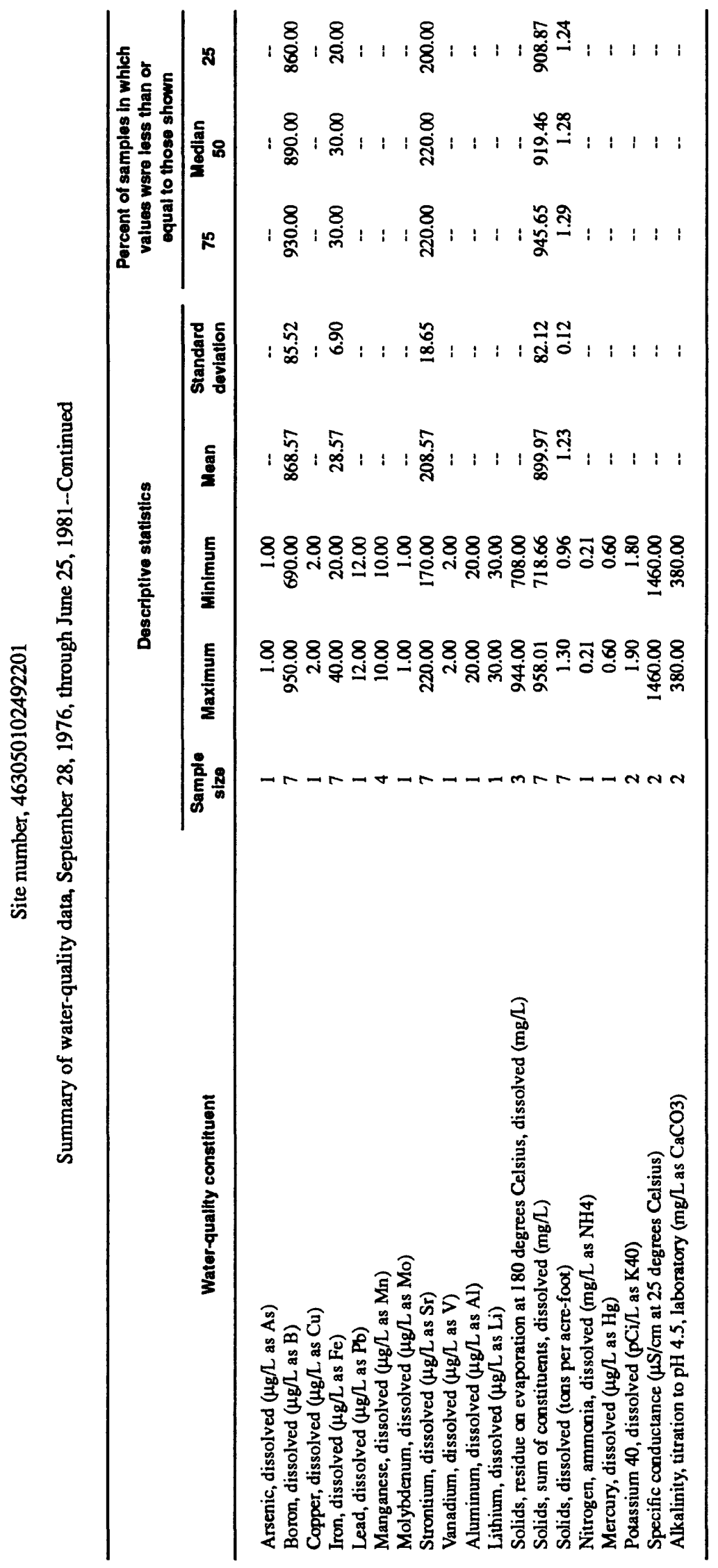




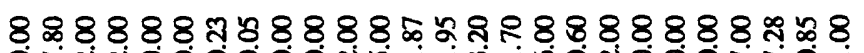

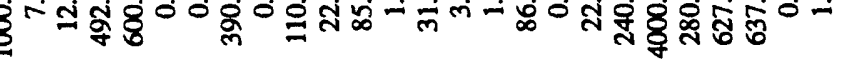

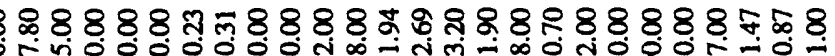

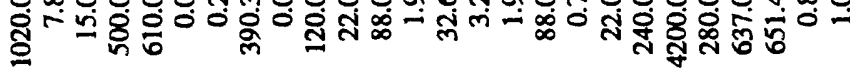

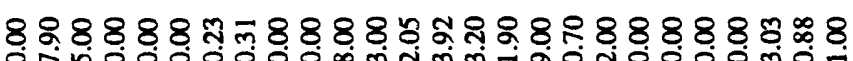

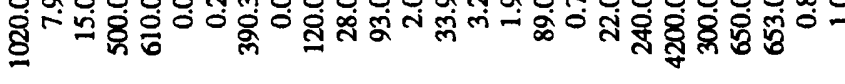

도

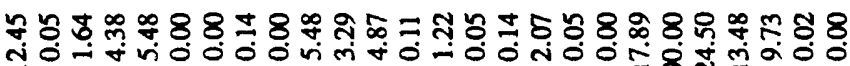

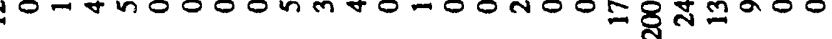

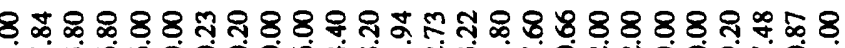

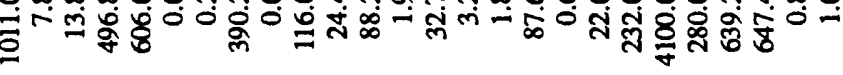

808888 웅 88 \%

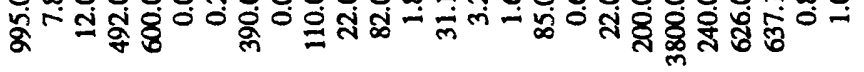

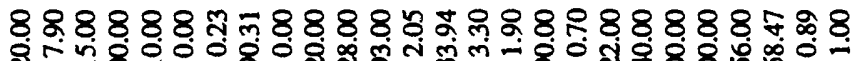

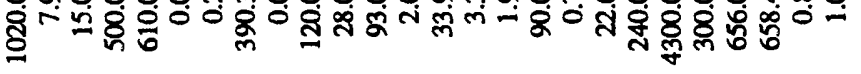

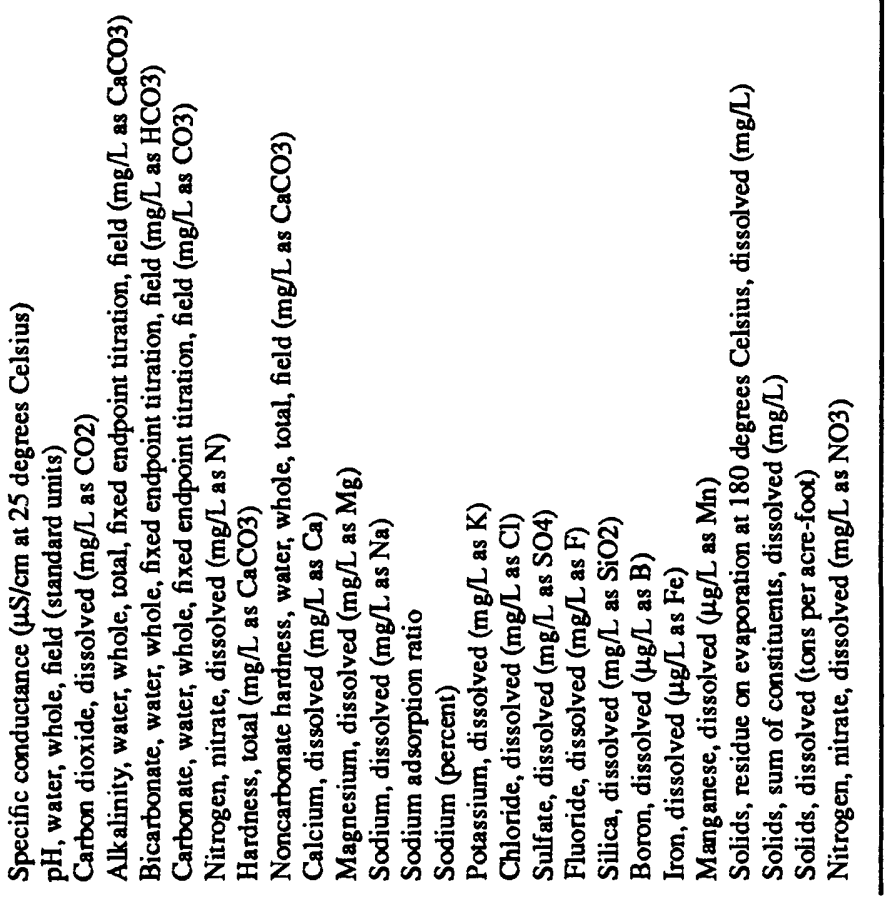




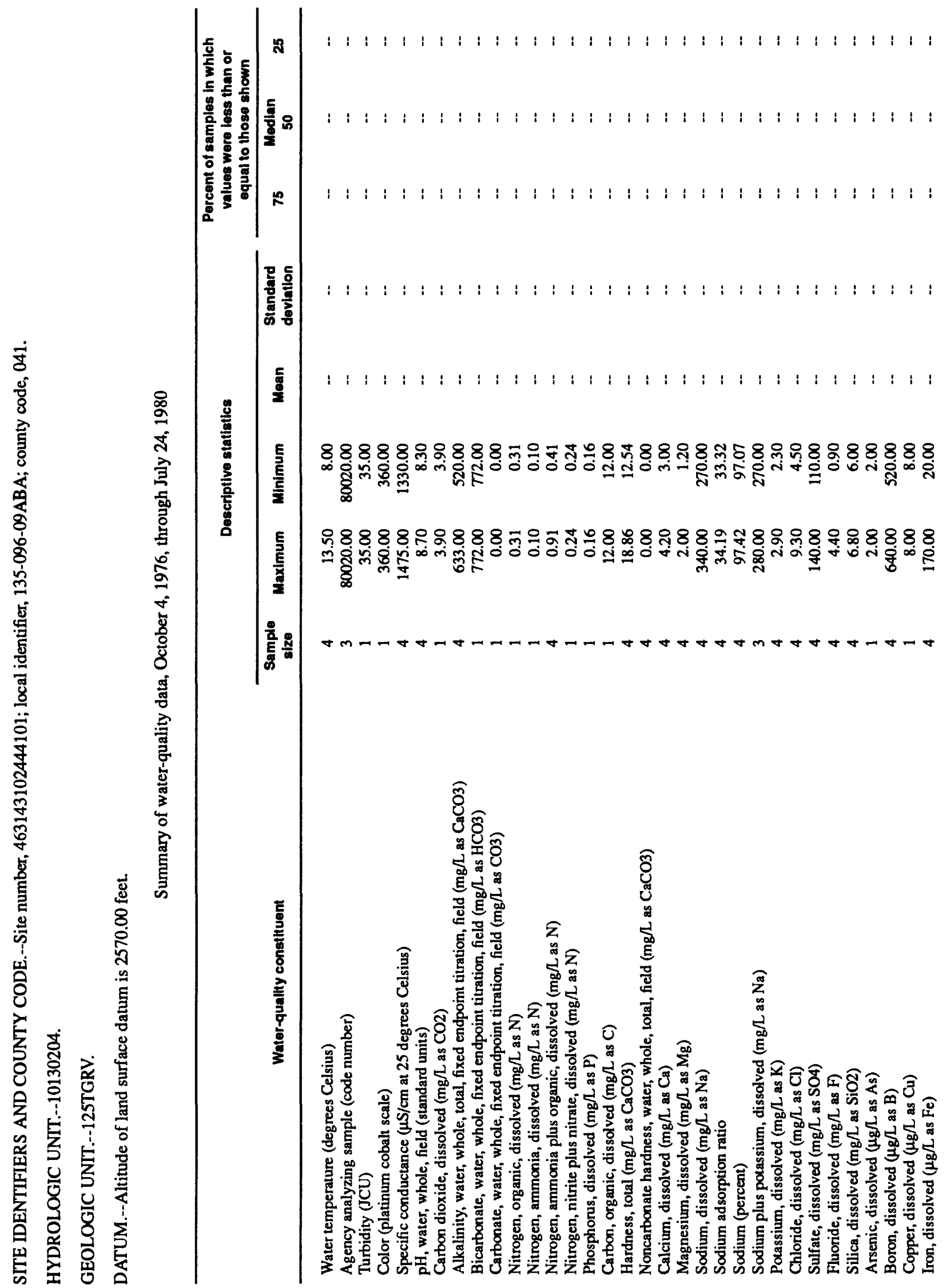




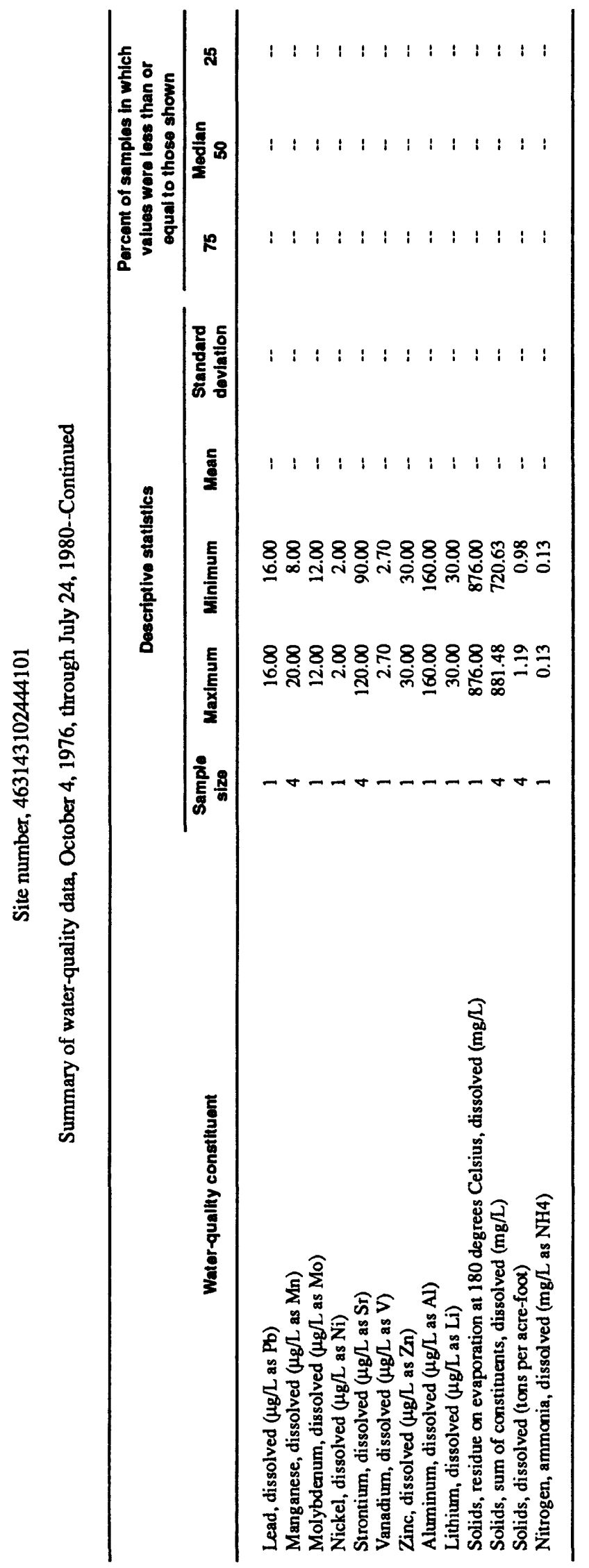




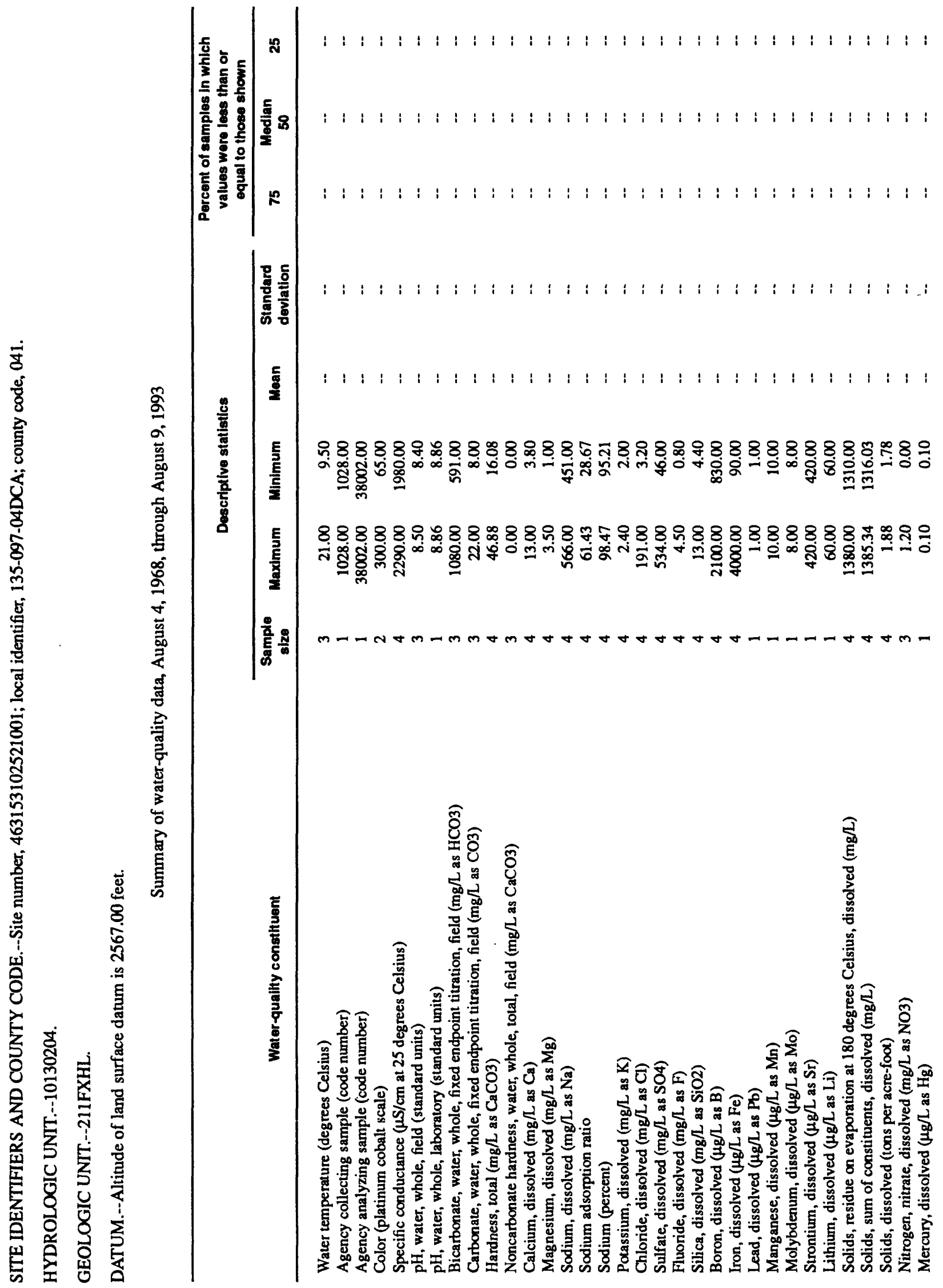




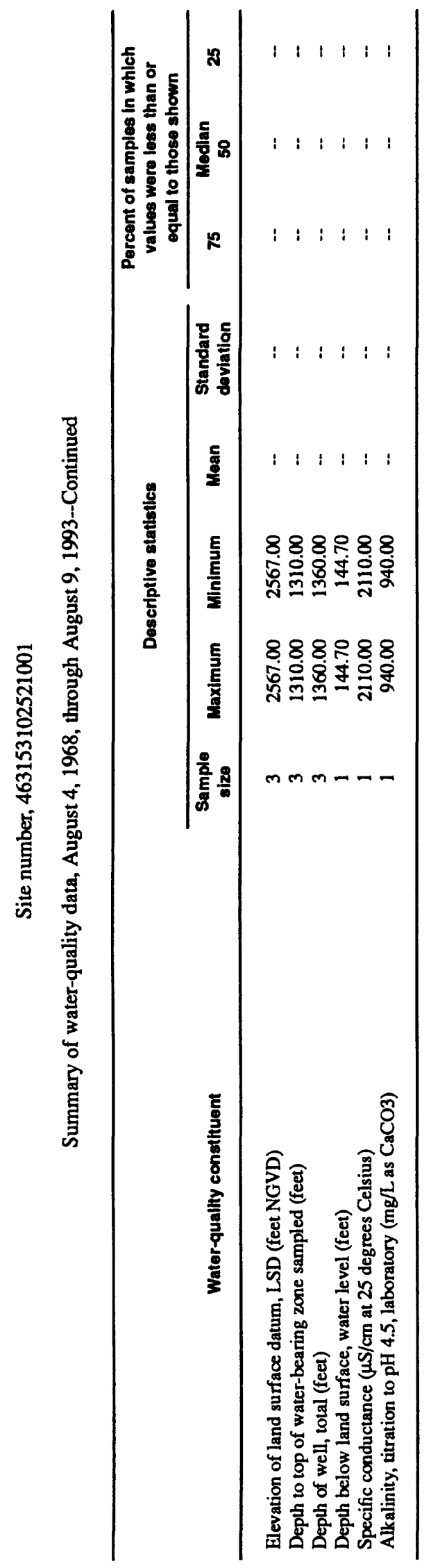




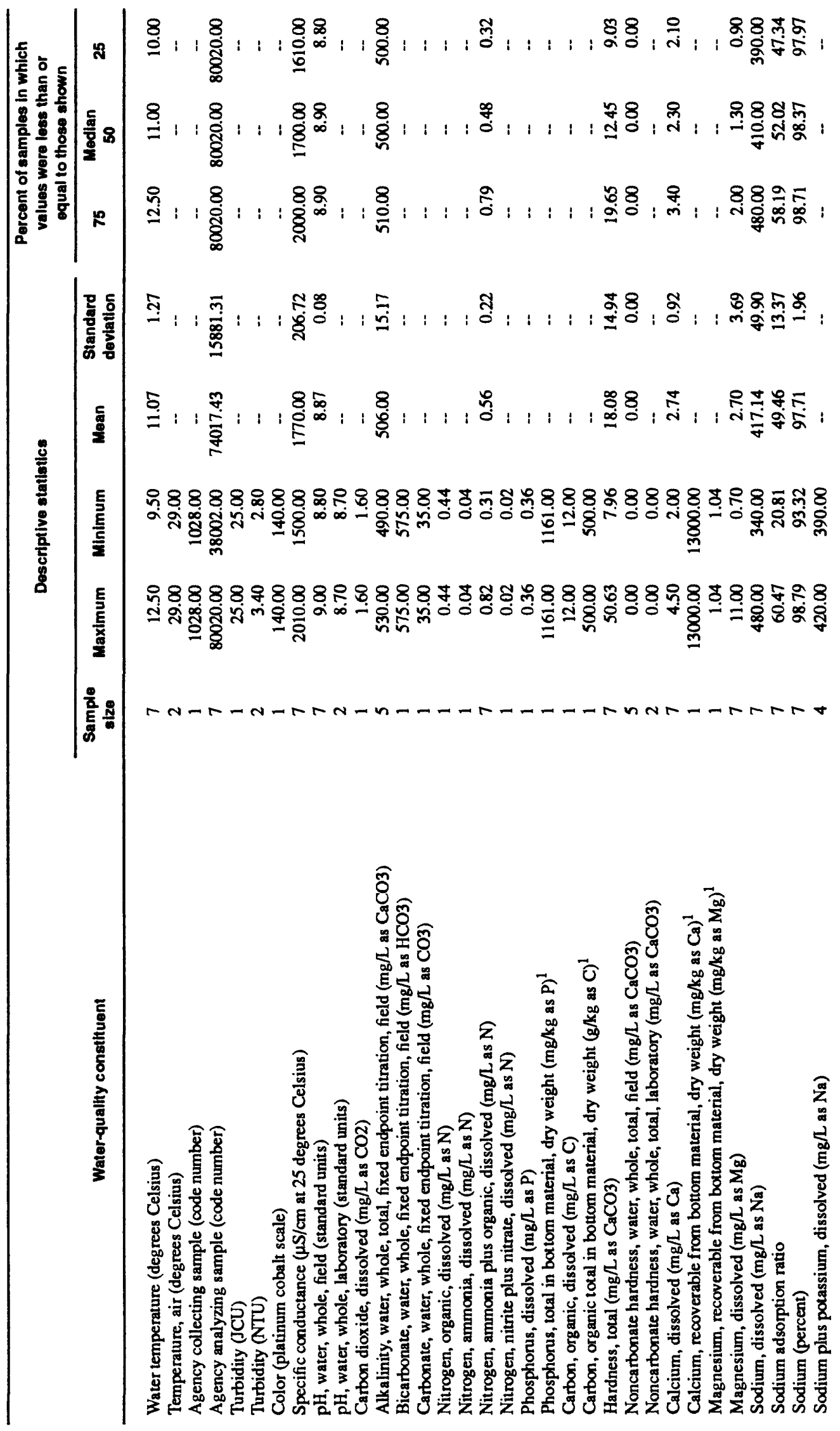




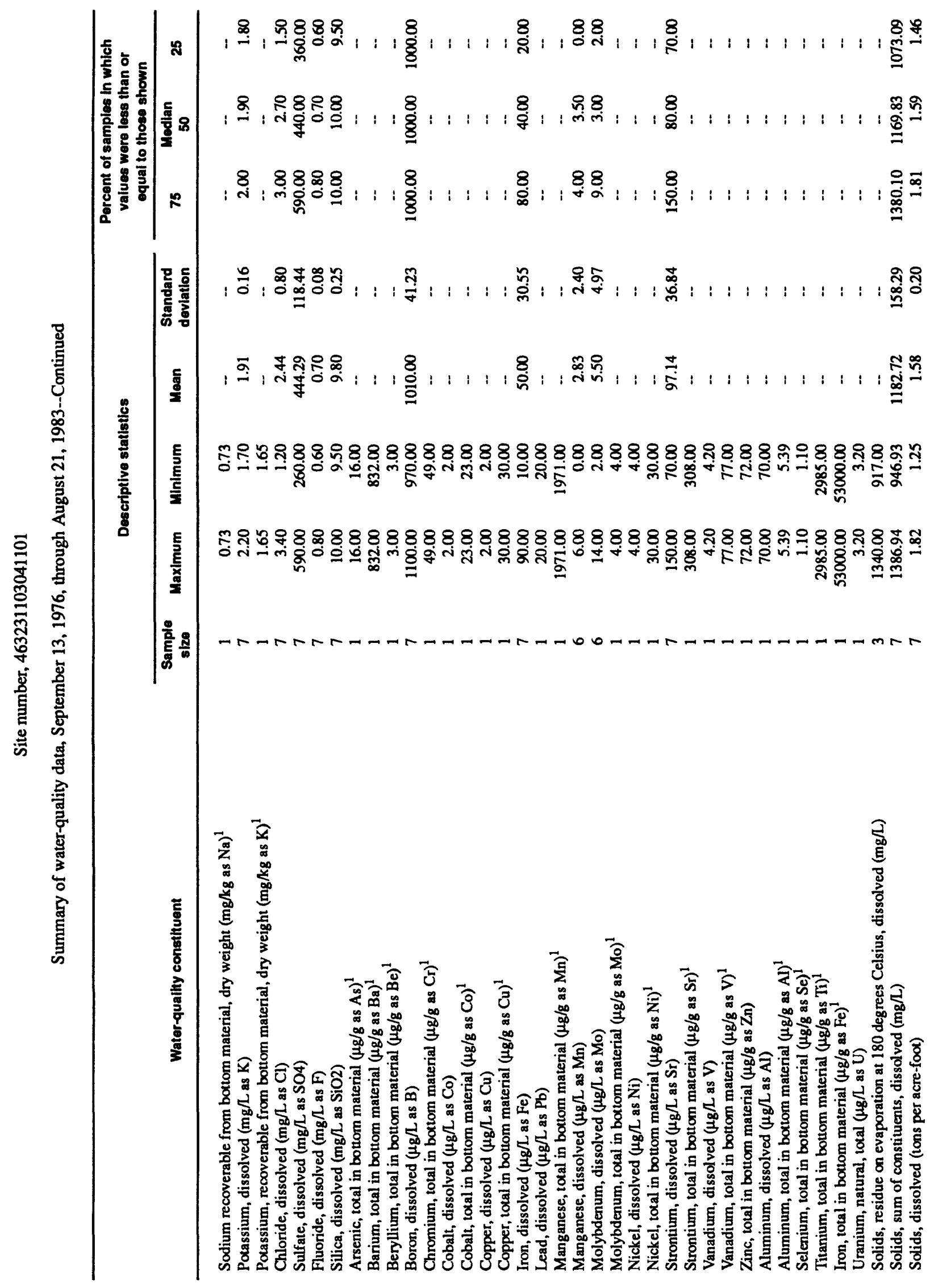




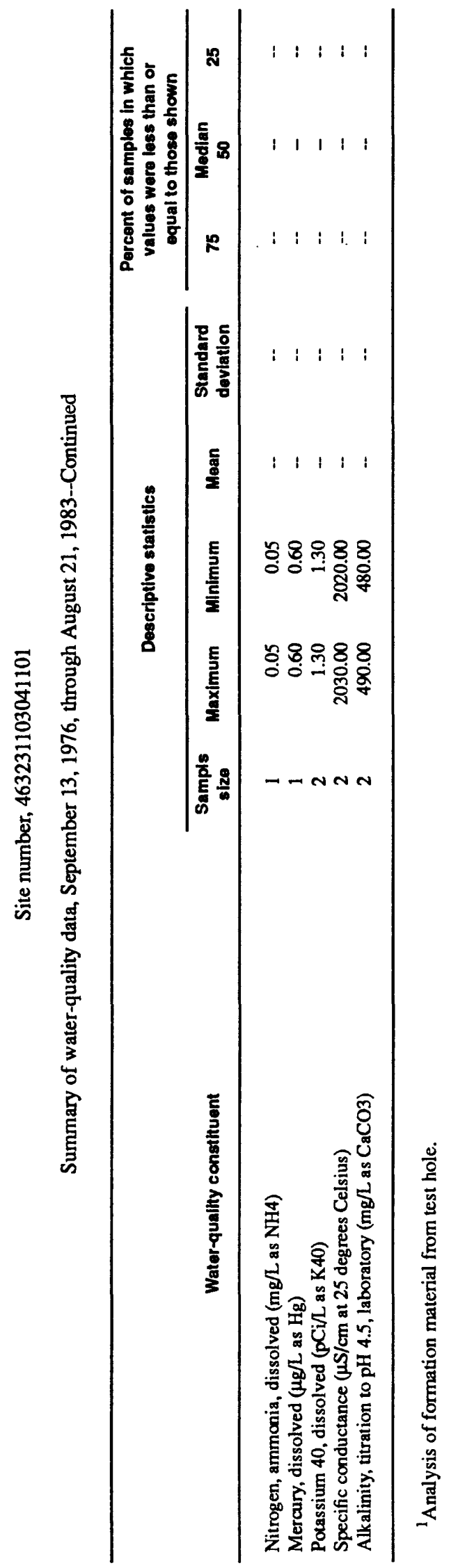




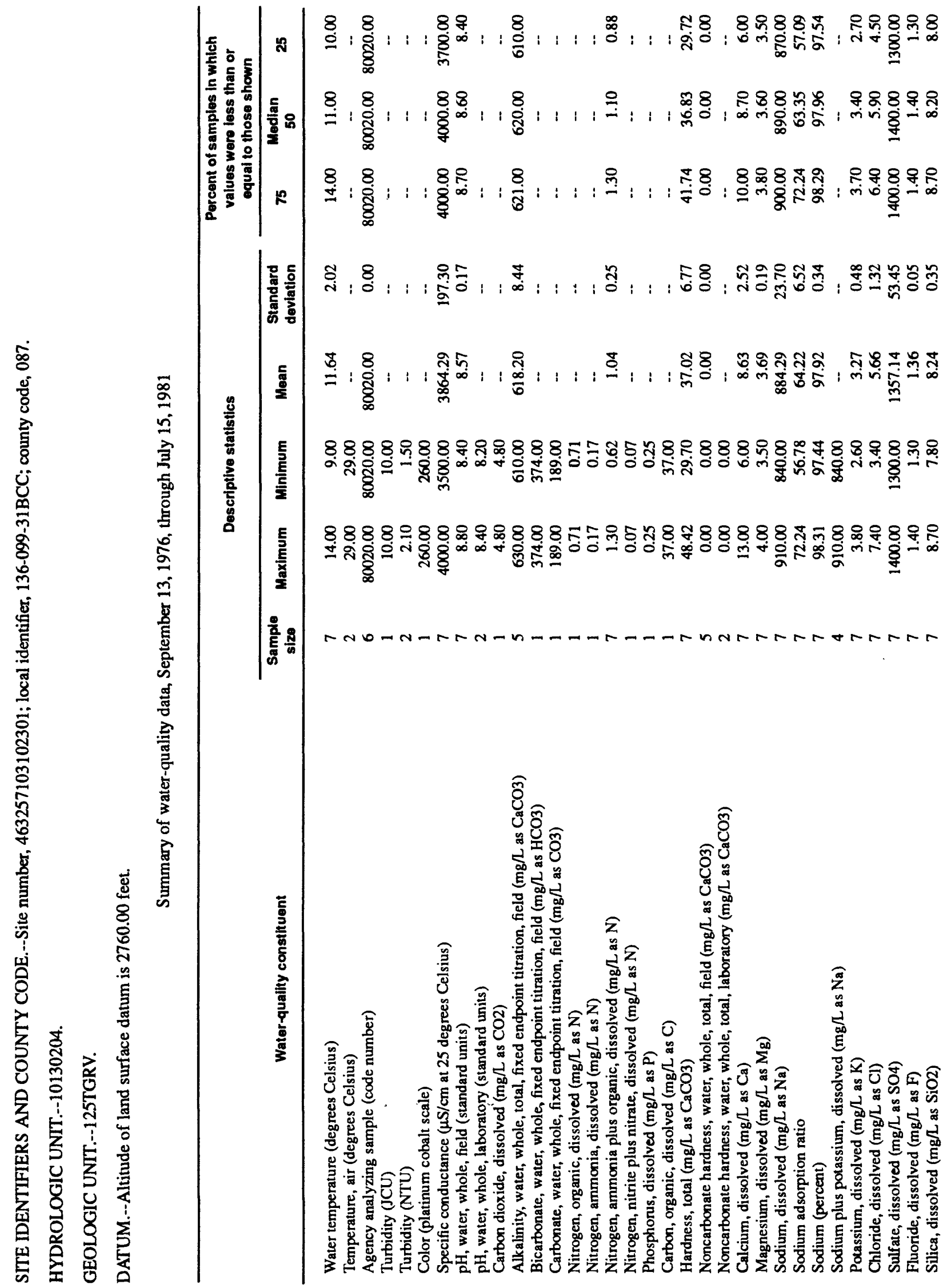




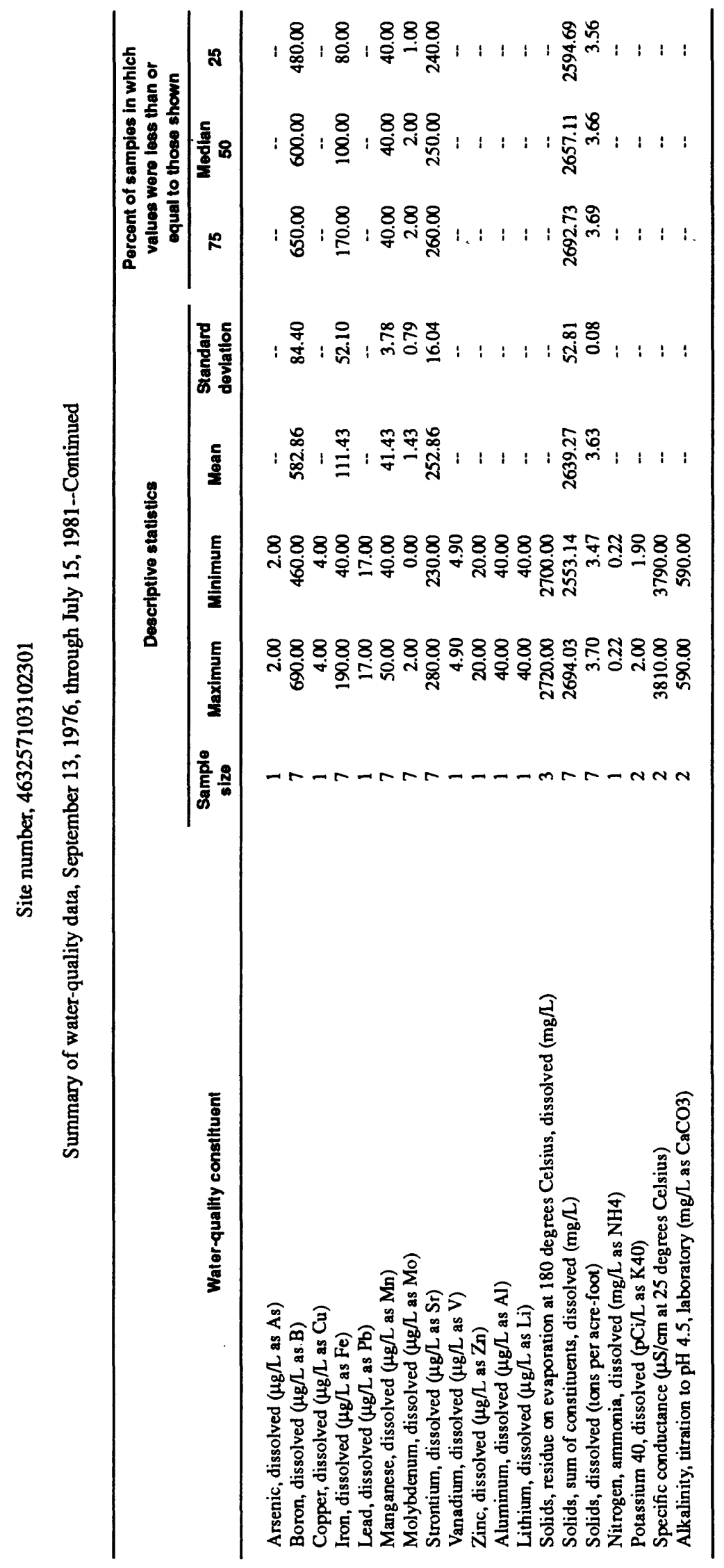




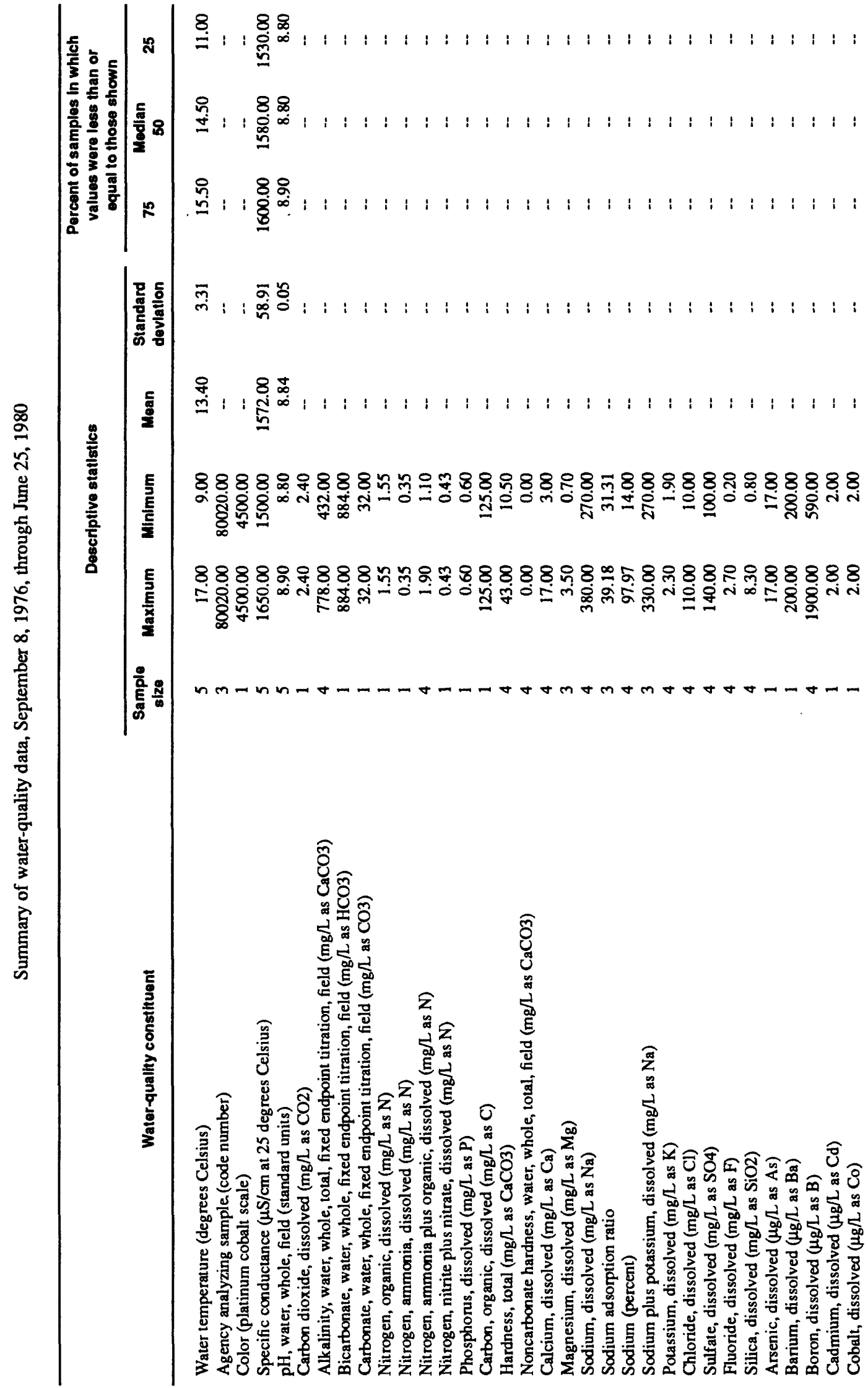




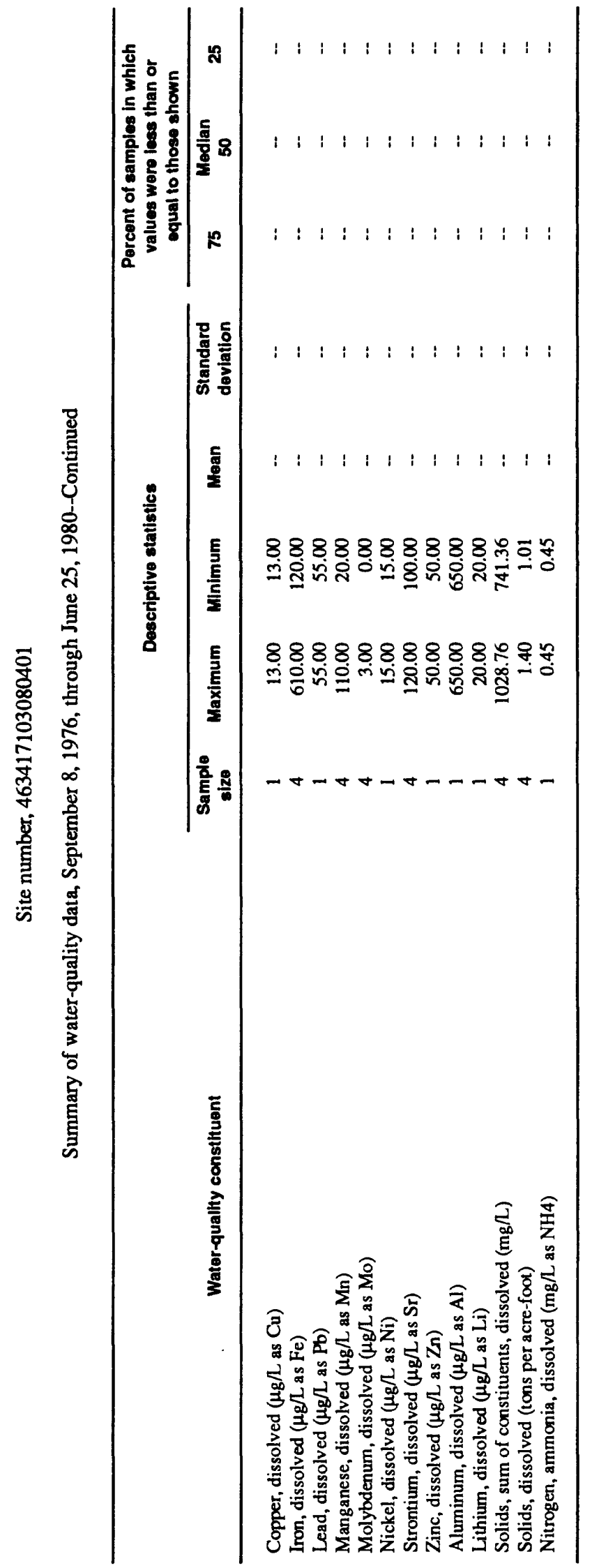




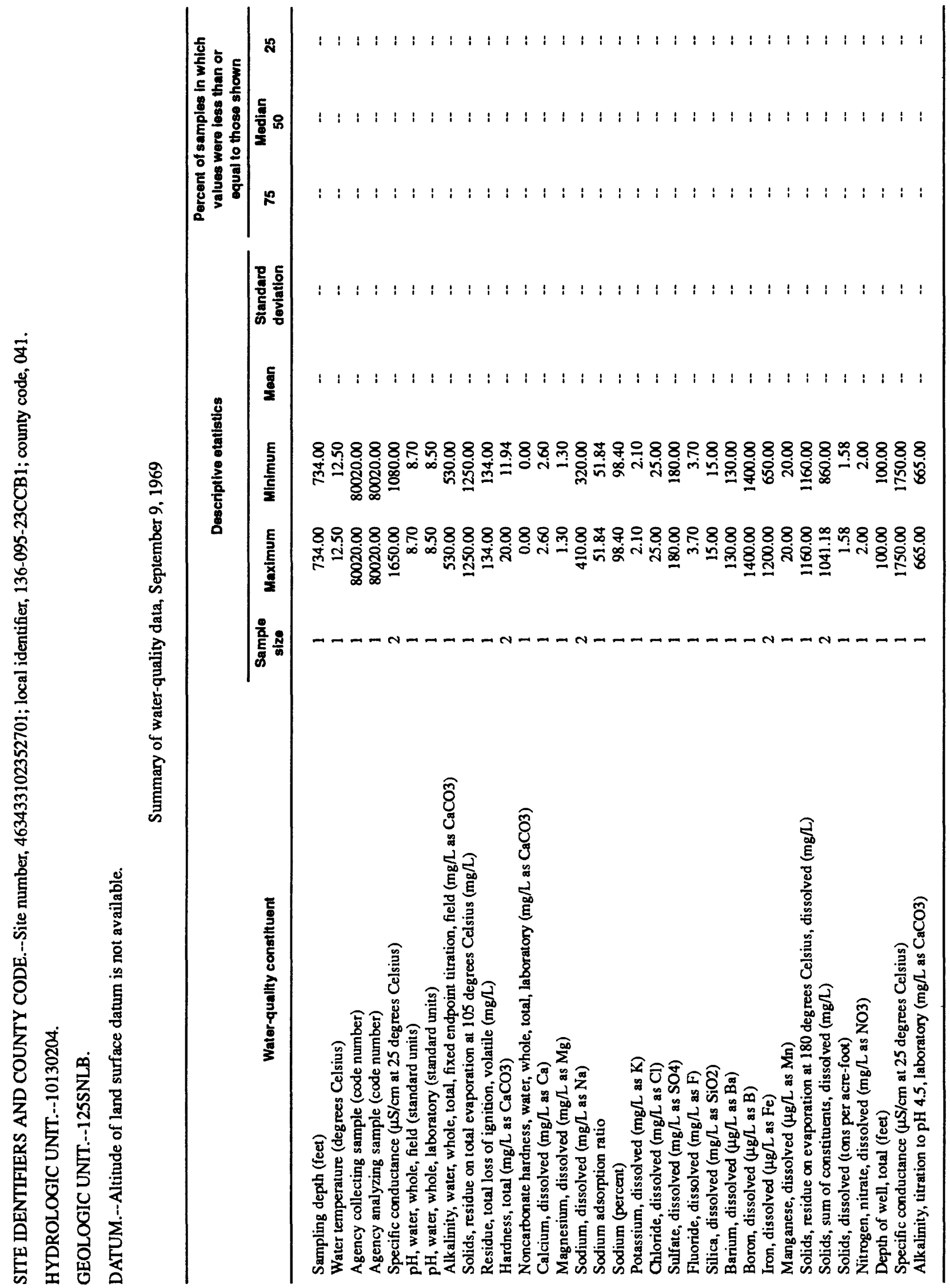




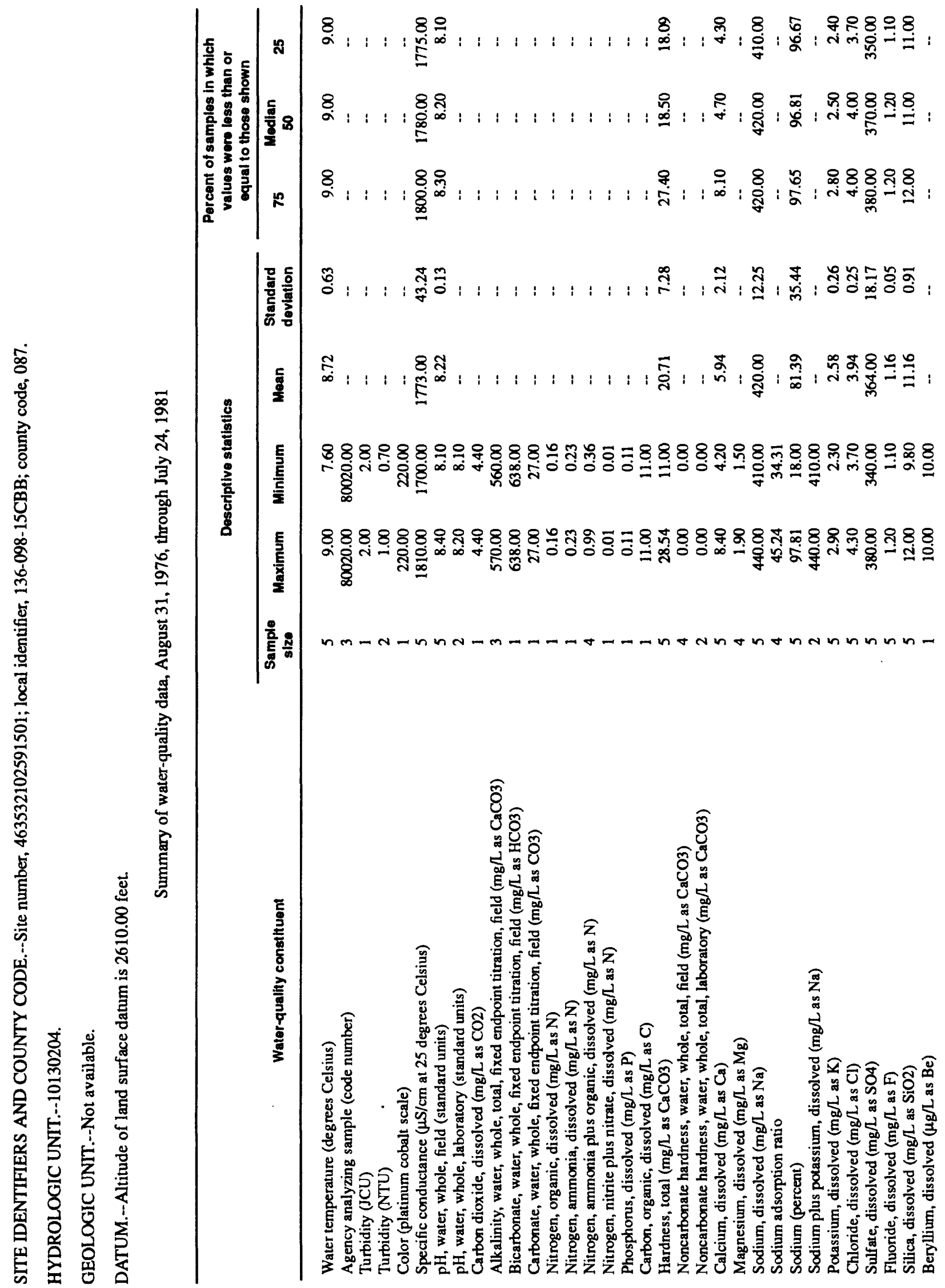




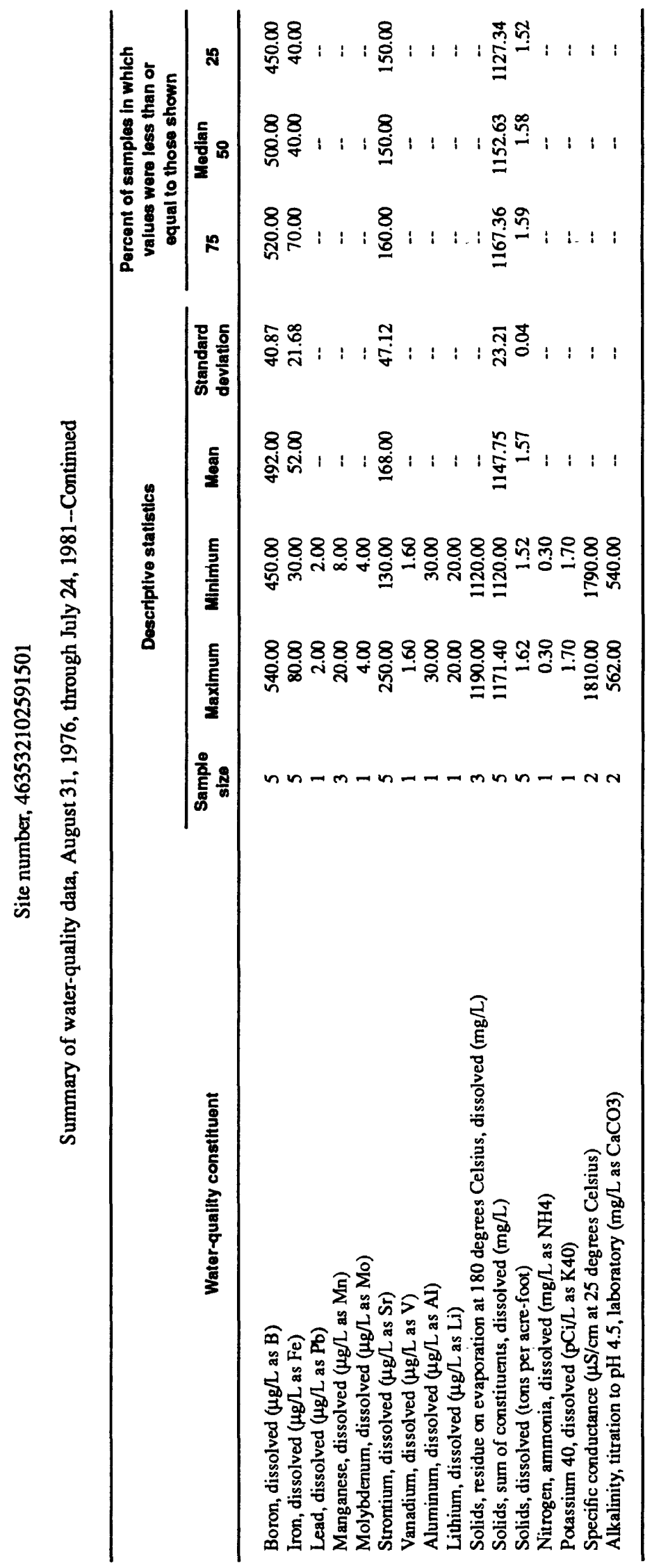




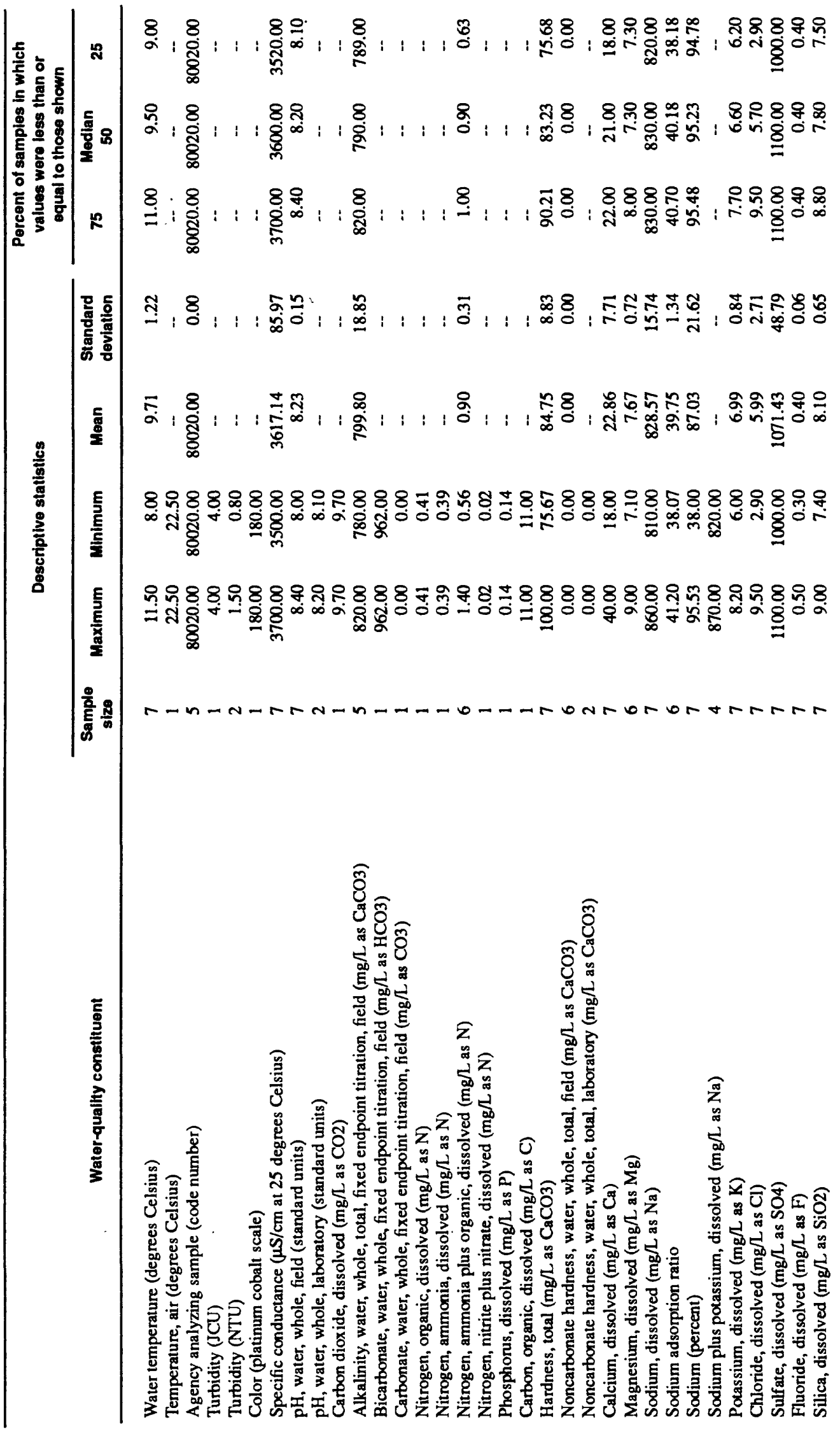




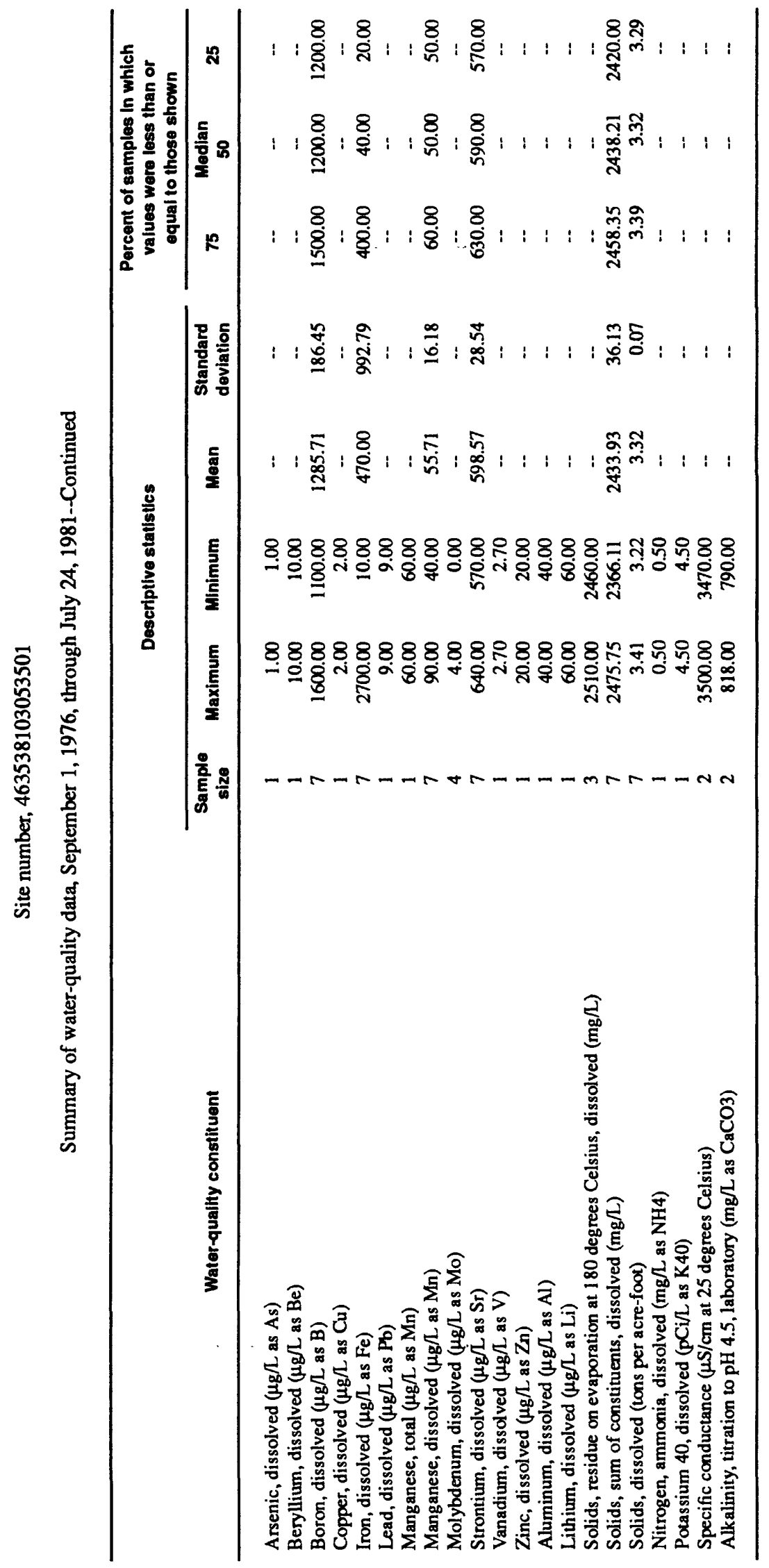




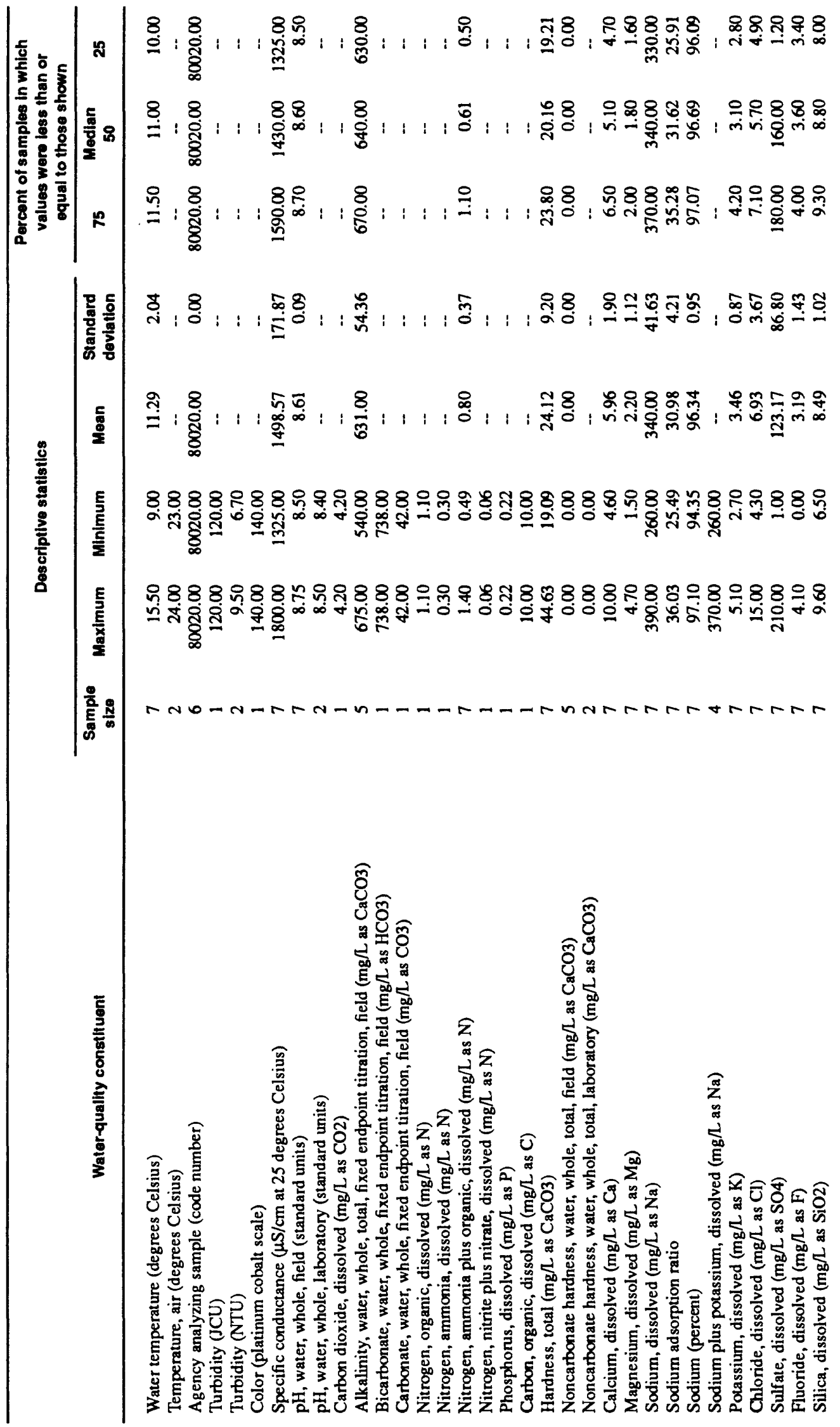




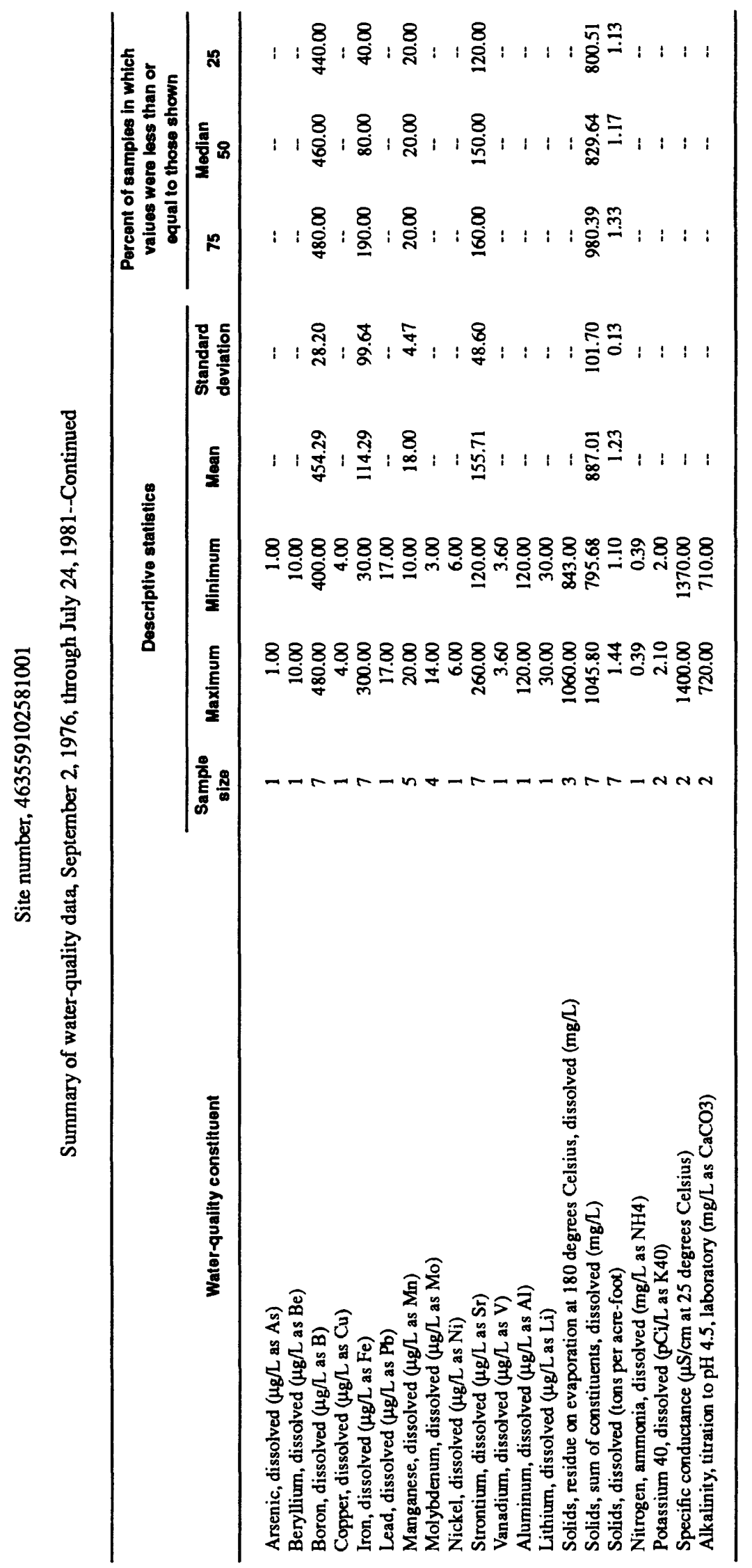


: 88 :

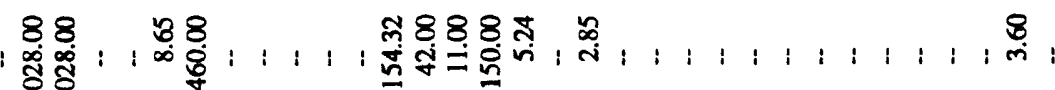

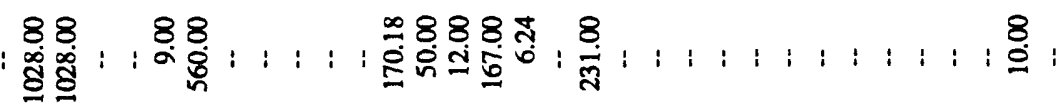

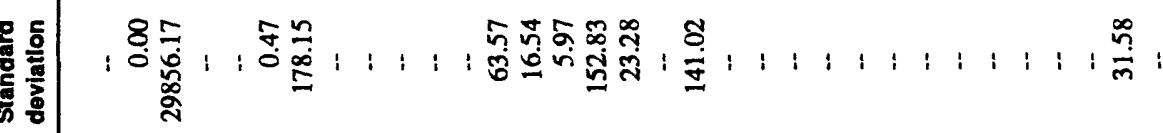

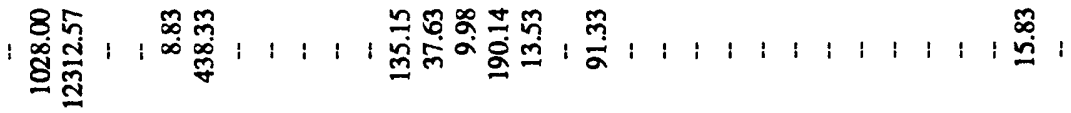

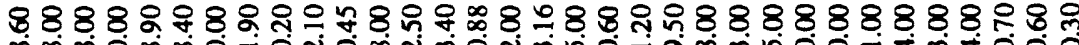

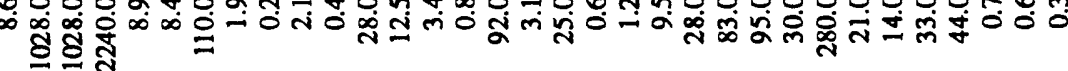

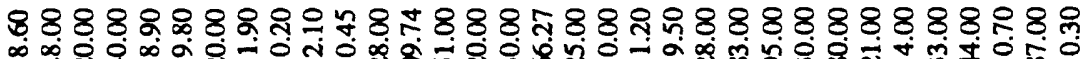

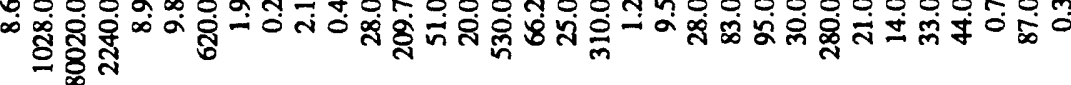

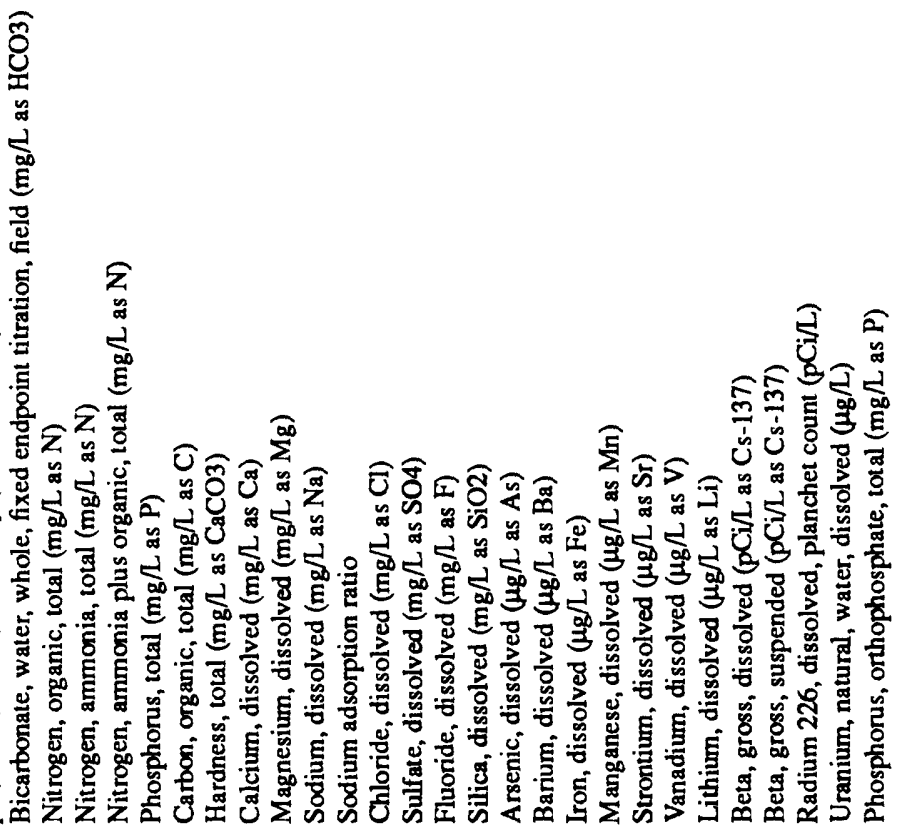

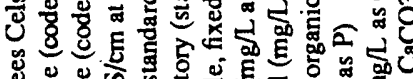

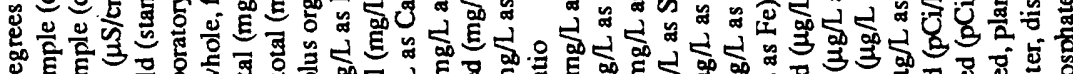

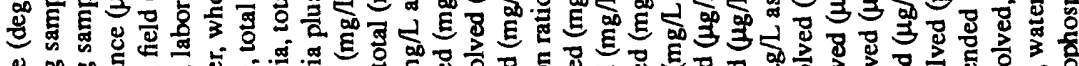

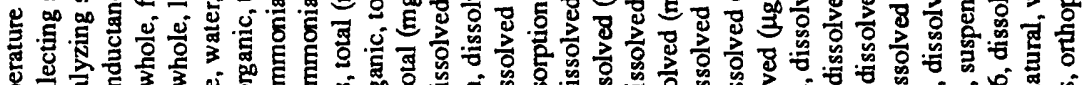

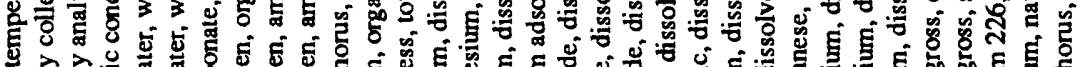

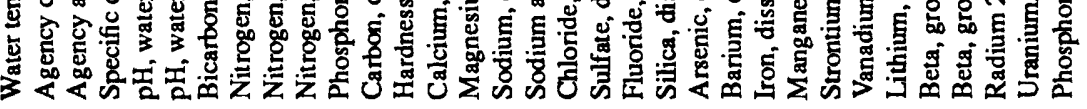




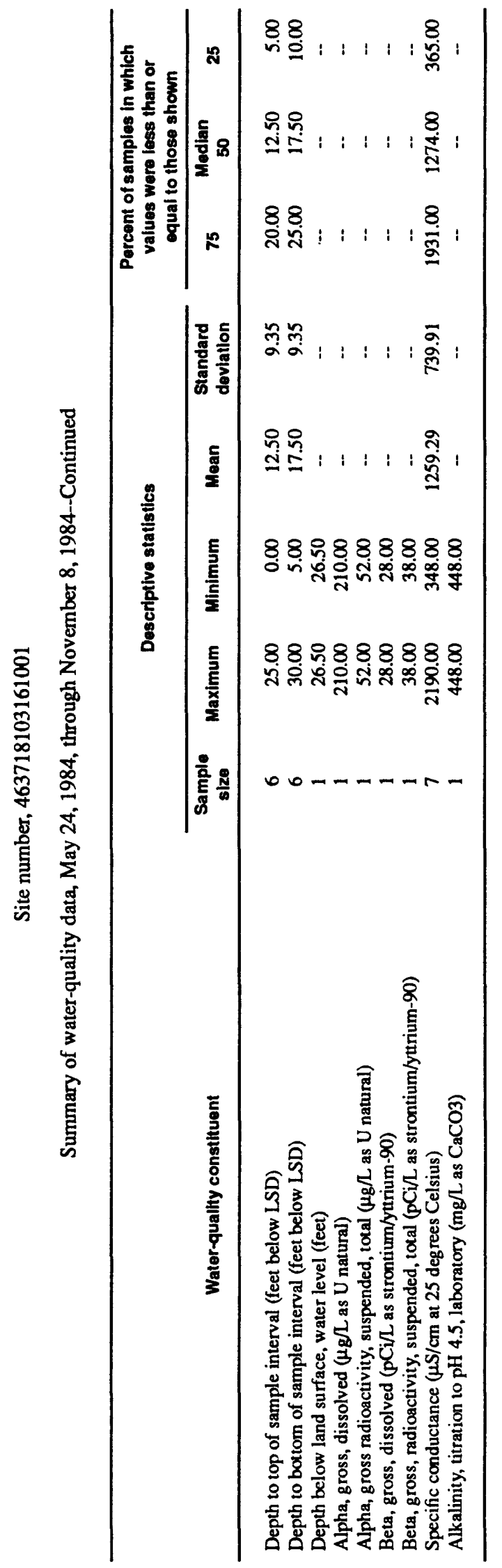




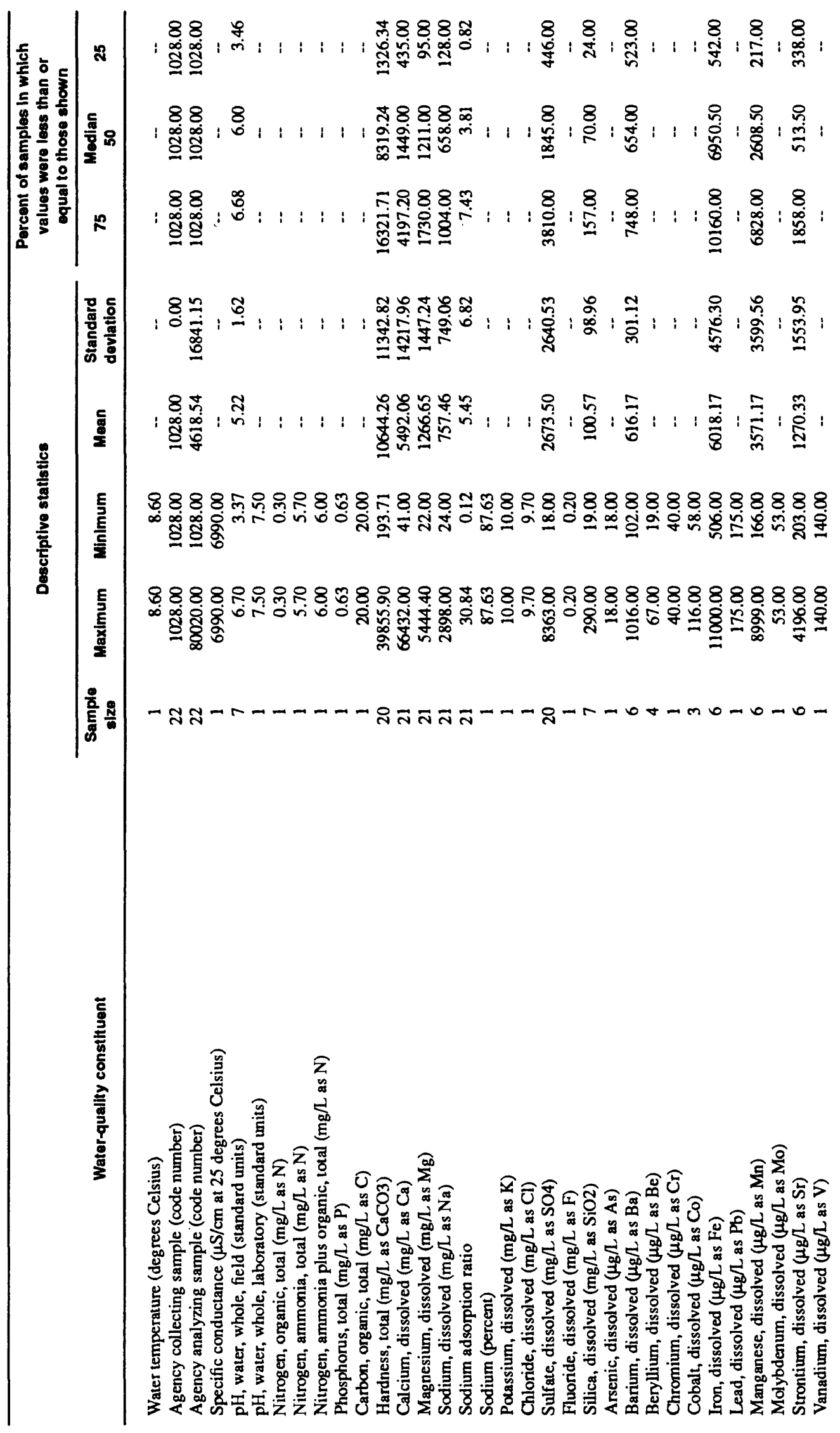




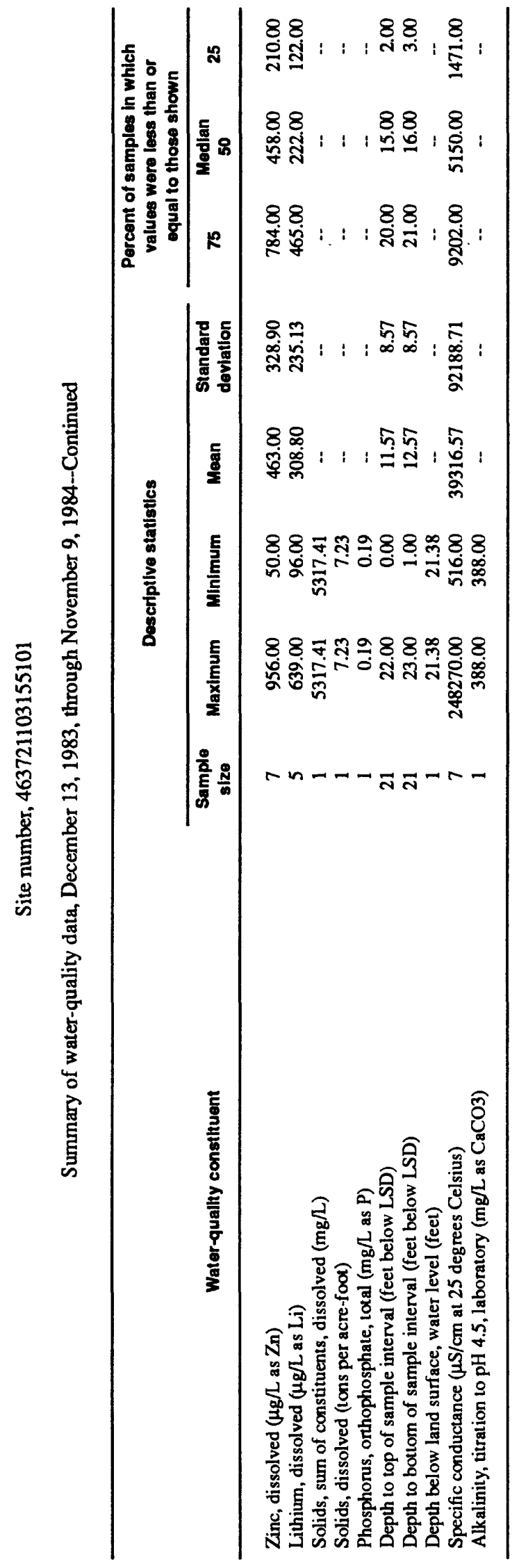




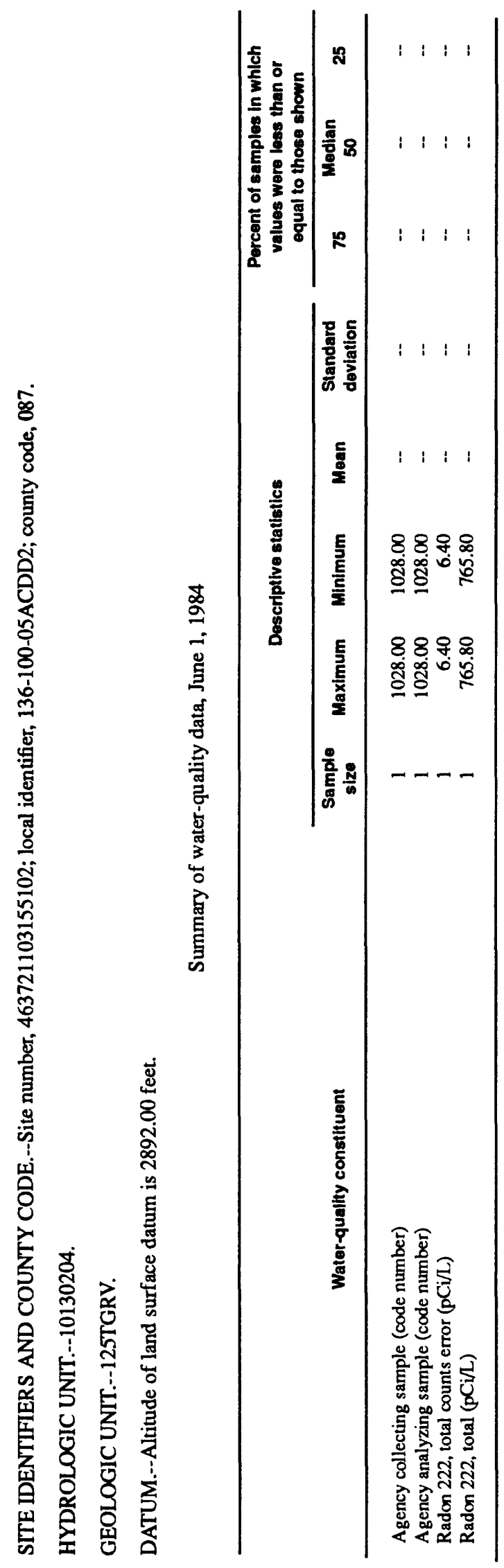




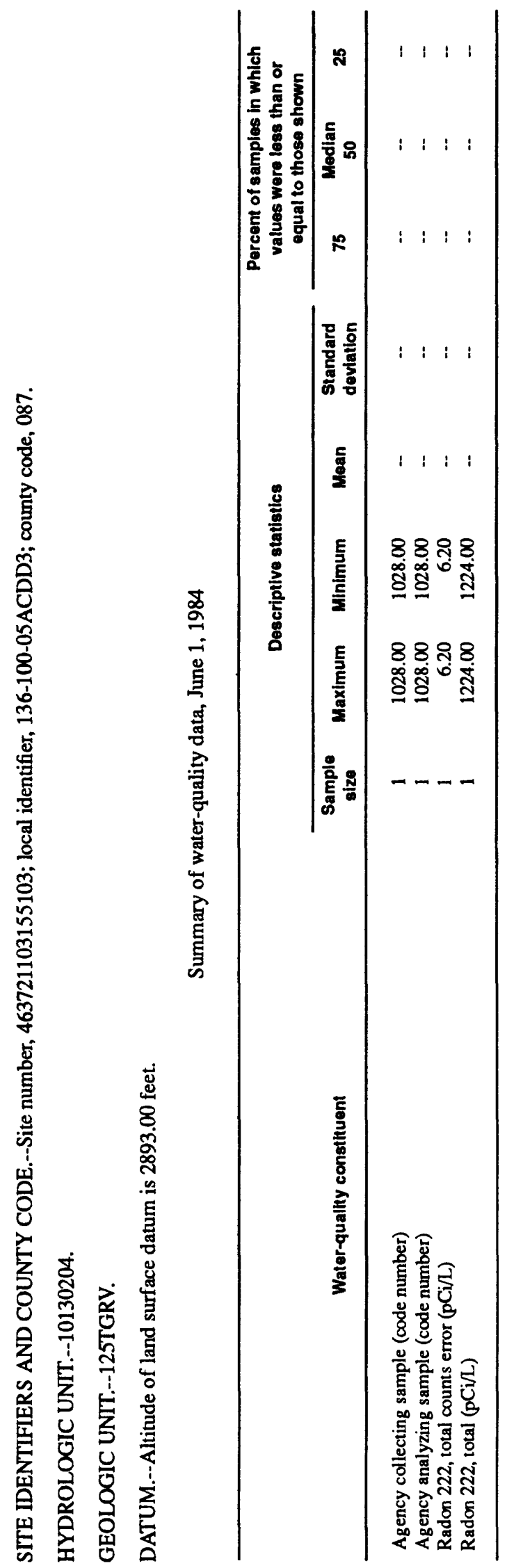




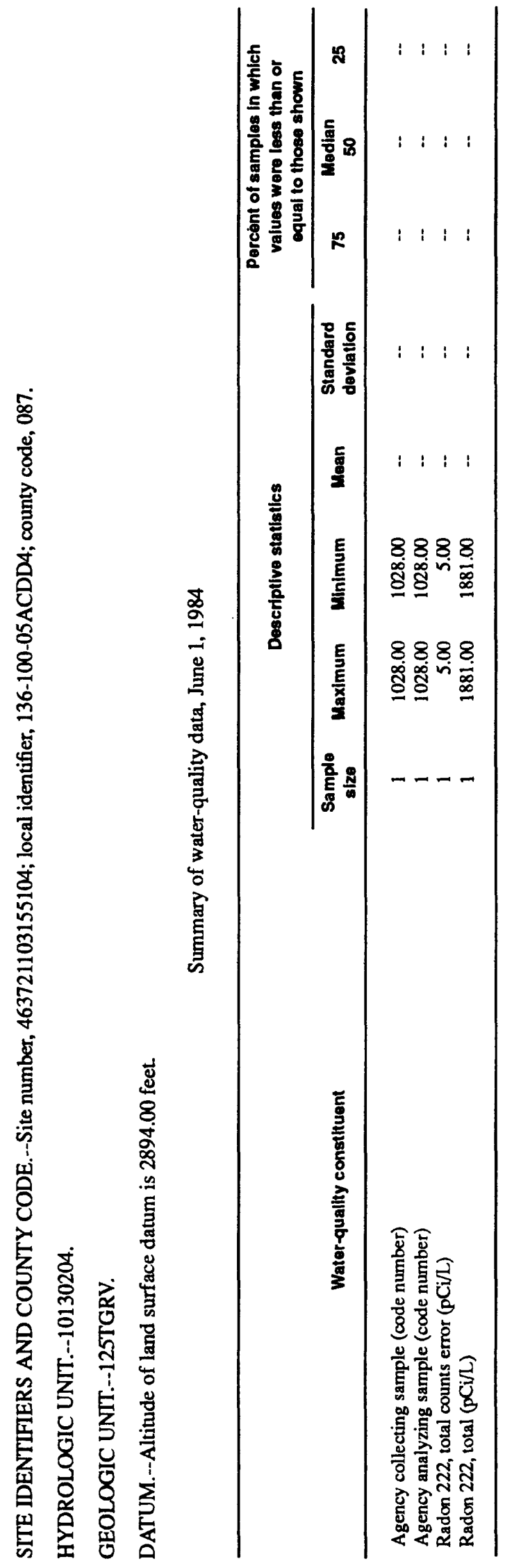




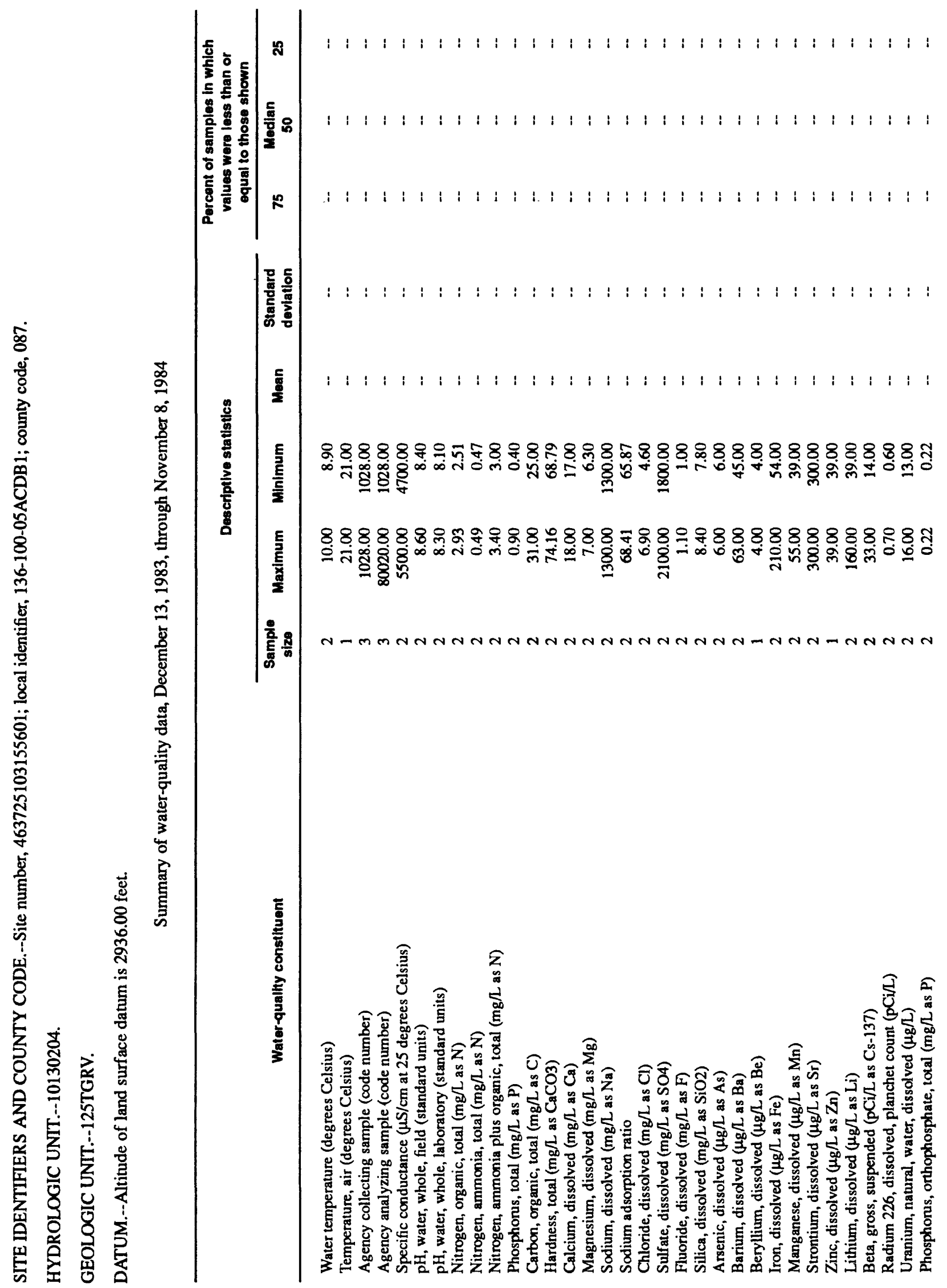




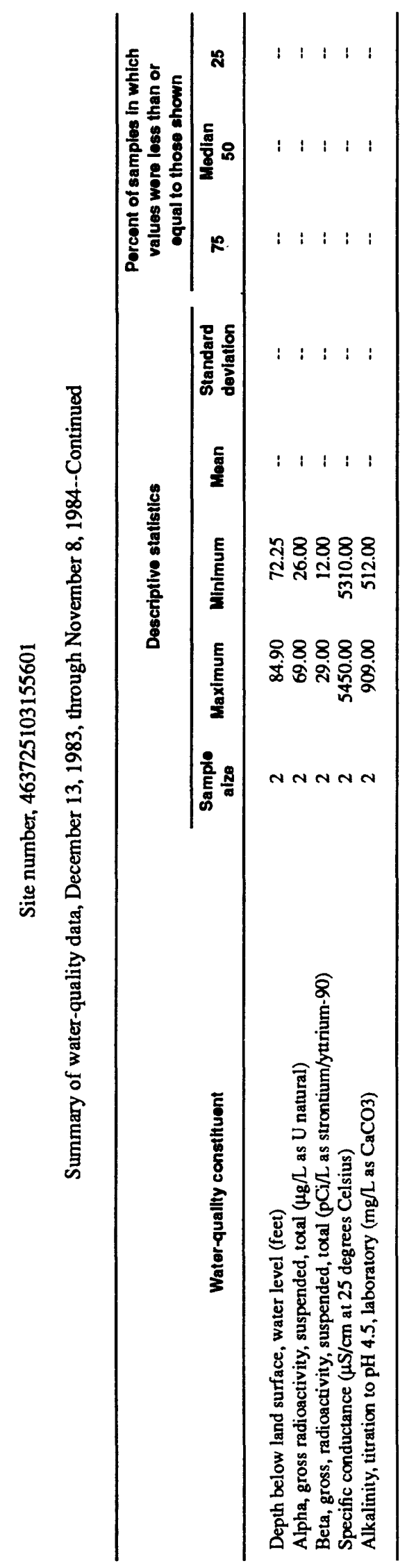




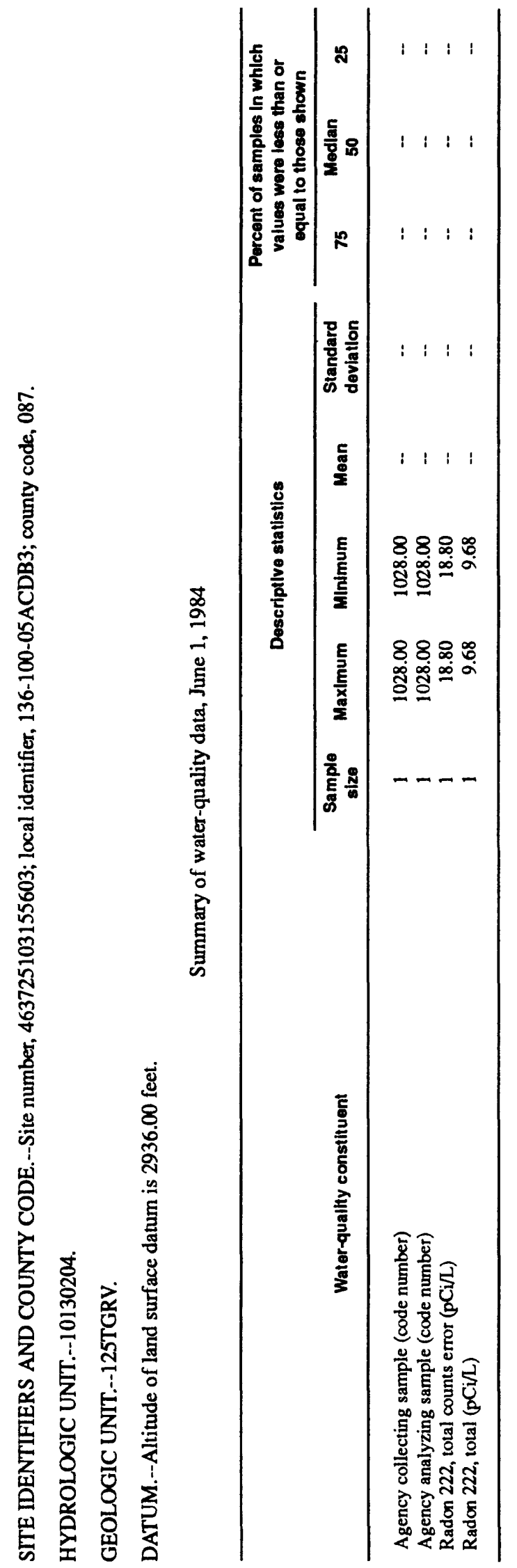




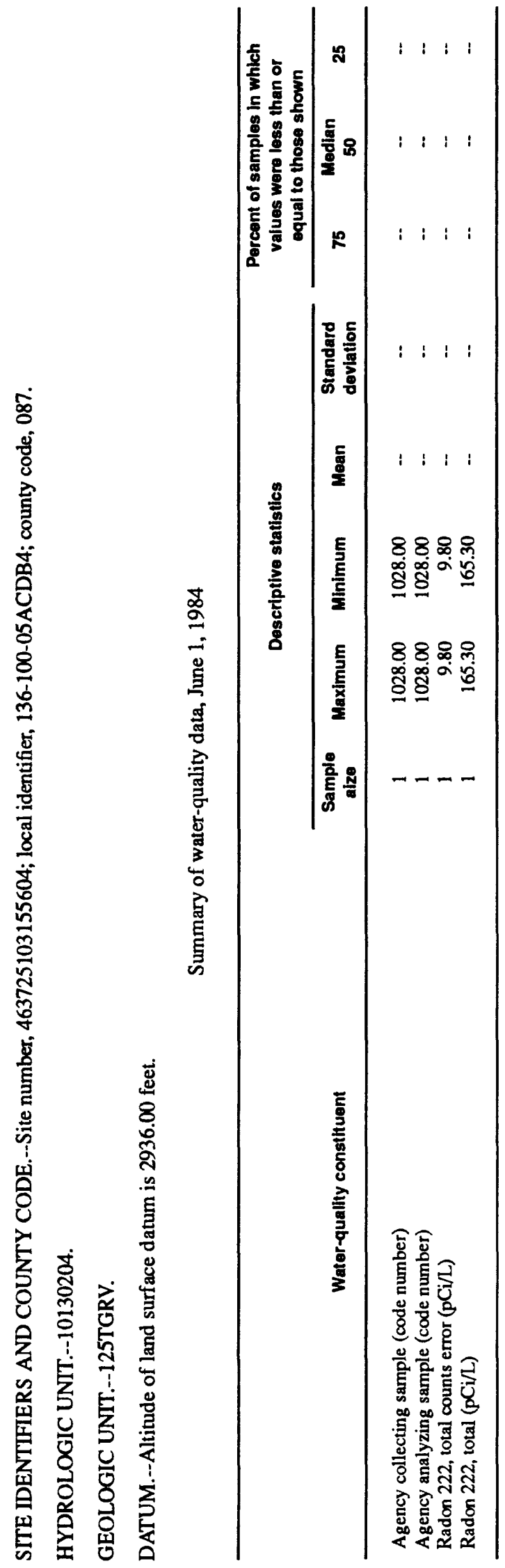




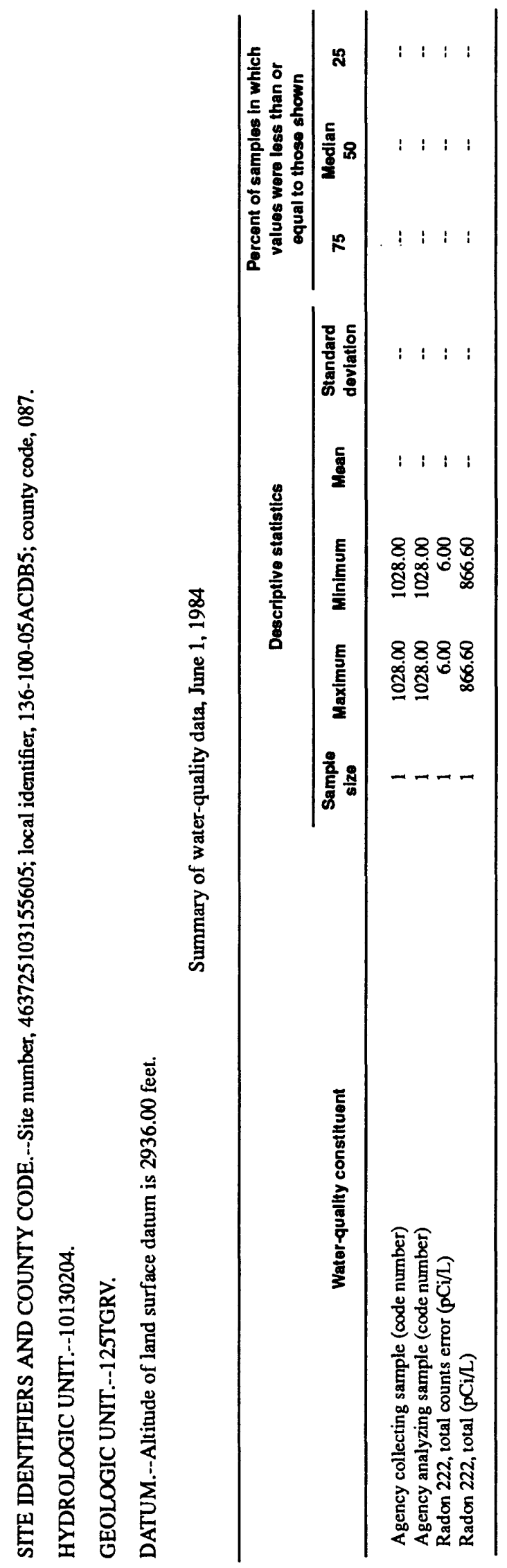




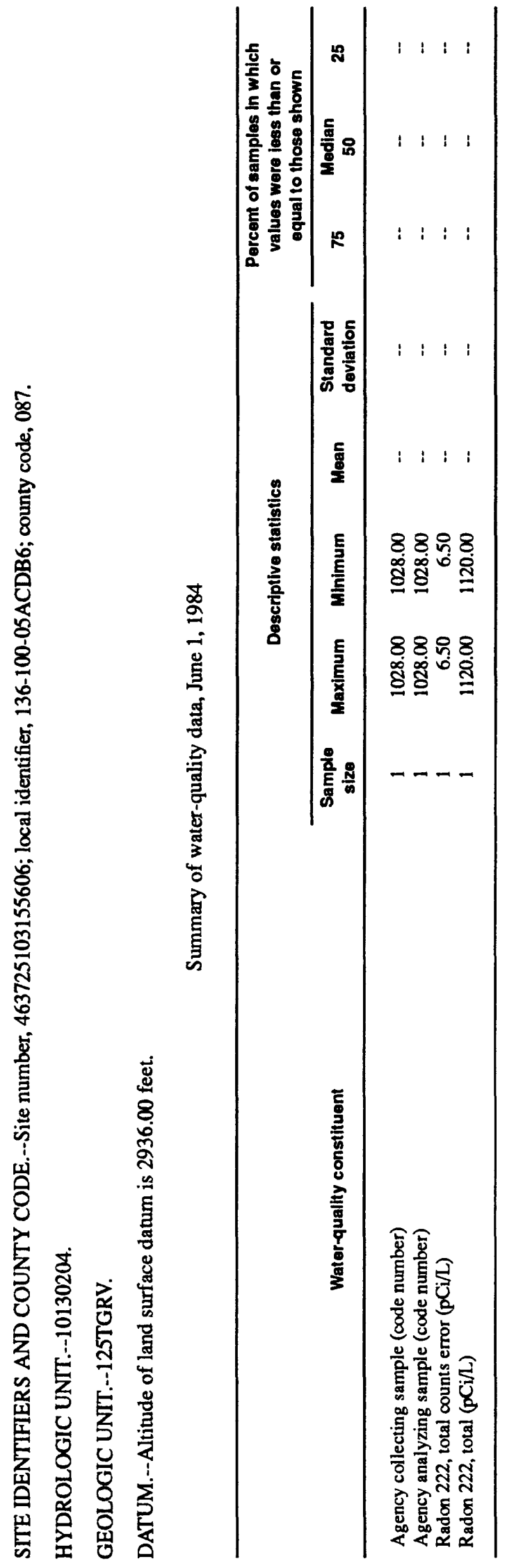




$$
\text { hin }
$$




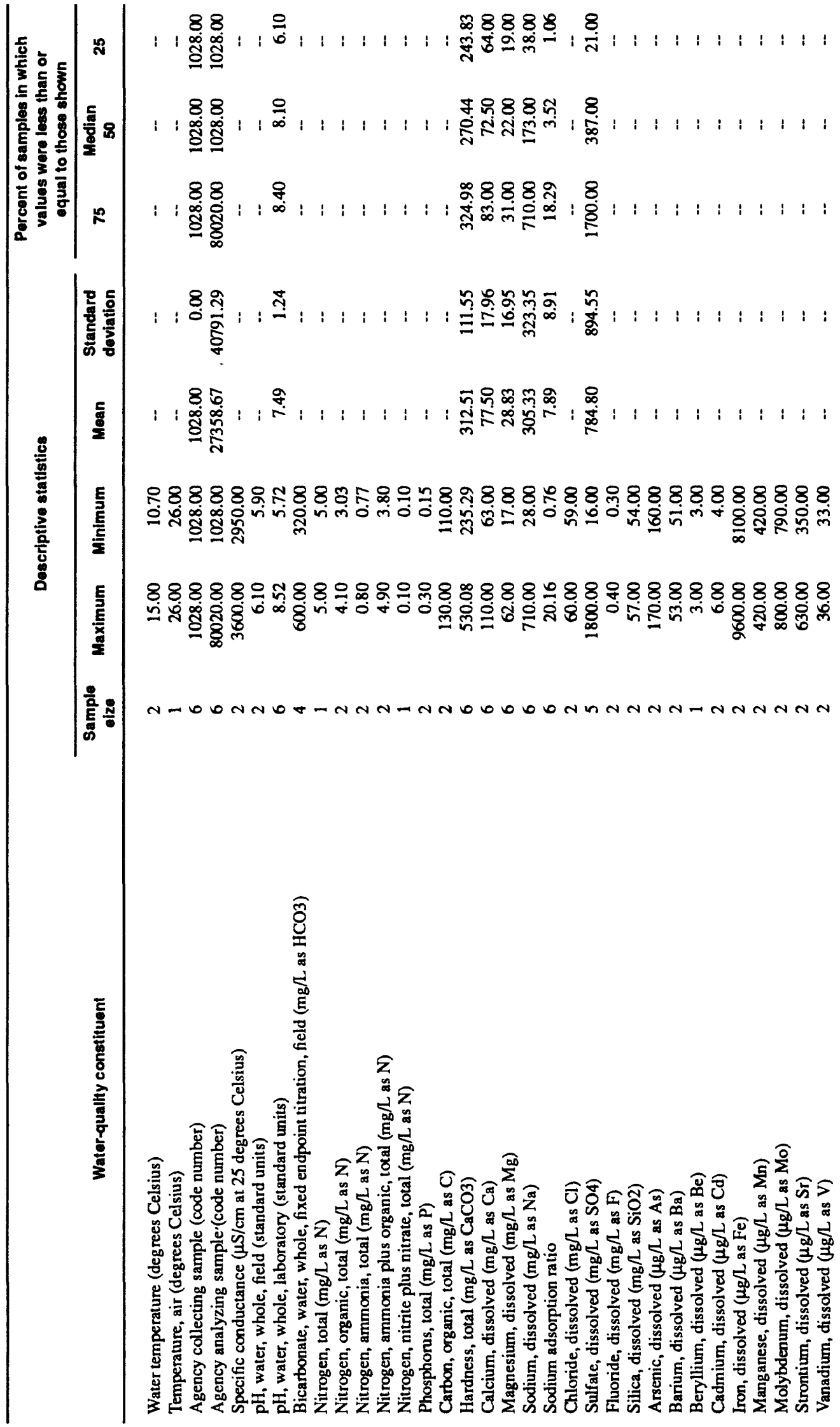




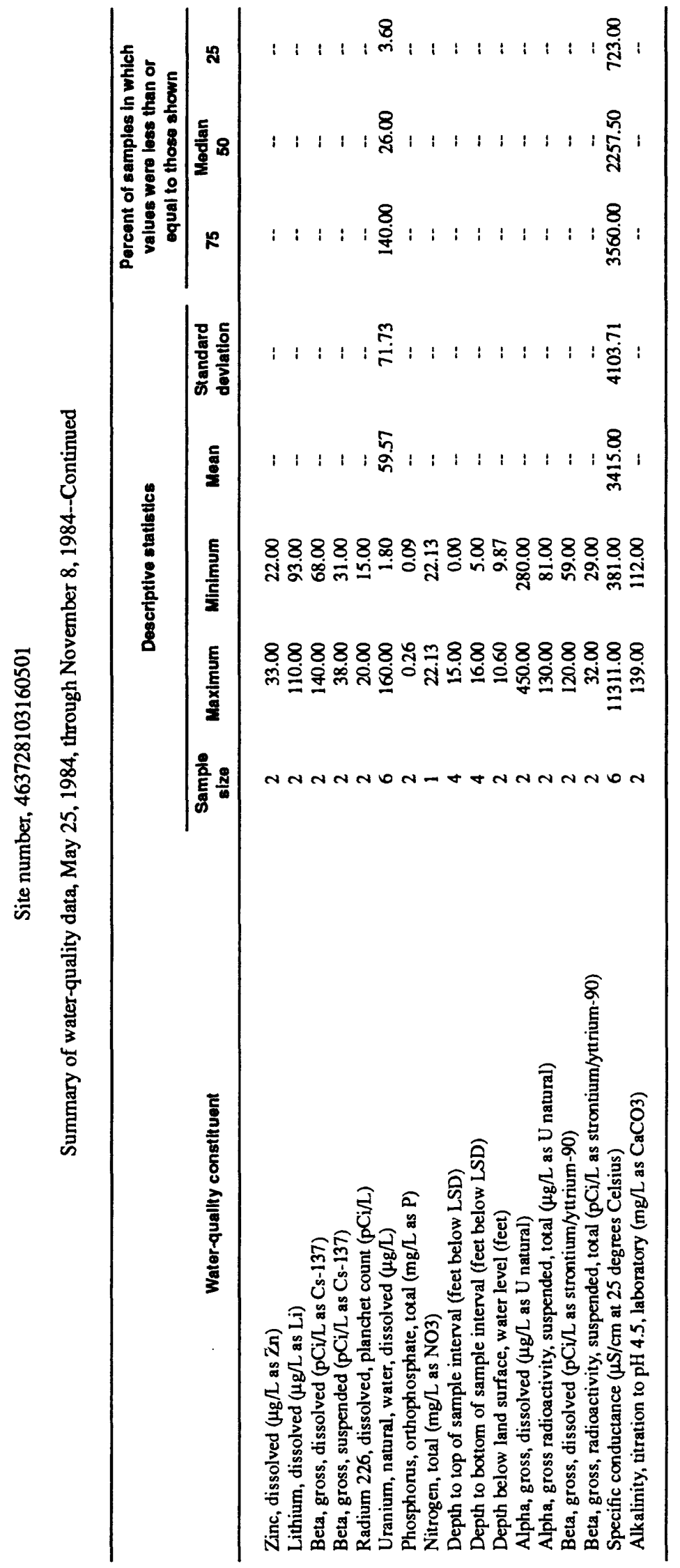




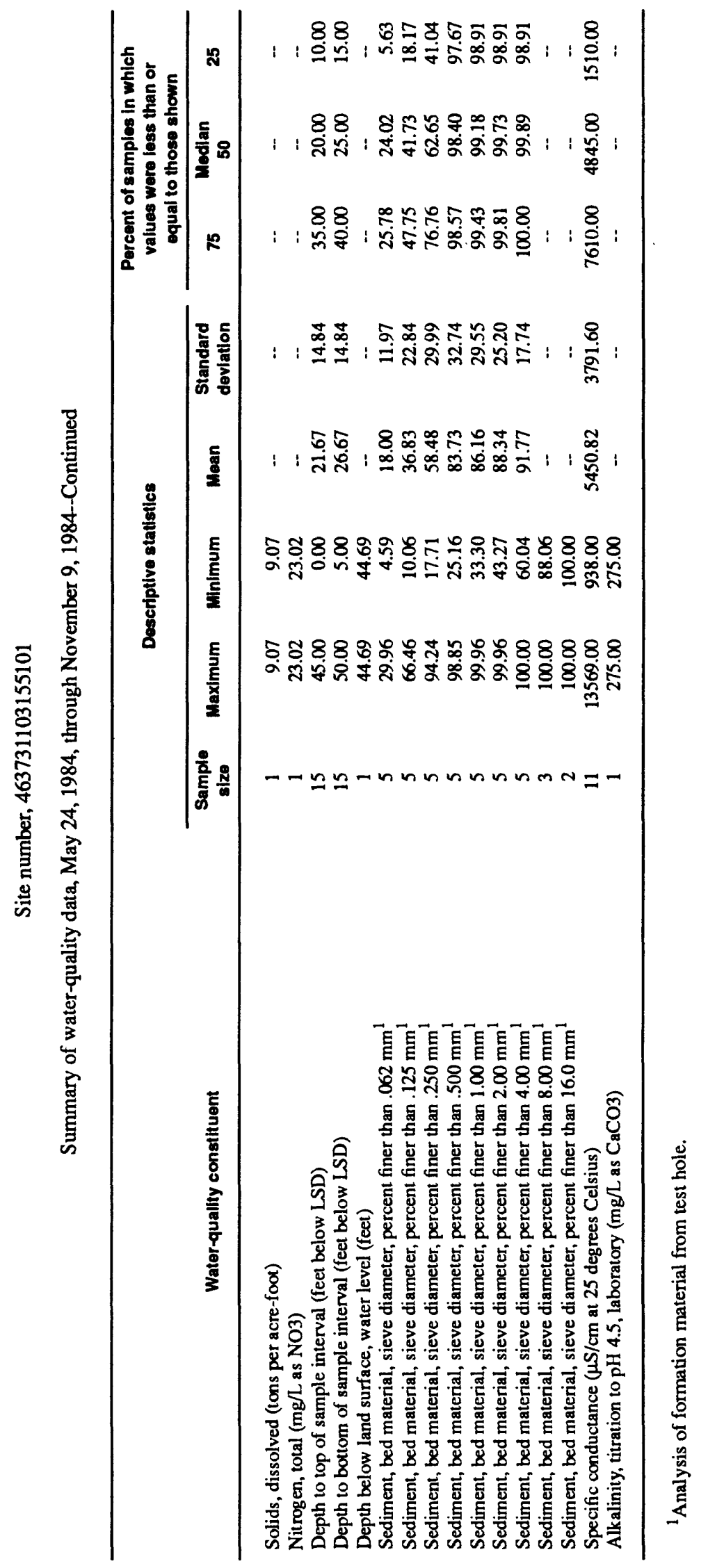




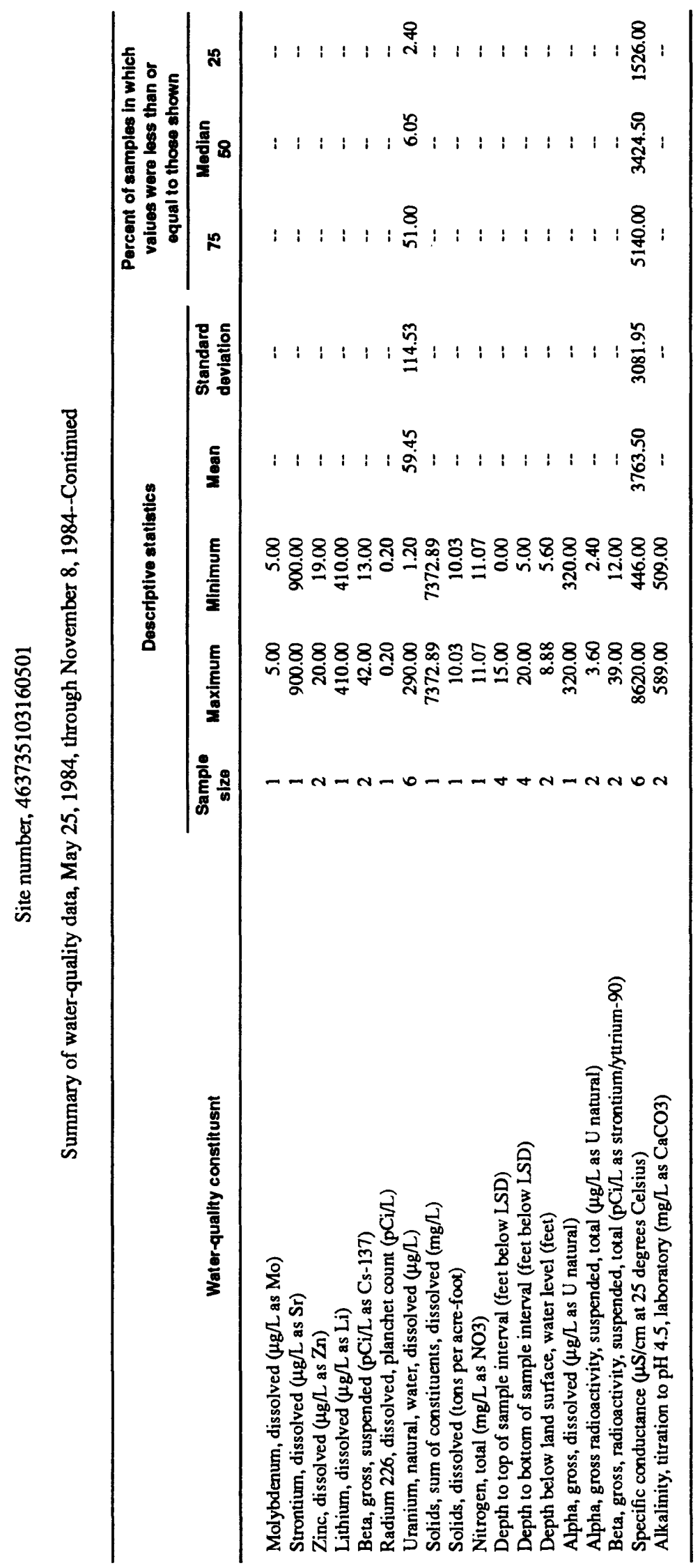




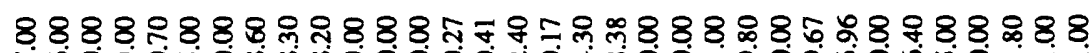
金通

ชิ

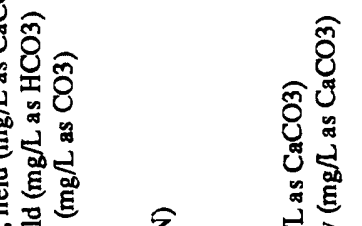

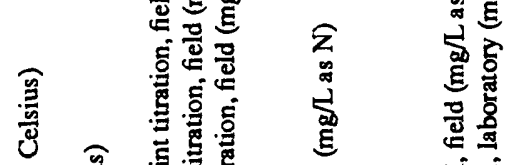

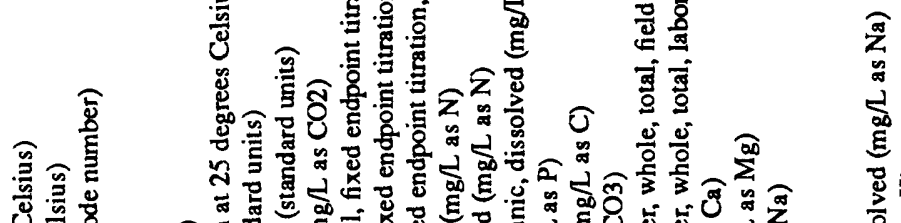

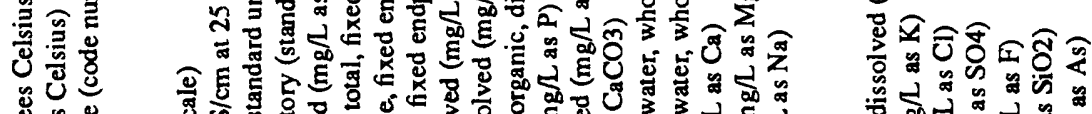

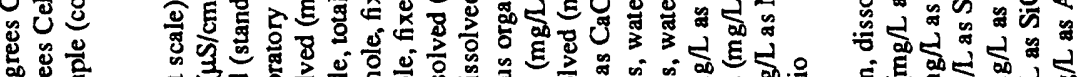

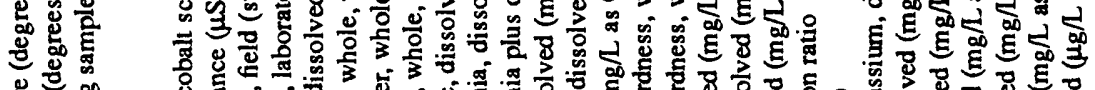

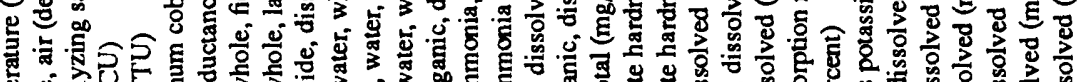

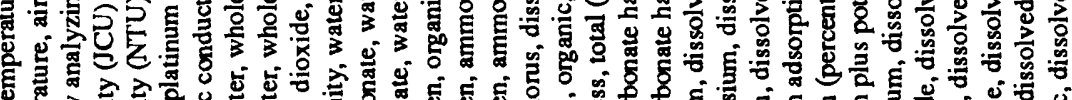

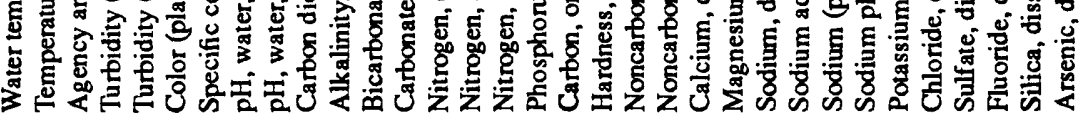




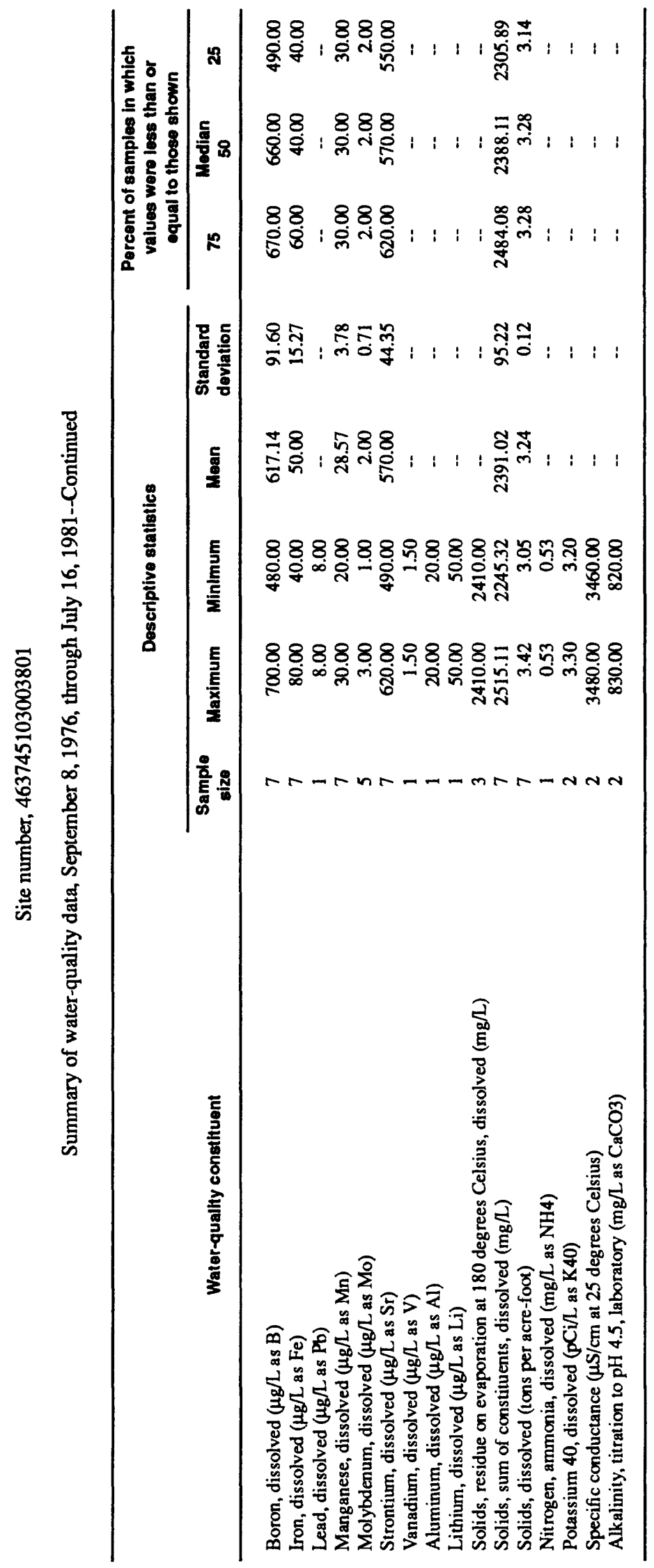



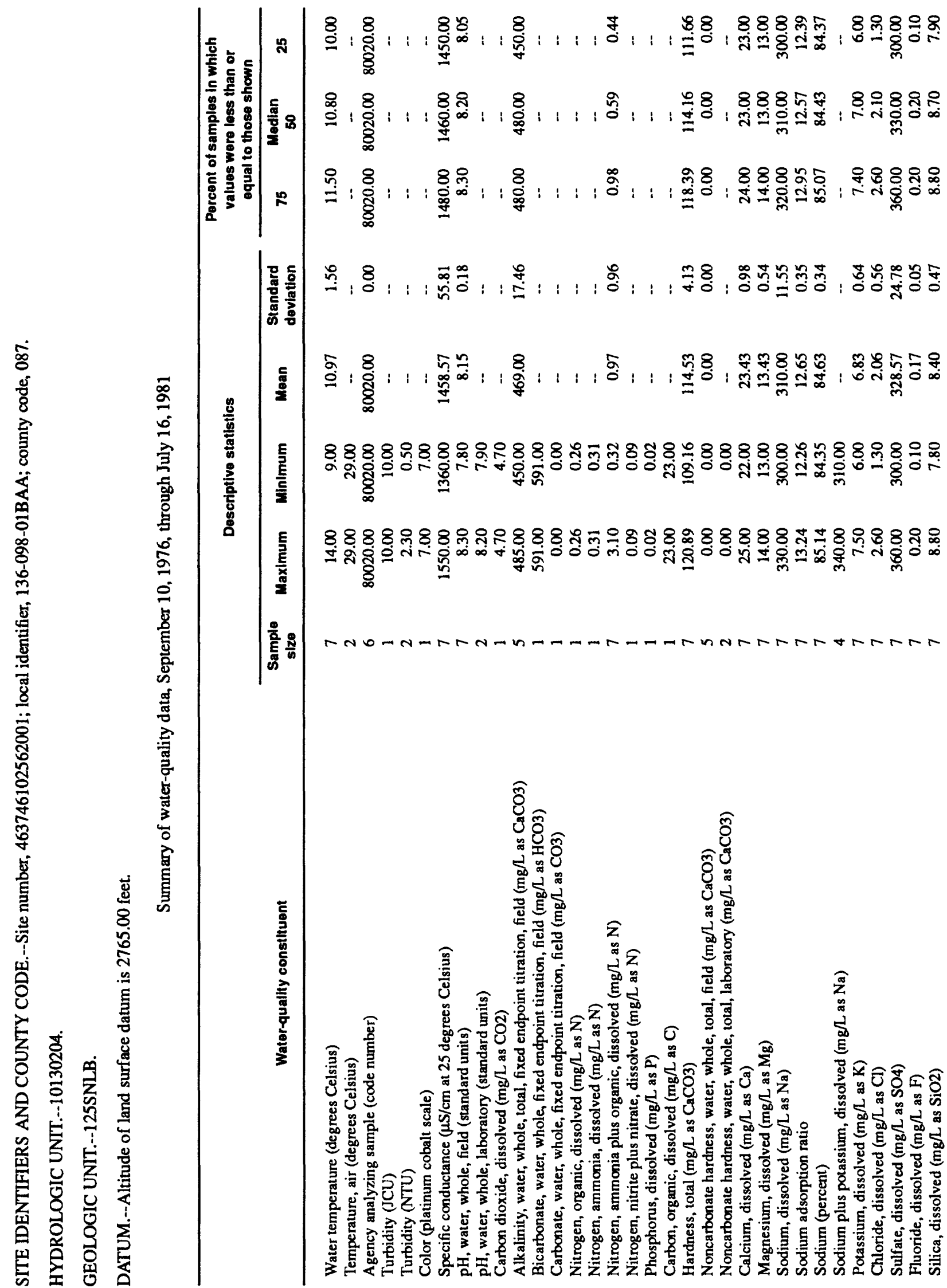

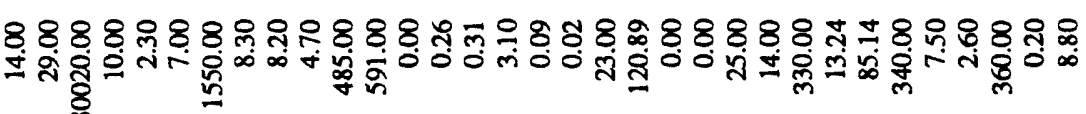

ชิ ซ్ల్

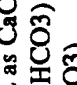

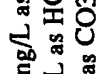

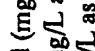

远尊

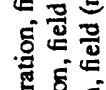

商

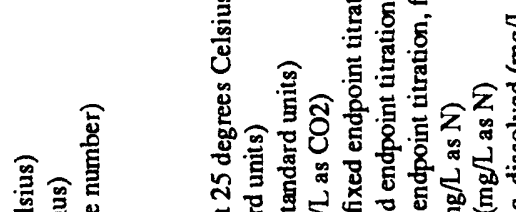

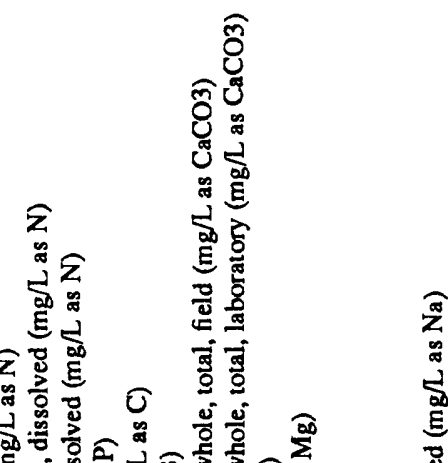

U⿺辶寸

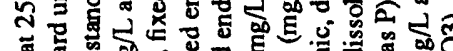

Q

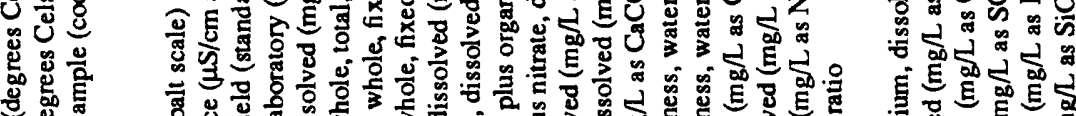

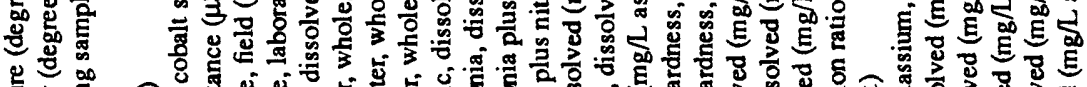

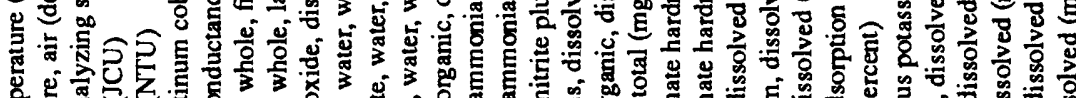

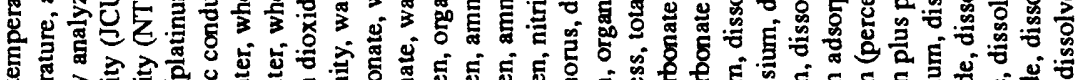

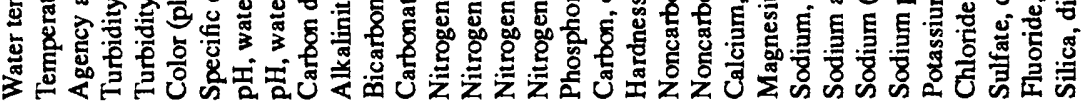




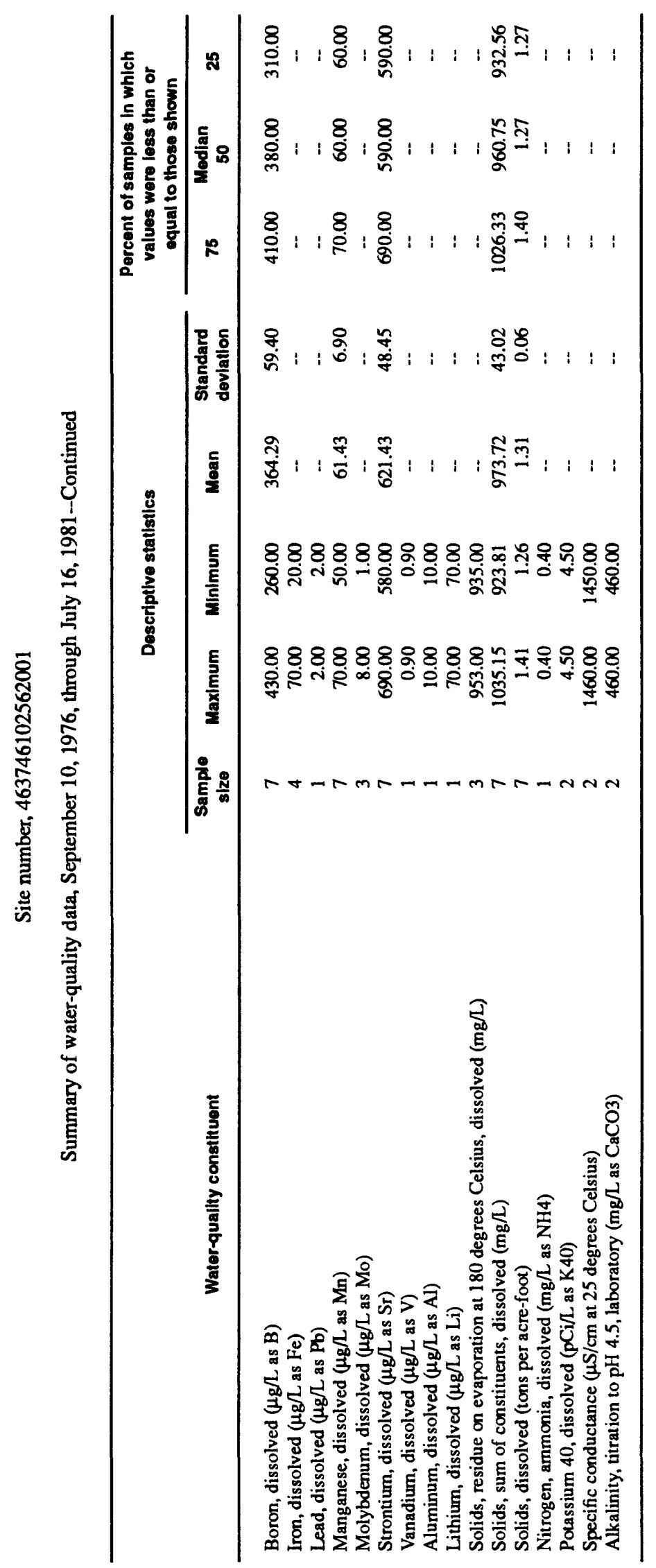


Table 6. Summary of daily ground-water level data obtained from the U.S. Geological Survey

\section{Symbols}

,-- no data

\section{County code}

011, Bowman County

\section{Geologic unit}

125 Paleocene

TRVL Tongue River-Ludlow Members of Fort Union Formation 
SITE IDENTIFIERS AND COUNTY CODE.--Site number, 460705103005301; local identifier, 130-099-01BBB;

county code, 011 .

HYDROLOGIC UNTT.--10130205.

GEOLOGIC UNIT.--125TRVL.

DATUM.-Altitude of land surface datum is 2768.00 feet.

Summary of daily ground-water level data, in feet below land surface datum, June 1974 through December 1976

\begin{tabular}{lcc}
\hline Month & Maximum & Minimum \\
\hline October & 25.63 & 24.20 \\
November & 25.53 & 24.14 \\
December & 25.44 & 24.06 \\
January & 25.24 & 23.47 \\
February & 25.47 & 23.38 \\
March & 25.48 & 23.56 \\
April & 25.10 & 22.36 \\
May & 24.61 & 22.81 \\
June & 25.06 & 23.42 \\
July & 25.68 & 23.00 \\
August & 26.09 & 24.11 \\
September & 26.23 & 24.33 \\
\hline
\end{tabular}

SITE IDENTIFIERS AND COUNTY CODE.--Site number, 461355103055701; local identifier, 131-099-19DDD; county code, 011.

HYDROLOGIC UNIT.--10130204.

GEOLOGIC UNIT.--125TRVL.

DATUM.-Altibude of land surface datum is 2775.00 feet.

Summary of daily ground-water level data, in feet below land surface datum, June 1974 through December 1976

\begin{tabular}{lcc}
\hline Month & Maximum & Minimum \\
\hline October & 24.89 & 23.54 \\
November & 26.33 & 23.66 \\
December & 24.30 & 23.59 \\
January & 24.16 & 24.03 \\
Febrairy & 24.23 & 24.12 \\
March & 24.28 & 24.04 \\
April & 23.69 & - \\
May & 23.48 & 23.22 \\
June & 23.33 & 19.91 \\
July & 23.32 & 20.00 \\
August & 23.56 & 20.28 \\
September & 23.69 & 20.40 \\
\hline
\end{tabular}

SITE IDENTIFIERS AND COUNTY CODE.--Site number, 461355103032801; local identifier, 131-099-21DDD; county code, 011.

HYDROLOGIC UNIT.--10130205.

GEOLOGIC UNIT.--Not available.

DATUM.--Altitude of land surface datum is not available.

Summary of daily ground-water level data, in feet below land surface datum, November 1974 through November 1976

\begin{tabular}{lcc}
\hline Month & Maximum & Minimum \\
\hline October & 47.25 & 45.67 \\
November & 47.31 & 35.46 \\
December & 46.86 & 35.46 \\
January & 35.62 & 35.46 \\
Febrairy & 47.80 & 35.54 \\
March & 48.05 & 36.92 \\
April & 48.62 & 37.13 \\
May & 48.89 & 37.07 \\
June & 48.45 & 38.50 \\
July & 47.63 & 39.50 \\
August & 51.01 & 42.93 \\
September & 51.01 & 45.52 \\
\hline
\end{tabular}


SITE IDENTIFIERS AND COUNTY CODE.-Site number, 461257103004902; local identifier, 131-099-36BBB2; county code, 011.

HYDROLOGIC UNTT.--10130205.

GEOLOGIC UNIT.--Not available.

DATUM.--Altitude of land surface datum is 2822.00 feet.

Summary of daily ground-water level data, in feet below land surface datum, June 1974 through December 1976

\begin{tabular}{lcc}
\hline Month & Maximum & Minimum \\
\hline October & 66.06 & 64.69 \\
November & 65.80 & 64.09 \\
December & 66.03 & 64.40 \\
January & 64.69 & 64.37 \\
Febrary & 64.83 & 64.36 \\
March & 64.75 & 64.23 \\
April & 64.92 & 63.58 \\
May & 65.19 & 61.99 \\
June & 65.26 & 61.21 \\
July & 64.79 & 61.74 \\
August & 65.10 & 62.51 \\
September & 66.06 & 56.97 \\
\hline
\end{tabular}


Table 7. Summary of periodic ground-water level data obtained from the U.S. Geological Survey

\section{Symbols}

,- no data

\section{County code}

01 , Adams County

011, Bowman County

037, Grant County

041, Hettinger County

059, Morton County

085, Sioux County

087, Slope County

\section{Geologlc unlt}

112 Pleistocene

125 Paleocene

$211 \quad$ Upper Cretaceous

BGFV Buried glaciofluvial deposits

EMCK Elm Creek aquifer

FXHL Fox Hills Sandstone

HLCK Hell Creek Formation

HRMN Harmon lignite aquifer

LDLW Ludlow Member of Fort Union Formation

LHCK Ludlow Member of Fort Union Formation-Hell Creek Formation

SNLB Sentinel Butte Member of Fort Union Formation

TGRV Tongue River Member of Fort Union Formation

TRVL Tongue River-Ludlow Members of Fort Union Formation 
SITE IDENTIFIERS AND COUNTY CODE.-Site number, 460047101333801; local identifier, 129-087-10BBC; county code, 037.

HYDROLOGIC UNIT.--10130205.

GEOLOGIC UNIT.--211FXHL.

DATUM.-Altitude of land surface daum is 2060.00 feet.

Summary of periodic ground-water level data, in feet below land surface datum, November 1972 through July 1989

\begin{tabular}{lccc}
\hline \multicolumn{1}{c}{ Month } & Sample size & Maximum & Minimum \\
\hline October & 2 & 107.71 & 107.55 \\
November & 6 & 107.70 & 107.00 \\
December & 11 & 107.52 & 105.54 \\
January & - & - & 107.43 \\
Febrary & 1 & 107.43 & - \\
March & - & - & 107.45 \\
April & 1 & 107.45 & 107.45 \\
May & 1 & 107.68 & 107.68 \\
June & 1 & 107.67 & 107.42 \\
July & 3 & 107.78 & 107.01 \\
August & 2 & -- & - \\
September & - & & \\
\hline
\end{tabular}

SITE IDENTIFIERS AND COUNTY CODE.--Site number, 460056102061001; local identifier, 129-091-07AAA1; county code, 001.

HYDROLOGIC UNTT.--10130205.

GEOLOGIC UNIT.--125LHCK.

DATUM.--Altitude of land surface datum is 2422.00 feet.

Summary of periodic ground-water level data, in feet below land surface datum, September 1971 through December 1993

\begin{tabular}{lccc}
\hline Month & Sample size & Maximum & Minimum \\
\hline October & 1 & 150.00 & 150.00 \\
November & 12 & 151.22 & 149.90 \\
December & 11 & 150.95 & 149.43 \\
January & 1 & 150.21 & 150.21 \\
February & 2 & 150.30 & 150.02 \\
March & 2 & 150.32 & 150.32 \\
April & 2 & 150.25 & 149.75 \\
May & 2 & 150.05 & 148.72 \\
June & 2 & 149.87 & 149.70 \\
July & 2 & 150.91 & 150.05 \\
August & 2 & 150.01 & 149.99 \\
September & 4 & 150.47 & 149.96 \\
\hline
\end{tabular}

SITE IDENTIFIERS AND COUNTY CODE.--Site number, 460056102061002; local identifier, 129-091-07AAA2; county code, 001 .

HYDROLOGIC UNIT.--10130205.

\section{GEOLOGIC UNIT.--125LDLW.}

DATUM.-Altitude of land surface datum is 2422.00 feet.

Summary of periodic ground-water level data, in feet below land surface datum, September 1971 through December 1993

\begin{tabular}{lccc}
\hline Month & Sample size & Maximum & Minimum \\
\hline October & 1 & 93.62 & 93.62 \\
November & 11 & 93.65 & 89.16 \\
December & 11 & 94.34 & 89.29 \\
January & 1 & 93.61 & 93.61 \\
February & 2 & 94.22 & 93.40 \\
March & 2 & 93.85 & 93.55 \\
April & 2 & 93.80 & 93.48 \\
May & 2 & 93.63 & 93.45 \\
June & 2 & 93.64 & 93.15 \\
July & 2 & 94.08 & 93.50 \\
August & 2 & 94.09 & 93.62 \\
September & 4 & 94.06 & 89.74 \\
\hline
\end{tabular}


SITE IDENTIFIERS AND COUNTY CODE.-Site number, 460239101135301; local identifier, 130-084-31AAA1; county code, 085 .

HYDROLOGIC UNIT.--10130206.

GEOLOGIC UNIT.--211HLCK.

DATUM.--Altitude of land surface datum is 2238.00 feet.

Summary of periodic ground-water level data, in feet below land surface datum, July 1973 through November 1981

\begin{tabular}{lccc}
\hline \multicolumn{1}{c}{ Month } & Sample size & Maximum & Minimum \\
\hline October & 2 & 311.00 & 171.76 \\
November & 5 & 311.94 & 185.23 \\
December & 10 & 312.30 & 172.22 \\
January & - & - & - \\
Febrary & 1 & 174.27 & 174.27 \\
March & - & - & - \\
April & - & - & - \\
May & - & 173.89 & 173.89 \\
June & 1 & 178.35 & 177.24 \\
July & 2 & 176.42 & 175.18 \\
August & 2 & -- & -- \\
September & -- & & - \\
\hline
\end{tabular}

SITE IDENTIFIERS AND COUNTY CODE.--Site number, 460239101135302; local identifier, 130-084-31AAA2; county code, 085 .

HYDROLOGIC UNIT.--10130206.

GEOLOGIC UNIT.--211HLCK.

DATUM.--Altitude of land surface datum is $2238.00 \mathrm{feet}$.

Summary of periodic ground-water level data, in feet below land surface datum, July 1973 through November 1989

\begin{tabular}{lccc}
\hline \multicolumn{1}{c}{ Month } & Sample size & Maximum & Minimum \\
\hline October & 2 & 165.56 & 164.35 \\
November & 6 & 165.34 & 162.89 \\
December & 10 & 165.60 & 163.52 \\
January & -- & -- & 165.60 \\
February & 1 & 165.60 & -- \\
March & - & -- & -- \\
April & -- & -- & - \\
May & -- & 165.37 & 165.37 \\
June & 1 & 165.72 & 163.26 \\
July & 3 & 166.55 & 165.73 \\
August & 2 & -- & -- \\
September & -- & & - \\
\hline
\end{tabular}

SITE IDENTIFIERS AND COUNTY CODE.--Site number, 460240101075501; local identifier, 130-084-36ABA; county code, 085 .

HYDROLOGIC UNIT.--10130206.

GEOLOGIC UNIT.--211FXHL.

DATUM.--Altitude of land surface datum is $2148.00 \mathrm{feet}$

Summary of periodic ground-water level data, in feet below land surface datum, November 1972 through November 1989

\begin{tabular}{lccc}
\hline \multicolumn{1}{c}{ Month } & Sample size & Maximum & Minimum \\
\hline October & 2 & 240.58 & 240.50 \\
November & 6 & 248.00 & 211.59 \\
December & 11 & 248.50 & 239.58 \\
January & -- & -- & - \\
February & 1 & 240.67 & 240.67 \\
March & - & -- & - \\
April & 1 & 240.60 & 240.60 \\
May & - & -- & -- \\
June & 1 & 240.75 & 240.75 \\
July & 1 & 241.20 & 241.20 \\
August & 2 & -240.97 & 240.22 \\
September & - & - & - \\
\hline
\end{tabular}


SITE IDENTIFIERS AND COUNTY CODE.--Site number, 460449101200701; local identifier, 130-085-17DAA1; county code, 085 .

HYDROLOGIC UNTT.--10130205.

GEOLOGIC UNIT.--211FXHL.

DATUM.--Altitude of land surface datum is 1910.00 feet.

Summary of periodic ground-water level data, in feet below land surface datum, October 1972 through December 1993

\begin{tabular}{lccc}
\hline Month & Sample size & Maximum & Minimum \\
\hline October & 3 & 22.16 & 22.43 \\
November & 6 & 22.25 & 21.20 \\
December & 16 & 22.34 & 21.11 \\
January & 3 & 22.46 & 21.44 \\
February & 6 & 22.03 & 21.31 \\
March & 3 & 22.63 & 21.19 \\
April & 3 & 22.19 & 21.38 \\
May & 70 & 22.29 & 2.19 \\
June & 1 & 22.70 & 21.46 \\
July & 5 & 21.68 \\
August & 7 & 21.26 \\
September & 2 & 20.90 \\
\hline
\end{tabular}

SITE IDENTIFIERS AND COUNTY CODE.--Site number, 460244101272701; local identifier, 130-086-28CCC1; county code, 085 .

HYDROLOGIC UNIT.--10130205.

GEOLOGIC UNIT.--211FXHL.

DATUM.-Altitude of land surface datum is 2062.00 feet.

Summary of periodic ground-water level data, in feet below land surface datum, July 1973 through December 1993

\begin{tabular}{lccc}
\hline \multicolumn{1}{c}{ Month } & Sample size & Maximum & Minimum \\
\hline October & 2 & 139.89 & 139.44 \\
November & 7 & 140.11 & 139.35 \\
December & 14 & 140.10 & 139.07 \\
January & - & - & - \\
February & 1 & 139.75 & 139.75 \\
March & - & - & - \\
April & -- & -- & - \\
May & - & -- & - \\
June & 1 & 139.84 & 139.84 \\
July & 3 & 140.10 & 139.37 \\
August & 2 & 140.13 & 139.40 \\
September & -- & - & -- \\
\hline
\end{tabular}

SITE IDENTIFIERS AND COUNTY CODE.--Site number, 460244101272702; local identifier, 130-086-28CCC2; county code, 085 .

HYDROLOGIC UNIT.--10130205.

GEOLOGIC UNIT.--211HLCK.

DATUM.--Altitude of land surface datum is 2062.00 feet.

Summary of periodic ground-water level data, in feet below land surface datum, July 1973 through December 1993

\begin{tabular}{lccc}
\hline \multicolumn{1}{c}{ Month } & Sample size & Maximum & Minimum \\
\hline October & 2 & 136.05 & 135.72 \\
November & 7 & 136.70 & 135.63 \\
December & 14 & 136.29 & 134.78 \\
January & - & - & - \\
February & 1 & 135.92 & 135.92 \\
March & - & - & - \\
April & - & - & - \\
May & - & -- & 136.05 \\
June & 1 & 136.19 & 136.12 \\
July & 2 & 136.30 & 136.00 \\
August & 2 & -- & - \\
September & -- & & - \\
\hline
\end{tabular}


SITE IDENTIFIERS AND COUNTY CODE.--Site number, 460159101500401; local identifier, 130-089-32DDA; county code, 037 .

HYDROLOGIC UNIT.--10130205.

GEOLOGIC UNIT.--211FXHL.

DATUM.--Altitude of land surface datum is $2165.00 \mathrm{feet}$

Summary of periodic ground-water level data, in feet below land surface datum, December 1972 through December 1981

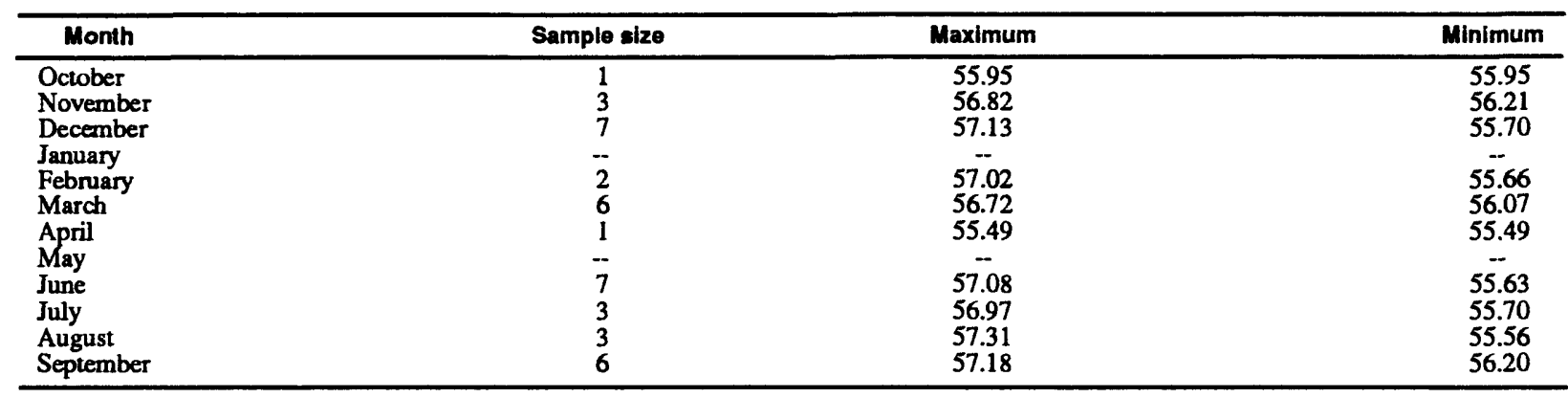

SITE IDENTIFIERS AND COUNTY CODE.-Site number, 460705103005301; local identifier, 130-099-01BBB; county code, 011.

HYDROLOGIC UNIT.--10130205.

GEOLOGIC UNIT.--125TRVL.

DATUM.--Altitude of land surface datum is 2768.00 feet.

Summary of periodic ground-water level data, in feet below land surface datum, October 1974 through July 1991

\begin{tabular}{lccc}
\hline Month & Sample size & Maximum & Minimum \\
\hline October & 2 & 25.60 & 25.53 \\
November & 6 & 25.50 & 24.25 \\
December & 3 & 25.39 & 23.97 \\
January & 2 & 25.10 & 23.97 \\
February & 2 & 25.29 & 23.45 \\
March & 2 & 25.43 & 23.68 \\
April & 2 & 23.88 & 23.46 \\
May & 1 & 23.93 & 23.93 \\
June & 2 & 24.15 & 23.51 \\
July & 4 & 28.18 & 23.61 \\
August & 4 & 27.86 & 24.25 \\
September & 4 & 25.96 & 24.45 \\
\hline
\end{tabular}

SITE IDENTIFIERS AND COUNTY CODE.--Site number, 460845101512001; local identifier, 131-089-30AAA; county code, 037 .

HYDROLOGIC UNIT.--10130205.

GEOLOGIC UNIT.--211FXHL.

DATUM.--Altitude of land surface datum is 2395.00 feet.

Summary of periodic ground-water level data, in feet below land surface datum, July 1973 through December 1992

\begin{tabular}{lccc}
\hline \multicolumn{1}{c}{ Month } & Sample size & Maximum \\
\hline October & 2 & 322.58 \\
November & 10 & 323.44 \\
December & 10 & 323.36 \\
January & -- & - & 321.90 \\
February & 1 & 321.62 & -31 \\
March & -- & -- & - \\
April & -- & 321.86 \\
May & - & 323.34 \\
June & 1 & 322.05 \\
July & 3 & -- \\
August & 2 & -- \\
September & -- & 321.86 \\
\hline
\end{tabular}


SITE IDENTIFIERS AND COUNTY CODE.--Site number, 461130102122601; local identifier, 131-092-05DDD; county code, 001.

HYDROLOGIC UNIT.--10130205.

GEOLOGIC UNIT.--Not available.

DATUM.--Altitude of land surface datum is not available.

Summary of periodic ground-water level data, in feet below land surface datum, October 1976 through December 1993

\begin{tabular}{lccc}
\hline Month & Sample size & Maximum & Minimum \\
\hline October & 1 & 72.45 & 72.45 \\
November & 10 & 73.23 & 70.97 \\
December & 5 & 73.29 & 70.86 \\
January & 1 & 72.25 & 72.25 \\
February & - & -- & 72.26 \\
March & 1 & 72.26 & 72.26 \\
April & 1 & 72.26 & 72.37 \\
May & 1 & 72.37 & 71.50 \\
June & 2 & --74 & 71.62 \\
July & - & 71.62 & 71.28 \\
August & 1 & 71.28 & \\
September & 1 & & 76 \\
\hline
\end{tabular}

SITE IDENTIFIERS AND COUNTY CODE.-Site number, 460908102282702; local identifier, 131-094-20CBC2; county code, 001.

HYDROLOGIC UNIT.--10130205.

\section{GEOLOGIC UNIT.--125LHCK.}

DATUM.-Altitude of land surface datum is 2500.00 feet.

Summary of periodic ground-water level data, in feet below land surface datum, September 1971 through December 1993

\begin{tabular}{lccc}
\hline Month & Sample size & Maximum & Minimum \\
\hline October & 1 & $\mathbf{8 5 . 3 1}$ & $\mathbf{8 5 . 3 1}$ \\
November & 13 & $\mathbf{8 5 . 9 0}$ & $\mathbf{8 4 . 8 4}$ \\
December & 10 & $\mathbf{8 5 . 5 3}$ & $\mathbf{8 5 . 1 1}$ \\
January & 1 & $\mathbf{8 5 . 4 1}$ & 85.41 \\
February & 2 & 85.50 & 85.35 \\
March & 2 & 85.70 & 85.44 \\
April & 1 & 85.38 & 85.38 \\
May & 2 & 85.30 & 85.10 \\
June & 2 & 85.19 & 85.18 \\
July & 2 & 85.97 & 85.45 \\
August & 2 & 85.47 & 85.42 \\
September & 4 & 85.62 & 85.29 \\
\hline
\end{tabular}

SITE IDENTIFIERS AND COUNTY CODE.--Site number, 460908102282703; local identifier, 131-094-20CBC3; county code, 001 .

HYDROLOGIC UNIT.--10130205.

\section{GEOLOGIC UNIT.--125LDLW.}

DATUM.--Altitude of land surface datum is 2500.00 feet.

Summary of periodic ground-water level data, in feet below land surface datum, September 1971 through December 1993

\begin{tabular}{lccc}
\hline Month & Sample size & Maximum & Minimum \\
\hline October & 1 & 63.90 & 63.90 \\
November & 13 & 69.50 & 62.29 \\
December & 10 & 69.49 & 61.92 \\
January & 1 & 65.84 & 65.84 \\
February & 2 & 63.93 & 63.89 \\
March & 2 & 64.09 & 64.01 \\
April & 1 & 64.05 & 64.05 \\
May & 2 & 64.04 & 63.65 \\
June & 2 & 63.86 & 63.77 \\
July & 2 & 64.23 & 63.90 \\
August & 2 & 64.30 & 64.04 \\
September & 4 & 66.80 & 62.08 \\
\hline
\end{tabular}


SITE IDENTIFIERS AND COUNTY CODE.--Site number, 461355103055701; local identifier, 131-099-19DDD; county code, 011.

HYDROLOGIC UNIT.--10130204.

GEOLOGIC UNIT.--125TRVL.

DATUM.-Altitude of land surface datum is 2775.00 feet.

Summary of periodic ground-water level data, in feet below land surface datum, March 1974 through July 1990

\begin{tabular}{lccc}
\hline Month & Sample size & Maximum & Minimum \\
\hline October & 1 & 23.60 & 23.60 \\
November & 4 & 24.05 & 20.42 \\
December & 3 & 24.04 & 23.47 \\
January & 2 & 24.15 & 24.01 \\
February & 2 & 24.14 & 19.65 \\
March & 2 & 24.23 & 23.58 \\
April & 1 & 20.05 & 20.05 \\
May & 3 & 25.85 & 20.60 \\
June & 2 & 23.24 & 20.01 \\
July & 4 & 25.59 & 20.05 \\
August & 4 & 26.35 & 20.30 \\
September & 4 & 22.42 & 10.90 \\
\hline
\end{tabular}

SITE IDENTIFIERS AND COUNTY CODE.--Site number, 461355103032801; local identifier, 131-099-21DDD; county code, 011.

HYDROLOGIC UNIT.--10130205.

GEOLOGIC UNIT.--Not available.

DATUM.-Altitude of land surface datum is not available.

Summary of periodic ground-water level data, in feet below land surface datum, March 1974 through December 1976

\begin{tabular}{lccc}
\hline Month & Sample size & Maximum & Minimum \\
\hline October & 2 & 45.77 & 34.81 \\
November & 4 & 46.74 & 35.46 \\
December & 3 & 46.45 & 35.55 \\
January & 2 & 47.12 & 35.46 \\
February & 2 & 47.63 & 35.65 \\
March & 2 & 47.89 & 37.28 \\
April & 1 & 48.18 & 48.18 \\
May & 2 & 48.72 & 37.05 \\
June & 2 & 48.45 & 38.72 \\
July & 2 & 46.92 & 40.91 \\
August & 2 & 45.67 & 43.37 \\
September & 2 & 43.73 & 43.16 \\
\hline
\end{tabular}

SITE IDENTIFIERS AND COUNTY CODE.--Site number, 460830103044504; local identifier, 131-099-29ADD4; county code, 011 .

HYDROLOGIC UNIT.--10130205.

GEOLOGIC UNIT.--125HRMN.

DATUM.--Altitude of land surface datum is 2796.00 feet.

Summary of periodic ground-water level data, in feet below land surface datum, September 1987 through August 1991

\begin{tabular}{lccc}
\hline \multicolumn{1}{c}{ Month } & Sample size & Maximum & Minimum \\
\hline October & - & - & - \\
November & - & - & - \\
December & - & - & - \\
January & - & - & - \\
February & - & - & - \\
March & - & - & - \\
April & - & - & - \\
May & - & - & - \\
June & - & -- & - \\
July & - & 61.51 & 58.28 \\
August & 3 & 58.79 & 55.32 \\
September & 2 & & \\
\hline
\end{tabular}


SITE IDENTIFIERS AND COUNTY CODE.--Site number, 461257103004902; local identifier, 131-099-36BBB2; county code, 011 .

HYDROLOGIC UNIT.--10130205.

GEOLOGIC UNIT.--Not available.

DATUM.-Altitude of land surface datum is 2822.00 feet.

Summary of periodic ground-water level data, in feet below land surface datum, October 1974 through December 1976

\begin{tabular}{lccc}
\hline Month & Sample size & Maximum \\
\hline October & 2 & 65.47 \\
November & 3 & 65.78 \\
December & 3 & 66.04 & 64.90 \\
January & 2 & 64.68 & 64.09 \\
February & 2 & 64.80 & 64.00 \\
March & 2 & 64.63 & 64.84 \\
April & 2 & 64.94 & 65.16 \\
May & 1 & 64.41 & 63.61 \\
June & 2 & 64.94 \\
July & 2 & 61.95 \\
August & 2 & 65.93 \\
September & 3 & 62.70 \\
\hline
\end{tabular}

SITE IDENTIFIERS AND COUNTY CODE.--Site number, 461344101072901; local identifier, 132-083-30BCB; county code, 037.

HYDROLOGIC UNIT. - 10130206.

GEOLOGIC UNIT.--112BGFV.

DATUM.--Altitude of land surface datum is $1814.00 \mathrm{feet}$.

Summary of periodic ground-water level data, in feet below land surface datum, November 1971 through December 1993

\begin{tabular}{lccc}
\hline Month & Sample size & Maximum & Minimum \\
\hline October & 2 & 25.81 & 25.31 \\
November & 8 & 26.20 & 25.10 \\
December & 16 & 26.45 & 24.65 \\
January & 1 & 25.63 & 25.63 \\
February & 3 & 25.68 & 25.20 \\
March & 1 & 25.52 & 25.52 \\
April & 2 & 25.43 & 25.06 \\
May & 1 & 25.10 & 25.10 \\
June & 3 & 25.87 & 24.89 \\
July & 3 & 25.80 & 25.28 \\
August & 4 & 25.95 & 25.31 \\
September & 1 & 25.40 & 25.40 \\
\hline
\end{tabular}

SITE IDENTIFIERS AND COUNTY CODE.--Site number, 461345101323801; local identifier, 132-087-27ADA; county code, 037.

HYDROLOGIC UNIT.--10130204.

GEOLOGIC UNIT.--211HLCK.

DATUM.--Altitude of land surface datum is $2010.00 \mathrm{feet}$.

Summary of periodic ground-water level data, in feet below land surface datum, November 1972 through December 1993

\begin{tabular}{|c|c|c|c|}
\hline Month & Sample size & Maximum & Minimum \\
\hline $\begin{array}{l}\text { October } \\
\text { November } \\
\text { December }\end{array}$ & $\begin{array}{r}2 \\
8 \\
15\end{array}$ & $\begin{array}{l}12.32 \\
12.65 \\
12.73\end{array}$ & $\begin{array}{l}10.97 \\
11.00 \\
10.54\end{array}$ \\
\hline January & -- & - & -- \\
\hline February & 1 & 10.81 & 10.81 \\
\hline March & -- & -- & -- \\
\hline April & 1 & 10.36 & 10.36 \\
\hline June & 1 & 11.07 & 11.07 \\
\hline July & 2 & 12.53 & 10.74 \\
\hline August & 3 & 11.28 & 10.71 \\
\hline September & -- & -- & - \\
\hline
\end{tabular}


SITE IDENTIFIERS AND COUNTY CODE.--Site number, 461542101535902; local identifier, 132-090-14AAB2; county code; 037.

HYDROLOGIC UNIT.--10130204.

GEOLOGIC UNIT.--125TGRV.

DATUM.--Altitude of land surface datum is 2340.00 feet.

Summary of periodic ground-water level data, in feet below land surface datum, July 1973 through December 1993

\begin{tabular}{lccc}
\hline \multicolumn{1}{c}{ Month } & Sample size & Maximum & Minimum \\
\hline October & 2 & 13.39 & 12.34 \\
November & 10 & 14.95 & 12.07 \\
December & 10 & 14.95 & 11.76 \\
January & -- & -- & 13.73 \\
February & 1 & 13.73 & - \\
March & -- & - & - \\
April & - & -- & 13.50 \\
May & - & 13.50 & 12.89 \\
June & 1 & 13.75 & 12.98 \\
July & 2 & 14.02 & -- \\
August & 3 & - & - \\
September & - & & - \\
\hline
\end{tabular}

SITE IDENTIFIERS AND COUNTY CODE.--Site number, 461315102034501; local identifier, 132-091-28DDD; county code, 041 .

HYDROLOGIC UNIT.--10130205.

GEOLOGIC UNIT.--211FXHL.

DATUM.-Altitude of land surface datum is 2469.00 feet.

Summary of periodic ground-water level data, in feet below land surface datum, September 1968 through December 1993

\begin{tabular}{lccc}
\hline Month & Sample size & Maximum & Minimum \\
\hline October & 2 & 289.90 & 289.70 \\
November & 14 & 291.62 & 289.47 \\
December & 14 & 295.54 & 289.40 \\
January & 1 & 289.73 & 289.73 \\
Febrary & 1 & 290.30 & 290.30 \\
March & 1 & 289.34 & 289.34 \\
April & 2 & 289.96 & 289.49 \\
May & 1 & 290.11 & 290.11 \\
June & 2 & 289.89 & 289.50 \\
July & 1 & 289.46 & 289.46 \\
August & 1 & 289.72 & 289.72 \\
September & 3 & 290.46 & 289.93 \\
\hline
\end{tabular}

SITE IDENTIFIERS AND COUNTY CODE.--Site number, 461348102121901; local identifier, 132-092-28BCB; county code, 041.

HYDROLOGIC UNIT.--10130205.

GEOLOGIC UNTT.-125TGRV.

DATUM.--Altitude of land surface datum is 2455.00 feet.

Summary of periodic ground-water level data, in feet below land surface datum, April 1977 through June 1981

\begin{tabular}{lccc}
\hline \multicolumn{1}{c}{ Month } & Sample size & Maximum & Minimum \\
\hline October & - & - & -- \\
November & - & -- & - \\
December & - & - & - \\
January & - & - & - \\
February & - & - & - \\
March & - & - & - \\
April & 1 & -0.92 & -0.92 \\
May & 1 & -1.49 & -0.89 \\
June & 1 & -1.89 & -1.49 \\
July & 1 & -- & -1.89 \\
August & -- & -- & -- \\
September & -- & - & - \\
\hline
\end{tabular}


SITE IDENTIFIERS AND COUNTY CODE.-Site number, 461452102194601; local identifier, 132-093-21BBB; county code, 041.

HYDROLOGIC UNIT.--10130205.

GEOLOGIC UNIT.--125TGRV.

DATUM.-Altitude of land surface datum is 2510.00 feet.

Summary of periodic ground-water level data, in feet below land surface datum, November 1976 through October 1981

\begin{tabular}{lccc}
\hline \multicolumn{1}{c}{ Month } & Sample elze & Maximum & Minimum \\
\hline October & - & - & -5.96 \\
November & 2 & 14.33 & 12.9 .14 \\
December & 1 & 14.14 & 14.70 \\
January & 1 & -- & 14.88 \\
February & -- & 14.88 & 13.79 \\
March & 1 & 13.79 & 13.80 \\
April & 1 & 13.80 & 12.79 \\
May & 1 & 13.89 & -- \\
June & 2 & -- & 12.57 \\
July & --57 & - \\
August & 1 & - & - \\
September & -- & & - \\
\hline
\end{tabular}

SITE IDENTIFIERS AND COUNTY CODE.--Site number, 461614102515202; local identifier, 132-097-07CAB2; county code, 001.

HYDROLOGIC UNIT.--10130205.

GEOLOGIC UNIT.--125LHCK.

DATUM.--Altitude of land surface datum is 2665.00 feet.

Summary of periodic ground-water level data, in feet below land surface datum, August 1971 through December 1993

\begin{tabular}{lccc}
\hline Month & Sample size & Maximum & Minimum \\
\hline October & 1 & 86.23 & 86.23 \\
November & 13 & 87.05 & 86.42 \\
December & 10 & 87.25 & 85.39 \\
January & 2 & 86.64 & 86.61 \\
February & 2 & 86.87 & 86.59 \\
March & 2 & 86.99 & 86.80 \\
April & 2 & 86.95 & 85.70 \\
May & 2 & 86.80 & 86.70 \\
June & 2 & 86.85 & 86.68 \\
July & 2 & 86.84 & 86.18 \\
August & 3 & 87.23 & 85.99 \\
September & 3 & 87.04 & 86.89 \\
\hline
\end{tabular}

SITE IDENTIFIERS AND COUNTY CODE.--Site number, 461614102515203; local identifier, 132-097-07CAB3; county code, 001.

HYDROLOGIC UNIT.--10130205.

GEOLOGIC UNIT.--125LDLW.

DATUM.--Altitude of land surface datum is 2665.00 feet.

Summary of periodic ground-water level data, in feet below land surface datum, August 1971 through December 1993

\begin{tabular}{lccc}
\hline Month & Sample size & Maximum & Minimum \\
\hline October & 1 & 15.55 & 15.55 \\
November & 13 & 15.48 & 13.52 \\
December & 10 & 15.74 & 13.11 \\
January & 2 & 15.82 & 15.49 \\
February & 2 & 15.88 & 15.31 \\
March & 2 & 15.55 & 15.42 \\
April & 2 & 15.65 & 15.20 \\
May & 2 & 15.33 & 15.31 \\
June & 2 & 15.42 & 15.11 \\
July & 2 & 15.54 & 15.50 \\
August & 3 & 15.83 & 15.44 \\
September & 3 & 15.57 & 13.70 \\
\hline
\end{tabular}


SITE IDENTIFIERS AND COUNTY CODE.--Site number, 46204310110210; local identifier, 133-083-07CCB1; county code, 037.

HYDROLOGIC UNIT.--10130206.

GEOLOGIC UNIT.--112BGFV.

DATUM.--Altitude of land surface datum is 1885.00 feet.

Summary of periodic ground-water level data, in feet below land surface datum, November 1971 through December 1993

\begin{tabular}{lccc}
\hline Month & Sample size & Maximum & Minimum \\
\hline October & 2 & 25.71 & 25.39 \\
November & 9 & 26.79 & 24.90 \\
December & 16 & 26.55 & 24.12 \\
January & 1 & 26.10 & 26.10 \\
February & 3 & 26.20 & 25.03 \\
March & 1 & 25.84 & 25.84 \\
April & 2 & 25.43 & 24.79 \\
May & 1 & 24.80 & 24.80 \\
June & 3 & 25.52 & 24.51 \\
July & 3 & 25.30 & 24.78 \\
August & 3 & 26.20 & 24.83 \\
September & 1 & 25.09 & 25.09 \\
\hline
\end{tabular}

SITE IDENTIFIERS AND COUNTY CODE.--Site number, 462005101080001; local identifier, 133-083-17DAA; county code, 037.

HYDROLOGIC UNIT.--10130206.

GEOLOGIC UNIT.--112BGFV.

DATUM.--Altimde of land surface datum is 1842.00 feet.

Summary of periodic ground-water level data, in feet below land surface datum, January 1971 through December 1993

\begin{tabular}{lccc}
\hline Month & Sample size & Maximum & Minimum \\
\hline October & 2 & 28.75 & 27.52 \\
November & 8 & 28.85 & 27.84 \\
December & 15 & 28.71 & 27.41 \\
January & 2 & 28.71 & 28.71 \\
February & 3 & 28.69 & 28.60 \\
March & 1 & 28.83 & 28.83 \\
April & 2 & 28.71 & 28.52 \\
May & 1 & 28.43 & 28.43 \\
June & 3 & 28.65 & 27.75 \\
July & 3 & 28.84 & 28.65 \\
August & 4 & 28.97 & 28.58 \\
September & 1 & 28.75 & 28.75 \\
\hline
\end{tabular}

SITE IDENTIFIERS AND COUNTY CODE.--Site number, 462103101180301; local identifier, 133-085-12AAD; county code, 037 .

HYDROLOGIC UNIT.--10130206.

GEOLOGIC UNIT.--211FXHL.

DATUM.--Altitude of land surface datum is 2020.00 feet.

Summary of periodic ground-water level data, in feet below land surface datum, October 1972 through December 1993

\begin{tabular}{lccc}
\hline Month & Sample size & Maximum & Minimum \\
\hline October & 3 & 183.00 & 180.13 \\
November & 5 & 180.54 & 178.20 \\
December & 16 & 182.50 & 178.82 \\
January & -- & - \\
February & 2 & 180.30 & 180.05 \\
March & -- & -- & - \\
April & 1 & 180.10 & 180.10 \\
May & - & -- & 180.12 \\
June & 1 & 180.18 & 179.55 \\
July & 4 & 180.43 & 180.34 \\
August & 2 & -- & -- \\
September & -- & -
\end{tabular}


SITE IDENTIFIERS AND COUNTY CODE.--Site number, 462139101514301; local identifier, 133-089-04DAD; county code, 037.

HYDROLOGIC UNIT.--10130204.

GEOLOGIC UNIT.--211HLCK.

DATUM.--Altitude of land surface datum is $2120.00 \mathrm{feet}$

Summary of periodic ground-water level data, in feet below land surface datum, November 1972 through November 1982

\begin{tabular}{lccc}
\hline \multicolumn{1}{c}{ Month } & Sample size & Maximum & Minimum \\
\hline October & 1 & 130.46 & 130.46 \\
November & 4 & 139.57 & 129.48 \\
December & 6 & 137.07 & 129.50 \\
January & - & -- & - \\
February & 1 & 131.24 & 131.24 \\
March & - & - & - \\
April & 1 & 129.52 & 129.52 \\
May & 1 & 129.64 & 129.64 \\
June & 3 & 134.90 & 129.76 \\
July & - & -- & - \\
August & 3 & 132.85 & 129.99 \\
September & - & - & -- \\
\hline
\end{tabular}

SITE IDENTIFIERS AND COUNTY CODE.--Site number, 461755102163001; local identifier, 133-092-29CCC1; county code, 041 .

HYDROLOGIC UNIT.--10130204.

GEOLOGIC UNIT.--125TGRV.

DATUM.--Altitude of land surface damum is 2572.00 feet.

Summary of periodic ground-water level data, in feet below land surface datum, November 1976 through June 1981

\begin{tabular}{lccc}
\hline \multicolumn{1}{c}{ Month } & Sample size & Maximum & Minimum \\
\hline October & -- & - & - \\
November & 2 & 105.35 & 104.04 \\
December & 1 & 104.09 & 104.09 \\
January & 1 & 106.09 & 106.09 \\
February & - & -- & 105.39 \\
March & 1 & 105.26 & 105.39 \\
April & 1 & 105.33 & 105.26 \\
May & 1 & 104.30 & 105.33 \\
June & 1 & 104.10 & 104.30 \\
July & 2 & -- & 103.36 \\
August & -- & - & - \\
September & - & - & - \\
\hline
\end{tabular}

SITE IDENTIFIERS AND COUNTY CODE.--Site number, 461747102513401; local identifier, 133-097-34BBB; county code, 041 .

HYDROLOGIC UNIT.--10130205.

GEOLOGIC UNIT.--125LDLW.

DATUM.--Altitude of land surface datum is $2733.00 \mathrm{feet}$

Summary of periodic ground-water level data, in feet below land surface datum, December 1967 through November 1993

\begin{tabular}{lccc}
\hline Month & Sample size & Maximum & Minimum \\
\hline October & 3 & 158.82 & 157.74 \\
November & 17 & 159.93 & 157.30 \\
December & 13 & 159.40 & 157.14 \\
January & 3 & 158.55 & 157.77 \\
February & 4 & 158.80 & 157.60 \\
March & 3 & 158.57 & 157.56 \\
April & 6 & 159.65 & 157.59 \\
May & 3 & 161.00 & 157.70 \\
June & 3 & 158.50 & 157.59 \\
July & 3 & 160.75 & 157.81 \\
August & 4 & 158.49 & 157.82 \\
September & 3 & 158.06 & 157.78 \\
\hline
\end{tabular}


SITE IDENTIFIERS AND COUNTY CODE.--Site number, 462747100564701; local identifier, 134-082-35DAA;

county code, 059 .

HYDROLOGIC UNIT.--10130206.

GEOLOGIC UNIT.--112EMCK.

DATUM.--Altitude of land surface datum is 1697.00 feet.

Summary of periodic ground-water level data, in feet below land surface datum, November 1973 through December 1993

\begin{tabular}{lccc}
\hline Month & Sample size & Maximum & Minimum \\
\hline October & 1 & 4.76 & 4.76 \\
November & 8 & 5.00 & 4.12 \\
December & 14 & 5.23 & 3.93 \\
January & -- & -- & - \\
February & 1 & 4.45 & 4.45 \\
March & 1 & 3.84 & 3.84 \\
April & - & -- \\
May & 2 & 3.93 & 3.43 \\
June & 1 & 4.81 & 4.81 \\
July & 1 & 4.15 & 4.15 \\
August & 2 & 4.53 & -15 \\
September & -- & -- & - \\
\hline
\end{tabular}

SITE IDENTIFIERS AND COUNTY CODE.--Site number, 462222100562701; local identifier, 134-082-36DCD; county code, 085 .

HYDROLOGIC UNIT.--10130206.

GEOLOGIC UNIT.--211FXHL.

DATUM.--Altitude of land surface datum is 1711.00 feet.

Summary of periodic ground-water level data, in feet below land surface datum, November 1971 through December 1993

\begin{tabular}{lccc}
\hline Month & Sample size & Maximum & Minimum \\
\hline October & 3 & 35.80 & 33.46 \\
November & 10 & 35.20 & 33.38 \\
December & 15 & 35.49 & 34.23 \\
January & 1 & 34.20 & 34.20 \\
February & 7 & 34.64 & 33.59 \\
March & 3 & 34.78 & 33.31 \\
April & 2 & 33.49 & 33.42 \\
May & 6 & 34.82 & 32.98 \\
June & 5 & 35.39 & 33.11 \\
July & 3 & 34.30 & 33.55 \\
August & 8 & 34.86 & 33.85 \\
September & 5 & 34.96 & 33.50 \\
\hline
\end{tabular}

SITE IDENTIFIERS AND COUNTY CODE.--Site number, 462216102090801; local identifier, 134-091-32CCC; county code, 041 .

HYDROLOGIC UNIT.--10130204.

GEOLOGIC UNIT.--125TGRV.

DATUM.--Altinude of land surface datum is 2378.00 feet.

Summary of periodic ground-water level data, in feet below land surface datum, November 1967 through November 1993

\begin{tabular}{lccc}
\hline Month & Sampie size & Maximum & Minimum \\
\hline October & 6 & 46.50 & 42.96 \\
November & 17 & 53.00 & 43.07 \\
December & 13 & 50.78 & 43.05 \\
January & 3 & 43.39 & 42.47 \\
February & 4 & 43.47 & 40.64 \\
March & 3 & 43.35 & 42.68 \\
April & 5 & 43.56 & 42.57 \\
May & 3 & 43.00 & 42.73 \\
June & 5 & 48.50 & 42.75 \\
July & 3 & 43.26 & 42.82 \\
August & 3 & 43.32 & 42.88 \\
September & 2 & 50.77 & 43.92 \\
\hline
\end{tabular}


SITE IDENTIFIERS AND COUNTY CODE.--Site number, 462218102130301; local identifier, 134-092-34DDC; county code, 041.

HYDROLOGIC UNIT.-10130204.

GEOLOGIC UNIT.--125TGRV.

DATUM.-Altitude of land surface datum is 2434.00 feet.

Summary of periodic ground-water level data, in feet below land surface datum, November 1968 through November 1993

\begin{tabular}{lccc}
\hline Month & Sample size & Maximum & Minimum \\
\hline October & 3 & 100.14 & 99.64 \\
November & 17 & 100.47 & 87.85 \\
December & 12 & 100.40 & 88.66 \\
January & 2 & 99.83 & 99.41 \\
February & 3 & 100.17 & 99.59 \\
March & 2 & 99.85 & 99.30 \\
April & 4 & 100.32 & 99.51 \\
May & 2 & 100.17 & 99.85 \\
June & 4 & 100.41 & 91.26 \\
July & 2 & 100.13 & 99.66 \\
August & 2 & 101.38 & 99.53 \\
September & 2 & 99.22 & 89.30 \\
\hline
\end{tabular}

SITE IDENTIFIERS AND COUNTY CODE.--Site number, 462429102191001; local identifier, 134-093-23ADD; county code, 041.

HYDROLOGIC UNIT.--10130204.

GEOLOGIC UNIT.--125TGRV.

DATUM.--Altitude of land surface datum is 2510.00 feet.

Summary of periodic ground-water level data, in feet below land surface datum, June 1969 through November 1993

\begin{tabular}{lccc}
\hline Month & Sample size & Maximum & Minimum \\
\hline October & 3 & 129.80 & 129.26 \\
November & 16 & 129.78 & 126.52 \\
December & 11 & 129.80 & 126.79 \\
January & 2 & 129.61 & 129.29 \\
February & 2 & 129.72 & 129.26 \\
March & 2 & 129.69 & 129.21 \\
April & 2 & 129.65 & 129.33 \\
May & 1 & 129.36 & 129.36 \\
June & 3 & 130.00 & 129.46 \\
July & 2 & 129.56 & 129.55 \\
August & 2 & 129.63 & 129.53 \\
September & 2 & 129.09 & 127.11 \\
\hline
\end{tabular}

SITE IDENTIFIERS AND COUNTY CODE.--Site number, 462632102462301; local identifier, 134-096-08BAB; county code, 041 .

HYDROLOGIC UNIT.--10130204.

GEOLOGIC UNIT.--125TGRV.

DATUM.--Altitude of land surface datum is 2575.00 feet.

Summary of periodic ground-water level data, in feet below land surface datum, October 1976 through June 1981

\begin{tabular}{lccc}
\hline Momth & Sample size & Maximum & Minimum \\
\hline October & 1 & 44.91 & 44.91 \\
November & 2 & 44.69 & 44.68 \\
December & 1 & 44.70 & 44.70 \\
January & 1 & 44.80 & 44.80 \\
February & - & -.64 & - \\
March & 1 & 44.64 & 44.64 \\
April & 1 & 44.67 & 44.67 \\
May & 1 & 44.70 & 44.70 \\
June & 1 & 45.15 & 4.89 \\
July & 1 & -5.15 \\
August & - & 44.61 & - \\
September & 1 & 44.61 \\
\hline
\end{tabular}


SITE IDENTIFIERS AND COUNTY CODE.--Site number, 462351103071801; local identifier, 134-099-21DCC; county code, 087.

HYDROLOGIC UNIT.--10130205.

GEOLOGIC UNIT.--125TRVL.

DATUM.--Altitude of land surface datum is $2885.00 \mathrm{feet}$.

Summary of periodic ground-water level data, in feet below land surface datum, January 1977 through December 1993

\begin{tabular}{lccc}
\hline Month & Sample size & Maximum & Minimum \\
\hline October & -- & - & - \\
November & 10 & 156.67 & 155.78 \\
December & 7 & 156.56 & 155.75 \\
January & 1 & 158.20 & 158.20 \\
February & 1 & 158.12 & 158.12 \\
March & -- & -- \\
April & 1 & 156.91 & 156.91 \\
May & -- & -- & - \\
June & - & -- & - \\
July & - & 158.02 & - \\
August & - & - & -- \\
September & - & & -- \\
\hline
\end{tabular}

SITE IDENTIFIERS AND COUNTY CODE.--Site number, 463143102444101; local identifier, 135-096-09ABA; county code, 041 .

HYDROLOGIC UNIT.--10130204.

GEOLOGIC UNIT.--125TGRV.

DATUM.--Altitude of land surface datum is $2570.00 \mathrm{feet}$

Summary of periodic ground-water level data, in feet below land surface datum, October 1976 through June 1981

\begin{tabular}{lccc}
\hline Month & Sample size & Maximum & Minimum \\
\hline October & 1 & 58.54 & 58.54 \\
November & 2 & 58.53 & 57.94 \\
December & 1 & 58.23 & 58.23 \\
January & 1 & 59.30 & 59.30 \\
February & - & - & - \\
March & 1 & 58.65 & 58.65 \\
April & 1 & 58.60 & 58.60 \\
May & 1 & 58.49 & 58.49 \\
June & 1 & 58.52 & 58.52 \\
July & 1 & 58.19 & 58.19 \\
August & - & -- & 57.86 \\
September & 1 & 57.86 & 57.86 \\
\hline
\end{tabular}

SITE IDENTIFIERS AND COUNTY CODE.--Site number, 462906102465201; local identifier, 135-096-30AAA; county code, 041 .

HYDROLOGIC UNIT.--10130204.

GEOLOGIC UNIT.--125TGRV.

DATUM.--Altitude of land surface datum is $2550.00 \mathrm{feet}$.

Summary of periodic ground-water level data, in feet below land surface datum, November 1976 through June 1981

\begin{tabular}{lccc}
\hline Month & Sample size & Maximum & Minimum \\
\hline October & 1 & 21.07 & 21.07 \\
November & 2 & 21.12 & 20.82 \\
December & 1 & 21.85 & 21.85 \\
January & 1 & 21.44 & 21.44 \\
February & -- & - & - \\
March & 1 & 21.55 & 21.55 \\
April & 1 & 21.58 & 21.58 \\
May & 1 & 21.70 & 21.70 \\
June & 1 & 22.41 & 22.41 \\
July & 1 & 21.80 & 21.80 \\
August & -- & --.35 & 20.35 \\
September & 1 & 20.35 & 2 \\
\hline
\end{tabular}


SITE IDENTIFIERS AND COUNTY CODE.--Site number, 463153102521001; local identifier, 135-097-04DCA; county code, 041 .

HYDROLOGIC UNIT.--10130204.

GEOLOGIC UNIT.--211FXHL.

DATUM.--Altitude of land surface datum is $2567.00 \mathrm{feet}$

Summary of periodic ground-water level data, in feet below land surface datum, September 1968 through November 1993

\begin{tabular}{lccc}
\hline Month & Sample size & Maximum & Minimum \\
\hline October & 5 & 143.58 & 142.79 \\
November & 15 & 144.83 & 142.67 \\
December & 10 & 143.24 & 142.51 \\
January & 4 & 143.45 & 142.56 \\
February & 6 & 144.37 & 142.76 \\
March & 18 & 144.50 & 142.49 \\
April & 5 & 143.44 & 142.81 \\
May & 8 & 144.45 & 142.49 \\
June & 18 & 144.86 & 142.39 \\
July & 4 & 143.26 & 142.80 \\
August & 9 & 144.86 & 142.73 \\
September & 19 & 144.60 & 142.76 \\
\hline
\end{tabular}

SITE IDENTIFIERS AND COUNTY CODE.--Site number, 463050102492201; local identifier, 135-097-14AAA;

county code, 041 .

HYDROLOGIC UNIT.--10130204.

GEOLOGIC UNIT.-- 125TGRV.

DATUM.--Altitude of land surface datum is not available.

Summary of periodic ground-water level data, in feet below land surface datum, October 1976 through June 1981

\begin{tabular}{lccc}
\hline Month & Sample size & Maximum & Minimum \\
\hline October & 1 & 27.57 & 27.57 \\
November & 2 & 27.57 & 27.36 \\
December & 1 & 28.02 & 28.02 \\
January & 1 & 27.65 & 27.65 \\
February & - & - & - \\
March & 1 & 27.53 & 27.53 \\
April & 1 & 27.72 & 27.72 \\
May & -- & - & 28.39 \\
June & 1 & 28.39 & 27.94 \\
July & 1 & 27.94 & -- \\
August & - & -- & -- \\
September & - & - & - \\
\hline
\end{tabular}

SITE IDENTIFIERS AND COUNTY CODE.--Site number, 462729102520101; local identifier, 135-097-33DDC; county code, 041 .

HYDROLOGIC UNIT.--10130204.

GEOLOGIC UNIT.--125TGRV.

DATUM.--Altitude of land surface datum is $2685.00 \mathrm{feet}$

Summary of periodic ground-water level data, in feet below land surface datum, October 1976 through October 1981

\begin{tabular}{lccc}
\hline Month & Sample size & Maximum & Minimum \\
\hline October & 1 & 62.79 & 62.79 \\
November & 2 & 63.99 & 63.40 \\
December & 1 & 69.28 & 69.28 \\
January & 1 & 64.72 & 64.72 \\
February & - & - & 65.69 \\
March & 1 & 65.69 & 66.25 \\
April & 1 & 66.25 & 65.81 \\
May & 1 & 65.81 & 67.79 \\
June & 2 & 70.09 & - \\
July & - & - & - \\
August & - & -- & 62.45 \\
September & 1 & 62.45 \\
\hline
\end{tabular}


SITE IDENTIFIERS AND COUNTY CODE.--Site number, 463746102562001; local identifier, 136-098-01BAA; county code, 087 .

HYDROLOGIC UNIT.--10130204.

GEOLOGIC UNIT.--125SNLB.

DATUM.-Altiude of land surface datum is 2765.00 feet.

Summary of periodic ground-water level data, in feet below land surface datum, September 1976 through July 1981

\begin{tabular}{lccc}
\hline \multicolumn{1}{c}{ Month } & Sample size & Maximum & Minimum \\
\hline October & - & -- & - \\
November & 2 & 133.03 & 132.35 \\
December & 1 & 131.32 & 131.32 \\
January & - & - & -- \\
February & - & -- & - \\
March & - & 132.94 & - \\
April & 1 & 132.69 & 132.94 \\
May & 1 & 131.72 & 132.69 \\
June & 1 & 131.47 & 131.72 \\
July & 1 & 132.44 & 131.47 \\
August & 1 & 132.95 & 132.44 \\
September & 1 & 132.95 \\
\hline
\end{tabular}

SITE IDENTIFIERS AND COUNTY CODE.--Site number, 463745103003801; local identifier, 136-098-05AAA;

county code, 087 .

HYDROLOGIC UNIT.--10130204.

GEOLOGIC UNIT.--125TGRV.

DATUM.-Altitude of land surface datum is $2715.00 \mathrm{feet}$.

Summary of periodic ground-water level data, in feet below land surface datum, September 1976 through July 1981

\begin{tabular}{lccc}
\hline \multicolumn{1}{c}{ Month } & Sample size & Maximum & Minimum \\
\hline October & - & -- & -- \\
November & 2 & 119.42 & 118.65 \\
December & 1 & 117.14 & 117.14 \\
January & 1 & 119.34 & 119.34 \\
February & - & -- & 119.14 \\
March & 1 & 119.14 & 119.21 \\
April & 1 & 119.21 & 119.17 \\
May & 1 & 119.17 & 117.94 \\
June & 1 & 117.94 & 117.02 \\
July & 2 & 119.07 & -- \\
August & - & 119.37 & 119.37 \\
Seplember & 1 & & \\
\hline
\end{tabular}

SITE IDENTIFIERS AND COUNTY CODE.--Site number, 463559102581001; local identifier, 136-098-15AAA; county code, 087 .

HYDROLOGIC UNIT.--10130204.

GEOLOGIC UNIT.--125TGRV.

DATUM.--Altimude of land surface datum is 2670.00 feet.

Summary of periodic ground-water level data, in feet below land surface datum, September 1976 through July 1981

\begin{tabular}{lccc}
\hline \multicolumn{1}{c}{ Month } & Sample size & Maximum & Minimum \\
\hline October & - & -- & -- \\
November & 2 & 76.05 & 75.84 \\
December & 1 & 73.99 & 73.99 \\
January & 1 & 77.05 & 77.05 \\
February & -- & -- \\
March & 1 & 76.03 & 76.03 \\
April & 1 & 75.71 & 75.71 \\
May & 1 & 75.90 & 75.90 \\
June & 1 & 74.58 & 74.58 \\
July & 1 & 71.81 & 71.81 \\
August & 1 & 75.87 & 75.87 \\
September & 1 & 74.70 & 74.70 \\
\hline
\end{tabular}


SITE IDENTIFIERS AND COUNTY CODE.--Site number, 463538103053501; local identifier, 136-099-15ADD; county code, 087.

HYDROLOGIC UNIT.--10130204.

GEOLOGIC UNIT.--125SNLB.

DATUM.-Altitude of land surface datum is 2700.00 feet.

Summary of periodic ground-water level data, in feet below land surface datum, September 1976 through July 1981

\begin{tabular}{lccc}
\hline \multicolumn{1}{c}{ Month } & Sample size & Maximum & Minimum \\
\hline October & - & - & - \\
November & 2 & 37.93 & 37.47 \\
December & 1 & 37.92 & 37.92 \\
January & - & - & -- \\
February & - & - & -- \\
March & - & 36.97 & 36.97 \\
April & 1 & 38.06 & 38.06 \\
May & 1 & 38.40 & 38.40 \\
June & 1 & 38.87 & 37.84 \\
July & 2 & -- & -- \\
August & - & 38.78 & 38.78 \\
September & -1 & & \\
\hline
\end{tabular}

SITE IDENTIFIERS AND COUNTY CODE.--Site number, 463417103080401; local identifier, 136-099-20DDD; county code, 087 .

HYDROLOGIC UNIT.--10130204.

GEOLOGIC UNIT.--125TGRV.

DATUM.--Altitude of land surface datum is 2700.00 feet.

Summary of periodic ground-water level data, in feet below land surface darum, September 1976 through July 1981

\begin{tabular}{lccc}
\hline \multicolumn{1}{c}{ Month } & Sampie size & Maximum & Minimum \\
\hline October & - & -- & -- \\
November & 2 & 41.44 & 41.18 \\
December & 1 & 41.45 & 41.45 \\
January & -- & -- & - \\
February & - & 41.04 & 41.04 \\
March & 1 & 40.98 & 40.98 \\
April & 1 & 41.03 & 41.03 \\
May & 1 & 41.57 & 41.17 \\
June & 2 & 41.48 & 41.48 \\
July & 1 & -- & - \\
August & - & 42.47 & 42.47 \\
September & 1 & & \\
\hline
\end{tabular}

SITE IDENTIFIERS AND COUNTY CODE.--Site number, 463257103102301; local identifier, 136-099-31BCC; county code, 087 .

HYDROLOGIC UNIT.--10130204.

GEOLOGIC UNTT.--125TGRV.

DATUM.--Altitude of land surface datum is 2760.00 feet.

Summary of periodic ground-water level data, in feet below land surface datum, September 1976 through July 1981

\begin{tabular}{lccc}
\hline Month & Sampie size & Maximum & Minimum \\
\hline October & - & -- & - \\
November & 2 & 45.25 & 45.19 \\
December & 1 & 46.29 & 46.29 \\
January & 1 & 45.28 & 45.28 \\
February & -- & -- & 45.26 \\
March & 1 & 45.26 & 45.38 \\
April & 1 & 45.38 & 45.49 \\
May & 1 & 45.49 & 45.93 \\
June & 1 & 45.93 & 4.86 \\
July & 2 & -- & - \\
August & -- & 45.04 & 45.04 \\
September & 1 & & \\
\hline
\end{tabular}


SITE IDENTIFIERS AND COUNTY CODE.--Site number, 463231103041101; local identifier, 136-099-36CCC; county code, 087.

HYDROLOGIC UNIT.--10130204.

GEOLOGIC UNIT.--125TGRV.

DATUM.--Altitude of land surface datum is $2705.00 \mathrm{feet}$.

Summary of periodic ground-water level data, in feet below land surface datum, September 1976 through July 1981

\begin{tabular}{lccc}
\hline \multicolumn{1}{c}{ Month } & Sample size & Maximum & Minimum \\
\hline October & - & - & - \\
November & 2 & 55.90 & 55.84 \\
December & 1 & 53.98 & 53.98 \\
January & - & - & - \\
February & - & - & - \\
March & - & - & 55.80 \\
April & 1 & 55.80 & 55.64 \\
May & 1 & 55.64 & 55.73 \\
June & 1 & 55.81 & 53.68 \\
July & 2 & - & - \\
August & -- & 55.80 & 55.80 \\
September & 1 & & \\
\hline
\end{tabular}

SITE IDENTIFIERS AND COUNTY CODE.--Site number, 463735103160501; local identifier, 136-100-05ABCC; county code, 087.

HYDROLOGIC UNTT.--10130204.

GEOLOGIC UNIT.--125TGRV.

DATUM.--Altitude of land surface datum is 2892.00 feet.

Summary of periodic ground-water level data, in feet below land surface datum, August 1984 through June 1988

\begin{tabular}{lccc}
\hline Month & Sample size & Maximum & Minimum \\
\hline October & 4 & 9.21 & 8.46 \\
November & 4 & 9.29 & 8.29 \\
December & 2 & 9.05 & 8.33 \\
January & 2 & 9.59 & 8.56 \\
February & 1 & 8.66 & 8.66 \\
March & 3 & 9.55 & 8.53 \\
April & 3 & 8.96 & 8.18 \\
May & 3 & 8.91 & 8.02 \\
June & 4 & 9.12 & 8.17 \\
July & 3 & 8.95 & 8.25 \\
August & 4 & 8.55 & 7.90 \\
September & 3 & 9.16 & 8.34 \\
\hline
\end{tabular}

SITE IDENTIFIERS AND COUNTY CODE.--Site number, 463731103155101; local identifier, 136-100-05ACAA; county code, 087.

HYDROLOGIC UNIT.--10130204.

GEOLOGIC UNIT.--125TGRV.

DATUM.--Altitude of land surface datum is 2907.00 feet.

Summary of periodic ground-water level data, in feet below land surface datum, August 1984 through June 1988

\begin{tabular}{lccc}
\hline Month & Sample size & Maximum & Minimum \\
\hline October & 4 & 44.76 & 33.01 \\
November & 4 & 44.80 & 32.19 \\
December & 2 & 44.31 & 31.67 \\
January & 2 & 44.59 & 44.16 \\
February & 1 & 44.22 & 47.22 \\
March & 3 & 44.77 & 31.28 \\
April & 3 & 44.50 & 44.17 \\
May & 3 & 45.03 & 4.25 \\
June & 4 & 44.59 & 31.47 \\
July & 3 & 44.92 & 43.91 \\
August & 4 & 44.76 & 39.02 \\
September & 3 & 44.47 & 35.25 \\
\hline
\end{tabular}


SITE IDENTIFIERS AND COUNTY CODE.--Site number, 463728103160501; local identifier, 136-100-05ACBC; county code, 087.

HYDROLOGIC UNTT.--10130204.

GEOLOGIC UNIT.--125TGRV.

DATUM.-Altitude of land surface datum is 2906.00 feet.

Summary of periodic ground-water level data, in feet below land surface datum, July 1984 through June 1988

\begin{tabular}{lccr}
\hline Month & Sample size & Maximum & Minimum \\
\hline October & 4 & 10.46 & 9.74 \\
November & 4 & 10.52 & 9.87 \\
December & 2 & 10.59 & 10.43 \\
January & 2 & 10.47 & 10.34 \\
February & 1 & 10.51 & 10.51 \\
March & 3 & 10.74 & 10.48 \\
April & 3 & 10.46 & 10.02 \\
May & 3 & 10.31 & 10.01 \\
June & 4 & 10.66 & 9.86 \\
July & 4 & 10.33 & 10.04 \\
August & 3 & 10.31 & 10.02 \\
September & 3 & 10.36 & 9.95 \\
\hline
\end{tabular}

SITE IDENTIFIERS AND COUNTY CODE.--Site number, 463725103155601; local identifier, 136-100-05ACDB1; county code, 087.

HYDROLOGIC UNTT.--10130204.

GEOLOGIC UNIT.--125TGRV.

DATUM.--Altitude of land surface datum is 2936.00 feet.

Summary of periodic ground-water level data, in feet below land surface darum, October 1984 through June 1988

\begin{tabular}{lccc}
\hline Month & Sample size & Maximum & Minimum \\
\hline October & 4 & 72.28 & 70.21 \\
November & 4 & 72.25 & 70.19 \\
December & 2 & 70.73 & 70.25 \\
January & 2 & 70.26 & 70.24 \\
February & 1 & 70.08 & 70.08 \\
March & 3 & 70.93 & 70.31 \\
April & 3 & 71.46 & 70.36 \\
May & 3 & 71.59 & 70.32 \\
June & 4 & 71.74 & 70.42 \\
July & 3 & 72.04 & 70.29 \\
August & 3 & 71.64 & 70.37 \\
September & 3 & 70.60 & 70.24 \\
\hline
\end{tabular}

SITE IDENTIFIERS AND COUNTY CODE.--Site number, 463725103155602; local identifier, 136-100-05ACDB2; county code, 087 .

HYDROLOGIC UNIT.--10130204.

GEOLOGIC UNIT.--125TGRV.

DATUM.--Altitude of land surface datum is 2936.00 feet.

Summary of periodic ground-water level data, in feet below land surface datum, July 1984 through June 1988

\begin{tabular}{lccc}
\hline Month & Sample size & Maximum & Minimum \\
\hline October & 4 & 61.55 & 60.17 \\
November & 4 & 61.22 & 60.26 \\
December & 2 & 60.76 & 60.61 \\
January & 2 & 60.65 & 60.25 \\
February & 1 & 60.68 & 60.68 \\
March & 3 & 60.73 & 60.39 \\
April & 3 & 60.71 & 60.20 \\
May & 3 & 60.73 & 60.05 \\
June & 4 & 60.73 & 59.98 \\
July & 4 & 69.43 & 60.12 \\
August & 3 & 60.69 & 59.07 \\
September & 3 & 60.73 & 60.20 \\
\hline
\end{tabular}


SITE IDENTIFIERS AND COUNTY CODE.--Site number, 463721103155101; local identifier, 136-100-05ACDD1; county code, 087 .

HYDROLOGIC UNIT.--10130204.

GEOLOGIC UNIT.--125TGRV.

DATUM.--Altitude of land surface datum is 2891.00 feet.

Summary of periodic ground-water level data, in feet below land surface datum, July 1984 through June 1988

\begin{tabular}{lccc}
\hline Month & Sample size & Maximum \\
\hline October & 4 & 21.66 \\
November & 4 & 21.38 & 20.13 \\
December & 2 & 20.23 & 20.09 \\
January & 2 & 20.28 & 20.05 \\
February & 1 & 20.11 & 20.15 \\
March & 3 & 20.31 & 20.11 \\
April & 3 & 20.12 & \\
May & 3 & 20.45 & 27.73 \\
June & 4 & 20.24 \\
July & 4 & 20.64 \\
August & 3 & 20.56 \\
September & 3 & 20.12 & 20.10 \\
\hline
\end{tabular}

SITE IDENTIFIERS AND COUNTY CODE.--Site number, 463718103161001; local identifier, 136-100-05CAAA; county code, 087 .

HYDROLOGIC UNIT.--10130204.

GEOLOGIC UNIT.--125TGRV.

DATUM.--Altitude of land surface datum is 2901.00 feet.

Summary of periodic ground-water level data, in feet below land surface datum, July 1984 through June 1988

\begin{tabular}{lccc}
\hline Month & Sample size & Maximum & Minimum \\
\hline October & 4 & 26.71 & 25.58 \\
November & 4 & 26.56 & 26.28 \\
December & 2 & 26.53 & 26.49 \\
January & 2 & 26.34 & 26.33 \\
February & 1 & 26.38 & 26.38 \\
March & 3 & 26.84 & 26.40 \\
April & 3 & 26.36 & 26.29 \\
May & 3 & 27.05 & 26.51 \\
June & 4 & 26.69 & 26.40 \\
July & 4 & 27.84 & 26.26 \\
August & 3 & 26.79 & 26.37 \\
September & 3 & 26.40 & 25.98 \\
\hline
\end{tabular}


Table 8. Summary of periodic ground-water level data obtained from the North Dakota State Water Commission [Latitude and longitude are shown in decimal degrees as obtained from the North Dakota State Water Commission]

LOCAL IDENTIFIER.--134-083-05DCC.

LOCATION.--Lat. 46.44465, long. - 101.14099.

DATUM.-Altiude of land surface datum is 1835.00 feet.

Summary of periodic ground-water level data, in feet below land surface datum, July 1990 through December 1994

\begin{tabular}{lccc}
\hline Month & Sample size & Maximum & Minimum \\
\hline April & 2 & 0.58 & 0.42 \\
May & 2 & 0.66 & 0.44 \\
June & 2 & 0.78 & 0.48 \\
July & 2 & 0.72 & 0.43 \\
August & 4 & 1.03 & 0.13 \\
September & 3 & 0.99 & 0.90 \\
October & 4 & 1.03 & 0.63 \\
November & 2 & 9.97 & 0.77 \\
Decermber & 3 & 0.93 & 0.68 \\
\hline
\end{tabular}

LOCAL IDENTIFIER.--134-083-17CCC.

LOCATION.--Lat. 46.41570, long. - 101.15139.

DATUM.--Altitude of land surface datum is $1825.00 \mathrm{feet}$.

Summary of periodic ground-water level data, in feet below land surface datum, July 1990 through December 1994

\begin{tabular}{lccc}
\hline Month & Sample size & Maximum & Minimum \\
\hline April & 2 & 5.26 & 4.57 \\
May & 2 & 5.23 & 4.51 \\
June & 2 & 5.21 & 4.45 \\
July & 2 & 5.11 & 5.00 \\
August & 4 & 5.32 & 4.75 \\
September & 3 & 5.26 & 4.74 \\
October & 4 & 5.38 & 4.43 \\
November & 2 & 5.33 & 4.49 \\
December & 3 & 5.26 & 4.40 \\
\hline
\end{tabular}

LOCAL IDENTIFIER.--134-083-17DDB1.

LOCATION.--Lat. 46.41748, long. - 101.13564.

DATUM.--Altitude of land surface datum is 1825.00 feet.

Summary of periodic ground-water level data, in feet below land surface datum, July 1990 through December 1994

\begin{tabular}{lccc}
\hline Month & Sample size & Maximum & Minimum \\
\hline April & 2 & 3.77 & 3.18 \\
May & 2 & 3.73 & 3.14 \\
June & 2 & 3.80 & 3.15 \\
July & 2 & 3.70 & 3.68 \\
August & 4 & 4.00 & 3.18 \\
September & 3 & 4.10 & 3.49 \\
October & 4 & 4.15 & 3.44 \\
November & 2 & 4.02 & 3.38 \\
December & 3 & 3.96 & 3.33 \\
\hline
\end{tabular}


LOCAL IDENTIFIER.-134-083-17DDB2.

LOCATION.--Lat. 46.41748, long. - 101.13564.

DATUM.-Altitude of land surface datum is 1825.00 feet

Summary of periodic ground-water level data, in feet below land surface datum, September 1992 through December 1994

\begin{tabular}{lccc}
\hline Month & Sample size & Maximum & Minimum \\
\hline April & 2 & 12.06 & 11.49 \\
May & 2 & 12.42 & 11.99 \\
June & 2 & 12.54 & 12.00 \\
July & 1 & 12.24 & 12.24 \\
August & 3 & 12.40 & 9.68 \\
September & 3 & 13.15 & 12.59 \\
October & 4 & 13.10 & 12.32 \\
November & 2 & 12.99 & 12.40 \\
December & 3 & 12.88 & 12.48 \\
\hline
\end{tabular}

LOCAL IDENTIFIER.--134-083-26BBA.

LOCATION.--Lat. 46.39953, long. -101.08588.

DATUM.--Altitude of land surface datum is 1785.00 feet.

Summary of periodic ground-water level data, in feet below land surface datum, July 1990 through December 1994

\begin{tabular}{lccr}
\hline Month & Sample size & Maximum & Minimum \\
\hline April & 2 & 9.19 & 9.06 \\
May & 2 & 9.70 & 9.55 \\
June & 2 & 10.02 & 9.69 \\
July & 2 & 10.01 & 9.59 \\
August & 4 & 10.57 & 8.50 \\
September & 3 & 10.46 & 10.32 \\
October & 4 & 10.60 & 9.93 \\
November & 2 & 10.51 & 10.15 \\
December & 3 & 10.45 & 10.05 \\
\hline
\end{tabular}

LOCAL IDENTIFIER.--134-084-11DDD.

LOCATION.-Lat. 46.43019, long. -101.19543.

DATUM.--Altitude of land surface datum is 1869.00 feet.

Summary of periodic ground-water level data, in feet below land surface datum, August 1992 through June 1993

\begin{tabular}{lccc}
\hline Month & Sample size & Maximum & Minimum \\
\hline April & 1 & 16.17 & 16.17 \\
May & 1 & 15.82 & 15.82 \\
June & 1 & 16.09 & 16.09 \\
August & 1 & 16.97 & 16.97 \\
September & 1 & 16.94 & 16.94 \\
October & 1 & 17.12 & 17.12 \\
November & 1 & 17.18 & 17.18 \\
December & 1 & 17.18 & 17.18 \\
\hline
\end{tabular}


LOCAL IDENTIFIER.-135-084-04DCC.

LOCATION.--Lat. 46.53168, long. -101.24545.

DATUM.--Altitude of land surface datum is 1874.90 feet.

Summary of periodic ground-water level data, in feet below land surface datum, August 1992 through December 1994

\begin{tabular}{lccc}
\hline Month & Sample size & Maximum \\
\hline April & 2 & 41.23 & Minimum \\
May & 2 & 41.17 & 39.98 \\
June & 2 & 41.22 & 39.85 \\
July & 1 & 41.22 & 49.87 \\
August & 4 & 41.43 & 41.58 \\
September & 3 & 41.59 & 40.48 \\
October & 4 & 41.56 & 41.51 \\
November & 2 & 40.22 \\
December & 3 & 40.17 \\
\hline
\end{tabular}

LOCAL IDENTIFIER.--135-084-16ABA.

LOCATION.--Lat. 46.51534, long. -101.24290.

DATUM.-Altitude of land surface datum is $1898.70 \mathrm{feet}$

Summary of periodic ground-water level data, in feet below land surface datum, August 1992 through December 1994

\begin{tabular}{lccc}
\hline Month & Sample size & Maximum & Minimum \\
\hline April & 2 & 56.80 & 55.89 \\
May & 2 & 56.74 & 55.69 \\
June & 2 & 56.79 & 55.72 \\
July & 1 & 56.77 & 56.77 \\
August & 4 & 56.93 & 56.15 \\
September & 3 & 57.03 & 56.22 \\
October & 4 & 57.10 & 55.89 \\
November & 2 & 57.04 & 55.89 \\
December & 3 & 56.97 & 55.78 \\
\hline
\end{tabular}

LOCAL IDENTIFIER.--135-084-16ABB.

LOCATION.--Lat. 46.51531, long. -101.24550.

DATUM.--Altiude of land surface datum is $1879.20 \mathrm{feet}$

Summary of periodic ground-water level data, in feet below land surface datum, August 1992 through December 1994

\begin{tabular}{lccc}
\hline Month & Sample size & Maximum & Minimum \\
\hline April & 2 & 29.87 & 28.99 \\
May & 2 & 29.84 & 29.09 \\
June & 2 & 29.85 & 29.31 \\
July & 1 & 30.14 & 30.14 \\
August & 5 & 30.48 & 29.43 \\
September & 3 & 30.32 & 29.58 \\
October & 4 & 30.22 & 29.14 \\
November & 2 & 30.10 & 29.12 \\
December & 3 & 30.04 & 29.02 \\
\hline
\end{tabular}


LOCAL IDENTIFIER.--135-084-21DDD1.

LOCATION.--Lat. 46.48827, long. -101.23752.

DATUM.-Altitude of land surface datum is 1865.90 feet.

Summary of periodic ground-water level data, in feet below land surface datum, August 1992 through December 1994

\begin{tabular}{lccc}
\hline Month & Sample size & Maximum & Minimum \\
\hline April & 2 & 20.58 & 19.50 \\
May & 2 & 20.45 & 19.36 \\
June & 2 & 20.31 & 19.30 \\
July & 1 & 20.15 & 20.15 \\
August & 5 & 24.83 & 17.43 \\
September & 3 & 19.68 & 17.39 \\
October & 4 & 23.25 & 19.45 \\
November & 2 & 21.77 & 19.34 \\
December & 3 & 21.38 & 19.35 \\
\hline
\end{tabular}

LOCAL IDENTIFIER.--135-084-21DDD2.

LOCATION.-Lat. 46.48827, long. -101.23752.

DATUM.-Altitude of land surface datum is 1866.30 feet.

Summary of periodic ground-water level data, in feet below land surface datum, August 1992 through December 1994

\begin{tabular}{lccc}
\hline Month & Sample size & Maximum & Minimum \\
\hline April & 2 & 7.47 & 6.52 \\
May & 2 & 7.34 & 6.54 \\
June & 2 & 7.44 & 6.52 \\
July & 1 & 7.40 & 7.40 \\
August & 4 & 7.93 & 6.33 \\
September & 3 & 7.97 & 7.05 \\
October & 4 & 7.97 & 6.81 \\
November & 2 & 7.87 & 6.85 \\
December & 3 & 7.85 & 6.75 \\
\hline
\end{tabular}

LOCAL IDENTIFIER.--135-084-26DAA1.

LOCATION.--Lat. 46.47912, long. -101.19561.

DATUM.--Altitude of land surface datum is 1857.50 feet.

Summary of periodic ground-water level data, in feet below land surface datum, November 1973 through December 1994

\begin{tabular}{lccc}
\hline Month & Sample size & Maximum \\
\hline February & 1 & 13.15 \\
March & 1 & 12.44 & 13.15 \\
April & 2 & 12.67 & 12.44 \\
May & 4 & 12.55 & 11.60 \\
June & 3 & 12.37 & 12.81 \\
July & 2 & 12.66 & 1.40 \\
August & 5 & 11.32 & 15 \\
September & 4 & 11.82 \\
October & 5 & 13.32 \\
November & 12 & 12.90 \\
December & 14 & 11.38 \\
\hline
\end{tabular}


LOCAL IDENTIFIER.--135-084-26DAA2.

LOCATION.--Lat. 46.47912, long. -101.19561.

DATUM.--Altitude of land surface datum is 1856.50 feet.

Summary of periodic ground-water level data, in feet below land surface datum, November 1973 through December 1994

\begin{tabular}{lccc}
\hline Month & Sample size & Maximum & Minimum \\
\hline February & 1 & 8.29 & 8.29 \\
March & 1 & 7.72 & 7.72 \\
April & 2 & 8.19 & 7.02 \\
May & 4 & 8.02 & 6.97 \\
June & 3 & 7.98 & 6.83 \\
July & 2 & 7.86 & 6.19 \\
August & 5 & 8.21 & 7.25 \\
September & 4 & 8.24 & 7.39 \\
October & 5 & 8.41 & 7.06 \\
November & 12 & 8.36 & 6.39 \\
December & 14 & 8.30 & 5.50 \\
\hline
\end{tabular}

LOCAL IDENTIFIER.--135-084-26DAA3.

LOCATION.--Lat. 46.47912, long. - 101.19561.

DATUM.--Altitude of land surface datum is $1856.90 \mathrm{feet}$.

Summary of periodic ground-water level data, in feet below land surface datum, November 1973 through December 1994

\begin{tabular}{lccc}
\hline Month & Sample size & Maximum & Minimum \\
\hline February & 1 & 5.54 & 5.54 \\
March & 1 & 4.44 & 4.44 \\
April & 2 & 5.19 & 3.44 \\
May & 4 & 5.05 & 2.56 \\
June & 3 & 5.18 & 4.14 \\
July & 2 & 5.02 & 2.81 \\
August & 5 & 6.11 & 3.32 \\
September & 4 & 6.30 & 3.77 \\
October & 5 & 6.37 & 4.24 \\
November & 12 & 6.13 & 3.43 \\
December & 13 & 6.13 & 1.77 \\
\hline
\end{tabular}

LOCAL IDENTIFIER.--136-097-32DDA2.

LOCATION.-Lat. 46.54569, long. - 102.88462.

DATUM.--Altitude of land surface datum is 2586.90 feet.

Summary of periodic ground-water level data, in feet below land surface datum, June 1994 through August 1994

\begin{tabular}{lccc}
\hline Month & Sample size & Maximum & Minimum \\
\hline June & 1 & 34.33 & 34.33 \\
July & 1 & 35.15 & 35.15 \\
August & 3 & 35.60 & 35.34 \\
\hline
\end{tabular}


LOCAL IDENTIFIER.--136-097-32DDA3.

LOCATION.--Lat. 46.54569, long. -102.88462.

DATUM.--Altitude of land surface datum is 2586.90 feet.

Summary of periodic ground-water level data, in feet below land surface datum, July 18, 1994

\begin{tabular}{lccc}
\hline Month & Sample size & Maximum & Minimum \\
\hline July & 1 & -1.84 & -1.84 \\
\hline
\end{tabular}

LOCAL IDENTIFIER.--136-097-32DDB.

LOCATION.--Lat. 46.54564, long. -102.88721.

DATUM.--Altitude of land surface datum is 2580.15 feet.

Summary of periodic ground-water level data, in feet below land surface datum, June 1994 through August 1994

\begin{tabular}{lccc}
\hline Month & Sample size & Maximum & Minimum \\
\hline June & 1 & 26.91 & 26.91 \\
July & 1 & 28.89 & 28.89 \\
August & 3 & 28.58 & 28.25 \\
\hline
\end{tabular}

LOCAL IDENTIFIER.--136-097-32DDD.

LOCATION.--Lat. 46.54388, long. - 102.88454.

DATUM.--Altiude of land surface datum is 2577.89 feet.

Summary of periodic ground-water level data, in feet below land surface datum, June 1994 through August 1994

\begin{tabular}{lccc}
\hline Month & Sample size & Maximum & Minimum \\
\hline June & 1 & 26.66 & 26.66 \\
July & 1 & 26.89 & 26.89 \\
August & 3 & 27.38 & 27.10 \\
\hline
\end{tabular}

LOCAL IDENTIFIER.--136-097-32DDDC.

LOCATION.--Lat. 46.54341, long. -102.88517.

DATUM.--Altitude of land surface datum is $2571.64 \mathrm{feet}$.

Summary of periodic ground-water level data, in feet below land surface datum, June 1994 through August 1994

\begin{tabular}{lccc}
\hline Month & Sample size & Maximum & Minimum \\
\hline June & 1 & 18.48 & 18.48 \\
July & 1 & 20.18 & 20.18 \\
August & 3 & 20.91 & 20.53 \\
\hline
\end{tabular}


Table 9. Summary of periodic ground-water quality data obtained from the North Dakota State Water Commission [Latitude and longitude are shown in decimal degrees as obtained from the North Dakota State Water Commission; $X$, sampled for one or more representative physical properties or constiments in category; --, no data]

\begin{tabular}{|c|c|c|c|c|c|c|c|}
\hline \multirow[b]{2}{*}{ Local IdentIfler } & \multirow[b]{2}{*}{ Latitudo } & \multirow[b]{2}{*}{ Longitude } & \multirow[b]{2}{*}{ Perlod of record } & \multirow[b]{2}{*}{ Sample slzo } & \multicolumn{3}{|c|}{$\begin{array}{l}\text { Category for whlch samples } \\
\text { were analyzed }\end{array}$} \\
\hline & & & & & $\begin{array}{c}\text { Physicai } \\
\text { proporties }\end{array}$ & $\begin{array}{l}\text { Major } \\
\text { lons' }\end{array}$ & $\begin{array}{c}\text { Trace } \\
\text { motals }\end{array}$ \\
\hline $129-085-26 \mathrm{CCC} 1$ & 45.9595 & -101.29078 & 1971 & 1 & $\mathbf{x}$ & $\mathbf{x}$ & -- \\
\hline 129-086-04CDD1 & 46.01732 & -101.449 & 1971 & 1 & $\mathbf{x}$ & $\mathbf{x}$ & -- \\
\hline 129-087-10BBC & 46.01564 & -101.54101 & $1972,73,78,81$ & 7 & $\mathbf{x}$ & $\mathbf{x}$ & .. \\
\hline 129-088-05DDD1 & 46.01766 & -101.70871 & 1973 & 1 & $\mathbf{x}$ & $\mathbf{x}$ & -- \\
\hline 129-088-05DDD2 & 46.01766 & -101.70871 & 1973 & 1 & $\mathbf{x}$ & $\mathbf{x}$ & -- \\
\hline 129-088-05DDD3 & 46.01766 & -101.70871 & 1973 & 1 & $\mathbf{x}$ & $\mathbf{x}$ & -- \\
\hline 129-088-31DDA & 45.94703 & -101.72954 & 1971 & 1 & $\mathbf{x}$ & $\mathbf{x}$ & - \\
\hline 129-089-18DDA & 45.99064 & -101.85423 & 1971 & 1 & $\mathbf{x}$ & $\mathbf{x}$ & -. \\
\hline $129-089-20 \mathrm{BCCl}$ & 45.98157 & -101.85159 & 1971 & 1 & $\mathbf{x}$ & $\mathbf{x}$ & -- \\
\hline $129-089-20 \mathrm{BCC} 2$ & 45.98157 & -101.85159 & 1971 & 1 & $\mathbf{x}$ & $\mathbf{x}$ &.- \\
\hline 129-089-24DDD2 & 45.97418 & -101.75011 & 1971 & 1 & $\mathbf{x}$ & $\mathbf{x}$ & -- \\
\hline $129-090-29 \mathrm{ACA}$ & 45.96898 & -101.96312 & 1971 & 1 & $\mathbf{x}$ & $\mathbf{x}$ & -- \\
\hline 129-091-07AAA1 & 46.01623 & -102.10331 & 1971 & 1 & $\mathbf{x}$ & $\mathbf{x}$ & -- \\
\hline 129-091-07AAA2 & 46.01623 & -102.10331 & $1971,79,93$ & 3 & $\mathbf{x}$ & $\mathbf{x}$ & $\mathbf{x}$ \\
\hline $129-091-08 \mathrm{CCC}$ & 46.00359 & -102.10088 & 1971 & 1 & $\mathbf{x}$ & $\mathbf{x}$ & -- \\
\hline 129-091-19BAA & 45.98723 & -102.11359 & 1971 & 1 & $\mathbf{x}$ & $\mathbf{x}$ & - \\
\hline $129-092-06 \mathrm{DDC}$ & 46.01813 & -102.23056 & 1970 & 1 & $\mathbf{x}$ & $\mathbf{x}$ & -- \\
\hline $129-092-26 \mathrm{CCB}$ & 45.96192 & -102.1629 & 1971 & 1 & $\mathbf{x}$ & $\mathbf{x}$ & -. \\
\hline $129-092-35$ & 45.95195 & -102.15385 & 1971 & 1 & $\mathbf{x}$ & $\mathbf{x}$ & -- \\
\hline 129-093-08СВB 1 & 46.00926 & -102.35015 & 1972 & 1 & $\mathbf{x}$ & $\mathbf{x}$ & -. \\
\hline 129-093-08CBB2 & 46.00926 & -102.35015 & 1972 & 1 & $\mathbf{x}$ & $\mathbf{x}$ & -- \\
\hline 129-093-28ABB2 & 45.97296 & -102.3189 & 1971 & 1 & $\mathbf{x}$ & $\mathbf{x}$ & -- \\
\hline 129-093-28ABB3 & 45.97296 & -102.3189 & 1971 & 1 & $\mathrm{x}$ & $\mathbf{x}$ & -- \\
\hline 130-084-31AAA 1 & 46.04445 & -101.23089 & 1973 & 1 & $\mathrm{x}$ & $\mathbf{x}$ & -- \\
\hline 130-084-36ABA & 46.04425 & -101.13213 & 1973 & 1 & $\mathbf{x}$ & $\mathbf{x}$ & -- \\
\hline 130-085-08DA & 46.0944 & -101.33597 & $1972-94$ & 52 & $\mathrm{X}$ & $\mathbf{x}$ & $\mathbf{x}$ \\
\hline 130-085-17DAA & 46.08082 & -101.33476 & $1972,73,81$ & 7 & $\mathrm{x}$ & $\mathbf{x}$ & -- \\
\hline $130-086-28 \mathrm{CCC} 1$ & 46.04632 & -101.45681 & 1973 & 1 & $\mathbf{x}$ & $\mathbf{x}$ & -- \\
\hline $130-086-28 C C C 2$ & 46.04632 & -101.45681 & 1973 & 1 & $\mathrm{x}$ & $\mathbf{x}$ & -- \\
\hline $130-086-28 \mathrm{CCC} 2$ & 46.04632 & -101.45681 & 1978 & 1 & $\mathbf{x}$ & $\mathbf{x}$ & -- \\
\hline $130-088-22 \mathrm{CDD}$ & 46.06101 & -101.67755 & 1971 & 1 & $\mathrm{x}$ & $\mathbf{x}$ & -- \\
\hline 130-89-32DDA & 46.03412 & -101.83314 & 1972,78 & 2 & $\mathbf{x}$ & $\mathbf{x}$ & -- \\
\hline $130-089-33$ ССВ & 46.03409 & -101.83072 & $1971-76$ & 30 & $\mathbf{x}$ & $\mathbf{x}$ & -- \\
\hline $130-090-04 \mathrm{BBD}$ & 46.1157 & -101.95282 & 1971 & 1 & $\mathrm{x}$ & $\mathbf{x}$ & -- \\
\hline 130-090-10DDD & 46.09038 & -101.91641 & 1971 & 1 & $\mathrm{x}$ & $\mathbf{x}$ & -- \\
\hline 130-091-09BAB & 46.10306 & -102.07466 & 1971 & 1 & $\mathbf{X}$ & $\mathbf{x}$ & -- \\
\hline 130-092-22CBВ & 46.06692 & -102.18369 & 1971 & 2 & $\mathbf{x}$ & $\mathbf{x}$ & -- \\
\hline $130-092-22 \mathrm{CCC}$ & 46.06151 & -102.18361 & 1971 & 2 & $\mathrm{x}$ & $\mathbf{x}$ & -. \\
\hline 130-092-27BBA2 & 46.05971 & -102.18101 & 1970 & 1 & $\mathrm{x}$ & $\mathbf{x}$ & -- \\
\hline 130-092-27BBA3 & 46.05971 & -102.18101 & 1972 & 1 & $\mathrm{X}$ & $\mathbf{x}$ & - \\
\hline 130-094-07DDD1 & 46.09082 & -102.47729 & 1972 & 1 & $\mathbf{x}$ & $\mathrm{x}$ & - \\
\hline 130-094-07DDD2 & 46.09082 & -102.47729 & 1972 & 1 & $\mathbf{x}$ & $\mathbf{x}$ & -- \\
\hline 130-094-31BCC & 46.04011 & -102.49535 & 1971 & 1 & $\mathrm{x}$ & $\mathbf{x}$ & - \\
\hline 130-095-28AAA & 46.05998 & -102.56029 & 1971 & 1 & $\mathrm{x}$ & $\mathbf{x}$ & -- \\
\hline $130-096-14 \mathrm{AAB}$ & 46.08902 & -102.6459 & 1971 & 1 & $\mathrm{x}$ & $\mathrm{x}$ & -- \\
\hline
\end{tabular}




\begin{tabular}{|c|c|c|c|c|c|c|c|}
\hline \multirow[b]{2}{*}{ Local identifier } & \multirow[b]{2}{*}{ Latitude } & \multirow[b]{2}{*}{ Longitude } & \multirow[b]{2}{*}{ Period of record } & \multirow[b]{2}{*}{ Sample size } & \multicolumn{3}{|c|}{$\begin{array}{l}\text { Category for which samplos } \\
\text { were analyzed }\end{array}$} \\
\hline & & & & & $\begin{array}{l}\text { Physical } \\
\text { properties }\end{array}$ & $\begin{array}{l}\text { Major } \\
\text { lons' }\end{array}$ & $\begin{array}{l}\text { Trace } \\
\text { motals }\end{array}$ \\
\hline 130-096-15BCC & 46.08354 & -102.68235 & 1971 & 1 & $\mathbf{X}$ & $\mathbf{X}$ & .. \\
\hline 131-084-03AAD & 46.20181 & -101.1684 & 1972,73 & 3 & $\mathbf{X}$ & $\mathbf{X}$ & -- \\
\hline $131-085-08 D D D 1$ & 46.17654 & -101.33462 & 1971 & 1 & $\mathbf{X}$ & $\mathbf{X}$ & - \\
\hline 131-085-32ADA & 46.12772 & -101.33467 & 1972 & 1 & $\mathbf{X}$ & $\mathbf{x}$ & - \\
\hline 131-086-04ADA & 46.20015 & -101.4387 & 1971 & 1 & $\mathbf{x}$ & $\mathbf{x}$ & -- \\
\hline 131-086-14DAB & 46.16761 & -101.39956 & 1980 & 1 & $\mathbf{x}$ & $\mathbf{X}$ & - \\
\hline $131-087-06 \mathrm{BCB} 1$ & 46.19988 & -101.6228 & 1971 & 1 & $\mathbf{x}$ & $\mathbf{X}$ & 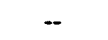 \\
\hline 131-087-28CDD & 46.13316 & -101.57385 & . $\quad 1971$ & 1 & $\mathbf{X}$ & $\mathbf{X}$ & - \\
\hline $131-087-32 C D C$ & 46.1188 & -101.5972 & 1971 & 1 & $\mathbf{X}$ & $\mathbf{X}$ & - \\
\hline 131-087-33CBA & 46.12417 & -101.57903 & 1971 & 1 & $\mathbf{X}$ & $\mathbf{X}$ & - \\
\hline 131-089-30AAA & 46.14644 & -101.85448 & 1973 & 1 & $\mathbf{x}$ & $\mathbf{x}$ & - \\
\hline $131-090-04 \mathrm{CAA} 2$ & 46.19731 & -101.94773 & 1971 & 1 & $\mathbf{x}$ & $\mathbf{X}$ & - \\
\hline $131-090-04 D D C 1$ & 46.19187 & -101.93992 & 1971 & 1 & $\mathbf{X}$ & $\mathbf{X}$ & - \\
\hline 131-091-10CCC & 46.17739 & -102.05879 & 1971 & 1 & $\mathbf{X}$ & $\mathbf{X}$ & - \\
\hline $131-091-13 \mathrm{CCB}$ & 46.16467 & -102.01711 & 1980 & 1 & $\mathbf{x}$ & $\mathbf{X}$ & - \\
\hline $131-091-15 \mathrm{CCC}$ & 46.16282 & -102.05881 & 1972 & 1 & $\mathbf{X}$ & $\mathbf{x}$ & -- \\
\hline 131-092-05DDD & 46.19188 & -102.20712 & 1988,93 & 2 & $\mathbf{x}$ & $\mathbf{X}$ & $\mathbf{x}$ \\
\hline 131-092-08BBA & 46.1901 & -102.22278 & 1970 & 1 & $\mathbf{x}$ & $\mathbf{X}$ & -- \\
\hline 131-093-21AAA1 & 46.1613 & -102.31101 & 1972 & 1 & $\mathbf{x}$ & $\mathbf{x}$ & -- \\
\hline 131-093-21AAA2 & 46.1613 & -102.31101 & 1972 & 1 & $\mathbf{x}$ & $\mathbf{x}$ & -- \\
\hline 131-093-21 AAA3 & 46.1613 & -102.31101 & 1972 & 1 & $\mathbf{X}$ & $\mathbf{x}$ & -- \\
\hline 131-094-07DAA & 46.18322 & -102.47747 & 1970 & 1 & $\mathbf{x}$ & $\mathbf{X}$ & -- \\
\hline $131-094-20$ & 46.15511 & -102.46567 & $1971-93$ & 59 & $\mathrm{X}$ & $\mathbf{x}$ & $\mathbf{x}$ \\
\hline $131-094-20 \mathrm{CBC} 1$ & 46.15236 & -102.4748 & 1971 & 1 & $\mathbf{x}$ & $\mathbf{x}$ & -- \\
\hline $131-094-20 \mathrm{CBC} 2$ & 46.15236 & -102.4748 & $1971,79,93$ & 4 & $\mathbf{X}$ & $\mathbf{x}$ & $\mathbf{x}$ \\
\hline $131-094-20 C B C 3$ & 46.15236 & -102.4748 & 1971 & 1 & $\mathbf{X}$ & $\mathbf{x}$ & - \\
\hline 131-095-15DCC & 46.16316 & -102.54754 & 1971 & 1 & $\mathbf{x}$ & $\mathbf{x}$ & -- \\
\hline 131-095-22BAA & 46.16137 & -102.55015 & 1971 & 1 & $\mathbf{X}$ & $\mathbf{x}$ & -- \\
\hline 131-095-27CCB & 46.13609 & -102.55798 & 1970 & 1 & $\mathbf{x}$ & $\mathbf{x}$ & -- \\
\hline 131-096-02DBD & 46.19579 & -102.64865 & 1971 & 1 & $\mathbf{X}$ & $\mathbf{x}$ & -- \\
\hline $131-096-15 \mathrm{CCC} 1$ & 46.16314 & -102.68219 & 1971 & 1 & $\mathbf{x}$ & $\mathbf{x}$ & -- \\
\hline $131-096-15 C C C 2$ & 46.16336 & -102.6824 & 1972 & 1 & $\mathbf{X}$ & $\mathbf{x}$ & - \\
\hline 131-096-18ADD & 46.17052 & -102.72681 & 1971 & 1 & $\mathbf{x}$ & $\mathbf{x}$ & -- \\
\hline 131-096-21DDA & 46.15054 & -102.68509 & 1971 & 1 & $\mathbf{x}$ & $\mathbf{x}$ & -- \\
\hline 131-096-27BCC2 & 46.14149 & -102.68245 & 1971 & 1 & $\mathbf{X}$ & $\mathbf{X}$ & -- \\
\hline 131-096-30DCD & 46.13434 & -102.73188 & 1971 & 1 & $\mathbf{X}$ & $\mathbf{X}$ & -- \\
\hline 131-098-01BCC4 & 46.1994 & -102.88999 & 1971 & 1 & $\mathbf{X}$ & $\mathbf{x}$ & -- \\
\hline $131-098-07 \mathrm{BCC}$ & 46.185 & -102.9936 & 1971 & 1 & $\mathbf{x}$ & $\mathbf{X}$ & -- \\
\hline $131-098-08 C D D$ & 46.17771 & -102.9656 & 1971 & 1 & $\mathbf{x}$ & $\mathbf{X}$ & - \\
\hline 131-098-23DAD1 & 46.15228 & -102.89253 & 1972 & 1 & $\mathbf{X}$ & $\mathbf{x}$ & -- \\
\hline 131-098-23DAD2 & 46.15228 & $-102.89253^{-}$ & 1972 & 1 & $\mathbf{x}$ & $\mathbf{X}$ & -- \\
\hline 131-099-04DAA & 46.19777 & -103.05865 & 1971 & 1 & $\mathbf{x}$ & $\mathbf{x}$ & -- \\
\hline 132-083-08DCD & 46.26313 & -101.09043 & 1972 & 3 & $\mathbf{x}$ & $\mathbf{x}$ & - \\
\hline 132-083-19CDC & 46.23432 & -101.11897 & 1971 & 1 & $\mathbf{x}$ & $\mathbf{x}$ & - \\
\hline 132-083-29BBB & 46.23247 & -101.10373 & 1971 & 1 & $\mathbf{X}$ & $\mathbf{X}$ & - \\
\hline $132-083-29 C C C$ & 46.21978 & -101.10359 & 1971,81 & 2 & $\mathbf{x}$ & $\mathbf{X}$ & -- \\
\hline 132-083-30ВСВ & 46.22891 & -101.12412 & 1971 & 1 & $\mathbf{x}$ & $\mathbf{X}$ & - \\
\hline 132-083-30DCC & 46.21982 & -101.11386 & 1972,73 & 3 & $\mathbf{X}$ & $\mathbf{X}$ & - \\
\hline 132-083-31DBA & 46.21083 & -101.11132 & 1971 & 1 & $\mathbf{X}$ & $\mathbf{X}$ & -- \\
\hline 132-084-01DAA & 46.28015 & -101.12668 & 1971 & 1 & $\mathbf{x}$ & $\mathbf{x}$ & - \\
\hline
\end{tabular}




\begin{tabular}{|c|c|c|c|c|c|c|c|}
\hline \multirow[b]{2}{*}{ Local identifier } & \multirow[b]{2}{*}{ Latitude } & \multirow[b]{2}{*}{ Longitude } & \multirow[b]{2}{*}{ Period of record } & \multirow[b]{2}{*}{ Sample size } & \multicolumn{3}{|c|}{$\begin{array}{l}\text { Category for which samples } \\
\text { were analyzed }\end{array}$} \\
\hline & & & & & $\begin{array}{l}\text { Physical } \\
\text { properties }\end{array}$ & $\begin{array}{l}\text { Major } \\
\text { lons' }\end{array}$ & $\begin{array}{l}\text { Trace } \\
\text { motals }\end{array}$ \\
\hline $132-084-06 \mathrm{CCC}$ & 46.27738 & -101.24874 & 1971 & 1 & $\mathbf{x}$ & $\mathbf{x}$ & - \\
\hline 132-084-12BAA & 46.27587 & -101.13701 & 1973 & 1 & $\mathbf{x}$ & $\mathbf{x}$ & - \\
\hline $132-084-12 C C D$ & 46.26316 & -101.14237 & 1972 & 1 & $\mathbf{x}$ & $\mathbf{x}$ & -- \\
\hline 132-084-16DAA & 46.25423 & -101.18945 & 1973 & 1 & $\mathbf{x}$ & $\mathbf{x}$ & -- \\
\hline $132-086-21 \mathrm{BCB}$ & 46.24357 & -101.45691 & 1971 & 1 & $\mathbf{x}$ & $\mathbf{x}$ & -- \\
\hline 132-086-24AAA & 46.24711 & -101.37619 & 1974 & 1 & $\mathbf{x}$ & $\mathbf{x}$ & -- \\
\hline 132-087-27ADA & 46.22889 & -101.54266 & $1972,73,78,81$ & 8 & $\mathbf{X}$ & $\mathbf{X}$ & -- \\
\hline $132-090-14 A A B 1$ & 46.26244 & -101.89812 & 1973 & 1 & $\mathbf{x}$ & $\mathbf{x}$ & - \\
\hline $132-090-14 \mathrm{AAB} 2$ & 46.26244 & -101.89812 & 1973,78 & 2 & $\mathbf{X}$ & $\mathbf{x}$ & - \\
\hline $132-091-14 \mathrm{AAB}$ & 46.26242 & -102.02213 & 1967 & 1 & $\mathbf{x}$ & $\mathbf{x}$ & - \\
\hline 132-091-28DDD & 46.22081 & -102.06141 & 1968,87 & 2 & $\mathbf{x}$ & $\mathbf{x}$ & -- \\
\hline 132-092-09AAA & 46.27698 & -102.18651 & 1967 & 4 & $\mathbf{X}$ & $\mathbf{X}$ & -- \\
\hline 132-092-21DDD & 46.23549 & -102.1865 & 1969 & 1 & $\mathbf{x}$ & $\mathbf{x}$ & -- \\
\hline 132-092-24AAA & 46.24784 & -102.12362 & 1969 & 1 & $\mathbf{X}$ & $\mathbf{x}$ & - \\
\hline 132-093-23BAB2 & 46.24821 & -102.28246 & 1967 & 1 & $\mathbf{x}$ & $\mathbf{x}$ & -- \\
\hline 132-093-28BCB2 & 46.23012 & -102.32929 & 1969 & 1 & $\mathbf{x}$ & $\mathbf{x}$ & -. \\
\hline 132-094-15BBB 1 & 46.26296 & -102.43319 & 1969 & 1 & $\mathbf{x}$ & $\mathbf{x}$ & -- \\
\hline 132-094-15BBB2 & 46.26296 & -102.43319 & 1969 & 1 & $\mathbf{X}$ & $\mathbf{X}$ & - \\
\hline $132-094-29 \mathrm{CCC}$ & 46.22119 & -102.4749 & 1969 & 1 & $\mathbf{x}$ & $\mathbf{x}$ & -- \\
\hline 132-095-20AAD & 46.24657 & -102.58179 & 1917 & 1 & $\mathbf{x}$ & $\mathbf{x}$ & - \\
\hline $132-096-22 \mathrm{ABC1}$ & 46.24654 & -102.67242 & 1972 & 1 & $\mathbf{X}$ & $\mathbf{X}$ & - \\
\hline $132-096-22 \mathrm{ABC} 2$ & 46.24654 & -102.67242 & 1972 & 1 & $\mathbf{X}$ & $\mathbf{X}$ & -- \\
\hline 132-096-23BBD & 46.24653 & -102.65938 & 1971 & 1 & $\mathbf{x}$ & $\mathbf{x}$ & - \\
\hline 132-096-26BBA2 & 46.23384 & -102.6593 & 1971 & 1 & $\mathbf{X}$ & $\mathbf{X}$ & -- \\
\hline $132-097-07 \mathrm{CAB}$ & 46.27 & -102.8643 & 1971 & 3 & $\mathbf{x}$ & $\mathbf{X}$ & - \\
\hline $132-097-07 \mathrm{CAB} 2$ & 46.27 & -102.8643 & 1971,79 & 2 & $\mathbf{x}$ & $\mathbf{x}$ & -- \\
\hline $132-097-07 \mathrm{CAB} 3$ & 46.27 & -102.8643 & 1971,92 & 2 & $\mathbf{X}$ & $\mathbf{x}$ & -- \\
\hline 132-097-07CAB4 & 46.27 & -102.8643 & 1972 & 1 & $\mathbf{X}$ & $\mathbf{X}$ & -- \\
\hline 132-097-08DAA & 46.27 & -102.831 & 1971,77 & 2 & $\mathbf{X}$ & $\mathbf{X}$ & -- \\
\hline 132-097-09AAA & 46.27727 & -102.81002 & 1971 & 1 & $\mathbf{x}$ & $\mathbf{x}$ & -- \\
\hline $132-097-09 \mathrm{CCC} 2$ & 46.26459 & -102.82839 & 1976 & 1 & $\mathbf{X}$ & $\mathbf{x}$ & -- \\
\hline $132-097-15 C B C$ & 46.25373 & -102.80742 & 1970 & 1 & $\mathrm{X}$ & $\mathbf{X}$ & -- \\
\hline 132-097-17ADD & 46.25733 & -102.83095 & 1976 & 1 & $\mathbf{X}$ & $\mathbf{x}$ & -- \\
\hline 132-097-23BAA & 46.24838 & -102.7787 & 1970 & 1 & $\mathbf{x}$ & $\mathbf{X}$ & -- \\
\hline 132-098-23CDD & 46.23558 & -102.90311 & 1970 & 1 & $\mathbf{X}$ & $\mathbf{x}$ & - \\
\hline 132-099-32DDC1 & 46.20667 & -103.0822 & 1971 & 1 & $\mathbf{x}$ & $\mathbf{X}$ & -- \\
\hline 132-099-32DDC2 & 46.20667 & -103.0822 & 1971 & $i$ & $\mathrm{x}$ & $\mathrm{x}$ & -. \\
\hline $132-100-14 A D B$ & 46.25912 & -103.14429 & 1970 & 1 & $\mathbf{X}$ & $\mathbf{X}$ & -- \\
\hline 132-101-10DDD & 46.26453 & -103.28721 & 1972 & 1 & $\mathbf{X}$ & $\mathbf{X}$ & -- \\
\hline $132-101-12 \mathrm{CCC}$ & 46.26449 & -103.26371 & 1971 & 1 & $\mathbf{X}$ & $\mathbf{x}$ & - \\
\hline 133-080-12DDD & 46.34318 & -100.67352 & 1971 & 1 & $\mathbf{x}$ & $\mathbf{X}$ & -- \\
\hline $133-082-01$ & 46.36416 & -100.9334 & $1970-72$ & 19 & $\mathrm{X}$ & $\mathbf{X}$ & -- \\
\hline 133-082-05ABC & 46.36874 & -101.01598 & 1972 & 1 & $\mathrm{X}$ & $\mathbf{x}$ & - \\
\hline 133-082-05DBA & 46.3633 & -101.01334 & 1972,73 & 2 & $\mathbf{x}$ & $\mathbf{x}$ & - \\
\hline 133-082-15ACD2 & 46.33604 & -100.97127 & 1972 & 1 & $\mathbf{x}$ & $\mathbf{X}$ & -- \\
\hline 133-082-29CDD & 46.2999 & -101.0181 & 1972 & 1 & $\mathbf{x}$ & $\mathbf{x}$ & -- \\
\hline 133-082-31DDA & 46.28713 & -101.02851 & 1972 & 1 & $\mathbf{x}$ & $\mathbf{x}$ & - \\
\hline 133-082-32BAD & 46.29627 & -101.01809 & 1972 & 1 & $\ddot{x}$ & $\ddot{x}$ & - \\
\hline 133-083-06CDD & 46.3578 & -101.16411 & 1971 & 1 & $\mathbf{x}$ & $\mathbf{X}$ & .. \\
\hline $133-083-07 \mathrm{CCB} 1$ & 46.34512 & -101.17198 & 1971,78 & 2 & $\mathbf{x}$ & $\mathbf{x}$ & -- \\
\hline
\end{tabular}




\begin{tabular}{|c|c|c|c|c|c|c|c|}
\hline \multirow[b]{2}{*}{ Local Identifier } & \multirow[b]{2}{*}{ Latitude } & \multirow[b]{2}{*}{ Longltude } & \multirow[b]{2}{*}{ Period of record } & \multirow[b]{2}{*}{ Sample size } & \multicolumn{3}{|c|}{$\begin{array}{l}\text { Category for which samples } \\
\text { were analyzed }\end{array}$} \\
\hline & & & & & $\begin{array}{l}\text { Physical } \\
\text { properties }\end{array}$ & $\begin{array}{l}\text { Major } \\
\text { ions' }\end{array}$ & $\begin{array}{l}\text { Trace } \\
\text { motals }\end{array}$ \\
\hline 133-083-12ADA1 & 46.35246 & -101.04931 & 1973 & 2 & $\mathbf{x}$ & $\mathbf{X}$ & -- \\
\hline 133-083-17DAA & 46.33437 & -101.13289 & 1971,78 & 2 & $\mathbf{X}$ & $\mathbf{X}$ & -. \\
\hline $133-083-21 \mathrm{ABB}$ & 46.32715 & -101.1198 & 1971 & 1 & $\mathrm{X}$ & $\mathbf{X}$ & -. \\
\hline 133-083-28AAB & 46.31269 & -101.11459 & 1971 & 1 & $\mathbf{X}$ & $\mathbf{X}$ & -- \\
\hline 133-083-28DCD & 46.29987 & -101.11726 & 1071 & 1 & $\mathbf{X}$ & $\mathbf{X}$ &.- \\
\hline $133-083-34 \mathrm{CBC}$ & 46.28902 & -101.10942 & 1971 & 1 & $\mathbf{x}$ & $\mathbf{X}$ & -. \\
\hline 133-084-01DCC & 46.3578 & -101.18239 & 1973 & 1 & $\mathbf{X}$ & $\mathbf{x}$ & -- \\
\hline $133-085-12 \mathrm{AAD}$ & 46.35421 & -101.29979 & 1972 & 2 & $\mathbf{X}$ & $\mathbf{X}$ & -- \\
\hline 133-089-04DAD & 46.36117 & -101.86112 & 1972 & 3 & $\mathbf{X}$ & $\mathbf{x}$ & -- \\
\hline I33-089-15BAA & 46.34123 & -101.85103 & 1970 & 1 & $\mathbf{x}$ & $\mathbf{X}$ & -- \\
\hline 133-089-32BDA2 & 46.29388 & -101.89356 & 1971 & 1 & $\mathrm{x}$ & $\mathbf{x}$ & -- \\
\hline $133-090-06 \mathrm{CC}$ & 46.35781 & -102.04431 & $1972-81$ & 40 & $x$ & $\mathbf{X}$ & -- \\
\hline 133-091-01BBD & 46.36773 & -102.06411 & 1967 & 1 & $\mathbf{x}$ & $\mathbf{X}$ &.- \\
\hline 133-091-24BAB & 46.32613 & -102.06096 & 1967 & 1 & $\mathbf{x}$ & $\mathbf{x}$ & -- \\
\hline $133-092-05 B$ & 46.36737 & -102.27218 & 1967 & 1 & $\mathbf{x}$ & $\mathbf{x}$ & -- \\
\hline 133-092-27ABB & 46.31184 & -102.22268 & 1969 & 1 & $\mathbf{X}$ & $\mathbf{x}$ & $\ldots$ \\
\hline 133-093-02AAB & 46.37024 & -102.32151 & 1967 & 1 & $\mathbf{x}$ & $\mathbf{x}$ & -- \\
\hline 133-093-05DCC & 46.35727 & -102.38949 & 1967 & 1 & $\mathbf{x}$ & $\mathbf{x}$ & -- \\
\hline 133-093-26AAA & 46.31228 & -102.31881 & 1967 & 1 & $\mathbf{x}$ & $\mathbf{x}$ & -- \\
\hline $133-094-04 \mathrm{D}$ & 46.35931 & -102.49036 & 1967 & 1 & $x$ & $\mathbf{x}$ & -- \\
\hline 133-094-12BDD & 46.34979 & -102.43406 & 1967 & 1 & $\mathbf{x}$ & $\mathbf{x}$ & -- \\
\hline $133-094-25 \mathrm{ACC}$ & 46.30633 & -102.43129 & 1969 & 1 & $\mathbf{X}$ & $\mathbf{X}$ & -- \\
\hline 133-095-11DDD2 & 46.34159 & -102.57052 & 1969 & 1 & $\mathrm{X}$ & $\mathbf{X}$ & -- \\
\hline 133-095-19DDD & 46.31246 & -102.65393 & 1969 & 1 & $\mathbf{x}$ & $\mathbf{x}$ & -. \\
\hline 133-095-26AAD2 & 46.30896 & -102.57011 & 1969 & 1 & $\mathbf{X}$ & $\mathbf{x}$ & -- \\
\hline $133-096-10 \mathrm{BBB}$ & 46.35354 & -102.73598 & 1967 & 1 & $\mathbf{x}$ & $\mathbf{x}$ & -- \\
\hline 133-096-28DAA2 & 46.30288 & -102.73773 & 1969 & 1 & $\mathbf{X}$ & $\mathbf{x}$ & -- \\
\hline 133-097-09AAA1 & 46.35312 & -102.86349 & 1971 & 1 & $\mathbf{x}$ & $\mathbf{X}$ & -- \\
\hline 133-097-09AAA2 & 46.35312 & -102.86349 & 1967,71 & 3 & $\mathbf{x}$ & $\mathbf{x}$ & -- \\
\hline 133-097-I1DDA2 & 46.34229 & -102.82154 & 1968 & 1 & $\mathbf{X}$ & $\mathrm{x}$ & -- \\
\hline 133-097-32DCC3 & 46.28243 & -102.89129 & 1967 & 1 & $\mathbf{X}$ & $\mathbf{x}$ & -- \\
\hline 133-097-34BBB & 46.29519 & -102.86018 & 1968 & 2 & $\mathbf{X}$ & $\mathbf{X}$ & -- \\
\hline 133-098-06DCC & 46.35342 & -103.03854 & 1975 & 1 & $\mathbf{x}$ & $\mathbf{x}$ & -- \\
\hline 133-098-14AAA & 46.33842 & -102.94691 & 1975 & 1 & $\mathbf{x}$ & $\mathbf{x}$ & -- \\
\hline 133-098-18DDD & 46.32455 & -103.03031 & 1975 & 1 & $\mathbf{x}$ & $\mathbf{x}$ & -- \\
\hline 133-098-19DDB & 46.31186 & -103.03263 & 1975 & 1 & $\mathbf{x}$ & $\mathbf{x}$ & -- \\
\hline $133-098-20 \mathrm{CBC}$ & 46.31376 & -103.02749 & 1975 & 1 & $\mathbf{X}$ & $\mathbf{X}$ & -- \\
\hline $133-098-21 \mathrm{~B}$ & 46.32039 & -103.00285 & $1972-94$ & 35 & $\mathbf{X}$ & $\mathbf{X}$ & $\mathbf{x}$ \\
\hline 133-098-32BCA & 46.29015 & -103.02452 & 1975 & 1 & $\mathbf{x}$ & $\mathbf{x}$ & -- \\
\hline $133-099-02 \mathrm{CBB}$ & 46.35852 & -103.09068 & 1974,75 & 2 & $\mathbf{x}$ & $\mathbf{x}$ & -- \\
\hline 133-099-20ADA & 46.31875 & -103.13448 & 1975 & 1 & $\mathbf{X}$ & $\mathbf{x}$ & -- \\
\hline 133-099-32BBA & 46.29342 & -103.14988 & 1974 & 1 & $\mathbf{X}$ & $\mathbf{x}$ & -- \\
\hline 133-100-08ADA & 46.34774 & -103.25958 & 1975 & 1 & $\mathbf{X}$ & $\mathbf{x}$ & -. \\
\hline 133-101-34DAA & 46.28628 & -103.34295 & 1975 & 1 & $\mathbf{x}$ & $\mathbf{x}$ & -- \\
\hline $134-079-07 \mathrm{BCB}$ & 46.43984 & -100.67073 & 1973 & 1 & $\mathbf{X}$ & $\mathbf{x}$ & -- \\
\hline 134-079-20AAB & 46.41457 & -100.63442 & 1975 & 1 & $\mathbf{X}$ & $\mathbf{x}$ & -- \\
\hline 134-080-16DAA & 46.42076 & -100.73606 & 1973 & 1 & $\mathbf{x}$ & $\mathbf{x}$ & -- \\
\hline 134-080-17DDD & 46.41553 & -100.7571 & 1974 & 1 & $\mathbf{x}$ & $\mathbf{x}$ & -- \\
\hline $134-080-23 \mathrm{BAB}$ & 46.41491 & -100.69791 & 1974 & 1 & $\mathrm{X}$ & $\mathrm{X}$ & -- \\
\hline $134-081-06 \mathrm{DAB} 1$ & 46.45008 & -100.90601 & 1972 & 1 & $\mathrm{x}$ & $\mathrm{x}$ & -- \\
\hline
\end{tabular}




\begin{tabular}{|c|c|c|c|c|c|c|c|}
\hline \multirow[b]{2}{*}{ Local Identifier } & \multirow[b]{2}{*}{ Latitude } & \multirow[b]{2}{*}{ Longitude } & \multirow[b]{2}{*}{ Period of record } & \multirow[b]{2}{*}{ Samplo size } & \multicolumn{3}{|c|}{$\begin{array}{l}\text { Catogory for which samples } \\
\text { were analyzed }\end{array}$} \\
\hline & & & & & $\begin{array}{l}\text { Physical } \\
\text { properties }\end{array}$ & $\begin{array}{l}\text { Major } \\
\text { Ions' }\end{array}$ & $\begin{array}{c}\text { Trace } \\
\text { motals }\end{array}$ \\
\hline 134-081-06DAB2 & 46.45008 & -100.90601 & 1972 & 1 & $\mathbf{x}$ & $\mathbf{X}$ & - \\
\hline 134-081-08DAD1 & 46.43382 & -100.88251 & 1972 & 1 & $\mathbf{x}$ & $\mathbf{X}$ & - \\
\hline 134-081-08DAD2 & 46.43382 & -100.88251 & 1972 & 1 & $\mathbf{X}$ & $\mathbf{X}$ & -- \\
\hline $134-081-30 \mathrm{BCD} 2$ & 46.39406 & -100.91897 & 1972 & 1 & $\mathbf{X}$ & $\mathbf{X}$ & - \\
\hline 134-082-01AAC & 46.45548 & -100.92676 & 1972 & 1 & $\mathbf{X}$ & $\mathbf{X}$ & -- \\
\hline $134-082-01 \mathrm{ACA}$ & 46.4537 & -100.92939 & 1972 & 1 & $\mathbf{X}$ & $\mathbf{X}$ & - \\
\hline 134-082-35DAA & 46.37779 & -100.94511 & 1972 & 1 & $\mathbf{X}$ & $\mathbf{x}$ & -- \\
\hline $134-082-36$ & 46.37865 & -100.93337 & $1970,71,90,93,94$ & 9 & $\mathbf{X}$ & $\mathbf{X}$ & $\mathbf{X}$ \\
\hline 134-082-36DCD & 46.37227 & -100.92956 & 1971,78 & 2 & $\mathbf{x}$ & $\mathbf{x}$ & - \\
\hline 134-083-05CCD 1 & 46.44464 & -101.14886 & 1972 & 1 & $\mathbf{X}$ & $\mathbf{X}$ & - \\
\hline $134-083-05 C C D 2$ & 46.44464 & -101.14886 & 1972 & 1 & $\mathbf{X}$ & $\mathbf{X}$ & -- \\
\hline $134-083-05 D C C$ & 46.44465 & -101.14099 & 1973,92 & 2 & $\mathbf{X}$ & $\mathbf{X}$ & - \\
\hline $134-083-17 \mathrm{CCC}$ & 46.4157 & -101.15139 & 1973,92 & 2 & $\mathrm{x}$ & $\mathbf{x}$ & - \\
\hline 134-083-17DBD & 46.41931 & -101.13826 & 1972 & I & $\mathbf{x}$ & $\mathbf{x}$ & -- \\
\hline 134-083-17DDB1 & 46.41748 & -101.13564 & 1973,92 & 2 & $\mathbf{x}$ & $\mathbf{x}$ & - \\
\hline 134-083-17DDB2 & 46.41748 & -101.13564 & 1973,92 & 2 & $\mathbf{X}$ & $\mathbf{X}$ & -- \\
\hline 134-083-23CAD & 46.40493 & -101.08067 & 1972 & 1 & $\mathbf{X}$ & $\mathbf{X}$ & -- \\
\hline 134-083-26BBA & 46.39953 & -101.08588 & 1973,92 & 2 & $\mathbf{x}$ & $\mathbf{x}$ & - \\
\hline 134-083-32AAA & 46.38497 & -101.13301 & 1973 & 1 & $\mathrm{X}$ & $\mathrm{X}$ & - \\
\hline 134-083-32AAA2 & 46.38497 & -101.13301 & 1973 & 1 & $\mathbf{X}$ & $\mathbf{X}$ & -- \\
\hline 134-084-01CDC & 46.44466 & -101.18764 & 1973 & 1 & $\mathbf{X}$ & $\mathbf{X}$ & -- \\
\hline 134-084-01CDC2 & 46.44466 & -101.18764 & 1973 & 1 & $\mathbf{x}$ & $\mathbf{x}$ & -- \\
\hline 134-084-03AAD & 46.45566 & -101.21651 & 1972 & 1 & $\mathbf{X}$ & $\mathbf{X}$ & - \\
\hline 134-084-03 ADC & 46.45202 & -101.21913 & 1975 & 1 & $\mathbf{X}$ & $\mathrm{X}$ & - \\
\hline $134-084-03 B$ & 46.45475 & -101.23093 & 1970 & 1 & $\mathbf{X}$ & $\mathbf{X}$ & - \\
\hline 134-084-03CBAI & 46.45021 & -101.23222 & 1975 & 1 & $\mathbf{x}$ & $\mathbf{x}$ & - \\
\hline 134-084-11DDD & 46.43019 & -101.19543 & 1973,92 & 2 & $\mathbf{X}$ & $\mathbf{X}$ & - \\
\hline 134-085-2IDAB3 & 46.40676 & -101.36514 & 1973 & 1 & $\mathrm{X}$ & $\mathrm{X}$ & - \\
\hline $134-089-27 \mathrm{ABCl}$ & 46.39791 & -101.8476 & 1972 & 1 & $\mathbf{X}$ & $\mathbf{x}$ & - \\
\hline 134-090-35DAC & 46.37518 & -101.94679 & 1972 & 1 & $\mathrm{X}$ & $\mathrm{x}$ & -- \\
\hline 134-090-35DBD & 46.37517 & -101.94941 & 1972 & 1 & $\mathbf{X}$ & $\mathbf{X}$ & -- \\
\hline $134-090-36 \mathrm{CBC}$ & 46.37519 & -101.94156 & 1972 & 1 & $\mathbf{X}$ & $\mathbf{x}$ & -- \\
\hline $134-091-06 D D C 2$ & 46.44361 & -102.15651 & 1970 & $i$ & $\mathrm{X}$ & $x$ & -- \\
\hline $134-091-32 \mathrm{CCC}$ & 46.37118 & -102.15051 & $1967,71,74,81,93$ & 5 & $\mathbf{X}$ & $\mathbf{X}$ & $\mathbf{X}$ \\
\hline 134-091-34DDD & 46.37136 & -102.09033 & 1969 & 1 & $\mathbf{x}$ & $\mathbf{x}$ & - \\
\hline $134-092-02 \mathrm{CCC}$ & 46.4439 & -102.21408 & 1969 & 1 & $\mathbf{x}$ & $\mathbf{x}$ & -- \\
\hline 134-092-34DDC & 46.37153 & -102.21854 & 1969,81 & 2 & $\mathbf{X}$ & $\mathbf{x}$ & -- \\
\hline 134-093-08DBB & 46.43509 & -102.38969 & 1969 & 1 & $\mathbf{X}$ & $\mathbf{x}$ & -- \\
\hline $134-093-12 C C C 2$ & 46.43007 & -102.31629 & 1969 & 1 & $\mathbf{X}$ & $\mathbf{x}$ & -- \\
\hline 134-093-23ADD & 46.40835 & -102.31883 & 1969,93 & 2 & $\mathbf{x}$ & $\mathbf{X}$ & $\mathbf{X}$ \\
\hline 134-093-35DCDI & 46.37205 & -102.32413 & 1967 & 1 & $\mathbf{X}$ & $\mathbf{X}$ & - \\
\hline 134-093-35DCD2 & 46.37205 & -102.32413 & 1967 & I & $\mathrm{x}$ & $\mathbf{x}$ & - \\
\hline 134-094-08DCC & 46.4288 & -102.51524 & 1968,69 & 2 & $\mathbf{X}$ & $\mathbf{X}$ & -. \\
\hline 134-094-32AAAI & 46.38357 & -102.50742 & 1967 & 1 & $\mathbf{X}$ & $\mathbf{X}$ & - \\
\hline 134-095-03DCC & 46.44297 & -102.60121 & 1967 & 1 & $\mathbf{X}$ & $\mathbf{X}$ & - \\
\hline 134-095-13AA & 46.42578 & -102.5523 & $1970-93$ & 56 & $\mathbf{x}$ & $\mathbf{X}$ & $\mathbf{X}$ \\
\hline 134-095-20AAA & 46.4121 & -102.63461 & 1967 & I & $\mathbf{X}$ & $\mathbf{x}$ & - \\
\hline $134-096-05 C \mathrm{CD} 2$ & 46.44217 & -102.77617 & 1969 & I & $\mathbf{X}$ & $\mathbf{X}$ & - \\
\hline 134-096-25BBB & 46.39745 & -102.69469 & 1969 & I & $\mathrm{x}$ & $\mathbf{X}$ & - \\
\hline $134-096-32 \mathrm{~A}$ & 46.37968 & -102.76394 & 1967 & 1 & $\mathrm{X}$ & $\mathrm{X}$ & -- \\
\hline
\end{tabular}




\begin{tabular}{|c|c|c|c|c|c|c|c|}
\hline \multirow[b]{2}{*}{ Local identifier } & \multirow[b]{2}{*}{ Latitude } & \multirow[b]{2}{*}{ Longitude } & \multirow[b]{2}{*}{ Period of record } & \multirow[b]{2}{*}{ Sample size } & \multicolumn{3}{|c|}{$\begin{array}{l}\text { Category for which samples } \\
\text { were analyzed }\end{array}$} \\
\hline & & & & & $\begin{array}{l}\text { Physical } \\
\text { properties }\end{array}$ & $\begin{array}{l}\text { Major } \\
\text { ions' }\end{array}$ & $\begin{array}{c}\text { Trace } \\
\text { metals }\end{array}$ \\
\hline $134-097-15 C C C 2$ & 46.41301 & -102.86132 & 1967 & 1 & $\mathbf{x}$ & $\mathbf{X}$ & - \\
\hline $134-097-22 \mathrm{BCC}$ & 46.40576 & -102.8613 & 1967 & 1 & $\mathbf{x}$ & $\mathbf{X}$ & -- \\
\hline 134-098-06DDA & 46.44201 & -103.03106 & 1975 & 1 & $\mathrm{X}$ & $\mathrm{X}$ & -- \\
\hline 134-098-27DAA & 46.3894 & -102.96867 & 1975 & 1 & $\mathrm{X}$ & $\mathbf{x}$ & -- \\
\hline 134-098-33BCC & 46.37552 & -103.0079 & 1975 & 1 & $\mathbf{x}$ & $\mathbf{x}$ & -- \\
\hline 134-099-21DCC & 46.39662 & -103.12251 & 1976 & 1 & $\mathbf{x}$ & $\mathbf{x}$ & - \\
\hline 134-100-07ADD2 & 46.43265 & -103.28054 & 1977 & 1 & $\mathbf{X}$ & $\mathbf{X}$ & - \\
\hline $134-100-10 \mathrm{AAD}$ & 46.4364 & -103.21783 & 1975 & 1 & $\mathbf{x}$ & $\mathbf{X}$ & -- \\
\hline $135-080-30 \mathrm{AAB}$ & 46.48617 & -100.78064 & 1973 & 1 & $\mathbf{X}$ & $\mathbf{X}$ & -- \\
\hline 135-080-32DDA & 46.46071 & -100.75711 & 1972 & 1 & $X$ & $\mathbf{X}$ & - \\
\hline 135-080-33DDA & 46.46059 & -100.73604 & 1973 & 1 & $\mathbf{x}$ & $\mathbf{x}$ & - \\
\hline 135-081-24DDD & 46.48799 & -100.799 & 1975 & 1 & $\mathbf{x}$ & $\mathbf{x}$ & - \\
\hline $135-082-30 \mathrm{CBB}$ & 46.47921 & -101.04697 & 1973 & 1 & $\mathbf{X}$ & $\mathbf{x}$ & -- \\
\hline $135-083-31 B C D$ & 46.46647 & -101.16946 & 1972 & 1 & $\mathrm{X}$ & $\mathbf{x}$ & -- \\
\hline 135-083-32CBB 1 & 46.46467 & -101.1515 & 1975,93 & 2 & $\mathbf{x}$ & $\mathbf{x}$ & -- \\
\hline 135-083-32CBB2 & 46.46467 & -101.1515 & 1975,93 & 2 & $\mathbf{X}$ & $\mathbf{x}$ & - \\
\hline $135-084-04 \mathrm{DCC}$ & 46.53168 & -101.24545 & 1973,92 & 2 & $\mathbf{x}$ & $\mathbf{x}$ & - \\
\hline $135-084-09 \mathrm{CCD}$ & 46.51705 & -101.2533 & 1974 & 2 & $\mathbf{x}$ & $\mathbf{X}$ & - \\
\hline 135-084-16AAA1 & 46.51539 & -101.2377 & 1974 & 1 & $\mathbf{x}$ & $\mathbf{x}$ &.- \\
\hline 135-084-16AAA2 & 46.51538 & -101.2377 & 1974 & 1 & $\mathbf{X}$ & $\mathbf{x}$ & -- \\
\hline 135-084-16AAA3 & 46.51538 & -101.2377 & 1974 & 1 & $\mathbf{x}$ & $\mathbf{x}$ & -- \\
\hline 135-084-16AAA4 & 46.51538 & -101.2377 & 1974 & 1 & $\mathbf{X}$ & $\mathbf{X}$ & - \\
\hline 135-084-16AAA5 & 46.51538 & -101.2377 & 1974 & 1 & $\mathbf{X}$ & $\mathbf{X}$ & -- \\
\hline 135-084-16AAA6 & 46.51538 & -101.2377 & 1974 & 1 & $\mathbf{x}$ & $\mathbf{x}$ & -- \\
\hline 135-084-16AAA7 & 46.51538 & -101.2377 & 1974 & 1 & $x$ & $\mathbf{x}$ & -- \\
\hline 135-084-16AAA8 & 46.51538 & -101.2377 & 1974 & 1 & $\mathbf{x}$ & $\mathbf{X}$ & - \\
\hline 135-084-16AAA9 & 46.51538 & -101.2377 & 1974 & 5 & $\mathrm{X}$ & $\mathbf{X}$ & - \\
\hline 135-084-16AAB & 46.51536 & -101.2403 & 1974,92 & 2 & $\mathbf{x}$ & $\mathbf{X}$ & -- \\
\hline 135-084-16AAD & 46.51357 & -101.2377 & 1974,93 & 2 & $\mathbf{x}$ & $\mathbf{X}$ & - \\
\hline 135-084-16ABA & 46.51534 & -101.2429 & $1974,92,94$ & 4 & $\mathbf{X}$ & $\mathbf{X}$ & - \\
\hline 135-084-21DDD1 & 46.48827 & -101.23752 & 1973 & 1 & $\mathbf{x}$ & $\mathbf{X}$ & - \\
\hline 135-084-21DDD2 & 46.48827 & -101.23752 & 1973,92 & 2 & $\mathrm{X}$ & $\mathbf{X}$ & -- \\
\hline $135-084-23 \mathrm{AAB}$ & 46.50079 & -101.19831 & 1972 & 1 & $\mathbf{X}$ & $\mathbf{X}$ & - \\
\hline 135-084-26DAA1 & 46.47912 & -101.19561 & 1973,93 & 2 & $\mathbf{x}$ & $\mathbf{X}$ & - \\
\hline 135-084-26DAA2 & 46.47912 & -101.19561 & 1973,92 & 2 & $\mathbf{X}$ & $\mathbf{x}$ & - \\
\hline 135-084-26DAA3 & 46.47912 & -101.19561 & 1973,92 & 2 & $\mathbf{x}$ & $\mathbf{x}$ & - \\
\hline $135-091-20 \mathrm{ACB}$ & 46.49606 & -102.14157 & 1969 & 1 & $\mathbf{X}$ & $\mathbf{X}$ & -- \\
\hline $135-091-28 C C B 3$ & 46.4744 & -102.13082 & 1969 & 1 & $\mathbf{X}$ & $\mathbf{X}$ & -- \\
\hline 135-093-01BCB & 46.54057 & -102.31629 & 1967 & 1 & $\mathbf{x}$ & $\mathbf{x}$ & -- \\
\hline $135-093-08 \mathrm{ABA}$ & 46.52916 & -102.38726 & 1969 & 1 & $\mathbf{x}$ & $\mathbf{X}$ & -- \\
\hline $135-093-12 \mathrm{CCC}$ & 46.51699 & -102.31639 & 1967 & 1 & $\mathbf{X}$ & $\mathbf{X}$ & -- \\
\hline $135-094-19 \mathrm{CCC}$ & 46.48647 & -102.54904 & 1967 & 1 & $\mathbf{x}$ & $\mathbf{X}$ & -- \\
\hline $135-094-28 \mathrm{CBB}$ & 46.47779 & -102.50489 & 1969 & 1 & $\mathbf{X}$ & $\mathbf{X}$ & -- \\
\hline $135-094-31 \mathrm{CCC}$ & 46.45747 & -102.54886 & 1969,71 & 2 & $\mathbf{x}$ & $\mathbf{X}$ & -- \\
\hline 135-095-19DDC2 & 46.48621 & -102.65904 & 1969 & 1 & $\mathbf{x}$ & $\mathbf{X}$ & -- \\
\hline
\end{tabular}




\begin{tabular}{|c|c|c|c|c|c|c|c|}
\hline \multirow[b]{2}{*}{ Local identifier } & \multirow[b]{2}{*}{ Latitude } & \multirow[b]{2}{*}{ Longitude } & \multirow[b]{2}{*}{ Period of record } & \multirow[b]{2}{*}{ Sample size } & \multicolumn{3}{|c|}{$\begin{array}{l}\text { Category for which samples } \\
\text { were analyzed }\end{array}$} \\
\hline & & & & & $\begin{array}{l}\text { Phyeical } \\
\text { properties }\end{array}$ & $\begin{array}{l}\text { Major } \\
\text { lons' }\end{array}$ & $\begin{array}{c}\text { Trace } \\
\text { metals }\end{array}$ \\
\hline 135-096-10BAA & 46.52754 & -102.72926 & 1969 & 1 & $\mathbf{x}$ & $\mathbf{x}$ & - \\
\hline $135-096-20 \mathrm{BBB} 2$ & 46.49833 & -102.77918 & 1969 & 1 & $\mathbf{x}$ & $\mathbf{x}$ & - \\
\hline 135-096-28BDA & 46.48038 & -102.75014 & 1969 & 1 & $\mathbf{x}$ & $\mathbf{x}$ & - \\
\hline $135-096-34 \mathrm{CB}$ & 46.46146 & -102.73572 & 1975,76 & 3 & $\mathbf{x}$ & $\mathbf{x}$ & - \\
\hline $135-097-04 \mathrm{ABB}$ & 46.54202 & -102.87152 & 1967 & 1 & $\mathbf{x}$ & $\mathbf{x}$ & - \\
\hline 135-097-04ADD & 46.53655 & -102.86383 & 1967 & 1 & $\mathbf{x}$ & $\mathbf{x}$ & - \\
\hline $135-097-04 \mathrm{CCA}$ & 46.53098 & -102.8797 & 1987 & 1 & $\mathbf{X}$ & $\mathbf{x}$ & -- \\
\hline 135-097-04DCA & 46.531 & -102.86924 & 1968,93 & 4 & $\mathbf{x}$ & $\mathbf{x}$ & $\mathbf{x}$ \\
\hline $135-097-07 \mathrm{~A}$ & 46.52442 & -102.91076 & 1970 & 2 & $\mathbf{x}$ & $\mathbf{x}$ & - \\
\hline 135-098-32DAD1 & 46.45835 & -103.0107 & 1975 & 1 & $\mathbf{x}$ & $\mathbf{x}$ & - \\
\hline 135-099-01DDD & 46.52712 & -103.05251 & 1974 & 1 & $\mathbf{x}$ & $\mathbf{x}$ & -- \\
\hline 135-101-26BAA & 46.48148 & -103.33266 & 1974 & 1 & $\mathbf{x}$ & $\mathbf{x}$ & - \\
\hline 136-083-10CDA & 46.60593 & -101.10181 & 1972 & 1 & $\mathbf{x}$ & $\mathbf{x}$ & -- \\
\hline 136-083-10CDD & 46.60412 & -101.10181 & 1972 & 1 & $\mathbf{x}$ & $\mathbf{x}$ & -- \\
\hline 136-083-19ADB1 & 46.58407 & -101.15688 & 1972 & 1 & $\mathbf{x}$ & $\mathbf{x}$ & -- \\
\hline 136-083-22BDD1 & 46.58243 & -101.10184 & 1972 & 1 & $\mathbf{x}$ & $\mathbf{x}$ & -. \\
\hline 136-083-22BDD3 & 46.58243 & -101.10184 & 1972 & 1 & $\mathbf{x}$ & $\mathbf{x}$ & - \\
\hline $136-083-22 C C B$ & 46.57698 & -101.10971 & 1972 & 1 & $\mathbf{x}$ & $\mathbf{X}$ & -- \\
\hline 136-083-22CDD3 & 46.57514 & -101.10182 & 1972 & 1 & $\mathbf{x}$ & $\mathbf{x}$ & -- \\
\hline 136-083-26DDC & 46.56068 & -101.07277 & 1972 & 1 & $\mathbf{x}$ & $\mathbf{x}$ & -- \\
\hline 136-083-32CDD1 & 46.54609 & -101.14372 & 1972 & 1 & $\mathbf{x}$ & $\mathbf{x}$ & -- \\
\hline 136-093-34BCD & 46.55316 & -102.35568 & 1969 & 1 & $\mathbf{x}$ & $\mathbf{x}$ & - \\
\hline 136-094-03DDD & 46.61755 & -102.46561 & 1968 & $i$ & $\mathbf{x}$ & $\mathbf{x}$ & - \\
\hline 136-094-33CВC & 46.54823 & -102.50495 & 1967 & 1 & $\mathbf{x}$ & $\mathbf{x}$ & - \\
\hline 136-096-19DDD & 46.57248 & -102.78157 & 1967 & 1 & $\mathbf{x}$ & $\mathbf{x}$ & -- \\
\hline 136-097-15DAD & 46.59105 & -102.84276 & 1967 & 1 & $\mathbf{x}$ & $\mathbf{x}$ & - \\
\hline 136-097-32CBB & 46.54915 & -102.90299 & 1967 & 1 & $\mathbf{x}$ & $\mathbf{x}$ & - \\
\hline 136-097-32DDA2 & 46.54569 & -102.88462 & 1994 & 1 & $\mathbf{x}$ & $\mathbf{x}$ & $\mathbf{x}$ \\
\hline $136-097-32 \mathrm{DDB}$ & 46.54564 & -102.88721 & 1994 & 1 & $\mathrm{X}$ & $\mathrm{X}$ & $\mathbf{X}$ \\
\hline 136-097-32DDD & 46.54388 & -102.88454 & 1994 & 1 & $\mathbf{x}$ & $\mathbf{x}$ & $\mathbf{x}$ \\
\hline 136-097-32DDDC & 46.54341 & -102.88517 & 1994 & 1 & $\mathbf{x}$ & $\mathbf{x}$ & $\mathbf{x}$ \\
\hline $136-098-31 \mathrm{ABB}$ & 46.55407 & -103.03914 & 1975 & 1 & $\mathbf{x}$ & $\mathbf{X}$ & - \\
\hline 136-098-33CCD & 46.54158 & -103.00518 & 1975 & 1 & $\mathbf{x}$ & $\mathbf{x}$ & -- \\
\hline 136-099-26DAD & 46.55953 & -103.07331 & 1976 & 1 & $\mathbf{x}$ & $\mathbf{x}$ & -- \\
\hline 136-100-31DDC1 & 46.5414 & -103.28324 & 1975 & 1 & $\mathrm{X}$ & $\mathbf{x}$ & -- \\
\hline 136-100-31DDC2 & 46.5414 & -103.28324 & 1977 & 1 & $\mathbf{x}$ & $\mathbf{x}$ & - \\
\hline $137-094-20 C C D 2$ & 46.65994 & -102.57567 & 1969 & 1 & $\mathbf{x}$ & $\mathbf{X}$ & - \\
\hline 137-094-32BBB 1 & 46.64355 & -102.57807 & 1969 & 1 & $\mathrm{X}$ & $\mathbf{x}$ & -- \\
\hline $137-095-28$ & 46.65169 & -102.67382 & 1967 & 1 & $\mathbf{x}$ & $\mathbf{x}$ & -- \\
\hline 137-097-31BDD & 46.63812 & -102.96922 & 1967 & 1 & $\mathbf{x}$ & $\mathbf{x}$ & - \\
\hline 137-099-24ABA & 46.67239 & -103.11143 & 1969 & 1 & $\mathrm{x}$ & $\mathbf{X}$ & - \\
\hline $130-083-28 \mathrm{DC}$ & 46.04701 & -101.07111 & 1965 & 1 & $\mathrm{X}$ & $\mathbf{x}$ & -- \\
\hline $130-084-13 A A$ & 46.08691 & -101.12813 & 1966 & 1 & $\mathrm{X}$ & $\mathbf{x}$ & - \\
\hline $130-090-09 \mathrm{AD}$ & 46.09857 & -101.93864 & 1967 & 1 & $\mathbf{x}$ & $\mathbf{x}$ & - \\
\hline $131-086-14$ & 46.16853 & -101.4061 & 1968 & 1 & $\mathbf{X}$ & $\mathbf{x}$ & -- \\
\hline $132-089-14 \mathrm{CBB}$ & 46.2548 & -101.78945 & 1968 & 1 & $\mathbf{x}$ & $\mathbf{x}$ & -- \\
\hline $132-089-22 \mathrm{CCCl}$ & 46.23498 & -101.81021 & 1968 & 1 & $\mathbf{x}$ & $\mathbf{x}$ & -- \\
\hline $132-090-31$ & 46.21268 & -101.9876 & 1964 & 1 & $\mathbf{X}$ & $\mathbf{X}$ & $\ldots$ \\
\hline $133-079-07$ & 46.34951 & -100.66183 & 1967 & 1 & $\mathrm{x}$ & $\mathbf{x}$ & - \\
\hline $133-082-07 \mathrm{CC}$ & 46.34427 & -101.04543 & 1969 & 1 & $\mathbf{X}$ & $\mathbf{x}$ & - \\
\hline
\end{tabular}




\begin{tabular}{|c|c|c|c|c|c|c|c|}
\hline \multirow[b]{2}{*}{ Local identifier } & \multirow[b]{2}{*}{ Latitude } & \multirow[b]{2}{*}{ Longlitude } & \multirow[b]{2}{*}{ Perlod of record } & \multirow[b]{2}{*}{ Sample eize } & \multicolumn{3}{|c|}{$\begin{array}{l}\text { Category for which samples } \\
\text { were analyzed }\end{array}$} \\
\hline & & & & & $\begin{array}{l}\text { Physical } \\
\text { properties }\end{array}$ & $\begin{array}{l}\text { Major } \\
\text { ions } 1\end{array}$ & $\begin{array}{l}\text { Trace } \\
\text { motals }\end{array}$ \\
\hline $133-085-06$ & 46.3643 & -101.41348 & 1966 & 1 & $\mathbf{x}$ & $\mathbf{x}$ & - \\
\hline $133-088-14 \mathrm{D}$ & 46.33174 & -101.7006 & 1965 & 1 & $\mathbf{x}$ & $\mathbf{X}$ & -- \\
\hline $133-092-12$ & 46.34873 & -102.18285 & 1967 & 1 & $\mathbf{x}$ & $\mathbf{X}$ & -- \\
\hline $133-092-16$ & 46.33464 & -102.24533 & 1966 & 1 & $\mathbf{x}$ & $\mathbf{x}$ & -- \\
\hline $133-093-02 \mathrm{BAD}$ & 46.3684 & -102.32934 & 1966 & 1 & $\mathbf{x}$ & $\mathbf{x}$ & -- \\
\hline 133-093-05AAD & 46.36816 & -102.38176 & 1965 & 1 & $\mathbf{x}$ & $\mathbf{X}$ & - \\
\hline $133-093-09$ & 46.34922 & -102.36975 & 1968 & 1 & $\mathbf{X}$ & $\mathbf{X}$ & -- \\
\hline $133-096-04$ & 46.36169 & -102.74798 & 1968 & 1 & $\mathbf{X}$ & $\mathbf{X}$ & -- \\
\hline $133-096-06 \mathrm{BB}$ & 46.36676 & -102.797 & 1967 & 1 & $\mathbf{x}$ & $\mathbf{X}$ & -. \\
\hline $134-079-16$ & 46.42247 & -100.61992 & 1967 & 1 & $\mathbf{x}$ & $\mathbf{x}$ & -- \\
\hline $134-089-35$ & 46.37912 & -101.82815 & 1968 & 1 & $\mathbf{x}$ & $\mathbf{x}$ & -- \\
\hline $134-090-13$ & 46.42141 & -101.93215 & 1967 & 1 & $\mathbf{x}$ & $\mathbf{x}$ & - \\
\hline $134-090-13$ & 46.42141 & -101.93215 & 1967 & 1 & $\mathbf{x}$ & $\mathbf{X}$ & -- \\
\hline $134-090-36 \mathrm{CBC}$ & 46.37519 & -101.94156 & 1966 & 1 & $\mathbf{X}$ & $\mathbf{x}$ & -- \\
\hline $134-090-36 \mathrm{CCB}$ & 46.37338 & -101.94157 & 1966 & 1 & $\mathbf{X}$ & $\mathbf{x}$ & -- \\
\hline $134-093-02 D C$ & 46.44545 & -102.32544 & 1967 & 1 & $\mathbf{x}$ & $\mathbf{x}$ & -- \\
\hline 134-097-34DAA & 46.37491 & -102.84289 & 1966 & 1 & $\mathrm{x}$ & $\mathbf{x}$ & - \\
\hline $134-099-14$ & 46.41748 & -103.08222 & 1967 & 1 & $\mathbf{x}$ & $\mathbf{x}$ & -- \\
\hline $135-083-34$ & 46.46564 & -101.10057 & 1965 & 1 & $\mathbf{x}$ & $\mathbf{x}$ & -- \\
\hline 135-084-16AAA1 & 46.51539 & -101.2377 & 1974 & 1 & $\mathbf{x}$ & $\mathbf{x}$ & -- \\
\hline $135-084-16 \mathrm{ABA}$ & 46.51534 & -101.2429 & 1974 & 1 & $\mathbf{x}$ & $\mathbf{x}$ & -- \\
\hline $135-084-16 \mathrm{ABB}$ & 46.51531 & -101.2455 & 1973 & 1 & $\mathbf{X}$ & $\mathbf{x}$ & - \\
\hline $135-096-22 \mathrm{DD}$ & 46.48687 & -102.72011 & 1984 & 1 & $\mathbf{X}$ & $\mathbf{x}$ & - \\
\hline $135-097-04$ & 46.53559 & -102.87303 & 1968 & 1 & $\mathbf{x}$ & $\mathbf{x}$ & - \\
\hline $136-094-04$ & 46.62321 & -102.496 & 1961 & 1 & $\mathbf{x}$ & $\mathbf{x}$ & -- \\
\hline $136-095-28 B B$ & 46.57056 & -102.63179 & 1966 & 2 & $\mathbf{x}$ & $\mathbf{x}$ & -- \\
\hline 136-100-31DDC1 & 46.5414 & -103.28324 & 1975 & 1 & $\mathbf{x}$ & $\mathbf{x}$ & -- \\
\hline $137-095-32$ & 46.63723 & -102.69482 & 1959 & 1 & $\ddot{x}$ & $\mathbf{x}$ & - \\
\hline
\end{tabular}

${ }^{1}$ Data consist of values for major cations and anions and common metals, such as iron and manganese, in solution.

${ }^{2}$ Data consist of values for trace metals. 